\title{
Multicomponent Case Weights for the 1985 Resample of the 1983 Pacific Northwest Residential Energy Survey
}
M. S. Klan
S. A. Shankle
M. A. Kellogg

June 1990

Prepared for the

Bonneville Power Administration

under a Related Services Agreement

with the U.S. Department of Energy

Contract DE-AC06-76RLO 1830

Pacific Northwest Laboratory

Operated for the U.S. Department of Energy

by Battelle Memorial Institute 


\title{
DISCLAIMER
}

This report was prepared as an account of work sponsored by an agency of the United States Government. Neither the United States Government nor any agency thereof, nor Battelle Memorial Institute, nor any of their employees, makes any warranty, expressed or implied, or assumes any legal liability or responsibility for the accuracy, completeness, or usefulness of any information, apparatus, product, or process disclosed, or represents that its use would not infringe privately owned rights. Reference herein to any specific commercial product, process, or service by trade name, trademark, manufacturer, or otherwise, does not necessarily constitute or imply its endorsement, recommendation, or favoring by the United States Covernment or any agency thereof, or Battelle Mernorial Institute. The views and opinions of authors expressed herein do not necessarily state or reflect those of the United States Government or any agency thereof.

\author{
PACIFIC NORTHWEST LABORATORY \\ operated by \\ BATTELLE MEMORIAL INSTITUTE \\ for the \\ UNITED STATES DEPARTMENT OF ENERGY \\ under Contract DE-AC06-76RLO 1830
}

Printed in the United States of America

Available to DOE and DOE contractors from the

Office of Scientific and Technical Information, P.O. Box 62, Oak Ridge, TN 37831; prices available from (615) 576-8401. FTS 626-8401.

Available to the public from the National Technical Information Service, U.S. Department of Commerce, 5285 Port Royal Rd., Springfield, VA 22161.

NTIS Price Codes, Microfiche A01

Printed Copy

\begin{tabular}{cr}
\hline Price Code & Page Range \\
\hline A02 & $1-10$ \\
A03 & $11-50$ \\
A04 & $51-75$ \\
A05 & $76-100$ \\
A06 & $101-125$ \\
A07 & $126-150$ \\
A08 & $151-175$ \\
A09 & $176-200$ \\
A10 & $201-225$ \\
A11 & $226-250$ \\
A12 & $251-275$ \\
A13 & $276-300$ \\
A14 & $301-325$
\end{tabular}

\begin{tabular}{cc}
\hline Price Code & Page Range \\
\hline A15 & $326-350$ \\
A16 & $351-375$ \\
A17 & $376-400$ \\
A18 & $401-425$ \\
A19 & $426-450$ \\
A20 & $451-475$ \\
A21 & $476-500$ \\
A22 & $501-525$ \\
A23 & $526-550$ \\
A24 & $551-575$ \\
A25 & $576-600$ \\
A99 & $601-$ Up
\end{tabular}


MULTICOMPONENT CASE WEIGHTS FOR

THE 1985 RESAMPLE OF THE 1983

PACIFIC NORTHWEST RESIDENTIAL

ENERGY SURVEY

M. S. Klan

S. A. Shankle

M. A. Kellogg

Manuscript Completed: September 1986

Date Published: June 1990

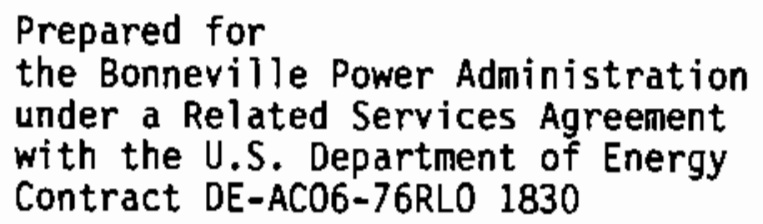

Prepared for the Bonneville Power Administration under a Related Services Agreement with the U.S. Department of Energy Contract DE-AC06-76RLO 1830

Pacific Northwest Laboratory Richland, Washington 99352 


\section{SUMMARY}

In this study a set of multicomponent case weights applicable to residential survey information were prepared for the Bonneville Power Administration (BPA) by the Pacific Northwest Laboratory (PNL). These case weights were prepared for the 1985 resample of respondents of an earlier BPA residential energy survey--the original 1983 survey and subsequent surveys administered to the 1985 PNHRES resample were designed to gather information from households concerning their use of energy and related data.

The PNWRES samples were drawn based on stratified random sampling techniques, that allow the survey results to represent the characteristics of the overall Pacific Northwest population of residential utility accounts. In order to determine the characteristics of the population, however, the survey results must be appropriately weighted. Case weights were developed for 1983 PNWRES by Lou Harris and Associates, Inc. This report briefly documents PNL's extension of the weighting methodology to the subsequent 1985 PNWRES resample, and describes the resulting caseweights generated by PNL. 


\section{CONTENTS}

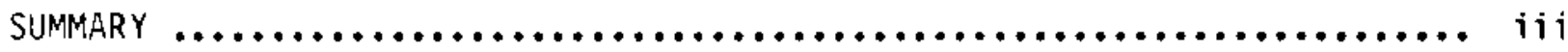

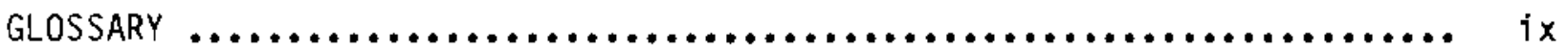

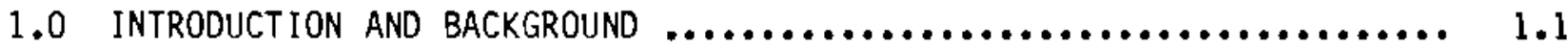

2.0 SAMPLING PLAN AND DEVIATIONS $\ldots \ldots \ldots \ldots \ldots \ldots \ldots \ldots \ldots \ldots \ldots \ldots \ldots \ldots \ldots \ldots \ldots$

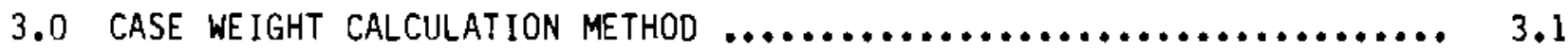

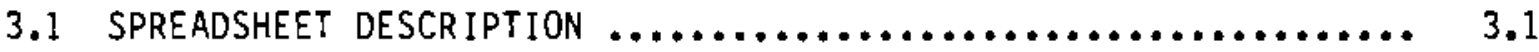

3.2 MODIFICATIONS OF ORIGINAL SPREADSHEET $\ldots \ldots \ldots \ldots \ldots \ldots \ldots \ldots ., 3.6$

4.0 CALCUlated CASEWEights: OVERALl RESAMPLE, ROSM, AND ROST $\ldots \ldots \ldots \ldots 4.1$

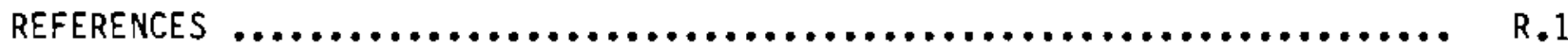
APPENDIX A - OVERALL RESAMPLE SPREADSHEET CONTENTS $\ldots \ldots \ldots \ldots \ldots \ldots \ldots \ldots$ A.1 APPENDIX B - ROSM RESPONDENTS SPREADSHEET CONTENTS $\ldots \ldots \ldots \ldots \ldots \ldots \ldots \ldots$ B.1 APPENDIX $C$ - ROST RESPONDENTS SPREADSHEET CONTENTS $\ldots \ldots \ldots \ldots \ldots \ldots \ldots \ldots$ c..

\section{TABLES}

2.1 Deviations from Sampling Plan for 1985 Resample of 1983 PNWRES ..... 2.2

3.1 Spreadsheet Definitions for Case Weight Calculations ............. 3.2

3.2 Structural Changes to Louis Harris Spreadsheet ................. 3.7

3.3 Nonstructural Changes to Louis Harris Spreadsheet $\ldots . . . \ldots \ldots \ldots . . .3 .8$

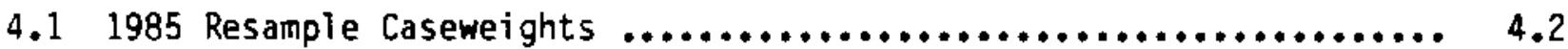



,

\author{
.
}




\section{ABBREVIATIONS}

BPA Bonneville Power Administration

MRR Meter Reading Rate

PNL Pacific Northwest Laboratory

PNWRES Pacific Northwest Residential Energy Survey

ROSM Residential Occupant Survey - Mail

s:

ROST Residential Occupant Survey - Telephone 
. 


\subsection{INTROOUCTION AND BACKGROUND}

In 1983, the Bonneville Power Administration (BPA) conducted the administration of the Pacific Northwest Residential Energy Survey (1983 PNWRES). The survey was fielded by Louis Harris and Associates, Inc. The PNWRES sample was designed to facilitate estimates of residential energy-related information for the Pacific Northwest through the use of stratified random sampling tech-

niques. However, because stratification results in disproportionate household selection across strata, the survey results must be multiplied by mathematical constants (weighted) on a case-by-case bas is to adjust for disproportionate selection. The weighted results can then be used to generate population estimates. The calculated mathematical constants used for weighting are called case weights.

For the 1983 PNWRES, Louis Harris and Associates prepared a set of ... multicomponent case weights which reallocated [the] sample as a function of cluster size, disproportionate probabilities of selection which occurred as a function of MRR [Meter Reading Route] size, and differential nonresponse. Case weight components were constructed for each stage of the sample selection process--individual unit, MRR, utility, substrata and strata. A final component was then devised in order to allow analysts to project sample estimates to the entire population or population subgroup (e.g., Western Oregon). (a)

In 1985, BPA staff reselected some of the 1983 PNWRES respondents for a new series of surveys. The households in the "1985 Resample" were surveyed in the Spring of that year with a questionnaire administered by mail. The Residential Occupant Survey - Mail (ROSM), implemented by Evaluation Research Corporation, obtained information about energy-related behavior, attitudes, socioeconomic information, and other factors. A similar survey--the Residential Occupant Survey - Telephone (ROST)--was also administered to the Resample households. ROST was codesigned by staff from BPA and the Pacific Northwest Laboratory (PNL), and was fielded by Columbia Research Center in the summer of

(a) Louis Harris and Associates, Inc. Pacific Northwest Residential Energy Consumption Survey: Construction of Multicomponent Case Weights for Regional Sample Data (Final Report). Submitted to the Bonneville Power Administration, Contract No. DE-AC79-83BP39950. July 29, 1984. 
1985. Thus, two companion surveys were administered to the occupants of dweilings identified in the 1985 Resample of 1983 PNWRES respondents.

This document briefiy describes the development of multicomponent case weights by PNL for the 1985 Resample. These Resample case weights result from a fairly straightforward extension of the methodology developed by Louis Harris and Associates for the 1983 PNkiRES. (a) Section 2.0 describes trie 1985 pian for resampling 1983 PNWRES along with deviations from that plan. Section 3.0 describes the specific method of case weight calculation implemented for the 1985 Resample. This section also documents specific changes made to the original spreadsheet used to calculate case weights by Louis Harris and Associates. The 1985 Resample case weights resulting from the calculations are shown in Section 4.0. A references section and appendices detailing the various spreadsheet contents follow Section 4.0 .

\footnotetext{
(a) Oetails concerning the 1983 PNwiRES (including sample design, questionnaire contents, analysis of results, and methodology/construction of case weights) can be found in documents prepared by Louis Harris and Associates. See the references section of this report.
} 


\subsection{SAMPLING PLAN AND DEVIATIONS}

The basic strategy of the 1985 Resample of 1983 PNWRES was to obtain a representative sample of 1983 PNWRES respondents with a smaller sample size than used for 1983 PNWRES. 1983 PNWRES was intended to be a representative sample for the residential utility accounts in the Pacific Northwest. For the 1985 Resample, a strategy was devised to sample from the 4703 respondents to 1983 PNWRES.

BPA's service area consists of six geographic/weather zones, referred to as geographic strata. Stratum 1 consists of Western Montana, stratum 2 consists of Idaho and small areas in Nevada, Utah, and Wyoming, stratum 3 consists of the portion of Washington east of the Cascade mountains, stratum 4 consists of Washington west of the Cascades, stratum 5 consists of Oregon east of the Cascades and a small portion of northern California, and stratum 6 is Oregon west of the Cascades. (a)

Substrata were constructed based upon the size of the residential population within each geographic stratum. Public utilities were then selected within substratum in proportion to the size of the residential population served. (b)

The sampling plan was to select two respondents from each meter reading route (MRR) in geographic strata 4 and 6 (West of the Cascade mountains) and 1 respondent from each MRR in geographic strata 1, 2, 3 and 5 (East of the Cascade mountains). There were some deviations from this strategy, as shown in Table 2.1 below. The actual sample drawn can be inferred from this sampling plan and the deviations from it. This is relevant to LOTUS spreadsheets used later to calculate the case weights (see especially column E, MRR SAMPLE DRAWN in Section 3.0 below).

(a) Louis Harris and Associates, Pacific Northwest Energy Consumption Survey: Sample Selection Activities, 1983. Page 3 and Table 2.

(b) Couis harris and Associates, Pacific Northwest Energy Consumption Survey: Construction of Mult icomponent Case Werghts for Regional Sample Data, 1984. Page 2. 
TABLE 2.1. Deviations from Sampling Plan for 1985 Resample of 1983 PNWRES

\begin{tabular}{|c|c|c|c|}
\hline Strata & Location & Utility & Deviations \\
\hline 1 & East & $\begin{array}{l}\text { Glacier Electric Coop., } \\
\text { ID \#6 }\end{array}$ & $\begin{array}{l}\text { MRRs } 6,7 \text { and } 8 \text { were not } \\
\text { resampled }\end{array}$ \\
\hline 1 & East & $\begin{array}{l}\text { Ravalli Co. } \\
\text { Electric Coop., ID } \# 7\end{array}$ & $\begin{array}{l}21983 \text { PNWRES respondents were } \\
\text { selected for MRRs } 1,3 \text {, and } 5\end{array}$ \\
\hline 2 & East & Fall River, ID 114 & MRR 2 was not resampled \\
\hline 2 & East & Lost River, ID \#21 & $\begin{array}{l}21983 \text { PNWRES respondents were } \\
\text { selected for MRR } 1\end{array}$ \\
\hline 2 & East & Weiser, ID \#22 & MRR I was not resampled \\
\hline 3 & East & Franklin Co. PUD, ID $\# 26$ & MRR 6 was not resampled \\
\hline 3 & East & Ferry Co. PUD, ID \#31 & MRR 3 was not resampled \\
\hline 4 & West & Clark Co. PUD, ID $\$ 37$ & $\begin{array}{l}11983 \text { PNWRES respondent } \\
\text { selected for MRR } 4 \text { and } 6\end{array}$ \\
\hline 4 & West & Tacoma, ID \#38 & $\begin{array}{l}11983 \text { PNWRES respondent } \\
\text { selected for MRR } 3 \text { and } 5\end{array}$ \\
\hline 5 & East & $\begin{array}{l}\text { Pacific Power and Light, } \\
\text { Oregon, East ID } \# 2\end{array}$ & MRR 14 was not resampled \\
\hline 5 & East & CP Nationa 1, ID $\# 47$ & $\begin{array}{l}\text { MRRs } 3,5 \text { and } 7 \text { were not } \\
\text { resampied }\end{array}$ \\
\hline 6 & West & Central Lincoln PUD, ID $\# 54$ & MRR 2 and 3 were not resamipled \\
\hline 6 & West & $\begin{array}{l}\text { Pacific Power and Light, } \\
\text { Oregon, West, ID } \$ 2\end{array}$ & $\begin{array}{l}11983 \text { PNWRES respondent } \\
\text { selected for MRR } 1 \text { and } 2\end{array}$ \\
\hline
\end{tabular}




\subsection{CASE WEIGHT CALCULATION METHOD}

Seven weights were calculated in the process of constructing multicomponent case weights. An individual unit weight, an MRR weight, and a utility weight were designed to weight observations on the basis of the average number of responses from MRRs within a utility. These weights are used to allocate observations of the survey respondents (achieved sample) within the targeted sample. A substrata and strata weight were constructed to reallocate the achieved sample proportionate to the actual total eligible population of the Pacific Northwest. The final weight was constructed to allow characteristics of the entire eligible population to be projected from sample estimates. This final weight is the product of the six weights described above.

The calculations used to generate the multicomponent case weights were done using LOTUS 1-2-3 spreadsheet software. Louis Harris and Associates' original spreadsheet was modified by PNL in order to generate case weights for the 1985 Resample. Column references in the text refer to columns in the LOTUS spreadsheet.

\subsection{SPREADSHEET DESCRIPTION}

Table 3.1 describes the LOTUS spreadsheet used to calculate the 1985 Resample case weights. The table describes the alphabetic column address of each spreadsheet column (COLUMN), whether or not a column has been modified since the Louis Harris version (MOD), the column heading or title (IITLE), a description of the column (DESCRIPTION) and a generalization of the formula used to generate the column (FORMULA).

The table contains a NO in the MOD column if a particular spreadsheet column definition has not been modified from the Louis Harris version, and a YES if it has been modified. In the FORMULA column of the table, a generalization of the spreadsheet formula is given. The word INPUT in the FORMULA column designates where a numeric value exists in the spreadsheet, as opposed to a formula. Capital letters in the FORMULA column refer to spreadsheet columns. An example of a spreadsheet column consisting of a formula is column $D$, UNABLE TD CONTACT. Column D contains the formula $E-L$, where $E$ refers to 
TABLE 3.1. Spreadsheet Definitions for Case Weight Calculations (1985 Resample)

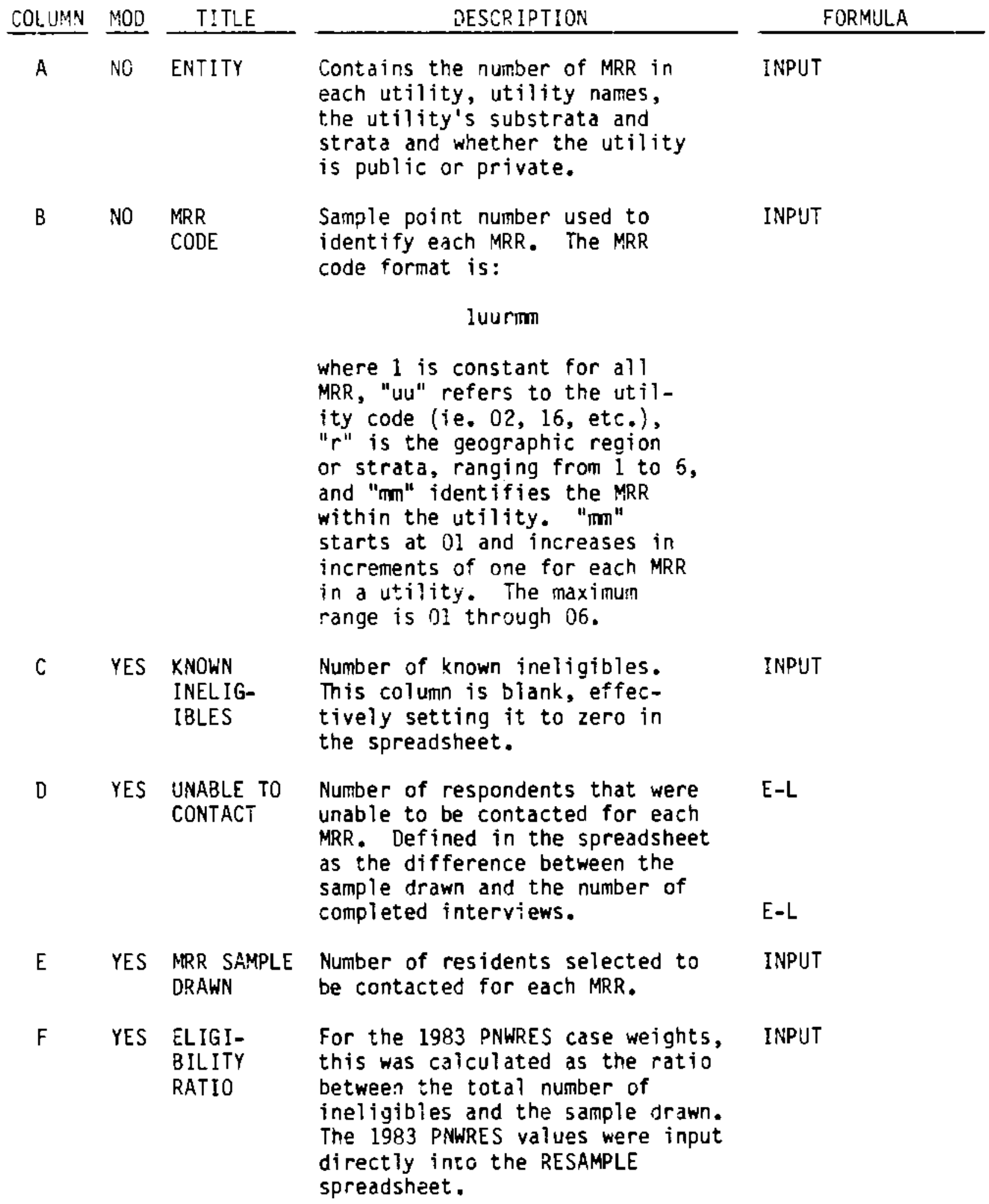


4
TABLE 3.1. (contd)

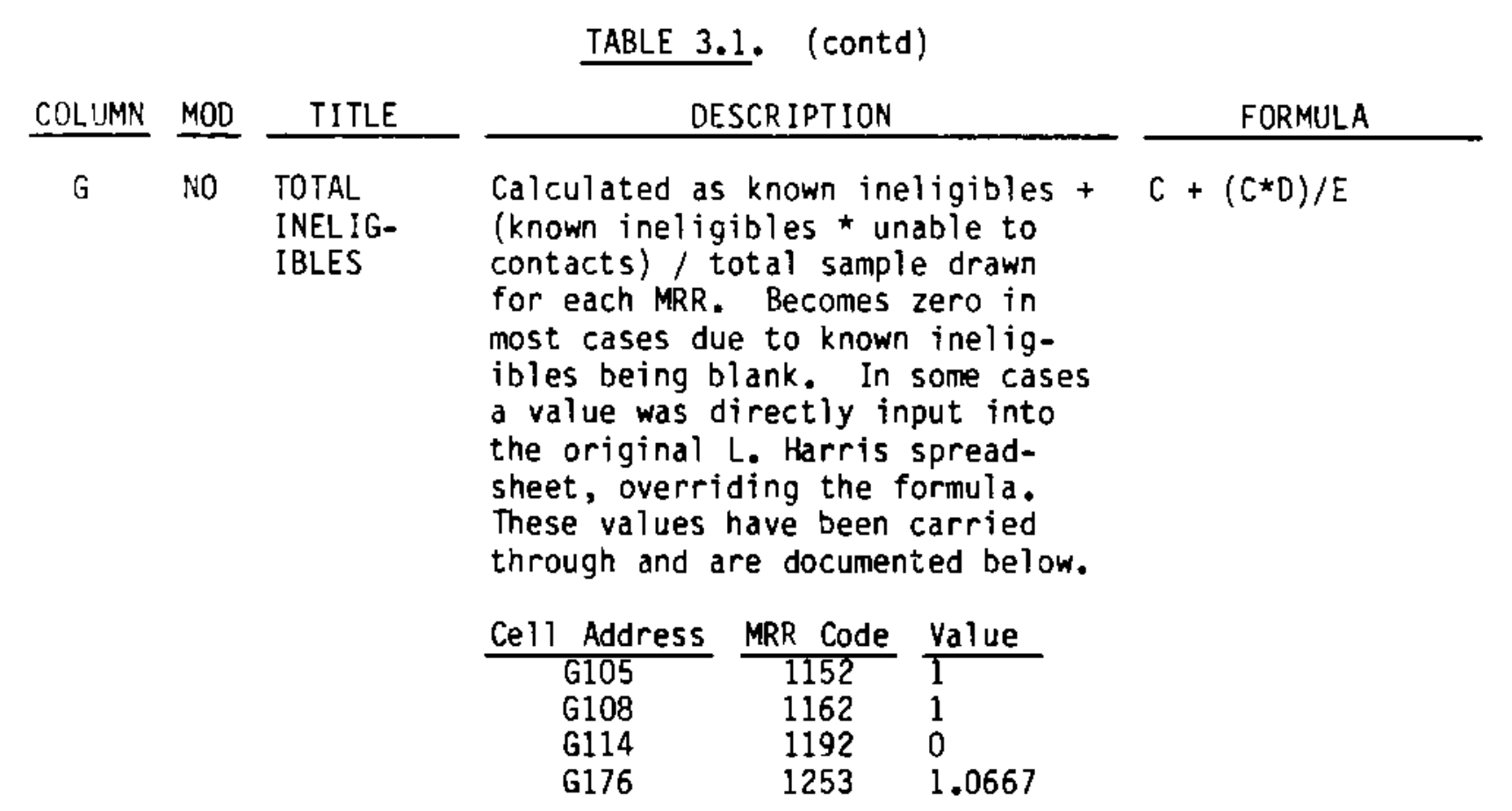

H NO TOTAL MRR Total number of customers

POPULATION serviced by each MRR. Values unchanged from 1983 PNWRES case weights spreadsheet.

I YES ELIGIBLE If there is at least one comMRR pleted interview for an MRR, this POPULATION column is the product of the eligibility ratio and the total MRR population. If there are no completed interviews for an MRR, this column is zero.

J NO OTHER TOTAL POPULATION

Total number of customers serviced by each utility, substrata and strata, reported by L. Harris as being according to data supplied by BPA.

$K \quad$ NO OTHER EL IGIBLE TOTAL POPULATION

Product of the eligibility ratio and the total number of customers serviced by each utility, sub-

$L$ YES COMPLETED INTERVIEWS
INPUT

IF $L>0$

THEN $F^{*} H$

ELSE 0

INPUT

$J \star F$
Total number of completed inter- INPUT views within an MRR. 
TABLE 3.1. (contd)

\begin{tabular}{|c|c|c|c|c|}
\hline COLLUMN & P10D & TITLE & DESCRIPTION & FORMULA \\
\hline M & YES & $\begin{array}{l}\text { AVERAGE } \\
\text { COMPLETES } \\
\text { PER MRR }\end{array}$ & $\begin{array}{l}\text { The ratio of the total number of } \\
\text { completed interviews to the total } \\
\text { number of MRRs with at least one } \\
\text { response. }\end{array}$ & $\begin{array}{l}\text { L809 / } 315 \\
\text { (equals } 1.2286 \text { ) }\end{array}$ \\
\hline N & YES & $\begin{array}{l}\text { IND IVIDUAL } \\
\text { WEIGHTS }\end{array}$ & $\begin{array}{l}\text { If there is at least one response } \\
\text { in an MRR, this column is calcu- } \\
\text { lated by dividing the average } \\
\text { completions per MRR by the number } \\
\text { of completed interviews, If } \\
\text { there are no completed "nterviews, } \\
\text { the column's value is zerc. }\end{array}$ & $\begin{array}{l}\text { IF L > } 0 \\
\text { THEN M/L } \\
\text { SLSE } 0\end{array}$ \\
\hline 0 & YES & $\begin{array}{l}\text { AVERAGE } \\
\text { POPULATION } \\
:\end{array}$ & $\begin{array}{l}\text { The eligible population of each } \\
\text { utility divided by the number of } \\
\text { MRR within the utility with at } \\
\text { least one response. }\end{array}$ & $\begin{array}{l}\text { I \{utility level\}/ } \\
\text { of MRR with at } \\
\text { least one response }\end{array}$ \\
\hline$p$ & YES & $\begin{array}{l}\text { MRR } \\
\text { WEIGHTS }\end{array}$ & $\begin{array}{l}\text { If the average oopulation of an } \\
\text { MRR is greater than zero, this } \\
\text { column is the eligible MRR popu- } \\
\text { iation divided by the average } \\
\text { popuiation. If the average popu- } \\
\text { lation is not greater than zero, } \\
\text { this column becomes zero. }\end{array}$ & $\begin{array}{l}\text { IF } 0>0 \\
\text { THEN I/0 } \\
\text { ELSE } 0\end{array}$ \\
\hline Q & NO & $\begin{array}{l}\text { UTIL ITY } \\
\text { WEIGHTS }\end{array}$ & $\begin{array}{l}\text { Eligible population for the util- } \\
\text { ity divided by the average popu- } \\
\text { lation for the utilities sampied } \\
\text { within substrata. }\end{array}$ & $k / 0$ \\
\hline$R$ & NO & $\begin{array}{l}\text { SUBSTRATA } \\
\text { WEIGHTS }\end{array}$ & $\begin{array}{l}\text { Weighted number of compietes } \\
\text { within substrata divided by the } \\
\text { sum of the weighted number of } \\
\text { compietes for each utility within } \\
\text { substrata. }\end{array}$ & $\begin{array}{l}\text { V\{substrata\} } \\
\text { U\{substrata\} }\end{array}$ \\
\hline$s$ & NO & $\begin{array}{l}\text { STRATA } \\
\text { WEIGHTS }\end{array}$ & $\begin{array}{l}\text { Weignted number of compietes } \\
\text { within strata divided by the sum } \\
\text { of the weighted number of com- } \\
\text { pletes for each substrata within } \\
\text { strata, }\end{array}$ & $\begin{array}{l}\text { V\{strata }\} \\
\text { U\{substrata\} }\end{array}$ \\
\hline
\end{tabular}


TABLE 3.1. (contd)

\begin{tabular}{|c|c|c|c|c|}
\hline COLUMN & MOD & TITLE & DESCR IPTION & FORMULA \\
\hline$T$ & NO & $\begin{array}{l}\text { WE IGHTED } \\
\text { COMPLETES } \\
\text { PER MRR }\end{array}$ & $\begin{array}{l}\text { The product of the number of com- } \\
\text { pleted interviews for each MRR, } \\
\text { the individual weight and the MRR } \\
\text { weight. }\end{array}$ & $L * N \star P$ \\
\hline U & NO & $\begin{array}{l}\text { WE IGHTED } \\
\text { COMPLETES } \\
\text { PER } \\
\text { UT ILITY }\end{array}$ & $\begin{array}{l}\text { The sum of the weighted number of } \\
\text { completes within each MRR sampled } \\
\text { times the utility weight. }\end{array}$ & $T\{u t i\}\}$ \\
\hline V & NO & $\begin{array}{l}\text { WE IGHTED } \\
\text { COMPLETES }\end{array}$ & $\begin{array}{l}\text { For substrata: } \\
\text { The product of the eligible popu- } \\
\text { lation for the substrata divided } \\
\text { by the eligible population for the } \\
\text { strata and the sum of the weighted } \\
\text { completes for each utility within } \\
\text { strata. } \\
\text { For strata: } \\
\text { The product of the eligible popu- } \\
\text { lation for the strata divided by } \\
\text { the total eligible population and } \\
\text { the sum of the weighted completes } \\
\text { for each utility sampled. }\end{array}$ & $\begin{array}{l}\text { (K\{substrata }\} \\
K\{\text { strata }) \\
\star U \text { [each utility } \\
\text { within strata\} }\end{array}$ \\
\hline$W$ & NO & $\begin{array}{l}\text { WEIGHTED } \\
\text { ELIGIBLE } \\
\text { POPULATION } \\
\text { PER STRATA }\end{array}$ & $\begin{array}{l}\text { The product of the weighted com- } \\
\text { pletes per strata divided by the } \\
\text { total weighted completes and the- } \\
\text { total eligible population. }\end{array}$ & $\begin{array}{l}\text { (V\{strata\}/ } \\
V\{\text { total }\}) \\
\star K \text { \{tota }\}\end{array}$ \\
\hline$x$ & NO & $\begin{array}{l}\text { SAMPLE } \\
\text { PROJECTED } \\
\text { WEIGHT }\end{array}$ & $\begin{array}{l}\text { Weighted eligible population per } \\
\text { strata divided by weighted com- } \\
\text { pletes per strata. }\end{array}$ & $w / v$ \\
\hline$Y$ & NO & $\begin{array}{l}\text { TOTAL } \\
\text { WEIGHT PER } \\
\text { MRR }\end{array}$ & $\begin{array}{l}\text { The product of the individual } \\
\text { weight, the MRR weight, the util- } \\
\text { ity weight, the substrata weight, } \\
\text { the strata weight and the sample } \\
\text { projected weight. }\end{array}$ & $\begin{array}{l}N\{M R R\} * \\
P\{M R R\} * \\
Q\{\text { uti }\} * \\
R\{\text { substrata }\} \\
\star S\{\text { strata }\} \\
\star X\{\text { strata }\end{array}$ \\
\hline$z$ & NO & $\begin{array}{l}\text { FINAL } \\
\text { WEIGHTED } \\
\text { MRR } \\
\text { POPULATION }\end{array}$ & $\begin{array}{l}\text { The product of the number of com- } \\
\text { pleted interviews per MRR and the } \\
\text { total weight per MRR. }\end{array}$ & $L * Y$ \\
\hline
\end{tabular}


column E, MRR SAMPLE DRAWN, and L refers to column L, COMPLETED INTERVIEWS. Specifically, cell 031 (column D, row 31) contains the result of the calculation E31-L31, read as the value of column $E$, row 31 minus the value of column L, row 31 .

The results of calculations performed at the MRR level are summed to the utility level, the substrata level, the strata level, the type of ownership (public utilities or private utilities), and to the population level. The level of aggregation referred to in a formula is designated with \{\} , for example, \{strata\}. An example of a formula with different levels of aggregation is column $Y$, TOTAL WEIGHT PER MRR, which contains the product of six weights at different levels of aggregation. The formula is $N\{M R R\} * P\{M R R\}$ *

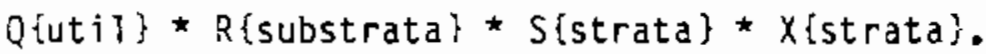

\subsection{MOOIFICATIONS OF ORIGINAL SPREADSHEET}

Various changes to the original spreadsheet developed for 1983 PNWRES were necessitated for the Resample. Some of the changes are more or less fundamental in nature, in that the changes were required to allow the spreadsheet's application to the 1985 Resample. The structural changes were required to accommodate the differences between the PNWRES and the 1985 Resample's sample sizes and different numbers of respondents in each MRR. Additionally, the spreadsheet had to be changed to allow for the possibility of an MRR having no responses. The structural changes are given in Table 3.2.

Other changes (Table 3.3 ) were made to the Louis Harris spreadsheet to correct computational and typographical errors (changes 1 and 2), as well as to make the spreadsheet internally consistent so as to allow its application to the resanple (change 3 ). In the latter case, the changes were required in order to avoid introducing errors into the calculations when applied to the 1985 Resample. 
TABLE 3.2. Structural Changes to Louis Harris Spreadsheet

$\frac{\text { COLUMN }}{C} \frac{\text { TITLE }}{\begin{array}{l}\text { KNOWN } \\ \text { INEL IGIBLES }\end{array}}$

CHANGE

Changed from a number obtained from the fielding of

1983 PNWRES to a blank column, functionally very similar to setting the column equal to zero. No known ineligibles were identified for the PNWRES resample.

D UNABLE TO

Changed from a number obtained from the fielding of

li 1983 PNWRES to equal the difference between the sample drawn and the number of completed interviews.

E MRR SAMPLE Changed to reflect the resample's sampling plan. DRAWN

F ELIGIBILITY RATIO

The values generated by the 1983 PNWRES spreadsheet were retained directly, replacing the formula used to calculate them. The formula would have generated a value of 1 in all cases.

I ELIGIBLE MRR Changed to equal zero if there are no completed interPOPULATION views for an MRR.

L COMPLETED Actual number of resample respondents for each MRR was INTERVIEWS input.

M AVERAGE Denominator changed from total number of MRRs to number COMPLETES of MRRs with at least one response.

PER MRR

$N$ INDIVIDUAL Changed to equal zero if the number of respondents in an WEIGHTS MRR (the denominator in the formula) equals zero.

0 AVERAGE Denominator changed from number of MRR in a utility to POPULATION number of MRR with at least one response.

P MRR WEIGHTS Changed to equal zero if the average population of an MRR (the denominator in the formula) equals zero. 
TABLE 3.3. Nonstructural Changes to Louis Harris Spreadsheet

CHANGE

1. Column $Y$, TOTAL WEIGHT PER MRR, changed from $N+P+Q+R+S+X$ to $N \star P \star Q \star R \star S \star X$ for the MRR 147501 through 147508 (ail the MRR in CP NATL).

2. Column B, MRR CODE, changed

\begin{tabular}{ll}
\multicolumn{1}{c}{ From } & $\frac{\text { To }}{143203}$ \\
143403 \\
134412 (first occurrence) & 134402 \\
103303 & 109303
\end{tabular}

3. Column $T$, WEIGHTED COMPLETES PER MRR, was changed from an input numeric constant to a formula for the utilities represented by the MRR codes 1152 , 1162,1182 and 1192 . 


\subsection{CALCULATED CASE WEIGHTS: OVERALL RESAMPLE, ROSM, AND ROST}

In order to calculate specific case weights for the 1985 Resample, the number of survey respondents must be identified. When calculating 1985 Resample case weights, individuals are identified as respondents if they responded to either the RDSM, or the ROST, or both. At the spreadsheet level, this transiates into the number of individuals in an MRR that responded to either the ROSM or the ROST or both. The 1983 PNWRES spreadsheet was adapted to calculate case weights for the 1985 Resample.

After all the structural changes had been made, application of the spreadsheet to the calculation of ROSM and ROST case weights required additional changes in three columns. The major changes were in column $L$, COMPLETED INTERVIEWS. The entries in this column had to be replaced with the number of individuals in each MRR that had responded to the individual surveys (ROSM or ROST). After column $L$ was filled in, the denominator in column $M$, AVERAGE COMPLETES PER MRR, had to be changed to the new total number of MRRs with at least one response. Similarly, the denominators in column 0 , AVERAGE POPULATIDN, had to be changed to the number of MRRs within a utility with at least one response. All other changes were automatically calculated by LOTUS.

Table 4.1 shows three sets of case weights for each observation (OBS), labeled according to PNWRES identification number (PNRES_IO) and meter reader route code (MRRCODE). The first set of case weights (ROSMWT) pertains to respondents of ROSM. The second set of case weights (ROSTWT) pertains to respondents of ROST. The combined ROSM/ROST resample case weights (RESAMWT) are given in the final column of the table. Also shown is the response status for each of the PNWRES households, where a "l" indicates a response was obtained, and a "." indicates a missing value for that survey. 
TABLE 4.1. 1985 Resampie Caseweights

\begin{tabular}{|c|c|c|c|c|c|c|c|c|}
\hline OBS & PNRES ID & MRRCODE & ROSM & ROSMWT & ROST & POSTWT & RESAMPLE & RESAMWT \\
\hline 1 & 1010 & 101101 & 1 & 1212.2 & 1 & 1019.3 & 1 & 940.2 \\
\hline 2 & 1043 & 101103 & 1 & 1880.2 & 1 & 1581.0 & 1 & 1458.3 \\
\hline 3 & 1059 & 101104 & . & 0.0 & 1 & 1351.6 & 1 & 1246.8 \\
\hline 4 & 1069 & 101105 & 1 & 836.9 & 1 & 703.7 & 1 & 649.1 \\
\hline 5 & 1097 & 101107 & 1 & 8302.7 & 1 & 6981.4 & 1 & 6439.9 \\
\hline 6 & 1115 & 101108 & 1 & 6741.8 & 1 & 5668.9 & 1 & $5229 . ?$ \\
\hline 7 & 1124 & 101109 & 1 & 6824.8 & 1 & 5738.8 & 1 & 5293.6 \\
\hline 8 & 1143 & 101110 & 1 & 7748.6 & 1 & 6515.5 & 1 & 6010.1 \\
\hline 9 & 1163 & 101111 & 1 & 5321.5 & - & 0.0 & 1 & 4127.6 \\
\hline 10 & 1177 & 101112 & - & 0.0 & 1 & 1974.9 & 1 & 1821.7 \\
\hline 11 & 1188 & 101113 & 1 & 2036.0 & 1 & 1712.0 & 1 & 1579.2 \\
\hline 12 & 1206 & 101114 & 1 & 361.3 & 1 & 303.8 & 1 & 280.2 \\
\hline 13 & 1242 & 102101 & 1 & 4053.5 & 1 & 2975.6 & 1 & 2851.6 \\
\hline 14 & 1268 & 102102 & 1 & 5333.9 & 1 & 3915.5 & 1 & 3752.3 \\
\hline 15 & 1269 & 102103 & - & 0.0 & 1 & 4162.3 & 1 & 3988.9 \\
\hline 16 & 1285 & 102104 & . & 0.0 & 1 & 1744.9 & 1 & 1672.2 \\
\hline 17 & 1317 & 102106 & 1 & 2604.8 & 1 & 1912.1 & $i$ & 1832.4 \\
\hline 18 & 1337 & 102107 & . & 0.0 & 1 & 2908.5 & $i$ & 2787.3 \\
\hline 19 & 1346 & 102108 & . & 0.0 & 1 & 3039.0 & 1 & 2912.4 \\
\hline 20 & 1371 & 102109 & 1 & 3845.6 & 1 & 2823.0 & 1 & 2705.3 \\
\hline 21 & 1398 & 102111 & . & 0.0 & 1 & 3541.7 & 1 & 3394.1 \\
\hline 22 & 1413 & 102112 & 1 & 3309.5 & 1 & 2429.5 & $i$ & 2328.2 \\
\hline 23 & 1429 & 102113 & 1 & 4469.9 & 1 & 3281.2 & 1 & 3144.5 \\
\hline 24 & 1440 & 102114 & 1 & 5269.0 & 1 & 3867.8 & 1 & 3706.7 \\
\hline 25 & 1456 & 102201 & 1 & 23515.7 & 1 & 19963.5 & 1 & 16978.9 \\
\hline 26 & 1479 & 102203 & - & 0.0 & 1 & 15022.5 & 1 & 12776.6 \\
\hline 27 & 1496 & 102204 & 1 & 10034.3 & . & 0.0 & 1 & 7245.0 \\
\hline 28 & 1509 & 102205 & • & 0.0 & 1 & 13790.6 & 1 & 11728.9 \\
\hline 29 & 1524 & 102206 & 1 & 12101.4 & 1 & 10273.4 & 1 & 8737.5 \\
\hline 30 & 1543 & 102207 & 1 & 11934.2 & 1 & 10131.5 & 1 & 8616.8 \\
\hline 31 & 1558 & 102208 & 1 & 11463.9 & 1 & 9732.2 & 1 & 8277.2 \\
\hline 32 & 1574 & 102301 & - & 0.0 & 1 & 18217.9 & 1 & 15744.2 \\
\hline 32 & 1598 & 102302 & 1 & 24975.3 & - & 0.0 & 1 & 15477.3 \\
\hline 34 & 1628 & 102304 & 1 & 9694.8 & 1 & 6951.9 & 1 & 6007.9 \\
\hline 35 & 1641 & 102305 & 1 & 16855.2 & 1 & 12086.5 & 1 & 10445.3 \\
\hline 36 & 1655 & 102306 & 1 & 11626.4 & 1 & 8337.0 & 1 & 7205.0 \\
\hline 37 & 1663 & 102307 & • & 0.0 & 1 & 8204.7 & 1 & 7090.6 \\
\hline 38 & 1675 & 102308 & 1 & 20423.1 & 1 & 14644.9 & 1 & 12656.3 \\
\hline 39 & 1689 & 102309 & . & 0.0 & 1 & 16056.5 & 1 & 13876.2 \\
\hline 40 & 1715 & 102310 & $\bullet$ & 0.0 & 1 & 6628.4 & 1 & 5728.4 \\
\hline 41 & 1742 & 102312 & 1 & 15651.3 & 1 & 11223.2 & 1 & 9699.2 \\
\hline 42 & 1754 & 102313 & 1 & 14579.2 & 1 & 10454.4 & 1 & 9034.8 \\
\hline 43 & 1771 & 102314 & . & 0.0 & 1 & 9969.1 & 1 & 8615.4 \\
\hline 44 & 1785 & 102315 & • & 0.0 & 1 & 10711.4 & 1 & 9256.9 \\
\hline 45 & 1810 & 102501 & 1 & 8008.2 & 1 & 7295.9 & 1 & 6420.4 \\
\hline 46 & 1830 & 102502 & • & 0.0 & 1 & 6218.8 & 1 & 5472.6 \\
\hline 47 & 1845 & 102503 & 1 & 9573.0 & . & 0.0 & 1 & 7674.9 \\
\hline
\end{tabular}


TABLE 4.1. (contd)

\begin{tabular}{|c|c|c|c|c|c|c|c|c|}
\hline OBS & PNRES IO & MRRCOOE & ROSM & ROSMWT & ROST & ROSTWT & RESAMPLE & RESAMWT \\
\hline 48 & 1863 & 102504 & 1 & 5654.0 & 1 & 5151.0 & 1 & 4532.9 \\
\hline 49 & 1879 & 102505 & 1 & 2279.8 & & 0.0 & 1 & 1827.8 \\
\hline 50 & 1885 & 102506 & 1 & 3822.5 & 1 & 3482.5 & 1 & 3064.6 \\
\hline 51 & 1914 & 102508 & & 0.0 & $i$ & 7386.3 & 1 & 6500.0 \\
\hline 52 & 1931 & 102509 & i & 4207.4 & 1 & 3833.1 & $i$ & 3373.2 \\
\hline 53 & 1956 & 102510 & 1 & 6982.1 & $i$ & 6361.0 & 1 & 5597.7 \\
\hline 54 & 1971 & 102511 & $i$ & 2744.5 & . & 0.0 & 1 & 2200.4 \\
\hline 55 & 1995 & 102513 & $\bullet$ & 0.0 & 1 & 1460.2 & 1 & 1285.0 \\
\hline 56 & 2024 & 102515 & $i$ & 2387.3 & 1 & 2175.0 & 1 & 1914.0 \\
\hline 57 & 2037 & 102516 & 1 & 7232.9 & 1 & 6589.5 & 1 & 5798.8 \\
\hline 58 & 2053 & 102601 & & 0.0 & 1 & 9169.9 & 1 & 8791.5 \\
\hline 59 & 2066 & 102602 & $i$ & 24842.5 & $i$ & 17785.1 & 1 & 17051.2 \\
\hline 60 & 2088 & 102603 & $i$ & 34763.1 & $i$ & 24887.3 & 1 & 23860.4 \\
\hline 61 & 2118 & 102605 & $i$ & 17312.8 & $i$ & 12394.5 & 1 & 11883.0 \\
\hline 62 & 2130 & 102606 & 1 & 12975.5 & $i$ & 9289.3 & 1 & 8906.0 \\
\hline 63 & 2136 & 102606 & 1 & 12975.5 & 1 & 9289.3 & $i$ & 8906.0 \\
\hline 64 & 2146 & 102607 & 1 & 48500.3 & $i$ & 34722.0 & 1 & 33289.3 \\
\hline 65 & 2163 & 102608 & . & 0.0 & 1 & 36199.8 & 1 & 34706.0 \\
\hline 66 & 2175 & 102609 & . & 0.0 & $i$ & 34447.9 & 1 & 33026.5 \\
\hline 67 & 2186 & 102610 & 1 & 8051.2 & 1 & 11528.0 & 1 & 5526.1 \\
\hline 68 & 2196 & 102610 & $i$ & 8051.2 & . & 11528.0 & 1 & 5526.1 \\
\hline 69 & 2205 & 102611 & 1 & 12160.2 & $i$ & 8705.6 & 1 & 8346.4 \\
\hline 70 & 2206 & 102611 & 1 & 12160.2 & 1 & 8705.6 & 1 & 8346.4 \\
\hline 71 & 2214 & 102612 & $i$ & 22534.2 & 1 & 32265.0 & $i$ & 15466.8 \\
\hline 72 & 2218 & 102612 & 1 & 22534.2 & . & 32265.0 & 1 & 15466.8 \\
\hline 73 & 2234 & 102613 & $\bullet$ & 0.0 & 1 & 39203.3 & 1 & 37585.6 \\
\hline 74 & 2250 & 102614 & 1 & 25909.4 & 1 & 1854 & 1 & 17783.5 \\
\hline 75 & 2266 & 102615 & 1 & 17518.9 & 1 & 12542.0 & 1 & 12024.5 \\
\hline 76 & 2270 & 102615 & 1 & 17518.9 & 1 & 12542.0 & 1 & 12024.5 \\
\hline 77 & 2274 & 102616 & 1 & 19973.0 & . & 0.0 & i & 13708.9 \\
\hline 78 & 2324 & 104101 & 1 & 1325.1 & 1 & 1309.5 & 1 & 1222.8 \\
\hline 79 & 2345 & 104102 & 1 & 1217.0 & 1 & 1202.6 & 1 & 1123.0 \\
\hline 80 & 2359 & 104103 & 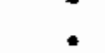 & 0.0 & 1 & 1403.1 & 1 & 1310.1 \\
\hline 81 & 2373 & 104104 & 1 & 1325.1 & & 0.0 & 1 & 1222.8 \\
\hline 82 & 2384 & 104105 & 1 & 1318.8 & 1 & 1303.3 & 1 & 1217.0 \\
\hline 83 & 2397 & 104106 & 1 & 1419.8 & 1 & 1403.1 & 1 & 1310.1 \\
\hline 84 & 2437 & 104108 & 1 & 1336.3 & i & 1320.5 & 1 & 1233.1 \\
\hline 85 & 2457 & 105102 & 1 & 525.0 & 1 & 525.0 & 1 & 489.5 \\
\hline 86 & 2477 & 105103 & 1 & 814.6 & 1 & 814.6 & l & 759.5 \\
\hline 87 & 2488 & 105104 & 1 & 943.7 & 1 & 943.7 & 1 & 879.9 \\
\hline 88 & 2498 & 105105 & 1 & 2347.8 & 1 & 2347.8 & 1 & 2189.0 \\
\hline 89 & 2515 & 105106 & 1 & 3062.4 & 1 & 3062.4 & 1 & 2855.2 \\
\hline 90 & 2540 & 105107 & 1 & 2603.0 & 1 & 2603.0 & 1 & 2426.9 \\
\hline 91 & 2564 & 106101 & • & 0.0 & 1 & 2288.3 & 1 & 2288.3 \\
\hline 92 & 2593 & 106103 & $i$ & 2982.3 & 1 & 2383.6 & 1 & 2383.6 \\
\hline 93 & 2621 & 106105 & 1 & 2161.5 & 1 & 1727.6 & 1 & 1727.6 \\
\hline 94 & 2681 & 107101 & 1 & 1033.3 & 1 & 857.1 & 1 & 857.1 \\
\hline
\end{tabular}


TABLE 4.1. (contd)

\begin{tabular}{|c|c|c|c|c|c|c|c|c|}
\hline OBS & PNRES ID & MRRCODE & ROSM & ROSMWT & ROST & ROSTWT & RESAMPLE & RESAMWT \\
\hline 95 & 2697 & 107101 & 1 & 1033.3 & 1 & 857.1 & 1 & 857.1 \\
\hline 96 & 2710 & 107102 & 1 & 1650.3 & 1 & 1368.8 & 1 & 1368 \\
\hline 97 & 2726 & 107103 & 1 & 2281.2 & 1 & 1892.1 & 1 & 1892 \\
\hline 98 & 2776 & 107105 & 1 & 681.4 & 1 & 565.2 & 1 & 565 \\
\hline 99 & 2781 & 107105 & 1 & 681.4 & 1 & 565.2 & 1 & 565 \\
\hline 100 & 2797 & 108201 & 1 & 13055.1 & 1 & 11631.3 & 1 & 9656 \\
\hline 101 & 2821 & 108202 & 1 & 13603.7 & 1 & 12120.0 & 1 & 10062 \\
\hline 102 & 2838 & 108203 & 1 & 15475.0 & 1 & 13787.3 & 1 & 11446 \\
\hline 103 & 2852 & 108204 & - & 0.0 & 1 & 17395.0 & 1 & 14441 . \\
\hline 104 & 2880 & 108206 & & 0.0 & 1 & 0.0 & 1 & 8846 \\
\hline 105 & 2886 & 108207 & 1 & 14906.7 & . & 1.3280 .9 & 1 & 11025 \\
\hline 106 & 2909 & 108208 & 1 & 12009.0 & 1 & 1.0699 .3 & 1 & 8882 \\
\hline 107 & 2921 & 108501 & 1 & 4195.9 & 1 & 3808.2 & 1 & 3264 \\
\hline 108 & 2930 & 108502 & 1 & 10239.9 & 1 & 9293.8 & 1 & 7966 \\
\hline 109 & 2965 & 108504 & 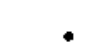 & 0.0 & 1 & 5969.7 & 1 & 5116 \\
\hline 110 & 3004 & 108506 & 1 & 3760.3 & 1 & 3412.8 & $i$ & 2925 \\
\hline 111 & 3006 & 108507 & 1 & 476.9 & 1 & 432.8 & 1 & 371 \\
\hline 112 & 3032 & 108508 & 1 & 7772.8 & $i$ & 7054.6 & $i$ & 6046 \\
\hline 113 & 3035 & 109201 & 1 & 14150.7 & 1 & 11734.9 & 1 & 10885 \\
\hline 114 & 3060 & 109202 & $i$ & 15442.9 & 1 & 12806.5 & $i$ & 11879 \\
\hline 115 & 3075 & 109203 & 1 & 8008.9 & . & 0.0 & 1 & 6160 \\
\hline 116 & 3105 & 109205 & 1 & 13461.0 & . & 0.0 & $i$ & 10354 \\
\hline 117 & 3112 & 109206 & 1 & 17986.1 & 1 & 14915.5 & $i$ & 13835 \\
\hline 118 & 3151 & 109302 & 1 & 16017.4 & 1 & 1.1554 .8 & 1 & 10604 \\
\hline 119 & 3159 & 109303 & . & 0.0 & 1 & 11646.5 & 1 & 10688 \\
\hline 120 & 3177 & 109304 & $i$ & 18068.3 & 1 & 1.3034 .3 & 1 & 11962 \\
\hline 121 & 3221 & 109307 & 1 & 14555.5 & 1 & 10500.2 & 1 & 9536 \\
\hline 122 & 3233 & 109308 & 1 & 16733.5 & 1 & 12071.4 & 1 & 11078 \\
\hline 123 & 3290 & 109312 & . & 0.0 & 1 & 5716.4 & 1 & 5246 \\
\hline 124 & 3334 & 109314 & 1 & 18496.3 & 1 & 13343.1 & 1 & 12245 \\
\hline 125 & 3337 & 109315 & 1 & 13676.4 & . & 0.0 & $i$ & 9054 \\
\hline 126 & 3379 & 111201 & 1 & 13490.5 & 1 & 1.2025 .4 & 1 & 10185 \\
\hline 127 & 3392 & 1112 & - & 0.0 & 1 & 18272.3 & 1 & 15476 \\
\hline 128 & 3397 & 111203 & 1 & 23163.9 & 1 & 20648.2 & 1 & 17488 \\
\hline 129 & 3434 & 111205 & 1 & 13431.2 & 1 & 1972.6 & I & 10140 \\
\hline 130 & 3443 & 111206 & 1 & 9001.6 & 1 & 8024.0 & 1 & 6796 \\
\hline 131 & 3458 & 111207 & 1 & 9962.3 & & 0.0 & 1 & \\
\hline 132 & 3481 & 111208 & - & 0.0 & $i$ & 7971.2 & $i$ & 6751 \\
\hline 133 & 3512 & 112202 & & 0.0 & 1 & 11746.5 & 1 & 11746 \\
\hline 134 & 3543 & 112204 & 1 & 21111.5 & 1 & 8008.4 & 1 & 8008 \\
\hline 135 & 3552 & ii 2205 & • & 0.0 & 1 & 7494.5 & 1 & \\
\hline 136 & 3566 & 112206 & & 0.0 & 1 & 3854.3 & 1 & 3854 \\
\hline 137 & 3586 & 113201 & 1 & 11045.8 & 1 & 3988.6 & 1 & \\
\hline 138 & 2601 & 113202 & $i$ & 11282.5 & 1 & 4074.1 & 1 & 4074 \\
\hline 139 & 3619 & 113203 & - & 0.0 & 1 & 4273.5 & 1 & 4273 \\
\hline 140 & 3674 & 114201 & 1 & 3765.9 & 1 & 1475.4 & 1 & \\
\hline & 3697 & 114203 & & 0.0 & & 4348.9 & 1 & 377 \\
\hline
\end{tabular}


TABLE 4.1. (contd)

\begin{tabular}{|c|c|c|c|c|c|c|c|c|}
\hline OBS & PNRES ID & MRRCODE & ROSM & ROSMWT & ROST & ROSTWT & RESAMPLE & RESAMWT \\
\hline 142 & 3737 & 114205 & 1 & 990.0 & 1 & 387.8 & 1 & 336.4 \\
\hline 143 & 3760 & 115201 & 1 & 2817.9 & 1 & 2453.9 & 1 & 2128.1 \\
\hline 144 & 3771 & 116201 & 1 & 2817.9 & $I$ & 2453.9 & 1 & 2128.1 \\
\hline 145 & 3788 & 118201 & 1 & 2817.9 & 1 & 2453.9 & 1 & 2128.1 \\
\hline 146 & 3802 & 119201 & 1 & 2817.9 & . & 0.0 & 1 & 2128.1 \\
\hline 147 & 3838 & 120202 & & 0.0 & 1 & 2453.9 & 1 & 2128.1 \\
\hline 148 & 3849 & 121201 & $i$ & 1608.6 & $i$ & 1887.3 & 1 & 1608.6 \\
\hline 149 & 3894 & 121204 & 1 & 1615.5 & 1 & 1895.4 & 1 & 1615.5 \\
\hline 150 & 3915 & 121205 & 1 & 1615.5 & 1 & 1895.4 & 1 & 1615.5 \\
\hline 151 & 3930 & 121206 & 1 & 1355.8 & . & 0.0 & 1 & 1355.8 \\
\hline 152 & 3958 & 122202 & 1 & 2329.2 & 1 & 2846.3 & 1 & 2329.2 \\
\hline 153 & 4070 & 123304 & 1 & 14520.9 & 1 & 14520.9 & 1 & 12487.7 \\
\hline 154 & 4128 & 124301 & 1 & 10237.2 & 1 & 9190.4 & 1 & 7731.7 \\
\hline 155 & 4132 & 124302 & 1 & 4849.7 & 1 & 4353.8 & 1 & 3662.8 \\
\hline 156 & 4157 & 124303 & 1 & 15736.4 & & 0.0 & 1 & 11885.1 \\
\hline 157 & 4165 & 124304 & $i$ & 20033.2 & $i$ & 17984.7 & 1 & 15130.2 \\
\hline 158 & 4185 & 124305 & & 0.0 & 1 & 19327.5 & 1 & 16259.9 \\
\hline 159 & 4193 & 125301 & $i$ & 12714.1 & 1 & 12714.1 & 1 & 10933.9 \\
\hline 160 & 4218 & 126301 & 1 & 2708.3 & $i$ & 1493.5 & 1 & 1493.5 \\
\hline 161 & 4233 & 126302 & 1 & 3828.1 & 1 & 2111.0 & 1 & 2111.0 \\
\hline 162 & 4257 & 126304 & 1 & 21282.9 & 1 & 11736.8 & 1 & 11736.8 \\
\hline 163 & 4268 & 126305 & 1 & 11776.4 & 1 & 6494.3 & 1 & 6494.3 \\
\hline 164 & 4305 & 127301 & - & 0.0 & 1 & 6950.8 & 1 & 6950.8 \\
\hline 165 & 4333 & 127303 & & 0.0 & 1 & 8443.9 & 1 & 8443.9 \\
\hline 166 & 4346 & 127304 & $i$ & 14728.3 & 1 & 7105.2 & 1 & 7105.2 \\
\hline 167 & 4363 & 127305 & $\bullet$ & 0.0 & 1 & 9988.5 & 1 & 9988.5 \\
\hline 168 & 4426 & 128303 & $i$ & 2717.0 & . & 0.0 & $i$ & 2234.4 \\
\hline 169 & 4440 & 128304 & 1 & 3734.9 & 1 & 3915.1 & 1 & 3071.5 \\
\hline 170 & 4451 & 130301 & . & 0.0 & 1 & 5091.3 & 1 & 3418.8 \\
\hline 171 & 4468 & 130302 & 1 & 3561.5 & 1 & 4288.7 & 1 & 2879.8 \\
\hline 172 & 4488 & & 1 & 4134.6 & & 0.0 & 1 & 3343.2 \\
\hline 173 & 4490 & 130304 & 1 & 4177.5 & $i$ & 5030.4 & 1 & 3377.9 \\
\hline 174 & 4513 & 1313 & . & 0.0 & $i$ & 1697.7 & 1 & 844.0 \\
\hline 175 & 4534 & 13130 & . & 0.0 & 1 & 1925.4 & 1 & 957.2 \\
\hline 176 & 4556 & 1313 & 1 & 1405.5 & - & 0.0 & 1 & 1281.6 \\
\hline 177 & 4584 & 1323 & 1 & 1796.3 & & 0.0 & 1 & 1313.3 \\
\hline 178 & 4592 & 132302 & 1 & 1322.2 & $i$ & 2009.7 & 1 & 966.7 \\
\hline 179 & 4605 & 133301 & 1 & 1895.6 & • & 0.0 & 1 & 1385.8 \\
\hline & 4627 & 133302 & 1 & 1222.9 & 1 & 2009.7 & 1 & 894.1 \\
\hline 181 & 4640 & 134401 & 1 & 39842.3 & 1 & 36435.2 & 1 & 36435.2 \\
\hline 18 & 4664 & 134403 & 1 & 15528.9 & 1 & 14200.9 & 1 & 14200.9 \\
\hline 183 & 4670 & 134403 & 1 & 15528.9 & $i$ & 14200.9 & 1 & 14200.9 \\
\hline 184 & 4692 & 134405 & 1 & 22077.2 & 1 & 20189.3 & 1 & 20189.3 \\
\hline 185 & 4702 & 134405 & 1 & 22077.2 & 1 & 20189.3 & 1 & 20189.3 \\
\hline 186 & 4713 & 134406 & 1 & 17462.2 & 1 & 15968.9 & 1 & 15968.9 \\
\hline 187 & 4719 & 134406 & 1 & 17462.2 & 1 & 15968.9 & 1 & 15968.9 \\
\hline 138 & 4725 & 134407 & 1 & 14215.1 & & 25998.9 & 1 & 12999.5 \\
\hline
\end{tabular}


TABLE 4.1. (contd)

\begin{tabular}{|c|c|c|c|c|c|c|c|c|}
\hline OBS & PNRES ID & MRRCODE & ROSM & ROSMWT & ROST & ROSTWT & RESAMPLE & RESAMWT \\
\hline 189 & 4730 & 134407 & 1 & 14215.1 & 1 & 25998.9 & 1 & 12999.5 \\
\hline 190 & 4744 & 134408 & & 0.0 & 1 & 16083.0 & $i$ & 16083.0 \\
\hline 191 & 4756 & 134409 & 1 & 70095.4 & 1 & 64101.3 & 1 & 64101.3 \\
\hline 192 & 4778 & 134410 & & 0.0 & $i$ & 24043.0 & $i$ & 24043.0 \\
\hline 19 & 4784 & 134411 & $i$ & 53963.1 & 1 & 49348.5 & 1 & 49348.5 \\
\hline 194 & 4804 & 134412 & $i$ & 22655.9 & 1 & 20718.5 & $i$ & 20718.5 \\
\hline $19 !$ & 4821 & 134413 & 1 & 25320.2 & $i$ & 23154.9 & 1 & 23154.9 \\
\hline 196 & 4827 & 134414 & $i$ & 13092.2 & 1 & 1972.6 & $i$ & 11972.6 \\
\hline 19 & 4836 & 134414 & 1 & 13092.2 & 1 & 11972.6 & $i$ & 11972.6 \\
\hline 198 & 4846 & 134415 & $i$ & 69529.4 & $i$ & 63583.7 & 1 & 63583.7 \\
\hline 19 & 4858 & 134416 & 1 & 23075.0 & 1 & 21101.8 & 1 & 21101.8 \\
\hline 20 & 4891 & 135402 & . & 0.0 & 1 & 60213.5 & 1 & 50887.0 \\
\hline 20 & 4902 & 135403 & 1 & 27182.6 & . & 0.0 & $i$ & 20013.9 \\
\hline 20 & 4906 & 135403 & 1 & 27182.6 & - & 0.0 & 1 & 20013.9 \\
\hline 20 & 4924 & 135404 & 1 & 34128.4 & & 59466.5 & 1 & 25127 . \\
\hline 20 & 4925 & 135404 & 1 & 34128.4 & $i$ & 59466.5 & 1 & 25127.8 \\
\hline 20 & $49 ? 9$ & 135405 & 1 & 56183.2 & $i$ & 48947.8 & 1 & 41366 . \\
\hline 20 & 4945 & 135406 & 1 & 37729.8 & 1 & 32870.9 & 1 & 27779.5 \\
\hline 20 & 4955 & 135406 & 1 & 37729.8 & 1 & 32870.9 & 1 & 27779.5 \\
\hline 20 & 4960 & 136401 & 1 & 15272.8 & $i$ & 3583.9 & 1 & 10940.4 \\
\hline 20 & 4983 & 136402 & $i$ & 35716.7 & . & 0.0 & $i$ & 25585.0 \\
\hline 210 & 4999 & 136403 & & 0.0 & $i$ & 38034.8 & $i$ & 30633.2 \\
\hline 21 & 5008 & 136404 & $i$ & 20506.8 & 1 & 36478.0 & 1 & 14689.7 \\
\hline 212 & 5018 & 136404 & 1 & 20506.8 & & 36478.0 & $i$ & 14689.7 \\
\hline 213 & 5032 & 136405 & . & 0.0 & I & 28083.5 & $i$ & 22518.4 \\
\hline 214 & 5044 & 136406 & $i$ & 38721.4 & $i$ & 34439.4 & 1 & 27737.4 \\
\hline 215 & 5057 & 137401 & 1 & 7241.6 & $i$ & 7241.6 & 1 & 6496.3 \\
\hline 216 & 5061 & 137401 & 1 & 7241.6 & $i$ & 7241.6 & $i$ & 6496.3 \\
\hline 217 & 5063 & 137402 & i & 19312.3 & 1 & 9656.2 & $i$ & 8662.4 \\
\hline 218 & 5069 & 137402 & . & 19312.3 & 1 & 9656.2 & 1 & 8562.4 \\
\hline 219 & 5086 & 137403 & - & 20446.7 & 1 & 10223.3 & 1 & 9171.2 \\
\hline 220 & 5087 & 137403 & 1 & 20446.7 & 1 & $\vdots 0223.3$ & 1 & 9171.2 \\
\hline 221 & 5105 & 137404 & 1 & 8645.0 & $i$ & 8645.0 & 1 & 7755. \\
\hline 22 & 5112 & 137405 & 1 & 28658.6 & 1 & 28558.6 & 1 & 25709 . \\
\hline 22 & 5141 & 138401 & & 23986.1 & 1 & $\therefore 4342.9$ & 1 & 13172 . \\
\hline 22 & 5144 & 138401 & $i$ & 28986.1 & 1 & $\lcm{4342.9}$ & 1 & 13172.5 \\
\hline 225 & 5154 & 138402 & 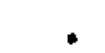 & 23203.9 & 1 & 21481.8 & 1 & 10544.8 \\
\hline 22 & 5165 & 138402 & 1 & 23203.9 & 1 & $\vdots 1481.8$ & 1 & 10544.8 \\
\hline 22 & 5173 & 138403 & 1 & 23047.1 & $i$ & 22808.4 & 1 & 20947.1 \\
\hline 228 & 5186 & 138404 & & 26499.3 & 1 & 25224.8 & $i$ & 12042 . \\
\hline 22 & 5194 & 138404 & 1 & 26499.3 & . & 26224.8 & 1 & 12042 . \\
\hline 23 & 5204 & 138405 & 1 & 21424.5 & & 0.0 & 1 & 19472.3 \\
\hline 23 & 5225 & 138406 & & 0.0 & $i$ & 22478.4 & 1 & 20644.1 \\
\hline 23 & 5246 & 139402 & 1 & 4170.5 & $i$ & 3770.6 & $i$ & 3770.6 \\
\hline 23 & 5257 & 139402 & i & 4170.5 & $i$ & 3770.6 & 1 & 3770.6 \\
\hline 23 & 5258 & 139403 & 1 & 2509.2 & 1 & 2268.6 & 1 & 2268.6 \\
\hline & 5272 & 139403 & 1 & 2509.2 & 1 & 2268.6 & $i$ & 2268 \\
\hline
\end{tabular}


TABLE 4.1. (contd)

\begin{tabular}{|c|c|c|c|c|c|c|c|c|}
\hline$\underline{O B S}$ & PNRES ID & MRRCODE & ROSM & ROSMWT & ROST & ROSTWT & RESAMPLE & RESAMWT \\
\hline 236 & 5287 & 139404 & 1 & 12224.1 & 1 & 11052.1 & 1 & 11052.1 \\
\hline 237 & 5293 & 139405 & $i$ & 2022.0 & 1 & 1828.2 & 1 & 1828.2 \\
\hline 238 & 5299 & 139405 & 1 & 2022.0 & $i$ & 1828.2 & 1 & 1828.2 \\
\hline 239 & 5308 & 139406 & 1 & 3452.8 & $i$ & 6243.4 & 1 & 3121.7 \\
\hline 240 & 5315 & 139406 & $i$ & 3452.8 & . & 6243.4 & $i$ & 3121.7 \\
\hline 241 & 5338 & 140402 & 1 & 5351.0 & 1 & 4726.9 & 1 & 4726.9 \\
\hline 242 & 5346 & 140402 & $i$ & 5351.0 & $i$ & 4726.9 & 1 & 4726.9 \\
\hline 243 & 5359 & 140403 & $\bullet$ & 0.0 & 1 & 6641.2 & 1 & 6641.2 \\
\hline 244 & 5367 & 140404 & $i$ & 4382.9 & 1 & 3871.7 & $i$ & 3871.7 \\
\hline 245 & 5378 & 140405 & $i$ & 4359.2 & 1 & 3850.8 & $i$ & 3850.8 \\
\hline 246 & 5397 & 140406 & 1 & 7466.5 & $i$ & 6595.6 & 1 & 6595.6 \\
\hline 247 & 5417 & 141401 & 1 & 2293.9 & 1 & 2979.9 & $i$ & 2158.2 \\
\hline 248 & 5422 & 141401 & 1 & 2293.9 & 1 & 2979.9 & 1 & 2158.2 \\
\hline 249 & 5428 & 141402 & . & 0.0 & 1 & 1989.8 & 1 & 1441.1 \\
\hline 250 & 5430 & 141402 & & 0.0 & 1 & 1989.8 & 1 & 1441.1 \\
\hline 251 & 5448 & 141403 & $i$ & 6494.6 & . & 0.0 & $i$ & 6110.3 \\
\hline 252 & 5467 & 141404 & $i$ & 3823.2 & 1 & 4966.5 & 1 & 3597.0 \\
\hline 253 & 5469 & 142401 & i & 1675.9 & 1 & 1675.9 & $i$ & 1425.6 \\
\hline 254 & 5470 & 142401 & 1 & 1675.9 & 1 & 1675.9 & 1 & 1425.6 \\
\hline 25 & 5491 & 142402 & 1 & 3335.8 & 1 & 3335.8 & 1 & 2837.6 \\
\hline 256 & 5507 & 142403 & 1 & 1675.9 & 1 & 1675.9 & 1 & 1425.6 \\
\hline 257 & 5510 & 142403 & 1 & 1675.9 & 1 & 1675.9 & 1 & 1425.6 \\
\hline 258 & 5514 & 142404 & 1 & 3351.8 & 1 & 1675.9 & 1 & 1425.6 \\
\hline 259 & 5515 & 142404 & & 3351.8 & 1 & 1675.9 & 1 & 1425.6 \\
\hline 260 & 5537 & 143401 & $i$ & 330.9 & 1 & 396.8 & 1 & 330.9 \\
\hline 261 & 5538 & 143401 & 1 & 330.9 & 1 & 396.8 & 1 & 330.9 \\
\hline 26 & 5551 & 143402 & 1 & 722.0 & . & 0.0 & 1 & 722.0 \\
\hline 263 & 5552 & 143402 & $i$ & 722.0 & & 0.0 & l & 722.0 \\
\hline 264 & 5566 & 143403 & 1 & 734.4 & 1 & 880.6 & 1 & 734.4 \\
\hline 265 & 5570 & 143403 & 1 & 734.4 & $i$ & 880.6 & 1 & 734.4 \\
\hline 266 & 5579 & 143404 & 1 & 648.0 & 1 & 777.0 & 1 & 648.0 \\
\hline 267 & 5586 & 143404 & $i$ & 648.0 & 1 & 777.0 & $i$ & 648.0 \\
\hline 268 & 5589 & 144401 & • & 1656.5 & 1 & 931.6 & 1 & 828.3 \\
\hline 2 & 5596 & 144401 & 1 & 1656.5 & 1 & 931.6 & 1 & 828.3 \\
\hline 270 & 5609 & 144402 & 1 & 600.5 & 1 & 675.5 & 1 & 600.5 \\
\hline 271 & 5615 & 144402 & 1 & 600.5 & 1 & 675.5 & 1 & 600.5 \\
\hline 272 & 5625 & 144403 & 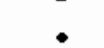 & 1780.4 & 1 & 1001.3 & 1 & 890.2 \\
\hline 273 & 5627 & 144403 & 1 & 1780.4 & 1 & 100 & 1 & 890.2 \\
\hline 274 & 5645 & 144404 & 1 & 1469.6 & 1 & 165 & 1 & 1469.6 \\
\hline 275 & 5656 & 145401 & 1 & 140 & 1 & 1633.3 & 1 & 1399.4 \\
\hline 276 & 5657 & 145401 & 1 & 1407.7 & 1 & 1633.3 & 1 & 1399.4 \\
\hline 277 & 5668 & 145402 & 1 & 1330.8 & 2 & 0.0 & 1 & 1322.9 \\
\hline 278 & 5691 & 145403 & $i$ & 405.7 & $i$ & 470.7 & 1 & 403.3 \\
\hline 279 & 5694 & 145404 & • & 0.0 & $i$ & 814.7 & $i$ & 698.0 \\
\hline 280 & 5717 & 146401 & 1 & 485.6 & $i$ & 242.8 & 1 & 209.0 \\
\hline 28 & 5721 & 146401 & • & 485.6 & 1 & 242.8 & 1 & 209.0 \\
\hline & 5723 & 146402 & 1 & 906.0 & & 1812.0 & 1 & 779.7 \\
\hline
\end{tabular}


TABLE 4.1. (contd)

\begin{tabular}{|c|c|c|c|c|c|c|c|c|}
\hline$O B S$ & PNRES ID & MRRCODE & ROSM & ROSMWT & ROST & ROSTWT & RESAMPLE & RESAMWT \\
\hline 283 & 5729 & 146402 & 1 & 906.0 & 1 & 1812.0 & 1 & 779.7 \\
\hline 284 & 5744 & 146403 & 1 & 529.5 & $i$ & 529.5 & 1 & 455.7 \\
\hline 285 & 5745 & 146403 & 1 & 529.5 & 1 & 529.5 & 1 & 455.7 \\
\hline 286 & 5758 & 146404 & 1 & 1455.6 & 1 & 1455.6 & 1 & 1252.6 \\
\hline 287 & 5782 & 147502 & 1 & 8080.6 & 1 & 7631.6 & 1 & 6541.4 \\
\hline 288 & 5820 & 147504 & $i$ & 2497.8 & $i$ & 2359.0 & 1 & 2022.0 \\
\hline 289 & 5888 & 148501 & 1 & 3519.9 & 1 & 2853.5 & 1 & 2634.5 \\
\hline 290 & 5906 & 148502 & 1 & 5044.1 & 1 & 4089.1 & 1 & 3775.2 \\
\hline 291 & 5931 & 148503 & 1 & 1142.5 & 1 & 926.2 & 1 & 855.1 \\
\hline 292 & 5943 & 148504 & - & 0.0 & 1 & 3902.4 & 1 & 3602.8 \\
\hline 293 & 5970 & 148505 & 1 & 2654.1 & 1 & 2151.6 & $i$ & 1986.5 \\
\hline 294 & 6000 & 149501 & 1 & 1790.0 & - & 0.0 & 1 & 1474.9 \\
\hline 295 & 6010 & 149502 & 1 & 2410.8 & $i$ & 1986.3 & $i$ & 1986.4 \\
\hline 29 & 6046 & 149504 & 1 & 3290.8 & 1 & 2711.3 & 1 & 2711.4 \\
\hline 297 & 6053 & 149505 & 1 & 3206.4 & 1 & 2641.8 & $i$ & 2641.9 \\
\hline 29 & 6098 & 149508 & 1 & 2712.2 & 1 & 2234.6 & 1 & 2234.7 \\
\hline 29 & 6118 & 150501 & 1 & 1632.5 & 1 & 1694.6 & $i$ & 1650.3 \\
\hline 30 & 6125 & 150502 & 1 & 1542.3 & 1 & 1600.9 & $i$ & 1564.8 \\
\hline 301 & 6151 & 150503 & 1 & 1361.8 & 1 & 1413.6 & 1 & 1381.6 \\
\hline 30 & 6172 & 150505 & 1 & 1569.6 & 1 & 1629.3 & 1 & 1592.5 \\
\hline 30 & 6183 & 150506 & - & 0.0 & 1 & 475.1 & 1 & 464.3 \\
\hline 30 & 6208 & 150507 & 1 & 745.4 & . & 0.0 & 1 & 756.3 \\
\hline 30 & 6223 & 150508 & 1 & 1004.7 & $i$ & 1042.9 & 1 & 1019.3 \\
\hline 306 & 6239 & 151501 & 1 & 1082.5 & 1 & 1082.5 & 1 & 996.2 \\
\hline 30 & 6243 & 151502 & 1 & 1377.6 & 1 & 1377.6 & $i$ & 1267.8 \\
\hline 308 & 6296 & 1515 & 1 & 2059.9 & 1 & 2059.9 & 1 & 1895.7 \\
\hline & 6303 & 151506 & 1 & 1471.4 & 1 & 1471.4 & 1 & 1354.1 \\
\hline 310 & 6317 & 151507 & 1 & 1267.6 & 1 & 1267.6 & 1 & 1166.6 \\
\hline & 6345 & 152601 & 1 & 20347.4 & 1 & 16003.3 & 1 & 14801.0 \\
\hline 31 & 6362 & 152602 & 1 & 34313.2 & . & 0.0 & I & 24959.8 \\
\hline 3. & 6379 & 152603 & 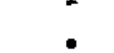 & 26047.2 & $i$ & 10243.1 & $i$ & 947 \\
\hline 31 & 6380 & 152603 & $i$ & 26047.2 & 1 & 10243.1 & $i$ & 9473.5 \\
\hline 31 & 6397 & 1526 & 1 & 18950.9 & 1 & 14904.9 & 1 & 13785.1 \\
\hline 31 & 6403 & 152604 & 1 & 18950.9 & 1 & 14904.9 & 1 & 13785.1 \\
\hline & 6407 & 1526 & 1 & 7215.0 & 1 & 5674.6 & 1 & 5248.3 \\
\hline & 6418 & 152605 & 1 & 7215.0 & 1 & 5674.6 & 1 & 5248.3 \\
\hline 31 & 6422 & 152606 & 1 & 12227.0 & 1 & 9616.6 & 1 & 8894.1 \\
\hline & 6428 & 1526 & 1 & 12227.0 & 1 & 9616.6 & 1 & 8894.1 \\
\hline & 6437 & 152607 & 1 & 17215.6 & • & 27080.3 & 1 & 12522.9 \\
\hline 32 & 6446 & 152607 & 1 & 17215.6 & 1 & 27080.3 & 1 & 12522.9 \\
\hline 32 & 6457 & 1526 & $\bullet$ & 23669.0 & $i$ & 9307.9 & 1 & 8608.6 \\
\hline 3? & 6460 & 152608 & $\mathrm{i}$ & 23669.0 & 1 & 9307.9 & 1 & 8608.6 \\
\hline 325 & 6465 & 152609 & 1 & 17170.0 & 1 & 27008.4 & 1 & 12489.7 \\
\hline 32 & 6472 & 152609 & 1 & 17170.0 & & 27008.4 & 1 & 12489.7 \\
\hline 32 & 6492 & 152610 & • & 0.0 & 1 & 32036.6 & 1 & 29629.7 \\
\hline 32 & 6506 & 152611 & . & 0.0 & 1 & 28229.6 & 1 & 26108.7 \\
\hline 329 & 6513 & 152612 & & 0.0 & 1 & 26290.1 & 1 & 24315.0 \\
\hline
\end{tabular}


TABLE 4.1. (contd)

\begin{tabular}{|c|c|c|c|c|c|c|c|c|}
\hline OBS & PNRES ID & MRRCODE & ROSM & ROSMWT & ROST & ROSTWT & RESAMPLE & RESAMWT \\
\hline 330 & 6525 & 152613 & 1 & 13297.6 & 1 & 20917.2 & 1 & 9672.8 \\
\hline 331 & 6527 & 152613 & $i$ & 13297.6 & $\bullet$ & 20917.2 & 1 & 9672.8 \\
\hline 332 & 6560 & 152615 & 1 & 32970.0 & 1 & 25931.0 & 1 & 23982.8 \\
\hline 333 & 6576 & 152616 & 1 & 18585.6 & 1 & 14617.6 & 1 & 13519.4 \\
\hline 334 & 6583 & 152616 & 1 & 18585.6 & 1 & 14617.6 & 1 & 13519.4 \\
\hline 335 & 6592 & 153601 & 1 & 4627.7 & 1 & 5391.5 & 1 & 4627.7 \\
\hline 336 & 6596 & 153601 & $i$ & 4627.7 & $i$ & 5391.5 & $i$ & 4627.7 \\
\hline 337 & 6603 & 153602 & 1 & 10589.2 & 1 & 12337.0 & 1 & 10589.2 \\
\hline 338 & 6621 & 153603 & 1 & 9185.8 & . & 0.0 & 1 & 9185.8 \\
\hline 339 & 6636 & 153604 & & 9411.7 & 1 & 5482.5 & 1 & 4705.8 \\
\hline 340 & 6642 & 153604 & 1 & 9411.7 & 1 & 5482.5 & 1 & 4705.8 \\
\hline 341 & 6646 & 153605 & 1 & 8499.0 & 1 & 9901.8 & 1 & 8499.0 \\
\hline 342 & 6660 & 153606 & $i$ & 4048.5 & 1 & 9433.4 & l & 4048.5 \\
\hline 343 & 6667 & 153606 & 1 & 4048.5 & - & 9433.4 & 1 & 4048.5 \\
\hline 344 & 6673 & 154601 & - & 3628.1 & 1 & 2112.9 & 1 & 1814.1 \\
\hline 345 & 6681 & 154601 & $i$ & 3628.1 & 1 & 2112.9 & 1 & 1814.1 \\
\hline 346 & 6718 & 154604 & 1 & 970.8 & 1 & 1130.7 & 1 & 970.8 \\
\hline 347 & 6721 & 154604 & 1 & 970.8 & 1 & 1130.7 & 1 & 970.8 \\
\hline 348 & 6751 & 154606 & & 4251.7 & 1 & 4952.1 & 1 & 2125.9 \\
\hline 349 & 6755 & 154606 & 1 & 4251.7 & & 4952.1 & 1 & 2125.9 \\
\hline 350 & 6771 & 155601 & . & 8538.3 & $i$ & 3571.4 & 1 & 3571.4 \\
\hline 351 & 6775 & 155601 & 1 & 8538.3 & 1 & 3571.4 & 1 & 3571.4 \\
\hline 352 & 6791 & 155602 & 1 & 10966.4 & $i$ & 4587.0 & $i$ & 4587.0 \\
\hline 353 & 6792 & 155602 & 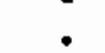 & 10966.4 & $i$ & 4587.0 & 1 & 4587.0 \\
\hline 354 & 6823 & 155605 & . & 0.0 & 1 & 8017.3 & 1 & 8017.3 \\
\hline 355 & 6838 & 155606 & 1 & 3850.4 & 1 & 3221.1 & 1 & 3221.1 \\
\hline & 6843 & 155606 & 1 & 3850.4 & 1 & 3221.1 & 1 & 3221.1 \\
\hline 357 & 6869 & 156602 & 1 & 2761.9 & & 4686.6 & 1 & 2343.3 \\
\hline 358 & 6880 & 1566 & 1 & 2761.9 & 1 & 4686 & 1 & 2343.3 \\
\hline 359 & 6883 & 156603 & 1 & 4874.0 & 1 & 4135.3 & 1 & 4135.3 \\
\hline 360 & 6913 & 1566 & 1 & 3847.0 & 1 & & 1 & 3263.9 \\
\hline 361 & 6931 & 156606 & 1 & 9312.8 & 1 & 3950.7 & 1 & 3950.7 \\
\hline 362 & 6933 & $156 t$ & & 931 & 1 & & 1 & 3950.7 \\
\hline 36 & 6950 & 157601 & 1 & 2943.0 & 1 & 2943.0 & 1 & 2943.0 \\
\hline 364 & 6952 & 157601 & 1 & 2943.0 & 1 & 2943.0 & 1 & 2943.0 \\
\hline 365 & 6965 & 1576 & 1 & 3399.1 & 1 & & 1 & 3399.1 \\
\hline 366 & 6967 & 1576 & 1 & 3399.1 & 1 & & 1 & 3399.1 \\
\hline 367 & 6985 & 157603 & 1 & 2345 & 1 & 2349.5 & 1 & 2349.5 \\
\hline & 6986 & & 1 & & 1 & & 1 & 2349.5 \\
\hline 36 & 6988 & 15760 & $i$ & 681 & $i$ & & 1 & 3405.1 \\
\hline 370 & 6990 & 1576 & 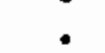 & 6810.3 & 1 & 3405.1 & 1 & 3405.1 \\
\hline 371 & 7006 & 158601 & 1 & 5199.7 & 1 & 5199.7 & 1 & 5199.7 \\
\hline 372 & 7018 & 15860 & 1 & 1626.6 & 1 & & 1 & 1626.6 \\
\hline 373 & 7020 & 158602 & 1 & 1626.6 & 1 & & 1 & 1626.6 \\
\hline 374 & 7049 & 158604 & 1 & 7828.1 & 1 & & 1 & 7828.1 \\
\hline 375 & 7067 & 159601 & 1 & 1130.0 & 1 & 1126.7 & 1 & 978.3 \\
\hline 376 & 7075 & 159601 & 1 & 1130.0 & 1 & 1126.7 & 1 & 978.3 \\
\hline
\end{tabular}


TABLE 4.1. (contd)

\begin{tabular}{|c|c|c|c|c|c|c|c|c|}
\hline OBS & PNRES ID & MRRCODE & ROSM & ROSMWT & ROST & ROSTWT & RESAMPLE & RESAMWT \\
\hline 377 & 7087 & 159602 & 1 & 1449.2 & 1 & 1445.D & 1 & 1254.6 \\
\hline 378 & 7091 & 159602 & 1 & 1449.2 & 1 & 1445.0 & 1 & 1254.6 \\
\hline 379 & 7094 & 159603 & 1 & 2293.84 & . & 0.00 & 1 & 1985.80 \\
\hline 380 & 7118 & 159604 & 1 & 2326.53 & 1 & 2319.81 & 1 & 2014.10 \\
\hline 381 & 7130 & 160601 & - & 0.00 & 1 & 3122.70 & 1 & 2656.53 \\
\hline 382 & 7143 & 160602 & & 2840.86 & 1 & 1159. & 1 & 986.61 \\
\hline 383 & 7151 & 160602 & 1 & 2840.86 & 1 & 1159.73 & 1 & 986.61 \\
\hline 384 & 7171 & 160604 & 1 & 778.76 & 1 & 635 & 1 & 540.91 \\
\hline 385 & 7175 & 160604 & 1 & 778.76 & 1 & 635.83 & 1 & 540.91 \\
\hline 386 & 8238 & 148507 & & D. 00 & 1 & 2846.14 & 1 & 2627.70 \\
\hline 387 & 8245 & i48508 & 1 & 3021.34 & $i$ & 2449.30 & 1 & 2261.31 \\
\hline
\end{tabular}




\section{REFERENCES}

Bronfman, B. H. and D. I. Lerman. 1985. Energy Efficiency in Northwest Homes Survey Summary Report. Sponsored by the Office of Conservation, Bonneville Power Administration, Portland, Oregon. Under Contract No. DE-AC79-83BP11542 with Evaluation Research Corporation, Portland, Oregon.

Columbia Research Center. 1985. Residential 0ccupant Survey--Telephone, Final Report. Submitted to Pacific Northwest Laboratories, Richland, Washington.

Darwin, R. F., D. L. Ivey, M. S. Klan, and B. L. Mohler. 1986. The Residential Occupant Survey--Telephone: Sumary Report. D0E/BP $1 \overline{3795-15}$, Bonneville Power Administration, Portland, Oregon.

Louis Harris and Associates, Inc. 1984. Pacific Northwest Residential Energy Consumption Survey: Construction of Multicomponent Case Weights for Regional Sample Data, Final Report. Submitted to the Bonneville Power Administration, Portland, Oregon, under Contract No. DE-AC79-83BP39950.

Louis Harris and Associates, Inc. 1984. Pacific Northwest Residential Energy Consumption Survey (1983): Field and Data Reduction Activities Final Report. Submitted to the Bonneville Power Administration, Portland, Oregon, pursuant to Contract No. DE-AC79-83BP39950.

Louis Harris and Associates, 1nc. 1984. Pacific Northwest Residential Energy Consumption Survey (1983): Fieldwork and Data Reduction Activities Report, Appendices. Submitted to the Bonneville Power Administration, Portland, Oregon, under Contract No. DE-AC79-83BP39950.

Louis Harris and Associates, Inc. 1983. Pacific Northwest Residential Energy Consumption Survey: Interviewer Debriefing. Submitted to the Bonneville Power Administration, Portland, Oregon, under Contract No. DE-AC79-83BP39950.

Louis Harris and Associates, Inc. 1984. Pacific Northwest Residential Energy Consumption Survey: Response Analys is Final Report. Submitted to the Bonneville Power Administration, Portland, Oregon, under Contract No. DE-AC79-83BP39950.

Louis Harris and Associates, Inc. 1983. Pacific Northwest Residential Energy Consumption Survey: Sample Selection Activities. Submitted to the Bonneville Power Administration, Portland, Oregon, under Contract No. DE-AC79-83BP39950. 
.

, 
APPENDIX A

OVERALL RESAMPLE SPREADSHEET CONTENTS 


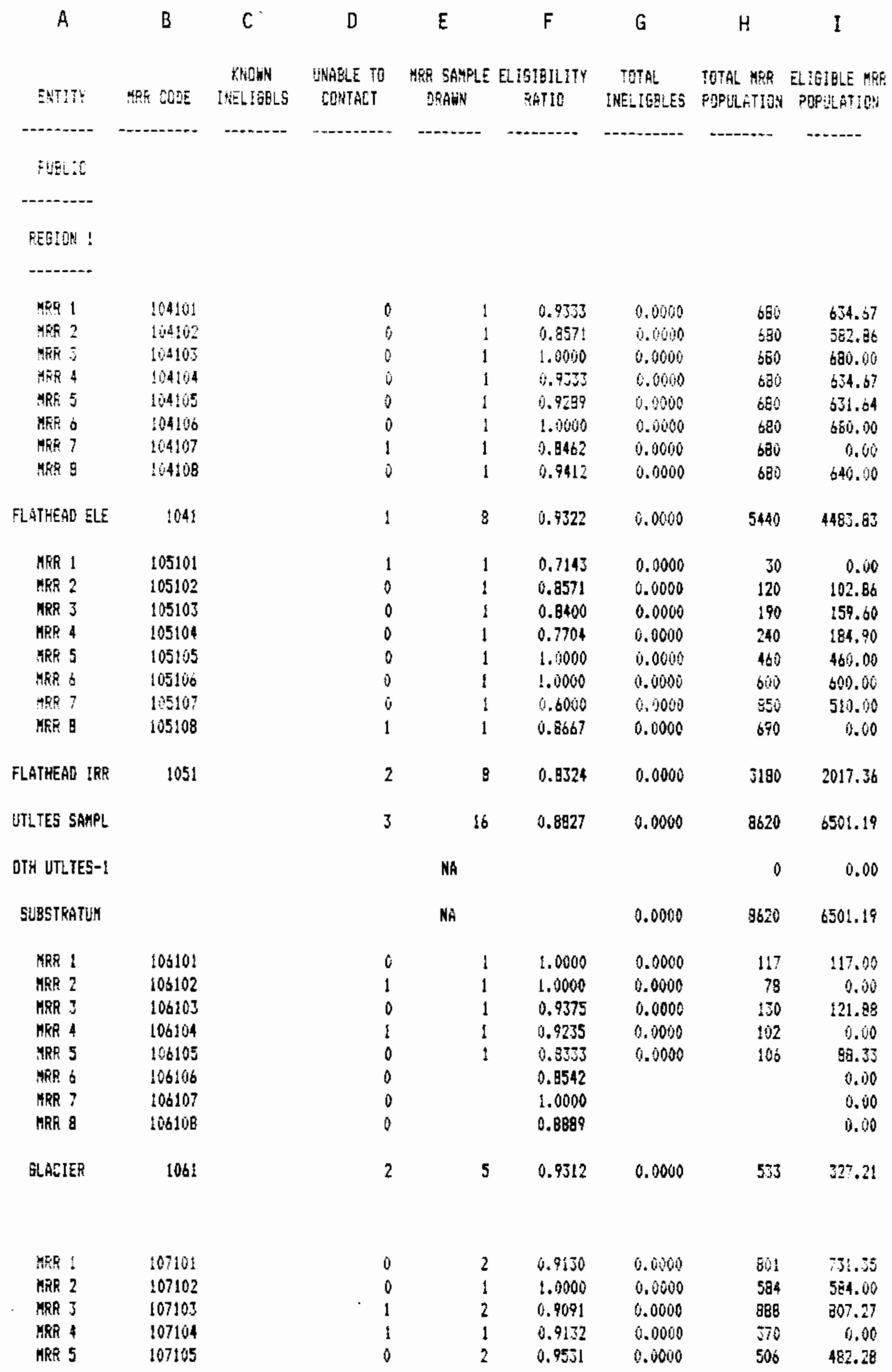

A. 1 


\begin{tabular}{|c|c|c|c|c|c|c|c|c|}
\hline ENTITY & WEF REE & $\begin{array}{c}\text { WMEWN } \\
\text { INEL:GELS }\end{array}$ & $\begin{array}{l}\text { SHABLE TO } \\
\text { CANTACT }\end{array}$ & $\begin{array}{c}\text { MRR FAMPLE } \\
\text { DRA }\end{array}$ & $\begin{array}{c}\text { Etigibiliti } \\
\text { RATIO }\end{array}$ & $\begin{array}{c}\text { TETAL } \\
\text { INELIEBLES }\end{array}$ & $\begin{array}{l}\text { TOTAL MGR } \\
\text { POPULATION }\end{array}$ & $\begin{array}{l}\text { ELIEISLE ME } \\
\text { POFELATIOH }\end{array}$ \\
\hline$\cdots+--n$ & $++\infty+\ldots+n$ & $\cdots$ & - & -------- & ------ & - & - & n........ \\
\hline nistits & n! & & 5 & $\mathrm{E}$ & 0.9578 & 9. boing & 319 & 5004,90 \\
\hline LTLTES SAML & & & 4 & 13 & 0.9347 & 0.0000 & 3652 & 2032.11 \\
\hline OTH WTLTES-? & & & & $\mathrm{KA}$ & & & & \\
\hline SUBSTYATUM & & & & $\mathrm{NA}$ & & 0.0000 & 3682 & 2032.11 \\
\hline STFATIM TOT & & & & $\mathrm{HA}$ & & 5.0000 & $12 \div / 2$ & 947.30 \\
\hline \multicolumn{9}{|l|}{ YESION 2} \\
\hline --------- & & & & & & & & \\
\hline 䩗 1 & 112201 & & 1 & 1 & 1. 1000 & 0.5000 & 148 & 0.60 \\
\hline Xen 2 & 112202 & & 0 & 1 & 1.0000 & 0.0000 & 256 & 256.10 \\
\hline x & 112205 & & $!$ & 1 & 0.8657 & 0.0000 & 276 & 0.00 \\
\hline $\mathrm{MEF} 4$ & 112204 & & 0 & 1 & 0.9333 & 6.9000 & 197 & {$[74.53$} \\
\hline ARR 5 & 112205 & & 0 & 1 & 0.9353 & 0.0000 & 175 & 163.33 \\
\hline MRR 6 & 112206 & & 0 & 1 & 1.0000 & 0.0000 & 84 & 84.00 \\
\hline TEAHO FALLS & 1122 & & 2 & $t$ & 0.9551 & 0.0000 & 1126 & 677.87 \\
\hline FE I & 113201 & & j & 1 & 0.7692 & j. & 130 & 200.50 \\
\hline$\triangle P R=$ & 113202 & & 0 & 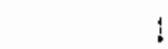 & $0.02 E 6$ & 6.0000 & $1: 0$ & 102,14 \\
\hline HKR 3 & 113203 & & 0 & 1 & 0.8571 & 0.0000 & 125 & 107.14 \\
\hline $\mathrm{NPS} 4$ & $\$ 13264$ & & 1 & 1 & 0.9289 & 0.6000 & 94 & 0.00 \\
\hline $\operatorname{MER} 5$ & 113205 & & 1 & 1 & 0.9231 & 0.0000. & 364 & 0.00 \\
\hline MRR 6 & 113206 & & 1 & 1 & 0.9333 & 0.6000 & 240 & 0.00 \\
\hline LONER YALLEY & 1132 & & 3 & 6 & 0.8921 & 0.0000 & 1083 & 309.29 \\
\hline JJLTES SAMFL & & & 5 & 12 & 0.9245 & 0.6000 & 2209 & 987,15 \\
\hline QTH נTLTES-J & & & 0 & $\mathrm{NA}$ & & & & \\
\hline SUBSTRATUN & & & & $\mathrm{NA}$ & & 0.0000 & 2209 & 987.15 \\
\hline 积F 1 & $\$ 14201$ & & 0 & 1 & 0.7145 & 0.0000 & 351 & 250.71 \\
\hline MER 2 & 114202 & & 0 & & & & & \\
\hline MRK 3 & $1: 4203$ & & 0 & $!$ & 1.0000 & 0.0000 & 739 & 739.60 \\
\hline MKR 4 & 114204 & & 1 & : & 0.3571 & 0.0000 & $i ! B$ & 0.90 \\
\hline MKR 5 & 114205 & & 0 & I & 0.8672 & 0.0000 & 76 & $65.7 !$ \\
\hline $\mathrm{M} F R$ is & 114200 & & 1 & 1 & $0.06 \dot{0} \overline{7}$ & 0.0060 & 399 & 0.00 \\
\hline FAL: RIVER & 1142 & & $?$ & 5 & 0.7279 & 4.6000 & 1573 & 1555.62 \\
\hline HRR ! & $! 15261$ & & 0 & $i$ & a. 9296 & 0.0000 & 174 & 103.21 \\
\hline EESTEND WUT & ! & & 3 & i & 0.9230 & 1.0600 & 179 & 160.21 \\
\hline MPR I & $: 16201$ & & 0 & 1 & 0.9333 & 0.0000 & 90 & 64.00 \\
\hline FARMERS ELE & 1162 & & 0 & 1 & 0.0353 & 1.0000 & 90 & 84.00 \\
\hline
\end{tabular}




\begin{tabular}{|c|c|c|c|c|c|c|c|c|}
\hline ENTITY & MAF CDDE & $\begin{array}{c}\text { KHOL } \\
\text { INELIGELS }\end{array}$ & $\begin{array}{l}\text { UNABLE TO } \\
\text { CONTACT }\end{array}$ & $\begin{array}{l}\text { ARR SAMPLE } \\
\text { ORAWH }\end{array}$ & $\begin{array}{c}\text { ELIGIEILITY } \\
\text { RATIO }\end{array}$ & $\begin{array}{c}\text { TDTAL } \\
\text { IHELIGELES }\end{array}$ & $\begin{array}{l}\text { TUTAL MKR } \\
\text { POPILATIOH }\end{array}$ & $\begin{array}{l}\text { ELIGIBLE YFA } \\
\text { POPULATIOH }\end{array}$ \\
\hline नीक & 11501 & - & $\overline{0}$ & 1 & is & 0.600 & 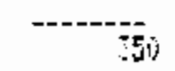 & 32.11 \\
\hline CIYEREIDE EL & 1152 & & 9 & 1 & 0.9299 & 0.1000 & 350 & 325.11 \\
\hline MFR ! & 119201 & & 0 & $!$ & 1.0000 & 0.0000 & 500 & 500.00 \\
\hline SOUTHS:DE EL & 1192 & & 0 & 1 & 1. 10000 & 0.0000 & 500 & 503.06 \\
\hline AFR 1 & 120201 & & 1 & 1 & 9.8578 & 0.0090 & 300 & 0.00 \\
\hline MRR 2 & $12020 \mathrm{z}$ & & $\hat{0}$ & 1 & i. 7245 & 0.0000 & $\$ 10$ & 224.59 \\
\hline BHITY LeF & 1202 & & 1 & 2 & 0.7934 & 0.0000 & 610 & 224.59 \\
\hline HINUSKA PFOJ & & & $b$ & 0.00 & 0.6971 & 2. 0000 & NA & HA \\
\hline WTLTES SAMPL & & & & $\sharp$ & 0.8095 & 2.0000 & 3402 & 2355.54 \\
\hline OTH UTLTES-4 & & & & NA & & & & \\
\hline SUBSTRATUH & & & & FA & & 2.0000 & 3402 & 2355.54 \\
\hline MRR I & $12120 !$ & & 1 & 2 & 0.9333 & 0.0000 & 232 & 216.53 \\
\hline HRR 2 & 121202 & & 1 & 1 & 0.8667 & 0.0000 & 233 & 0.00 \\
\hline MRR 3 & $\$ 21203$ & & 1 & 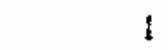 & 0.7867 & 0.0000 & 232 & 0.00 \\
\hline MRP 4 & 121204 & & 0 & 1 & 0.9333 & 0.0000 & 233 & 217,47 \\
\hline ARF 5 & 121205 & & 0 & 1 & 0.9533 & 0.10000 & 233 & $217.4 ?$ \\
\hline MES & 121266 & & 0 & 1 & U. 7867 & 0.0000 & 232 & 187.51 \\
\hline LOST RIVER & $12 ! 2$ & & 3 & 7 & 0.6733 & 0.0000 & $! 395$ & 833.97 \\
\hline MRR ! & 122201 & & 0 & & 0.8578 & & & 0.00 \\
\hline MRR 2 & 122202 & & 0 & 1 & 0.9289 & 0.0000 & 129 & 119.83 \\
\hline MRR 3 & 122203 & & 1 & 1 & 1.0000 & 0.0000 & 123 & 0.00 \\
\hline MRR 4 & 122204 & & 1 & 1 & 0.9333 & 0.0000 & 166 & 0.00 \\
\hline WRR 5 & 122205 & & 1 & t & 1.0000 & 0.0000 & 104 & 0.00 \\
\hline akR 6 & 122206 & & $t$ & 1 & 0.9286 & 0.0000 & 169 & 0.00 \\
\hline WEISER & 1222 & & 4 & 5 & 0.9416 & 0.0000 & 691 & $119.8 \mathrm{~J}$ \\
\hline UTLTES SAKPZ & & & & 12 & 0.9073 & 0.0000 & 2096 & 950.80 \\
\hline GTH UTLTES-4 & & & & MA & & & & \\
\hline SJASTRATUH & & & & MA & & 0.0000 & 2006 & 953.90 \\
\hline STRATUA TDT & & & & NA & & 2.0000 & 7697 & 1296.49 \\
\hline \multicolumn{9}{|l|}{ REGIGN 3} \\
\hline \multicolumn{9}{|l|}{-..-- } \\
\hline MER : & 123301 & & 1 & 1 & 0.9344 & $\dot{0}, \dot{0} 00 \dot{0}$ & 92 & 0.00 \\
\hline SIRR 2 & $\{23302$ & & 1 & 1 & 0.9231 & 0.0000 & 242 & 0.00 \\
\hline MRe 3 & 125393 & & 1 & 1 & 0.7333 & 1.0000 & 819 & 9.00 \\
\hline RRR 4 & 123304 & & 0 & 1 & 0.9244 & 0.0000 & 628 & 590.55 \\
\hline MRR 5 & 123305 & & 1 & $i$ & 0.7704 & 0.0000 & 675 & 0.00 \\
\hline
\end{tabular}

A. 3 


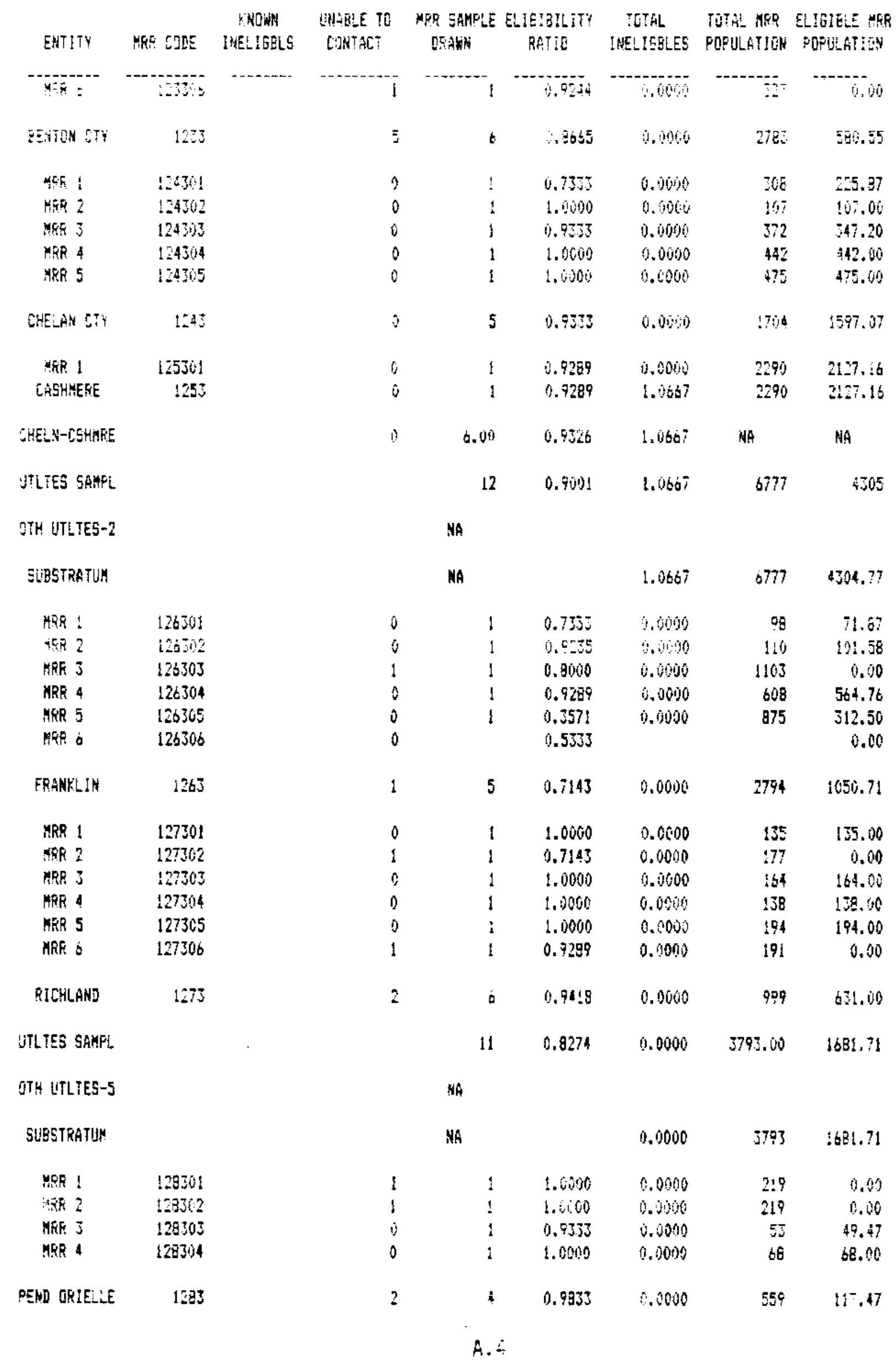




\begin{tabular}{|c|c|c|c|c|c|c|c|c|}
\hline ENIITY & MFR CODE & $\begin{array}{c}\text { KWOWN } \\
\text { IHEL:'GBLS }\end{array}$ & $\begin{array}{l}\text { WNAELE TO } \\
\text { CONTACT }\end{array}$ & $\begin{array}{l}\text { MRR SAMPLE } \\
\text { DRGAN }\end{array}$ & $\begin{array}{l}\text { ELIGIBILITY } \\
\text { RATIL }\end{array}$ & $\begin{array}{c}\text { TOTAL } \\
\text { IHELI I }\end{array}$ & $\begin{array}{l}\text { TOTAL MFR } \\
\text { PEPULATION }\end{array}$ & $\begin{array}{l}\text { ELIGIELE MEF } \\
\text { POP ILLTIES }\end{array}$ \\
\hline ----n-... & - & $\cdots+\cdots$ & --1-n-n- & $---\cdots$ & ----n- & --1-nen & $---+\cdots$ & $\cdots$ \\
\hline ME 1 & 150301 & & i & 1 & S. $560 \%$ & G.0000 & إ! & 248.53 \\
\hline ast? & 130302 & & 0 & i & 1).9259 & 0.9000 & 286 & 249.54 \\
\hline $\mathrm{MES} 3$ & 130303 & & 0 & $i$ & $\therefore .000$ & 0.0000 & 289 & 257.617 \\
\hline MFE 4 & 130404 & & $\hat{0}$ & $\mathrm{f}$ & 1.0000 & 0.10000 & 292 & 202.06 \\
\hline WERA IRR & 1303 & & 0 & 4 & 0.9535 & 0.0000 & 329 & 1175.48 \\
\hline ITLTES SAMPL & & & & 8 & 0.9677 & 0.0000 & SEE & $: 242.94$ \\
\hline STH UTLTES-3 & & & & $N A$ & & & & \\
\hline SUBSTRATUM & & & & NA & & 0.0000 & $B 88$ & 1242.94 \\
\hline MFE 1 & 131301 & & 0 & 1 & 0.5000 & 0.0000 & 404 & 242.40 \\
\hline HRP 2 & 131302 & & 0 & i & 0.6805 & 0.0000 & 404 & 274.71 \\
\hline MRR 3 & 131302 & & 0 & & 0.3889 & & & 0.00 \\
\hline MRE 4 & 131304 & & 0 & 1 & 0.9111 & 0.0000 & 404 & 368.09 \\
\hline FERRY CTY & 1313 & & D) & 3 & 0.7669 & 0.0000 & 1212 & 885.40 \\
\hline MKR ! & 132301 & & 0 & 1 & 1.0000 & 0.0000 & 267 & 267.00 \\
\hline MRR 2 & 132302 & & 0 & 1 & 0.7333 & 0.6000 & 268 & 195.53 \\
\hline WESPEEE & 1323 & & 1) & 2 & i. 8567 & 0.0000 & 55 & $46: 53$ \\
\hline MRR 1 & 133301 & & 0 & 1 & 0.9333 & 0.0000 & 93 & 86.20 \\
\hline MRR 2 & 133302 & & 0 & 1 & 1.0000 & 0.0000 & 56 & 56.50 \\
\hline COGLEE DAH & 1333 & & 0 & 2 & 0.9655 & 0.0000 & 149 & 142.80 \\
\hline NSPA.H-COELEE & & & 4 & 4.00 & 0.9153 & 0.0000 & & \\
\hline UTLTES SAIPL & & & & 7 & 0.5437 & 0.0000 & 1896 & 1491.73 \\
\hline DTH UTLTES-4 & & & & HA & & & & \\
\hline SUASTRATUM & . & & & NA & & 0.0000 & 1896 & 2491.73 \\
\hline STRATUH TOT & & & & NA & & 1.0667 & 13354 & 8721.16 \\
\hline \multicolumn{9}{|l|}{ RESION 4} \\
\hline 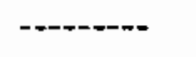 & & & & & & & & \\
\hline SRR 1 & 135401 & & 2 & 2 & 1.0000 & 0.0000 & 399 & 0.00 \\
\hline MRR 2 & 135402 & & 1 & 2 & 1.0000 & 0.0000 & 405 & $40 J .00$ \\
\hline MER j & 135403 & & 0 & $\hat{2}$ & 1.0000 & 0.6000 & 317 & 317,0 \\
\hline MKR 4 & 135404 & & 0 & 2 & 1.0000 & 0.0000 & 398 & 393.60 \\
\hline MAR 5 & 155405 & & 1 & 2 & 0.8667 & 0.0000 & 378 & 327.60 \\
\hline MRR 6 & 135406 & & 0 & 2 & 1.0000 & 0.0000 & 440 & 440.00 \\
\hline
\end{tabular}

A. 5 


\begin{tabular}{|c|c|c|c|c|c|c|c|c|}
\hline EHT? & WRE SJIE & $\begin{array}{c}\text { KuIWW } \\
\text { MELISES } 5\end{array}$ & $\begin{array}{l}\text { WNAELE TO } \\
\text { CLNTACT }\end{array}$ & $\begin{array}{c}\text { RFE GAMFLE } \\
\text { ORALN }\end{array}$ & $\begin{array}{c}\text { ELteIHILITY } \\
\text { FATIO }\end{array}$ & $\begin{array}{l}\text { TCTAL } \\
\text { INEL IGELEE }\end{array}$ & $\begin{array}{l}\text { TOTAL KRE } \\
\text { POTELLATIOH }\end{array}$ & $\begin{array}{l}\text { ELIGIBLE AER } \\
\text { PCFELATION }\end{array}$ \\
\hline ETTE & 25 & & $\cdots \cdot$ & 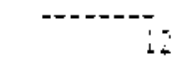 & $\theta_{2}=7$ & a 10 & $2=5$ & $19 \mathrm{E}$ \\
\hline Wif ! & $50+i$ & & $!$ & ? & . onot & 20000 & 89 & $8 \overline{0} .00$ \\
\hline Mes: & $135 \mathrm{~d} 12$ & & ! & 2 & 0.9333 & 0.0800 & $22:$ & 208.13 \\
\hline Nat 3 & $13 b 403$ & & 1 & 2 & 0.538 & $\therefore 0000$ & 467 & $: 47.70$ \\
\hline * 4 & 136404 & & $\therefore$ & 2 & 1.0000 & 19.0000 & 239 & 259.67 \\
\hline HR 5 & 130415 & & 1 & 2 & 1.0000 & 0.0000 & 184 & 184.60 \\
\hline MRR 6 & 136406 & & 1 & 2 & 9.9786 & (1). (1)000 & 243 & 225.54 \\
\hline Stomin:SH & 1364 & & 5 & 12 & 0.9647 & 0.0000 & 1245 & 1194.98 \\
\hline $\begin{array}{l}\text { WTLTES SAAFL } \\
\text { QTH UTLTES- }\end{array}$ & & & & 24 & 0.9711 & 0.1600 & 3580 & 3080.58 \\
\hline SUESTRATLA & & & & NA & & 0.0000 & 3590 & 3090.58 \\
\hline MRR ! & 137401 & & 0 & 2 & 1.0000 & 0.0000 & 255 & 259.60 \\
\hline WR 2 & 137402 & & 0 & 2 & 0.0053 & 0.0000 & 850) & 344.02 \\
\hline ARR 3 & 137403 & & 0 & 2 & 0.9244 & 0.0000 & 394 & 304.23 \\
\hline GRR 4 & 137404 & & 0 & 1 & 0.6875 & 0.5000 & 224 & 154.60 \\
\hline 5 & 137405 & & 1 & 2 & 0.9133 & 0.0000 & 559 & 510.52 \\
\hline$M R R$ & $\$ 37406$ & & 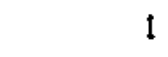 & 1 & 0.9231 & 0.0000 & 364 & 0.00 \\
\hline CLARE CTY & 1374 & & 2 & 10 & 0.9994 & 0.6000 & 2179 & 860.77 \\
\hline XFt 1 & 13001 & & 6 & 2 & 0.7033 & 5.1000 & 134 & $17 ! .73$ \\
\hline FRP 2 & $13840 ?$ & & 0 & 2 & 0.9239 & 0.0000 & 148 & 137.48 \\
\hline HKA 3 & 158403 & & 0 & ! & 0.9289 & 0.0000 & 147 & 136.55 \\
\hline MER A & 138404 & & 0 & 2 & 1.0000 & 0.0000 & 157 & 157.00 \\
\hline MRR 5 & 138405 & & 0 & 1 & 0.9333 & 0.0000 & 136 & 126.93 \\
\hline MRE \& & 138406 & & 1 & 2 & 0.351 & 0.0000 & $15 i$ & 134.57 \\
\hline TACOMA & 1584 & & 1 & 10 & 0.9295 & 0.0000 & 929 & 204.26 \\
\hline !HLIES SAMEL & & & & 20 & 0.7144 & 0.0000 & 3108 & 2495.03 \\
\hline UTH UTLTES-2 & & & & $\mathrm{kA}$ & & & & \\
\hline SUBSTRATIH & & & & NA & & 0.0000 & 3108 & 2495.03 \\
\hline MER I & 139401 & & 2 & 2 & 0.9789 & 0.0060 & 418 & 0.00 \\
\hline MRR 2 & 154402 & & 0 & 2 & 1.0000 & 0.9000 & 363 & 363.00 \\
\hline$M P R 3$ & 139403 & & 0 & 2 & 0.8667 & 0.0000 & 252 & 218.10 \\
\hline MRF 4 & 139404 & & $i$ & 2 & 1.0000 & 0.6000 & $5 j 2$ & 532.10 \\
\hline KaR 5 & 139405 & & 0 & 2 & 0.5060 & 0.0600 & 220 & $176 .: 0$ \\
\hline MPF $\dot{a}$ & 139406 & & 0 & 2 & 0.7551 & 0.0000 & 398 & 300.53 \\
\hline HASON CTY-3 & 1394 & & 3 & 12 & ن. $\varepsilon ? 21$ & 0.0000 & 2183 & 1589.95 \\
\hline Marin! & $140+41$ & & $\Xi$ & 2 & $(.0000)$ & 4.0600 & $\$ 19$ & $i 1.0 \mathrm{u}$ \\
\hline ARR 2 & 140402 & & 0 & 2 & 1.7530 & 0.0000 & 903 & 662.20 \\
\hline MRE 3 & 140403 & & i & 2 & 0.8499 & 0.01000 & 548 & 465.19 \\
\hline MRR 4 & 140404 & & $i$ & 2 & 5.8000 & 0.0000 & 339 & 271.20 \\
\hline MSR 5 & $1+13405$ & & $!$ & 2 & 0.9533 & 0.1000 & $2 \mathrm{eq}$ & 269.75 \\
\hline
\end{tabular}

A. 6 


\begin{tabular}{|c|c|c|c|c|c|c|c|c|}
\hline EHTITY & YAR CDDE & $\begin{array}{c}\text { XNDWN } \\
\text { IHELIGBLS }\end{array}$ & $\begin{array}{l}\text { UHABLE i] } \\
\text { [CNTACT }\end{array}$ & $\begin{array}{l}\text { MFR SAMFLE } \\
\text { ORÉAN }\end{array}$ & $\begin{array}{l}\text { ELIGIEILITY } \\
\text { FATIO }\end{array}$ & $\begin{array}{c}\text { TOTAL } \\
\text { INELIGELES }\end{array}$ & $\begin{array}{l}\text { TUTAL NRR } \\
\text { PEPILATIOK }\end{array}$ & $\begin{array}{l}\text { ELIEIOLE MFR } \\
\text { POPIULATIOH }\end{array}$ \\
\hline 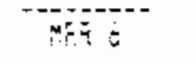 & tqujo & --.-- & $!$ & 2 & 0.840 & 1000 & 55 & 452,40 \\
\hline CLALLAH CTY & 1404 & & $b$ & 12 & 0.8593 & 0.13000 & $30 \hat{40}$ & 2130.32 \\
\hline UTLTES SANPU. & & & & 24 & 4.9755 & 0.0000 & 5231 & 3720.26 \\
\hline OTH UTLTES-J & & & & MA & & & & \\
\hline SUESTKATUK & & & & NA & & 0.0000 & 5231 & 3720.26 \\
\hline MRE 1 & $14140 !$ & & 0 & 2 & 0.9567 & 0.0000 & 4.32 & 374.40 \\
\hline WRE 2 & 141402 & & 0 & 2 & 1.9000 & 1,0000 & 250 & 250.00 \\
\hline HER 3 & 141403 & & 1 & 2 & 1.0000 & 0.0000 & 530 & 530.00 \\
\hline MER 4 & 141404 & & 1 & 2 & 1.0000 & 0.0000 & 312 & 312.60 \\
\hline ELHHURST RUT & 1414 & & 2 & 8 & 0.9667 & 0.0000 & 1524 & 1460.40 \\
\hline HER I & 142401 & & 0 & 2 & 0.9533 & 0.0000 & 1250 & 1160.67 \\
\hline KRR 2 & 142402 & & $!$ & 2 & 0.9289 & 0.0000 & 1250 & 1162.11 \\
\hline MRR 3 & 142403 & & 0 & 2 & 0.9333 & 0.0000 & 1250 & 1166.67 \\
\hline MRR 4 & 142404 & & 0 & 2 & 0.9333 & 0.0000 & 1250 & 1166.67 \\
\hline ORCAS FWK & $: 424$ & & 1 & 8 & 0.9322 & 0.9000 & 5060 & 4661.11 \\
\hline UTLTES SAMFL & & & & 16 & 2.9494 & 0.0000 & 6524 & 8127.51 \\
\hline DTH UTLTES-3 & & & & NA & & & & \\
\hline SUASTKATUH & & & & HA & & 0.0000 & 6524 & 6127.51 \\
\hline MRR ! & 143401 & & 0 & 2 & 0.9286 & 0,0000 & 77 & 71.50 \\
\hline HAR 2 & 143402 & & 0 & 2 & 0.9296 & 0.0000 & 168 & 156.00 \\
\hline MRR 3 & 163403 & & 0 & 2 & 0.9333 & 0.0000 & 170 & 158.67 \\
\hline AlRR 4 & 143404 & & 0 & 2 & 1.0000 & 0.0000 & 140 & 140.00 \\
\hline PARKLAKD LLP & 1434 & & 0 & $B$ & $0.948 ?$ & 0.0000 & 555 & 526.17 \\
\hline MRR 1 & 144401 & & 0 & 2 & 0.9231 & 0.0000 & 127 & $117.2 \mathrm{X}$ \\
\hline HRR 2 & 244402 & & 0 & 2 & 1.0000 & 0.0000 & 85 & 85.00 \\
\hline HRP 3 & 144403 & & 0 & 2 & $0.933 ?$ & 0.0000 & 135 & 136.00 \\
\hline hîR 4 & $\{44404$ & & 1 & 2 & 1.0000 & 0.0000 & 104 & 104.20 \\
\hline SKALANIA & $: 444$ & & 1 & B & 0.9655 & 0.0000 & 451 & 452.23 \\
\hline WTLTES SAKPL & & & & 16 & 0.9569 & 0.0000 & 1006 & 958.40 \\
\hline DTH UTLTES-2 & & & & NA & & & & \\
\hline SUASTRATLM & & & & WA & & 0.6000 & 1006 & 95.40 \\
\hline MFR 1 & 145401 & & 0 & 2 & 1.0000 & 0.0000 & 421 & 421.00 \\
\hline SRR 2 & 145402 & & 1 & 2 & 1.0000 & 0.0000 & 199 & 199.00 \\
\hline MRT 3 & 145403 & & 1 & 2 & 0.9333 & 0.0000 & 65 & 60.67 \\
\hline
\end{tabular}




\begin{tabular}{|c|c|c|c|c|c|c|c|c|}
\hline ENTIY & WFF CODE & $\begin{array}{c}\text { KHLWK } \\
\text { IHE: ISBLS }\end{array}$ & $\begin{array}{l}\text { DHABEE TO } \\
\text { CANFACT }\end{array}$ & $\begin{array}{c}\text { MEF SRMPLS } \\
\text { OSA }\end{array}$ & $\begin{array}{c}\text { ELIGISI:ITY } \\
\text { BAIIO }\end{array}$ & $\begin{array}{c}\text { TOTAL } \\
\text { DUELIGELES }\end{array}$ & 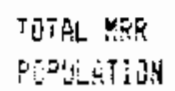 & $\begin{array}{l}\text { ELIGISIE KRR } \\
\text { PUTLATION }\end{array}$ \\
\hline 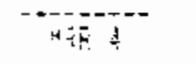 & 14:301 & & 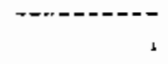 & 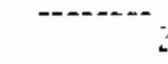 & 唡 & ang? & 5 & 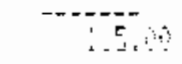 \\
\hline [Ho MITJGL & $: 454$ & & $\because$ & $\bar{a}$ & $\therefore 9873$ & $\therefore .000$ & ) & 79.67 \\
\hline$F F$ & $1464 \div !$ & & i & 2 & 0,735 & 9. 19064 & $\because 2$ & $5.8 \hat{0}$ \\
\hline ? & $14 t 412$ & & 0 & 2 & 50900 & 1.000 & 197 & 197.150 \\
\hline MFR 3 & 1464013 & & $i$ & 2 & $0,929 \mathrm{~g}$ & 0.0000 & 124 & 11.1 .14 \\
\hline MET 4 & $14,4 \pi 14$ & & 1 & 2 & 0.7500 & 0.0000 & 211 & $15 E .25$ \\
\hline 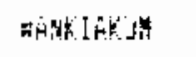 & 1464 & & 1 & 8 & 0.8519 & 5.0000 & 5014 & 523.19 \\
\hline UTLTES SANPL & & & & io & 0.9211 & 0,0000 & 1394 & 178.86 \\
\hline JTH UTLTES-5 & & & & $N A$ & & & & \\
\hline GUBSTFATIM & & & & NA & & 0.0000 & 1394 & 1308.96 \\
\hline STEATUH TOT & & & & $\mathrm{NA}$ & & 0.9000 & 5083 & 17590.63 \\
\hline \multicolumn{9}{|l|}{ REGICN 5} \\
\hline \multicolumn{9}{|l|}{-nen } \\
\hline$M R$ & $4850 !$ & & $\theta$ & 1 & 0.750 & 0.0000 & 594 & $\$ 79.60$ \\
\hline MFA ? & 148502 & & $\hat{\imath}$ & 1 & 0.7073 & 60004 & $E 1 E$ & 328.45 \\
\hline 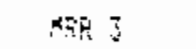 & 495 & & $i$ & $\mathrm{i}$ & 1.8616 & $1, .760)$ & 155 & $\{+2.16$ \\
\hline AkP 4 & $14 E_{504}$ & & 0 & 1 & 1.0000 & 0.0000 & 579 & 599.60 \\
\hline MER 5 & 143505 & & 0 & $!$ & 0.9975 & 0.0000 & 368 & $37 \% .27$ \\
\hline$A P R$ b & 148506 & & ! & 1 & 1.0000 & 0.0000 & 1058 & ij, 00 \\
\hline MRt 7 & $\{48507$ & & 0 & 1 & 0.9375 & 0.8000 & 466 & $4 J 6.8 B$ \\
\hline MRP 8 & $i 48508$ & & is & 1 & 0.7800 & 0.0000 & 482 & 375.96 \\
\hline MIUSTATE & 1485 & & 1 & 8 & $0.8 B 22$ & 0.0000 & 4540 & 2949.93 \\
\hline SFP: & $! 4750 !$ & & 0 & 1 & $0.773 \mathrm{z}$ & 0.0000 & Ji: & 59.40 \\
\hline MR 2 & $14 \div 512$ & & 6 & 1 & 1.8000 & 0.2000 & 80 & 80.60 \\
\hline MR & $14950 \tau$ & & 1 & 1 & 0.9335 & 0.0000 & 102 & 0.30 \\
\hline $\tan 4$ & 149504 & & 0 & : & 0.8067 & $0.0(00)$ & 126 & 109.20 \\
\hline Whe 5 & 149505 & & 0 & 1 & 0.0333 & 0.0000 & 114 & 105.40 \\
\hline Mak 6 & 140506 & & 1 & 1 & 0.9333 & 0.5000 & 82 & 9.100 \\
\hline K59 Y & 149507 & & 1 & $\mathrm{i}$ & 1.3289 & 0.0000 & 106 & 0.20 \\
\hline KKRE & 149508 & & 0 & i & 1.0000 & 0.0000 & 90 & 0.00 \\
\hline MPATI:LA & 1495 & & $?$ & 3 & $0,0 \div 21$ & 0.0000 & $8 \div:$ & 425,00 \\
\hline WTLTES SAHFL- & & & & 16 & 0.8580 & 0.0000 & 5781 & 5394,07 \\
\hline DTH UTLTES-2 & & & & Vh & & & & \\
\hline 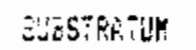 & & & & in & & 8.6000 & 5391 & 3074.95 \\
\hline MRR ! & $15) 50 !$ & & 0 & 1 & 1.0006 & $0.00 \mathrm{pin} 0$ & 199 & 199.00 \\
\hline FRR 2 & 1E050: & & 0 & $!$ & $\therefore 8000$ & 0.0000 & 188 & 162.00 \\
\hline $\operatorname{Meq}=$ & 150503 & & $\hat{y}$ & 1 & $\therefore 0900$ & 0.0000 & lóo & 150.00 \\
\hline
\end{tabular}




\begin{tabular}{|c|c|c|c|c|c|c|c|c|}
\hline ENTITY & MRR SODE & $\begin{array}{c}\text { KNOWN } \\
\text { INELISBLS }\end{array}$ & $\begin{array}{l}\text { LNAELE TO } \\
\text { COHTAET }\end{array}$ & $\begin{array}{l}\text { MRR SAMPLE } \\
\text { DFAUN }\end{array}$ & $\begin{array}{c}\text { ELIGIBILITY } \\
\text { RATIO }\end{array}$ & $\begin{array}{c}\text { TOTAL } \\
\text { INELLIGELES }\end{array}$ & $\begin{array}{l}\text { TOTAL MRR } \\
\text { PSFLLATIITN }\end{array}$ & $\begin{array}{l}\text { ELIGIELE MRR } \\
\text { POPULATIG： }\end{array}$ \\
\hline $4=4$ & IE & ------- & i & i & $2 \mathrm{C}_{2}$ & $0.010 i$ & 190 & $\cdots$ \\
\hline MER 5 & 1505 & & i & i & 1.955 & 0.0000 & 205 & 191.33 \\
\hline Mes 6 & 15050 & & 0 & 1 & 0.7557 & 0.10000 & 71 & 55.79 \\
\hline MRR 7 & 150507 & & 0 & 1 & 0.6444 & 0.10000 & 141 & 90.97 \\
\hline$M R R$ & [5josve & & 0 & 1 & 0.7335 & 0.0000 & 167 & 122.47 \\
\hline CELUMETA & 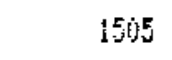 & & 1 & 8.00 & 0.8704 & 0.0000 & 1267 & 5013.45 \\
\hline HRR : & $\$ 51501$ & & D & 1 & 0.9396 & 0.0000 & 103 & 95.64 \\
\hline MEF 2 & 151.502 & & 0 & 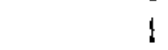 & 0.8571 & 0.60150 & 142 & $12 ! .71$ \\
\hline MRR j & $: 5 ! 503$ & & 1 & 1 & 1.0060 & 3.0000 & 112 & 0.00 \\
\hline MRR 4 & E:504 & & $i$ & $i$ & 1.0000 & 0.0000 & 134 & 0.90 \\
\hline MES 5 & 151505 & & 0 & 1 & 1.0000 & 0.0000 & 182 & 102.60 \\
\hline MEF 6 & 151505 & & 0 & 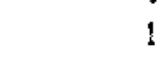 & 1.6000 & 0.0000 & 130 & 130.00 \\
\hline MFG 7 & 151507 & & 0 & 1 & 1.0000 & 0.10000 & 112 & 112.00 \\
\hline ARR 8 & 151508 & & 1 & 1 & 1.0000 & 0.0000 & 99 & 0.00 \\
\hline MLTH-FREEHTR & 1515 & & 3 & B & 0.9741 & 0.0000 & 1014 & $64: .36$ \\
\hline UTLTES SAKPL & & & & 16 & 0.9253 & 0.0000 & 2281 & 1654.81 \\
\hline DTH SAMPL-5 & & & & NA & & & & \\
\hline SLESTRATUM & & & & $\mathrm{NA}$ & & 0.0000 & 2281 & 16EA.B1 \\
\hline STRATA IDT & & & & NA & & 0.0000 & 7662 & 5049.73 \\
\hline \multicolumn{9}{|l|}{ REG!ON 6} \\
\hline -...-...-- & & & & & & & & \\
\hline MRE ! & 153,3601 & & 0 & 2 & 0.9289 & 0.0000 & 2741 & 2540.08 \\
\hline MRR 2 & 153602 & & 1 & 2 & 1,0000 & 0.0000 & 2913 & 2913.00 \\
\hline MRP 3 & 153603 & & 1 & 2 & 0.9367 & 0.0000 & 3020 & 2526.94 \\
\hline MRR 4 & 153604 & & 0 & 2 & 0.9333 & 0.0000 & 2774 & 2589.07 \\
\hline MPR 5 & 153605 & & 1 & 2 & 1.0000 & 0.0000 & 2538 & 2338.00 \\
\hline MRR 6 & 153606 & & $\theta$ & 2 & 0.9235 & 0.0000 & 2412 & 2227.41 \\
\hline EUGENE WTR & 1536 & & 3 & 12 & 0.9384 & 0.0000 & 16198 & 15140.50 \\
\hline MER 1 & \pm 54601 & & 0 & 2 & 0.9289 & 0.0000 & 192 & 179.35 \\
\hline MFR 2 & 154502 & & 0 & & 0.5833 & & & 0.00 \\
\hline MER 3 & 154603 & & 0 & & 0.8929 & & & 0.70 \\
\hline RRP 4 & 154604 & & 0 & 2 & 0.7515 & 0.0000 & 127 & 95,44 \\
\hline MRR 5 & $\{54605$ & & 2 & 2 & 0.7289 & 0.0000 & 126 & 0.00 \\
\hline MRR 6 & 154606 & & 0 & 2 & 1.0000 & 0.0000 & 209 & 209.60 \\
\hline CNTRL-LACLN & 1546 & & 2 & 9 & 0.8553 & 0.0000 & 664 & $4 E 2.78$ \\
\hline UTLTES EAMP: & & & & 20 & $\dot{0} .8953$ & 0.0000 & 16362 & $1=623 .: B$ \\
\hline OTH UTLTES-O & & & & MA & & & & \\
\hline SLESTRATUA & & & & HA & & 0.0000 & 16862 & 15623.28 \\
\hline HFR 1 & $15560 !$ & & 0 & 2 & 0.9244 & 0.0000 & 379 & 350.36 \\
\hline
\end{tabular}

A. 9 


\begin{tabular}{|c|c|c|c|c|c|c|c|c|}
\hline ENTITY & MRF CCEE & $\begin{array}{c}\text { KNJWN } \\
\text { INEL! } 98 L 5\end{array}$ & $\begin{array}{l}\text { RAEEEE TS } \\
\text { COHTACT }\end{array}$ & $\begin{array}{l}\text { MFR SAHFLE } \\
\text { DAALAR }\end{array}$ & $\begin{array}{c}\text { ELIGIJILITY } \\
\text { FSTIO }\end{array}$ & $\begin{array}{l}\text { TOTAL } \\
\text { INEL!GGLES }\end{array}$ & $\begin{array}{l}\text { TDTAL MER } \\
\text { FUFULATIONW }\end{array}$ & $\begin{array}{l}\text { ELIGIELE MEP } \\
\text { PBPLEATINH }\end{array}$ \\
\hline 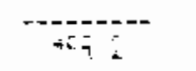 & 列E & - - + & 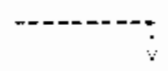 & ; & L. & $b_{1}$ & $\cdots+15$ & 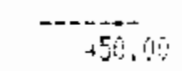 \\
\hline YH 3 & $i=500$ & & $i$ & 2 & 1.6010 & 0.40 & $\equiv$ & 16 \\
\hline NER + & 155 & & 2 & 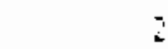 & 1. & 1. 100 & 访 & a.jo \\
\hline$k 5$ & $15=0.5$ & & 1 & 2 & 0.8367 & 0.0000 & 470 & 392.27 \\
\hline 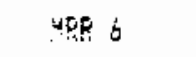 & 155006 & & $\theta$ & 2 & 1. 19050 & 0.0000 & E1s & 310.60 \\
\hline SPCINGFIELD & 1556 & & 5 & 12 & 0.9616 & 0.0000 & 2206 & 1509.63 \\
\hline A 1 & Essbul & & ? & 2 & 0.8489 & 0.0000 & 53 & 0.00 \\
\hline MFin? & {$[50602$} & & 9 & 2 & 0.9333 & 9,13060 & 56 & 63.47 \\
\hline MPS ? & 15050 & & $!$ & 2 & 0.9000 & 1.0000 & 70 & 5.60 \\
\hline $\operatorname{man} 4$ & 156604 & & 2 & 2 & 0.9333 & 0.0000 & $: 57$ & 0,10 \\
\hline MRE & 156605 & & 1 & 2 & 0.8667 & 6.7000 & 51 & 44.20 \\
\hline SP? 6 & {$[5660)$} & & $\therefore$ & 2 & 1.0000 & 5.6000 & $100^{7}$ & 107,00 \\
\hline EOKCLUAERS & 1500 & & 6 & 12 & 0.8970 & 0.6000 & 516 & $27 i, 67$ \\
\hline UTLTES SRMPL & & & & 24 & 0.97291 & 0.0000 & 2712 & 1790.30 \\
\hline OIK UTLTES-2 & & & & ${ }^{*} A^{\prime}$ & & & & \\
\hline SUESTRATUK & & & & NA & & 0.0000 & $271 ?$ & 1780.30 \\
\hline ME I & 157001 & & 3 & 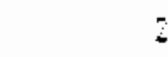 & 0.9239 & 0.0000 & 107 & 93.53 \\
\hline 铅 2 & 157602 & & 0 & 2 & 5.9184 & 0.0000 & 125 & 54.90 \\
\hline MRR 3 & 157603 & & 0 & 2 & V. 8265 & 0.6000 & 96 & 79.35 \\
\hline MRS \& & 157604 & & 0 & 2 & 1.0000 & 0.0000 & $: 15$ & 115.00 \\
\hline ASHELANO & 1576 & & 0 & 日 & 0.9186 & 0.0000 & 443 & $40.5 J$ \\
\hline ART 1 & $15860 !$ & & 1 & 2 & 0.8667 & 0.0000 & 105 & 91.00 \\
\hline ARR 2 & 158602 & & 0 & 2 & 0.9333 & 0.0000 & 61 & 50.93 \\
\hline RRR 3 & 158603 & & 2 & 2 & 0.8667 & 0.0000 & 152 & 0.00 \\
\hline HES 4 & 586604 & & $!$ & 2 & 1.0000 & 0.0000 & 137 & 137.00 \\
\hline FOSESST GROVE & 1596 & & 4 & 8 & 0.9167 & 0.0000 & 455 & 294.93 \\
\hline UTLTES SARPL & & & & 16 & 0.9176 & 0.0000 & 598 & 695.47 \\
\hline OTH UTL:TES-4 & & & & if & & & & \\
\hline SUBETRATUM & & & & NA & & $0.00 \% 0$ & 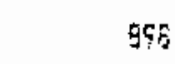 & 695.47 \\
\hline $\operatorname{HRR} 1$ & 159601 & & 0 & 2 & $0 . \$ 184$ & 0.9000 & 45 & 413.27 \\
\hline MeR 2 & $15 \% 602$ & & 0 & 2 & 1.0000 & $0.600 \hat{\mathrm{y}}$ & 530 & 535.00 \\
\hline HRR 3 & {$[5960\}$} & & 1 & 2 & 0.9578 & 0.3000 & 489 & 415,45 \\
\hline KR 4 & $15960 \hat{4}$ & & 1 & 2 & 0.9299 & $\dot{8} .0000$ & 458 & $425.4 j$ \\
\hline SEFHLO-LAGE & 1896 & & : & b & 0.9238 & $i .6 i v 0$ & 1927 & $: 758.15$ \\
\hline MRR ! & 160601 & & i & 2 & ن. .8578 & 0.0000 & 438 & 375.71 \\
\hline MER 2 & 160602 & & 0 & 2 & 0.9535 & 0,5100 & 299 & 279.97 \\
\hline MES 3 & 100,003 & & 2 & $i$ & 0.9239 & 0.350 & 413 & 4.00 \\
\hline
\end{tabular}




\begin{tabular}{|c|c|c|c|c|c|c|c|c|}
\hline EMITY & WR CODE & $\begin{array}{c}\text { YNOEN } \\
\text { IMELIGELS }\end{array}$ & $\begin{array}{l}\text { JNARELE TO } \\
\text { CONTACT }\end{array}$ & $\begin{array}{l}\text { MER SAMPLE } \\
\text { DRÁHN }\end{array}$ & $\begin{array}{c}\text { ELIFIBILITY } \\
\text { RAIIO }\end{array}$ & $\begin{array}{l}\text { TOTAL } \\
\text { IHELIGBLES }\end{array}$ & $\begin{array}{l}\text { TDTAL MRR } \\
\text { FOFLLLGTION }\end{array}$ & $\begin{array}{l}\text { ELIGIBLE HRR } \\
\text { PLPULAT!CH }\end{array}$ \\
\hline ME 4 & atoit & & $i$ & 2 & 1.0619 & 0. & 153 & 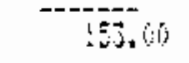 \\
\hline MC世MGIJH & 1606 & & 3 & $\hat{y}$ & 0.9300 & 0.0000 & 1303 & 607.77 \\
\hline UTLTES SAMMPL & & & & 16 & 0.9270 & 0.0000 & 220 & 2595.92 \\
\hline OTH UTLTES-4 & & & & NA & & & & \\
\hline SUEETRATUM & & & & $N A$ & & 0.10000 & 3230 & 2595.92 \\
\hline STEATUM TET & & & & NA & & 1). 0100 & 23702 & 20072.77 \\
\hline FUELIC TOT & & & & NA & & 3.0007 & 85560 & $65 E 64.29$ \\
\hline \multicolumn{9}{|l|}{ PRIVATE } \\
\hline \multicolumn{9}{|l|}{ REGION I } \\
\hline 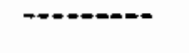 & & & & & & & & \\
\hline MRR 1 & 101101 & & 0 & $!$ & 1.0000 & 0.0000 & 73 & 73.00 \\
\hline KRR 2 & 101102 & & 1 & 1 & 0.7333 & 0.0000 & 55 & 0.00 \\
\hline HRR 3 & 101103 & & 0 & 1 & 0.9578 & 0.0000 & 132 & $113.2 \mathrm{~J}$ \\
\hline NhR 4 & 101104 & & 0 & 1 & 0.8000 & 0.0000 & 121 & 96.80 \\
\hline$M B R 5$ & 101105 & & 0 & 1 & 0.9335 & 0.0000 & 54 & 50.40 \\
\hline MGR 6 & 101100 & & ! & 1 & 1. 90000 & 0.09010 & 156 & 0.60 \\
\hline MRR 7 & 101107 & & 0 & 1 & 1.0000 & 0.0000 & 500 & 500.00 \\
\hline MRR 8 & $10 t 108$ & & 0 & 1 & $0.933 \mathrm{~J}$ & 0.0000 & 435 & 406.00 \\
\hline HRR 9 & 101109 & & 0 & 1 & 1.0000 & 0.0000 & 411 & $\$ 11.00$ \\
\hline MRR 10 & 101110 & & 0 & 1 & 0.8578 & 0.0000 & 544 & 466.03 \\
\hline MRR II & 101111 & & 0 & 1 & 0.9289 & 0.0000 & 345 & 320.47 \\
\hline HRR 12 & 101112 & & 0 & 1 & 0.9244 & 0.0000 & $15 J$ & 141.44 \\
\hline MFR IS & 101113 & & 0 & 1 & 0.9289 & 0.0000 & 132 & 122.61 \\
\hline MRR 14 & 101114 & & 0 & $t$ & 0.8367 & 0,0000 & 26 & 21.76 \\
\hline MRR 15 & 101115 & & 1 & 1 & 0.9296 & 0.0000 & 358 & 0.00 \\
\hline MER 16 & 101116 & & 1 & 1 & 0.7551 & 0.0000 & 167 & 0.00 \\
\hline $\begin{array}{l}\text { MDNTANA FWR } \\
\text { SUBSTRATUM }\end{array}$ & 1011 & & 4 & 16 & 0.8998 & 0.0000 & 3662 & 2723.33 \\
\hline
\end{tabular}

\begin{tabular}{|c|c|}
\hline ARR 1 & 102101 \\
\hline MER 2 & 102102 \\
\hline WfR 3 & 102103 \\
\hline MRR 4 & 102104 \\
\hline KRP 5 & 102105 \\
\hline MER of & 102106 \\
\hline$M G R 7$ & $1021 \div 7$ \\
\hline IARR B & 102108 \\
\hline HRR 9 & 102109 \\
\hline ARR 10 & $102: 10$ \\
\hline MRR 11 & 102111 \\
\hline
\end{tabular}




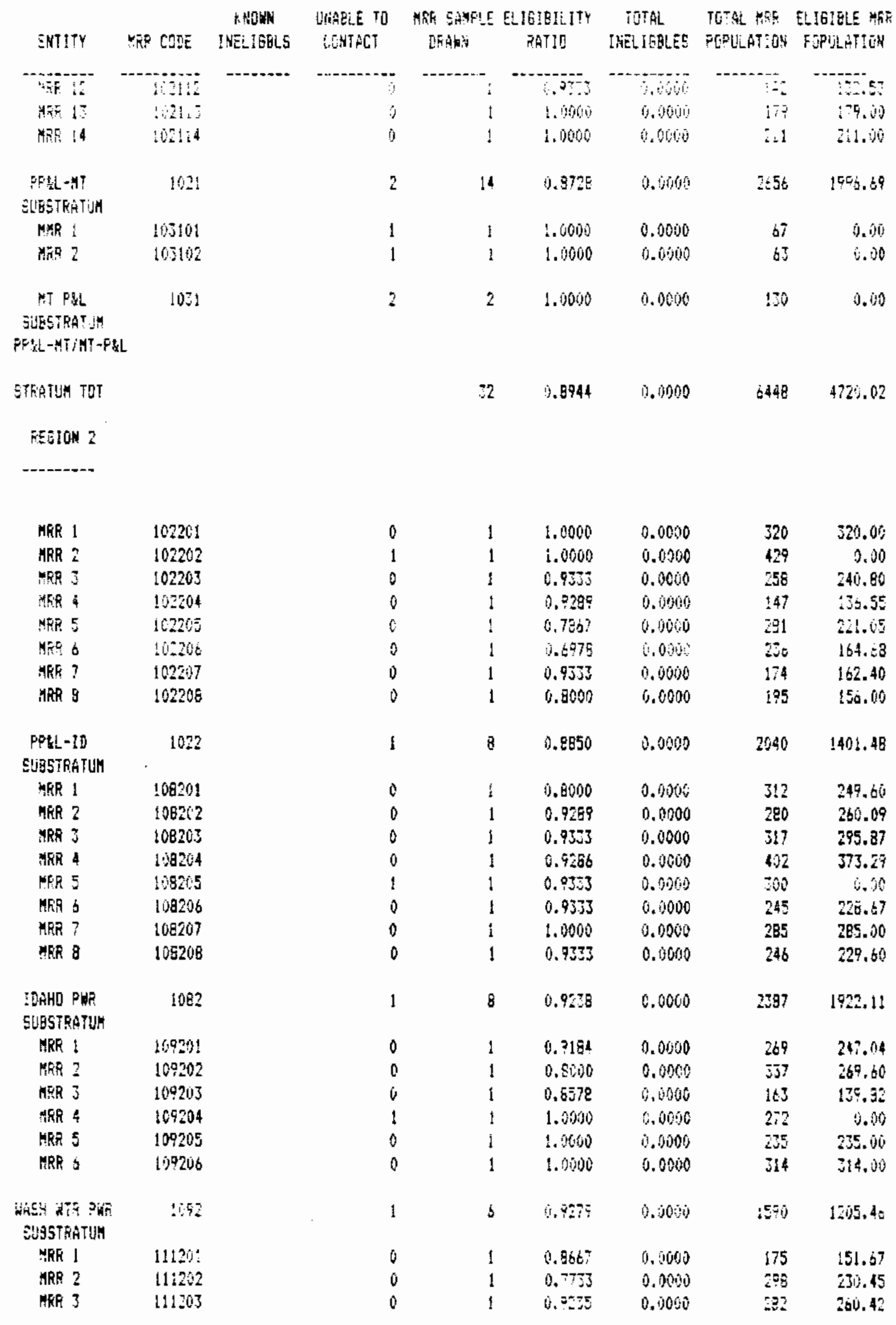

A. 12 


\begin{tabular}{|c|c|c|c|c|c|c|c|c|}
\hline EMT:TY & KRE OUE & $\begin{array}{c}\text { P.SDUN } \\
\text { MELI6 }\end{array}$ & $\begin{array}{l}\text { UNABLE TO } \\
\text { CONTACT }\end{array}$ & $\begin{array}{l}\text { MRR SAMPIEE } \\
\text { DRANHA }\end{array}$ & $\begin{array}{c}\text { ELIGIBILITY } \\
\text { FATIO }\end{array}$ & $\begin{array}{c}\text { TOTAL } \\
\text { INELISELES }\end{array}$ & $\begin{array}{l}\text { TETAL MRR } \\
\text { POPLLATION }\end{array}$ & $\begin{array}{l}\text { ELIGIBLE YRR } \\
\text { POPILATIOM }\end{array}$ \\
\hline G & 1: & & $\overline{1}$ & - & 9.950 & (1) & 48 & 6.69 \\
\hline MEP $\bar{Z}$ & $: \vdots 1: 5$ & & 0 & $i$ & 1.3000 & 4.9010 & 151 & 551.00 \\
\hline MER i & 11120 & & 0 & $i$ & 0.7300 & 0.0000 & 138 & 101.24 \\
\hline MR ? & 111207 & & 0 & 1 & 0.0733 & 6.00000 & 120 & 112.00 \\
\hline MaR 8 & 111208 & & 0 & 1 & 8.8667 & 0.0060 & 116 & 169.57 \\
\hline $\begin{array}{c}\text { UTAH DUL } \\
\text { SLESTRATUYH }\end{array}$ & 1112 & & $!$ & 8 & $0.851 t$ & 0.0000 & 1712 & 1107.27 \\
\hline STRATIH TQT & & & & 30 & 1.9975 & 0.0000 & 7729 & 5636.31 \\
\hline \multicolumn{9}{|l|}{ REGIGY J } \\
\hline \multicolumn{9}{|l|}{--------- } \\
\hline MRR 1 & 102301 & & 0 & 1 & 1.0000 & 0.0000 & 413 & 413.00 \\
\hline MTR 2 & 1023012 & & 0 & 1 & 1.0000 & 0.0060 & 406 & 400,60 \\
\hline MRE 3 & 102303 & & 1 & $!$ & 1.0000 & 0.0000 & 291 & 0.00 \\
\hline MER 4 & 102304 & & 0 & 1 & 9.8000 & 0.0000 & 197 & 157.60 \\
\hline MiR 5 & 102305 & & 0 & 1 & 1.0000 & 0.0000 & 274 & 274.00 \\
\hline KRR B & 102306 & & 0 & 1 & 1.0000 & 0.0000 & 199 & 189.00 \\
\hline MRR 7 & 102307 & & 0 & $t$ & 1.0000 & 0.0000 & 186 & 186.00 \\
\hline MRR B & 102308 & & 0 & 1 & 1.0000 & 0.0000 & 332 & 332.00 \\
\hline MRR 9 & $\$ 02309$ & & 0 & 1 & 0.9333 & 9.0000 & 390 & 364.00 \\
\hline MRR 10 & 102310 & & 0 & 1 & 0.9333 & 0.0000 & $16 !$ & 150.27 \\
\hline MET 11 & 102311 & & 1 & 1 & 19.8667 & 0.0060 & 212 & 9.00 \\
\hline MFR 12 & 102312 & & 0 & 1 & 0.9296 & 0.0000 & 274 & 254.43 \\
\hline HRR 13 & $: 02313$ & & 0 & $!$ & 1.0000 & 0.0000 & 237 & 237.00 \\
\hline HRR 14 & 102314 & & 0 & I & 1.0000 & 0.0000 & 226 & 226.00 \\
\hline MRR 15 & 102315 & & 0 & 1 & 0.7733 & 0.0000 & 314 & 242.83 \\
\hline MRR 16 & 102316 & & 1 & 1 & 1.0000 & 0.0000 & 373 & 0.00 \\
\hline$P P E L-N A$ & 1023 & & 3 & 16 & $0.952 !$ & 0.0000 & 4475 & 3432 \\
\hline \multicolumn{9}{|l|}{ SUBSTRATUH } \\
\hline MRR I & 109301 & & 1 & 1 & 0.9333 & 0.0000 & 63 & 0.60 \\
\hline MRR 2 & 1093002 & & 0 & 1 & 1.0000 & 0.0000 & 252 & 252.00 \\
\hline MRR 3 & 109013 & & 0 & $!$ & 1.0000 & 0.0000 & 254 & 254.10 \\
\hline MRF 4 & 109304 & & 0 & 1 & 6.8667 & 0.0000 & 328 & 284.27 \\
\hline MRR 5 & 109305 & & 1 & 1 & 0.8667 & 0.0000 & 172 & 0.00 \\
\hline MRF : & 109306 & & 1 & 1 & 1.0000 & 0.0000 & 357 & 0.00 \\
\hline MRR 7 & 109307 & & 0 & 1 & 1.0000 & 0.0000 & 229 & 229.00 \\
\hline Int 8 & to930E & & 0 & 1 & 0.7333 & 0.0000 & 359 & 263.27 \\
\hline MFR 9 & 109309 & & 1 & 1 & 1.0000 & 0.6000 & 272 & 0.00 \\
\hline KRR 10 & 109310 & & 1 & 1 & 0.9311 & 0.0000 & 404 & 0.00 \\
\hline $\operatorname{MRR} 11$ & 109311 & & 1 & 1 & 0.9333 & 0.0000 & 424 & 0.00 \\
\hline ARR 12 & 109312 & & 0 & $\downarrow$ & 0.9235 & 0.0000 & 135 & 124.67 \\
\hline MRR 13 & 109313 & & 1 & 1 & 1.0000 & 0.0000 & 323 & 0.00 \\
\hline MRR 14 & 109314 & & 0 & ! & 1.0000 & 0.0000 & 291 & 291.00 \\
\hline MRR 15 & 109315 & & 0 & 1 & 0.9235 & 0.0000 & 253 & 213.17 \\
\hline MER I6 & 109316 & & 1 & \pm & 0.7733 & 0.0000 & 197 & 0.00 \\
\hline $\begin{array}{l}\text { WASH WAT PHR } \\
\text { SWBSTRATUAM }\end{array}$ & 1093 & & $B$ & 16 & 0.9234 & 0.0000 & 4289 & 1515.37 \\
\hline STRAFUM TOT & & & & 32 & 0.9378 & 0.0000 & 4617 & 5345.49 \\
\hline
\end{tabular}




\begin{tabular}{|c|c|c|c|c|c|c|c|c|}
\hline EN:ITY & MFE COLE & $\begin{array}{c}\text { KNLWH } \\
\text { TWE!ISELLS }\end{array}$ & $\begin{array}{l}\text { WHABLE TI } \\
\text { CONTACT }\end{array}$ & $\begin{array}{l}\text { MEA SAMFLE } \\
\text { DFAHN }\end{array}$ & $\begin{array}{c}\text { SLIOIFILIYY } \\
\text { FAHII }\end{array}$ & $\begin{array}{l}\text { TOTAL } \\
\text { INE:IGELES }\end{array}$ & $\begin{array}{l}\text { TOTAL MFR } \\
\text { POPLILATISN }\end{array}$ & $\begin{array}{l}\text { ELIGIGLE MRR } \\
\text { OPHHATIDN }\end{array}$ \\
\hline \multicolumn{9}{|l|}{ 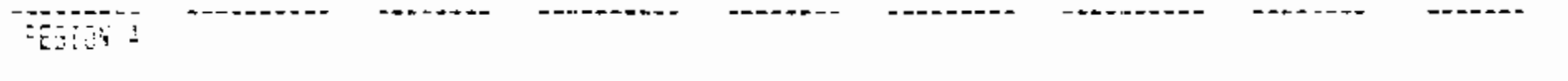 } \\
\hline - & & & & & & & & \\
\hline MEF： & 134401 & & 1 & 2 & 1). $92 \mathrm{E} 6$ & 0.9010 & 344 & 317.95 \\
\hline 4982 & $154+102$ & & 3 & 2 & 1. $(4, \mathrm{i}, \mathrm{i})$ & ijoot & 299 & $i, 00$ \\
\hline Whe & 134403 & & 0 & 2 & 1.0000 & 0.0000 & 290 & 245.60 \\
\hline MPR 4 & 134404 & & 2 & 2 & 0.9235 & j. $\$ 100$ & 262 & 1. .00 \\
\hline 5 & 134405 & & i) & 2 & 1.0000 & 0.6000 & 354 & 354.60 \\
\hline MES is & 154486 & & 0 & 2 & 1.2990 & 0.0060 & 290 & 20,0 \\
\hline Mat 7 & $i 3440 ?$ & & 0 & ? & 0.8667 & 0.0000 & 353 & 227.43 \\
\hline 498 & 174408 & & 1 & 2 & 1.0000 & $0.60 \mathrm{Hi}$ & 171 & 191.00 \\
\hline MRR 9 & 1344199 & & $!$ & $z$ & 0.9267 & i. 10000 & 205 & 561.78 \\
\hline Mล 10 & 134410 & & 1 & 2 & i. $92 \mathrm{Bg}$ & 0.0000 & 227 & 210.79 \\
\hline WE 11 & $13441 !$ & & 1 & 2 & 0.9244 & 0.6000 & 409 & 732.54 \\
\hline MEF 12 & 134412 & & 1 & 2 & 0.7600 & 0.170100 & 239 & 151.64 \\
\hline MFR 13 & 134413 & & 1 & 2 & 1.0000 & 0.0000 & 203 & 203.60 \\
\hline MES :4 & 134414 & & $\theta$ & 2 & 0.9289 & 0.0000 & 236 & $20 \% .97$ \\
\hline ARR : $: 5$ & $1344 ! 5$ & & 1 & 2 & 0.9244 & 0.0046 & 5iv & 557.44 \\
\hline MRR 16 & 134416 & & $!$ & 2 & 1.0000 & 1.0000 & 185 & 185.00 \\
\hline 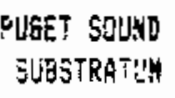 & 1344 & & 13 & 32 & 0.9441 & 0.0000 & 4948 & 4115.77 \\
\hline STSATH TOT & & & & 32 & $0.944:$ & 0.0000 & 4948 & $411 \%, 77$ \\
\hline
\end{tabular}

SEGION 5

\begin{tabular}{|c|c|}
\hline MRR ! & 10250 \\
\hline MVR 2 & 1025 \\
\hline KRR 3 & 1025 \\
\hline 筷 & $10 z$ \\
\hline Kลू 5 & t02 \\
\hline $\operatorname{MRE} 6$ & 102 \\
\hline MiR 7 & 102 \\
\hline MRP 8 & \\
\hline IRP 7 & \\
\hline MFR 10 & 102 \\
\hline$M F R$ & 102 \\
\hline MRR :2 & 10 \\
\hline 49 ! 13 & 102 \\
\hline KRF 14 & 102 \\
\hline MRK 15 & $102 x$ \\
\hline Hikg ib & 102 \\
\hline 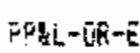 & \\
\hline CUESTKATU & \\
\hline SRR I & 109 \\
\hline$M R P 2$ & 10,8 \\
\hline 解 & 165 \\
\hline RR 4 & 108 \\
\hline MER 5 & 108 \\
\hline MRR 6 & 108 \\
\hline $\operatorname{MRP} 7$ & 108 \\
\hline
\end{tabular}

\begin{tabular}{|c|c|c|c|c|c|}
\hline 0 & 1 & 0.9333 & 0.0000 & $36 ?$ & 338.80 \\
\hline 0 & 1 & 0.9286 & 0.00010 & 311 & 298.79 \\
\hline 0 & 1 & 1.0000 & 0.0000 & $\$ 05$ & 405.00 \\
\hline 0 & 1 & 0.5667 & 0.0000 & 276 & 239.27 \\
\hline 0 & 1 & 0.6939 & 0.3000 & 158 & 96.45 \\
\hline 0 & 1 & 0.7156 & 13.0000 & 226 & 161.72 \\
\hline 1 & 1 & 0.8400 & 0,0000 & 276 & 0.00 \\
\hline 0 & 1 & $\therefore .0700$ & 0.0000 & 243 & 343.00 \\
\hline 0 & 1 & 1.0000 & 0.0000 & 178 & 173.00 \\
\hline 0 & $t$ & 0.9289 & 0.0060 & 319 & 295.39 \\
\hline 0 & 1 & 0.9289 & 0.0000 & 125 & $1: 6.11$ \\
\hline 1 & 1 & 0.9231 & $\$, 0000$ & 229 & 0.00 \\
\hline 0 & 1 & 0.9389 & 0.0000 & 73 & 67.91 \\
\hline 0 & & 8.4000 & & & 9.90 \\
\hline 0 & 1 & 1.0902 & 0.0000 & 101 & 101.60 \\
\hline 0 & 1 & 1. 0000 & 9.0000 & 305 & 306.90 \\
\hline 2 & 15 & 0.8797 & 0.0800 & $3=68$ & 2977.20 \\
\hline 0 & 1 & 1.0000 & 9.0000 & 193 & $18 \mathrm{BJ}, \dot{0}$ \\
\hline 0 & 1 & 0.7333 & ij, 90,0 & 89 & +46.61 \\
\hline 1 & 1 & 0.349 & 0.0500 & 171 & 5010 \\
\hline 0 & $!$ & 0.3067 & 0. 0060 & 31 & 280.57 \\
\hline 1 & $!$ & 0.9333 & 0.0000 & 209 & 0.10 \\
\hline 0 & $!$ & 1.0000 & 0.0000 & 164 & 104.00 \\
\hline 0 & 1 & 0.8000 & 19.9000 & 20 & 20.30 \\
\hline
\end{tabular}




\begin{tabular}{|c|c|c|c|c|c|c|c|c|}
\hline E.TITY & MRR SDDE & $\begin{array}{c}\text { INOWN } \\
\text { IMELIGSLS }\end{array}$ & $\begin{array}{l}\text { JWABLE TO } \\
\text { CONTACT }\end{array}$ & $\begin{array}{l}\text { MER SAMPLE } \\
\text { DFAGN }\end{array}$ & $\begin{array}{c}\text { ELIGIEILITY } \\
\text { FATIO }\end{array}$ & $\begin{array}{l}\text { TOFAL } \\
\text { IHELIGELES }\end{array}$ & $\begin{array}{l}\text { TOTAL MES } \\
\text { PGFULATIEA }\end{array}$ & $\begin{array}{l}\text { ELIGIELE MGR } \\
\text { PIPHLATION }\end{array}$ \\
\hline 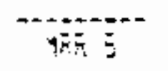 & ngiv & 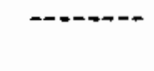 & 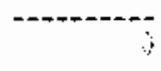 & : & $1.00 \%$ & o. & 375 & 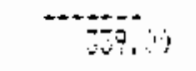 \\
\hline 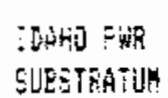 & 1055 & & 2 & 8 & 1.6739 & 6.0010 & $0 \overline{5} 2$ & 144.37 \\
\hline सQT 1 & $14750 !$ & & 1 & 1 & 0.9235 & 6.060 & 523 & $1 \%, 00$ \\
\hline MiR 2 & 147502 & & 0 & 1 & 0.7039 & 0.0000 & 847 & 647.11 \\
\hline$M P R \quad 3$ & 147503 & & 0 & & 0.6173 & & & 0.00 \\
\hline Mfin 4 & 147504 & & 0 & 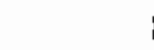 & 1. & 0.0000 & 200 & 200.00 \\
\hline MA 5 & $1475 \div 5$ & & 0 & & 1). 1407 & & & 10.00 \\
\hline MFE & 147506 & & I & 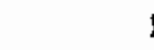 & 1). .9289 & 0.0000 & 206 & 0.00 \\
\hline 7 & 147507 & & 9 & & 1.0900 & & & 0.60 \\
\hline ARE a & $14750 \mathrm{E}$ & & 1 & . & 4.573? & 0.3000 & 232 & 1.60 \\
\hline $\begin{array}{c}\text { CF NATNL } \\
\text { SUBSTRATUM }\end{array}$ & 1475 & & 3 & 5 & 0.7410 & 0.0000 & 2008 & 847.01 \\
\hline STEATUM TOT & & & & 28 & i).8444 & 0,0000 & 7728 & 5224.54 \\
\hline
\end{tabular}

REGION $b$

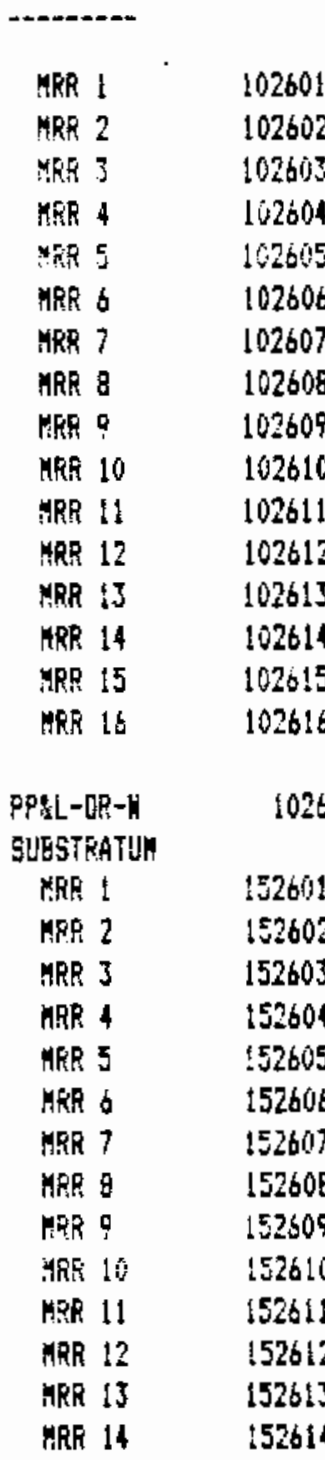




\begin{tabular}{|c|c|c|c|c|c|c|c|c|}
\hline ENIT & TRS COQE & $\begin{array}{c}\text { KNowN } \\
\text { INEL_igeLs }\end{array}$ & $\begin{array}{l}\text { WNABLE TO } \\
\text { CINTALT }\end{array}$ & $\begin{array}{c}\text { WTR EAMPLE } \\
\text { DRAWN }\end{array}$ & $\begin{array}{l}\text { ELIGIE!LITY } \\
\text { RATIO }\end{array}$ & $\begin{array}{l}\text { TOTAL } \\
\text { INELIGRLES }\end{array}$ & $\begin{array}{l}\text { TOTÁE MFP } \\
\text { PIOPILATIJH }\end{array}$ & $\begin{array}{l}\text { ELIGIELE YOS } \\
\text { POPULTI:XN }\end{array}$ \\
\hline 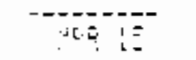 & 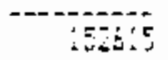 & & $\cdots$ & $\stackrel{?}{2}$ & 1.190 & 460 & Bi & 51.8 \\
\hline 6 & 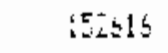 & & $\therefore$ & ? & 1.000 & o. onto & 47 & 457.50 \\
\hline $\begin{array}{l}\text { FRT EEH ELE } \\
\text { SUESTRHTH }\end{array}$ & 1526 & 0 & 1) & 32 & 0.95 .50 & 9.6000 & 5418 & $50 \mathrm{iv} . \mathrm{EL}$ \\
\hline STEPTIAH IGT & & 0 & 0 & 62 & 1.9315 & 6.0000 & 9691 & 8525.52 \\
\hline FETIATE TDT & & 0 & 0 & NA & & 0.0000 & $4 ! 161$ & 356.3 .65 \\
\hline ERAND TYT & & 0 & 6 & $\begin{array}{r}218 \\
324.00 \\
542.00\end{array}$ & $\begin{array}{l}0.9032 \\
0.9079 \\
0.9060\end{array}$ & $\begin{array}{l}3.0667 \\
91.9000 \\
3.0667\end{array}$ & $12 j 721$ & 0.9447 .54 \\
\hline
\end{tabular}

A. ?6 


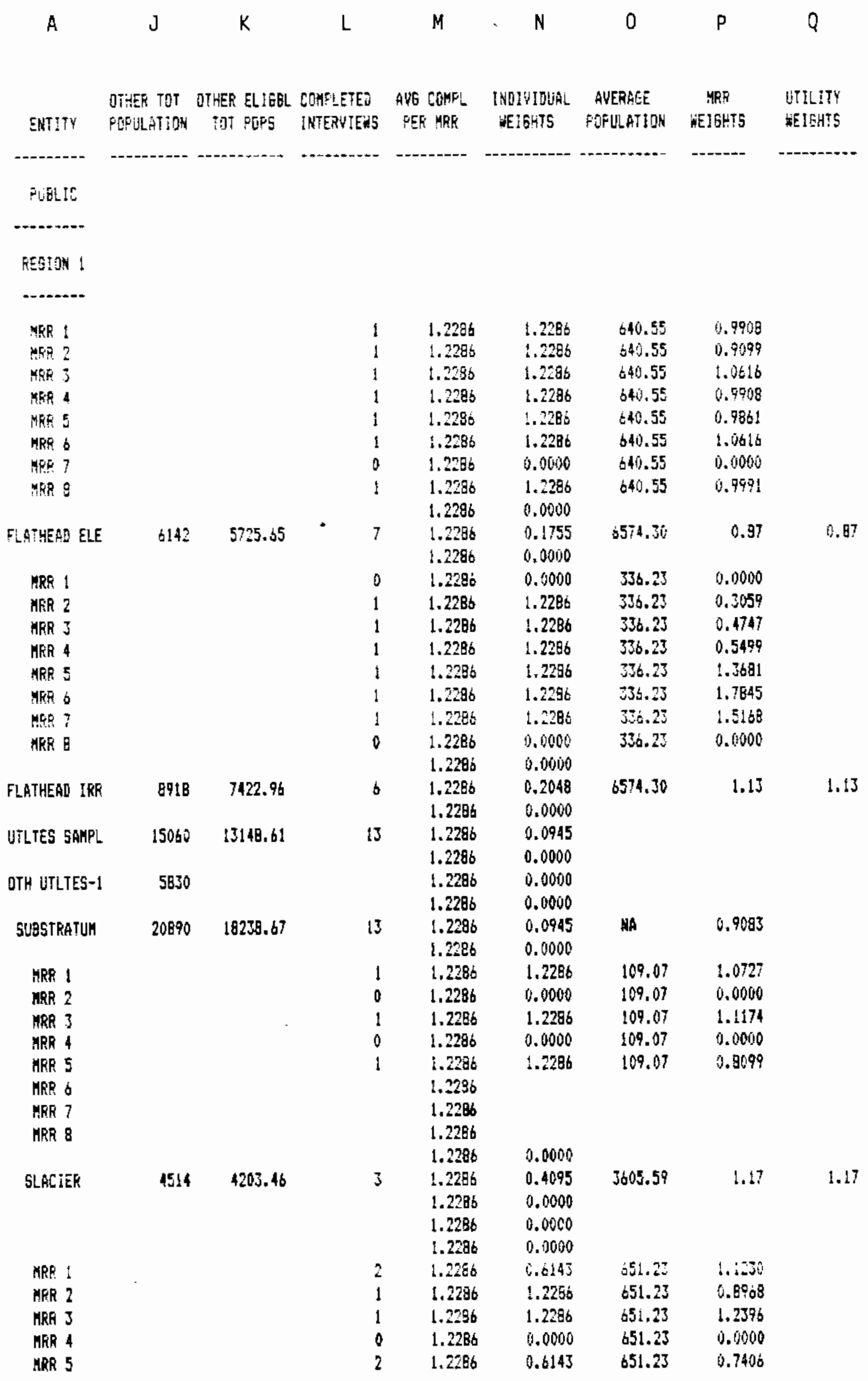

\section{A. 17}




\begin{tabular}{|c|c|c|c|c|c|c|c|c|}
\hline ENTITY & $\begin{array}{l}\text { DTHES TUT } \\
\text { PLIFJLATIEW }\end{array}$ & $\begin{array}{c}\text { OTHER ES1SBL } \\
\text { TOT FOPS }\end{array}$ & $\begin{array}{l}\text { CEMPLETED } \\
\text { INTERUIEHE }\end{array}$ & $\begin{array}{l}\text { AWG CEHF } \\
\text { PET MRP }\end{array}$ & $\begin{array}{l}\text { INDWIDUAL } \\
\text { NE?GHTS }\end{array}$ & $\begin{array}{l}\text { AVERGGE } \\
\text { PLFULALITON }\end{array}$ & $\begin{array}{c}\text { MRG } \\
\text { WEIGHTS }\end{array}$ & $\begin{array}{l}\text { ITILITY } \\
\text { WESENTS }\end{array}$ \\
\hline - & 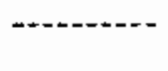 & 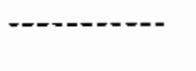 & - & 1.286 & पiva & ----n-- -- & $-\cdots--$ & \\
\hline \multirow[t]{2}{*}{ GIUALL! } & 3207 & 397.71 & $b$ & 1.2226 & 0.2045 & 505.59 & 0.88 & 9.87 \\
\hline & & & & 1.2286 & 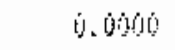 & & & \\
\hline \multirow[t]{2}{*}{ UTLTES GAHEL } & $772 !$ & $721: .17$ & 9 & 1.2286 & 0.1305 & & & \\
\hline & & & & 1.2286 & 1.0000 & & & \\
\hline \multirow[t]{2}{*}{ OTH U UTES-2 } & 5668 & & & 1.2286 & 0. 0000 & & & \\
\hline & & & & 1.2286 & 0.0000 & & & \\
\hline \multirow[t]{2}{*}{ SUESTKATUM } & 13399 & 15504.90 & 9 & 1.2386 & 0.1365 & (k & 1.1728 & \\
\hline & & & & 1.2296 & 9.5000 & & & \\
\hline \multirow[t]{2}{*}{ STKATUH TOT } & 34279 & 30743.58 & 22 & 1.2236 & 0.0558 & NA & 1). 17 & \\
\hline & & & & 1.2286 & 0.0000 & & & \\
\hline \multirow[t]{3}{*}{ REGIOH 2} & & & & 1.2280 & 0.2000 & & & \\
\hline & & & & 1.2296 & 12.6000 & & & \\
\hline & & & & 1.2286 & 0.0000 & & & \\
\hline MRE i & & & 0 & $\{.228 \dot{0}$ & 0.0000 & 169.47 & 0.0400 & \\
\hline Mnk 2 & & & 1 & 1.2296 & 1.2286 & 169.47 & 1.5106 & \\
\hline$M E E^{\prime} ?$ & & & 0 & 1.220s & 0.1000 & 250,47 & 0.0000 & \\
\hline MRR 4 & & & 1 & 1.2296 & 1.2286 & 169.47 & 1.9299 & \\
\hline SRR 5 & & & 1 & 1.2286 & $1.22 \mathrm{Bb}$ & 169.47 & 0.96 .38 & \\
\hline \multirow[t]{2}{*}{ MER E } & & & 1 & 1.2286 & 1.2286 & 169.47 & 0.4957 & \\
\hline & & & & 1.2286 & 0.0000 & & & \\
\hline \multirow[t]{2}{*}{ IDAHO FALLS } & 15243 & 14557.92 & 4 & 1.2396 & 0.3071 & 11128.21 & 1.31 & 1.31 \\
\hline & & & & $i .286$ & 0.5000 & & & \\
\hline MRP 1 & & & 1 & 1.2866 & i. 2296 & 195.16 & 0.9700 & \\
\hline 4 4 ? & & & $i$ & $\therefore .2286$ & 1.2385 & 050 & 6. 9978 & \\
\hline AR 3 & & & 1 & $1.228 \dot{b}$ & 1.2286 & 103.10 & 1.6593 & \\
\hline MRR 4 & & & 0 & 1.2286 & 0.0000 & 103.10 & 0.0000 & \\
\hline HRF 5 & & & 0 & 1.2286 & 0.0000 & 103.10 & 9.5000 & \\
\hline \multirow[t]{2}{*}{ MRR E } & & & 0 & 1.2286 & 0.0000 & 103.10 & $0.0000^{\circ}$ & \\
\hline & & & & 1.2296 & 0.0000 & & & \\
\hline \multirow[t]{2}{*}{ LDHER WQLLEY } & 8650 & 7698.51 & 3 & 1.2286 & 0.4095 & $11: 28.21$ & 0.69 & 0.69 \\
\hline & & & & 1.2286 & 0.5000 & & & \\
\hline \multirow[t]{2}{*}{ UTLTES SHHFL } & 23973 & 22256.43 & 7 & $1.22 \mathrm{Bg}$ & 0.1755 & & & \\
\hline & & & & 1.2286 & 0.0000 & & & \\
\hline \multirow[t]{2}{*}{ OTH UTLTES-3 } & 22722 & & & $1.22 \mathrm{ES}$ & 0.0000 & & & \\
\hline & & & & 1.2206 & 0.0000 & & & \\
\hline \multirow[t]{2}{*}{ SUESTRATUH } & 46595 & 13439.80 & 7 & 1.2286 & 0.1755 & NA & 1.7374 & \\
\hline & & & & 1.2206 & 0.0000 & & & \\
\hline MEA 1 & & & 1 & 1.2286 & 1.2286 & 351.97 & $0.7: 25$ & \\
\hline MER 2 & & & & 1.2286 & & 351.97 & & \\
\hline MFE 3 & & & 1 & $1.2280^{\circ}$ & 1.2296 & $55 ! .37$ & 2.1202 & \\
\hline Heg 4 & & & 0 & 1.2286 & 0.0050 & 351.37 & 0.0000 & \\
\hline MRE 5 & & & 1 & 1.2296 & 1.3286 & 351.87 & 0.1873 & \\
\hline \multirow[t]{2}{*}{ MRR is } & & & 0 & i. 7886 & 0.0060 & 351.97 & 0.0090 & \\
\hline & & & & 1.2286 & 0.1000 & & & \\
\hline \multirow[t]{2}{*}{ FALL GIVER } & 4844 & 3501.92 & $\mathfrak{J}$ & 1.2286 & 0.4965 & 3825.90 & 0.72 & $0 . \%$ \\
\hline & & & & 1.2286 & 0.0000 & & & \\
\hline AES 1 & & & 1 & 1.2266 & i. 2265 & 166.21 & 1. 5000 & \\
\hline \multirow[t]{2}{*}{ 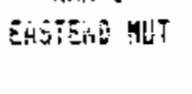 } & $\mathrm{NA}$ & $\mathrm{NA}$ & 1 & 1.2280 & 1.2200 & 1273.30 & พิ艹 & th \\
\hline & & & & 1.2286 & 46090 & & & \\
\hline \multirow{3}{*}{$\begin{array}{c}\text { MRE I } \\
\text { FGFMERE ElE }\end{array}$} & & & 1 & $1.22 E 5$ & 1.2256 & 54.00 & a. $0(i i j)$ & \\
\hline & NA & NA & 1 & 1,2286 & 1.2096 & 1275.39 & $N$ & $\mathrm{NA}$ \\
\hline & & & & 1.2280 & $0 . j 000$ & & & \\
\hline
\end{tabular}




\begin{tabular}{|c|c|c|c|c|c|c|c|c|}
\hline ERITIY & $\begin{array}{l}\text { DTHEF TOT } \\
\text { EOPULATIOH }\end{array}$ & $\begin{array}{c}\text { OTHER ELIEBL } \\
\text { IOT POFS }\end{array}$ & $\begin{array}{l}\text { COHPLETED } \\
\text { ENTERVIEAS }\end{array}$ & $\begin{array}{l}\text { AVG CUKAL } \\
\text { PER KRR }\end{array}$ & $\begin{array}{l}\text { ENDIV!DUAL } \\
\text { WEIGHTS }\end{array}$ & $\begin{array}{l}\text { AVERAEE } \\
\text { POFIJEATIOK }\end{array}$ & $\begin{array}{l}\text { MRR } \\
\text { WEIGRTS }\end{array}$ & $\begin{array}{l}\text { WElLITY } \\
\text { WEIEHTS }\end{array}$ \\
\hline 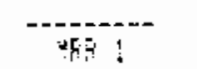 & & & 1 & and & 1.25: & 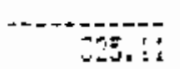 & $\cdots$ & \\
\hline Frgergide EL & NA & WA & 1 & $\begin{array}{l}1.3256 \\
1.2800\end{array}$ & $\begin{array}{l}1.228 t \\
0.600 t\end{array}$ & 1275.5 & ift & mis \\
\hline sing 1 & & & 1 & 1.2286 & 1.2296 & 500.60 & 1,5000 & \\
\hline SQUTHSIDE SI & NA & $\because A$ & 1 & $\begin{array}{l}1.2286 \\
1.2286\end{array}$ & $\begin{array}{l}1.2280 \\
0.6006\end{array}$ & $\{275.36\}$ & $\mathrm{BH}$ & SA \\
\hline MER 1 & & & 0 & 1.2286 & 0.6000 & 224.59 & 0.0000 & \\
\hline MFF 2 & & & 1 & $1.22 \mathrm{eb}$ & 1.2296 & 234.59 & 1.0000 & \\
\hline !JHITY LEP & NA & NA & 1 & $\begin{array}{l}1.2285 \\
1.2285\end{array}$ & $\begin{array}{l}1.2260 \\
0.0000\end{array}$ & 1275.30 & $\mathrm{NA}$ & HA \\
\hline HINOEKA FROJ & 4526,00 & 4149.88 & ith & $\begin{array}{l}1.2286 \\
1.2286 \\
1.2206\end{array}$ & $\begin{array}{l}0.9000 \\
0.0000 \\
0.0000\end{array}$ & 3875.90 & 1.18 & 1.08 \\
\hline IJTLES SAMYPL & 9470.00 & 7651.80 & $B$ & $\begin{array}{l}1.2286 \\
1.2286\end{array}$ & $\begin{array}{l}0.1536 \\
0.0000\end{array}$ & & & \\
\hline OTH UTLTES $\rightarrow 4$ & 10366 & & & $\begin{array}{l}1.2286 \\
1.2286\end{array}$ & $\begin{array}{l}0.13000 \\
19.0000\end{array}$ & & & \\
\hline SUESTEATUH & 19836 & 16027.58 & $B$ & $\begin{array}{l}1.2286 \\
1.2286\end{array}$ & $\begin{array}{l}0.1530 \\
0.0000\end{array}$ & NA & 0.5735 & \\
\hline MRE 1 & & & 1 & $1.228 \mathrm{gs}$ & 1.2286 & 200.49 & $\{.0396$ & \\
\hline KRR 2 & & & 0 & 1.2296 & 0.0000 & 208.49 & 0.0000 & \\
\hline $\operatorname{MER} 5$ & & & 0 & $\$ .2286$ & 0.0000 & 209.49 & 0.0000 & \\
\hline MPR 4 & & & 1 & 1.2286 & 1.2286 & 208.49 & 1.0430 & \\
\hline MRR 5 & & & 1 & 1.2206 & 1.7286 & 208.49 & 1.0430 & \\
\hline 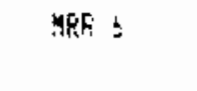 & & & 1 & $\begin{array}{l}1.2386 \\
1.2286\end{array}$ & $\begin{array}{l}1.2286 \\
0.0000\end{array}$ & 208.49 & 0.0754 & \\
\hline LOST RIVER & 1398 & 1220.92 & 4 & $\begin{array}{l}1.2286 \\
1.2286\end{array}$ & $\begin{array}{l}0.3071 \\
0.0000\end{array}$ & 1528.49 & 0.80 & 0.80 \\
\hline MRR 1 & & & & 1.2286 & & & & \\
\hline HFR 2 & & & 1 & 1.2286 & 1.2286 & {$[19.83$} & 1.0000 & \\
\hline MRR 3 & & & 0 & 1.2286 & 0.0000 & 119.83 & 0.0000 & \\
\hline ARR 4 & & & 0 & 1.2286 & 0.0000 & 119.83 & 0.0000 & \\
\hline AKR 5 & & & 0 & 1.2286 & 0.0000 & 119.83 & 0.0000 & \\
\hline MFR b & & & 0 & $\begin{array}{l}1.2286 \\
1.2286\end{array}$ & $\begin{array}{l}0.0000 \\
0.0000\end{array}$ & 119.83 & 0.0000 & \\
\hline HEISER & 1950 & 1836.07 & 1 & $\begin{array}{l}1.2286 \\
1.22 \mathrm{Bb}\end{array}$ & $\begin{array}{l}1.2286 \\
0.0000\end{array}$ & 1528.49 & 1.20 & 1.20 \\
\hline UTLTES SAMPL & 3348 & 3056.99 & 5 & $\begin{array}{l}1.2296 \\
1.2286\end{array}$ & $\begin{array}{l}0.2457 \\
0.0000\end{array}$ & & & \\
\hline OTH UTLIES-4 & 5988 & & & $\begin{array}{l}1.2285 \\
1.2286\end{array}$ & $\begin{array}{l}0.0000 \\
0.0000\end{array}$ & & & \\
\hline SUBSTKATWH & 9336 & 8524.50 & 5 & $\begin{array}{l}1.2286 \\
1.2286\end{array}$ & $\begin{array}{l}0.2457 \\
0.1000\end{array}$ & HA & 0.5668 & \\
\hline STRATUH TOT & 75767 & 67941.88 & 20 & $\begin{array}{l}1.2286 \\
1.2286\end{array}$ & $\begin{array}{l}0.0614 \\
0.0000\end{array}$ & NA & 0.36 & \\
\hline REEIOH 3 & & & & $\begin{array}{l}1.2286 \\
1.2296 \\
1.2296\end{array}$ & $\begin{array}{l}0.0000 \\
0.0070 \\
0.6000\end{array}$ & & & \\
\hline YKF 1 & & & 0 & 1.2296 & 0.0000 & 580.55 & 0.6000 & \\
\hline $\operatorname{MRR} 2$ & & & 0 & 1.2266 & 0.0000 & 560.55 & 6. 00000 & \\
\hline MRR 3 & & & 0 & 1.2286 & 0.0000 & 580.55 & 0.0000 & \\
\hline RRR 4 & & & 1 & 1.2286 & 1.2286 & 580.55 & 1.0000 & \\
\hline MRR 5 & & & 0 & 1.2286 & 0.0000 & 580.55 & 13.0000 & \\
\hline
\end{tabular}




\begin{tabular}{|c|c|c|c|c|c|c|c|c|}
\hline ENTITY & $\begin{array}{l}\text { BTHE TET } \\
\text { OPLLATISM }\end{array}$ & 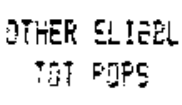 & $\begin{array}{l}\text { AAFIETED } \\
\text { IHTEGIENS }\end{array}$ & $\begin{array}{l}\text { AVG ERES } \\
\text { PEP MFR }\end{array}$ & $\begin{array}{l}\text { WDIWIDLS } \\
\text { HElgHTS }\end{array}$ & $\begin{array}{l}\text { AVERAGE } \\
\text { FMFGLATIGN }\end{array}$ & $\begin{array}{c}\text { MRR } \\
\text { HEIGHTS }\end{array}$ & $\begin{array}{l}\text { MILETY } \\
\text { WETGHTE }\end{array}$ \\
\hline 政 & --1 & - & 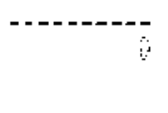 & $\begin{array}{l}1.256 \\
1.2256\end{array}$ & \begin{tabular}{l}
- \\
\hdashline \\
$\dot{v} \cdot \mathrm{seng}$
\end{tabular} & 酒, & bu & $\cdots$ \\
\hline 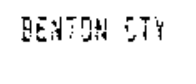 & 20631 & 23075.83 & ؛ & $\begin{array}{l}1.2286 \\
1.2255\end{array}$ & $\begin{array}{l}1.7756 \\
0.7000\end{array}$ & 316412.72 & 1.77 & 1.67 \\
\hline ME! ! & & & 1 & 1.5266 & 1.2285 & 315.41 & 8.7071 & \\
\hline MRE ? & & & 1 & $1.28 s$ & 1.2280 & 319,41 & 0.3755 & \\
\hline 䛲 3 & & & $!$ & $1.22 B \mathrm{~b}$ & 1.2286 & 31541 & 1.0870 & \\
\hline MAP A & & & 1 & 1.2286 & 1.2290 & 317.41 & 1.3838 & \\
\hline MER & & & $!$ & $\begin{array}{l}1.2286 \\
1.2286\end{array}$ & $\begin{array}{l}1.2296 \\
i .0000\end{array}$ & 315.11 & $1 .: 571$ & \\
\hline CHELAN STI & 勘 & 0.90 & 5 & $\begin{array}{l}1.2250 \\
1.2286\end{array}$ & $\begin{array}{l}\text { 6.2457 } \\
\text { ט. } 0 \text { (rivo }\end{array}$ & is & NA & $N B$ \\
\hline MRE ! & & & 1 & 1.2286 & 1.2286 & 2127.16 & 1. 10000 & \\
\hline CASHMERE & NA & 0.00 & 1 & $\begin{array}{l}1.2206 \\
1.2286\end{array}$ & $\begin{array}{l}1.2285 \\
\dot{0} .2000\end{array}$ & NA & $N A$ & HA \\
\hline CHELKK-CSHMRE & 21605.60 & 20204.61 & 0.00 & $\begin{array}{l}1.2266 \\
1.2286\end{array}$ & $\begin{array}{l}0.2048 \\
0.6000\end{array}$ & 21540.22 & 0.93 & 0.93 \\
\hline VİLTES SAMPL & 49296 & $432 B O$ & 7 & $\begin{array}{l}1.2286 \\
1.2286\end{array}$ & $\begin{array}{l}0.1755 \\
0.0000\end{array}$ & & & \\
\hline OTH UTLTES-2 & $38 B 45$ & & & $\begin{array}{l}1.2286 \\
1.2286\end{array}$ & $\begin{array}{l}0.0000 \\
0.0000\end{array}$ & & & \\
\hline SUESTEATUH & 87141 & 78091.37 & 7 & $\begin{array}{l}1.22 \mathrm{Bb} \\
1.2 \approx \mathrm{Bb}\end{array}$ & $\begin{array}{l}0.1755 \\
0.5900\end{array}$ & RA & 2.0647 & \\
\hline AhR I & & & 1 & 1.2200 & 1.2280 & 262.68 & 0.278 & \\
\hline MPR 2 & & & 1 & 1.2286 & $i, 229 t$ & 76.2 .9 & 0.3967 & \\
\hline 期 3 & & & 0 & 1.2286 & 0.0000 & 262.68 & 0. v้งต์ & \\
\hline MRR 4 & & & 1 & 1.2286 & 1.2296 & 262.38 & 2.1500 & \\
\hline MFR 5 & & & 1 & 1.2286 & $1.2280^{\circ}$ & 262.69 & 1.1597 & \\
\hline MRP $E$ & & & & $\begin{array}{l}1.2296 \\
1.2286\end{array}$ & 0.0000 & & & \\
\hline FRAKKLIN & 11623 & 8.506 .34 & 4 & $\begin{array}{l}1.2286 \\
1.2286\end{array}$ & $\begin{array}{l}0.3071 \\
0.00000\end{array}$ & 10332.55 & 0.80 & 0.50 \\
\hline MER ! & & & 1 & 1.2286 & 1.2286 & 157.75 & 0.8558 & \\
\hline MRT 2 & & & 0 & 1.2286 & 0,0000 & 157.75 & 0.0000 & \\
\hline$\times E:$ & & & $!$ & 1.2296 & 1.2285 & 157.75 & 1.0396 & \\
\hline He 4 & & & 1 & $1.228 \mathrm{~s}$ & 1.2286 & 157.75 & 0.9748 & \\
\hline MRR 5 & & & 1 & 1.2286 & $1.228 \mathrm{~b}$ & 17.75 & 1.2299 & \\
\hline$M R R$ & & & 0 & $\begin{array}{l}1.2296 \\
1.2286\end{array}$ & $\begin{array}{l}0.0000 \\
0.0000\end{array}$ & 157.75 & 0.0000 & \\
\hline 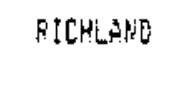 & 13123 & 12359.75 & 4 & $\begin{array}{l}1.22966 \\
1.2296\end{array}$ & $\begin{array}{l}0.3071 \\
0.0000\end{array}$ & 10332.55 & $! .20$ & 1.30 \\
\hline UTLTES SAMPL & 24751.00 & 20665.09 & 3 & $\begin{array}{l}1.2206 \\
1.2286\end{array}$ & $\begin{array}{l}0.1536 \\
0.0000\end{array}$ & & & \\
\hline OTA ITILTES-5 & 40314 & & & $\begin{array}{l}1.2286 \\
1.2586\end{array}$ & $\begin{array}{l}0.000 \hat{0} \\
0.0000\end{array}$ & & & \\
\hline SUESTRATUN & 65065 & 54324.04 & B & $\begin{array}{l}1.2296 \\
1.2280^{\circ}\end{array}$ & $\begin{array}{l}0.1536 \\
0.0900\end{array}$ & SA & 1.1972 & \\
\hline MhR I & & & 0 & 1.2796 & 0.0000 & 58.73 & 0.0000 & \\
\hline$H R P=$ & & & $\psi$ & 1.2286 & $6.00(1)$ & 58.7 & 0.600 & \\
\hline ASE 3 & & & 1 & 1.2280 & $1.22 E S$ & 59.7 & 0.8432 & \\
\hline MEE 4 & & & $i$ & $\begin{array}{l}1.2296 \\
1.22 B 6\end{array}$ & $\begin{array}{l}1.7286 \\
0.0000\end{array}$ & 55.73 & 1.1579 & \\
\hline PEND DRIELLLE & 3838 & 3774.03 & 2 & 1.2200 & $0.614]$ & 45.20 & 0.90 & $\therefore 80$ \\
\hline
\end{tabular}




\begin{tabular}{|c|c|c|c|c|c|c|c|c|}
\hline & $\begin{array}{l}\text { DTHEF TOT } \\
\text { FTOOH ATIOH }\end{array}$ & $\begin{array}{c}\text { OTHEX ELIGEL } \\
\text { TAT FES }\end{array}$ & CQMPLETEQ & AVG CONPL & $\begin{array}{l}\text { INOIVIDJAL } \\
\text { SIE IGHTC }\end{array}$ & $\begin{array}{l}\text { AVEFAGE } \\
\text { FOPII GTIOK }\end{array}$ & $\begin{array}{c}\text { ARR } \\
\text { HEIGHTS }\end{array}$ & GILITY \\
\hline EM!ITY & & & & & & & & \\
\hline - & n--- & $\cdots$ & & 人. & 6. 060 & - & $\cdots-$ & \\
\hline HeP 1 & & & 1 & 1.2286 & 1.2286 & 281,37 & $\therefore 650$ & \\
\hline MRE 2 & & & 1 & $1.22 \mathrm{st}$ & 1.2336 & $281 . \overline{27}$ & EE4 & \\
\hline MRE $j$ & & & ! & 1.280 & 1.2286 & 281.37 & 1.0271 & \\
\hline WR 4 & & & $\vdots$ & 1.2286 & 1.2236 & 281.37 & 1.0378 & \\
\hline & & & & 1.2256 & 0.0000 & & & \\
\hline VERA IRA & 4856 & 4630.37 & 4 & 1.2286 & 0.3071 & 4202.20 & 1.10 & 1.10 \\
\hline & & & & 1.2786 & 0.0000 & & & \\
\hline WTLIES SAHPL & 8694 & 8404.40 & 0 & $1.228 \mathrm{o}$ & 0.2048 & & & \\
\hline & & & & 1.2286 & 0.0000 & & & \\
\hline BTH UILTES-3 & 10257 & & & 1.2200 & 0.0000 & & & \\
\hline & & & & 1.2286 & 0.0000 & & & \\
\hline SUESTRATUH & 18957 & 18325.54 & 6 & 1.2286 & 0.2048 & $\mathrm{HA}$ & 0.5208 & \\
\hline & & & & $1.22 B 6$ & 0.0000 & & & \\
\hline MhR 1 & & & $!$ & 1.2286 & 1.2286 & 295.13 & 0.8213 & \\
\hline 柾民 2 & & & 1 & 1.2286 & 1.2296 & 295.13 & 0.9315 & \\
\hline MRR 3 & & & & 1.2286 & & 295.13 & & \\
\hline MRR 4 & & & 1 & 1.2286 & 1.2286 & 295.13 & 1.2472 & \\
\hline & & & & 1.2286 & 0.0000 & & & \\
\hline FESRY CTY & 1746 & 1339.01 & 3 & 1.2286 & 0,4095 & 1412.23 & 0.95 & 0.95 \\
\hline & & & & 1.2286 & 0.0000 & & & \\
\hline MRR ! & & & 1 & 1.2286 & 1.2286 & $23: .77$ & 1.1520 & \\
\hline KEP 2 & & & 1 & 1.2286 & 1.2286 & 231.77 & 0.8460 & \\
\hline & & & & 1.2286 & 6.0000 & & & \\
\hline HESEEEE & is & NAP & 2 & 1.2296 & 9.5143 & $\mathrm{HA}$ & NA & is \\
\hline & & & & 1.2286 & 0.0000 & & & \\
\hline KRF 1 & & & 1 & 1.2286 & 1.2286 & 71.40 & 1.2157 & \\
\hline MRR 2 & & & 1 & 1.2286 & 1.2286 & 71.40 & 0.7843 & \\
\hline & & & & 1.2286 & 0.0000 & & & \\
\hline COULEE DAH & NA & HA & 2 & 1.2286 & 0.6143 & MA & NA & NA \\
\hline & & & & 1.2286 & 0.0000 & & & \\
\hline KSFLH-COULEE & 1623.00 & 1485.46 & & 1.2286 & 0.0000 & 1412.23 & 1.05 & 1.05 \\
\hline & & & & 1.2286 & 0.0000 & & & \\
\hline UTLTES SAMPL & 3369.00 & 2924.46 & $i$ & 1.2286 & 0.1755 & & & \\
\hline & & & & 1.2286 & 0.0000 & & & \\
\hline GTH UĨLTES-4 & 5747 & & & 1.2786 & 0.0600 & & & \\
\hline & & & & 1.2286 & 0.0000 & & & \\
\hline SUESTRATIM & 9116 & 7642.57 & 7 & 1.2296 & 0.1755 & NA & 0.1911 & \\
\hline & & & & $1.228 \mathrm{Bb}$ & 0.0000 & & & \\
\hline SIRATLA TOT & 180279 & 158383.51 & 29 & 1.2286 & 0.0439 & NA & 0.60 & \\
\hline & & & & 1.2286 & 0.0000 & & & \\
\hline & & & & 1.2286 & 0.0000 & & & \\
\hline RESION 4 & & & & 1.2286 & 0.0000 & & & \\
\hline & & & & 1.2296 & 0.0000 & & & \\
\hline & & & & 1.2286 & 0.0000 & & & \\
\hline & & & & 1.2286 & 0.6000 & & & \\
\hline$M R R 1$ & & & 0 & 1.2286 & 0.0000 & 377.12 & 0.0000 & \\
\hline Ma 2 & & & 1 & 1.2286 & 1.2286 & 377.12 & 1.0686 & \\
\hline $\operatorname{Mag} j$ & & & 2 & $1.2=96$ & U.614J & 377.12 & $0.94: 5$ & \\
\hline MEP 4 & & & 2 & $1.328 b$ & 0.6143 & $77 .: 2$ & 1.0054 & \\
\hline HeR 5 & & & 1 & 1.2208 & 1.2286 & 37.12 & 0.9697 & \\
\hline MRR o & & & 2 & 1.2286 & 0.6143 & 377.12 & 1.1067 & \\
\hline & & & & 1.2266 & 0.0000 & & & \\
\hline
\end{tabular}




\begin{tabular}{|c|c|c|c|c|c|c|c|c|}
\hline E!!T!ד & $\begin{array}{l}\text { QTHEA TOI } \\
\text { POPILATIQH }\end{array}$ & $\begin{array}{c}\text { OTYEF ELIGÉL } \\
\text { TOT POPS }\end{array}$ & $\begin{array}{l}\text { COMPLETED } \\
\text { TMTEFVIEHS }\end{array}$ & $\begin{array}{l}\text { AVG CDLSE } \\
\text { PER HRK }\end{array}$ & $\begin{array}{l}\text { INEIH? VLAL } \\
\text { HEIGHTE }\end{array}$ & $\begin{array}{l}\text { AVERASE } \\
\text { PDFLLATICH }\end{array}$ & $\begin{array}{c}\text { MRK } \\
\text { BEIGHTE }\end{array}$ & 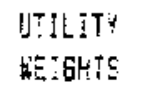 \\
\hline 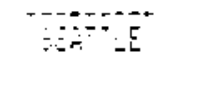 & itipi & 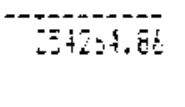 & 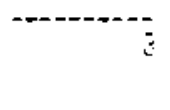 & $\begin{array}{l}1.2750 \\
1.250\end{array}$ & $\begin{array}{l}0.05 \\
0.000\end{array}$ & botitis? & $\therefore 27$ & 1.22 \\
\hline a & & & 1 & i. 200 & 1.288 & 179.10 & $\therefore 44 \div 9$ & \\
\hline MER 2 & & & 1 & $1,22 \mathrm{E}$ & 1.2536 & 179.16 & 1.0456 & \\
\hline$\triangle F$ & & & 1 & $1.2 \div 86$ & 1.2236 & 159.15 & 1.2512 & \\
\hline MCR 4 & & & 2 & 1.2286 & 0.9145 & 195.16 & 1.2000 & \\
\hline YRK 5 & & & 1 & 1.2286 & 1.2286 & 199.16 & 0.9239 & \\
\hline WF 6 & & & 1 & $\begin{array}{l}1.2286 \\
1.2286\end{array}$ & $\begin{array}{l}1.2256 \\
0.00000\end{array}$ & 199.16 & 1.1300 & \\
\hline SNDKDWISh' & 175507 & $1317 \% 4.40$ & 7 & $\begin{array}{l}1.2786 \\
1.286\end{array}$ & $\begin{array}{l}9.1: 35 \\
0.1060\end{array}$ & 192494.63 & 0.68 & 9.06 \\
\hline $\begin{array}{l}\text { ZLFES SAREL } \\
\text { ETH UIITES- }\end{array}$ & 395685 & 384989.26 & 15 & $\begin{array}{l}1.286 t \\
1.2286\end{array}$ & $\begin{array}{l}0.0049 \\
0.0000\end{array}$ & & & \\
\hline SLESTRATUA & 395685 & 384989.26 & 15 & $\begin{array}{l}1.2286 \\
1.2286\end{array}$ & $\begin{array}{l}0.0819 \\
0.00100\end{array}$ & $N A$ & 2.8275 & \\
\hline MER 1 & & & 2 & $1.22 \mathrm{Bb}$ & 0.6145 & 326.15 & $0.791 \hat{0}$ & \\
\hline $\mathrm{MEF} 2$ & & & 2 & $1.22 \mathrm{Bb}$ & 0.6143 & 326.15 & 1.9549 & \\
\hline MES 3 & & & 2 & 1.2286 & 0.6143 & 526.15 & 1.1167 & \\
\hline MRR 1 & & & 1 & 1.2286 & 1.2296 & 326.15 & 0.4722 & \\
\hline MRR 5 & & & 1 & 1.2266 & $1.22 \mathrm{eb}$ & 326.15 & 1.5353 & \\
\hline ARP 6 & & & 0 & $\begin{array}{l}1.2286 \\
1.2296\end{array}$ & $\begin{array}{l}0.0000 \\
0.0000\end{array}$ & 326.15 & 0.00000 & \\
\hline CXFAC CT & 74392 & 66836.32 & 8 & $\begin{array}{l}1.258 t \\
\vdots .235 t\end{array}$ & $\begin{array}{l}0.1536 \\
0.9050\end{array}$ & 78377.27 & 3.85 & 0.85 \\
\hline $\tan 1$ & & & 2 & $\therefore 22 \mathrm{gt}$ & 0.5147 & 244.04 & 1.1922 & \\
\hline He 1 & & & 2 & 1.2286 & 0.6145 & 244.04 & 0.9544 & \\
\hline ykE 3 & & & 1 & 1.2296 & 1.2286 & 144.i)4 & 0.9480 & \\
\hline 螒长 1 & & & 2 & 1.2286 & 0.6143 & 144.64 & 1.0899 & \\
\hline GR 5 & & & 1 & 1.2206 & 1.2286 & 144.04 & 0.8812 & \\
\hline AFR $b$ & & & 1 & $\begin{array}{l}1.2586 \\
1.2286\end{array}$ & $\begin{array}{l}1.22 \mathrm{a} 6 \\
0.0000\end{array}$ & 144.04 & 0.9342 & \\
\hline TACOAA & 96758 & 89918.16 & 9 & $\begin{array}{l}1.2286 \\
1.2286\end{array}$ & $\begin{array}{l}0.1365 \\
0.1000\end{array}$ & 78377.24 & 1.15 & 1.15 \\
\hline UTLTES SAMPL & 171130 & 156754.48 & 17 & $\begin{array}{l}1.2296 \\
1.2286\end{array}$ & $\begin{array}{l}0.0723 \\
0.0000\end{array}$ & & & \\
\hline OTH tiTitss-2 & 6.3267 & & & $\begin{array}{l}1.2286 \\
1.2286\end{array}$ & $\begin{array}{l}0.9000 \\
0.0000\end{array}$ & & & \\
\hline SUESTRATIHA & 234397 & 214706.93 & 17 & $\begin{array}{l}1.22 E 6 \\
1.2286\end{array}$ & $\begin{array}{l}0.0723 \\
0.0000\end{array}$ & NA & 1.5107 & \\
\hline MPR I & & & 0 & 1.2286 & 0.0015 & 317.99 & 0.0000 & \\
\hline 将R 2 & & & 2 & 1.2296 & 0.6143 & 317.99 & 1.1416 & \\
\hline MRA J & & & 2 & 1.2205 & 0.6143 & 317.99 & 0.6868 & \\
\hline IRR 4 & & & 1 & 4.2286 & $1.2 \% 06$ & 317.99 & 1.6730 & \\
\hline MRR 5 & & & 2 & 1.2250 & $0.614 j$ & 317.99 & 0.55 .5 & \\
\hline MRR & & & 2 & $\begin{array}{l}1.3296 \\
1.2296\end{array}$ & $\begin{array}{l}0.614 \pi \\
0.0000\end{array}$ & 317.99 & 0.9451 & \\
\hline YASOA CTY -3 & 16233 & 14486.45 & 7 & $\begin{array}{l}1.2256 \\
1.2296\end{array}$ & $\begin{array}{l}0.1365 \\
0.0000\end{array}$ & 17507.72 & 1.04 & 5.04 \\
\hline Mht & & & $\checkmark$ & 1.2586 & (1. 10000 & 425,06 & . .890 & \\
\hline HRR 2 & & & 2 & 1.2296 & $0.6 \mathrm{id} \bar{z}$ & 420.00 & 1.354 & \\
\hline Wfen 3 & & & 1 & $\vdots .2285$ & 1.2226 & 40.06 & 1.018 & \\
\hline PRR 4 & & & $!$ & 1.2286 & 1.2266 & 426.06 & 0.6305 & \\
\hline HKR 5 & & & 1 & 1.2926 & 1.7236 & 900.00 & 0.0351 & \\
\hline
\end{tabular}




\begin{tabular}{|c|c|c|c|c|c|c|c|c|}
\hline E!dTITY & $\begin{array}{l}\text { DIHER TOT } \\
\text { EOP!IETION }\end{array}$ & $\begin{array}{c}\text { DTHER ELIGEL } \\
\text { TDT POPS }\end{array}$ & $\begin{array}{l}\text { COMPLETED } \\
\text { INTEPUIENS }\end{array}$ & $\begin{array}{l}\text { ANG COHFL } \\
\text { PET MRR }\end{array}$ & $\begin{array}{l}\text { INDTVIDUAL } \\
\text { AEISHTS }\end{array}$ & $\begin{array}{l}\text { AVERAGE } \\
\text { PIIPULATION }\end{array}$ & $\begin{array}{c}\text { HRR } \\
\text { HEIEHTS }\end{array}$ & $\begin{array}{l}\text { UTILITY } \\
\text { HEISHTS }\end{array}$ \\
\hline 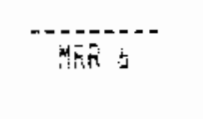 & $----1-1$ & - & 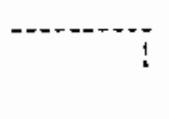 & $\begin{array}{l}1.280 \\
1.2280\end{array}$ & 19. & 46.100 & ..5845 & \\
\hline CLALLFIS CTY & $: 55: B$ & 13733.99 & 6 & $\begin{array}{l}1.2286 \\
1.2286\end{array}$ & $\begin{array}{l}0.2048 \\
0.0000\end{array}$ & 15967,72 & 0.96 & $i i_{0}=0$ \\
\hline UTLIES SAHPL & 31751 & 27615.44 & 15 & $\begin{array}{l}1.2056 \\
1.2286\end{array}$ & $\begin{array}{l}1.0919 \\
0.6000\end{array}$ & & & \\
\hline סTH UTLTES-3 & 40669 & & & $\begin{array}{l}1.2286 \\
1.2286\end{array}$ & $\begin{array}{l}0.00000 \\
0.0000\end{array}$ & & & \\
\hline SUASTFATUM & 72420 & 63447.48 & 15 & $\begin{array}{l}1.2286 \\
1.2286\end{array}$ & $\begin{array}{l}0.0819 \\
0.0000\end{array}$ & NA & $0.497 b$ & \\
\hline 政 1 & & & 2 & 1.2286 & 0.5143 & $366.6 \hat{0}$ & 1.0213 & \\
\hline MER 2 & & & $i$ & 1.2286 & 0.3143 & 366.60 & 0.6819 & \\
\hline HFR J & & & 1 & 1.2296 & 1.228 & 366.60 & 1.4457 & \\
\hline \multirow[t]{2}{*}{ MEN 4} & & & 1 & $\therefore .2286$ & 1.2296 & 366.60 & 0.8511 & \\
\hline & & & & 1.2286 & 0.0000 & & & \\
\hline \multirow[t]{2}{*}{ ELMHURST MUT } & 7225 & 6984.17 & 6 & 1.2286 & 0.2048 & 5845.01 & 1.19 & 1.19 \\
\hline & & & & 1.2286 & 0.0000 & & & \\
\hline MRF 1 & & & 2 & $1.22 \mathrm{Bb}$ & 0.6143 & 1165.28 & 1.0012 & \\
\hline MRR 2 & & & 1 & $1.22 \mathrm{Bb}$ & 1.2286 & 1165.28 & 0.9964 & \\
\hline MRR 3 & & & 2 & $1.22 \mathrm{Bb}$ & 0.6143 & 1165.26 & 1,0012 & \\
\hline \multirow[t]{2}{*}{ MRR 4} & & & 2 & $1.22 B 6$ & 0.6143 & 1165.28 & 1.0012 & \\
\hline & & & & 1.2286 & 0.0000 & & & \\
\hline \multirow[t]{2}{*}{ ORCAS PNH } & $504 \overline{8}$ & 4705.86 & 7 & 1.2256 & 0.1755 & 5845.01 & $0.9 !$ & 0.91 \\
\hline & & & & 1.2286 & 0.0000 & & & \\
\hline \multirow[t]{2}{*}{ WTLTES SAMPL } & 12273 & 11690.02 & 13 & 1.2236 & 0.0945 & & & \\
\hline & & & & 1.2296 & 0.0000 & & & \\
\hline \multirow[t]{2}{*}{ OTH UILTES-3 } & 17435 & & & 1.2296 & 0.0000 & & & \\
\hline & & & & 1.2286 & 0.0000 & & & \\
\hline \multirow[t]{2}{*}{ SUESTRATHH } & 29708 & 28296.85 & 13 & 1.2286 & 0.0945 & $N A$ & 0.2774 & \\
\hline & & & & 1.2286 & 0.0000 & & & \\
\hline MeR I & & & 2 & 1.2296 & 0.6143 & 131.54 & 0.5436 & \\
\hline WRR 2 & & & 2 & 1.2286 & 0.6143 & $13 t .54$ & 1.1859 & \\
\hline MRR J & & & 2 & 1.2286 & 0.6143 & 131.54 & 1.2062 & \\
\hline \multirow[t]{2}{*}{ Wein 4} & & & 2 & $\{.2286$ & 0.6143 & 131.54 & 1.0643 & \\
\hline & & & & 1.2256 & 0.0000 & & & \\
\hline \multirow[t]{2}{*}{ PARKLANE LQP } & 2794 & 2649.48 & 8 & 1.2286 & 0.1536 & 2995.91 & 0.99 & 0.89 \\
\hline & & & & 1.2286 & 0.0000 & & & \\
\hline MRR 1 & & & 2 & 1.2286 & 0,6143 & 108.06 & $1.0 B 49$ & \\
\hline MRR 2 & & & 2 & 1.2286 & 0.6143 & 108.06 & 0.7966 & \\
\hline$M R O J$ & & & 2 & 1.2286 & $0.614 \mathrm{~J}$ & 108.06 & 1.1600 & \\
\hline \multirow[t]{2}{*}{ IFR 4} & & & 1 & 1.2296 & 1.2296 & 108.06 & 0.9624 & \\
\hline & & & & 1.2286 & 0,0000 & & & \\
\hline \multirow[t]{2}{*}{ SKAMANIA } & 3441 & 3322.34 & 7 & 1.2286 & 0.1755 & 2985.91 & 1.11 & 1.11 \\
\hline & & & & 1.2286 & 0.0000 & & & \\
\hline \multirow[t]{2}{*}{ UTLTES SAMPL } & 6235 & 5971.83 & 15 & 1.2296 & $0.08 \div 9$ & & & \\
\hline & & & & 1.2296 & 0.0000 & & & \\
\hline \multirow[t]{2}{*}{ OTH JTLTES-2 } & 5227 & & & 1.2286 & 0.0000 & & & \\
\hline & & & & 1.2286 & 0,0000 & & & \\
\hline \multirow[t]{2}{*}{ SLESTRATEK } & 11402 & 10576.20 & 15 & 1.286 & $0.85: 9$ & NÀ & 0.1676 & \\
\hline & & & & 1.2286 & 0.00000 & & & \\
\hline HRR I & & & 2 & 1.2286 & 0.6143 & 196.42 & 2.1434 & \\
\hline MRR 2 & & & 1 & 1.2286 & 1.2286 & 196.42 & 1.0132 & \\
\hline MRR 3 & & & 1 & 1.2286 & 1.2286 & 196.42 & 0.3089 & \\
\hline
\end{tabular}




\begin{tabular}{|c|c|c|c|c|c|c|c|c|}
\hline EMTITY & $\begin{array}{l}\text { DTHER TDT } \\
\text { PUP!JLAT TAN }\end{array}$ & $\begin{array}{c}\text { OTHEK SLIGAL } \\
\text { TSTSPS }\end{array}$ & $\begin{array}{l}\text { EEMPLETED } \\
\text { IMTEFUIERS }\end{array}$ & $\begin{array}{l}\text { AWG COMPL } \\
\text { PES MFE }\end{array}$ & $\begin{array}{l}\text { IWHivinINL } \\
\text { WEIGHTS }\end{array}$ & $\begin{array}{l}\text { ANERAGE } \\
\text { PHPULATISA }\end{array}$ & $\begin{array}{c}\text { MPF } \\
\text { idEIGHTS }\end{array}$ & $\begin{array}{l}\text { WTLETY } \\
\text { WE:GHTS }\end{array}$ \\
\hline 464 & $\cdots$ & & $\vec{i}$ & $\begin{array}{l}1.280 \\
1.2 \pm 80\end{array}$ & $\begin{array}{l}0.2200 \\
0.3600\end{array}$ & 975 & 0.540 & $\cdots$ \\
\hline 3HiT H!TUA & 1032 & $\{.24 .47$ & 5 & $\begin{array}{l}1.256 \\
1.2296\end{array}$ & $\begin{array}{l}0.2457 \\
0.0000\end{array}$ & 1456.22 & $! .12$ & 1.12 \\
\hline MPT! & & & $?$ & 1.2286 & $0.614 \mathrm{Z}$ & 130.80 & 0.4057 & \\
\hline MRP 2 & & & 2 & 1.2586 & 0.6143 & 130.80 & 1.5001 & \\
\hline RRR J & & & 2 & 1.2286 & 0.6143 & 130.80 & 0.8805 & \\
\hline MER 4 & & & 1 & $\begin{array}{l}1.2286 \\
1.2296\end{array}$ & $\begin{array}{l}1.7286 \\
0.01010\end{array}$ & 170.90 & 1.2099 & \\
\hline WANSIARL & $15 ! 2$ & 1288.00 & 7 & $\begin{array}{l}1.2286 \\
1.2296\end{array}$ & $\begin{array}{l}0.1755 \\
0.6000\end{array}$ & 1452.23 & 0.86 & $0.8 \mathrm{e}$ \\
\hline UTLTES SAMPL & 3134 & 2912.47 & 12 & $\begin{array}{l}1.2286 \\
1.2286\end{array}$ & $\begin{array}{l}0.1024 \\
0.0000\end{array}$ & & & \\
\hline DTH HTLTES-5 & 7009 & & & $\begin{array}{l}1.22 B C \\
1.22 B 6\end{array}$ & $\begin{array}{l}0.0000 \\
0.6000\end{array}$ & & & \\
\hline SUJESTRATUA & 90173 & 9364.26 & 12 & $\begin{array}{l}1.2286 \\
1.2286\end{array}$ & $\begin{array}{l}3.1024 \\
0.0000\end{array}$ & NA & 0.0918 & \\
\hline STRATUM TOZ & 753845 & 711778.89 & 87 & $\begin{array}{l}1.2286 \\
1.2286\end{array}$ & $\begin{array}{l}0.0141 \\
0.0000\end{array}$ & NA & 1.36 & \\
\hline REGION 5 & & & & $\begin{array}{l}1.2286 \\
1.2286 \\
1.2286\end{array}$ & $\begin{array}{l}0.0000 \\
0.0000 \\
0.0000\end{array}$ & & & \\
\hline MRR : & & & $!$ & 1.2285 & 1.2256 & 421.42 & 1.0393 & \\
\hline MRE 2 & & & 1 & $\therefore .2286$ & 1.2280 & 421.42 & 1,4594 & \\
\hline MAE 3 & & & 1 & $\therefore .3286$ & 1.2866 & $421 .+2$ & 1.3375 & \\
\hline HRF 1 & & & : & 1.2286 & $1.22 \mathrm{Bb}$ & 221.42 & 1.4214 & \\
\hline MFR 5 & & & 1 & 1.2286 & 1.2286 & 421.42 & $0.7 \mathrm{B37}$ & \\
\hline MRR 6 & & & 0 & 1.2286 & 0.0000 & 421.42 & 0.0000 & \\
\hline HRR 7 & & & 1 & 1.2286 & 1.2286 & 421.42 & 1.0367 & \\
\hline NRR \& & & . & 1 & $\begin{array}{l}1.2286 \\
1.2286\end{array}$ & $\begin{array}{l}1.2286 \\
0.0000\end{array}$ & 421.42 & 0.5972 & \\
\hline MIOSTATE & 7797 & 6878.58 & 7 & $\begin{array}{l}1.2286 \\
1.2286\end{array}$ & $\begin{array}{l}0.1755 \\
0.0600\end{array}$ & 6437.72 & 1.07 & 1.07 \\
\hline MAR 1 & & & 1 & 1.2286 & 1.2286 & 89.00 & 0.6674 & \\
\hline MRP 2 & & & $f$ & 1.2286 & 1.2296 & 59.90 & 0.8999 & \\
\hline $4 R R 3$ & & & 0 & $1.22 B 6$ & 0.0000 & 85.00 & 0.3000 & \\
\hline MRR 4 & & & 1 & 1.2286 & 1.2282 & 65.00 & 1.2270 & \\
\hline ARE 5 & & & 1 & 1.2286 & 1.2206 & 89.00 & 1.1955 & \\
\hline IRR 6 & & & 0 & 1.2286 & 0.0000 & 69.00 & 0,0000 & \\
\hline MRT̃ 7 & & & 0 & 1.2286 & 0.0600 & 89.00 & 0.0000 & \\
\hline GRR 8 & & & 1 & $\begin{array}{l}1.2286 \\
1.2286\end{array}$ & $\begin{array}{l}1.2286 \\
0.00000\end{array}$ & 89.00 & 1.0112 & \\
\hline WHATILLA & 6546 & 5996.86 & 5 & $\begin{array}{l}1.2286 \\
1.2286\end{array}$ & $\begin{array}{l}0.2457 \\
0.0600\end{array}$ & 6437.72 & 0.93 & 0.93 \\
\hline UTLTES SAMPL & 14343 & 12875.44 & 12 & $\begin{array}{l}1.2286 \\
1.2286\end{array}$ & $\begin{array}{l}0.1024 \\
0.0000\end{array}$ & & & \\
\hline DTH UTLTES-2 & $1773 !$ & & & $\begin{array}{l}1.2286 \\
1.2296\end{array}$ & $\begin{array}{l}0.0000 \\
0.0000\end{array}$ & & & \\
\hline SUESTRETLE & 32074 & 2879.23 & 12 & $\begin{array}{l}1.2236 \\
1.2296\end{array}$ & $\begin{array}{l}0.1024 \\
0.0000\end{array}$ & HA & 1.2505 & \\
\hline MR 1 & & & 1 & 1.7786 & 1.2286 & 144.78 & 1.3745 & \\
\hline ה & & & 1 & 1.2226 & 1.2296 & 94.75 & 1.2985 & \\
\hline ARR 3 & & & 1 & 1.2298 & 1.2280 & 244.78 & 1.1466 & \\
\hline
\end{tabular}




\begin{tabular}{|c|c|c|c|c|c|c|c|c|}
\hline EHTITY & $\begin{array}{l}\text { DTHER TOT } \\
\text { POFILATIGN }\end{array}$ & $\begin{array}{c}\text { BTHER ELIEEL } \\
\text { TOT POFS }\end{array}$ & $\begin{array}{l}\text { COKPIETED } \\
\text { INTERVIEHS }\end{array}$ & $\begin{array}{l}\text { AVG COMPL } \\
\text { PER MRE }\end{array}$ & $\begin{array}{l}\text { INEIVIBUAL } \\
\text { NEIGHTS }\end{array}$ & $\begin{array}{l}\text { AVERAGE } \\
\text { EOFULATIGA }\end{array}$ & $\begin{array}{c}\text { MRR } \\
\text { WEIGHTS }\end{array}$ & $\begin{array}{l}\text { WTILITY } \\
\text { HEIEHTS }\end{array}$ \\
\hline 4ft 4 & -------- & - - & 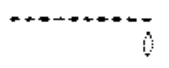 & $1.2 . \overline{s i}$ & $0.0 \mathrm{ji}) \mathrm{ij0}$ & 144.5 & i. (bing) & \\
\hline Ning 5 & & & 1 & 1,2286 & 1.2286 & 144,78 & 1.3216 & \\
\hline SARR D & & & 1 & 1.2286 & 1.2286 & 144.70 & (1. 7853 & \\
\hline KEF 7 & & & 1 & 1.2296 & 1.2206 & 144.78 & 0.6276 & \\
\hline \multirow[t]{2}{*}{ HER S } & & & 1 & 1.2280 & 1.2280 & 144.78 & 0.9454 & \\
\hline & & & & 1.2286 & 0.0000 & & & \\
\hline \multirow[t]{2}{*}{ COLUMEIA } & 2855 & 2502.23 & 7 & 1.2286 & 0.1755 & 2635.29 & 0.95 & 0.95 \\
\hline & & & & 1.2286 & 0,0000 & & & \\
\hline MFA 1 & & & 1 & 1.2286 & 1.2276 & 129.77 & 0.7456 & \\
\hline MKR 2 & & & 1 & 1.2206 & 1.2286 & $12 \mathrm{~B} .27$ & 0.9489 & \\
\hline ARR 3 & & & 0 & 1.2286 & 0.0600 & 128.27 & 0.0000 & \\
\hline MER 4 & & & 0 & 1.2286 & 0.0000 & 128.27 & 0.0000 & \\
\hline MRR 5 & & & 1 & 1.2286 & 1.2286 & 128.27 & 1.4189 & \\
\hline MKR 6 & & & 1 & 1.2286 & 1.2386 & 125.27 & 1.6175 & \\
\hline HRO 7 & & & 1 & 1.2286 & 1.2286 & 128.27 & 0.3731 & \\
\hline \multirow[t]{2}{*}{ MER } & & & 0 & 1.2286 & 0.0000 & 128.27 & 0.0000 & \\
\hline & & & & 1.2296 & 0.0000 & & & \\
\hline \multirow{2}{*}{ HLTN-FREENTR } & 2848 & 2774.34 & 5 & 1.2286 & 0.2457 & 2638.29 & 1.05 & 1.05 \\
\hline & & & & 1.2286 & 0.0000 & & & \\
\hline \multirow[t]{2}{*}{ UTLTES SAMPL } & 5703 & 5276.57 & 12 & 1.2286 & 0.1024 & & & \\
\hline & & & & 1.2286 & 0.0000 & & & \\
\hline \multirow[t]{2}{*}{ OTH SAHPL-5 } & 10634 & & & 1.2286 & 0.0000 & & & \\
\hline & & & & 1.2286 & 0.0000 & & & \\
\hline \multirow[t]{2}{*}{ SUASTRATUH } & 16337 & 15115.44 & 12 & 1.2286 & 0.1024 & 21553.93 & 0.6955 & \\
\hline & & & & 1.2296 & 0.0000 & & & \\
\hline \multirow[t]{2}{*}{ STRATA FUT } & 48411 & 43907,67 & 24 & 1.2286 & 0.0512 & NA & 0.19 & \\
\hline & & & & 1.2286 & 0.0000 & & & \\
\hline \multirow{2}{*}{ REBION 6} & & & & 1.2286 & 0.0000 & & & \\
\hline & & & & 1.2286 & 0.0000 & & & \\
\hline -......... & & & & 1.2296 & 0.0000 & & & \\
\hline KRR I & & & 2 & 1.2286 & 0.6143 & 2523.42 & 1.0090 & \\
\hline MRR 2 & & & 1 & 1.2286 & 1.2286 & 2523.42 & 1.1544 & \\
\hline MRR 3 & & & 1 & 1.2386 & 1.2286 & 2523.42 & 1.0014 & \\
\hline MRR 4 & & & 2 & 1.2296 & 0.6143 & 2523.42 & 1.0260 & \\
\hline MRP 5 & & & $!$ & 1.2286 & 1.2286 & 2523.42 & 0.9265 & \\
\hline \multirow[t]{2}{*}{ MRR 6} & & & 2 & 1.2296 & 0.6143 & 2523.42 & 0.9827 & \\
\hline & & & & 1.2286 & 0.0000 & & & \\
\hline \multirow[t]{2}{*}{ EUGEHE HTR } & 54494 & 5115.31 & 9 & 1.2286 & 0.1365 & 34692.59 & 1.47 & 1.47 \\
\hline & & & & 1.2286 & 0.0000 & & & \\
\hline$H R R$ & & & 2 & $\$ .2286$ & 0.6143 & 160.93 & 1.1062 & \\
\hline HRR 2 & & & & 1.2296 & & 160.93 & & \\
\hline MRR 3 & & & & 1.2286 & & 160.93 & & \\
\hline MRR 4 & & & 2 & 1.2286 & 0.6143 & 160.93 & 0.5930 & \\
\hline MRR 5 & & & 0 & 1.2286 & 0.0000 & 160.93 & 0.0000 & \\
\hline \multirow[t]{2}{*}{ MKR 6} & & & 2 & 1.2286 & 0.6143 & 160.97 & 1.2987 & \\
\hline & & & & 1.2286 & 0.0000 & & & \\
\hline \multirow[t]{2}{*}{ CKTRL-LHCLA } & 21337 & 18249.67 & 6 & 1.2286 & 0.2049 & 34692.59 & 0.53 & 0.53 \\
\hline & & & & 1.2286 & 0.0000 & & & \\
\hline UTLIES SAMFL & 75831 & 69385.18 & 15 & 1.2285 & 0.3619 & & & \\
\hline OTH UTLTES-1) & & & & 1.2286 & 0.0000 & & & \\
\hline \multirow[t]{2}{*}{ SUBSTRATUH } & 75831 & 64859.45 & 15 & 1.2296 & 0.0819 & NA & 1.1889 & \\
\hline & & & & 1.2286 & 0.0000 & & & \\
\hline MRR I & & & 2 & $\begin{array}{r}1.2286 \\
-\end{array}$ & 0.6143 & 377.41 & 0.9283 & \\
\hline
\end{tabular}

A. 25 


\begin{tabular}{|c|c|c|c|c|c|c|c|c|}
\hline EATITY & $\begin{array}{l}\text { UTHER TUT } \\
\text { FOFULATIGN }\end{array}$ & $\begin{array}{c}\text { BTHER ELIGGL } \\
\text { TOT FOES }\end{array}$ & $\begin{array}{l}\text { ELHPLETED } \\
\text { IHTEFVIENS }\end{array}$ & $\begin{array}{c}\text { AVS EOMFL } \\
\text { PES MAR }\end{array}$ & $\begin{array}{l}\text { THOIWIDUAL } \\
\text { HEIGHTE }\end{array}$ & $\begin{array}{l}\text { AUEFAGE } \\
\text { FSOPLLATIOI }\end{array}$ & $\begin{array}{c}\text { MER } \\
\text { NEIJHTS }\end{array}$ & $\begin{array}{l}\text { WTILITY } \\
\text { HEIEHTS }\end{array}$ \\
\hline 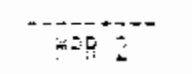 & $\cdots$ & 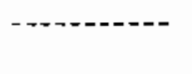 & 2 & ذ5ت5 & i. & 779.41 & 1.190 & \\
\hline Nä & & & ì & 1.25itet & 6. & (7) & ofo & \\
\hline YFi 4 & & & 0 & 1.2236 & 0.6000 & -77.41 & 6.00100 & \\
\hline 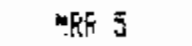 & & & $!$ & 1.2296 & 1.7286 & 377.41 & 1.0420 & \\
\hline $\operatorname{sen} 6$ & & & 2 & $\$ .2286$ & 0.0145 & $37 ? .41$ & דירוּם & \\
\hline & & & & 1.526 & i. 3600 & & & \\
\hline SPFINGFIELO & 15520 & 17808.53 & 7 & 1.2206 & 9.1755 & $145 \overline{\mathrm{B}} .01$ & $1.2 !$ & 1.21 \\
\hline & & & & 1,2280 & 0.0000 & & & \\
\hline rifa 1 & & & $1)$ & 1.2296 & 0.0060 & 07.67 & 0. $0 \hat{1006}$ & \\
\hline ARR 2 & & & 2 & 1.2298 & 0.6143 & 67.67 & {$[! .9379$} & \\
\hline : & & & 1 & 1.2280 & $\{.7286$ & 67.67 & 0.5276 & \\
\hline Nes 4 & & & 0 & 1.2286 & $1.206 \mathrm{v}$ & 57.67 & 0.0000 & \\
\hline MER 5 & & & 1 & 1.2736 & 1.2296 & 57.67 & 0.6532 & \\
\hline MRR o & & & 2 & 1.2286 & 0.6143 & 67.67 & 1.5813 & \\
\hline & & & & 1.2266 & $0.000 \%$ & & & \\
\hline CINSULERS & 12893 & 11565.50 & 6 & 1.2286 & 0.2048 & 14587.01 & 1. .79 & 0.79 \\
\hline & & & & 1,2286 & 0.0000 & & & \\
\hline UT: & 31413 & 29374.03 & $\{3$ & $1.22 \mathrm{~B} 6$ & 0.0945 & & & \\
\hline & & & & 1.2286 & 0.0060 & & & \\
\hline DTH UTLTES-2 & 22874 & & & 1.2286 & 0.0000 & & & \\
\hline & & & & 1.2286 & 0.0000 & & & \\
\hline SLESTRATUM & 54287 & 50763.31 & 13 & 1.2286 & 9.0945 & HA & 1.2122 & \\
\hline & & & & 1.2286 & 0.0600 & & & \\
\hline HER: & & & 2 & 1.2250 & 9.6143 & 102.13 & 0.9731 & \\
\hline Whe 2 & & & 2 & 1.2386 & 0.6143 & 102.13 & 1.1240 & \\
\hline MFR 3 & & & 2 & 1.2286 & 0.6143 & 102.17 & 0.7769 & \\
\hline MRR \& & & & 2 & 1.2286 & 0.5143 & 102.13 & 1.1260 & \\
\hline & & & & 1.2286 & 0.0000 & & & \\
\hline ASHLAND & 5714 & 5249.05 & 8 & 1.2286 & 0.1536 & 4979.44 & 1.65 & 1.05 \\
\hline & & & & $1.229 t$ & 0.0000 & & & \\
\hline MRR I & & & 1 & 1.2286 & 1.2286 & 94.98 & $0.958 \mathrm{i}$ & \\
\hline HER 2 & & & 2 & 1.2206 & 0.6143 & 94.99 & 0.5994 & \\
\hline SRF 3 & & & 0 & 1.2286 & 0.0000 & 94,98 & 0.0000 & \\
\hline $\operatorname{MER} 4$ & & & 1 & 1.2286 & $1.228 \mathrm{~b}$ & 94.96 & 1.4424 & \\
\hline & & & & 1.2296 & 0.0000 & & & \\
\hline FUREST SRDVE & 5158 & 4709.87 & 4 & 1.2290 & $0.3 \hat{7} 71$ & 4979.44 & 1.95 & 0.75 \\
\hline & & & & 1.2286 & 0.6000 & & & \\
\hline UTLIES SAMPL & 10852 & $995 B .8 B$ & 12 & 1.2286 & 0.1024 & & & \\
\hline & & & & 1.2286 & 0.0000 & & & \\
\hline OTH UTLTES-4 & 33252 & & & 1.2286 & 0.0000 & & & \\
\hline & & & & 1.2286 & 0.0000 & & & \\
\hline SUESTRATUH & $44 ! 04$ & 40474.26 & 12 & 1.2280 & 0.1024 & $\mathrm{NA}$ & 1.0951 & \\
\hline & & & & 1.2296 & (1.0000) & & & \\
\hline URR： & & & 2 & 1.2286 & 0.6143 & $4 d 7.04$ & 0.7245 & \\
\hline MEP 1 & & & 2 & 1.3286 & 0.6147 & 447.04 & 1.1850 & \\
\hline HER I & & & 1 & 1.2256 & 1.2286 & 447.04 & 0.9363 & \\
\hline MPE \& & & & 1 & 1.2286 & 1.2296 & 447.04 & 0.9517 & \\
\hline & & & & 1.2796 & 0.0000 & & & \\
\hline CLEEALY-LANE & 2528 & 2575.39 & $\dot{0}$ & $1.2 \mathrm{KD}$ & औ. & $278.0 ?$ & $\therefore 65$ & $\therefore .05$ \\
\hline & & & & 1,2280 & 0.00000 & & & \\
\hline MRR I & & & 1 & 1.2286 & 1.2286 & 269.26 & 1.3953 & \\
\hline 绿 2 & & & 2 & 1.2280 & 0.0143 & 269.26 & 1.0364 & \\
\hline MFF 3 & & & 0 & 1.786 & 0.6000 & 269.26 & 0.0000 & \\
\hline
\end{tabular}




\begin{tabular}{|c|c|c|c|c|c|c|c|c|}
\hline EkTITY & $\begin{array}{l}\text { OTHEF TUT } \\
\text { POPULGTJUH }\end{array}$ & $\begin{array}{c}\text { OTHER ELIGBL } \\
\text { TOT POPS }\end{array}$ & $\begin{array}{l}\text { CUHELETED } \\
\text { INTEEVIEHS }\end{array}$ & $\begin{array}{l}\text { SVE CEMPL } \\
\text { PEF MPF }\end{array}$ & $\begin{array}{l}\text { INDIVIDUAL } \\
\text { WE! IHTS }\end{array}$ & $\begin{array}{l}\text { AVEFAGE } \\
\text { POPULATICH }\end{array}$ & $\begin{array}{c}\text { MRT } \\
\text { HEIEHTS }\end{array}$ & $\begin{array}{l}\text { UTILITY } \\
\text { HEIEHTS }\end{array}$ \\
\hline 4 & - & n.t. & $\cdots$ & $\begin{array}{l}t .2296 \\
1.2286\end{array}$ & 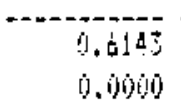 & 209.26 & $\therefore .562$ & \\
\hline nidthilTH & $25: 99$ & 2100,87 & 5 & $\begin{array}{l}1.2286 \\
1.2286\end{array}$ & $\begin{array}{l}0.2457 \\
0.00(t)\end{array}$ & 2218.13 & 1). 55 & 0.05 \\
\hline UT: _ES SAMPL & 4767 & 4436.26 & 11 & $\begin{array}{l}1.2286 \\
1.2286\end{array}$ & $\begin{array}{l}0.1117 \\
0.00100\end{array}$ & & & \\
\hline UTH UTLTES-4 & $105: 1$ & & & $\begin{array}{l}1.2296 \\
1.2286\end{array}$ & $\begin{array}{l}0.0000 \\
0.0000\end{array}$ & & & \\
\hline SUESTRATUN & 15295 & 14177.13 & 11 & $\begin{array}{l}1.2286 \\
1.2286\end{array}$ & $\begin{array}{l}0.1117 \\
0.0000\end{array}$ & NA & 0.3840 & \\
\hline STRATUH TOT & 189520 & 170274.15 & $5 !$ & $\begin{array}{l}1.2286 \\
1.2286\end{array}$ & $\begin{array}{l}0.0241 \\
0.0000\end{array}$ & HA & 0.56 & \\
\hline FUBLIC TOT & 1282101 & 1183079.68 & 232 & $\begin{array}{l}1.2286 \\
1.2286\end{array}$ & $\begin{array}{l}0.0053 \\
0.0000\end{array}$ & & 1.0000 & \\
\hline PRIVATE & & & & $\begin{array}{l}1.2286 \\
1.2286\end{array}$ & $\begin{array}{l}0.0000 \\
0.0000\end{array}$ & & & \\
\hline RESION! & & & & $\begin{array}{l}1.2286 \\
1.2286 \\
1.2286\end{array}$ & $\begin{array}{l}0.0000 \\
0.0000 \\
0.0000\end{array}$ & & & \\
\hline HRR ! & & & 1 & 1.2286 & 1.2286 & 226.94 & 0.3217 & \\
\hline MRF 2 & & & 0 & 1.2286 & 0.0000 & 226.94 & 0.0000 & \\
\hline Heg 3 & & & 1 & 1.2286 & 1.2286 & 226.74 & 0.4989 & \\
\hline $\operatorname{ARR} 4$ & & & 1 & 1.2296 & 1.2286 & 226.94 & $i, 4265$ & \\
\hline MFE 5 & & & 1 & 1.2286 & 1.2286 & 226.94 & 1). 2221 & \\
\hline MER o & & & j & $1.22 E_{6}$ & 0.0000 & 235.94 & 0.0000 & \\
\hline MKR ? & & & 1 & $1.22 \mathrm{gc}$ & 1.2236 & 226.94 & 2.2072 & \\
\hline MRR 9 & & & \pm & 1.2286 & 1.2286 & 226.94 & 1.7890 & \\
\hline MRR 9 & & & 1 & 1.2286 & 1.2286 & 226.94 & $1.81: 0$ & \\
\hline SRR 10 & & & 1 & 1.2286 & 1.2286 & 226.94 & 2.6561 & \\
\hline HRR II & & & 1 & 1.2286 & $1.22 \mathrm{Bb}$ & 226.94 & $1 . \$ 121$ & \\
\hline MTR 12 & & & 1 & $1.22 \mathrm{Bb}$ & 1.2296 & 226.94 & 0.6232 & \\
\hline MRR 13 & & & 1 & 1.2286 & $1.22 \mathrm{~B} 6$ & 226.94 & 0.5403 & \\
\hline MRR 14 & & & 1 & 1.2286 & $1.22 \mathrm{Bt}$ & 226.94 & 0.0959 & \\
\hline ARR 15 & & & 0 & 1.2286 & 0.0000 & 226.94 & 0.0000 & \\
\hline GRR it & & & 0 & $\begin{array}{l}1.2286 \\
1.2286\end{array}$ & $\begin{array}{l}0.0000 \\
0.0000\end{array}$ & 226.94 & 0.9000 & \\
\hline $\begin{array}{l}\text { HONTANA PHR } \\
\text { SUBSTRATUM }\end{array}$ & 55982 & 50372.31 & 12 & $\begin{array}{l}1.2286 \\
1.2286 \\
1.2286 \\
1.2286 \\
1.2286 \\
1.2296\end{array}$ & $\begin{array}{l}0.1024 \\
0.0000 \\
0.0000 \\
0.0000 \\
0.0000 \\
0.00000\end{array}$ & 23384.01 & 1.0000 & 1.00 \\
\hline KRR 1 & & & 1 & 1.2286 & 1.2286 & 166.39 & 0.9756 & \\
\hline WFR 2 & & & 1 & 1.2286 & 1.2286 & 166.39 & 1.2837 & \\
\hline MRE J & & & 1 & $1.22 \mathrm{gb}$ & 1.2286 & 166.39 & $\vdots .3647$ & \\
\hline MRR 4 & & & 1 & 1.2296 & 1.2286 & 166.39 & 0.5721 & \\
\hline MRR 5 & & & 0 & 1.2286 & 0.0000 & 166.39 & 0.0000 & \\
\hline MRR 6 & & & 1 & 1.2286 & 1.2280 & 266.35 & 0.6259 & \\
\hline FE? & & & 1 & 1.2256 & 1.2280 & .56 .39 & 2.7536 & \\
\hline MRR B & & & 1 & 1.2286 & 1.2296 & 166.39 & 0.9964 & \\
\hline MPR 9 & & & 1 & 1.2286 & 1.2286 & 160.39 & 0.9255 & \\
\hline KER 10 & & & 0 & 1.2286 & 0.0000 & 166.39 & 0.0000 & \\
\hline HRR 11 & & & 1 & $\begin{array}{l}\quad 1.2286 \\
\text { A. } 27\end{array}$ & 1.2286 & 166.39 & 1.1612 & \\
\hline
\end{tabular}




\begin{tabular}{|c|c|c|c|c|c|c|c|c|}
\hline ENTITY & $\begin{array}{l}\text { QTHER TOT } \\
\text { FIFULATISUV }\end{array}$ & $\begin{array}{c}\text { STHER ELIGBL } \\
\text { IOT FLPS }\end{array}$ & $\begin{array}{l}\text { COHFIETED } \\
\text { INTEEVIENS }\end{array}$ & $\begin{array}{l}\text { AVG COMEL } \\
\text { FER MRR }\end{array}$ & $\begin{array}{l}\text { INEYYIDUAL } \\
\text { HEIGHTS }\end{array}$ & $\begin{array}{l}\text { AHERAGE } \\
\text { POFULAT:SWH }\end{array}$ & $\begin{array}{c}\text { MET } \\
\text { WEISHTE }\end{array}$ & $\begin{array}{l}\text { DTILITY } \\
\text { HETGHTS }\end{array}$ \\
\hline 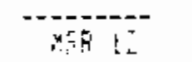 & $\cdots$ & -- & $\bar{\vdots}$ & 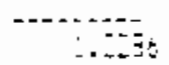 & 1.28 & 60 & 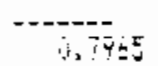 & \\
\hline ME & & & 1 & $1.22 \mathrm{Et}$ & 1.2258 & 180.74 & 1,1728 & \\
\hline STR 14 & & & 1 & 1.2285 & 1.2256 & 150.59 & \pm .2081 & \\
\hline & & & & 1.2256 & 0.0000 & & & \\
\hline Dofl-mi & 21927 & 19135.75 & 12 & 1,2200 & 0.1024 & 7869.67 & $1.0 \%$ & 1.6000 \\
\hline SUEETRETUK & & & & 1.2286 & 决政 & & & \\
\hline Nhts: & & & 0 & 1.2295 & 0.0000 & 0.00 & 0.0000 & \\
\hline Mât 2 & & & 0 & $1.22 \mathrm{Bo}$ & 0,0000 & $0 \overline{0} .00$ & ن. $0 \mathrm{sen}$ & \\
\hline & & & & 1. 2296 & 0.0000 & & & \\
\hline M! QRL & 641 & 641.60 & i) & $1.22 \mathrm{e}$ & 1). $06 \mathrm{ph}$ & 9859.87 & 1.100 & 1.6060 \\
\hline GHESTAA'DM & & & & 1.2286 & 9.0000 & & & \\
\hline$E P \mathrm{~S}_{-}-\mathrm{MT} / M T-P$ & & 19779.75 & & $1.228 \mathrm{~s}$ & 0,0000 & & & \\
\hline & & & & 1.2286 & 0.2000 & & & \\
\hline 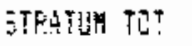 & 78550 & 70152.04 & 24 & 1.2296 & 0.0512 & NA & 4.3115 & \\
\hline & & & & 1.2256 & 0.0000 & & & \\
\hline REOIJi 2 & & & & 1.2286 & 0.0000 & & & \\
\hline 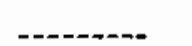 & & & & 1.2286 & 0.0000 & & & \\
\hline & & & & 1.2286 & 0.9000 & & & \\
\hline & & & & 1.2286 & 0.0000 & & & \\
\hline Men 1 & & & 1 & 1.2286 & 1.2286 & 200.21 & 1.5983 & \\
\hline MER 2 & & & $\theta$ & 1.2296 & 0.0000 & 200.21 & 0.0000 & \\
\hline FPQ 3 & & & 1 & $t .2286$ & 1.2286 & 200.21 & 1.2027 & \\
\hline$M 9$ & & & $!$ & 1.3296 & $1.2 \% 66$ & 200.21 & $6,682 \hat{v}$ & \\
\hline Hash 5 & & & 1 & 1.2296 & 1.2296 & 200.21 & $1.104 !$ & \\
\hline 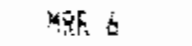 & & & 1 & 1.2666 & 1.2286 & $200.2 !$ & 0.8225 & \\
\hline MER 7 & & & 1 & 1.2286 & 1.2286 & 200.21 & O. B11! & \\
\hline MRE 8 & & & 1 & 1.2286 & 1.2286 & 200.21 & 0.7792 & \\
\hline & & & & $1.22 \mathrm{E} 6$ & 0.0000 & & & \\
\hline FPLL-10 & 6655 & 5889.68 & 7 & 5.2280 & 0.1755 & 69049.52 & 1.00 & 1.0000 \\
\hline SUBSTRATHM & & & & 1.2286 & 0.0000 & & & \\
\hline ARP L L & & & 1 & 1.2236 & 1.2280 & 274.59 & 0.9090 & \\
\hline KRR 2 & & & 1 & $1.228 \mathrm{~s}$ & $1.228{ }^{\circ}$ & 274.59 & 0.9472 & \\
\hline NAR 3 & & & 1 & $1.22 \mathrm{~B} 6$ & 1.2286 & 274.59 & 1.0775 & \\
\hline AFR 4 & & & 1 & 1.2886 & 1.2296 & 274.59 & 1.3594 & \\
\hline MRP 5 & & & 0 & $1.228 \mathrm{c}$ & 7. .0000 & 274.59 & 0,9000 & \\
\hline MRE 6 & & & 1 & 1.2256 & 1.2286 & $274 .{ }^{2} ?$ & 0.6328 & \\
\hline $\operatorname{MRR} 7$ & & & 1 & 1.2296 & $1.22 B 6$ & 274.37 & 1.0379 & \\
\hline ARR B & & & 1 & 1.2286 & 1.2296 & 274.59 & $0.93 \$ 2$ & \\
\hline & & & & 1.2286 & 0.0000 & & & \\
\hline IOALAR PUA & 202649 & 187209.182 & 7 & 1.2286 & 0.1755 & 89049.52 & 1.00 & 1.0000 \\
\hline SIBSTKitidH & & & & 1.2286 & 0.0000 & & & \\
\hline MRR 1 & & & 1 & 1.2286 & $1.22 \mathrm{~B} 6$ & $24 \div .09$ & 1.0247 & \\
\hline$M R R 2$ & & & 1 & 1.2296 & 1.2286 & 241.09 & 1.1182 & \\
\hline MER 3 & & & f & 1.2280 & $i .2286$ & $24: .09$ & 0.5799 & \\
\hline$M R \&$ & & & 0 & 1.2286 & 1).0000 & 241.09 & 9.0060 & \\
\hline MER 5 & & & 1 & $1.22 \theta t$ & 1.2786 & 241.07 & 0,9747 & \\
\hline Wek b & & & 1 & 1.2386 & 1.2336 & $24 i .09$ & 1,3024 & \\
\hline & & & & 1.2285 & 0.06150 & & & \\
\hline FHSI BTR FLR & 50727 & 34470.73 & $\xi$ & $1 . \therefore \theta_{0}$ & 2.2457 & 29647. 22 & 1.00 & $\$ .0510$ \\
\hline SUSSTKRTLN & & & & 1.2236 & 0.0000 & & & \\
\hline N 1 & & & $!$ & 1.2296 & 1.2296 & 158.18 & $0.559 \mathrm{~g}$ & \\
\hline MER 2 & & & 1 & 1.2250 & 1.2286 & 159.18 & 1.4569 & \\
\hline$Y 43$ & & & 1 & 1.3296 & 1. 2286 & 158.13 & 1.8463 & \\
\hline
\end{tabular}




\begin{tabular}{|c|c|c|c|c|c|c|c|c|}
\hline ERTITY & $\begin{array}{l}\text { UTHER TJT } \\
\text { PIQPijLAṬIOH }\end{array}$ & $\begin{array}{c}\text { OTHER ELIGBL } \\
\text { TGT PrgS }\end{array}$ & $\begin{array}{l}\text { COMPLETED } \\
\text { IHTEPVIEHS }\end{array}$ & $\begin{array}{l}\text { AVG COHPL } \\
\text { PER HRR }\end{array}$ & $\begin{array}{l}\text { IWDIVIOJAL } \\
\text { WEIGHTS }\end{array}$ & $\begin{array}{l}\text { AHERAGE } \\
\text { PGFULATIOH }\end{array}$ & $\begin{array}{c}\text { GRR } \\
\text { WEISHFS }\end{array}$ & $\begin{array}{l}\text { UILITif } \\
\text { WEIETST }\end{array}$ \\
\hline - & & & 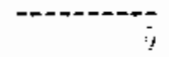 & 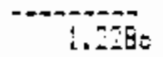 & 4.6190 & $1: 58: 8$ & is ibing & \\
\hline MPE $E$ & & & $!$ & 1. $2 * \overline{8}$ & 1,270 & 158.13 & $0.95 \mathrm{do}$ & \\
\hline Hats & & & 1 & 1.2286 & $1.225 b$ & 159.18 & 0.6398 & \\
\hline BER 7 & & & 1 & 1.2296 & 1.2286 & 159.18 & 0.7080 & \\
\hline \multirow[t]{2}{*}{ MEg 8} & & & 1 & 1.2286 & 1.2286 & 159.18 & 0.5356 & \\
\hline & & & & 1.2286 & 0.0000 & & & \\
\hline JTAH PLL & 33205 & $28608.9 ?$ & 7 & $1.22 \mathrm{~B} 6$ & 0.1755 & 69049.52 & 1.60 & 1.1000 \\
\hline SIJBSTRATU & & & & 1.2286 & i. .0000 & & & \\
\hline \multirow[t]{2}{*}{ STRATUA TOT } & 301236 & 276198.10 & 26 & 1.2266 & 0,0473 & NA & 1.13 & \\
\hline & & & & 1.2286 & 0.10000 & & & \\
\hline \multirow[t]{3}{*}{ REGIOH? } & & & & 1.2796 & 0.0000 & & & \\
\hline & & & & 1.2286 & 0.0000 & & & \\
\hline & & & & 1.2286 & 0.0000 & & & \\
\hline MRR ! & & & 1 & $1.22 \mathrm{~B} 6$ & 1.2286 & 254.01 & 1.5643 & \\
\hline MRR 2 & & & 1 & 1.2286 & 1.2286 & 244.61 & 1.5578 & \\
\hline $\operatorname{Mag} 3$ & & . & 0 & $1.22 \mathrm{~B} 6$ & 0.6000 & 264.11 & 10.0000 & \\
\hline MRK 4 & & & 1 & $1.22 \mathrm{~B}$ & 1.2206 & 264,01 & 0.5969 & \\
\hline MRR 5 & & & 1 & 1.2286 & 1.2286 & 254.01 & 1.0378 & \\
\hline MRR b & & & 1 & 1.2286 & 1.2286 & 264.01 & 0.7159 & \\
\hline MRR 7 & & & 1 & 1.2286 & 1.2286 & 264.01 & 0.7045 & \\
\hline HRR g & & & 1 & 1.2286 & 1.2286 & 264.01 & 1.2575 & \\
\hline MRR 9 & & & 1 & 1.2286 & 1.2296 & 264.01 & 1.3787 & \\
\hline MSR 10 & & & 1 & 1.2286 & 1.2296 & $264.0 !$ & 0.5642 & \\
\hline MRR II & & & 0 & 1.2296 & 0.0000 & $264.0 !$ & 0.0000 & \\
\hline MPR 12 & & & 1 & 1.2296 & 1.2796 & 264.01 & 0.9657 & \\
\hline MRR IJ & & & 1 & 1.2286 & 1.2296 & 264.01 & 0.9977 & \\
\hline MRR 14 & & & 1 & 1.2286 & 1.2286 & 264.01 & 0.8560 & \\
\hline MRR 15 & & & 1 & 1.22 日6 & 1.2286 & 264.01 & 0.9198 & \\
\hline \multirow[t]{2}{*}{$\operatorname{MRR} 16$} & & & 0 & 1.2286 & 0.0000 & 264.01 & 0.0000 & \\
\hline & & & & 1.2286 & 0.0000 & & & \\
\hline PPEL-NA & 81702 & 77789.54 & 13 & 1.2286 & 0.0945 & 105676.38 & 1.00 & 1.0000 \\
\hline SUBSTRATLM & & & & 1.2286 & 0.0000 & & & \\
\hline MRR L & & & 0 & 1.2296 & 0.0000 & $239 . ! 7$ & 0.0000 & \\
\hline MRR 2 & & & 1 & 1.2286 & 1.2286 & 239.17 & 1.0536 & \\
\hline MRR 3 & & & $!$ & 1.2296 & 1.2296 & 279.17 & 1.0620 & \\
\hline MRR 4 & & & 1 & 1.2286 & 1.2286 & 234.17 & 1.1855 & \\
\hline HER 5 & & & 0 & 1.2286 & 0.0000 & 239.17 & 0.1000 & \\
\hline FRR o & & & 0 & $1.22 B 6$ & 0.0000 & 239.17 & 0.0000 & \\
\hline MRR 7 & & & 1 & 1.2286 & 1.2286 & 239.17 & 0.9575 & \\
\hline ARR 8 & & & 1 & 1.2296 & 1.2296 & 239.17 & $1 .: 007$ & \\
\hline HRR 9 & & & 0 & 1.2286 & 0.0000 & 239.17 & 0.0000 & \\
\hline $\operatorname{MRR} 10$ & & & 0 & 1,2286 & 0,0000 & 239.17 & 0.0000 & \\
\hline MRR 11 & & & 0 & 1.2286 & 0,0000 & 279.17 & 0.0000 & \\
\hline HRR 12 & & & 1 & 1.2296 & 1.2296 & 239.17 & 0.5213 & \\
\hline MRG 13 & & & 9 & 1.2286 & 0.0000 & 239.17 & 0.0000 & \\
\hline MRR I4 & & & 1 & 1.2296 & 1.2286 & 239.17 & 1.2167 & \\
\hline HRR 15 & & & 1 & 1.2296 & 1.2286 & 259.17 & $0.89 \%$ & \\
\hline \multirow[t]{2}{*}{ MRP 16} & & & 0 & 1.2286 & 0.0000 & 239.17 & 0.0000 & \\
\hline & & & & 1.2286 & 0. viviti & & & \\
\hline \multirow{2}{*}{$\begin{array}{l}\text { WASH WAT PWR } \\
\text { SUSSTGATUM }\end{array}$} & 144643 & 133564.21 & 日 & 1.2296 & 0.1536 & 105676.3 & 1.60 & 1.0000 \\
\hline & & & & 1.2296 & 0.0000 & & & \\
\hline \multirow[t]{2}{*}{ STRATIMI TDT } & 226345 & 211352.75 & $2 !$ & 1.2286 & 0.0585 & $\mathrm{HA}$ & 1.07 & $\cdot$ \\
\hline & & & & t. 2296 & 0.0000 & & & \\
\hline
\end{tabular}




\begin{tabular}{|c|c|c|c|c|c|c|c|c|}
\hline EVTITY & $\begin{array}{l}\text { BFHER TOT } \\
\text { PEPLLATIGN }\end{array}$ & $\begin{array}{c}\text { OTHER ELIGEL } \\
\text { TST PCFS }\end{array}$ & $\begin{array}{l}\text { COHPLETED } \\
\text { IHTEFVIEHS }\end{array}$ & $\begin{array}{l}\text { AVE COHOL } \\
\text { PER XRE }\end{array}$ & $\begin{array}{l}\text { INOIVIDLAA } \\
\text { WEIEHTS }\end{array}$ & $\begin{array}{l}\text { AUEPAGE } \\
\text { EDPULATIDN }\end{array}$ & $\begin{array}{c}\text { Men } \\
\text { MEIJATS }\end{array}$ & $\begin{array}{l}\text { DTILITY } \\
\text { XEIGHTS }\end{array}$ \\
\hline 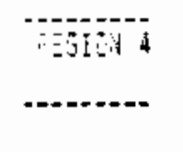 & --- - & - & -------- & $\begin{array}{l}1.29 \\
1.2866 \\
1.296\end{array}$ & 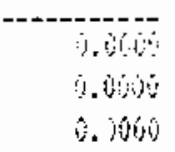 & & -- & \\
\hline ARR I & & & 1 & 1.2286 & $\{.2286$ & 293.84 & 1.9871 & · \\
\hline $4 F 2 ?$ & & & 0 & 1.2206 & 0.6700 & 295.84 & 1.900 & \\
\hline YRE & & & 2 & 1.2966 & 0.6143 & 295.87 & $1,34,4$ & \\
\hline$M F i R 4$ & & & 0 & 1.2296 & 0.0000 & 293.84 & i. & \\
\hline MRR 5 & & & 2 & 1.7286 & $0,5 ! 43$ & 295,84 & 1.2647 & \\
\hline Mat i & & & 2 & 1.296 & $0,614:$ & 275.34 & 0.7529 & \\
\hline MRE 7 & & & 2 & 1.2236 & 0.6143 & 245.84 & 9.7757 & \\
\hline Mō日 & & & 1 & 1.2680 & 1.290 & 273.64 & $0.47 \%$ & \\
\hline MER ? & & & 1 & 1.2296 & 1.2286 & 293.94 & $1.0: 25$ & \\
\hline ARE 10 & & & 1 & 1.2296 & 1.2286 & 293.34 & 0.7175 & \\
\hline MER II & & & 1 & 1.2296 & 1.3256 & 297.84 & 1.1724 & \\
\hline 秘至 12 & & & 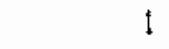 & 1.2236 & 1.2286 & 293.34 & Nogez & \\
\hline MRR 13 & & & 1 & 1.2296 & 1.2286 & 293.84 & 0.6908 & \\
\hline ngp 14 & & & $\hat{\imath}$ & 1.2296 & $0.514 \xi$ & 293.94 & $0.71 \div 4$ & \\
\hline ARS 15 & & & $!$ & 1.2206 & 1.2296 & 293.34 & 1.5971 & \\
\hline BRE 16 & & & 1 & $\begin{array}{l}1.22866 \\
1.2286\end{array}$ & $\begin{array}{l}1.2206 \\
0.0000\end{array}$ & 253.84 & 0.6790 & \\
\hline $\begin{array}{l}\text { PUEET SOLUNO } \\
\text { CLBSTRATLH }\end{array}$ & 496995 & 469232.46 & 19 & $\begin{array}{l}1.22866 \\
1.2286\end{array}$ & $\begin{array}{l}0.0647 \\
0.0000\end{array}$ & 469232.46 & 1.00 & 1.3000 \\
\hline ETEATUS TOT & 496995 & $4672 \div 2.46$ & 19 & $\begin{array}{l}1.2296 \\
1.2286\end{array}$ & $\begin{array}{l}0.0647 \\
0.1000\end{array}$ & $N A$ & 5.57 & \\
\hline gegian s & & & & $\begin{array}{l}1.2286 \\
1.3286 \\
1.2286\end{array}$ & $\begin{array}{l}0.0000 \\
0.0000 \\
0.0000\end{array}$ & & & \\
\hline MRR I & & & 1 & 1.2286 & 1.2296 & 225.94 & 1.4995 & \\
\hline ARR 2 & & & $!$ & 1.2296 & 1.2236 & 225.94 & $! .279 !$ & \\
\hline MRR J & & & 1 & 1.2286 & 1.2286 & $22 \pm .94$ & 1.7925 & \\
\hline$M R A$ & & & 1 & 1.2286 & 1.2286 & 225.94 & 1.0597 & \\
\hline MRR 5 & & & 1 & 1.2296 & 1.2286 & 225.54 & 0.4769 & \\
\hline MER 6 & & & 1 & 1.2286 & 1.2236 & 225.94 & 0.7157 & \\
\hline MaR ? & & & 0 & 1. 2296 & 0.0000 & $2 m 5 . ? 4$ & D. & \\
\hline MPS 8 & & & 1 & 1.2786 & 1.2286 & 225,94 & i.E13! & \\
\hline MkP 9 & & & 1 & $1.22 \%$ & 1.2296 & 235.94 & 0.7678 & \\
\hline MRR 10 & & & 1 & 1.2286 & 1.2296 & 275.94 & $1.307 t$ & \\
\hline ARR II & & & 1 & 1.2286 & 1.2286 & 325.94 & 0.5134 & \\
\hline MER !2 & & & 0 & 1.2286 & 0.0000 & 225.94 & 0.0060 & \\
\hline AKA IJ & & & $t$ & 1.2258 & 1.7286 & 225.94 & 0.501 & \\
\hline AFP 14 & & & & 1.2286 & & 225.74 & & \\
\hline VER 15 & & & 1 & 1.2286 & 1.3286 & 325.94 & 19.4470 & \\
\hline ARE is & & & 1 & $\begin{array}{l}1.2230 \\
1.2296\end{array}$ & $\begin{array}{l}1.2296 \\
0.0000\end{array}$ & 235.94 & $1.354 ?$ & \\
\hline$P P X_{L}-O R-E$ & 71835 & 63192.59 & 15 & 1.2296 & 0.0945 & :9972.i11 & $\therefore .00$ & $\therefore .0,000$ \\
\hline SLESTRATUH & & & & 1.2296 & $0.6 \times 00$ & & & \\
\hline$M G R$ & & & $!$ & 1.2296 & 1.2250 & 210.34 & 0.7624 & \\
\hline 492 & & & $\vdots$ & 1.2236 & $1.2 n \mathrm{~Eb}^{2}$ & 340.04 & $! .8 \pm 1.5$ & \\
\hline औरूก & & & $i^{2}$ & 1.2296 & 0.0000 & 240.94 & $\therefore 900$ & \\
\hline$M R$ & & & 1 & 1.2296 & 1.2285 & 249.4 & 1.1951 & \\
\hline MRP 5 & & & 0 & 1.2536 & $0.000 \hat{\mathrm{s}}$ & 24010.04 & 0.0000 & \\
\hline MRR is & & & $!$ & $1.22 \mathrm{gt}_{\mathrm{t}}$ & 1.206 & 270.04 & 0.6352 & \\
\hline TRR 7 & & & 1 & 1.2986 & 1.2290 & 340,04 & 0.0567 & \\
\hline
\end{tabular}




\begin{tabular}{|c|c|c|c|c|c|c|c|c|}
\hline ENTITY & $\begin{array}{l}\text { OTHER TOT } \\
\text { PGEULATIOH }\end{array}$ & $\begin{array}{c}\text { OTHER ELIGEL } \\
\text { POT PDFS }\end{array}$ & $\begin{array}{l}\text { COMPLETED } \\
\text { INTERUIENS }\end{array}$ & $\begin{array}{l}\text { AVE COMPL } \\
\text { PER MRF }\end{array}$ & $\begin{array}{l}\text { IHOIVIDUAL } \\
\text { WEIGHTS }\end{array}$ & $\begin{array}{l}\text { AVERAGE } \\
\text { POPILLATION }\end{array}$ & $\begin{array}{l}\text { MRR } \\
\text { WE!GHTS }\end{array}$ & $\begin{array}{l}\text { DTILITY } \\
\text { DEIGHTS }\end{array}$ \\
\hline 4 & & & $!$ & $\begin{array}{l}1.2955 \\
1.2286\end{array}$ & $\begin{array}{l}1.585 \\
0.6000\end{array}$ & 24.64 & 1.412 & . \\
\hline 106+0 PUR & 1675 & 10203.36 & 8 & 1.2286 & 0.2048 & 29472.01 & 1.60 & 1.000 \\
\hline 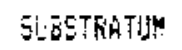 & & & & 1.2286 & 0,0000 & & & \\
\hline MFR ! & & & 0 & 1.2280 & 0,0000 & 423.51 & 0.10000 & \\
\hline 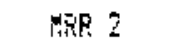 & & & 1 & 1.2286 & 1.2296 & $423.5 !$ & 1.5278 & \\
\hline MAR $=$ & & & & $1.2280^{\circ}$ & & 423.51 & & \\
\hline MRF 4 & & & 1 & 1.2286 & 1.2286 & 423.51 & 0.4722 & \\
\hline MFO 5 & & & & 1.2296 & & 423.51 & & \\
\hline ARR 6 & & & 0 & 1.2296 & 0.0000 & 423.51 & 6. 60000 & \\
\hline MRE 7 & & & & 1.2286 & & 423.51 & & \\
\hline HFR 8 & & & 0 & 1.2286 & 0.6000 & 425.51 & 0.60000 & \\
\hline & & & & 1.2286 & 0.0000 & & & \\
\hline OF NATHL & 22295 & 16520.09 & 2 & 1.2286 & 0.6143 & 29072.01 & 1.00 & 1.00 \\
\hline EJJASTRATUH & & & & $1.22 a 6$ & 0.60000 & & & \\
\hline STRATUM TOT & 105805 & 89916.03 & 21 & 1.2286 & 0.0585 & NA & 0.46 & \\
\hline & & & & 1.2286 & 0.00015 & & & \\
\hline REGION 6 & & & & 1.2286 & 0.0000 & & & \\
\hline ……-- & & & & $\$ .2286$ & 0.0000 & & & \\
\hline & & & & 1.2286 & 0.0000 & & & \\
\hline MRR！ & & & 1 & $\$ .2286$ & 1.2286 & 234.85 & 0.3969 & \\
\hline IRR 2 & & & 1 & 1.2286 & 1.2286 & 234.85 & 0.7599 & \\
\hline MER 3 & & & 1 & 1.2286 & 1.2286 & 234.95 & 1.0773 & \\
\hline HFF 4 & & & 0 & 1.2286 & 0.0000 & 234.85 & 0.0000 & \\
\hline $\operatorname{MRE} 5$ & & & 1 & 1.2296 & 1.2280 & 234.95 & 4.5355 & \\
\hline HRR 6 & & & 2 & 1.2286 & 0.6143 & 234.95 & 0.8042 & \\
\hline IFR 7 & & & 1 & 1.2286 & 1.2286 & 234.85 & 1.5030 & \\
\hline MFR 9 & & & 1 & 1.2286 & 1.2286 & 234.85 & 1.5670 & \\
\hline IRR 9 & & & 1 & 1.2286 & 1.2286 & 234.85 & 1.4911 & \\
\hline ARR 10 & & & 2 & 1.2286 & 0.6143 & 234.85 & 0.4990 & \\
\hline ARR 11 & & & $z$ & 1.2286 & 0.6143 & 234.85 & 6.7537 & \\
\hline HRR $1 ?$ & & & 2 & $1.228 b$ & 0.0143 & 234.85 & 1.3967 & \\
\hline MFR 13 & & & 1 & 1.2286 & 1.2286 & 234.85 & 1.6970 & \\
\hline MER 14 & & & 1 & 1.2286 & 1.2296 & 234.85 & 0.8029 & \\
\hline MEP: I5 & & & 2 & 1,2286 & 0.6143 & 234.85 & 1.0858 & \\
\hline MRR 16 & & & 1 & 1.2296 & 1.2286 & 234.85 & 0.6190 & \\
\hline & & & & 1.2286 & 0.0000 & & & \\
\hline PP\&L-OR-N & 270237 & $245757.4 b$ & 20 & $1.22 \mathrm{Eo}$ & 0.0614 & 332225.74 & 1.00 & 1.0000 \\
\hline SUGSTRATUH & & & & 1.2286 & 0.0000 & & & \\
\hline MRR！ & & & 1 & $1.22 \mathrm{Bb}$ & 1.2296 & 333.39 & 0.6683 & \\
\hline MRR 2 & & & 1 & 1.2286 & 1.2286 & 373.39 & 1.1269 & \\
\hline FRP 3 & & & 2 & 1.2236 & 0.6143 & 333.39 & 0.8555 & \\
\hline SFR 4 & & & 2 & 1.2286 & 0.6143 & 333.39 & 1.2448 & \\
\hline MFR 5 & & & 2 & 1.2286 & 0.6143 & 353.39 & 0.4739 & \\
\hline MRR 6 & & & 2 & $\$ .2286$ & 0.6143 & 355.79 & 0.9031 & \\
\hline MRR 7 & & & 2 & 1.2296 & 0.6143 & 333.39 & 1.1508 & \\
\hline MRR 8 & & & 2 & 1.2286 & 0.6143 & 333.37 & 0.7774 & \\
\hline MRT 9 & & & 2 & 1.2286 & 0.6143 & 353.59 & 1,1278 & \\
\hline Wh 10 & & & 1 & 1.2286 & 1.2886 & 335.39 & 1.3778 & \\
\hline MRR 11 & & & 1 & 1.2286 & 1.2286 & 33.39 & 1.1788 & \\
\hline MRR 12 & & & 1 & 1.2286 & 1.2296 & 327.59 & 1.0978 & \\
\hline MRR 13 & & & 2 & 1.2286 & 0.6143 & 333.39 & 0.8735 & \\
\hline YRR 14 & & & 0 & 1.2286 & 0.0000 & 333.59 & $0.00 \mathrm{ij}$ & \\
\hline
\end{tabular}




\begin{tabular}{|c|c|c|c|c|c|c|c|c|}
\hline ENTIT & $\begin{array}{l}\text { DTHES TIT } \\
\text { FOPULAION }\end{array}$ & $\begin{array}{c}\text { OTHEF EDIEBi } \\
\text { TOT FOES }\end{array}$ & $\begin{array}{l}\text { CORPLETES } \\
\text { INTERUIEIKS }\end{array}$ & $\begin{array}{l}\text { AVE CLMEL } \\
\text { SER MARE }\end{array}$ & $\begin{array}{l}\text { FNDIVIELAL } \\
\text { HEIGHTS }\end{array}$ & $\begin{array}{l}\text { AVERAGE } \\
\text { FGPIJLAIIOW }\end{array}$ & $\begin{array}{c}\text { Mer } \\
\text { hEIGHTS }\end{array}$ & $\begin{array}{l}\text { UTILITY } \\
\text { WEIOHTE }\end{array}$ \\
\hline 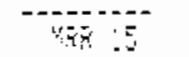 & & & s. & 1,28 & $\therefore 3$ & 4 & X. E & \\
\hline MET Is & & & $\Sigma$ & $\begin{array}{l}1.3250 \\
1.380\end{array}$ & $\cos 143$ & 73.79 & 1,2008 & \\
\hline $\begin{array}{l}\text { DOT EEM ESE } \\
\text { SUESTHATLN }\end{array}$ & 49156 & 415444,01 & 24 & & & 332225.74 & 1.00 & .. \\
\hline 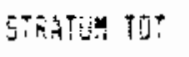 & 79973 & $664451.4 \bar{t}$ & 44 & & & NA & 2.30 & \\
\hline COEHTE TQT & 1913304 & $: 781303$ & 155 & & & 170150 & 1.6 & \\
\hline GPAlt TET & 79049 & 296483 & 387 & & & 2964753 & .60 & \\
\hline
\end{tabular}




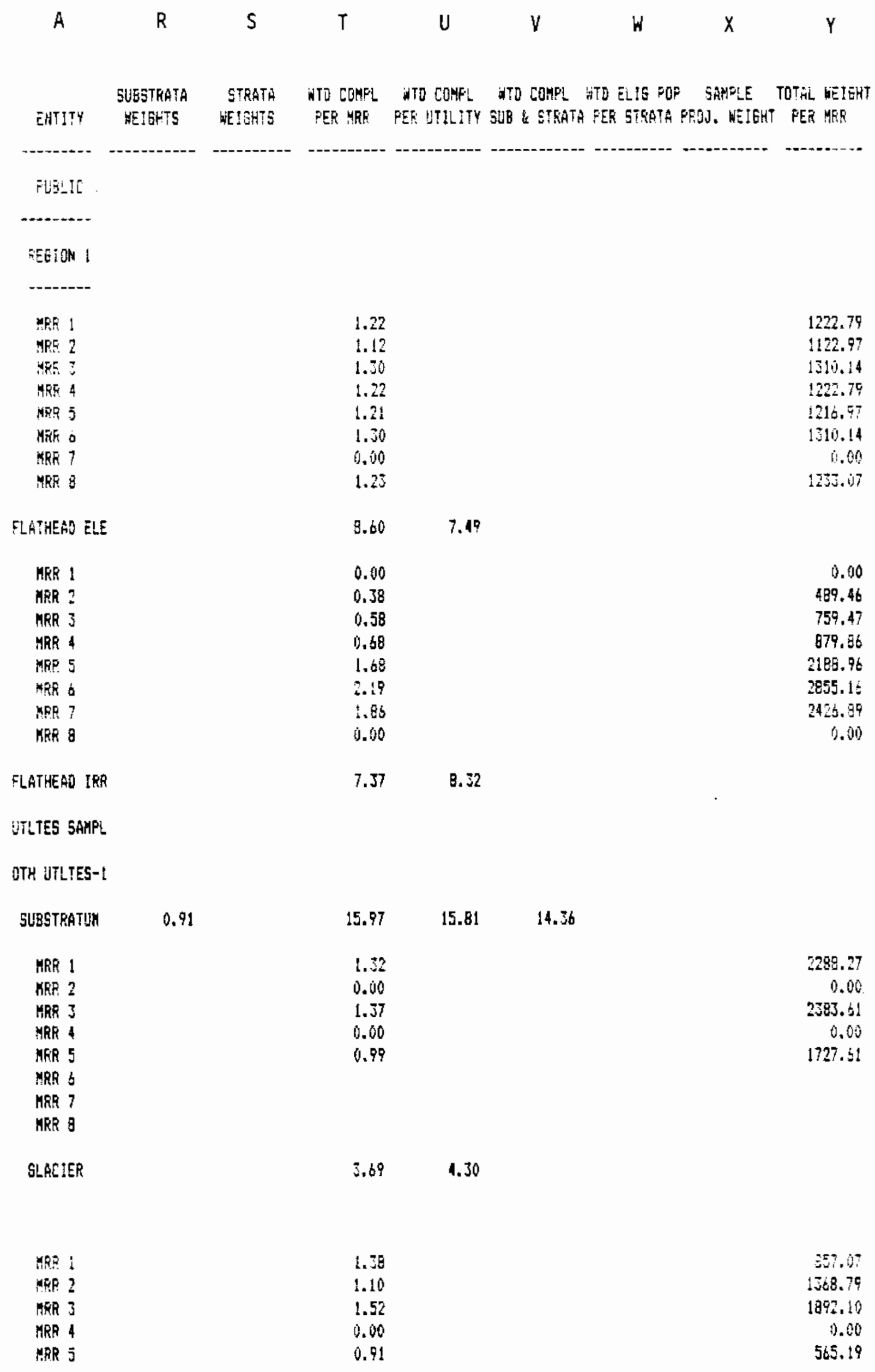




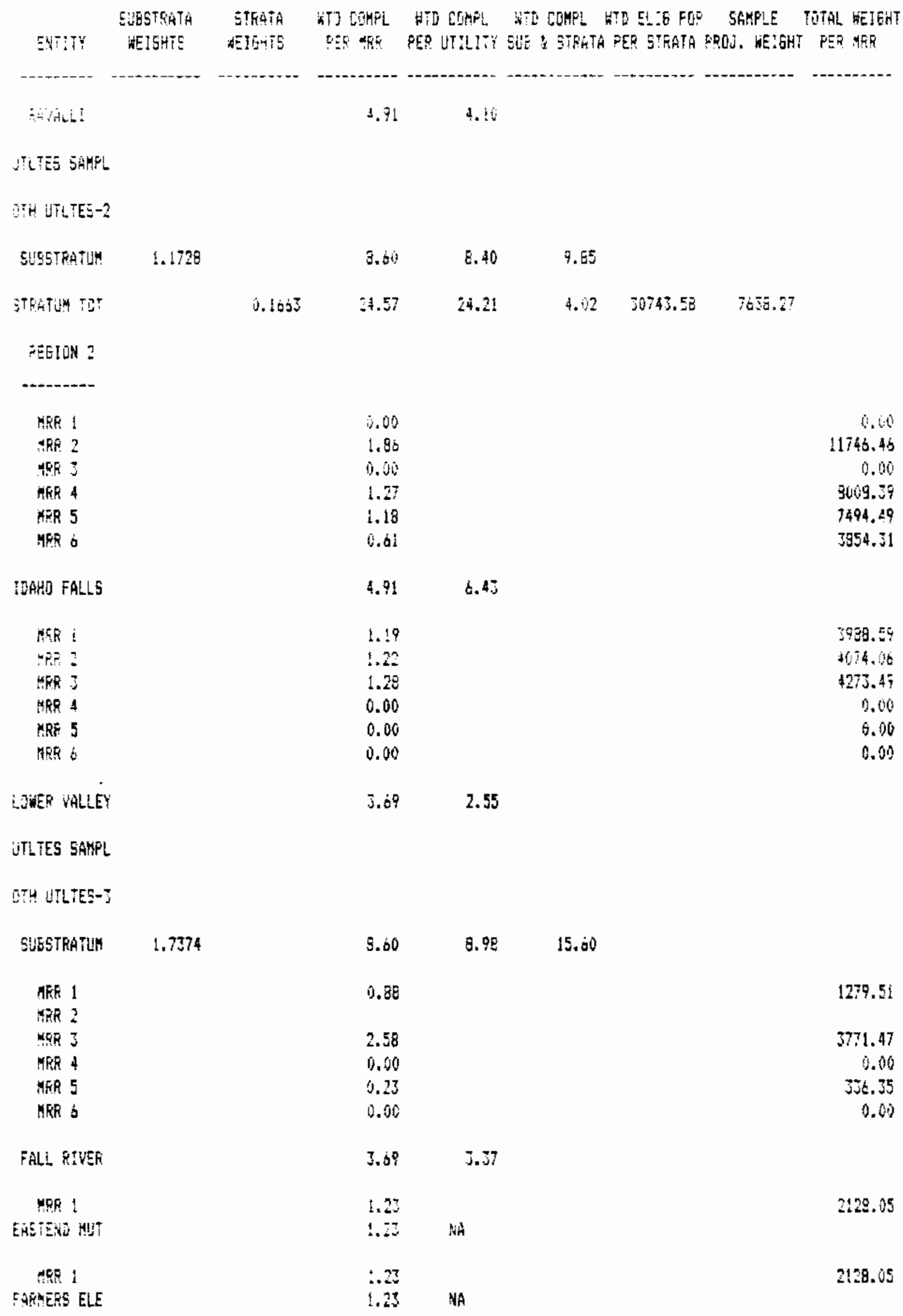




\begin{tabular}{|c|c|c|c|c|c|c|c|}
\hline EATITY & $\begin{array}{l}\text { 5UGSTKATA } \\
\text { WEIGHTS }\end{array}$ & $\begin{array}{r}\text { STAATA } \\
\text { WEIGHFS }\end{array}$ & $\begin{array}{l}\text { WTD COMPL } \\
\text { PEF MRR }\end{array}$ & $\begin{array}{l}\text { HTD COHPL } \\
\text { PER UTILITY }\end{array}$ & $\begin{array}{l}\text { ATD COMEL } \\
\text { SLLE : STEATE }\end{array}$ & $\begin{array}{l}\text { HTD ELIG FAP SAHPLE } \\
\text { PER STRATA FQOU. WEIGHT }\end{array}$ & $\begin{array}{l}\text { WUAL HEIGHT } \\
\text { QER KAF }\end{array}$ \\
\hline सER ! & (1) & 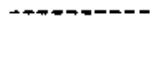 & $1 . \bar{j}$ & 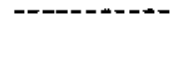 & 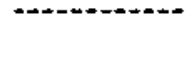 & 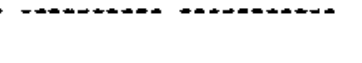 & 210.05 \\
\hline FUVESIOE EL & & & 1.23 & $\mathrm{NA}$ & & & \\
\hline MRR 1 & & & $1.2 \mathrm{~J}$ & & & & 2125.05 \\
\hline SEUTHSIDE EL & & & 1.23 & NA & & & \\
\hline ARR! & & & 0.00 & & & & 0.00 \\
\hline MRR 2 & & & 1.23 & & & & 2128.15 \\
\hline WNITY L LPP & & & 1.23 & NA & & & \\
\hline MINDQKA PFOJ & & & 6.14 & $\dot{3.66}$ & & & \\
\hline \multicolumn{8}{|l|}{ JTLLTES SAMPL } \\
\hline \multicolumn{8}{|l|}{ OTH UTLTES-4 } \\
\hline SUESTRATUH & 0.5735 & & 9.83 & 10.04 & 5.70 & & \\
\hline IIRR 1 & & & 1.28 & & & & 1608.55 \\
\hline ARR 2 & & & 0.00 & & & & 0.00 \\
\hline MRR 3 & & & 0.00 & & & & 0.00 \\
\hline MRR 4 & & & t.28 & & & & 1615.49 \\
\hline HER 5 & & & 1.28 & & & & 1515.49 \\
\hline MAR of & & & 1.05 & & & & $\therefore E=: 3$ \\
\hline LOST RIVER & & & 4.91 & 3.93 & & & \\
\hline \multicolumn{8}{|l|}{ MRR I } \\
\hline MRR 2 & & & 1.23 & & & & 2329.19 \\
\hline MRR 3 & & & 0.00 & & & & 0.20 \\
\hline MRR 4 & & & 0.00 & & & & 0.00 \\
\hline MRR 5 & & & 0.00 & & & & 0,00 \\
\hline MRR 6 & & & 0.00 & & & & (1) \\
\hline WEISER & & & $1.2 \mathrm{~J}$ & 1.48 & & & \\
\hline \multicolumn{8}{|l|}{ UTLTES SAMPL } \\
\hline \multicolumn{8}{|l|}{ OTH UTLTES-4 } \\
\hline SUESTRATUA & 0.5668 & & 6.14 & 5.40 & 3.06 & & \\
\hline STRATUL" TOT & & 0.3646 & 24.57 & 24.42 & 8.99 & 67991.88 & \\
\hline \multicolumn{8}{|l|}{ RESI bn 3} \\
\hline --ns-s- & & & & & & & \\
\hline HRR? ! & & & 0.60 & & & & i. .1$)$ \\
\hline MER 2 & & & 0.00 & & & & $x_{2} 60$ \\
\hline MGR 3 & & & 9.90 & & & & 1,30 \\
\hline IRR 4 & & & 1.23 & & & & 12487.73 \\
\hline MRR 5 & & & 0.00 & & & & 0.60 \\
\hline
\end{tabular}

A. 35 


\begin{tabular}{|c|c|c|c|c|c|c|}
\hline E:T!Ty & $\begin{array}{l}\text { SUESTEATIA } \\
\text { WETEHTS }\end{array}$ & $\begin{aligned} \text { CTKATA } \\
\text { WETEHTS }\end{aligned}$ & TOS CFME & $\begin{array}{l}\text { WTE CUMPL } \\
\text { PER LTILJTY }\end{array}$ & 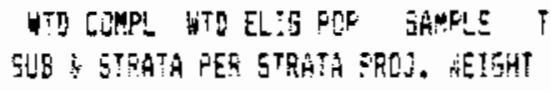 & 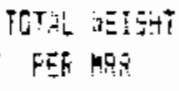 \\
\hline 将 & -⿻------ & 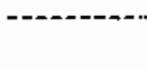 & , ili & - n-n-n & n-men & $\therefore$ \\
\hline EENT Cit & & & 1.23 & $1.8 !$ & & \\
\hline WF 1 & & & 1.57 & & & $773: 7 !$ \\
\hline MFE? & & & $i, \$ 1$ & & & $\dot{s e n}=15$ \\
\hline HRR 3 & & & 1.34 & & & 11385.11 \\
\hline MRR 4 & & & 1.70 & & & 15170.24 \\
\hline YXE 5 & & & 1.83 & & & 1455,28 \\
\hline CHELAY RTY & & & 5.14 & :iA & & \\
\hline MFR I & & & 1.23 & & & 50733,74 \\
\hline CASSMERE & & & 1.23 & $B A$ & & \\
\hline CHELH-CSHMRE & & & 7.37 & ל. 98 & & \\
\hline \multicolumn{7}{|l|}{ IJTLTES SAYPL } \\
\hline \multicolumn{7}{|l|}{ QTH UTLTES-2 } \\
\hline SLEBTRATJH & 2.3647 & & 8.00 & 8.19 & 16.91 & \\
\hline MRR I & & & 4.34 & & & 2445.35 \\
\hline 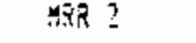 & & & 0.48 & & & 2161,84 \\
\hline MER j & & & 0.00 & & & 0.0 \\
\hline NRR 4 & & & 2.64 & & & $\$ 1730.73$ \\
\hline MeR 5 & & & 1.46 & & & 5494.37 \\
\hline HFR $S$ & & & & & & \\
\hline FRANKLIM & & & $4.9:$ & 3.95 & & \\
\hline MRR : & & & 1.05 & & & 8950.75 \\
\hline MER 2 & & & 0.00 & & & 8 \\
\hline IRR 3 & & & $\therefore .79$ & & & $84+3, ? !$ \\
\hline WRO 4 & & & 1.97 & & & $7: 12.24$ \\
\hline MiR 5 & & & $1 . \overline{3} !$ & & & 79:3. $=32$ \\
\hline NRR 6 & & & 0.00 & & & 3.6 \\
\hline RICHLANO & & & 4.91 & 5.83 & & \\
\hline \multicolumn{7}{|l|}{ WTLIES SAHPL } \\
\hline \multicolumn{7}{|l|}{ UTH WTLIES-5 } \\
\hline SLEETRATUK & 1.1972 & & 9.83 & 9.83 & 11.77 & \\
\hline PR ! & & & $5, i(i)$ & & & 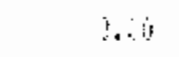 \\
\hline 你 2 & & & 30 & & & a \\
\hline WhR 3 & & & 1.03 & & & 234.38 \\
\hline $\operatorname{MRR} 4$ & & & $1.4 ?$ & & & 571.52 \\
\hline PENC ORIELLE & & & 2.46 & 2.21 & & \\
\hline
\end{tabular}




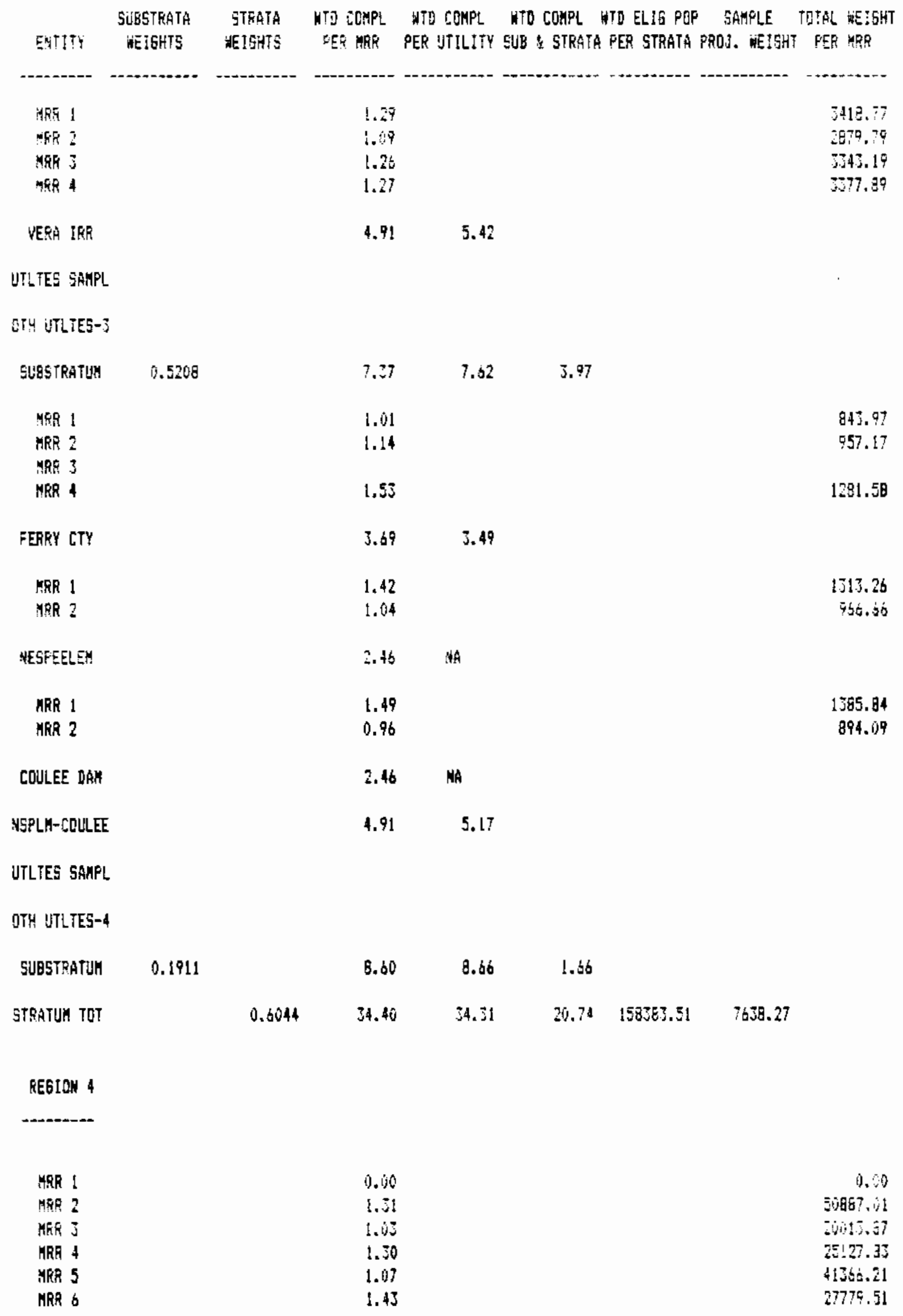




\begin{tabular}{|c|c|c|c|c|c|c|}
\hline ENT:TYY & $\begin{array}{l}\text { SLGESTRATA } \\
\text { WE:AHTS }\end{array}$ & $\begin{array}{l}\text { STFATA } \\
\text { WETGHTE }\end{array}$ & WT COHPL & 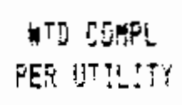 & 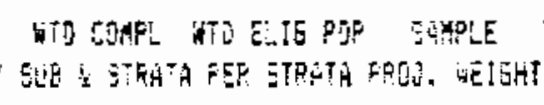 & $\begin{array}{l}\text { TOTAL WEIETT } \\
\text { T FEF HPF }\end{array}$ \\
\hline 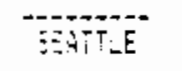 & - & & 0.1 & जi: & & \\
\hline 释 1 & & & 1.55 & & & $199+4.41$ \\
\hline$M 2$ & & & 1.28 & & & $255 \div 5.40$ \\
\hline YER: & & & 1.54 & & & 3052 \\
\hline ARt 4 & & & 1.17 & & & 14587.00 \\
\hline MRR 5 & & & 1.14 & & & $226: 9.38$ \\
\hline MRR G & & & 2.59 & & . & 27757.37 \\
\hline ENütonts? & & & 7.37 & 5.01 & & \\
\hline \multicolumn{7}{|l|}{$\begin{array}{l}\text { UTLTES SAMYPL } \\
\text { DTH UTLTES-T }\end{array}$} \\
\hline SLESTRATUN & 2.8275 & & $13.5 !$ & $1: 12$ & $37.1 \mathrm{j}$ & \\
\hline YTR I & & & 3.97 & & & $3496 . J 5$ \\
\hline KGP? & & & $(1.2)$ & & & 92.36 \\
\hline 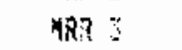 & & & 1.37 & & & स:1.:18 \\
\hline$P R R+$ & & & .05 & & & 7755.20 \\
\hline ARR 5 & & & 1.92 & & & 25709.10 \\
\hline MRR 6 & & & 0.60 & & & 9.50 \\
\hline CLAPK CTY & & & $5 .: 4$ & 5.24 & & \\
\hline WFE! & & & $\therefore 36$ & & & ה \\
\hline MRR 2 & & & $\therefore .17$ & & & 10544.79 \\
\hline ARR 3 & & & 1.16 & & & 20917.08 \\
\hline MR 4 & & & 1.34 & & & 12042.37 \\
\hline MER 5 & & & $\therefore 08$ & & & 17472.33 \\
\hline FRR 6 & & & 1.15 & & & 20644.06 \\
\hline IACOHA & & & $\therefore .37$ & 8.46 & & \\
\hline
\end{tabular}

UTLTES SAMPL

OTH UTLTES-2

SUESTRATIM $\quad 1.5107 \quad 1 \% .51 \quad 13.70 \quad 30.69$

MFR!

0.00

9.00

1.40

3770.51

0.94

278.50

$\therefore 46$

(1) 5.2 .5

(I. $\mathrm{e} \theta$

․:-3.:7?

MeR 5

IRR 6

l. 16

$\therefore . .72$

AASON CTY-J

ALR!

$61 .: 4 \quad 0.40$

MRR ?

i. . . . i

i. 80

$1.5 !$

1.5

6.78

4726.80

5641.20

MPR 4

5.75

28. 7.73

7.56

A. 38 


\begin{tabular}{|c|c|c|c|c|c|c|}
\hline ENTIT & $\begin{array}{l}\text { SUESTFATH } \\
\text { HE!GHTS }\end{array}$ & $\begin{array}{l}\text { STFATA } \\
\text { WEIEHTS }\end{array}$ & $\begin{array}{l}\text { WTE EOHFL } \\
\text { FEF HRF }\end{array}$ & $\begin{array}{l}\text { HTD CEMEL } \\
\text { PES UTILIT' }\end{array}$ & $\begin{array}{l}\text { WTO COMFL WTO ELIT POP SAKFLE T } \\
\text { SHB \& STRATA FER STRATA FRUS. DEISHT }\end{array}$ & $\begin{array}{l}\text { TOFAL WEIEHT } \\
\text { PEF MAK }\end{array}$ \\
\hline 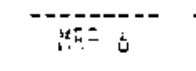 & 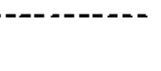 & 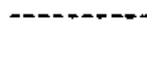 & 45 & & 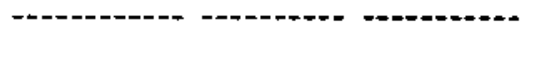 & WE \\
\hline CLiLify CIY & & & E.14 & 5.89 & & \\
\hline \multicolumn{7}{|l|}{ IT:TES SAMP! } \\
\hline \multicolumn{7}{|l|}{ JTH URLTES-3 } \\
\hline ELGETRATIH & 1). 4976 & & 12.29 & 12.24 & 6.11 & \\
\hline MR: & & & 1.25 & & & 2159.19 \\
\hline YPR 2 & & & 0.84 & & & $144 ! .10$ \\
\hline MER J & & & 1.78 & & & 6110.27 \\
\hline MGR 4 & & & 1.05 & & & 3596.99 \\
\hline ELMHURST MUT & & & 4.91 & 5.87 & & \\
\hline KRR I & & & 1.23 & & & $\{425.57$ \\
\hline MRK 2 & & & 1.22 & & & 2937.56 \\
\hline MRR 3 & & & 1.23 & & & 1425.57 \\
\hline MFR 4 & & & 1.23 & & & 1425.57 \\
\hline ORCAS PNR & & & $4.9 !$ & 3.96 & & \\
\hline \multicolumn{7}{|l|}{ IJTLTES SAMEL } \\
\hline \multicolumn{7}{|l|}{ OTH UTLIES-J } \\
\hline SUESTRATUN & 0.2774 & & 9.83 & 9.83 & 2.73 & \\
\hline KRR I & & & 0.67 & & & 330.93 \\
\hline MRR 2 & & & 1.46 & & & 722.03 \\
\hline MRR 3 & & & 1.48 & & & 734.37 \\
\hline MRR 4 & & & 1.31 & & & 647.98 \\
\hline PARKLAND L\&P & & & 4.91 & 4.36 & & \\
\hline MRR ! & & & 1.33 & & & 828.26 \\
\hline MRR 2 & & & 0.97 & & & 600.54 \\
\hline MRE 3 & & & 1.43 & & & 590.21 \\
\hline MKR 4 & & & 1.18 & & & 1469.56 \\
\hline SKAHANIA & & & 4.91 & 5.47 & & \\
\hline \multicolumn{7}{|l|}{ UTLTES SAMFL } \\
\hline \multicolumn{7}{|l|}{ CTH UILTES-2 } \\
\hline SJECTRATUH & 9.1076 & & 9.83 & 5.85 & 1.06 & \\
\hline *RR I & & & 2.63 & & & 1399.38 \\
\hline RRP 2 & & & 1.24 & & & 1322.93 \\
\hline HFi 3 & & & 0.38 & & & 403.31 \\
\hline
\end{tabular}




\begin{tabular}{|c|c|c|c|c|c|c|c|}
\hline E:TाTY & $\begin{array}{l}\text { SEBCTAATH } \\
\text { MEIGATSS }\end{array}$ & $\begin{array}{l}\text { STRATA } \\
\text { WEIENTS }\end{array}$ & $\begin{array}{l}\text { HTE EOKFI } \\
\text { FER ARK }\end{array}$ & $\begin{array}{l}\text { WTO COAF! } \\
\text { FEE 'TILITY }\end{array}$ & $\begin{array}{l}\text { WTE COAPL A } \\
\text { SUE S STATA }\end{array}$ & 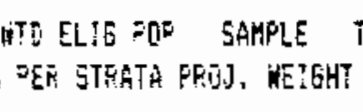 & $\begin{array}{l}\text { TUTAL WEIGH? } \\
\text { PER MER }\end{array}$ \\
\hline 4 & - non & - & $j$ & & ............ & & 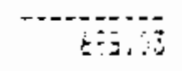 \\
\hline COF MUTSRL & & & 4.91 & 5.18 & & & \\
\hline MCE ! & & & .50 & & & & 209.96 \\
\hline WER $?$ & & & 1.85 & & & & 779.66 \\
\hline Whe 3 & & & 1.08 & & & & $4 \overline{5} 5.69$ \\
\hline SAF 4 & & & 1.49 & & & & 1252.37 \\
\hline 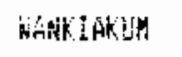 & & & $4.9 !$ & 4.35 & & & \\
\hline \multicolumn{8}{|l|}{ JTLISS SANPL } \\
\hline \multicolumn{8}{|l|}{ DTA HTLTES-5 } \\
\hline SUBSTEATUA & 0.0918 & & 9.63 & 9.83 & 0.90 & & \\
\hline STRATUS TOT & & 1.3587 & 68.80 & 88.59 & 93.19 & 311776.89 & \\
\hline \multicolumn{8}{|l|}{ REE!ON 5} \\
\hline \multicolumn{8}{|l|}{-........... } \\
\hline $\operatorname{xin} 1$ & & & 1.28 & & & & 2634.47 \\
\hline AES 2 & & & $\therefore 5$ & & & & 8775.34 \\
\hline Yhe & & & 3.41 & & & & EES. \\
\hline MRR A & & & 1.75 & & & & 3662.85 \\
\hline KRF 5 & & & 10.96 & & & & 1986.47 \\
\hline MFR 6 & & & 0.00 & & & & 0.00 \\
\hline MRR 7 & & & 1.27 & & & & 2627.70 \\
\hline 4 SE & & & 1.10 & & & & 2261.31 \\
\hline MIDState & & & 8.60 & 9.19 & & & \\
\hline MRR 1 & & & 1). 82 & & & & 1474.57 \\
\hline MR 2 & & & 1.10 & & & & 9905.35 \\
\hline ARR 3 & & & 1.00 & & & & $\dot{v}, 00$ \\
\hline MRR 4 & & & 1.51 & & & & $271 . .3 B$ \\
\hline SRS 5 & & & 1.47 & & & & 2641.85 \\
\hline MG 6 & & & 0.00 & & & & 0.00 \\
\hline MR 7 & & & 0.00 & & & & 9.00 \\
\hline $\mathrm{HER}$ & & & 1.24 & & & & 2234.65 \\
\hline WMATILLA & & & 5.14 & 5.72 & & & \\
\hline
\end{tabular}

WTLIES SAMPL

ETH UTLTES-2

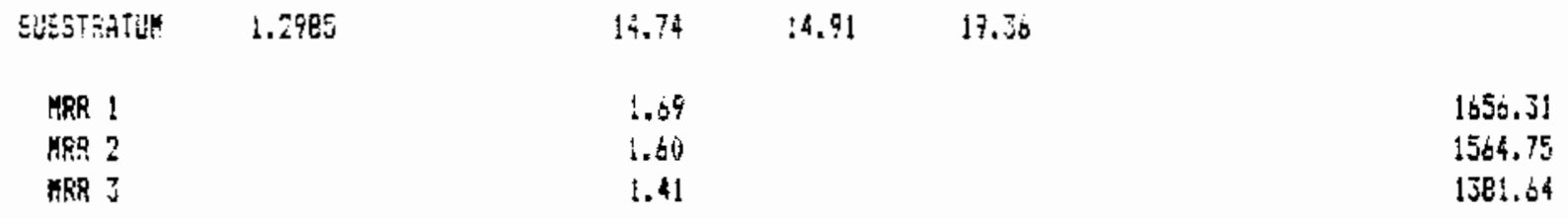




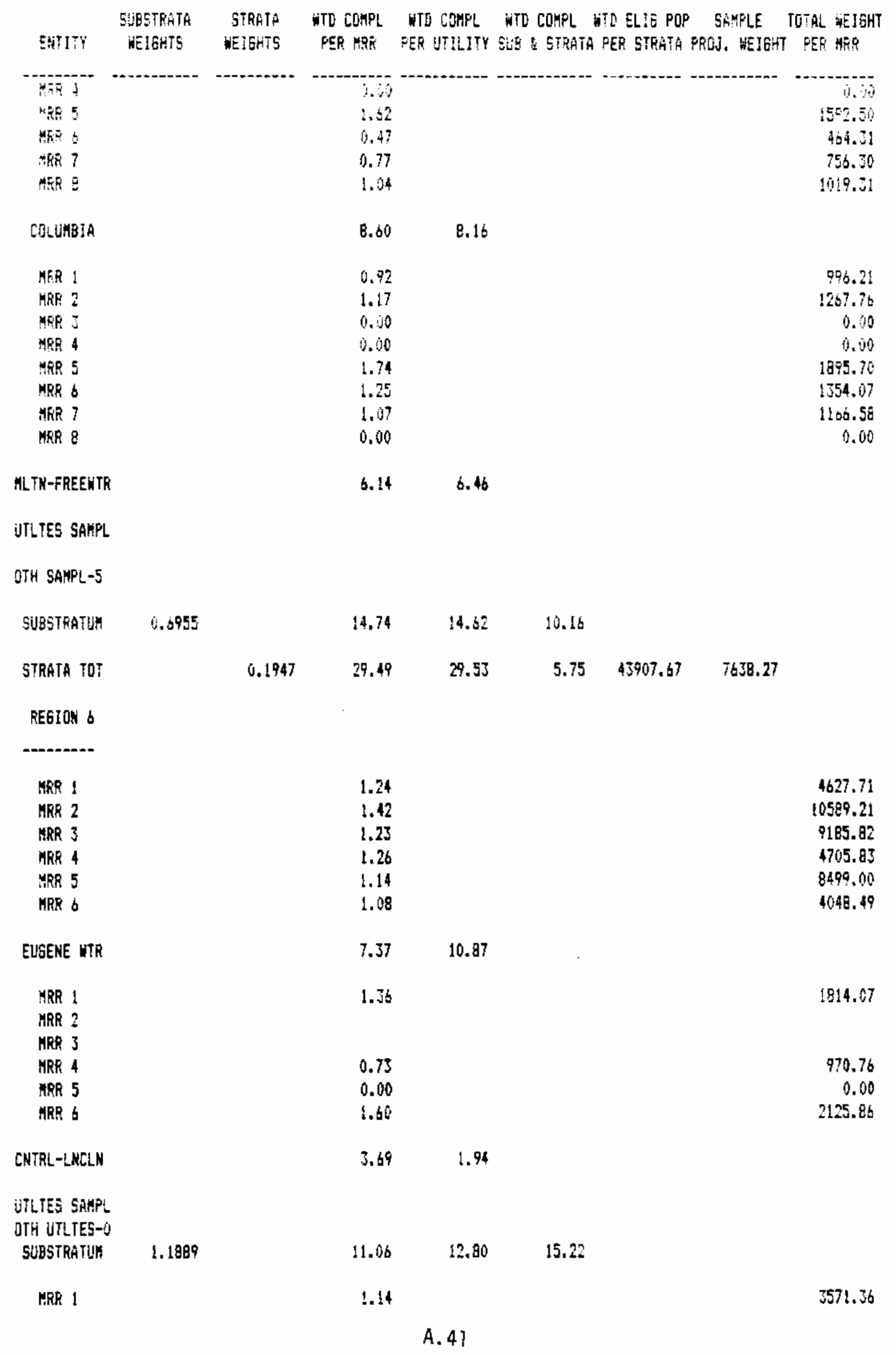




\begin{tabular}{|c|c|c|c|c|c|c|c|}
\hline Entity & $\begin{array}{l}\text { SUESTEATA } \\
\text { HEIGHTS }\end{array}$ & $\begin{array}{l}\text { STRATA } \\
\text { WEIGHTS }\end{array}$ & $\begin{array}{l}\text { WIS CDKLL } \\
\text { P:R KARR }\end{array}$ & $\begin{array}{l}\text { HTO SCMF! } \\
\text { PER DTILITY }\end{array}$ & 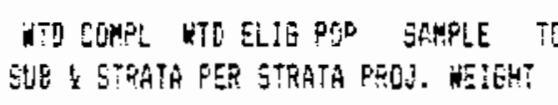 & $\begin{array}{l}\text { TETPL WE!GHT } \\
\text { CER MPR }\end{array}$ & \\
\hline पद ? & - & (n) & 1.45 & 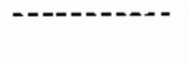 & . & 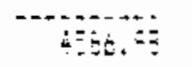 & \\
\hline ME: & & & 1,06 & & & $\therefore 6$ & \\
\hline 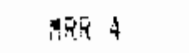 & & & 3.10 & & & 0.6 & \\
\hline Ma 5 & & & 1.28 & & & 8917,33 & • \\
\hline MFE: $=$ & & & 1.03 & & & 3221.69 & \\
\hline SPEINGE IELD & & & 4.91 & 5.96 & & & • \\
\hline 跀 1 & & & 0.60 & & & 0.60 & \\
\hline$M R_{R} 2$ & & & 1.15 & & & 2343.32 & \\
\hline 3 & & & 1.02 & & & 4135.27 & \\
\hline MkR 4 & & & 9.00 & & & 0.60 & \\
\hline MAR 5 & & & 0.80 & & & 3263.91 & \\
\hline MF & & & 1.94 & & & 3950.66 & . \\
\hline CONGUHERS & & & 4.91 & 3.87 & & & \\
\hline \multicolumn{8}{|l|}{ UTLIES SAHPL } \\
\hline \multicolumn{8}{|l|}{ DTTH UTLTES-2 } \\
\hline SUDETRATUK & t.2122 & & 9.83 & 9.83 & 11.91 & & $i$ \\
\hline $4 R_{R} 1$ & & & $! .2 \hat{0}$ & & & $5+42.9 t$ & \\
\hline MRE 2 & & & 1.38 & & & $3599 . j 9$ & \\
\hline MRR 3 & & & 0.95 & & & 2349.45 & \\
\hline AFR 4 & & & $1.3 \mathrm{~B}$ & & & 3405.13 & \\
\hline ASHLANO & & & 4.91 & 5.18 & & & \\
\hline MRR！ & & & 1.18 & & & 5199.71 & \\
\hline ARR $?$ & & & 0.74 & & & 1626.57 & \\
\hline MRR 3 & & & 6.00 & & & 0.00 & \\
\hline HRA 4 & & & 1.77 & & & 7829.13 & \\
\hline FOREST ERDVE & & & 3.69 & 3.99 & & & \\
\hline \multicolumn{8}{|l|}{ ITLTES SAHPL } \\
\hline QTH UFLIES-4 & & & & & & & $=$ \\
\hline SUBSTRATUL & 1.0961 & & 8.60 & 8.67 & 9.50 & & \\
\hline MEP 1 & & & 1.14 & & & 979.25 & \\
\hline MRR 2 & & & 1.46 & & & 1254.58 & \\
\hline MPR ? & & & 1.55 & & & 1595.80 & \\
\hline MRE 4 & & & 1.17 & & & 2014.10 & \\
\hline BLĂLHLY-LANE & & & 4.91 & 5.17 & & & 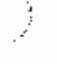 \\
\hline MER 1 & & & 1.71 & & & 2556.53 & \\
\hline WRR 2 & & & $!, 27$ & & & Gr.j! & \\
\hline $\mathrm{MKR} 3$ & & & 0.00 & & & $\therefore, 0$ & \\
\hline & & & & A. 42 & & & \\
\hline
\end{tabular}




\begin{tabular}{|c|c|c|c|c|c|c|c|c|}
\hline ENTITY & $\begin{array}{l}\text { SUESTRATA } \\
\text { LEIEHTS }\end{array}$ & $\begin{array}{l}\text { STRATA } \\
\text { WEIGHTS }\end{array}$ & $\begin{array}{l}\text { WTD COMPL } \\
\text { FER MFR }\end{array}$ & $\begin{array}{l}\text { WTD COMPL } \\
\text { PER UTLITY }\end{array}$ & $\begin{array}{l}\text { WTO COMPL } \\
\text { SHB \& STRATR }\end{array}$ & $\begin{array}{l}\text { KTD ELIG POH } \\
\text { PEL STRATA }\end{array}$ & $\begin{array}{l}\text { P SAKELE T } \\
\text { PFOJ. HEIGHT }\end{array}$ & $\begin{array}{l}\text { TOTAL HEIGUT } \\
\text { PER MRK }\end{array}$ \\
\hline 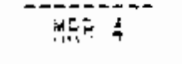 & 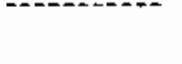 & 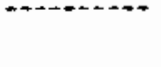 & 1.75 & 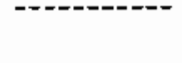 & . & & & $=t i d i$ \\
\hline MONmádTi & & & 3.69 & 3.49 & & & & \\
\hline \multicolumn{9}{|l|}{ UTL汭 SAMPL } \\
\hline \multicolumn{9}{|l|}{ Пт: :1FLIES-4 } \\
\hline SUBSTFATUK & 1.3840 & & 8.60 & 8.66 & 3.33 & & & \\
\hline EFATUM TUT & & $0.557 \%$ & 38.09 & 39.96 & 22.29 & 170274.15 & 938.27 & \\
\hline PHELIC TOT & & 1.00 & 219.91 & 221.01 & 154.89 & 1183079.68 & 7635.27 & \\
\hline \multicolumn{9}{|l|}{ ERIVATE } \\
\hline \multicolumn{9}{|l|}{ FETIN 1} \\
\hline \multicolumn{9}{|l|}{ - } \\
\hline HRR 1 & & & 0.40 & & & & & 940.23 \\
\hline MRR 2 & & & 0.00 & & & & & 0.00 \\
\hline MRR 3 & & & 0.01 & & & & & 1458.34 \\
\hline MRE 4 & & & 0.52 & & & & & 1246.77 \\
\hline ARF 5 & & & 0.27 & & & & & 649.14 \\
\hline 期保 6 & & & 0.00 & & & & & 0.00 \\
\hline MRR 7 & & & 2.71 & & & & & 6439.91 \\
\hline MRR 6 & & & 2.20 & & & & & 5229.20 \\
\hline HRR 9 & & & 2.22 & & & & & 5293.60 \\
\hline MRR 10 & & & 2.53 & & & & & 6010.12 \\
\hline MRR 11 & & & 1.73 & & & & & 4127.55 \\
\hline MRR 12 & & & 0.77 & & & & & 1821.72 \\
\hline KaR 13 & & & 0.66 & & & & & 1579.24 \\
\hline MRR 14 & & & 0.12 & & & & & 280.20 \\
\hline MRR 15 & & & 0.00 & & & & & 0.00 \\
\hline MRP 16 & & & 0.00 & & & & & 0.00 \\
\hline MONTANA PHR & & & 14.74 & 14.74 & & & & \\
\hline SUESTRATUM & 1.00 & & & & & & & \\
\hline
\end{tabular}

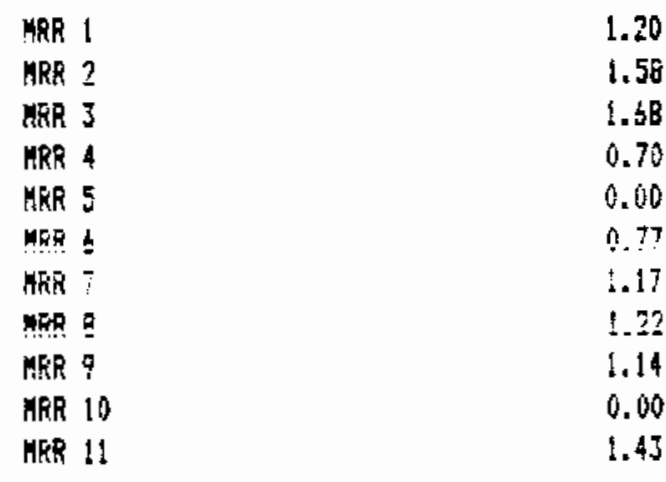

2851.61 3752.34 7989. 91 1672.16 0.00 1832.45 278.32 2912.38 2705.34 0.00 3394.12 


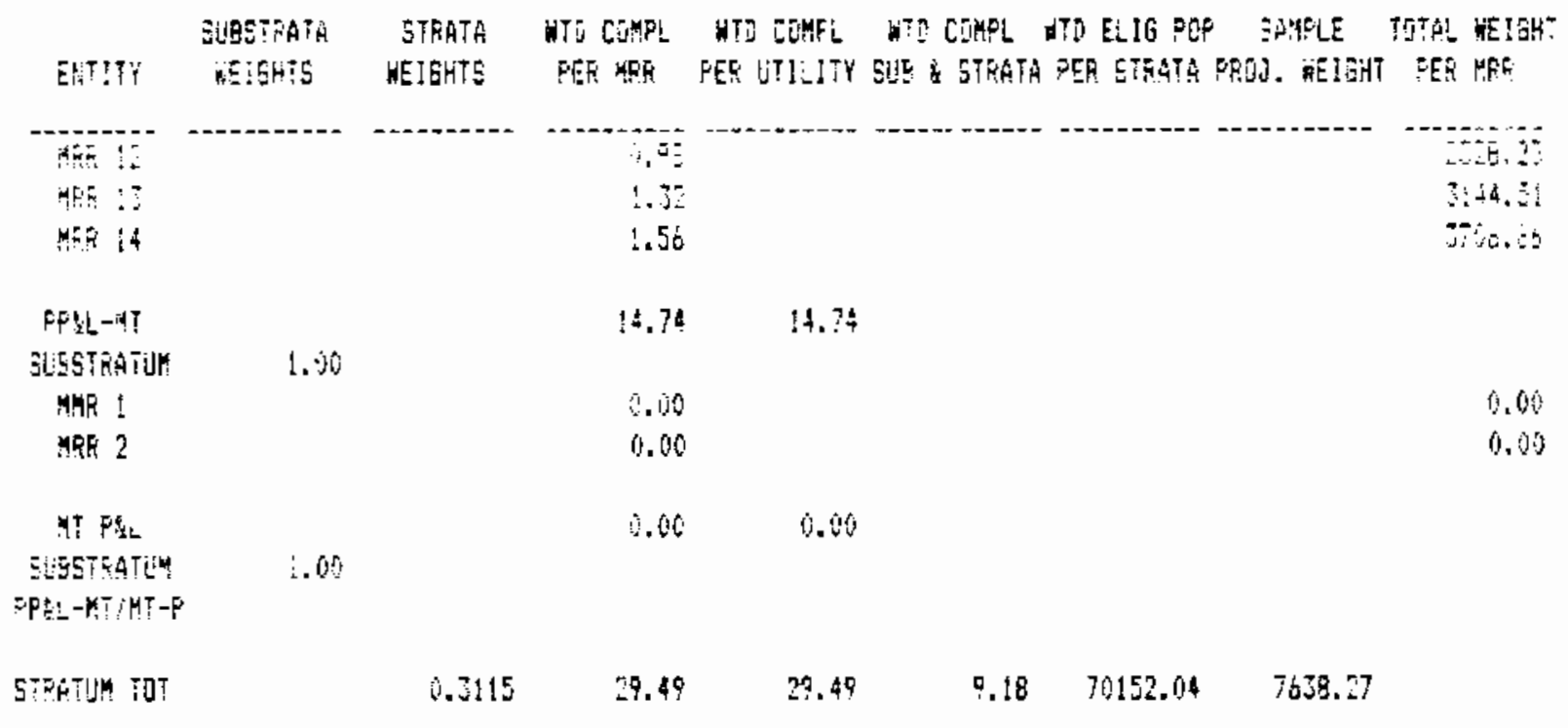

REg 2

\begin{tabular}{|c|c|c|c|c|}
\hline HAR I & & 1.96 & & 16978.91 \\
\hline MRR 2 & & 0.00 & & 0.60 \\
\hline MKR 3 & & 1.48 & & 12776.03 \\
\hline MPS 4 & & 0.94 & & 724504 \\
\hline MEF & & 1.36 & & $11735.8 \overline{4}$ \\
\hline$Y$ F & & ! It! & & 9727.54 \\
\hline $\operatorname{MrR} 7$ & & $1.00^{\circ}$ & & 8616.80 \\
\hline MRR 8 & & 0.96 & & 3277.22 \\
\hline $\begin{array}{l}\text { GFEL-ID } \\
\text { UESTRATUH }\end{array}$ & & 8.60 & 9.60 & \\
\hline UESTRATUH & 1.00 & & & \\
\hline MER 1 & & 1.12 & & 9656.33 \\
\hline MRR 2 & & 1.16 & & 10062.12 \\
\hline MeR 3 & & 1.32 & & 11446.56 \\
\hline KRR 4 & & 1.67 & & 14441.39 \\
\hline MPE 5 & & 0.00 & & 0.00 \\
\hline$M$ M 6 & & 1.02 & & $8 R 46.48$ \\
\hline ARR 7 & & 1.28 & & 11025.36 \\
\hline MRR $\mathrm{g}$ & & 1.05 & & 8EB2.59 \\
\hline $\begin{array}{l}\text { QAHO PWP } \\
\text { JBESTRATUH }\end{array}$ & 1.00 & 8.60 & 8.50 & \\
\hline MPR ! & & 1.26 & & 10885,13 \\
\hline SPR ? & & $\$ .87$ & & 11979.14 \\
\hline MRR? & & 0.71 & & 6160.66 \\
\hline VIRT 4 & & 4,00 & & 0.00 \\
\hline WR 5 & & 1.20 & & 10354.59 \\
\hline AEF 6 & & 1.60 & & 13635.49 \\
\hline SN & & 0.14 & 0.14 & \\
\hline ZESTRATUH & 1.00 & & & \\
\hline Ya! ! & & 1.18 & & 10185. \\
\hline ARR 2 & & 1.75 & & 45476.55 \\
\hline MRR 3 & & 2.02 & & 17488.91 \\
\hline
\end{tabular}




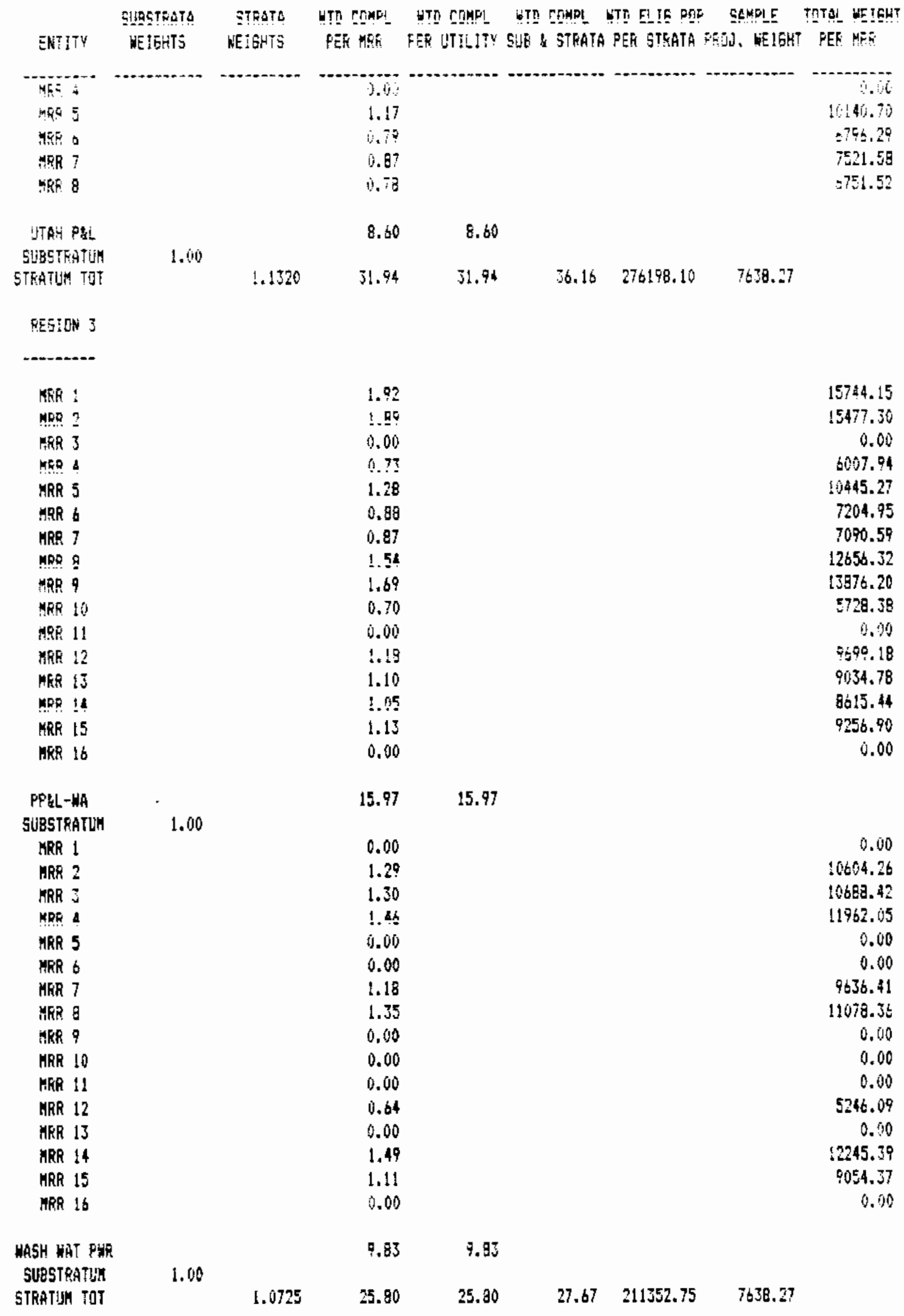




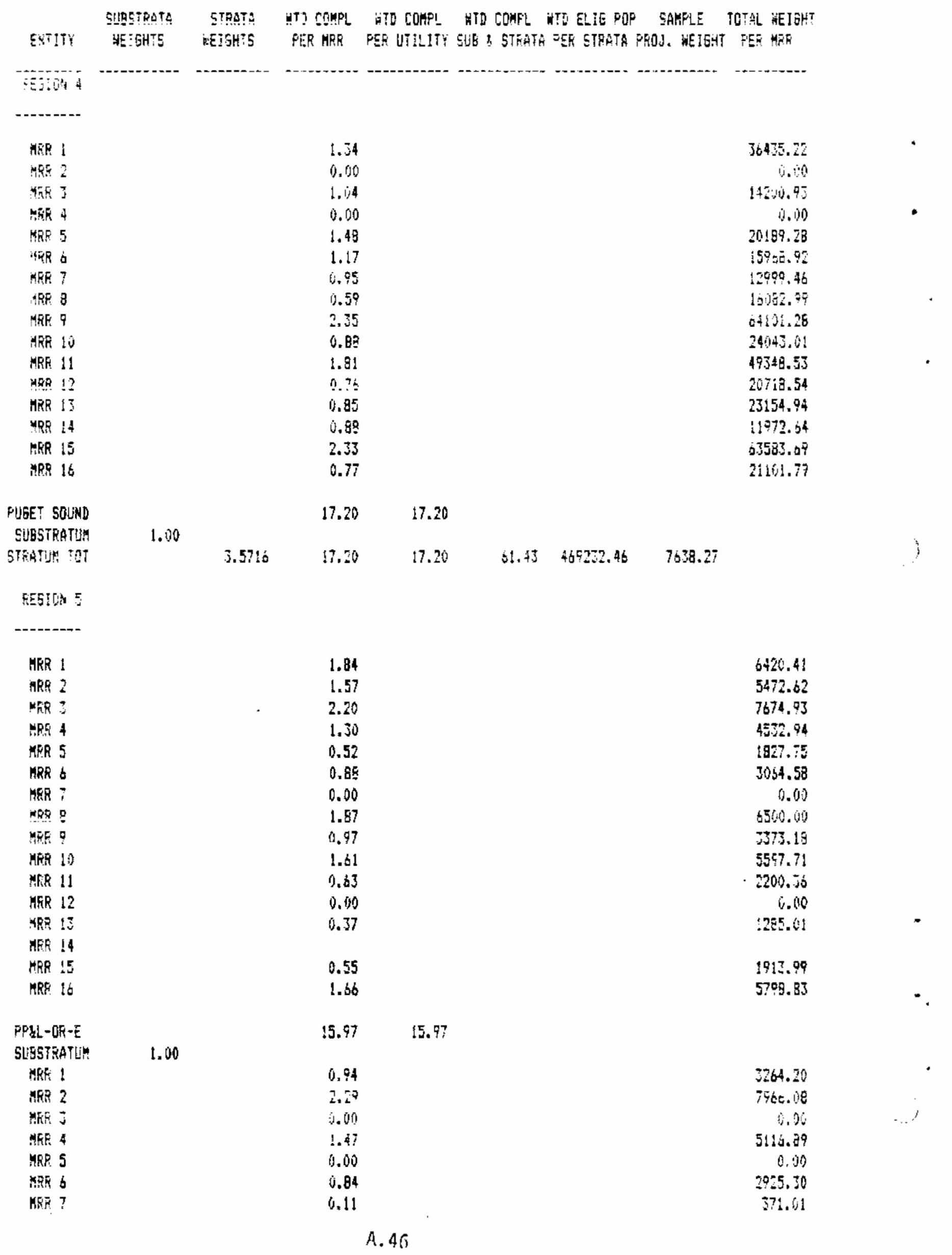




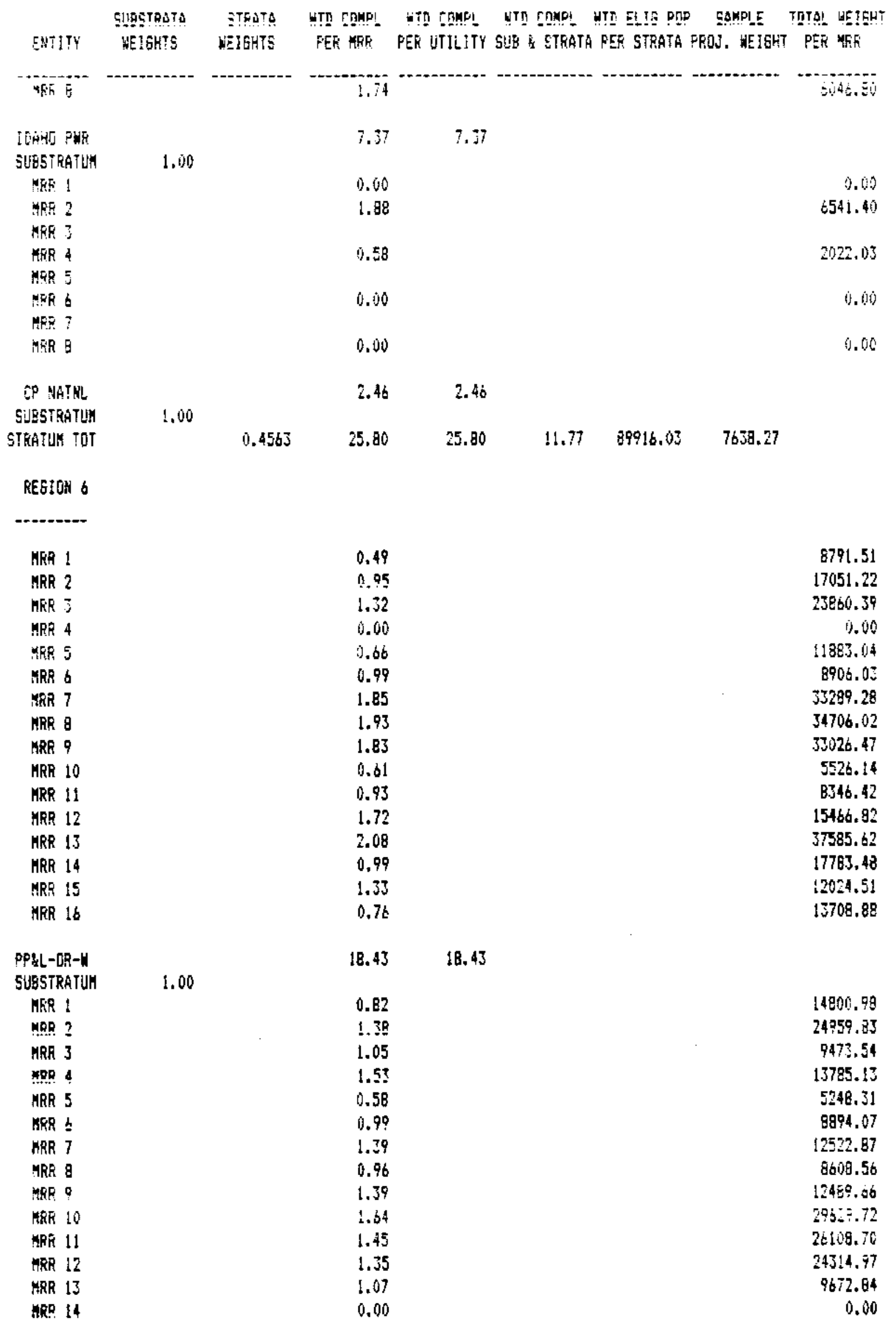




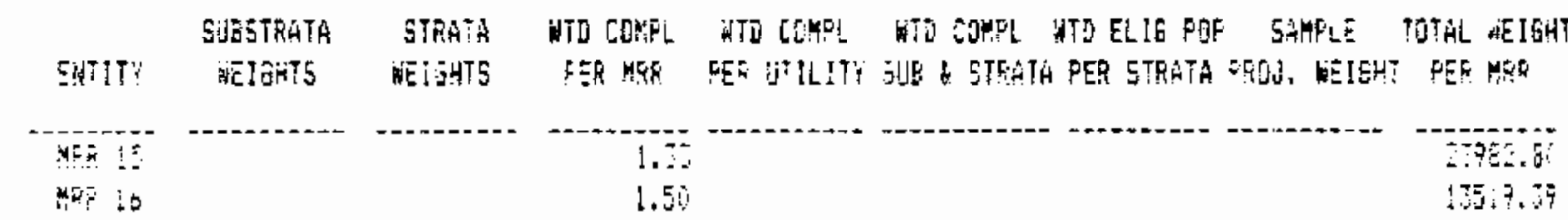

CORT BEN ELE

SUESTARTLA

STRATIM TET

ETUETE TIT

GPANAT TUT

1.00

$18.43 \quad 18.43$

$2.30 \%$ 35.56 35.6t B6.79 60445:.47 768.27

$\begin{array}{llllll}1.0000 & 167.09 & 167.09 & 235.21 & 179130 \% .65 & 7638.27\end{array}$

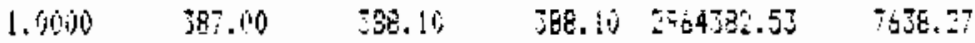




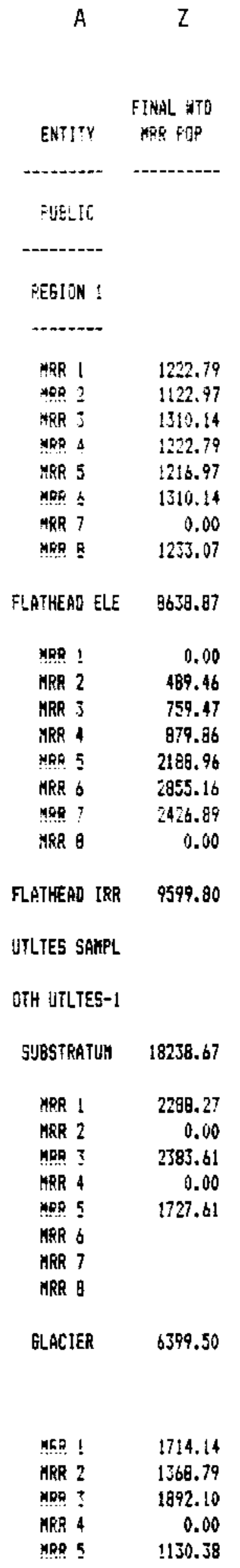




\begin{tabular}{|c|c|}
\hline EMTIT! & 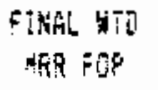 \\
\hline$-+n-1$ & $\cdots$ \\
\hline Mivfloci & $6160 .+1$ \\
\hline UTLIES SAMPI. & \\
\hline STH UTHES-2 & \\
\hline SLESTRATUH & \pm 2504.90 \\
\hline STRHTLY TOT & $3074 \vec{J} .5 \mathrm{~B}$ \\
\hline TESIOA 2 & \\
\hline$---\infty-\infty$ & \\
\hline MER I & i). 00 \\
\hline SRR 2 & 11746.46 \\
\hline $40 ?$ & 2.00 \\
\hline $\operatorname{MR} P$ & 9000.39 \\
\hline APP 5 & 7494.49 \\
\hline MER b & 3854.31 \\
\hline IDHHO FAL!S & 3110.3 .65 \\
\hline Men I & 3988.59 \\
\hline MRG ? & 3074.96 \\
\hline MPS ? & 4273.49 \\
\hline MRR 4 & 0.00 \\
\hline MKR 5 & 0.00 \\
\hline Mak 6 & 0.00 \\
\hline LOKER WALLEY & 12336.15 \\
\hline UTLIES SAMPE & \\
\hline 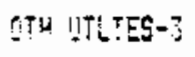 & \\
\hline SUESTKATIUH & 43430.80 \\
\hline SRR 1 & 1279.51 \\
\hline AKR 2 & \\
\hline ARR 3 & 3771.47 \\
\hline MRR 4 & 0.00 \\
\hline MEK 5 & 356.35 \\
\hline MRA 6 & ) .00 \\
\hline
\end{tabular}

FALL RIVER 53B7. 33

4月⿰ 12129.05

EASTENO WU 2126.05

HFi 1 2120.05

FAKNERS ELE 2125.15 


\begin{tabular}{|c|c|}
\hline ENTITY & $\begin{array}{l}\text { FINAL NTO } \\
\text { MER POP }\end{array}$ \\
\hline 45R! & $296.0 \mathrm{j}$ \\
\hline RIUEREIIE S & 2129.05 \\
\hline MRR! & 2128.05 \\
\hline SOUTHSIDE E! & 2128.15 \\
\hline WRR 1 & 10.00 \\
\hline MOR 2 & 2128.05 \\
\hline IjtilTY LSF & $2128.0 \mathrm{~S}$ \\
\hline ALSODKA PROJ & 10649.25 \\
\hline \multicolumn{2}{|l|}{ UTLTES SAMPL } \\
\hline \multicolumn{2}{|l|}{ nI4 HI!TES-4 } \\
\hline SUBSTRATUAH & 16027.58 \\
\hline MPP！ & 1608.55 \\
\hline HRR 2 & 0.00 \\
\hline ARR 3 & 0.00 \\
\hline 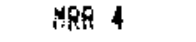 & 1615.49 \\
\hline ARR 5 & 1615.49 \\
\hline MRR 6 & 1355.78 \\
\hline LOST RIVER & 6195.31 \\
\hline \multicolumn{2}{|l|}{ HRR! } \\
\hline MRR 2 & 2329.19 \\
\hline MPR? ? & 0.00 \\
\hline MRR 4 & 0.00 \\
\hline$M R P 5$ & 0.00 \\
\hline MRR 6 & 0.00 \\
\hline AEISER & 2329.19 \\
\hline \multicolumn{2}{|l|}{ UTLTES SAHPL } \\
\hline \multicolumn{2}{|l|}{ OTH UTLTES-4 } \\
\hline SUBSTRATUH & 6524.50 \\
\hline STRATUH TOT & 67991.88 \\
\hline \multicolumn{2}{|l|}{ REEION 3} \\
\hline \multicolumn{2}{|l|}{--...-- } \\
\hline AKR I & 0.60 \\
\hline MRR 2 & 0.00 \\
\hline MRR 3 & 0.00 \\
\hline MRR 4 & 12487.75 \\
\hline MRR 5 & 0.00 \\
\hline
\end{tabular}




\begin{tabular}{|c|c|}
\hline EMTITY & $\begin{array}{l}\text { FINGL WTD } \\
\text { MRK FOP }\end{array}$ \\
\hline 10 & $y_{1}$ \\
\hline 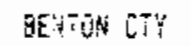 & $1240 \% .79$ \\
\hline 㢳 1 & 7751.71 \\
\hline MRF ? & 360,75 \\
\hline 46 & 11895.11 \\
\hline RP 4 & 15130.24 \\
\hline $\sin 5$ & .6259 .88 \\
\hline DHELAK ITV & 54667.70 \\
\hline Yise & 10735.94 \\
\hline CHEHMEKE & 10933.74 \\
\hline CHELAS-CSHANRE & 65003.64 \\
\hline LILTES SAMPL & \\
\hline OTH UFLTES-2 & \\
\hline SIEESTRATLH & 75091.37 \\
\hline Trô i & 1493.51 \\
\hline 준 2 & 2111,94 \\
\hline MRR 3 & 0,010 \\
\hline MeR 4 & 11736.75 \\
\hline "RR I & 6494.27 \\
\hline$M R R \quad b$ & \\
\hline FrANKLIV & 21935.57 \\
\hline MRR : & 8950.78 \\
\hline पRR ? & 0.00 \\
\hline $400 ?$ & 0443.71 \\
\hline HE & 7105.24 \\
\hline PPR 5 & 9938.5 \\
\hline MRR is & 0.00 \\
\hline RICHEANO & 32488.46 \\
\hline BPLTES SAMPL & \\
\hline DTH dİtTES-5 & \\
\hline SU8STEATUK & 54324.04 \\
\hline$M R R !$ & 0.50 \\
\hline $\operatorname{Min} 2$ & 4,100 \\
\hline 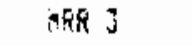 & 2234.58 \\
\hline HeP 4 & $307: .22$ \\
\hline
\end{tabular}

PEWD GRIELLE 5505.90 


\begin{tabular}{|c|c|}
\hline & FINAL HTD \\
\hline EKTITY & MES POP \\
\hline$-\cdots+\infty+\infty$ & -------- \\
\hline HRA 1 & $34: 5.77$ \\
\hline WR $?$ & 2879.79 \\
\hline MRR 3 & 334.19 \\
\hline WhE \& & 357.29 \\
\hline VERA IRR & 13119.64 \\
\hline UTITES SAHPL & \\
\hline OTH ITLIES-3 & \\
\hline SUESTRATUM & 18325.54 \\
\hline MRE I & 945.77 \\
\hline MRR 2 & 957.17 \\
\hline $\mathrm{KRR} J$ & \\
\hline MRR 4 & $1281.5 \mathrm{~B}$ \\
\hline FERRY CTY & 3082.72 \\
\hline MRR ! & 1313.26 \\
\hline $\operatorname{RRP} 2$ & 966.66 \\
\hline NESPEELEY & 2279.92 \\
\hline MRR I & 1385.84 \\
\hline MRK 2 & 894.39 \\
\hline COULEE DAH & 2279.92 \\
\hline NSPLM-COULEE & 4559.85 \\
\hline LITLTES SAMPL & \\
\hline OTH UTLTES-4 & \\
\hline SUASTRATUH & 7642.57 \\
\hline STRATIM TOT & $1583 \bar{B} 3.51$ \\
\hline REEIPN 4 & \\
\hline - & \\
\hline MRR ! & 0.00 \\
\hline Nak 2 & 50887.01 \\
\hline 战 3 & 40027.74 \\
\hline Hath 4 & 50255.65 \\
\hline $\operatorname{MRR} 5$ & 41366.21 \\
\hline $\operatorname{MRR} 6$ & 55559.01 \\
\hline
\end{tabular}




\begin{tabular}{|c|c|}
\hline ENTITY & $\begin{array}{l}\text { TINAL KTD } \\
\text { MRE FOF }\end{array}$ \\
\hline 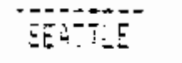 & 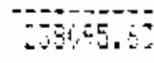 \\
\hline 掓! & $\mid(554), 4$ \\
\hline MEP 2 & $255 B C, 60$ \\
\hline 3 & 30.516 \\
\hline MER 4 & 29979.31 \\
\hline MES 5 & $226 ! 8.38$ \\
\hline MFe 6 & 27737.57 \\
\hline EkijHoni sh & 146993.63 \\
\hline $\begin{array}{l}\text { ITELTES SAMPL } \\
\text { DTH WRLTES-O }\end{array}$ & \\
\hline BLETRATIM & 384984.26 \\
\hline MEF I & 12992.45 \\
\hline ME? & 17324.73 \\
\hline STR 5 & 18342.35 \\
\hline NRR 1 & 7755.30 \\
\hline IRT 5 & 25709.10 \\
\hline MRR 6 & 0.60 \\
\hline ZLAEK CTY & 92124.13 \\
\hline$M g !$ & 26344.93 \\
\hline MFR 2 & $21039.5 ?$ \\
\hline MFE 3 & 20947.08 \\
\hline MFi 4 & 24084.74 \\
\hline MER 5 & 19472.33 \\
\hline STR & 20644.06 \\
\hline TACOUHA & 132582.70 \\
\hline HTLIES SAAPL & \\
\hline CTH UTLESA-2 & \\
\hline ZUBSTRATUY & 214706.83 \\
\hline MRE： & 0.00 \\
\hline MQ $?$ & 7541.22 \\
\hline MRF 3 & 4537.20 \\
\hline FRA 4 & 11052.15 \\
\hline MKR $\overline{7}$ & 3656.35 \\
\hline AR 6 & 6243.44 \\
\hline HASON CTYYZ & $33(131) .35$ \\
\hline MST ! & $0.0 \hat{6}$ \\
\hline MP ? & 9453.76 \\
\hline MER J & $6 c+1,20$ \\
\hline 4 & $387: .73$ \\
\hline the 5 & 3850.79 \\
\hline
\end{tabular}




\begin{tabular}{|c|c|}
\hline Extlit & $\begin{array}{l}\text { FINAL HTD } \\
\text { MER FOF }\end{array}$ \\
\hline dats & 450.65 \\
\hline ELALLAH CTY & \\
\hline WTLTES SAMFL & \\
\hline UTH UTLTES-? & \\
\hline SUESTEATUH & $6344 J .4 B$ \\
\hline MEF 1 & 4316.39 \\
\hline MRE ? & 2892.20 \\
\hline MFE 3 & 6110.27 \\
\hline Mre 4 & 7596.99 \\
\hline ELHHUKST HUT & 16905.86 \\
\hline $\operatorname{HRE} 1$ & 2851.14 \\
\hline HER 2 & 2937.56 \\
\hline MRR 3 & 2951.14 \\
\hline MRR 4 & 2851.14 \\
\hline ORCAS PWR & 11390.99 \\
\hline UTLEES SAMPL & \\
\hline OTH UTLTES-3 & \\
\hline SUESTRATUH & 28296.85 \\
\hline HRR ! & 661.86 \\
\hline MRR 2 & 1444.06 \\
\hline MRR 3 & 1468.75 \\
\hline MRR 4 & 1295.95 \\
\hline PARKLLAHJ LEP & 4870.63 \\
\hline MRR I & 1656.51 \\
\hline MRR 2 & 1201.08 \\
\hline MRR 3 & 1780.42 \\
\hline MRR 4 & 1469.56 \\
\hline SKAMANIÁ & 6107.57 \\
\hline UTLIES SAMPL & \\
\hline DTH UTLTES-2 & \\
\hline SUESTRATHA & 20979.20 \\
\hline MRP I & 2798.77 \\
\hline HRR 2 & 1322.93 \\
\hline HRR 3 & 403.31 \\
\hline
\end{tabular}




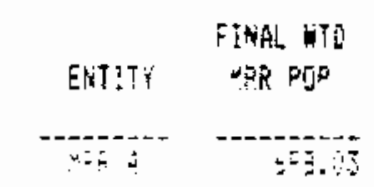

jof Ality

MP ! 417.57

45. $2 \quad 1555.31$

MFO $\mathrm{APR}$ Q11.39

ARR 4 1252.5?

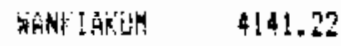

TLES SAMP!

-:ㅂ UTL!ES-5

SURSTRATLK 9J64.26

STKATUM TOT T11778.89

GEGIGN 5

\begin{tabular}{|c|c|}
\hline WER I & 2654.47 \\
\hline Mint 2 & 3175.24 \\
\hline MER & 855. 18 \\
\hline 散 4 & 3602.95 \\
\hline 船 5 & 1986.47 \\
\hline $\operatorname{MRR} 6$ & 0.00 \\
\hline HRE 7 & 2027.70 \\
\hline MRR B & 2261.31 \\
\hline MIDSTATE & 17743.12 \\
\hline ARin & 1474.37 \\
\hline FRO ? & 1986.36 \\
\hline Mex 3 & D. \\
\hline NER 4 & 2711.38 \\
\hline MER 5 & 2641.85 \\
\hline$M C Q$ & 0.60 \\
\hline MER 7 & 0.00 \\
\hline MRP E & 2234.65 \\
\hline JMATILLA & 11049.11 \\
\hline :TLLIES SAMP & \\
\hline JH JTLTES-2 & \\
\hline ELEETHATH & 26792.25 \\
\hline MR 1 & 1656.31 \\
\hline MER 2 & 1564.75 \\
\hline HFE 3 & 1381.64 \\
\hline
\end{tabular}




\begin{tabular}{|c|c|}
\hline ENTITY & $\begin{array}{l}\text { FINAL WTO } \\
\text { MQR POF }\end{array}$ \\
\hline यदि & 10 \\
\hline 파 5 & 1592.54 \\
\hline MSK 5 & 464,31 \\
\hline $\operatorname{mar} 7$ & 756.30 \\
\hline MRK & \1919.31 \\
\hline COLUMBIA & 8435.12 \\
\hline MFR I & 996.21 \\
\hline$M R 2$ & $\$ 267.76$ \\
\hline$M R$ & 0.00 \\
\hline MER 4 & 0.00 \\
\hline HRF 5 & 1895.70 \\
\hline MRR $E$ & 1354.07 \\
\hline KES 7 & 1166.58 \\
\hline MRR 8 & 0.00 \\
\hline HLTN-FREEKTR & 6680.32 \\
\hline
\end{tabular}

OTH SAAPL-S

\begin{tabular}{|c|c|}
\hline SLESTRATIHY & $15 t 15.44$ \\
\hline STRÁTA TOT & 43907.67 \\
\hline \multicolumn{2}{|l|}{ REGIOH 6} \\
\hline \multicolumn{2}{|l|}{-......-- } \\
\hline MRR 1 & 9255.42 \\
\hline MRR 2 & 10589.21 \\
\hline HRR J & 9185.82 \\
\hline MRR 4 & 9411.66 \\
\hline MRE 5 & 8499.00 \\
\hline MRF 6 & 8096.98 \\
\hline EUGENE NTR & 55038.08 \\
\hline MRR I & 3628.14 \\
\hline MRR 2 & \\
\hline MRR 3 & \\
\hline $\operatorname{MR} 4$ & 1941.51 \\
\hline MRR 5 & 0.00 \\
\hline MRR b & 4251.73 \\
\hline CNTRL-LNCLN & 9921.37 \\
\hline \multicolumn{2}{|l|}{$\begin{array}{l}\text { UTLTES SAMFL } \\
\text { DTH UTLTES-O }\end{array}$} \\
\hline SUYSTRATUK & 64859.45 \\
\hline MiR ! & 7142.73 \\
\hline
\end{tabular}

\section{A. 57}




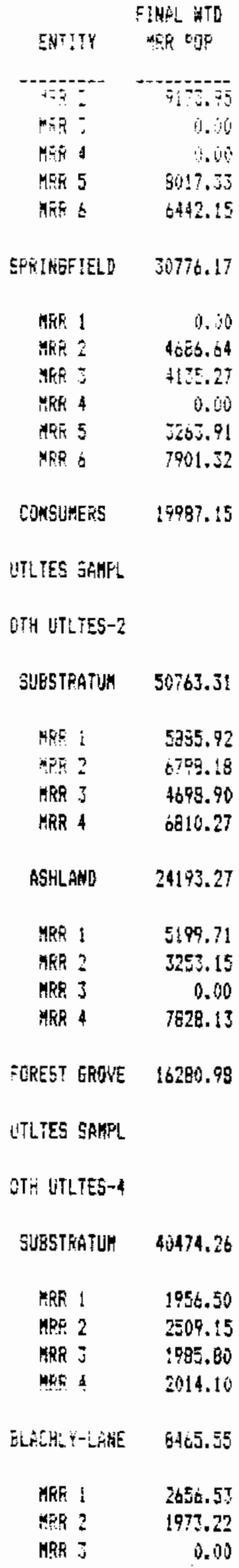




\begin{tabular}{|c|c|}
\hline ENitT & $\begin{array}{l}\text { FINAL NTD } \\
\text { MRR POP }\end{array}$ \\
\hline $4=4$ & 1681.83 \\
\hline Manugilty & 5711.58 \\
\hline WTLTES SAMPL & \\
\hline OFH UTLTES-4 & \\
\hline SUESTEAFUH & 14177.13 \\
\hline STRATU TOT & 170274.15 \\
\hline PUgLIC TGT & 1183079.68 \\
\hline PRIVATE & \\
\hline FEGION & \\
\hline --------.- & \\
\hline MRR I & 940.23 \\
\hline MRR 2 & 0.00 \\
\hline MRR 3 & 1458.34 \\
\hline iri 4 & 1246.77 \\
\hline MFE 5 & 249.14 \\
\hline Af & 0.00 \\
\hline HRR 7 & 6439.91 \\
\hline HER B & 5229.20 \\
\hline FRP 9 & 5293.60 \\
\hline SRA 10 & 6010.12 \\
\hline MRR 11 & 4127.55 \\
\hline MRR 12 & 1821.72 \\
\hline HRE 13 & 1579.24 \\
\hline MRR 14 & 280.20 \\
\hline MRE 15 & 0.00 \\
\hline$M R T$ : 6 & 0.00 \\
\hline $\begin{array}{l}\text { MBNTANA PNR } \\
\text { SLBSTRATUM }\end{array}$ & 35076.02 \\
\hline
\end{tabular}

$\begin{array}{lr}\text { MRR 1 } & 2851.61 \\ \text { HRR 2 } & 3752.34 \\ \text { MRR 3 } & 3988.91 \\ \text { HRR 4 } & 1672.16 \\ \text { MRP. 5 } & 0.00 \\ \text { HRR 6 } & 1932.45 \\ \text { HFR 7 } & 2787.32 \\ \text { MRR 9 } & 3912.38 \\ \text { MRR 9 } & 2705.34 \\ \text { MRR 10 } & 0.00 \\ \text { MRR 11 } & 3394.12\end{array}$




\begin{tabular}{|c|c|}
\hline EITTTY & $\begin{array}{c}\text { FINAL WTO } \\
\text { YART FOP }\end{array}$ \\
\hline $\begin{array}{lll}0 \\
\cdots\end{array}$ & 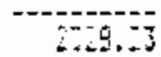 \\
\hline PES & 3144.51 \\
\hline $\operatorname{Mg} 14$ & 3706.00 \\
\hline $\cos _{\hat{L} L-M T}$ & 35676.07 \\
\hline 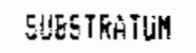 & \\
\hline MMR I & 0.60 \\
\hline$M F R 2$ & 0.00 \\
\hline 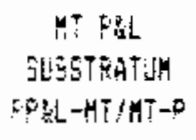 & 4.100 \\
\hline STEATUM TOF & 70152.04 \\
\hline SEGION 2 & \\
\hline - & \\
\hline MRR I & 16978.91 \\
\hline $\operatorname{MPR} 2$ & 0.60 \\
\hline MAg 3 & 12776.67 \\
\hline $\operatorname{MaP} 4$ & 7245.04 \\
\hline Tike 5 & 11729.89 \\
\hline 1486 & 8737.54 \\
\hline Mak 7 & 8610.80 \\
\hline TRR 8 & $B 277.22$ \\
\hline PFELL-1D & 74361.03 \\
\hline SUSSTRATLH & \\
\hline 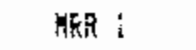 & 9656.33 \\
\hline MER 1 & 10062.12 \\
\hline HPR ? & 11446.26 \\
\hline MG 4 & 14441.j9 \\
\hline MER 5 & 0.00 \\
\hline MRP 6 & $9846.4 B$ \\
\hline MER 7 & 11025.86 \\
\hline KRR 8 & 8882.50 \\
\hline IMAHO : & 74361.03 \\
\hline SUESTKATUM & \\
\hline MRR I & 10EE5.13 \\
\hline XKR 2 & 11879.14 \\
\hline MRR 3 & 6160.66 \\
\hline MRR 4 & 0.60 \\
\hline Whit 5 & 10354.59 \\
\hline MRE 3 & 13835.49 \\
\hline $\begin{array}{l}\text { MAEA ИTF FUK } \\
\text { SUESTRATLK }\end{array}$ & 53115.62 \\
\hline HR 1 & 10135.48 \\
\hline$M R R 2$ & 15676.58 \\
\hline MER $J$ & 17429.91 \\
\hline
\end{tabular}




\begin{tabular}{|c|c|}
\hline EMTITY & $\begin{array}{c}\text { F:AAL WTD } \\
\text { WAR FOE }\end{array}$ \\
\hline यदि 4 & 3.5 \\
\hline सF 5 & $\operatorname{lig} 49,70$ \\
\hline YFA & 6796.29 \\
\hline MRR ? & 7521.58 \\
\hline the 8 & 6751,5 \\
\hline $\begin{array}{c}\text { UTAH PUL } \\
\text { SUBSTRATUH }\end{array}$ & 74361.03 \\
\hline STEATIMH TQT & 276178.10 \\
\hline
\end{tabular}

FeEton 3

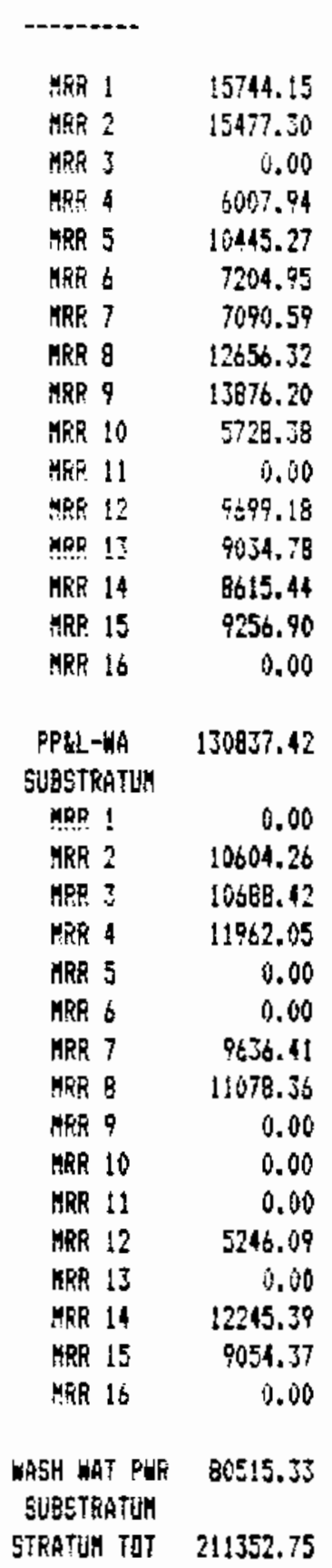




\begin{tabular}{|c|c|}
\hline ENHT & 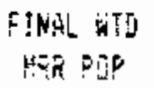 \\
\hline \multicolumn{2}{|l|}{ 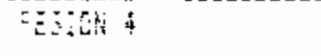 } \\
\hline \multicolumn{2}{|l|}{-----... } \\
\hline Mha 1 & 30435.22 \\
\hline 2 & 1.00 \\
\hline WEE 3 & 2404.57 \\
\hline MKP 4 & 0.00 \\
\hline WPF 5 & 4078.56 \\
\hline ang & 31977.95 \\
\hline H. & 25998.73 \\
\hline $\operatorname{ARP} 8$ & $10,08.09$ \\
\hline Whe ? & 64101.28 \\
\hline MRF 10 & 24043.015 \\
\hline HFa 11 & 49348.53 \\
\hline ing 12 & 20719.54 \\
\hline MPR 13 & $23 ! 54.94$ \\
\hline W? I & 23045.27 \\
\hline MR 15 & 65583.59 \\
\hline$A R=16$ & 21101.79 \\
\hline FIGET SDUND & 469232.46 \\
\hline SLESTRATLM & \\
\hline ETFETHA TQT & 469232,46 \\
\hline
\end{tabular}

I5sich 5

\begin{tabular}{|c|c|}
\hline HRR! & 6420.41 \\
\hline HER 2 & 5472.62 \\
\hline MRR 3 & 7674.93 \\
\hline $\mathrm{R} R \mathrm{R}^{4}$ & $\$ 532.94$ \\
\hline 5 & 1827.75 \\
\hline Kat & 3064.58 \\
\hline MER ? & 0.010 \\
\hline HF? 3 & 6500,00 \\
\hline Wha & 373.19 \\
\hline Mgh $1:$ & 5597,71 \\
\hline WRR $1 !$ & 27.60. उद \\
\hline MEE 12 & 0.00 \\
\hline KFR IS & 1285,01 \\
\hline ARE 14 & \\
\hline ARR 15 & 1513.99 \\
\hline tha lit & 5795.83 \\
\hline FigL-0R-E & 55462.31 \\
\hline SLESTRATLA & \\
\hline BnR ! & 3264.20 \\
\hline MEत 2 & 7900.08 \\
\hline At: & i. .90 \\
\hline Mra 4 & 5110.89 \\
\hline AGR 5 & 0.00 \\
\hline MRR i & 2925.30 \\
\hline MRE 7 & 371.0 \\
\hline
\end{tabular}




\begin{tabular}{|c|c|}
\hline EUTIYY & $\begin{array}{l}\text { FINAL WTO } \\
\text { ARR PGE }\end{array}$ \\
\hline 6 & 5040.90 \\
\hline $\begin{array}{l}\text { DEAHE PAK } \\
\text { SUBSTRATUK }\end{array}$ & 25090.30 \\
\hline we ! & 0.00 \\
\hline $\begin{array}{l}\text { MER } 2 \\
\text { MRR } 3\end{array}$ & 6541.40 \\
\hline $\begin{array}{l}\text { MFR } 4 \\
\text { MRR } 5\end{array}$ & 2022.03 \\
\hline $\begin{array}{l}\text { HaR } 6 \\
\text { HRR } 7\end{array}$ & 0.00 \\
\hline MRE & 0.90 \\
\hline $\begin{array}{l}\text { CP MATNL } \\
\text { SUIESTRATUM }\end{array}$ & 8563.43 \\
\hline $\begin{array}{l}\text { STRATUM TOT } \\
\text { REGION } 6\end{array}$ & 89916.03 \\
\hline -.....-- & \\
\hline HRR ! & 8791.51 \\
\hline MRR 2 & $17051.2 z$ \\
\hline MRR 3 & 23860.39 \\
\hline MRE 4 & 0.00 \\
\hline MPE 5 & 11863.04 \\
\hline RR 6 & 17812.05 \\
\hline HRR 7 & 33289.28 \\
\hline HRR g & 34706.02 \\
\hline MRR 9 & 33026.47 \\
\hline MRR 10 & 11052.28 \\
\hline MRR II & 16692.84 \\
\hline MRR 12 & 30933.63 \\
\hline MRR 13 & 37585.62 \\
\hline MRR 14 & 17783.48 \\
\hline MRP 15 & 24049.01 \\
\hline MRR 16 & 1708.88 \\
\hline PPLL-GR-M & 552225.74 \\
\hline SJESTRATUM & \\
\hline MR 1 & 14800.98 \\
\hline MRR 2 & 24959.83 \\
\hline HIR 3 & 18947.08 \\
\hline KRR 4 & 27570.25 \\
\hline ARA 5 & 10496.63 \\
\hline ARA 6 & 17788.14 \\
\hline HRR 7 & 25045.75 \\
\hline MRF 8 & 17217.13 \\
\hline 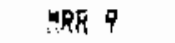 & 24979.31 \\
\hline Hen 100 & 29629.72 \\
\hline$M R$ & 20108.70 \\
\hline Whe 12 & 24314.97 \\
\hline MRR IJ & 19345.68 \\
\hline HRR 14 & 0.60 \\
\hline
\end{tabular}

A. 63 


\begin{tabular}{|c|c|}
\hline ETITY & $\begin{array}{l}\text { FINAL WTD } \\
\text { WRR FOP }\end{array}$ \\
\hline 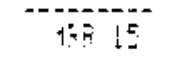 & 8580 \\
\hline ment 16 & 700.78 \\
\hline $\begin{array}{c}\text { FORT GEN ELE } \\
\text { OUESTAATLR }\end{array}$ & 332225,74 \\
\hline 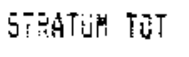 & 0004451.47 \\
\hline SPIAATE TOT & 1781302.5 \\
\hline Kint & 904 \\
\hline
\end{tabular}

A. 64 
APPENDIX B

ROSM RESPONDENTS SPREADSHEET CONTENTS 


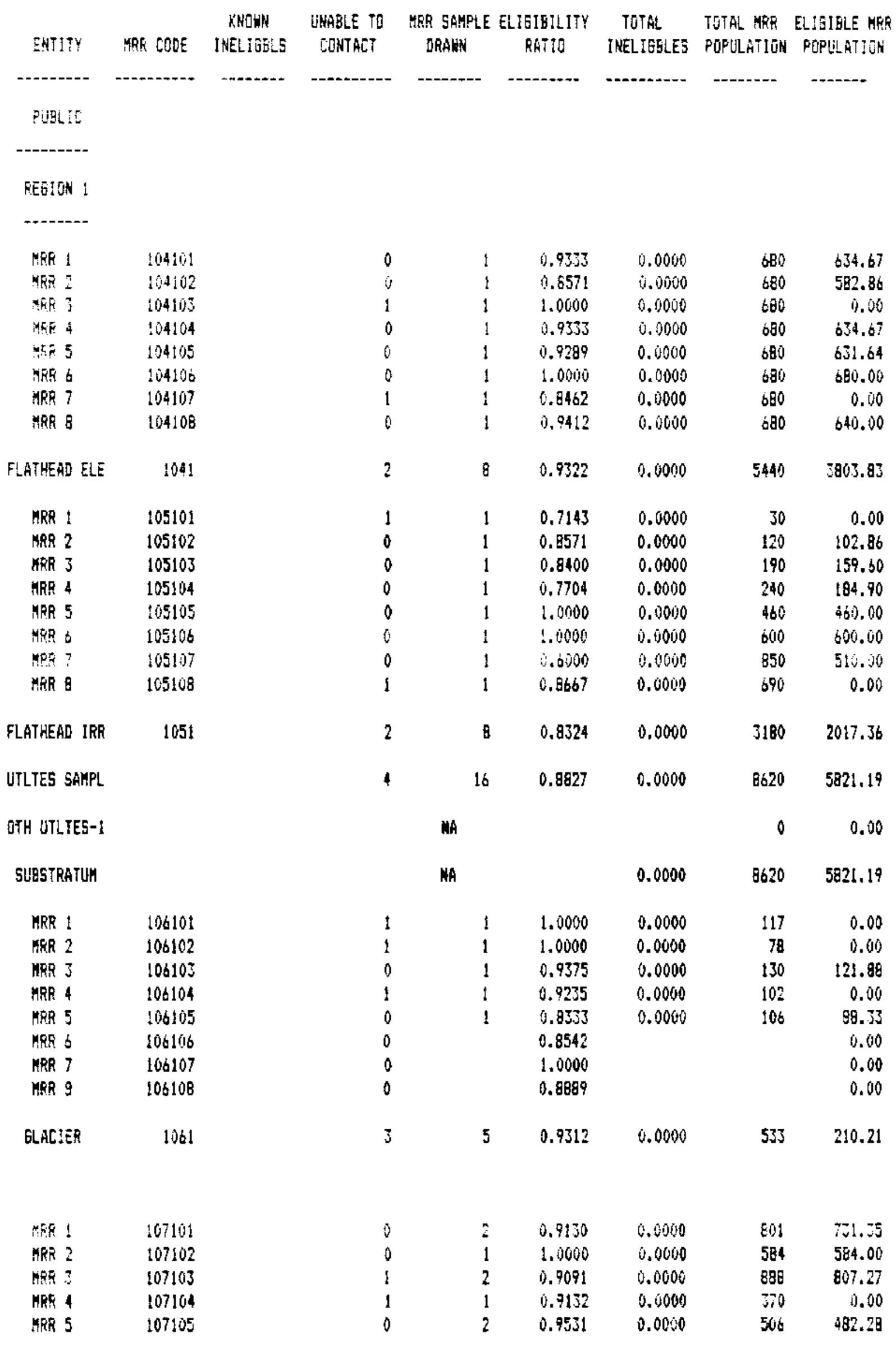




\begin{tabular}{|c|c|c|c|c|c|c|c|c|}
\hline ENTITY & WRK COSE & $\begin{array}{c}\text { RNOLN } \\
\text { INELIGBLE }\end{array}$ & $\begin{array}{l}\text { WREELE TO } \\
\text { SDNTACT }\end{array}$ & $\begin{array}{c}\text { MER SAMPLE } \\
\text { DRAMHN }\end{array}$ & $\begin{array}{c}\text { ELIGIELITYY } \\
\text { FAIIO }\end{array}$ & $\begin{array}{c}\text { TDTAL } \\
\text { INELIGE:ES }\end{array}$ & $\begin{array}{l}\text { TITAL MRR } \\
\text { PJEULATIOM }\end{array}$ & 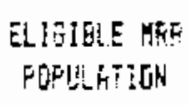 \\
\hline - n- & 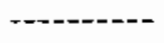 & -n. & 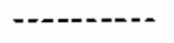 & +-ne-ce- & - & -..-- & -..n..... & $---\cdot-$ \\
\hline SHALI & $37 !$ & & $\because$ & 5 & 6,479 & ลकण & 3144 & 26094.50 \\
\hline UTLTES SAMFL & & & 5 & 13 & 0.5347 & 0.10000 & 3692 & $231 \pm .11$ \\
\hline פTH IITLIES-2 & & & & HA & & & & \\
\hline SUASTRAT!UH & & & & $\mathrm{HA}$ & & 0.6000 & 3682 & 2815.11 \\
\hline 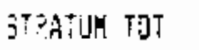 & & & & HA & & 0,0000 & $1330 ?$ & 8435.30 \\
\hline \multicolumn{9}{|l|}{ REGION 2} \\
\hline -...--- & & & & & & & & \\
\hline 短R： & 112201 & & 1 & 1 & 1.0000 & 1,0000 & 148 & 0.30 \\
\hline IRR 2 & 112202 & & $i$ & t & 1.0000 & 0.0000 & 256 & 0.70 \\
\hline MPE 3 & 112203 & & ! & $!$ & 0.9667 & 6,8000 & 276 & 7.20 \\
\hline Mkit 4 & $\$ 12204$ & & $\dot{v}$ & 1 & 0.9733 & 0.0000 & 187 & 174.53 \\
\hline NRR 5 & 112265 & & 1 & 1 & 0.9353 & 0.0000 & 175 & 0.00 \\
\hline MER \& & 112206 & & 1 & 1 & 1.6000 & 0.0000 & 84 & 0.00 \\
\hline ILAHO FALLS & 1122 & & 5 & $b$ & 0.9551 & 0,0000 & 1126 & 174.53 \\
\hline MER: & $\$ 17201$ & & 0 & 1 & 0.7652 & 0.000 & 130 & 190,00 \\
\hline Mer $?$ & 11302 & & רִ & $i$ & 1. 9286 & $0.600 \%$ & $11 \%$ & 192.14 \\
\hline met 3 & 113205 & & $!$ & 1 & 0.3571 & 0.0000 & 125 & 0.00 \\
\hline MGR 4 & 113204 & & 1 & 1 & 0.9289 & 0.0000 & 94 & 0.00 \\
\hline Min 5 & 113205 & & $!$ & 1 & $0.923 \mathrm{t}$ & 0.0000 & 384 & 0.00 \\
\hline MRR 6 & 113206 & & 1 & 1 & 0.9333 & 0.0000 & 240 & 0.00 \\
\hline LTHER VALLEY & 1152 & & 4 & $b$ & 0.8921 & 0.0000 & 1083 & 202.14 \\
\hline UTLTES GAMPL & & & 9 & 12 & 0.9245 & 0.0000 & 2209 & 376.68 \\
\hline OTH UTLTES-3 & & & 0 & NA & & & & \\
\hline SUZSTRATUH & & & & $\mathrm{NA}$ & & 6. 90000 & 2209 & $57 \dot{0.68}$ \\
\hline$M R R \perp$ & 114201 & & $\hat{0}$ & 1 & 0.7143 & 0.00000 & 551 & 350.71 \\
\hline MRR 2 & 114202 & & 0 & & & & & \\
\hline MRR 3 & 114203 & & 1 & $i$ & 1.2000 & 5.0000 & 739 & 8.61 \\
\hline KRR 4 & 114204 & & 1 & 1 & $0.557 i$ & 0.10000 & 118 & 0.0 \\
\hline MR 5 & 114275 & & 0 & : & 0.5672 & 0.00100 & 76 & 65.91 \\
\hline MER S & 114206 & & $t$ & ! & 4. & 0.0600 & 399 & 0,00 \\
\hline FALL RIVER & 1142 & & 3 & $\overline{5}$ & 0.7229 & 0.190100 & 1673 & 315.62 \\
\hline MRP 1 & $41520 !$ & & 0 & $!$ & $0.0=36$ & 0.0010 & 179 & 56.21 \\
\hline ESSENO MT & 1152 & & $j$ & : & 4.928 & 1.0900 & 97 & 60.21 \\
\hline MER I & 116201 & & 0 & 1 & 0.9333 & 0.0000 & 90 & 84.90 \\
\hline FAROEXS ELE & 1162 & & 0 & $i$ & 0.97 .33 & 1.0000 & 30 & 34.60 \\
\hline
\end{tabular}




\begin{tabular}{|c|c|c|c|c|c|c|c|c|}
\hline ENT!TY & MRR COOE & $\begin{array}{c}\text { KNOWN } \\
\text { IMELIGBLS }\end{array}$ & $\begin{array}{l}\text { INNAELE TO } \\
\text { CONTACY }\end{array}$ & $\begin{array}{l}\text { MRR SAMPLE } \\
\text { DKAWN }\end{array}$ & $\begin{array}{l}\text { ELIGIEILITY } \\
\text { RATIO }\end{array}$ & $\begin{array}{c}\text { TOIAAL } \\
\text { INELIGGLES }\end{array}$ & $\begin{array}{l}\text { TOTAL MRR } \\
\text { PDPULATIOM }\end{array}$ & $\begin{array}{l}\text { ELIGIBLE KRE } \\
\text { POPULATISN }\end{array}$ \\
\hline 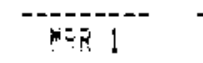 & 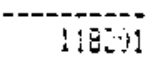 & $\cdots+\cdots+$ & 8 & 1 & 0.7259 & 40 & 70 & 32 \\
\hline FTVESIEE EL & $11: 2$ & & $\hat{s}$ & 1 & 0.9289 & 11. 1960 & $35 \hat{0}$ & $20.1 \pm$ \\
\hline MRR I & 119201 & & 0 & 1 & 1.0000 & 0.6000 & 500 & 5013,00 \\
\hline SQITHEIDE SL & 1192 & & $\hat{\theta}$ & 1 & 1.0000 & 1. कộ & 500 & 500.00 \\
\hline MRR I & 120201 & & 1 & 1 & 0.8578 & 0.0000 & 300 & 0.00 \\
\hline MRR 2 & $12 ; 202$ & & 1 & 1 & 0.7245 & 0.0000 & 310 & 0.50 \\
\hline WRITY LLF & 1202 & & 2 & $z$ & 0.7934 & 0.0000 & $\$ 10$ & 0.00 \\
\hline MiNODKA PROJ & & & 6 & 6.00 & 0.9971 & 2.0000 & NA & NA \\
\hline UTLTES SAKF! & & & & 11 & 0.8095 & 2.0000 & 3402 & 1391.95 \\
\hline OTH UTLTES-4 & & & & NA & & & & \\
\hline SUBSTKATUA & & & & MA & & 2.0000 & 3402 & 1391.95 \\
\hline KRR I & $12 ! 201$ & & 1 & 2 & $0.933 \mathrm{~J}$ & 0.0000 & 232 & 216.53 \\
\hline HRR 2 & 121202 & & 1 & 1 & 0.3667 & 0.0000 & 2.33 & 0,00 \\
\hline KE⿱ & 121203 & & 1 & 1 & 0.7867 & 0.0000 & 232 & 0.00 \\
\hline HFP A & 121204 & & 0 & 1 & 0.9733 & 6,0000 & 233 & 217.47 \\
\hline$M R R$ & 121205 & & 0 & 1 & 0.9333 & 0.0000 & 233 & 217.47 \\
\hline MAR & $2: 206$ & & 0 & 1 & 4.7867 & 0.0000 & 232 & $: 82.5 !$ \\
\hline LUST RIVER & 1212 & & 3 & 7 & $0.873 \mathrm{~J}$ & 0.0000 & 1395 & 833.97 \\
\hline MER 1 & 122201 & & 0 & & 0.8578 & & & 0.00 \\
\hline MRR 2 & 122202 & & 0 & 1 & 0.9289 & 0.0000 & 129 & 119.83 \\
\hline MRR 3 & 122203 & & 1 & 1 & 1.0000 & 0.0000 & 123 & 0.00 \\
\hline MRR 4 & 122204 & & 1 & 1 & 0.9333 & 0.0000 & 166 & 0.00 \\
\hline MRR 5 & 122205 & & 1 & 1 & 1.0000 & 0.0000 & 104 & 0.00 \\
\hline$M R R 6$ & 122206 & & 1 & 1 & 0.9286 & 0.0000 & 169 & 0.00 \\
\hline WEISER & 1222 & & 4 & 5 & 0.9416 & 0,0000 & 691 & 119.83 \\
\hline UTLJES SAHPL & & & & 12 & 0.9073 & 0.0000 & 2086 & 953.80 \\
\hline OTH UTLTES-4 & & & & $\mathrm{NA}$ & & & & \\
\hline SUBSTRATUH & & & & NA & & 0.0000 & $20 \mathrm{Bb}$ & 953.80 \\
\hline STRATUR TOT & & & & $\mathrm{NA}$ & & 2.0000 & 7697 & 2762.42 \\
\hline \multicolumn{9}{|l|}{ REGIDN 3} \\
\hline ---------- & & & & & & & & \\
\hline MRE 1 & 12301 & & 1 & 1 & 0.9244 & 0.8000 & 92 & $\dot{4} .00$ \\
\hline RRR 2 & 123302 & & 1 & 1 & 0.9231 & 0.0000 & 242 & 0.00 \\
\hline ARR 3 & 123303 & & 1 & 1 & $0.753 \mathrm{~J}$ & 0.6000 & 819 & $\$ .00$ \\
\hline RRR 4 & 123304 & & 0 & 1 & 0.7244 & 0.0000 & $62 \mathrm{P}$ & 580.55 \\
\hline MRA 5 & 123305 & & 1 & 1 & 0.7704 & 0.0000 & 675 & 0.00 \\
\hline
\end{tabular}




\begin{tabular}{|c|c|c|c|c|c|c|c|c|}
\hline ENTITY & MER CIE & $\begin{array}{c}\text { KNOHA } \\
\text { INELILBLS }\end{array}$ & $\begin{array}{l}\text { EWHELE TO } \\
\text { COHTACT }\end{array}$ & $\begin{array}{c}\text { WRE SAYFLE } \\
\text { DRAWWN }\end{array}$ & $\begin{array}{c}\text { EISEEILITY } \\
\text { SAT!S }\end{array}$ & $\begin{array}{c}\text { TOTAL } \\
\text { INELIGELES }\end{array}$ & $\begin{array}{l}\text { TOTAL WRR } \\
\text { PDPILATIUN }\end{array}$ & $\begin{array}{l}\text { ELIGIGLE KRP } \\
\text { PGFULATION }\end{array}$ \\
\hline 40 & 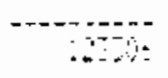 & $\cdots$ & ; & , & 8,894 & 40 & $\cdots$ & $\cdots$ \\
\hline SEMTN CT: & 228 & & $\mathbf{s}$ & z & i. $36 t 5$ & 3.5000 & 2783 & 5 E⿰彳亍J.55 \\
\hline AF ! & 1.471 & & 0 & i & 0.7933 & 0.0000 & 308 & 225.87 \\
\hline Mas ? & 124302 & & i & $!$ & 1.0002 & 0.0000 & 107 & 107.09 \\
\hline Fin 3 & $: 24305$ & & 0 & 1 & 0.9333 & 0.0000 & 372 & 347.20 \\
\hline MFE : & 124304 & & 0 & : & 1. 10000 & 0.1000 & 442 & $\$ 42.00$ \\
\hline N 5 & 124305 & & 1 & 1 & 1.9000 & 0.0000 & 475 & 0.20 \\
\hline SHELAH CTY & 1243 & & : & 5 & 0.9733 & 0.0000 & 1774 & 1122.07 \\
\hline 啙！ & $1: 5 j 01$ & & $\hat{0}$ & $\mathbf{j}$ & 1.9289 & 0.0000 & 2290 & 2127.16 \\
\hline CHSHEESE & 1253 & & 0 & 1 & 0.9289 & 1.0657 & 2290 & 2127.16 \\
\hline CHELH-CSHMRE & & & 1 & 6.00 & 0.7326 & 1.0667 & NA & NA \\
\hline UTLTES SAHPL & & & & 12 & 0.9001 & 1.0667 & 6777 & 3030 \\
\hline DTH UTLIES-Z & & & & NA & & & & \\
\hline SIBSTRATUK & & & & $N_{A}$ & & 1.0667 & 6777 & 3929.77 \\
\hline Ma ! & $12630 !$ & & j) & 1 & 0.7333 & 0.0000 & 98 & 74.37 \\
\hline Mepi & 126019 & & 8 & 1 & 0.7355 & 0.000 & 210 & 494.53 \\
\hline MEA 3 & 120305 & & 1 & 1 & 0.8000 & 0.0000 & 1103 & 0.00 \\
\hline$A T R 4$ & 126504 & & 0 & 1 & 0.7289 & 0.0000 & 608 & 564.76 \\
\hline IRR 5 & 126305 & & 0 & 1 & 0.3571 & 0.0000 & 875 & 312.50 \\
\hline MaR 6 & 126306 & & 0 & & 0.5333 & & & 0.00 \\
\hline FEAHKLIN & 1235 & & 1 & 5 & 0.7143 & 0.0000 & 2794 & 1050.71 \\
\hline MKR I & 127301 & & 1 & 1 & 1.0000 & 0.0090 & 135 & 0.00 \\
\hline MRR 2 & $: 27302$ & & 1 & 1 & $0.714 ?$ & 0.0000 & 177 & 0.00 \\
\hline MPR? & 127303 & & 1 & $!$ & 1.0000 & 0.0000 & 164 & 0.90 \\
\hline 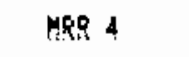 & 127304 & & 0 & 1 & 1.0000 & 0.1000 & 138 & 138.6 \\
\hline HKR $\Xi$ & 127305 & & \pm & $t$ & 1.00010 & 0.0060 & 194 & 0.100 \\
\hline MRR 6 & 127306 & & 1 & 1 & 0.9289 & 3.0000 & 191 & 0.60 \\
\hline FICHLAND & 1273 & & $\xi$ & 6 & 0.0418 & 0.0060 & 999 & 138.20 \\
\hline URLTES SAKFL & & & & 11 & 0.6274 & 0.0000 & 7793.00 & it88.?1 \\
\hline LTH UTLTEG-5 & & & & NA & & & & \\
\hline SUESTRATUM & & & & NA & & 0.0060 & 3793 & 1168.7 \\
\hline texin ! & 128301 & & 1 & 1 & 1.0000 & 0.600 & 210 & 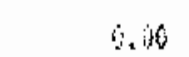 \\
\hline YKE 2 & 12502 & & 1 & 1 & 1. 3010 & 3600 & 215 & 0.00 \\
\hline FiR $j$ & 129303 & & $\hat{v}$ & 1 & $0.73 \mathrm{jz}$ & 0.6000 & 58 & 49.47 \\
\hline ARA A & 129504 & & 0 & ! & 1.0060 & 0.0000 & 68 & 68.00 \\
\hline FEND ORIELLE & 1253 & & 2 & 4 & 0.9833 & 0.9000 & 359 & 117.47 \\
\hline
\end{tabular}

B. 4 


\begin{tabular}{|c|c|c|c|c|c|c|c|c|}
\hline 대TITY & MES COQE & $\begin{array}{c}\text { KNDHN } \\
\text { IHELL IGELS }\end{array}$ & $\begin{array}{l}\text { LUABELE TO } \\
\text { COHTACT }\end{array}$ & $\begin{array}{l}\text { MFR SAMPLE } \\
\text { TFAHN }\end{array}$ & $\begin{array}{c}\text { ELIGIBILITY } \\
\text { R.RTI0 }\end{array}$ & $\begin{array}{c}\text { TDTAL } \\
\text { I MELLIGQGLES }\end{array}$ & $\begin{array}{l}\text { TOTAL MPR } \\
\text { PLPLLATIOH }\end{array}$ & $\begin{array}{l}\text { ELIGIELE Wh } \\
\text { PGPLLGT IU: }\end{array}$ \\
\hline --------- & -..---- & …..... & -------- & -------- & - - & .............. & n....... & -.-... \\
\hline MRP 1 & 130301 & & 1 & $\mathrm{t}$ & 0.8667 & 0.0000 & 341 & id \\
\hline MR 2 & 130302 & & $j$ & 1 & 4.9289 & 0.0000 & 258 & $24 \varepsilon .94$ \\
\hline MPE 3 & 130305 & & 0 & i & 1.0000 & 0.0000 & 289 & 289.110 \\
\hline NFR 4 & 133304 & & 0 & $!$ & 1.0000 & 0.0000 & 292 & 292.00 \\
\hline VERA IRE & 1303 & & 1 & 4 & 0.9535 & 0.0000 & 329 & 829.94 \\
\hline UTLTEE SAMPL & & & & 8 & 0.7677 & 0.0000 & 868 & 947.41 \\
\hline DTH UTLTES-3 & & & & IA & & & & \\
\hline SUESTRATUK & & & & NA & & 0.0000 & 388 & 947.41 \\
\hline MRR I & 131301 & & $t$ & 1 & 0.6000 & 0.0000 & 404 & 0.20 \\
\hline MRR 2 & 131302 & & 1 & 1 & 0.6805 & 0.6000 & 404 & 0.00 \\
\hline MRA 3 & 131303 & & 0 & & पे. 8в99 & & & 0.00 \\
\hline MRR \& & 131304 & & 0 & 1 & $0.911 !$ & 0.0000 & 404 & 360.09 \\
\hline FERRY CTY & $13: 3$ & & 2 & 3 & 0.7669 & 0.0000 & $12: 2$ & 368.09 \\
\hline MRR 1 & 132301 & & 0 & 1 & 1.0000 & 0.0000 & 267 & 267.00 \\
\hline MPR 2 & 132302 & & 0 & 1 & 0.7333 & 0.0000 & 268 & 196.53 \\
\hline NESPEELEY & 1223 & & 0 & $z$ & 0.5667 & 5.9000 & 535 & 463.53 \\
\hline MFR 1 & 133301 & & 0 & 1 & 0.9333 & 0.0000 & 93 & 86.90 \\
\hline MRA 2 & 133302 & & 0 & $i$ & 1.0000 & 0.0000 & 56 & 56.00 \\
\hline COULEE DAK & 1333 & & 0 & 2 & 0.9855 & 0.0000 & 149 & 142.80 \\
\hline HSPLM-CQULEE & & & 4 & 4.00 & 0.9153 & 0.0000 & & \\
\hline UTLTES SAMPL & & & & 7 & 0.8437 & 0.0000 & 1896 & 974.12 \\
\hline OTH UTLTES-4 & & & & $\mathrm{NA}$ & & & & \\
\hline SUGSTRATUM & & & & NA & & 0.0000 & 1896 & 974.42 \\
\hline STRATUM TIT & & & & WA & & 1.0667 & 13354 & 6940.32 \\
\hline \multicolumn{9}{|l|}{ REEIAH 4} \\
\hline -------- & & & & & & & & \\
\hline MRR ! & 135401 & & 2 & 2 & 1.0040 & 0.0000 & 399 & 0.60 \\
\hline MRR 2 & 135402 & & 2 & 2 & 1.0000 & 0.0000 & 403 & 0,00 \\
\hline HER J & 125403 & & 0 & 2 & 1.0000 & $9.00 \% 0$ & 317 & 317,0 \\
\hline MRA 4 & 135404 & & 0 & 2 & 1. 10000 & 0.0000 & 598 & 395.00 \\
\hline MRR 5 & 135405 & & 1 & 2 & 0.8667 & 0.6000 & 878 & 327.60 \\
\hline MkR 6 & 135406 & & 0 & 2 & 1.6000 & 0.0000 & 440 & 440.00 \\
\hline
\end{tabular}

B. 5 


\begin{tabular}{|c|c|c|c|c|c|c|c|c|}
\hline ENTITY & MRT SODE & $\begin{array}{c}\text { KMTWN } \\
\text { INELSGELS }\end{array}$ & $\begin{array}{c}\text { LMMREE TI } \\
\text { CIHTACT }\end{array}$ & $\begin{array}{c}\text { PGE SAMFLE } \\
\text { OLFA }\end{array}$ & $\begin{array}{c}\text { ELIE:B!LITH } \\
\text { HTIIJ }\end{array}$ & $\begin{array}{l}\text { VUTAL } \\
\text { INELIG9LES }\end{array}$ & $\begin{array}{l}\text { TUTAL YRR } \\
\text { POPIJETION }\end{array}$ & $\begin{array}{l}\text { ELIGIJLE } \\
\text { POPLAT }\end{array}$ \\
\hline 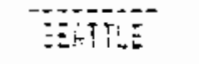 & 1 & & $\bar{F}$ & 12 & .93 & 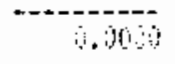 & 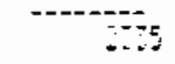 & 108,20 \\
\hline अन्दा & 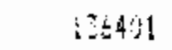 & & ! & 2 & 1.100 & 16.3000 & 97 & 89.30 \\
\hline$\therefore 2$ & 130402 & & ! & 2 & 0.9333 & i. $.90(5 i 9$ & 223 & 20.13 \\
\hline Q & 150413 & & 2 & 2 & 0.9737 & 0.0000 & 267 & 0.90 \\
\hline NRE 4 & i36404 & & 0 & 2 & 1.0000 & 4.0000 & 37 & 257.30 \\
\hline MFR 5 & 136405 & & 2 & 2 & 1.0000 & 0.0000 & 184 & 0.80 \\
\hline MRR $b$ & 136406 & & 1 & 2 & 0.9206 & 0.0000 & 243 & 225.64 \\
\hline SHEHIMI SH & $13 \leq 4$ & & 7 & 12 & 0.9647 & 0.0000 & 1245 & 761.78 \\
\hline DTLTES CAMEL & & & & 24 & 0,4711 & 0.6000 & 3590 & 2244.39 \\
\hline OTH ATLIES-O & & & & $N A$ & & & & \\
\hline SUESTRATIM & & & & NA & & 0.0000 & 3590 & 2244.38 \\
\hline MRE 1 & 137401 & & 0 & 2 & 1.0000 & 0.0000 & 258 & 259.00 \\
\hline MRF ? & $15: 402$ & & 1 & 2 & 0.9653 & 1,0000 & 380 & 344.172 \\
\hline 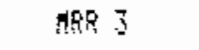 & 177403 & & 1 & 2 & 0.9244 & 12.2060 & 304 & 364.23 \\
\hline MR 4 & 137404 & & 0 & 1 & 4.6875 & 0.0000 & 224 & 158.60 \\
\hline MAS 5 & 137405 & & 1 & 2 & 0.9133 & 0.0000 & 559 & 510.52 \\
\hline MRR B & 137406 & & 1 & 1 & 0.9231 & 0.10000 & 364 & 9.60 \\
\hline CLARK STY & $: 374$ & & 4 & 10 & 3. 8994 & 1.0000 & 2179 & thith \\
\hline NRF 1 & $13 B 461$ & & 1 & 2 & $89070^{\circ}$ & $1 . \sin 0$ & 184 & 171.73 \\
\hline $\mathrm{ARR} Z$ & 138402 & & 1 & 2 & 6.9289 & 0.0000 & 148 & 657.48 \\
\hline HRR 3 & $13 B 40 \mathrm{~J}$ & & 0 & 1 & 0.9289 & 0.0000 & 147 & 136.55 \\
\hline MRR 4 & 138404 & & 1 & 2 & 1.0000 & 0.0000 & 157 & $15 \% .60$ \\
\hline Whe 5 & 138405 & & 0 & 1 & 0.9333 & 0.0000 & 136 & 126.93 \\
\hline MFR 6 & 138406 & & 2 & 2 & 0.8571 & 0.0000 & 157 & 0.60 \\
\hline TACONA & 1394 & & 5 & 10 & 0.9295 & 0.0000 & 929 & 729.69 \\
\hline UTLIES SFRFL & & & & 20 & 0.9144 & 0.0000 & 3108 & 2360.46 \\
\hline GTH UILTES-2 & & & & NA & & & & \\
\hline SUESTRATUM & & & & $N A$ & & 0.6000 & $310 \mathrm{~B}$ & 2550.46 \\
\hline MER 1 & 139401 & & 2 & 2 & 0.9289 & 0,0600 & 418 & 6.00 \\
\hline MER 2 & 139402 & & 8 & 2 & 1.0000 & 6,0000 & 343 & 357.60 \\
\hline MRR J & 139403 & & 0 & 2 & 0.8667 & 0.0000 & 252 & 218.40 \\
\hline$M R R \perp$ & 139404 & & 1 & 2 & 1.0060 & 0.10000 & 532 & $52 x+60$ \\
\hline Ma 5 & 139405 & & 0 & 2 & 0.8000 & 0.00010 & 220 & 170.60 \\
\hline Mat b & 139406 & & ) & 2 & 0.755 & 0.0000 & 398 & $30.5 J$ \\
\hline MASJN CTY-Z & 1394 & & 3 & 12 & 0.9921 & $0.060)$ & 2183 & 1589.93 \\
\hline Akx! & $1 4 \longdiv { 4 0 1 }$ & & 2 & 2 & $\therefore .0000$ & 4. 5000 & 419 & $\therefore$ \\
\hline$M H_{n}$ Z 2 & {$\left[4 i^{4} i^{2}\right.$} & & 0 & 2 & 0.7735 & 0.5000 & 9013 & $\Delta E^{2}=70$ \\
\hline MRR 3 & $: 40403$ & & 2 & 2 & 0.8487 & 0,0000 & 548 & 6.00 \\
\hline $4 P R 4$ & 140404 & & 1 & 2 & 0.8000 & 0.0000 & 337 & $27: .20$ \\
\hline AFR 5 & $1+10405$ & & 1 & 2 & 0.9530 & 0.0010 & 289 & 269,73 \\
\hline
\end{tabular}




\begin{tabular}{|c|c|c|c|c|c|c|c|c|}
\hline Ex:T ITY & HGS OOE & 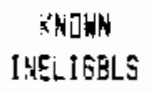 & $\begin{array}{l}\text { UWABLE TO } \\
\text { CONTACT }\end{array}$ & $\begin{array}{c}\text { HRR SAMFIE } \\
\text { DEAWN }\end{array}$ & $\begin{array}{c}\text { ELIGIEILITY } \\
\text { FATIO }\end{array}$ & $\begin{array}{c}\text { TOTAL } \\
\text { INELLISGSES }\end{array}$ & $\begin{array}{l}\text { TUTAL MPR } \\
\text { PCPULATIOUN }\end{array}$ & $\begin{array}{l}\text { ELIGIDLE AFR } \\
\text { PLPULATIOA }\end{array}$ \\
\hline do: & is:40 & - & & $\because$ & 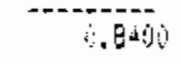 & 0.3000 & $5 j$ & $4 E \div 60$ \\
\hline CLALLAM CTY & $: \$ 04$ & & $?$ & 12 & 6.8573 & 0.9000 & 3048 & $1665:=3$ \\
\hline LTLTES SAMPI & & & & 24 & 9.9753 & 0.0000 & 5231 & 3255.06 \\
\hline UTH UTLTES-3 & & & & NA & & & & \\
\hline SLACTFATUM & & & & $\mathrm{NA}$ & & 0.5000 & 5231 & 3555.06 \\
\hline NFR 1 & 141401 & & $\hat{0}$ & 2 & 0.8667 & 0. .0000 & 432 & 374.40 \\
\hline Het 2 & 141402 & & 2 & 2 & 1,3000 & 1). 1000 & 250 & 0.00 \\
\hline SRR 3 & 141403 & & 1 & 2 & 1.0000 & $\$ .0000$ & 530 & 530.00 \\
\hline MRR 4 & 141404 & & 1 & 2 & 1.0000 & 0.0000 & 312 & 312.00 \\
\hline ELMHURET MUT T & 1414 & & 4 & 8 & 0.9667 & 0.0000 & .524 & 1216.40 \\
\hline $\mathrm{MKA} 1$ & 142401 & & 0 & 2 & 0.9333 & 0.0000 & 1250 & 1166.67 \\
\hline MRA 2 & 142402 & & 1 & 2 & 0.9289 & 0.0000 & 1250 & 1161.11 \\
\hline MRR 3 & 142403 & & 0 & 2 & 0.9333 & 0.0000 & 1250 & 1166.67 \\
\hline MER 4 & 142404 & & 1 & 2 & 0.9333 & 0.0000 & 1250 & 1166.67 \\
\hline DRCAS PHR & 1424 & & 2 & B & 0.9322 & 0.0000 & 5000 & 4661.11 \\
\hline JTLTES SAMPL & & & & 16 & 0.9494 & 0.0050 & j524 & $5877.5 !$ \\
\hline DTH UTLTES-J & & & & $N A$ & & & & \\
\hline SUBSTRATUJH & & & & औA & & 0.0000 & 6524 & 5877.51 \\
\hline MPR 1 & 143401 & & 0 & 2 & 0.9286 & 0.0000 & 77 & 71.50 \\
\hline MRR 2 & 143402 & & 0 & 2 & 0.9286 & 0.0000 & 168 & 156.00 \\
\hline MRR 3 & 143405 & & 0 & 2 & 0.9353 & 0.0000 & 170 & 158.67 \\
\hline MER 4 & 143404 & & 0 & 2 & 1.0000 & 0.0000 & 140 & 140.00 \\
\hline PARKLAND L LP & $\{434$ & & 0 & 8 & 0.9483 & 0.0000 & 555 & 526.17 \\
\hline MRP I & 144401 & & 1 & 2 & 0,9231 & 0.0000 & 127 & 117.23 \\
\hline MKR 2 & 144402 & & 0 & 2 & 1.0000 & 0.0000 & 85 & 85.00 \\
\hline MRR j & 144403 & & 1 & 2 & 0.0533 & 0.0000 & 135 & 126.00 \\
\hline MRR 4 & 144404 & & 1 & 2 & 1.0600 & 0.0000 & 104 & 104.00 \\
\hline SKAMANIA & 1444 & & 3 & 8 & 0.9655 & 0.0000 & 451 & 452.23 \\
\hline UTLTES SAMPL & & & & 16 & 0.9569 & 0.0000 & 1006 & 958.40 \\
\hline OTH UTLTES-2 & & & & NA & & & & \\
\hline SUBETRATLH & & & & NA & & 0.2000 & 1606 & 95.40 \\
\hline MRR 1 & {$[45401$} & & 0 & 2 & 1.0000 & 0.0000 & 421 & 421.00 \\
\hline ARR 2 & 145402 & & 1 & 2 & 1.0000 & 0.0000 & 199 & 199.00 \\
\hline MER 3 & 145403 & & 1 & 2 & 0.9733 & 0.0000 & 65 & 60.67 \\
\hline
\end{tabular}




\begin{tabular}{|c|c|c|c|c|c|c|c|c|}
\hline$E n^{\top} ! 1$ & MFR CORE & $\begin{array}{c}\text { KHIWH } \\
\text { INELISBLS }\end{array}$ & $\begin{array}{l}\text { UHAELE TO } \\
\text { DONTACT }\end{array}$ & 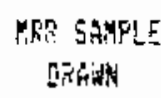 & $\begin{array}{c}\text { E ELIGLBILIT } \\
\text { PRTLE }\end{array}$ & $\begin{array}{l}\text { PDTAL } \\
\text { INELIGELES }\end{array}$ & $\begin{array}{l}\text { TOTAL WEP } \\
\text { PQPULATION }\end{array}$ & $\begin{array}{l}\text { ELIGILE MRE } \\
\text { PLPULFITS }\end{array}$ \\
\hline Mrk :- & 15404 & - & 2 & & $=$ & n & 10 & i. \\
\hline 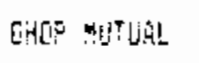 & $! 154$ & & 4 & & 9.9853 & 10000 & 700 & 680.57 \\
\hline YFe 1 & $: 464 ! 1$ & & 1 & & 0.7373 & 0.10000 & 72 & 52,80 \\
\hline 2 & $34: 400^{2}$ & & 0 & & 1. $i 0100$ & 0.1006 & 197 & 197.04 \\
\hline Mip 3 & 146403 & & 0 & & 0.9285 & 0.0000 & 124 & 115.14 \\
\hline$A F R A$ & 146414 & & 1 & & 0.7500 & 0.0000 & 211 & 158.25 \\
\hline WANY I H. UH & 1464 & & 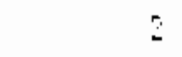 & & 0.9515 & 1.0090 & 504 & 523.19 \\
\hline LTLES SAMFL & & & & 16 & 0.9211 & $0.0: 00$ & 1394 & 1203.96 \\
\hline THW UTITES-5 & & & & $H A$ & & & & \\
\hline SEETRATLK & & & & $N A$ & & 0.6000 & 1394 & 1203.86 \\
\hline STRATUA TUT & & & & MA & & 0.0000 & 20845 & 15895.67 \\
\hline \multicolumn{9}{|l|}{ REGION 5} \\
\hline -..- & & & & & & & & \\
\hline SES 1 & $1425\rceil 1$ & & 0 & & 0.7560 & 0.0000 & 584 & 438.00 \\
\hline wet 2 & $i 4850$ & & 0 & & 0.7673 & 0.0060 & 619 & 627.66 \\
\hline HFA 3 & 148503 & & 0 & & ti. 8616 & 0.000 & 165 & it2. is \\
\hline MRE 4 & 148504 & & 1 & & 1.1000 & 0.0000 & 599 & 0.00 \\
\hline MER 5 & $14 E 505$ & & 0 & & 0.8975 & 0.0000 & 368 & 330.37 \\
\hline HRR 6 & 148506 & & 1 & & 1.0000 & 0.0000 & 1058 & 0.00 \\
\hline MRR 7 & 148507 & & 1 & & 0.9375 & 0.0000 & 466 & 8.00 \\
\hline MER A & $148508^{\circ}$ & & 0 & & 0.7800 & 0.0000 & \&E? & 375.96 \\
\hline MIDSTATE & 1495 & & 3 & & 0.8822 & 0.0000 & 4540 & 1914.05 \\
\hline MER 1 & 149501 & & 0 & & 0.7333 & 0.0000 & 91 & 59.40 \\
\hline $\operatorname{man} 2$ & $140519 ?$ & & 1 & & 1,0000 & 0.10000 & 80 & 80.00 \\
\hline$M R R 3$ & 149503 & & 1 & & 0.9733 & 0,0000 & 162 & 0.90 \\
\hline MES 4 & 149504 & & 0 & & 0.8667 & 0.0000 & 126 & 109.20 \\
\hline MRT 5 & 149505 & & 0 & & 0.9333 & 0.0000 & 114 & 106.40 \\
\hline MRR i & $\{49506$ & & 1 & & 0.0733 & 0.0000 & $8 ?$ & 0.00 \\
\hline MRP 7 & 149507 & & $i$ & & 0.9294 & 0.6000 & 106 & 0.90 \\
\hline HER 8 & 145508 & & 0 & & 1.0000 & 0.0000 & 90 & 90.00 \\
\hline MHATILLÂ & 1405 & & 3 & & $0.916 !$ & 0.0000 & 341 & 445.50 \\
\hline LTLIES SAYPL & & & & 16 & 0.8980 & 0.0000 & 5381 & 2359.05 \\
\hline GTH UTLTES-2 & & & & MA & & & & \\
\hline ELSSTATIA & & & & A & & 0.5000 & EJE1 & 35.65 \\
\hline MRE 1 & 150501 & & 0 & & 1.0000 & 0.0000 & 199 & 199.00 \\
\hline MRA 2 & 15,502 & & 0 & & 1.0000 & 0,0000 & 188 & 198.00 \\
\hline MRE 3 & 1505,13 & & 0 & & 1.10000 & 0.0000 & 166 & 156.00 \\
\hline
\end{tabular}

B. 8 


\begin{tabular}{|c|c|c|c|c|c|c|c|c|}
\hline EHTITY & MRR CODE & $\begin{array}{c}\text { KMOHN } \\
\text { IMELIBELS }\end{array}$ & $\begin{array}{l}\text { UNAELE TO } \\
\text { CONTACT }\end{array}$ & $\begin{array}{c}\text { MRF SAHPLE } \\
\text { DRAWN }\end{array}$ & $\begin{array}{l}\text { ELIEIBILITY } \\
\text { RATIO }\end{array}$ & $\begin{array}{l}\text { TOTAL } \\
\text { INEL IGBLES }\end{array}$ & $\begin{array}{l}\text { TOTAL MRF } \\
\text { PGPULATION }\end{array}$ & $\begin{array}{l}\text { ELIGIELE RRR } \\
\text { PIPULATIDN }\end{array}$ \\
\hline$x_{4}$ & 60054 & .......... & 1 & ${ }_{1}$ & \%86 & 0.6500 & $17 i$ & ---o- \\
\hline WhE & :5:5 & & 0 & $!$ & 0.933 & 1.0000 & $20^{5}$ & 191.33 \\
\hline What & $+505 i d 0$ & & 1 & 1 & 0.7857 & 0.6000 & 71 & 6.60 \\
\hline MER ? & $15050 ?$ & & $\hat{0}$ & $i$ & 0.6444 & 0.0000 & 141 & 90.87 \\
\hline MEF 3 & 150,008 & & 0 & 1 & 0.7353 & 0.00000 & 167 & $: 22.47$ \\
\hline COLUMEIA & 4505 & & 2 & E. 00 & 0.8764 & 0.0000 & 1267 & 957.67 \\
\hline MSR ! & !5!5.5! & & 0 & 1 & 0.9296 & 0,0000 & 103 & 95.64 \\
\hline MER 2 & 151502 & & 0 & 1 & 0.8571 & 0.0000 & 142 & 121.71 \\
\hline$M R R 3$ & 151503 & & ! & 1 & 1.0000 & 0.0000 & 112 & 0.00 \\
\hline MFR A & 151504 & & 1 & 1 & 1.0000 & 0.0000 & 134 & 0.00 \\
\hline MER 5 & 151505 & & 0 & 1 & 1.0000 & 0.0000 & 192 & LB2.00 \\
\hline WRR b & 151506 & & 0 & 1 & 1.0000 & 0.0000 & 130 & 130.00 \\
\hline MRt 7 & 151507 & & 0 & 1 & 1.0000 & 0.0000 & 112 & 112.00 \\
\hline MRR 8 & 151508 & & ! & 1 & 1.0060 & 0.0000 & 99 & 0.00 \\
\hline HLTN-FREENTA & 1515 & & 3 & 8 & 0.9741 & 0.0000 & 1014 & 641.36 \\
\hline UTLTES SAKPL & & & & 16 & $0.925 \mathrm{~J}$ & 0.0000 & 2281 & 1590.02 \\
\hline OTH SAMPL-5 & & & & NA & & & & \\
\hline SUBSTRATUK & & & & NA & & 0.0000 & 2281 & 1599,02 \\
\hline STRATA TOT & & & & HA & & 0.0000 & 7662 & 3958.07 \\
\hline \multicolumn{9}{|l|}{ REGION 6} \\
\hline \multicolumn{9}{|l|}{-.......- } \\
\hline MRg 1 & 153601 & & 0 & 2 & 0.9289 & 0.0000 & 2741 & 2546.08 \\
\hline MRR 2 & 153602 & & 1 & 2 & 1.0000 & 0.0000 & 2913 & 2913.00 \\
\hline MRR 3 & 153603 & & 1 & 2 & 0.8367 & 0.0000 & 3020 & 2526.94 \\
\hline MRR 4 & 153604 & & 1 & 2 & 0.9335 & 0.0000 & 2774 & 2589.07 \\
\hline MRR 5 & 153605 & & 1 & 2 & 1.0000 & 0.0000 & $235 \mathrm{~B}$ & $233 B .60$ \\
\hline ARR 6 & 153606 & & 0 & 2 & 0.9235 & 0.0000 & 2412 & 2227.41 \\
\hline ELGEANE UTK & 1536 & & 4 & 12 & 0.9384 & 0.0000 & 16198 & 15140.50 \\
\hline MRR 1 & $\lfloor 5460 !$ & & 1 & 2 & 0.9289 & 0.0000 & $19 ?$ & 178.35 \\
\hline MAR 2 & 154602 & & 0 & & 0.5835 & & & 0.00 \\
\hline MRR 3 & 154603 & & 0 & & 0.8929 & & & 0.00 \\
\hline $\operatorname{MrR} 4$ & {$[54604$} & & 0 & 2 & 0.7515 & 0.0000 & 127 & 95.44 \\
\hline MRR 5 & 154605 & & 2 & 2 & 0.9289 & 0.0000 & 136 & 0.00 \\
\hline HRA 5 & 154606 & & 1 & 2 & 1.0000 & 0.0000 & 209 & 209.00 \\
\hline CNTRL-LNCLN & 1546 & & 4 & 8 & 0.8553 & 0.0000 & 664 & 462.78 \\
\hline WITES SRMEL & & & & 20 & 6.8907 & 0.0000 & 10852 & 15625,28 \\
\hline OTH UTLTES-O & & & & HA & & & & \\
\hline SUESTRATUH & & & & HA & & 0.0000 & 16862 & 15623.28 \\
\hline HRR 1 & 155801 & & 1 & 2 & 0.9244 & 0.0000 & 379 & 350.36 \\
\hline & & & & B. 9 & & & & \\
\hline
\end{tabular}




\begin{tabular}{|c|c|c|c|c|c|c|c|c|}
\hline EnT!Ty & ARE CODE & $\begin{array}{c}\text { X'NOHN } \\
\text { IMELIGSLS }\end{array}$ & $\begin{array}{c}\text { WNABEE TO } \\
\text { COHTACT }\end{array}$ & $\begin{array}{c}\text { MFF SAMFIE } \\
\text { DRAUN }\end{array}$ & $\begin{array}{c}\text { ELIGIBLL:TY } \\
\text { RHTIO }\end{array}$ & $\begin{array}{c}\text { TO? मैL } \\
\text { THELIGGLES }\end{array}$ & $\begin{array}{l}\text { TOTAL FRR } \\
\text { ODFULATIJH }\end{array}$ & $\begin{array}{l}\text { ELIGIELE MER } \\
\text { POPULATIOH }\end{array}$ \\
\hline सरें & 28 & --...-.- & 1 & $\because$ & 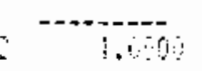 & 0.0 & 45 & 450 \\
\hline$A F=?$ & $\therefore 5=6 i_{2}^{*}$ & & 8 & 7 & 1.0000 & 0,600 & 55 & 1,60 \\
\hline जिए स & 155 oit & & 2 & 2 & 1. 0000 & $(1,0) 00$ & 76 & 6.6 \\
\hline Wer 5 & $1556 \%$ & & $\because$ & 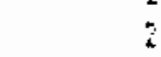 & 0.9367 & D. 0000 & 470 & $j_{2} 0$ \\
\hline As & $155 \div 06$ & & $\hat{\mathrm{u}}$ & 2 & 1.0000 & 0.0060 & $3 ! 5$ & 316.10 \\
\hline SRRINGFIELD & 1556 & & 8 & 12 & 0.9616 & 4.0000 & 22010 & 1116.36 \\
\hline He 1 & 156601 & & 2 & 2 & $0,84 B C$ & 1.0000 & 53 & b. $\mathrm{v} \theta$ \\
\hline MFP : & $15 c 001 ;$ & & 0 & 2 & 0,933 & 0.0000 & 38 & 63.47 \\
\hline MES 3 & 156003 & & 1 & 2 & 0.8000 & 0.0000 & 79 & 56.00 \\
\hline MRP 4 & 1565194 & & $\hat{2}$ & 2 & 0.9333 & 0.6000 & 157 & 0.00 \\
\hline HRR 5 & $: 56005$ & & 1 & 2 & 0.3667 & 0.0000 & $5 i$ & 44.20 \\
\hline MFR o & $1=6606$ & & 1 & 2 & 1.0000 & 0.0000 & 107 & 107.00 \\
\hline CDREUHERS & 1566 & & 7 & 12 & 0.8970 & 0.0006 & 506 & 270.67 \\
\hline WTLTES SAHPL & & & & 24 & $0.92 \% 1$ & $0.0000^{\circ}$ & 2712 & 1397.03 \\
\hline DTH UTLTES-2 & & & & MA & & & & \\
\hline SUBSTRATUM & & & & NA & & 0.0000 & 2712 & 1387.05 \\
\hline Yage : & 1376,1 & & $\tilde{z}$ & : & 0.7289 & 0.0090 & 107 & 99,39 \\
\hline $\mathrm{Han} 2$ & $157 \pm 07$ & & 0 & 2 & 0.5184 & 0.0000 & $12 \bar{j}$ & 114,90 \\
\hline $\mathrm{NRR} 3$ & 157603 & & 0 & 2 & 0.9205 & 0.0000 & 96 & 79.35 \\
\hline $\mathrm{MRF} 4$ & 157604 & & 1 & 2 & f.0000 & 0.0000 & 115 & 115.00 \\
\hline ASHLAND & 1576 & & $t$ & 8 & 0.9186 & 0.0000 & 443 & 409.53 \\
\hline MER I & 159601 & & 1 & 2 & 0.8667 & 0.0000 & 105 & 91,00 \\
\hline MRE ? & 159602 & & 0 & 2 & 0.9333 & 0.0000 & 61 & 56.93 \\
\hline hRR 3 & 158603 & & 2 & 2 & 0.8667 & 0.0000 & 152 & 0.40 \\
\hline KKR 4 & 156004 & & $!$ & 2 & 1.0000 & 0.0600 & 137 & 137.00 \\
\hline SOREST GRQVE & 1586 & & 4 & 8 & 0.9167 & 6.0000 & 455 & 284.53 \\
\hline UTLTEE SAHFL & & & & 16 & 0.9176 & 0.0000 & 898 & 693.47 \\
\hline STH UTLTES-4 & & & & MA & & & & \\
\hline SLESTRATUH & & & & 料 & & 0.0000 & 898 & 693.47 \\
\hline XRO 1 & $25960 !$ & & 0 & 2 & 0.9194 & 0.0000 & 450 & $41: 77$ \\
\hline Ma? & 155602 & & 0 & ? & 1.0000 & 0.10000 & 530 & $3 \mathrm{sic}^{\circ}$ \\
\hline $\mathrm{MER} 3$ & 159603 & & 1 & 2 & 0. $B^{5} 7 \mathrm{~B}$ & 0.0000 & 469 & 419,45 \\
\hline FGE 4 & 159604 & & 1 & 2 & 0.9289 & 0.0000 & 459 & 425.43 \\
\hline ELATHLY-LANE & 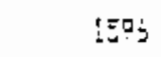 & & 2 & : & 0.9238 & 6.600 & 197 & 1788.15 \\
\hline MRR 1 & 160601 & & 2 & 2 & 0.8579 & 0,0000 & 4.38 & 0.60 \\
\hline HRR 2 & 100002 & & 1 & 2 & 0.9353 & $10.500 \mathrm{NO}$ & 299 & 270.07 \\
\hline ARR 3 & 160603 & & 2 & 2 & 0.9289 & 0,0000 & $4: 3$ & 9.40 \\
\hline
\end{tabular}




\begin{tabular}{|c|c|c|c|c|c|c|c|c|}
\hline Ekitiy & MRS CODE & $\begin{array}{c}\text { MNOWM } \\
\text { INELIGELS }\end{array}$ & $\begin{array}{l}\text { UNABLE TO } \\
\text { COMTACT }\end{array}$ & $\begin{array}{l}\text { MKR SAMPLE } \\
\text { DRAANA }\end{array}$ & $\begin{array}{l}\text { ELIGIBLITY } \\
\text { RATIO }\end{array}$ & $\begin{array}{c}\text { TOZAL } \\
\text { TMELIG8LES }\end{array}$ & $\begin{array}{l}\text { TOTAL HFR } \\
\text { POPLLLAT:OH }\end{array}$ & $\begin{array}{l}\text { ELIEIBDE MES } \\
\text { POPUATIDS }\end{array}$ \\
\hline$M=94$ & 100064 & n.......... & i & ; & $\therefore 000$ & 5.mo & 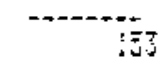 & [5. \\
\hline Minmolith & 1806 & & 5 & 8 & 19.9300 & 0.10000 & 1303 & 43.157 \\
\hline UTLTES SAHEL & & & & 16 & 0.9270 & 0.0000 & 3230 & 2220.22 \\
\hline OTH UTLTES-4 & & & & NA & & & & \\
\hline SUESTEATUM & & & & HA & & 0.0000 & 3230 & 2220.22 \\
\hline STRATUM TQT & & & & $\mathrm{NA}$ & & 4.0000 & 23702 & 19924.00 \\
\hline PUBLIC :OT & & & & NA & & 3.0667 & 85560 & 58080.70 \\
\hline \multicolumn{9}{|l|}{ PRIVATE } \\
\hline \multicolumn{9}{|l|}{ RESION! } \\
\hline ------- & & & & & & & & \\
\hline ARR 1 & 101101 & & 0 & 1 & 1.0000 & 0.0000 & 73 & 73.00 \\
\hline MRR 2 & 101102 & & $\mathrm{f}$ & $!$ & 0.7333 & 0.0000 & 55 & 0.00 \\
\hline MRR 3 & 101103 & & 0 & 1 & $0.857 \mathrm{~g}$ & 0.0000 & 132 & 113.23 \\
\hline MRR 4 & 101104 & & 1 & 1 & 0.8000 & 0.0000 & 121 & 0.00 \\
\hline HFR 5 & 101105 & & 0 & $i$ & 0.9333 & i. .0000 & 54 & 50.40 \\
\hline MFP 6 & $10 ! 106$ & & 1 & 1 & 1.0000 & $0.900 \%$ & 156 & 0.90 \\
\hline MRR 7 & 101107 & & 0 & 1 & 1.0000 & 0.0000 & 500 & 500.00 \\
\hline MRR 8 & $16110 \mathrm{~B}$ & & 0 & 1 & 0.9333 & 0.0000 & 135 & 406.00 \\
\hline MRR 9 & 101109 & & 0 & 1 & 1.0000 & 0.0000 & 411 & $\$ 11.00$ \\
\hline MRR 10 & 101110 & & 0 & 1 & 0.8578 & 0.0000 & 544 & 466.63 \\
\hline MRR II & 101111 & & 0 & 1 & 0.9289 & 0.0000 & 345 & 320.47 \\
\hline MRR 12 & 101112 & & 1 & 1 & 0.9244 & 0.0000 & 153 & 0.60 \\
\hline MRR is & 101113 & & 0 & $i$ & 0.9289 & 0.0000 & 132 & 122.61 \\
\hline MRR 14 & 101114 & & 0 & $i$ & 0.8367 & 0.0000 & 26 & 21.76 \\
\hline MRR 15 & 101115 & & 1 & 1 & 0.9286 & 0.0000 & 358 & 0.00 \\
\hline MRR 16 & 101116 & & 1 & 1 & 0.7551 & 0.0000 & 167 & 0.60 \\
\hline $\begin{array}{l}\text { MONTAHA PWR } \\
\text { SUIGTRATUH }\end{array}$ & 1011 & & 6 & 16 & 0.8998 & 0.0000 & 3662 & 2435.09 \\
\hline
\end{tabular}

\begin{tabular}{|c|c|}
\hline HRR I & $10210 !$ \\
\hline MRR 2 & 102102 \\
\hline KRR 3 & 102103 \\
\hline MRR 4 & 102104 \\
\hline Yop 5 & 102105 \\
\hline ذ) & 102106 \\
\hline MRP ? & 102107 \\
\hline MRR B & 102108 \\
\hline MRE 9 & 102109 \\
\hline HRR 10 & 102110 \\
\hline Wak 11 & 102111 \\
\hline
\end{tabular}




\begin{tabular}{|c|c|c|c|c|c|c|c|c|}
\hline ENTITY & MES [UDE & $\begin{array}{c}\text { BNOLW } \\
\text { DNELIGELS }\end{array}$ & $\begin{array}{l}\text { BNIELE TD } \\
\text { CENTACT }\end{array}$ & $\begin{array}{c}\text { MRE SAMFLE } \\
\text { WFARN }\end{array}$ & $\begin{array}{l}\text { ELIBIGILITY } \\
\text { RATIO }\end{array}$ & $\begin{array}{c}\text { TDTAL } \\
\text { INELLIGELES }\end{array}$ & $\begin{array}{l}\text { TUTAL MER } \\
\text { PIFILATIION }\end{array}$ & $\begin{array}{l}\text { ELIGIELE KRF } \\
\text { FIPIJEATIJN }\end{array}$ \\
\hline 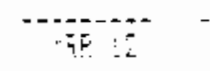 & - & $\cdots$ & 6 & ! & 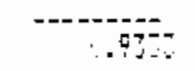 & 0.90 & 14 & $-7=0$ \\
\hline YSP & เ经:5 & & $\hat{v}$ & 1 & $1.0 \% 00$ & $0,0 \%$ & 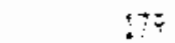 & 17.6 \\
\hline पit it & $1: 2114$ & & 0 & $!$ & 1.000 & 0.5000 & $2: 1$ & $21 . .0$ \\
\hline 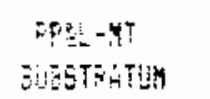 & 1021 & & $\ddot{i}$ & $: 4$ & 3.6728 & 0.9000 & 2655 & 1.56 .77 \\
\hline Mtis： & 103101 & & 1 & 1 & 1.0000 & 0.5000 & 67 & $1 . \mathrm{in}$ \\
\hline MRP 2 & 103102 & & 1 & I & 1.9000 & 1.00000 & 63 & 0.66 \\
\hline 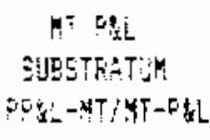 & $103 !$ & & 2 & 2 & 1.0000 & 5.0000 & 130 & 0.00 \\
\hline STFATSH TOT & & & & 32 & $0.9 \div 44$ & 0.0000 & $\$ 448$ & 3541.86 \\
\hline REGION 2 & & & & & & & & \\
\hline -------- & & & & & & & & \\
\hline MRF! & $10220 \mathrm{i}$ & & 0 & i & 1.5000 & 0.0000 & 320 & 320.00 \\
\hline SRF 2 & 102202 & & 1 & : & 1.0000 & 0.0000 & 429 & 0.60 \\
\hline MEP $Z$ & 102205 & & 1 & $t$ & 0.9333 & 0.0000 & 258 & 0.00 \\
\hline WFE 4 & 102294 & & 0 & 1 & 0.9289 & 6.00000 & 147 & 136.55 \\
\hline$M E=$ & $1020^{5}$ & & 1 & 1 & 0.7967 & 1.2000 & $22 !$ & 0.60 \\
\hline 495 & 102200 & & 0 & $!$ & 9.4973 & 1.0000 & 236 & $! 64.65$ \\
\hline ARE 7 & 102207 & & 0 & 1 & 0.9333 & 10.0000 & 174 & $16 ?+40$ \\
\hline SFR S & 102200 & & 0 & 1 & 0.8000 & 0.0000 & 105 & 156.000 \\
\hline 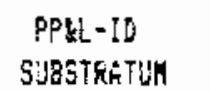 & 1022 & & 3 & 8 & 0.8850 & 0.0000 & 2040 & 939.62 \\
\hline MFR 1 & $10820 !$ & & 0 & 1 & 0.8000 & 0.0000 & 312 & 249.60 \\
\hline MR⿴ 2 & 108202 & & 0 & 1 & 0.9289 & 0.0000 & 280 & 260.69 \\
\hline MRR 3 & 108203 & & 0 & 1 & 0.9333 & 0.0000 & $31 ?$ & 295.97 \\
\hline MFF 4 & 108204 & & 1 & $!$ & 0.9286 & 0.6000 & 402 & 0.00 \\
\hline MRR 5 & 109215 & & 1 & 1 & 0.9333 & 0.0000 & 300 & 0.60 \\
\hline ARP 6 & 108206 & & 1 & ! & 0.9835 & 1,00000 & 245 & 5,00 \\
\hline MET T & $1082 i j 7$ & & 0 & 1 & 1.0000 & 0.0000 & 285 & 285.60 \\
\hline MFA $\mathrm{B}$ & j38209 & & 0 & 1 & 0.9335 & 0.10000 & 246 & 229.60 \\
\hline $\begin{array}{l}\text { IEAHO PAR } \\
\text { SUSSTRATEM }\end{array}$ & 1082 & & 3 & 8 & 0.9238 & 0.0000 & 2387 & 1320.10 \\
\hline MER I & 109201 & & 0 & 1 & 0.9184 & 9,0000 & 265 & 247.04 \\
\hline ART 2 & $10920 ?$ & & 0 & 1 & 0.9000 & 0.0900 & 537 & 269.60 \\
\hline MaF 3 & 109203 & & 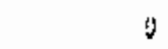 & 1 & 0.8578 & 0.0000 & 163 & 170.82 \\
\hline Ms? 4 & 109204 & & 1 & 1 & 1.00010 & 0.9000 & $2 \pi 2$ & i. \\
\hline MRR 5 & 109205 & & 0 & 1 & 1.0000 & 0.0000 & 235 & 235.100 \\
\hline MER i & 109206 & & 0 & : & 1.0800 & 0.0000 & 314 & 314.00 \\
\hline 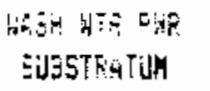 & 4642 & & 1 & $\vdots$ & 0.579 & $1 . \operatorname{sen}$ & 1500 & $1: C 5 .+c$ \\
\hline MRR I & $11+201$ & & 0 & $!$ & $0.866 i$ & 0.0000 & 175 & 151.67 \\
\hline MaR 2 & $1 i 1202$ & & 1 & 1 & 0.7733 & 0.0000 & 293 & $\hat{0} .90$ \\
\hline MRR 3 & 111203 & & 0 & i & 0.0235 & 0.0000 & $28 ?$ & 250.42 \\
\hline
\end{tabular}




\begin{tabular}{|c|c|c|c|c|c|c|c|c|}
\hline ENTITY & MAR CQDE & $\begin{array}{c}\text { VNOWN } \\
\text { IHELIGBLS }\end{array}$ & $\begin{array}{l}\text { UMABLE TO } \\
\text { CENTACT }\end{array}$ & $\begin{array}{c}\text { GRR SAMPLE } \\
\text { DRAWN }\end{array}$ & $\begin{array}{c}\text { ELIGIBILITY } \\
\text { RATIO }\end{array}$ & $\begin{array}{c}\text { TUTAL } \\
\text { IHELISBBLES }\end{array}$ & $\begin{array}{l}\text { FUTAL MAF } \\
\text { PUPULAIIOK }\end{array}$ & $\begin{array}{l}\text { ELIGIBLE YRR } \\
\text { POPLLATION }\end{array}$ \\
\hline 4 & .104 & & $!$ & ! & 0.390 & 3.06 & 47 & 10 \\
\hline MAR 5 & $1: 1205$ & & 0 & 1 & 1.100 & 0.000 & $\$ 51$ & $15 ! .00$ \\
\hline AF & 111200 & & 0 & i & 0.7333 & 0.10000 & 138 & 101.20 \\
\hline $\operatorname{men} 7$ & 111207 & & 0 & 1 & 0.9333 & 0.1000 & 120 & 112.00 \\
\hline$M R E$ & $11: 208$ & & $!$ & 1 & 0.8667 & 0.0000 & 116 & 0.00 \\
\hline $\begin{array}{l}\text { UTAH FHL } \\
\text { SLGSTRATUH }\end{array}$ & $\$ 112$ & & 3 & B & 0.8616 & 0.0000 & 1712 & 776.29 \\
\hline STRRTUM TOT & & & & 30 & 0.8975 & 0.0000 & 7729 & 4241.52 \\
\hline
\end{tabular}

REGiô:

\begin{tabular}{|c|c|c|c|c|c|c|c|}
\hline MRR I & 102301 & 1 & 1 & 1.0000 & 0.0000 & 413 & 0.00 \\
\hline MRR ? & $102 J^{0} 2$ & 0 & 1 & 1.6000 & 0.10000 & 406 & 406.00 \\
\hline MRR J & 102303 & 1 & 1 & 1.0000 & 0.0000 & 291 & 0.60 \\
\hline MRR 4 & 102304 & 0 & 1 & 0.8000 & 0.5000 & 197 & 157.60 \\
\hline MRR 5 & 102305 & 0 & 1 & 1.0000 & 0.0000 & 274 & 274.00 \\
\hline MRR 6 & 102306 & 0 & 1 & 1.0000 & 0.0000 & 189 & 189.00 \\
\hline KRR 7 & 102307 & 1 & 1 & 1.0000 & 0.0000 & 186 & 0.60 \\
\hline MRR B & 102308 & 0 & 1 & 1,0000 & 0.0000 & 332 & 332.00 \\
\hline MRR 9 & 102309 & 1 & 1 & 0.9333 & 0.0000 & 390 & 0.00 \\
\hline MRR 10 & 102310 & 1 & 1 & 0.9335 & 0.00010 & 161 & 0.60 \\
\hline KRR II & 102311 & 1 & 1 & 0.8667 & 0.0090 & 212 & 0.00 \\
\hline NEK 12 & 102312 & 0 & $i$ & 0.9286 & 0.0000 & 274 & 254.43 \\
\hline MRR 13 & 102313 & 0 & 1 & 1.0000 & 0.0000 & 237 & 237.00 \\
\hline MRR 14 & 102314 & 1 & ! & 1.0000 & 0.0000 & 226 & 0.00 \\
\hline MFR 15 & 102315 & 1 & 1 & 0.7733 & 0.0000 & 314 & 0.00 \\
\hline MRR 16 & 102316 & 1 & 1 & 1.0500 & 0.0000 & 373 & 0.00 \\
\hline PPLL-YHA & 1023 & 9 & 16 & 0.9521 & 0.0000 & 4475 & 1850 \\
\hline \multicolumn{8}{|l|}{ SUBSTRATUM } \\
\hline MRR 1 & 109301 & 1 & 1 & 0.9333 & 0.0000 & 63 & 0.00 \\
\hline MRR 2 & 109302 & 0 & 1 & 1.0000 & 0.0000 & 252 & 252.00 \\
\hline HRR 3 & 109303 & \{ & 1 & 1.0000 & 0.0000 & 254 & 0,00 \\
\hline MER 4 & 109304 & 0 & 1 & 0.9667 & 0.0000 & 329 & 284.27 \\
\hline MRR 5 & 109305 & 1 & 1 & 0.8667 & 0.0000 & 172 & 0.00 \\
\hline Her 6 & 109306 & 1 & 1 & 1.0000 & 0.0000 & 357 & 0.00 \\
\hline MRR 7 & 109307 & 0 & 1 & 1.0000 & 0.0000 & 229 & 229.00 \\
\hline HRF 8 & 109300 & 0 & ! & 0.7335 & 0.0060 & 359 & 263.27 \\
\hline BRR 9 & 109309 & 1 & 1 & 1.0000 & 0.0000 & 272 & 0.00 \\
\hline MRR 10 & 109310 & 1 & 1 & 0.9311 & 0.0000 & 404 & 0.00 \\
\hline MRR 11 & 109311 & 1 & 1 & 0.9333 & 0.00010 & 124 & $\dot{0} .60$ \\
\hline MRA 12 & 109312 & 1 & $i$ & 0.9235 & 0.0000 & 135 & 0.00 \\
\hline MRR IJ & 109213 & 1 & $i$ & 1.0000 & 0.0000 & 323 & 0.00 \\
\hline Hen 14 & 109314 & 0 & $i$ & 1.0000 & 0.0000 & 291 & 291.00 \\
\hline MRR 15 & 109315 & 0 & 1 & 0.9235 & 0.0000 & 233 & 215.17 \\
\hline SHeR 16 & 109316 & $!$ & 1 & 0.7733 & 0.0000 & 193 & 0.00 \\
\hline $\begin{array}{l}\text { WASH WAT PWE } \\
\text { SUESTEATUM }\end{array}$ & $: 093$ & 10 & 16 & 0.9234 & 0.0000 & 4289 & 1534.70 \\
\hline STRATUK TOT & & & 32 & 0.9578 & 0.0000 & 4617 & 3384.73 \\
\hline
\end{tabular}




\begin{tabular}{|c|c|c|c|c|c|c|c|c|}
\hline ENTITY & MER SME & 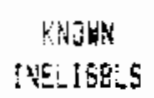 & $\begin{array}{l}\text { HAELE TO } \\
\text { CONTEST }\end{array}$ & $\begin{array}{l}\text { MFR SAMPLE } \\
\text { DRAWH }\end{array}$ & $\begin{array}{l}\text { ELIBIEILITY } \\
\text { RMIIO }\end{array}$ & $\begin{array}{l}\text { TOTAL } \\
\text { IMEL IEELES }\end{array}$ & $\begin{array}{l}\text { TETAL MER } \\
\text { PUPULATIOH }\end{array}$ & $\begin{array}{l}\text { ELIGIBLE } \\
\text { POPLATAIBN }\end{array}$ \\
\hline 影的 & ............ & ......... & -- & --.. - - & - nnon & $+\cdots$ & & \\
\hline \multicolumn{9}{|l|}{-..n- } \\
\hline MRE : & 174401 & & 1 & 2 & 0.3296 & 3.0000 & $: 44$ & 319.43 \\
\hline परद 2 & $1-+60^{2}$ & & 2 & 2 & 1.000 & 3.9000 & $29 \varphi$ & 800 \\
\hline nif & 03447 & & n & 2 & 1.0000 & $1.000 \%$ & 249 & $249 . j 0$ \\
\hline nRo 4 & 134404 & & 2 & 2 & 6.9235 & 120060 & 262 & 0.60 \\
\hline MER 5 & 134405 & & 0 & 3 & 1.0000 & 0.0000 & 354 & 354,00 \\
\hline$M 9$ & 134406 & & 0 & 2 & 1.9000 & 0.6000 & 260 & $2 B 6.50$ \\
\hline Whe? & $1: 4407$ & & 0 & 2 & 0.8667 & 10000 & 253 & 227.93 \\
\hline Whis & 174408 & & $z$ & 2 & 1.10000 & 0.00017 & $14 !$ & 0.90 \\
\hline MEF S & 134099 & & $!$ & 2 & 0.9289 & 0.6000 & 605 & 561.98 \\
\hline $\operatorname{MRR} 10$ & 134410 & & 3 & 2 & 0.9296 & 0.0600 & 27 & 0.00 \\
\hline MPR II & $134 d 11$ & & 1 & 2 & 0.9244 & 0.01000 & $46 \bar{B}$ & 432.84 \\
\hline KRR IZ & $1344 ! 2$ & & 1 & 2 & 0.7600 & 0,00 ing & 39 & 181.64 \\
\hline atk 13 & $1744 ! 3$ & & 1 & 2 & 1.0000 & 0.00000 & 200 & 203.00 \\
\hline MदP 14 & $1344 ! 4$ & & ? & 2 & 0.9289 & 0.0000 & 226 & 294.97 \\
\hline MRR 15 & 134415 & & 1 & 2 & 0.9244 & 0.6000 & 603 & $55 i .44$ \\
\hline PRE If & 134416 & & $!$ & 2 & 1.0000 & 0.0000 & 185 & 285.00 \\
\hline $\begin{array}{l}\text { PISGET SOUND } \\
\text { SUBSTEATEM }\end{array}$ & 1344 & & 15 & 32 & 0.9441 & 0.0000 & $\$ 948$ & 8761.99 \\
\hline STEATH TH TH & & & & 32 & $7.944 i$ & 0.6500 & 4948 & 5761.99 \\
\hline
\end{tabular}

PESTON

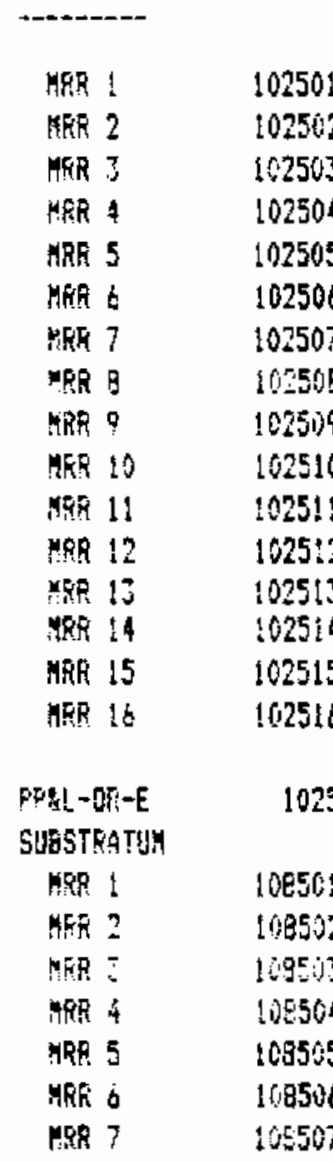

\begin{tabular}{|c|c|c|c|c|c|}
\hline 0 & 1 & 0.9333 & 0.0000 & 363 & 338.80 \\
\hline 1 & 1 & 0.9286 & 0.0000 & 311 & 0.00 \\
\hline 0 & 1 & 1.0000 & 0.0000 & 41.5 & 405.10 \\
\hline 0 & 1 & 0.9667 & 0.1000 & 275 & 239.20 \\
\hline 0 & 1 & 0.6939 & 0.0060 & 1.37 & 96.45 \\
\hline 0 & $t$ & 0.7150 & $j .0000$ & 226 & $: 61.72$ \\
\hline$!$ & 1 & 0.8400 & 0.0000 & 276 & 0.00 \\
\hline 1 & $!$ & 1.0000 & $\dot{0} .0000$ & 343 & 0.10 \\
\hline$\theta$ & 1 & 1.6900 & 0.0000 & 178 & 179.00 \\
\hline 0 & 1 & 0.9289 & 0.0000 & 319 & $299^{\circ} .37$ \\
\hline 0 & $!$ & 0.9289 & 0.6000 & 125 & 116,11 \\
\hline 1 & 1 & 0.9231 & 0.0000 & 228 & 0.00 \\
\hline 1 & $i$ & 0.9299 & 0.5000 & 73 & 0.00 \\
\hline 0 & & $0 . \$ 1000$ & & & 0.02 \\
\hline 0 & 1 & 1.0000 & 0.0000 & 101 & $10 ! .00$ \\
\hline 0 & 1 & 1.0090 & 0.6000 & Júb & 306.00 \\
\hline 5 & 15 & 0.8797 & 1). 0000 & 3668 & 2257.66 \\
\hline 0 & $!$ & 1.0000 & 0.6000 & 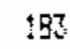 & 183.00 \\
\hline D & 1 & 0.7333 & 0.1000 & 519 & 465.60 \\
\hline 1 & $!$ & 19.6499 & 3.6000 & $10:$ & 0.00 \\
\hline 1 & 1 & 1).8ct7 & U. 1000 & 31 & 5.90 \\
\hline 1 & 1 & 0.9373 & 0.0000 & 209 & 0.00 \\
\hline i) & $!$ & 1.0000 & 0.0000 & 104 & 164.60 \\
\hline 0 & $!$ & 0.8000 & 0.0000 & $2 \mathrm{t}$ & $2 j .80$ \\
\hline
\end{tabular}




\begin{tabular}{|c|c|c|c|c|c|c|c|c|}
\hline ENTITY & MRR SODE & $\begin{array}{c}\text { KHOWN } \\
\text { INELI GBLS }\end{array}$ & $\begin{array}{l}\text { UNAELE TO } \\
\text { CONTACT }\end{array}$ & $\begin{array}{c}\text { FR SAMFLE } \\
\text { BRANN }\end{array}$ & $\begin{array}{l}\text { ELIGIBHLITY } \\
\text { RATIO }\end{array}$ & $\begin{array}{c}\text { TOTRL } \\
\text { IMELIGELES }\end{array}$ & $\begin{array}{l}\text { TOTAL MER } \\
\text { POPILATION }\end{array}$ & $\begin{array}{l}\text { ELIGIBLE NGK } \\
\text { POPULATION }\end{array}$ \\
\hline 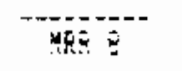 & 1085 & & 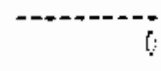 & 1 & 1.0500 & $\therefore 000$ & 3 & 399,00 \\
\hline $\begin{array}{l}\text { ILAHE FWR } \\
\text { SLESTEATUH }\end{array}$ & 1095 & & 3 & $g$ & 0.5739 & 0.0010 & 2052 & 115.40 \\
\hline MFR 1 & $14750:$ & & $!$ & j & 1. .9235 & 0.0000 & 523 & 11,60 \\
\hline MFiR 2 & 147502 & & 0 & 1 & 0.7639 & 0,0500 & 847 & $547.0 !$ \\
\hline MRR 3 & 147503 & & 0 & & 0.6173 & & & 0.60 \\
\hline HeR 4 & 147504 & & 0 & i & 1.0000 & 0.0000 & 200 & 200.00 \\
\hline MRF 5 & 147505 & & 0 & & 0.1467 & & & 0.00 \\
\hline MRR 6 & 147506 & & 1 & $!$ & 0.9289 & 0.6000 & 206 & 0.60 \\
\hline AfiR 7 & 147507 & & 0 & & 1.0000 & & & 0.00 \\
\hline NFF & 147508 & & 1 & 1 & 0.5733 & 7). 0000 & 232 & 0.00 \\
\hline $\begin{array}{l}\text { CP NATNL } \\
\text { SUESTRATUH }\end{array}$ & 1475 & & $?$ & 5 & 0.7410 & 0.0000 & 2008 & 847.01 \\
\hline STRATUM TOT & & & & $2 B$ & 0.8444 & 0.0000 & 7728 & 4258.08 \\
\hline
\end{tabular}

REgion 6

\begin{tabular}{|c|c|}
\hline MRR I & 102601 \\
\hline HRR 2 & 102602 \\
\hline MRP 3 & 102603 \\
\hline MRR 4 & 102604 \\
\hline MRR 5 & 102605 \\
\hline ARR 6 & 102606 \\
\hline MRR 7 & 102607 \\
\hline MRR 8 & 102608 \\
\hline MRR 9 & 102609 \\
\hline MRR 10 & 102610 \\
\hline MRR 11 & 102611 \\
\hline MRR 12 & 102612 \\
\hline MRP 13 & 102613 \\
\hline MRR 14 & 102614 \\
\hline MRR 15 & 102615 \\
\hline$M R R 16$ & 102616 \\
\hline PPLL-OR-N & 1026 \\
\hline \multicolumn{2}{|l|}{ SUESTRATUK } \\
\hline MFR ! & 152601 \\
\hline MRR 2 & 152602 \\
\hline MRR 3 & 152603 \\
\hline veth 4 & 152604 \\
\hline MRR 5 & 152605 \\
\hline$M R R$ i & 152606 \\
\hline MRR 7 & 152607 \\
\hline MRR B & 152609 \\
\hline MER 9 & 152609 \\
\hline MRE 10 & 152510 \\
\hline WRR II & 152611 \\
\hline MRR 12 & 152612 \\
\hline ARR 13 & 152613 \\
\hline MRR 14 & 152614 \\
\hline
\end{tabular}




\begin{tabular}{|c|c|c|c|c|c|c|c|c|}
\hline EMTIT: & MRR CODE & $\begin{array}{c}\text { KNOWN } \\
\text { IMELiGELS }\end{array}$ & $\begin{array}{l}\text { IMABLE TO } \\
\text { CON IACT }\end{array}$ & $\begin{array}{c}\text { MAF SAWFLE } \\
\text { DRALHN }\end{array}$ & $\begin{array}{c}\text { ELI6IgILITY } \\
\text { SATIO }\end{array}$ & $\begin{array}{c}\text { TUTAL } \\
\text { THELIGELES }\end{array}$ & $\begin{array}{l}\text { TDTAL IRR } \\
\text { POPULATION }\end{array}$ & $\begin{array}{l}\text { ELIGIBLE MRE } \\
\text { PUPULATIOH }\end{array}$ \\
\hline peo & 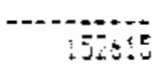 & $-\cdots-\cdots$ & ! & $-{ }_{2}$ & 1.8000 & 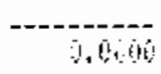 & l & 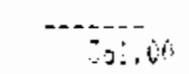 \\
\hline MFE 10 & $: E 25 i s$ & & i & 2 & 1. 1000 & 0.1000 & 407 & 95,0 \\
\hline $\begin{array}{l}\text { PIRT SEN ELE } \\
\text { SUESTAAUM }\end{array}$ & 1526 & 0 & 13 & 32 & 0.9530 & 0.0000 & 5418 & 3795.81 \\
\hline STEATUA TOT & & 0 & & 62 & 0.9315 & i. $\dot{0} 000$ & 9691 & $E 108.57$ \\
\hline ERIUTEE TOT & & $\theta$ & & NA & & 0.0000 & $41 j 61$ & 25376.75 \\
\hline HEABD TDT & & 0 & & $\begin{array}{r}248 \\
324.00 \\
542.00\end{array}$ & $\begin{array}{l}0.9032 \\
0.9079 \\
0.9060\end{array}$ & $\begin{array}{l}3.6667 \\
0.6000 \\
3.0667\end{array}$ & 126721 & 83457.53 \\
\hline
\end{tabular}




\begin{tabular}{|c|c|c|c|c|c|c|c|c|}
\hline A & $\mathrm{J}$ & K & $\mathbf{L}$ & $M$ & N & 0 & $P$ & $Q$ \\
\hline ENTITY & $\begin{array}{l}\text { OTHER TOT } \\
\text { POPULATION }\end{array}$ & $\begin{array}{c}\text { OTHER ELIGBL } \\
\text { TDT YOPS }\end{array}$ & $\begin{array}{l}\text { COMPLETED } \\
\text { INTERVIENS }\end{array}$ & $\begin{array}{l}\text { AVE CUNFL } \\
\text { PER MRR }\end{array}$ & $\begin{array}{l}\text { INOIVIDUA: } \\
\text { WEIGHTS }\end{array}$ & $\begin{array}{l}\text { AVERAGE } \\
\text { FDFiLATIEN }\end{array}$ & $\begin{array}{c}\text { MRR } \\
\text { WEIEHTS }\end{array}$ & $\begin{array}{l}\text { UTILITY } \\
\text { WEIGHTS }\end{array}$ \\
\hline \multicolumn{9}{|l|}{ F]ELII } \\
\hline \multicolumn{6}{|l|}{ PEgIQN } & & & \\
\hline \multicolumn{9}{|l|}{------- } \\
\hline 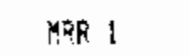 & & & 1 & 1.2047 & 1.2047 & 633.97 & 1.0011 & \\
\hline MRR 2 & & & $i$ & 1.2047 & 1.2047 & 653.97 & 0.9194 & \\
\hline$M F R$ & & & 0 & 1.2047 & 0.0000 & 633.97 & 0.0000 & \\
\hline MRE 4 & & & 1 & 1.2047 & 1.2047 & 633.97 & $\$ .0011$ & \\
\hline MES 5 & & & 1 & 1.2047 & 1.2047 & $633.9 ?$ & 0.9963 & \\
\hline MrF $b$ & & & 1 & 1.2047 & 1.2047 & 633.97 & 1.0726 & \\
\hline MRE 7 & & & 0 & 1.2047 & 0.0000 & 633.97 & 0.0000 & \\
\hline \multirow[t]{2}{*}{ MRR B } & & & 1 & 1.2047 & 1.2047 & 653.97 & 1.0095 & \\
\hline & & & & 1.2047 & 0.0000 & & & \\
\hline \multirow[t]{2}{*}{ FLATHEAD ELE } & $\$ 142$ & 5725.65 & $b$ & 1.2047 & 0.2008 & 6574.30 & 0.87 & 0.87 \\
\hline & & & & 1.2047 & 1). 1000 & & & \\
\hline MRR 1 & & & 0 & 1.2047 & 0.0000 & 336.23 & 0.0000 & \\
\hline MFR 2 & & & 1 & 1.2047 & 1.2047 & 336.23 & 0.3059 & \\
\hline MRR 3 & & & 1 & 1.2047 & 1.2047 & 336.23 & 0.4747 & \\
\hline MRR 4 & & & 1 & 1.2047 & 1.2047 & 336.23 & 0.5499 & \\
\hline MRR 5 & & & 1 & 1.2047 & 1.2047 & 336.23 & 1.3681 & \\
\hline MER i & & & 1 & 1.2047 & 1.2047 & 336.23 & 1.7845 & \\
\hline GRR 7 & & & 1 & 1.2047 & 1.2047 & 336.23 & 1.5168 & \\
\hline \multirow[t]{2}{*}{$\operatorname{MAR} \theta$} & & & 0 & 1.2047 & 0.0000 & 336.23 & 0.0000 & \\
\hline & & & & 1.2047 & 0.0000 & & & \\
\hline \multirow[t]{2}{*}{ FLATYEAD IRA } & $89 ! 8$ & 7422.96 & 6 & 1.2047 & $0.200 \mathrm{~B}$ & 6574.30 & 1.13 & 1.13 \\
\hline & & & & 1.2047 & 0.0000 & & & \\
\hline \multirow[t]{2}{*}{ UTLTES SAMPL } & 15060 & 13140.61 & 12 & 1.2047 & 0.1004 & & & \\
\hline & & & & 1.2047 & 0.0000 & & & \\
\hline \multirow{2}{*}{ OTH UTLTES-1 } & 5830 & & & 1.2047 & 0.0000 & & & \\
\hline & & & & 1.2047 & 0.0000 & & & \\
\hline \multirow[t]{2}{*}{ SUQSTRATUK } & 20890 & 18238.67 & 12 & 1.2047 & 0.1004 & MA & 0.8735 & \\
\hline & & & & 1.2047 & 0.0000 & & & \\
\hline MRR 1 & & & 0 & 1.2047 & 0.0000 & 105.10 & 0.0000 & \\
\hline MRR 2 & & & 0 & 1.2047 & 0.0000 & 105.10 & 0.0000 & \\
\hline MRR 3 & & & 1 & 1.2047 & 1.2047 & 105.10 & 1.1596 & \\
\hline PRR & & & 0 & 1.2047 & 0.0000 & 105.10 & 0.0000 & \\
\hline MRR 5 & & & 1 & 1.2047 & 1.2047 & 105.10 & 0.8404 & \\
\hline MRR 6 & & & & 1.2047 & & & & \\
\hline MRR 7 & & & & $\$ .2047$ & & & & \\
\hline \multirow[t]{2}{*}{ IRR } & & & & 1.2047 & & & & \\
\hline & & & & 1.2047 & 0.0000 & & & \\
\hline \multirow[t]{4}{*}{ BLACIER } & 4514 & 4203.46 & 2 & 1.2047 & 1). 6024 & 3605.59 & 1.17 & 1.17 \\
\hline & & & & 1.2047 & 0.0000 & & & \\
\hline & & & & 1.2047 & 0.0000 & & & \\
\hline & & & & 1.2047 & 0.0000 & & & \\
\hline MFR ! & & & 2 & 1.2047 & 0.5024 & 051.23 & 1.1200 & \\
\hline MRR 2 & & & $t$ & 1.2047 & 1.2047 & 651.23 & 0.8958 & \\
\hline MRR 3 & & & 1 & 1.2047 & 1.2047 & 651.23 & 1.2296 & \\
\hline MRR 4 & & & 0 & 1,2047 & 0.0000 & 651.23 & 0,0000 & \\
\hline \multirow[t]{2}{*}{ MRR 5} & & & 2 & 1.2047 & 0.6024 & 651.23 & 0.7406 & \\
\hline & & & & B. 17 & & & & \\
\hline
\end{tabular}




\begin{tabular}{|c|c|c|c|c|c|c|c|c|}
\hline ENTITY & $\begin{array}{l}\text { DFHER TUT } \\
\text { PUPULALIIEN }\end{array}$ & $\begin{array}{c}\text { OTHER ELIGEL } \\
\text { TOT ETJPS }\end{array}$ & $\begin{array}{l}\text { COMPIETED } \\
\text { INTERUIEHS }\end{array}$ & $\begin{array}{l}\text { AHS COKPL } \\
\text { PER MRR }\end{array}$ & $\begin{array}{l}\text { INDIVIפUAL } \\
\text { HEISHTS }\end{array}$ & $\begin{array}{l}\text { FNERAGE } \\
\text { FOPULATISK }\end{array}$ & $\begin{array}{c}\text { MRR } \\
\text { WEIEHTS }\end{array}$ & $\begin{array}{l}\text { UTILITY } \\
\text { WEIGHTS }\end{array}$ \\
\hline -------- & --- & -............ & - & 1.248 & 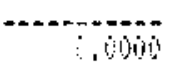 & + & ----- & 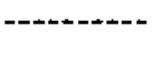 \\
\hline GHALLI & 5607 & 3907.7 & $b$ & 1.2647 & 1.2000 & 36.5 .59 & 6.53 & 0.53 \\
\hline \multirow[t]{2}{*}{ JULIES SAMPL } & 7721 & 7211.17 & 8 & 1.2047 & $0.55 i t$ & & & \\
\hline & & & & 1.2047 & 0.0000 & & & \\
\hline \multirow[t]{2}{*}{ JTH UTLTES-2 } & 5608 & & & 1.2047 & 0.9906 & & & \\
\hline & & & & 1.2047 & 19.6000 & & & \\
\hline \multirow[t]{2}{*}{ SUESTRATIIM } & 13389 & 12504.90 & B & 1.2047 & 0.1506 & NA & 1.2678 & \\
\hline & & & & 1.5047 & 0.0000 & & & \\
\hline \multirow[t]{2}{*}{ STEATLY TOT } & 34279 & $30743.5 \mathrm{E}$ & 80 & 1.2047 & 0.5602 & NA & 0.15 & \\
\hline & & & & 1.2047 & 0.0000 & & & \\
\hline \multirow{3}{*}{ REGIOK 2} & & & & $\therefore .2047$ & 0.0000 & & & \\
\hline & & & & 1.2047 & 0.0000 & & & \\
\hline & & & & 1.2047 & 0.0000 & & & \\
\hline 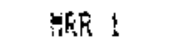 & & & 0 & 1.2047 & 0.0000 & 174.53 & 0.0000 & \\
\hline ARR 2 & & & 0 & 1.2047 & 3.0000 & 174.53 & 0.6000 & \\
\hline MRR j & & & 0 & 1.2047 & 0.0000 & 174.53 & 0.0000 & \\
\hline MER 4 & & & $!$ & 1.2047 & 1.2047 & 174.53 & 1.0000 & \\
\hline MRR 5 & & & 0 & 1.2047 & 0.0000 & 174.53 & 0.0000 & \\
\hline \multirow[t]{2}{*}{ aRR 6} & & & 0 & 1.2047 & 0.0000 & 174.53 & 0. 0000 & \\
\hline & & & & 1.2047 & 0.6050 & & & \\
\hline \multirow[t]{2}{*}{ IDAHO FALLS } & 15243 & 14557.92 & $\mathbf{I}$ & 1.2047 & 1.2047 & 11128.21 & 1.31 & 1.31 \\
\hline & & & & 1.2047 & 0.0000 & & & \\
\hline 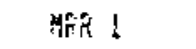 & & & $:$ & 1.2047 & $\therefore .2047$ & $\$ 6 ! .07$ & 6.9894 & \\
\hline NFR 2 & & & 1 & 1.2047 & 1.2047 & t01.017 & 1.0106 & \\
\hline MRR 3 & & & 0 & 1.2047 & 0.0000 & 101.07 & 0.0000 & \\
\hline MRF 4 & & & 0 & 1.2047 & 0.0000 & 101.07 & 0.0000 & \\
\hline NRR 5 & & & 0 & 1.2647 & 0.0000 & 101.07 & 0.0000 & \\
\hline \multirow[t]{2}{*}{ :FF' 6} & & & 0 & 1.2047 & 0.0000 & 101.07 & 0.0000 & \\
\hline & & & & 1.2047 & 0.0000 & & & \\
\hline \multirow[t]{2}{*}{ JHER VALLEY } & 9630 & 7699.51 & 2 & 1.2047 & 0.6024 & $11: 26.21$ & 0.69 & 0.59 \\
\hline & & & & 1.2047 & 0.0000 & & & \\
\hline \multirow[t]{2}{*}{ JFLIES SAMPL } & 23873 & 22256.43 & 3 & t.2047 & 0.4016 & & & \\
\hline & & & & 1.2047 & 0.0000 & & & \\
\hline \multirow[t]{2}{*}{ DTY UTLTES-3 } & 22722 & & & 1.2047 & 0.0000 & & & \\
\hline & & & & 1.2047 & 0.0000 & & & \\
\hline \multirow[t]{2}{*}{ SUSSTRATUM } & 46595 & 43439.80 & 3 & 1.2047 & 0.4016 & $\mathrm{NA}$ & 3.1467 & \\
\hline & & & & 1.2047 & 0.0000 & & & \\
\hline MRR I & & & 1 & 1.2047 & 1.2047 & 159.31 & 1.5837 & \\
\hline WRR 2 & & & & 1.2047 & & 158.31 & & \\
\hline ARR 3 & & & 0 & 1.2047 & 0.0000 & 158.31 & 0.0900 & \\
\hline HRR 4 & & & 0 & 1.2047 & 0.0000 & 158.31 & 0.0000 & \\
\hline MRR 5 & & & 1 & 1.2047 & 1.2047 & 158.31 & 6.4163 & \\
\hline \multirow[t]{2}{*}{ MER 6} & & & 0 & 1.2047 & 0.0000 & 158.31 & 0.0000 & \\
\hline & & & & 1.2047 & 1.0000 & & & \\
\hline \multirow[t]{2}{*}{ FALL RIVER } & 4844 & 3501.72 & 2 & 1.2047 & 0.6024 & 3825.90 & 1). .92 & 0.92 \\
\hline & & & & 1.2047 & 0.0000 & & & \\
\hline YF 1 & & & 1 & 1.2047 & 1.2047 & 160.21 & 1.0000 & \\
\hline \multirow[t]{2}{*}{ ESETE Kit } & $N A$ & NA & $!$ & 1.2047 & 2.2047 & 1775.71 & $\mathrm{~N} / \mathrm{H}$ & 织 \\
\hline & & & & 1.2047 & 0.0000 & & & \\
\hline RRR ! & & & $:$ & $\{.2047$ & 1.2047 & 84.00 & 1.0000 & \\
\hline \multirow{2}{*}{ FARAERS ELE } & NA & *A & 1 & 1.2947 & 1.2047 & 125.30 & NA & HA \\
\hline & & & & 1.2047 & 0.0000 & & & \\
\hline
\end{tabular}




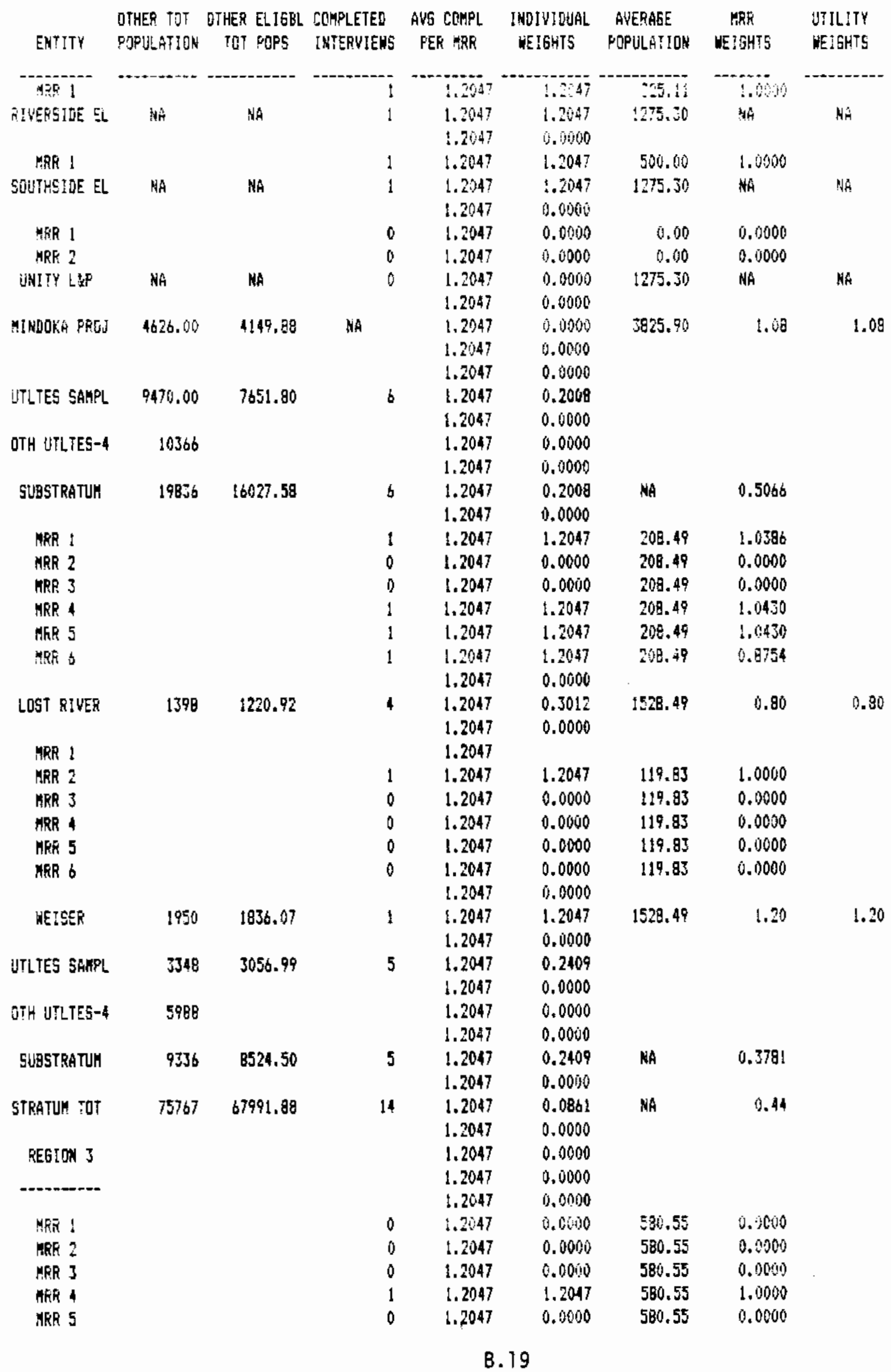




\begin{tabular}{|c|c|c|c|c|c|c|c|c|}
\hline$E \mathrm{NiT} \mathbf{i}^{\top}$ & $\begin{array}{l}\text { GTHER TTI } \\
\text { GOPULATISN }\end{array}$ & $\begin{array}{c}\text { DTHEF ELIGEL } \\
\text { TOT FOFS }\end{array}$ & $\begin{array}{l}\text { CORELETED } \\
\text { THEYVIEWS }\end{array}$ & $\begin{array}{l}\text { SUG CUMFL } \\
\text { PES MRT }\end{array}$ & $\begin{array}{l}\text { TOIVIDLAS } \\
\text { NEIGHTS }\end{array}$ & $\begin{array}{l}\text { AUERAGE } \\
\text { PSPILATIOK }\end{array}$ & $\begin{array}{c}\text { KFF } \\
\text { HEIGHTS }\end{array}$ & $\begin{array}{l}\text { WTLITY } \\
\text { WEITHTS }\end{array}$ \\
\hline 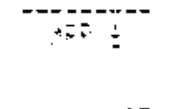 & - - n. & - & 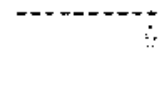 & $\begin{array}{l}1.947 \\
1.3097\end{array}$ & O. & 5 & 1. thon & \\
\hline QETTEA CTY & 2001 & 23075.53 & $\vdots$ & $\begin{array}{l}1.2047 \\
1.2047\end{array}$ & $\begin{array}{l}1.2047 \\
0.9006\end{array}$ & $2104 \div=2$ & $1.0 ?$ & 1.67 \\
\hline कीजि 1 & & & 1 & 1.2047 & 0.2047 & 780.52 & (9).855 & \\
\hline 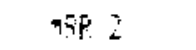 & & & : & 1.2047 & 1.2047 & 360.32 & 0.3814 & \\
\hline$M F \bar{n}$ & & & 1 & 1.2047 & 1.2047 & 280.52 & 1.2777 & \\
\hline MFE 4 & & & 1 & 1.2047 & 1.2047 & 280.52 & 1.5757 & \\
\hline Min & & & 0 & $\begin{array}{l}1.2047 \\
1.2047\end{array}$ & $\begin{array}{l}0.6000 \\
0.01000\end{array}$ & 250.52 & 0.0000 & \\
\hline CHELAY ZT & NA & 0.90 & 4 & $\begin{array}{l}\because .547 \\
1.2047\end{array}$ & $\begin{array}{l}10.3012 \\
0.05000\end{array}$ & NA & NA & NA \\
\hline 路 1 & & & 1 & 1.2047 & 1.2047 & 2127.18 & $\therefore .0000$ & \\
\hline CASHERERE & Nसे & 0.00 & 1 & $\begin{array}{l}1.2047 \\
1.2047\end{array}$ & $\begin{array}{l}1.2047 \\
0.6000\end{array}$ & NA & NA & $Y A$ \\
\hline CHE!H-LSHMRE & 21365.00 & $30204.6 !$ & 5.00 & $\begin{array}{l}1.2047 \\
1.2047\end{array}$ & $\begin{array}{l}0.2499 \\
0.6000\end{array}$ & 21640.22 & 0.93 & 0.93 \\
\hline UFLIES SAMPL & 48296 & 43280 & 6 & $\begin{array}{l}1.2047 \\
1.2047\end{array}$ & $\begin{array}{l}0.2000 \\
0.00100\end{array}$ & & & \\
\hline QTH UITLTES-? & 38845 & & & $\begin{array}{l}1.2047 \\
1.2047\end{array}$ & $\begin{array}{l}0.0000 \\
0.0000\end{array}$ & & & \\
\hline SUBSTRATLM & 87141 & 79091.37 & b & $\begin{array}{l}1.2047 \\
1.2047\end{array}$ & $\begin{array}{l}0.2008 \\
0.0000\end{array}$ & HA & 1.7543 & \\
\hline MRR ! & & & $\mathrm{i}$ & $\$ .2047$ & 1.2047 & 262.58 & 0.2736 & \\
\hline yen 2 & & & $!$ & 1.2047 & 1.2047 & 762.60 & 0.3867 & \\
\hline ARR 3 & & & 0 & 1.2047 & 0.0000 & 203.68 & 0.0006 & \\
\hline MRR 4 & & & 1 & 1.2047 & 1.2047 & 262.68 & 2.1500 & \\
\hline MRA 5 & & & 1 & 1.2047 & 1.2047 & 262.68 & 1.1897 & \\
\hline MRR 6 & & & & $\begin{array}{l}1.2047 \\
1.2047\end{array}$ & 0.0000 & & & \\
\hline FRANKLIN & 1623 & 8306.34 & 4 & $\begin{array}{l}1.2047 \\
1.2047\end{array}$ & $\begin{array}{l}0.3012 \\
0.0060\end{array}$ & 10332.55 & 0.80 & 0.80 \\
\hline MFR ! & & & 0 & 1.2047 & 0.0000 & 135.00 & 0.0000 & \\
\hline MRR Z & & & 0 & 1.2047 & 0.0010 & 178.00 & 0.90000 & \\
\hline$M R R$ & & & 0 & 1.2047 & 0.0000 & 138.00 & 0.0000 & \\
\hline MRP 4 & & & $\underline{1}$ & 1.2047 & 1.2047 & 138.00 & 1.0000 & \\
\hline 为 5 & & & 0 & 1.2047 & 0.0000 & 138.00 & 0.0000 & \\
\hline MRR is & & & 0 & $\begin{array}{l}1.2047 \\
1.2047\end{array}$ & $\begin{array}{l}0.00000 \\
0.0000\end{array}$ & 138.60 & 0.0000 & \\
\hline B!CHLANO & $13: 23$ & 12359.75 & $!$ & $\begin{array}{l}1.2047 \\
1.2047\end{array}$ & $\begin{array}{l}1.2047 \\
0.6000\end{array}$ & $: 0332.55$ & 1.20 & 1.20 \\
\hline UTLTES SAMPL & 24751.00 & 20665.09 & 5 & $\begin{array}{l}1.2047 \\
1.2047\end{array}$ & $\begin{array}{l}0.2409 \\
0.0000\end{array}$ & & & \\
\hline OTH UPLIES-5 & 40314 & & & $\begin{array}{l}1.2047 \\
1.2047\end{array}$ & $\begin{array}{l}0.0000 \\
0.0000\end{array}$ & & & \\
\hline SLESTRATH & 65065 & 54324.04 & 5 & $\begin{array}{l}1.2047 \\
1.2047\end{array}$ & $\begin{array}{l}0.2409 \\
0.0900\end{array}$ & $A A$ & $1.5 \mathrm{dt} 3$ & \\
\hline MRR 1 & & & 0 & 1.7047 & 0.0000 & 于ิे.73 & 7.0000 & \\
\hline NES : & & & 0 & 1.2947 & 0.1000 & 58.78 & 0.310 & \\
\hline KAR I & & & 1 & 1.5027 & 1.20147 & 58.73 & 0.8422 & \\
\hline MRT 4 & & & $:$ & $\begin{array}{l}1.2047 \\
1.2047\end{array}$ & $\begin{array}{l}1.2047 \\
0.0900\end{array}$ & 5.75 & 1.1578 & \\
\hline PEND QRIELLE & 3838 & 7774.08 & 2 & 1.2047 & 0.6024 & 4202.20 & 0.70 & 0.90 \\
\hline
\end{tabular}




\begin{tabular}{|c|c|c|c|c|c|c|c|c|}
\hline ENTITY & $\begin{array}{l}\text { OTHEK TOT } \\
\text { POPELATIOK }\end{array}$ & $\begin{array}{c}\text { OTHER EiIGBL } \\
\text { TOT FOPS }\end{array}$ & $\begin{array}{l}\text { COHPLEIED } \\
\text { INTERYIEHS }\end{array}$ & $\begin{array}{l}\text { AVG CONFL } \\
\text { PER MRR }\end{array}$ & $\begin{array}{l}\text { INDIVIDUAL } \\
\text { WEIGHTS }\end{array}$ & $\begin{array}{l}\text { AVERAGE } \\
\text { POPILATION }\end{array}$ & $\begin{array}{c}\text { MRR } \\
\text { HEISHTS }\end{array}$ & $\begin{array}{l}\text { ITILITY } \\
\text { WEIGRTS }\end{array}$ \\
\hline - - - & $\cdots$ & -- & $\cdots$ & $\therefore, 2647$ & $0,0.60$ & s & ….... & \\
\hline ME 1 & & & 0 & $0.204 ?$ & 0.0000 & 270.65 & 0,0000 & \\
\hline MQR 2 & & & 1 & 1.2047 & 1.2047 & 276.65 & D. $\$ 999$ & \\
\hline MRR I & & & 1 & 1.2047 & 1.2047 & 276.65 & 1.0447 & \\
\hline NRE 4 & & & 1 & 1.2047 & 1.2047 & 274.65 & 1.0555 & \\
\hline & & & & 1.2047 & 0.0000 & & & \\
\hline VERÁ IFR & 4856 & 4630.37 & 3 & 1.2047 & 0.4016 & 4202.20 & 1.10 & 1.10 \\
\hline & & & & 1.2047 & 0.0000 & & & \\
\hline UTLIES SAMFL & 3694 & 8404.10 & 5 & 1.2047 & 0.2409 & & & \\
\hline & & & & 1.2047 & 0.0000 & & & \\
\hline DTH UTLTES-J & 10253 & & & 1.2047 & 0.0000 & & & \\
\hline & & & & 1.2047 & 0.0000 & & & \\
\hline SUBSTEATUH & $19 \div 57$ & 18325.54 & 5 & 1.2047 & 0.2409 & NA & 0.4627 & \\
\hline & & & & 1.2047 & 0.0000 & & & \\
\hline 能 1 & & & 0 & 1.2047 & 0.0000 & 368.09 & 0.0000 & \\
\hline MRP 2 & & & 0 & 1.2047 & 0.0000 & 368.09 & 0.0000 & \\
\hline HR 3 & & & & 1.2047 & & 368.69 & & \\
\hline MRR 4 & & & 1 & 1.2047 & 1.2047 & 368.09 & 1.0000 & \\
\hline & & & & 1.2047 & 0.0000 & & & \\
\hline FERRY CTY & 1746 & 1339.01 & $!$ & 1.2047 & 1.2047 & 1412.23 & 0.95 & 6.95 \\
\hline & & & & 1.2047 & 0.0000 & & & \\
\hline MRR ! & & & 1 & 1.2047 & 1.2047 & 231.77 & 1.1520 & \\
\hline MEP 2 & & & 1 & 1.2047 & 1.2047 & 231.77 & 0.3480 & \\
\hline & & & & 1.2047 & 0.0000 & & & \\
\hline KESPEEIEH & KA & $N A$ & 2 & 1.2047 & 0.6024 & NA & WA & NA \\
\hline & & & & 1.2047 & 0.0000 & & & \\
\hline HRR I & & & 1 & 1.2047 & 1.2047 & 71.40 & 1.2157 & \\
\hline ARR 2 & & & 1 & 1.2047 & 1.2047 & 71.40 & 0.7843 & \\
\hline & & & & 1.2047 & 0.0000 & & & \\
\hline CDULEE DAY & NA & NA & 2 & 1.2047 & 0.6024 & NA & WA & NA \\
\hline & & & & 1.2047 & 0.0000 & & & \\
\hline NSFLH-COULEE & 1623.00 & 1485.46 & & 1.2047 & 0.0000 & 1412.23 & 1.05 & 1.05 \\
\hline & & & & 1.2047 & 0.0000 & & & \\
\hline UTLTES SAMPL & 3369.00 & $2 \mathrm{~B} 24.46$ & 5 & 1.2047 & 0.2409 & & & \\
\hline & & & & 1.2047 & 0.0000 & & & \\
\hline OTH UTLTES-4 & 5747 & & & 1.2047 & 0.00010 & & & \\
\hline & & & & 1.2047 & 0.0000 & & & \\
\hline SUASTRATUM & 9116 & 7642.57 & 5 & 1.2047 & 0.2409 & NA & 0.1910 & \\
\hline & & & & 1.2047 & Ù.0090 & & & \\
\hline STRATUH TOT & 180279 & $1583 \mathrm{~g} .5 !$ & 21 & 1.2047 & 0.0574 & NA & 0.56 & \\
\hline & & & & 1.2047 & 0.0000 & & & \\
\hline & & & & 1.2047 & 0.0000 & & & \\
\hline REGION 4 & & & & 1.2047 & 0.0000 & & & \\
\hline 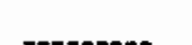 & & & & 1.2047 & 0.0000 & & & \\
\hline & & & & 1.2047 & 0.0060 & & & \\
\hline & & & & 1.2047 & 0.0000 & & & \\
\hline MRS 1 & & & 0 & 1.2047 & 0.6000 & 370.65 & 0.0000 & \\
\hline MSR 2 & & & 0 & 1.2047 & 0.0000 & 370.65 & 0.0000 & \\
\hline$M R E$ & & & 2 & $1.20 \div 7$ & 0.6024 & 370.65 & 0.8553 & \\
\hline MRR 4 & & & 2 & 1.2047 & 0.6024 & 370.65 & 1.0735 & \\
\hline MRR 5 & & & 1 & 1.2047 & 1.2047 & 370.65 & 0.8839 & \\
\hline HRR $b$ & & & 2 & 1.2047 & 0.6024 & 370.65 & 1.1871 & \\
\hline & & & & 1.2047 & 0.0000 & & & \\
\hline
\end{tabular}




\begin{tabular}{|c|c|c|c|c|c|c|c|c|}
\hline EnTITy & $\begin{array}{l}\text { DTHER TOT } \\
\text { PEPULATIOHY }\end{array}$ & $\begin{array}{c}\text { OTHER ELIGEL } \\
\text { TQT CDPS }\end{array}$ & $\begin{array}{l}\text { COYPLETED } \\
\text { IHTERVIEHS }\end{array}$ & $\begin{array}{l}\text { AVE COMPL } \\
\text { PER ARR }\end{array}$ & $\begin{array}{l}\text { INDIVIOUAL } \\
\text { WEIGHJS }\end{array}$ & $\begin{array}{l}\text { AYEFAGE } \\
\text { POFULATION }\end{array}$ & $\begin{array}{l}\text { MRR } \\
\text { HEITHTS }\end{array}$ & $\begin{array}{l}\text { MTILITY } \\
\text { WEIEHTE }\end{array}$ \\
\hline 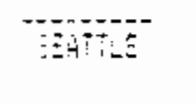 & - & 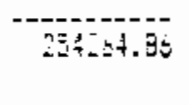 & $i$ & $\begin{array}{l}1.2047 \\
1.3047\end{array}$ & $\begin{array}{l}0.12 \mathrm{i} \\
1.10060\end{array}$ & U594.6j & 1.28 & 28 \\
\hline ME: & & & 1 & 1.2047 & $\therefore .2047$ & 196,44 & $0.467 \mathrm{~s}$ & \\
\hline MER $?$ & & & $i$ & 1.2047 & 1.2647 & 190.44 & 1.1929 & \\
\hline 453 & & & i) & 1.2047 & 0.9000 & 1901.44 & 0.0000 & \\
\hline 4 & & & 2 & 1.2047 & 0.6024 & 150.44 & 1.2550 & \\
\hline thiti 5 & & & 0 & 1.2047 & 0.0000 & 190.44 & 0.0000 & \\
\hline Wh & & & 1 & $\begin{array}{l}1.2047 \\
1.2047\end{array}$ & $\begin{array}{l}1.3047 \\
9.6000\end{array}$ & 190.44 & $1.184 B$ & \\
\hline 5MOHAMISH & 135507 & 130724.40 & 5 & $\begin{array}{l}1.2047 \\
1.2047\end{array}$ & $\begin{array}{l}0.2409 \\
0.69610\end{array}$ & 192494.63 & 0.68 & 0.58 \\
\hline $\begin{array}{l}\text { UTLTES SAMPL } \\
\text { OTH UILTES-iJ }\end{array}$ & 305585 & 354989.26 & $! 2$ & $\begin{array}{l}1.2047 \\
1.2047\end{array}$ & $\begin{array}{l}0.1004 \\
0.0000\end{array}$ & & & \\
\hline SUBSTRATUM & $395 t 85$ & 384989.26 & 12 & $\begin{array}{l}1.2047 \\
1.2047\end{array}$ & $\begin{array}{l}0.1001 \\
0.0000\end{array}$ & WA & 3.2947 & \\
\hline WFP: 1 & & & 2 & 1.2047 & 0.6024 & 326.15 & 0.7910 & \\
\hline$M F \% 2$ & & & ! & 1.2047 & 1.2047 & 326.15 & 1.0548 & \\
\hline WR 3 & & & 1 & 1.2047 & 1.2047 & 326.15 & 1.1167 & \\
\hline HFi 4 & & & 1 & 1.2047 & 1.2047 & 326.15 & 0.4722 & \\
\hline YRR 5 & & & 1 & 1.2047 & 1.2047 & 326.15 & 1.5653 & \\
\hline MPR G & & & 0 & $\begin{array}{l}1.2047 \\
1.2047\end{array}$ & $\begin{array}{l}0.0000 \\
0.0000\end{array}$ & 326.15 & 0.0000 & \\
\hline CLAFK LTY & 74392 & 66830.32 & $b$ & $\begin{array}{l}1.2047 \\
1.2047\end{array}$ & $\begin{array}{l}0.2008 \\
0.5000\end{array}$ & 78377.24 & 0.85 & 9.85 \\
\hline Map 1 & & & 1 & 1.2047 & 1.2647 & 145.74 & 1.1768 & \\
\hline MFE 2 & & & 1 & 1.2047 & 1.2047 & 145.94 & 0.9420 & \\
\hline Hin 3 & & & 1 & 1.2047 & 1.2047 & 145.94 & 0.9356 & \\
\hline MRR 4 & & & 1 & 1.2047 & 1.2017 & 145.94 & 1.0758 & \\
\hline HGR 5 & & & 1 & 1.2047 & 1.2047 & 145.94 & 0.8699 & \\
\hline AfS 6 & & & 0 & $\begin{array}{l}1.2047 \\
1.2047\end{array}$ & $\begin{array}{l}0.0000 \\
0.0000\end{array}$ & 145.94 & 0.0000 & \\
\hline TACOMA & $9673 B$ & 89918.16 & 5 & $\begin{array}{l}1.2047 \\
1.2047\end{array}$ & $\begin{array}{l}0.2409 \\
0.0000\end{array}$ & 78377.24 & 1.15 & 1.15 \\
\hline UTLTES SAMF! & 171130 & 156754.19 & 11 & $\begin{array}{l}1.2047 \\
1.2047\end{array}$ & $\begin{array}{l}0.1095 \\
0.0000\end{array}$ & & & \\
\hline BFH UTLTES-? & 63267 & & & $\begin{array}{l}1.2047 \\
1.2047\end{array}$ & $\begin{array}{l}0.0000 \\
i, .0000\end{array}$ & & & \\
\hline SUBSTRA?UM & 234397 & 214706.93 & 11 & $\begin{array}{l}1.2047 \\
1.2047\end{array}$ & $\begin{array}{l}0.1095 \\
0.0000\end{array}$ & NA & 1.4700 & \\
\hline MRP 1 & & & D & 1.2047 & 0,0000 & 317.99 & 0.0000 & \\
\hline MRR 2 & & & 2 & 1.2047 & 0.6024 & 317.99 & 1.1416 & \\
\hline MFP 3 & & & 2 & 1.2047 & $0 . \dot{6} \times 24$ & 317.99 & $0, \dot{0} 838$ & \\
\hline INRR 4 & & & $i$ & 1.2047 & 1.2047 & 317.59 & 1.6730 & \\
\hline MRF 5 & & & 2 & 1.3047 & 0.6024 & $31 ? .99$ & 0.5535 & \\
\hline 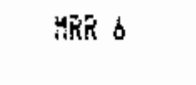 & & & 2 & $\begin{array}{l}1.2047 \\
1.2047\end{array}$ & $\begin{array}{l}0.6024 \\
0.0000\end{array}$ & 317.97 & 0.9451 & \\
\hline MASDN CTY-Z & 16233 & 14481.15 & 9 & $\begin{array}{l}1.2047 \\
1.2047\end{array}$ & $\begin{array}{l}0.1339 \\
0.0000\end{array}$ & 13907.72 & 1.64 & 1.04 \\
\hline kn 1 & & & 0 & 1.2047 & $0.0,00$ & 410.28 & (6000) & \\
\hline HRF 2 & & & 2 & 1.2047 & 3.6024 & 416.23 & 1.5907 & \\
\hline$M P 3$ & & & 0 & 1.2647 & 0.0000 & 414.28 & 0.6000 & \\
\hline ARR 4 & & & 1 & 1.2947 & $1,2047^{\circ}$ & 416.38 & 0.6515 & \\
\hline MRR 5 & & & 1 & 1.2047 & 1.2047 & $4 ! 6.28$ & 0.3480 & \\
\hline & & & & B. 22 & & & & \\
\hline
\end{tabular}




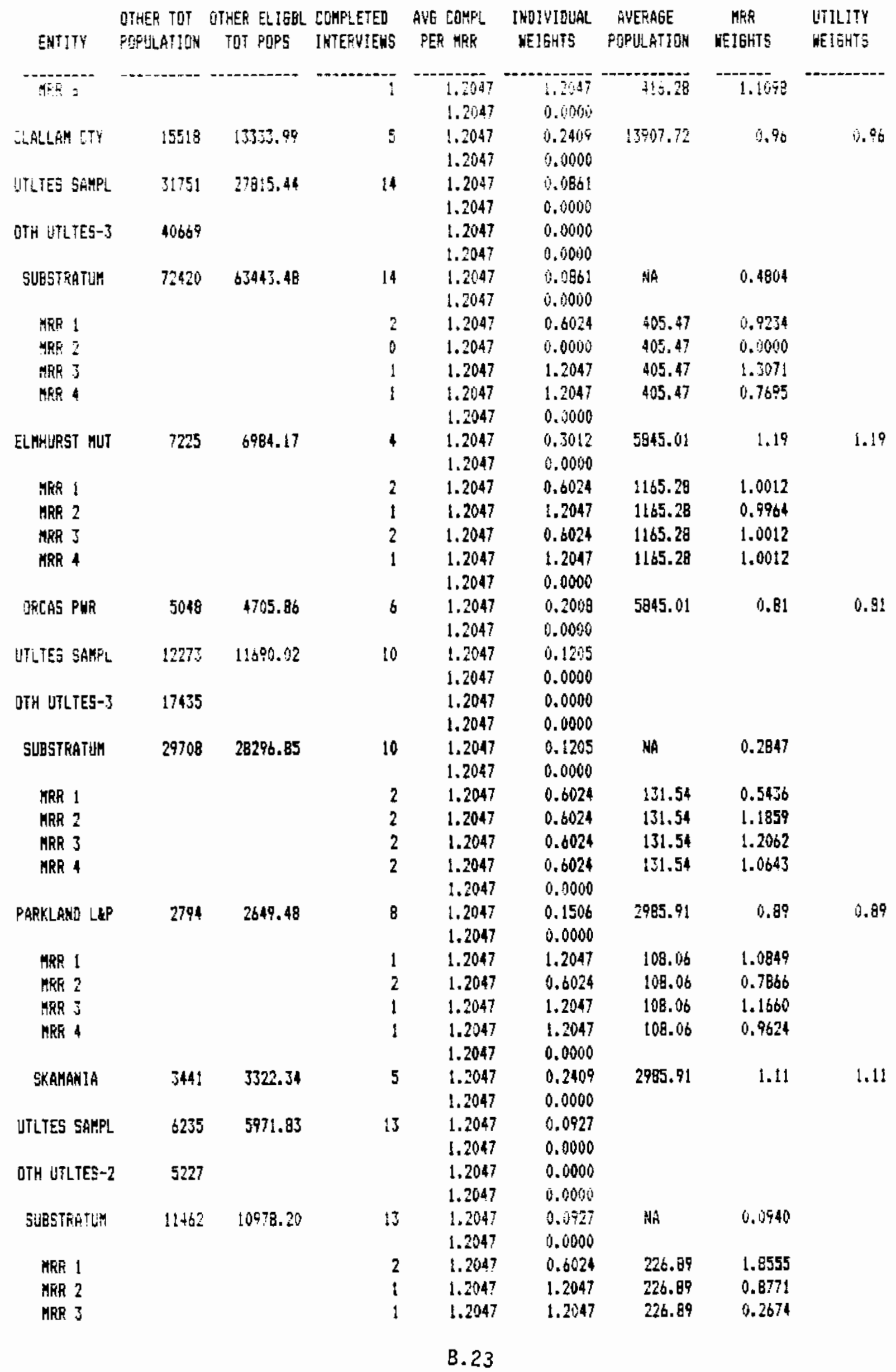




\begin{tabular}{|c|c|c|c|c|c|c|c|c|}
\hline EN:ITY & $\begin{array}{l}\text { STHER TET } \\
\text { EOPILAT:BN }\end{array}$ & $\begin{array}{c}\text { DTHER E!IGLL } \\
\text { TOT F[.5 }\end{array}$ & $\begin{array}{l}\text { CDIFLETED } \\
\text { INTEFVIFHS }\end{array}$ & $\begin{array}{l}\text { AVG COHEL } \\
\text { PES MEF }\end{array}$ & $\begin{array}{l}\text { INOIVIGLAL } \\
\text { HEIGHTS }\end{array}$ & $\begin{array}{l}\text { AUFFAEE } \\
\text { FOFUUATION }\end{array}$ & $\begin{array}{c}\text { HRR } \\
\text { WEIGHTS }\end{array}$ & $\begin{array}{l}\text { UTILITY } \\
\text { DEIGHTS }\end{array}$ \\
\hline 4 & -..-- & --.- & ! & $\begin{array}{l}1.507 ? \\
1.2047\end{array}$ & 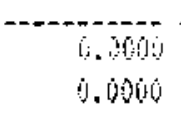 & 326.59 & s.molis & $A+\cdots$ \\
\hline 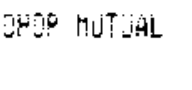 & ! $5:$ & $1024.4 \%$ & 4 & $\begin{array}{l}1.2047 \\
1.2047\end{array}$ & $\begin{array}{l}0.812 \\
0.0009\end{array}$ & 1450.73 & 1.12 & 1.12 \\
\hline MFn : & & & 1 & 1.2047 & $\therefore .2047$ & 130.80 & 0.4037 & \\
\hline WhR ? & & & 2 & 1.2047 & 0.0024 & 130.30 & $1.50 b 1$ & \\
\hline NKR 3 & & & 2 & 1.2047 & 6.6022 & 130.90 & 0.8803 & \\
\hline Nas 4 & & & 1 & $\begin{array}{l}1.2047 \\
1.2047\end{array}$ & $\begin{array}{l}1.2047 \\
0.9000\end{array}$ & 130.80 & 1.2099 & \\
\hline NAKIAKM & 1512 & 1288.00 & $b$ & $\begin{array}{l}1.2047 \\
1.2047\end{array}$ & $\begin{array}{l}0.2008 \\
0.0000\end{array}$ & 1456.23 & 0.98 & 0.88 \\
\hline ITIES SAMFL & 3104 & 2912.47 & $11)$ & $\begin{array}{l}1.2047 \\
1.2047\end{array}$ & $\begin{array}{l}0.1205 \\
0.0000\end{array}$ & & & \\
\hline DTH UTLEES-S & $700 \%$ & & & $\begin{array}{l}1.2047 \\
1.2047\end{array}$ & $\begin{array}{l}0.0000 \\
9.6090\end{array}$ & & & \\
\hline SUYSTKATUH & 10173 & 9364.26 & 10 & $\begin{array}{l}1.2047 \\
1.2047\end{array}$ & $\begin{array}{l}0.1205 \\
i .0000\end{array}$ & $\mathrm{NAT}$ & 0.0931 & \\
\hline STRATUH YOT & 753845 & 711778.99 & 70 & $\begin{array}{l}1.2047 \\
1.2047\end{array}$ & $\begin{array}{l}0.9172 \\
0.0000\end{array}$ & NA & 1.25 & \\
\hline REGION E & & & & $\begin{array}{l}1.2047 \\
1.2047 \\
1.2047\end{array}$ & $\begin{array}{l}0.00000 \\
0.0600 \\
0.00000\end{array}$ & & & \\
\hline 4901 & & & 1 & $\{.2047$ & 1.2047 & 38.81 & 1.1442 & \\
\hline $\mathrm{H}_{\mathrm{R}} \mathrm{R}$ & & & $!$ & 1.2047 & 1.2047 & $302.8 !$ & 1.6396 & \\
\hline Min & & & 1 & 1.2647 & 1.2047 & 382.81 & 1.3714 & \\
\hline WRF 4 & & & 0 & 1.2047 & 0.0000 & 382.81 & 0.0000 & \\
\hline MFE 5 & & & 1 & 1.2047 & 1.2047 & 382.81 & 6.9627 & \\
\hline MER & & & 0 & 1.2047 & 0.1000 & $3 \mathrm{E} 2.81$ & 0.0000 & \\
\hline MRF 7 & & & 0 & 1.2047 & 0.0000 & 382.81 & 0.0000 & \\
\hline MPR $B$ & & & 1 & $\begin{array}{l}1.2047 \\
1.2047\end{array}$ & $\begin{array}{l}1.2047 \\
0.0000\end{array}$ & $387.8 !$ & 0.9921 & \\
\hline HIDSTATE & 7797 & 6973.58 & 5 & $\begin{array}{l}1.2047 \\
1.2047\end{array}$ & $\begin{array}{l}0.2409 \\
0.0000\end{array}$ & 6437.72 & 1.67 & 1.07 \\
\hline PRR i & & & 1 & 1.2047 & 1.2047 & 89.60 & 0.3574 & \\
\hline HRP ? & & & 1 & 1.2047 & 1.3047 & 89.00 & 1. 9989 & \\
\hline Mes ? & & & 0 & 1.2047 & 0.0000 & 89.010 & $0.0 \% 00$ & \\
\hline Mps 4 & & & 1 & $\$ .2047$ & 1.2047 & 89.00 & 1.2270 & \\
\hline MER 5 & & & $!$ & 1.2047 & 1.2047 & 89.00 & 1.1955 & \\
\hline MFR 6 & & & 0 & 1.2047 & 0.0000 & 89.00 & 0.6000 & \\
\hline Hep 7 & & & 0 & 1.2047 & 0. 10000 & 89.00 & 0.0000 & \\
\hline MFR B & & & 1 & $\begin{array}{l}1.2047 \\
1.2047\end{array}$ & $\begin{array}{l}1.2047 \\
0.10000\end{array}$ & 89.00 & 1.0112 & \\
\hline UAATILLA & 3546 & 5996.86 & 5 & $\begin{array}{l}1.2047 \\
1.2047\end{array}$ & $\begin{array}{l}0.2409 \\
0.0000\end{array}$ & 6437.72 & 0.73 & 0.97 \\
\hline JTLTES SAMFL & 14343 & 12875.44 & 10 & $\begin{array}{l}1.2047 \\
1.2047\end{array}$ & $\begin{array}{l}0.1205 \\
6.0000\end{array}$ & & & \\
\hline DFH UTLIES-2 & 17731 & & & $\begin{array}{l}1.2047 \\
1.204 ?\end{array}$ & $\begin{array}{l}0.0000 \\
0.0000\end{array}$ & & & \\
\hline 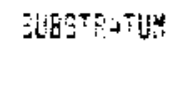 & 22074 & 2074.23 & 1i) & $\begin{array}{l}1.2447 \\
1.2047\end{array}$ & 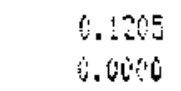 & Ne. & 1.777 & \\
\hline MRR ! & & & 1 & 1.2947 & 1.2047 & 150.61 & 1.2468 & \\
\hline MKE 2 & & & 1 & $\{.2047$ & 1.2047 & :59.6! & 1.1779 & \\
\hline HF 3 & & & 1 & 1.2047 & 1.2047 & 155.81 & 1.0400 & \\
\hline
\end{tabular}




\begin{tabular}{|c|c|c|c|c|c|c|c|c|}
\hline ENTITY & $\begin{array}{l}\text { BTHER TOT } \\
\text { POPULATION }\end{array}$ & $\begin{array}{c}\text { OTHER ELISBL } \\
\text { TIT FOFS }\end{array}$ & $\begin{array}{l}\text { COHFLETED } \\
\text { INTERUIEHS }\end{array}$ & $\begin{array}{c}\text { AVG COMFL } \\
\text { PES MGR }\end{array}$ & $\begin{array}{l}\text { INDIVIOUALL } \\
\text { WEIGHTS }\end{array}$ & $\begin{array}{l}\text { AVERAGE } \\
\text { POPULATTIOK }\end{array}$ & $\begin{array}{c}\text { MRR } \\
\text { REIEHTS }\end{array}$ & $\begin{array}{l}\text { UTILITY } \\
\text { HEIEHTE }\end{array}$ \\
\hline+4 & & & i) & 1.2047 & $0 ., 200$ & 159.61 & 8 & \\
\hline 4 & & & 1 & 1.2047 & 1.247 & 159.61 & 1.1097 & \\
\hline XRE & & & 0 & 1.2047 & 0.5000 & $: 50.61$ & 5 \% & \\
\hline MRR 7 & & & 1 & $\{.2047$ & 1.2047 & $159.6 !$ & 0.5653 & \\
\hline \multirow[t]{2}{*}{ MEF 8} & & & 1 & 1.2047 & 1.2047 & 159.61 & 0.7073 & \\
\hline & & & & 1.2047 & 0.0000 & & & \\
\hline \multirow[t]{2}{*}{ COLUHEIA } & 2855 & 2502.23 & 6 & 1.2047 & 0.2008 & 2638.29 & 0.95 & 0.95 \\
\hline & & & & 1.2047 & 0.0000 & & & \\
\hline MF 1 & & & $!$ & 1.2047 & 1.2047 & 129.27 & 1). 745 & \\
\hline MRR 2 & & & 1 & 1.2047 & 1.2047 & 128.27 & 0.9489 & \\
\hline WRP 3 & & & 0 & 1.2047 & 0.0000 & 129.27 & 9. 000 & \\
\hline MER 4 & & & 0 & 1.2047 & 0,0000 & 129.27 & 0.0010 & \\
\hline MFA 5 & & & 1 & 1.2047 & 1.2047 & 128.27 & 1.4189 & \\
\hline MRR b & & & 1 & 1.2047 & 1.2047 & 128.27 & 1.0135 & \\
\hline MAf 7 & & & 1 & 1.2047 & 1.2047 & 128.27 & 0.8731 & \\
\hline \multirow[t]{2}{*}{$M$} & & & 0 & $\{.2047$ & 0.0000 & 128.27 & 0.0000 & \\
\hline & & & & 1.2047 & 0.0000 & & & \\
\hline \multirow[t]{2}{*}{ MLIN-FREENTR } & 2848 & 2774.34 & 5 & 1.2047 & 0.2409 & 2638.29 & 1.05 & 1.05 \\
\hline & & & & 1.2047 & 0.0000 & & & \\
\hline \multirow[t]{2}{*}{ UTLTES SARPL } & 5703 & 5276.57 & 11 & 1.2047 & 0.1095 & & & \\
\hline & & & & 1.2047 & 0.0000 & & & \\
\hline \multirow[t]{2}{*}{ OTH SAMPL-5 } & 10634 & & & 1.2047 & 0.0000 & & & \\
\hline & & & & 1.2047 & 0.0000 & & & \\
\hline \multirow[t]{2}{*}{ SUBSTRATUH } & 16357 & 15115,44 & 11 & 1.2047 & 0.1045 & 21953.93 & 0.6597 & \\
\hline & & & & $\{.2047$ & 0.8000 & & & \\
\hline \multirow[t]{2}{*}{ STRATA TQT } & $494 ! !$ & 43907.67 & 21 & 1.2047 & 0.0574 & HA & 0.18 & \\
\hline & & & & 1.2047 & 0.0000 & & & \\
\hline \multirow[t]{3}{*}{ REGION 6} & & & & 1.2047 & 0.0000 & & & \\
\hline & & & & $\{.2047$ & 0.0000 & & & \\
\hline & & & & 1.2047 & 0.0000 & & & \\
\hline MRR 1 & & & 2 & 1.2047 & 0.6024 & 2523.42 & 1.0090 & \\
\hline MRR 2 & & & 1 & 1.2047 & 1.2047 & 2523.42 & 1.1544 & \\
\hline KRR 3 & & & 1 & 1.2047 & 1.2047 & 2523.42 & 1.0014 & \\
\hline RRR 4 & & & 1 & 1.2047 & 1.2047 & 2523.42 & 1.0260 & \\
\hline MRR 5 & & & 1 & 1.2047 & 1.2047 & 2523.42 & 0.9265 & \\
\hline \multirow[t]{2}{*}{ ARR 6} & & & 2 & 1.2047 & 0.6024 & 2523.42 & 0.8927 & \\
\hline & & & & 1.2047 & 0.0000 & & & \\
\hline \multirow[t]{2}{*}{ EUGENE XTR } & 54494 & 51135.31 & 日 & 1.2047 & 0.1506 & 34692.59 & 1.47 & 1.47 \\
\hline & & & & 1.2047 & 0.0000 & & & \\
\hline KRR 1 & & & 1 & 1.2047 & 1.2047 & 160.93 & 1.1782 & \\
\hline M 2 & & & & 1.2047 & & 160.93 & & \\
\hline MRR 3 & & & & 1.2047 & & 160.93 & & \\
\hline MRR 4 & & & 2 & 1.2947 & 0.6024 & 160.93 & 0.5930 & \\
\hline MRR 5 & & & 0 & 1.2047 & 0.0000 & 160.93 & 0.0000 & \\
\hline \multirow[t]{2}{*}{ MRR 6} & & & 1 & 1.2047 & 1.2047 & 160.93 & 1.2967 & \\
\hline & & & & 1.2047 & 0.0000 & & & \\
\hline \multirow[t]{2}{*}{ CNTRL-LMCLN } & 21337 & 18249.87 & 4 & 1.2047 & 0.3012 & 34692.59 & 0.53 & 0.53 \\
\hline & & & & 1.3047 & 0.0000 & & & \\
\hline WTLTES SAMLL & 75931 & 69385.18 & 12 & 1.2047 & 0.1004 & & & \\
\hline OTH UTLTES-O & & & & 1.2047 & 0.0000 & & & \\
\hline \multirow[t]{2}{*}{ SLIBSTRATUK } & $7593 i$ & 64859.45 & 12 & 1.2047 & 0.1004 & $\mathrm{NA}$ & 1.1100 & \\
\hline & & & & 1.2047 & 0.0000 & & & \\
\hline MRR I & & & $!$ & 1.2047 & 1.2047 & 3.2 .12 & 0.9415 & \\
\hline
\end{tabular}




\begin{tabular}{|c|c|c|c|c|c|c|c|c|}
\hline ENT!iy & 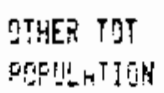 & $\begin{array}{c}\text { QTHER E: IGEL } \\
\text { TET POPS }\end{array}$ & $\begin{array}{l}\text { SOHPL CTED } \\
\text { IHEFUIEWE }\end{array}$ & $\begin{array}{l}\text { AVG COHFL } \\
\text { YER WRF }\end{array}$ & $\begin{array}{l}\text { INDIWIOUAL } \\
\text { SEISHTS }\end{array}$ & $\begin{array}{l}\text { MHEPATE } \\
\text { PIIFULATTIOK }\end{array}$ & $\begin{array}{c}\text { PEE } \\
\text { WEIEHTS }\end{array}$ & $\begin{array}{l}\text { L'TIITY } \\
\text { WElGHTS }\end{array}$ \\
\hline 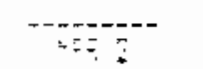 & & & 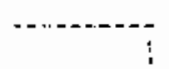 & 1,347 & 1.747 & $37, .2$ & 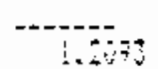 & $\cdots$ \\
\hline$p+3$ & & & 0 & 1.3047 & $i_{0}+0.00$ & $\because 7 \div .12$ & 1.600? & \\
\hline$M R_{4}$ & & & $\hat{0}$ & 1.2017 & în. & 79.12 & 0.0000 & \\
\hline MR 5 & & & i) & 1.2047 & 0.0000 & 512.12 & 0.6000 & \\
\hline MFP o & & & 5 & 1.2047 & 0.6024 & 372.12 & 0.8492 & \\
\hline & & & & 1.2547 & 5.00ivo & & & \\
\hline SPRINGF IELD & 9530 & $17809 .=3$ & 4 & $1.204 \mathrm{~T}$ & 0.3012 & $\{4687.0\}$ & 1.21 & $\{.21$ \\
\hline & & & & 1.7047 & 0.0000 & & & \\
\hline Mhi I & & & $i$ & 1.204? & ij.0000 & 67.57 & 0.0000 & \\
\hline Fff 2 & & & $\bar{z}$ & 1.2047 & 0.6924 & 67.57 & 0.9379 & \\
\hline $40 \% 3$ & & & 1 & 1.3947 & 1.2047 & 67.67 & 0.9276 & \\
\hline Nhet 4 & & & 0 & 1.3047 & D.0000 & 67.67 & 0.0000 & \\
\hline 所] 5 & & & 1 & 1.2047 & 1.2047 & $6: 57$ & 0.6532 & \\
\hline MRR d & & & $\vdots$ & 1.2047 & 1.2647 & 67.67 & 1.5813 & \\
\hline & & & & 1.2047 & 0.0000 & & & \\
\hline CDNEUHERS & 12893 & 11565.50 & 5 & 1.2047 & 0.2409 & 14687,01 & 9.79 & 0.79 \\
\hline & & & & 1.2047 & 0.0300 & & & \\
\hline BTLIES SAMPL & $3141 j$ & 29374.03 & 9 & 1.2047 & 0.1339 & & & \\
\hline & & & & 1.2047 & 0.0000 & & & \\
\hline OTH JT!TES-2 & 22874 & & & 1.2047 & 0.0000 & & & \\
\hline & & & & 1.2047 & 0.0000 & & & \\
\hline SUgETRATUM & 54287 & 50763.31 & 9 & 1.2047 & 0.1339 & NA & 1.3339 & \\
\hline & & & & 1.2447 & 0.0000 & & & \\
\hline WE 1 & & & 2 & 1.2047 & 0.6024 & 102.13 & 0.9751 & \\
\hline MSE 2 & & & $?$ & 1.2047 & $0.6 \times 24$ & 102.53 & 1.9240 & \\
\hline GRK J & & & 2 & 1.2047 & 0.6024 & 102.13 & 0.7768 & \\
\hline MFG 4 & & & 1 & 1.2047 & 1.2047 & 102.13 & 1.1260 & \\
\hline & & & & 1.2047 & 0.6000 & & & \\
\hline ASHLAND & 5714 & 5249.05 & 7 & 1,2047 & 0.1721 & 4979.44 & 1.05 & 1.05 \\
\hline & & & & t.2047 & 0.0000 & & & \\
\hline 飘 1 & & & $!$ & 1.2047 & \pm .2047 & 94.98 & 0.7581 & \\
\hline AhF 2 & & & 2 & 1.2047 & 0.6024 & 94.98 & 0.5994 & \\
\hline SER 3 & & & 0 & 1.2047 & 0.0000 & 94.98 & 0.0000 & \\
\hline HRF 4 & & & 1 & 1.2047 & 1.2047 & 94.98 & 1.4424 & \\
\hline & & & & $\{.2047$ & 0.0060 & & & \\
\hline FEFEST GROUE & 5138 & 4709.83 & 4 & 1.2647 & $0.30: 2$ & 4977.44 & 0.95 & 0.95 \\
\hline & & & & 1.2047 & 0.90000 & & & \\
\hline UTLTES SAHP! & 10852 & 9958.08 & $! 1$ & $! .2047$ & 0.1095 & & & \\
\hline & & & & 1.2047 & 0.0000 & & & \\
\hline OTH U:-TES-4 & 5.3252 & & & 1.2047 & 0.0090 & & & \\
\hline & & & & 1.2047 & 0.0000 & & & \\
\hline SURSTRATUA & 44104 & 40474.26 & 11 & 1.2047 & $0 .: 095$ & $\mathrm{NA \hat {H }}$ & 1.0235 & \\
\hline & & & & 1.2047 & 0.00000 & & & \\
\hline 触 1 & & & 2 & $\vdots .7047$ & 0.6 .224 & 947.94 & 0.9245 & \\
\hline PR 2 & & & 2 & 1.2047 & 0.6024 & $447+014$ & 1.1856 & \\
\hline$M R R$ & & & 1 & $1.204 ?$ & 1.2047 & 447.04 & 0.9383 & \\
\hline MKR 4 & & & 1 & 1.2047 & $1.204 \pi$ & 447.04 & 0.9517 & \\
\hline & & & & $i .2) 47$ & 0.0000 & & & \\
\hline 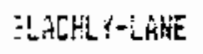 & 253 & 2355.70 & $b$ & 1.3047 & 0.2000 & 2213.13 & 1.85 & 1.5 \\
\hline & & & & 1.2647 & 0. 6090 ? & & & \\
\hline KKR I & & & $\theta$ & 1.2047 & 0.0070 & 215.63 & 0.2000 & \\
\hline MRR 2 & & & 1 & 1.2047 & 1.2047 & 200.03 & $\$ .2918$ & \\
\hline MSR 3 & & & 0 & 1.2017 & $0.51,00$ & 216,03 & 0.0000 & \\
\hline
\end{tabular}




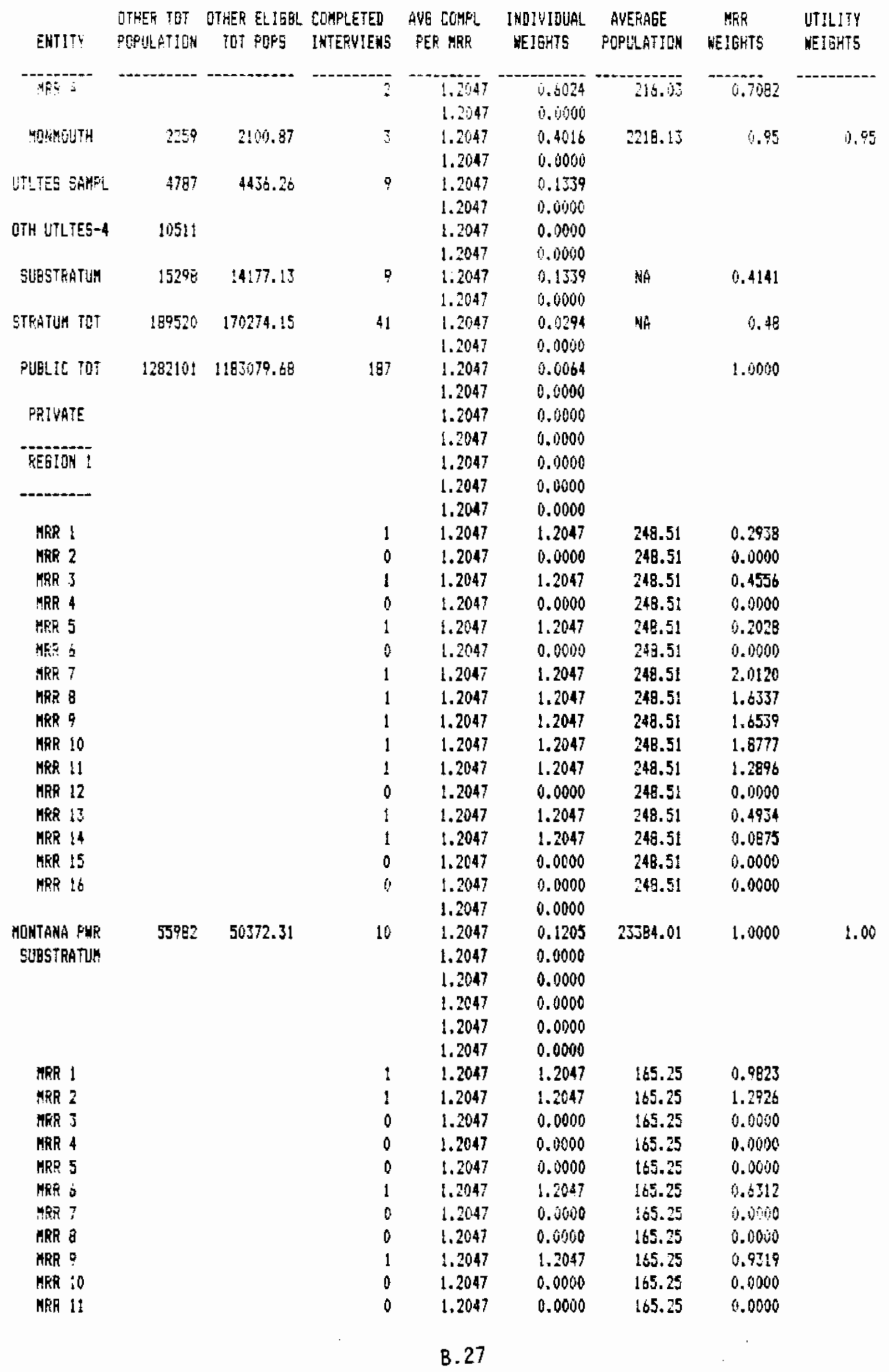




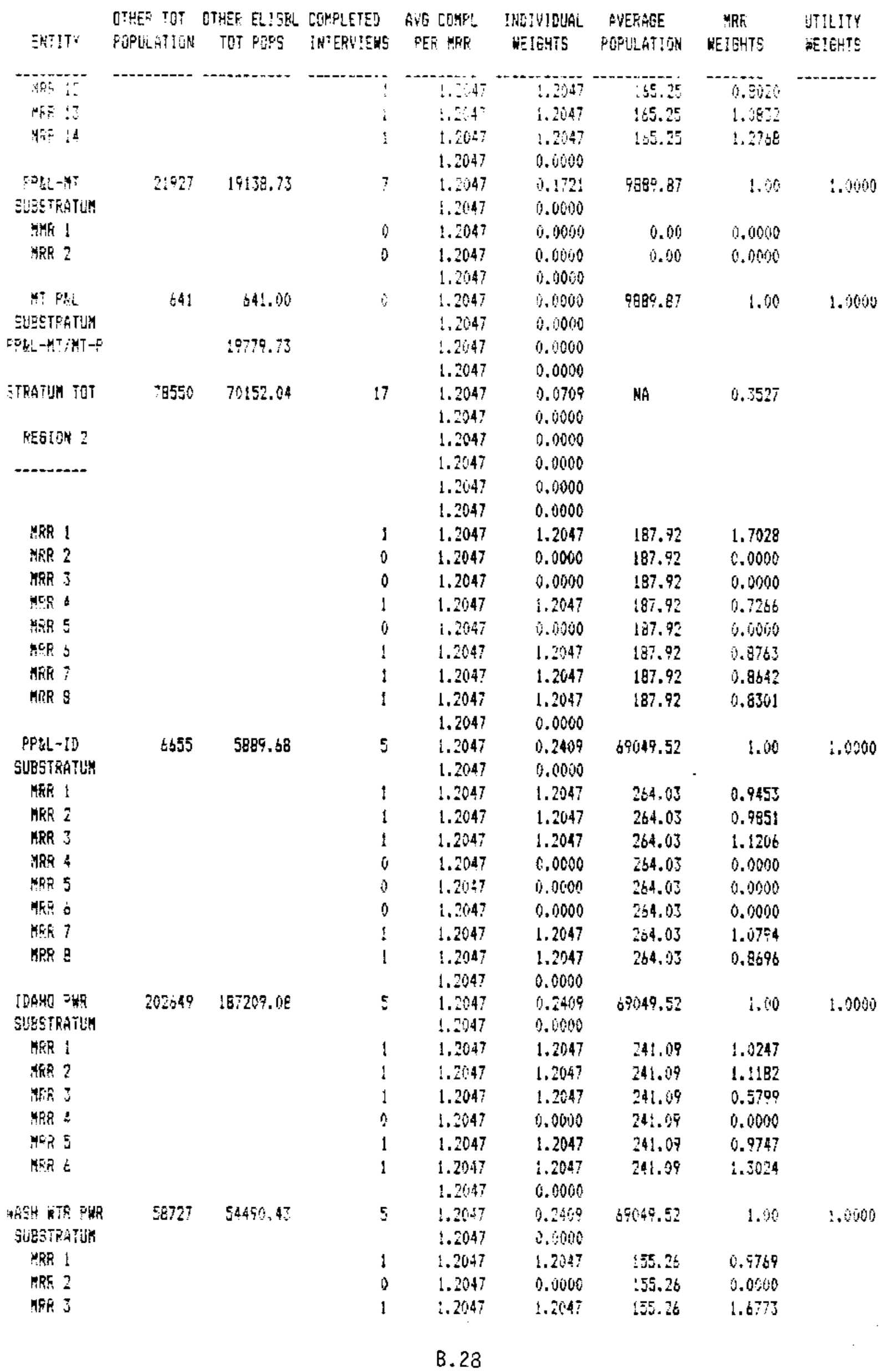




\begin{tabular}{|c|c|c|c|c|c|c|c|c|}
\hline E:HItiy & $\begin{array}{l}\text { OTHER TOT } \\
\text { POP!DATIUN }\end{array}$ & $\begin{array}{c}\text { OTHER ELIELL } \\
\text { TGT POPS }\end{array}$ & $\begin{array}{l}\text { COAPLETED } \\
\text { INTERVIENS }\end{array}$ & $\begin{array}{l}\text { AVE COMFL } \\
\text { PEF MFR }\end{array}$ & $\begin{array}{l}\text { INDIVIDUAL } \\
\text { WE!BHTS }\end{array}$ & $\begin{array}{l}\text { AVERLGE } \\
\text { FOPULATIOH }\end{array}$ & $\begin{array}{c}\text { MER } \\
\text { WEIGHTS }\end{array}$ & $\begin{array}{l}\text { STILITY } \\
\text { WEIGHTS }\end{array}$ \\
\hline 45e & & & 0 & 1.2 .47 & 3.6000 & 155.28 & 0.0010 & \\
\hline MRF 5 & & & 1 & 1.2047 & 1.2047 & 155.26 & 0.9726 & \\
\hline M & & & $t$ & 1.2047 & 1.2647 & $! 53.26$ & 0.6516 & \\
\hline MER 7 & & & 1 & 1.2047 & 1.2047 & 155.20 & 0.7214 & \\
\hline \multirow[t]{2}{*}{ KER B } & & & 0 & 1.2047 & 0.0000 & 155.26 & 0.6000 & \\
\hline & & & & 1.2047 & 0.0000 & & & \\
\hline UTAH PUL & 33205 & 28608.92 & 5 & 1.2047 & 0.2409 & 69049.52 & 1.00 & 1.0000 \\
\hline SUESTRATUM & & & & 1.2047 & 0.0000 & & & \\
\hline STSATIN TOT & 301236 & 276198.10 & 20 & 1.2047 & 0.0602 & HA & 1.18 & \\
\hline \multirow{3}{*}{ SEGION 3} & & & & $\begin{array}{l}1.2047 \\
1.2047\end{array}$ & $\begin{array}{l}0.0000 \\
0.0000\end{array}$ & & & \\
\hline & & & & 1.2047 & 0.0000 & & & \\
\hline & & & & 1.2047 & 0.0000 & & & \\
\hline MRR 1 & & & 0 & 1.2047 & 0.0000 & 264.29 & 0.0000 & \\
\hline MRE 2 & & & 1 & 1.2047 & 1.2047 & 264.29 & 1.5362 & \\
\hline MRR 3 & & & 0 & 1.2047 & 0.0000 & 264.29 & 0.0000 & \\
\hline Yef 4 & & & $!$ & 1.2047 & 1.2047 & 264.29 & 0.5963 & \\
\hline ARR 5 & & & 1 & 1.2047 & 1.2047 & 261.29 & 1.0367 & \\
\hline MPR \& & & & 1 & 1.2047 & 1.2047 & 264.29 & $0.715 t$ & \\
\hline HRR 7 & & & 0 & 1.2047 & 0.0000 & 264.29 & 0.0000 & \\
\hline KRR B & & & 1 & 1.2047 & 1.2047 & 264.29 & 1.2562 & \\
\hline MRR 9 & & & 0 & 1.2047 & 0.0000 & 264.29 & 0.0000 & \\
\hline NRR 10 & & & 0 & 1.2047 & 0.0000 & 264.29 & 0.0000 & \\
\hline Meg 11 & & & 0 & 1.2047 & 0.0000 & 264.24 & 0.0000 & \\
\hline H4 12 & & & $\downarrow$ & 1.2047 & 1.2047 & $2: 4.29$ & 0.96 .27 & \\
\hline MKS 13 & & & $t$ & 1.2047 & 1.2047 & 264.79 & 0.3967 & \\
\hline NRR 14 & & & 0 & 1.2047 & 0.0000 & 264.29 & 0.0000 & \\
\hline HRA 15 & & & 0 & 1.2047 & 0.0000 & 264.29 & 0.0000 & \\
\hline \multirow[t]{2}{*}{ MRR 16} & & & 0 & 1.2047 & 0.0000 & 264.24 & 0.0000 & \\
\hline & & & & 1.2047 & 0.0000 & & & \\
\hline \multirow{2}{*}{$\begin{array}{c}\text { PPKLL-KA } \\
\text { SUESTRATUK }\end{array}$} & 81702 & 77788.54 & 7 & $\{.2047$ & 0.1721 & 105676.38 & 1.00 & 1.0000 \\
\hline & & & & 1.2047 & 0.0000 & & & \\
\hline MRR : & & & 0 & 1.2047 & 0.0000 & 255.78 & 0.0000 & \\
\hline MRE 2 & & & $!$ & 1.2047 & 1.2047 & 255.78 & 0.9852 & \\
\hline MRR 3 & & & 0 & 1.2047 & 0.0000 & 255.78 & 0.0000 & \\
\hline MRP 4 & & & 1 & 1.2047 & 1.2047 & 255.78 & 1.1114 & \\
\hline ARR 5 & & & 0 & 1.2047 & 0.0000 & 255.78 & 0.0000 & \\
\hline RRR 6 & & & 0 & 1.2047 & 0.6000 & 255.78 & 0.0000 & \\
\hline HRR 7 & & & 1 & 1.2047 & 1.2047 & 255.78 & 0.8953 & \\
\hline$M R P$ & & & 1 & 1.2047 & 1.2047 & 255.78 & 1.0293 & \\
\hline MRR 9 & & & $\hat{0}$ & 1.2047 & 0.0000 & 255.78 & 0.0000 & \\
\hline KRR 10 & & & 0 & 1.2047 & 0.0000 & 255.78 & 0.0000 & \\
\hline MRR 11 & & & 0 & 1.2047 & 0.0000 & 255.78 & 0.0000 & \\
\hline IRR 12 & & & 0 & 1.2047 & 0.0000 & $2 \mathrm{Ex} .7 \mathrm{~B}$ & 0.0000 & \\
\hline MRR 13 & & & 0 & 1.2047 & 0.0000 & 255.78 & 0.10000 & \\
\hline MER 14 & & & 1 & 1.2047 & 1.2047 & 255.78 & 1.1377 & \\
\hline HRR 15 & & & 1 & 1.2047 & 1.2047 & 255.78 & 0.8412 & \\
\hline \multirow[t]{2}{*}{ MRR 16} & & & 0 & 1.2047 & 0.0000 & 255.78 & 0.0000 & \\
\hline & & & & 1.2047 & 0.0000 & & & \\
\hline \multirow{4}{*}{$\begin{array}{l}\text { WASK WAT PHR } \\
\text { SLBSTRATLM } \\
\text { STERTUH TOT }\end{array}$} & 144643 & 133564.21 & 6 & 1.2047 & 0.2000 & 105676.38 & 1.00 & 1.0000 \\
\hline & & & & 1.2047 & 0.0000 & & & \\
\hline & 226345 & 211352.75 & 13 & $\{.2047$ & 0.0927 & NA & 1.39 & \\
\hline & & & & 1.2047 & 0.6000 & & & \\
\hline & & & & & & & & \\
\hline
\end{tabular}




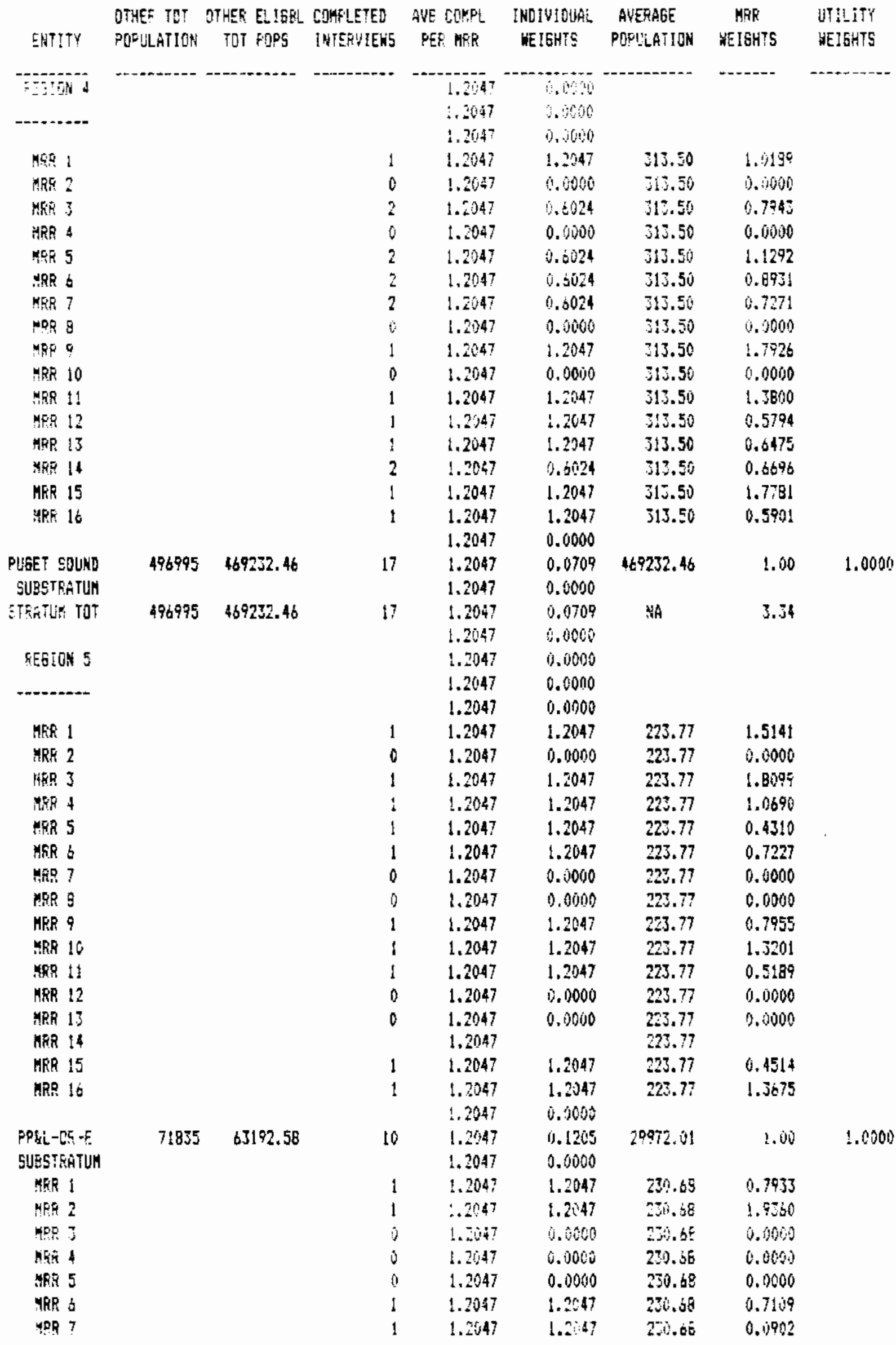




\begin{tabular}{|c|c|c|c|c|c|c|c|c|}
\hline ENTIT: & $\begin{array}{l}\text { OTHER TOT } \\
\text { ORPLLAT!ON }\end{array}$ & $\begin{array}{c}\text { OTHEP ELIGE } \\
\text { TOT FOPS }\end{array}$ & $\begin{array}{l}\text { COHPLETED } \\
\text { IHTERWIEWS }\end{array}$ & $\begin{array}{l}\text { AVG COAFL } \\
\text { PER MPR }\end{array}$ & $\begin{array}{l}\text { INOIVIDUAL } \\
\text { HEJEHTS }\end{array}$ & $\begin{array}{l}\text { AUERAGE } \\
\text { PAPULATION }\end{array}$ & $\begin{array}{c}\text { NER } \\
\text { HEIGHTS }\end{array}$ & $\begin{array}{l}\text { WTILITY } \\
\text { WEIGHTS }\end{array}$ \\
\hline 35 & & & $i$ & $\begin{array}{l}1.2047 \\
1.2647\end{array}$ & $\begin{array}{l}2.877 \\
6.0000\end{array}$ & $27 i d .68$ & 1.4096 & \\
\hline $\begin{array}{l}\text { GAHE = } \\
\text { SULSTKATIJ* }\end{array}$ & 11675 & 10200.36 & 5 & $\begin{array}{l}1.2047 \\
1.2047\end{array}$ & $\begin{array}{l}0.7409 \\
0.0000\end{array}$ & 29072,01 & 1.90 & 1.6000 \\
\hline HR ! & & & 0 & 1.2047 & 0.0600 & 423.51 & 0.0000 & \\
\hline MRR Z & & & 1 & 1.2047 & 1.2047 & 423.51 & 1,5278 & \\
\hline MRR 3 & & & & $\{.2047$ & & 423.51 & & \\
\hline MPF 4 & & & 1 & 1.2047 & 1.2047 & $423.5 !$ & 0.4722 & \\
\hline $\operatorname{MPF} 5$ & & & & 1.2047 & & 423.51 & & \\
\hline MAT O & & & 0 & 1.2047 & 0.0000 & 423.51 & 6.0000 & \\
\hline HRF? & & & & 1.2047 & & 423.51 & & \\
\hline MRP & & & 0 & 1.2047 & 0.0000 & 423.51 & 9.0000 & \\
\hline & & & & 1.2047 & 0.0000 & & & \\
\hline CP RATNL & 22295 & 16520.69 & 2 & 1.2047 & 0.6024 & 29972.01 & 1.60 & 1.00 \\
\hline SUESTRATLH & & & & 1.2047 & 0.0000 & & & \\
\hline STKATLM TOT & 105805 & 89916.03 & 17 & 1.2047 & 0.0709 & NA & 0.45 & \\
\hline & & & & 1.2047 & 0.0000 & & & \\
\hline REEIOK 6 & & & & 1.2047 & 0.0000 & & & \\
\hline-1 & & & & 1.2047 & 0.0000 & & & \\
\hline & & & & 1.2047 & 0.0000 & & & \\
\hline MER ! & & & 0 & 1.2047 & 0.0000 & 210.25 & 0.0000 & \\
\hline MRR 2 & & & 1 & 1.2047 & 1.2047 & 210.25 & 0.8599 & \\
\hline MFR 3 & & & 1 & 1.2047 & 1.2047 & 210.25 & 1.2053 & \\
\hline MRP 4 & & & 0 & 1.2047 & 0.0000 & 210.25 & u. 0000 & \\
\hline KFR 5 & & & 1 & 1.2047 & 1.2047 & 210.25 & 0.5997 & \\
\hline MRR io & & & 2 & 1.2047 & 0.5024 & 210.25 & 0.8993 & \\
\hline HRR 7 & & & 1 & 1.2047 & 1.2047 & 210.25 & $1.67 \theta 8$ & \\
\hline MRR 8 & & & 0 & 1.2047 & 0,0000 & 210.25 & 0.0000 & \\
\hline GRR 9 & & & 0 & 1.2047 & 0.0000 & 210.25 & 0.00010 & \\
\hline WR 10 & & & 2 & 1.2047 & 0.6024 & 210.25 & 0.5574 & \\
\hline MRR 11 & & & 2 & $\{.2047$ & 0.6024 & 210.25 & 0.8419 & \\
\hline ARR 12 & & & 2 & 1.2047 & 0.6024 & 210.25 & 1.5600 & \\
\hline HRR 13 & & & 0 & 1.2047 & 0.0000 & 210.25 & 0.0000 & \\
\hline MKR I4 & & & 1 & 1,2047 & 1.2047 & 210.25 & 0.8969 & \\
\hline MRE 15 & & & 2 & 1.2047 & 0.6024 & 210.25 & 1.2128 & \\
\hline MRR 16 & & & 1 & 1.2047 & 1.2047 & 210.25 & 0.6914 & \\
\hline & & & & 1.2047 & 0.0000 & & & \\
\hline$P P L L L A R-W$ & 270237 & 245957.46 & 16 & 1.2047 & 0.0753 & 332725.74 & 1.00 & 1.0000 \\
\hline SUBSTKATUH & & & & $\{.2047$ & 0.0000 & & & \\
\hline MRR 1 & & & 1 & 1.2047 & $\$ .2047$ & 316.32 & 0.7043 & \\
\hline ARR 2 & & & 1 & 1.2047 & 1.2047 & 316.32 & 1.1978 & \\
\hline HRE 3 & & & 1 & 1.2047 & 1.2047 & 316.32 & 0.9016 & \\
\hline MRR 4 & & & 2 & 1.2047 & 0.6024 & 316.32 & 1.3120 & \\
\hline MKR 5 & & & 2 & 1.2047 & 0.6024 & 316.32 & 0.4995 & \\
\hline MRR b & & & 2 & 1.2047 & 0.5024 & 316.32 & 0.8465 & \\
\hline MRP 7 & & & 2 & 1.2047 & 0.6024 & 316.32 & 1.1918 & \\
\hline MRR 9 & & & 1 & 1.2047 & 1.2047 & 316.32 & 0.8193 & \\
\hline MFR & & & 2 & 1.2047 & 0.6024 & 316.32 & 1.1687 & \\
\hline MER 10 & & & 0 & 1.2047 & 1. 11000 & 316.32 & 0.0600 & \\
\hline MRR 11 & & & 0 & 1.2047 & ง. 0900 & 316.32 & 0.0000 & \\
\hline WRR 12 & & & 0 & 1.2047 & 0.0000 & 316.32 & 0.0000 & \\
\hline WRR 13 & & & 2 & $1.20 \mathrm{~d} 7$ & 0.6024 & 316.32 & 0.9206 & \\
\hline MPR 14 & & & 0 & 1.2047 & 0.0000 & 316.32 & 0.0000 & \\
\hline
\end{tabular}




\begin{tabular}{|c|c|c|c|c|c|c|c|c|}
\hline ENIITY & $\begin{array}{l}\text { QTHER TOT } \\
\text { FOPULA:ION }\end{array}$ & $\begin{array}{l}\text { OFHER ELIGSL } \\
\text { TUT FOFS }\end{array}$ & $\begin{array}{l}\text { COMPLETEO } \\
\text { INEPUIEHS }\end{array}$ & $\begin{array}{l}\text { AWG COMF- } \\
\text { FET MRF }\end{array}$ & $\begin{array}{l}\text { INOWVIJUAR } \\
\text { WEIGHTS }\end{array}$ & $\begin{array}{l}\text { WWERASE } \\
\text { POFULATIDH }\end{array}$ & $\begin{array}{c}\text { MFR } \\
\text { WEISHTS }\end{array}$ & $\begin{array}{l}\text { UTHLITY } \\
\text { WEIGHTS }\end{array}$ \\
\hline 40 & $\cdots$ & י----- & $\overline{1}$ & 7. & 4.46 & 30.3 & $1.14 ! 2$ & $\cdots+\infty$ \\
\hline ffe lo & & & 2 & $\begin{array}{l}1.2047 \\
1.2047\end{array}$ & $\therefore$ sint & 315.32 & $1.20 t ?$ & \\
\hline $\begin{array}{l}\text { FUAT GEN ELE } \\
\text { SUESTKATIUH }\end{array}$ & 439136 & 418494.111 & 19 & $\begin{array}{l}1.2047 \\
1.2047\end{array}$ & & 32225.74 & 1.60 & 1.0000 \\
\hline STRHTIN TOT & 709772 & 564451.47 & 35 & $\begin{array}{l}1.2047 \\
1.2047\end{array}$ & & !s & 2.47 & \\
\hline FRINATE TOT & 1919304 & 1781303 & 149 & & & 1791303 & 1.00 & \\
\hline SFAsid Yor & 3200405 & 2964383 & 34 & & & 2964393 & 1.00 & \\
\hline
\end{tabular}




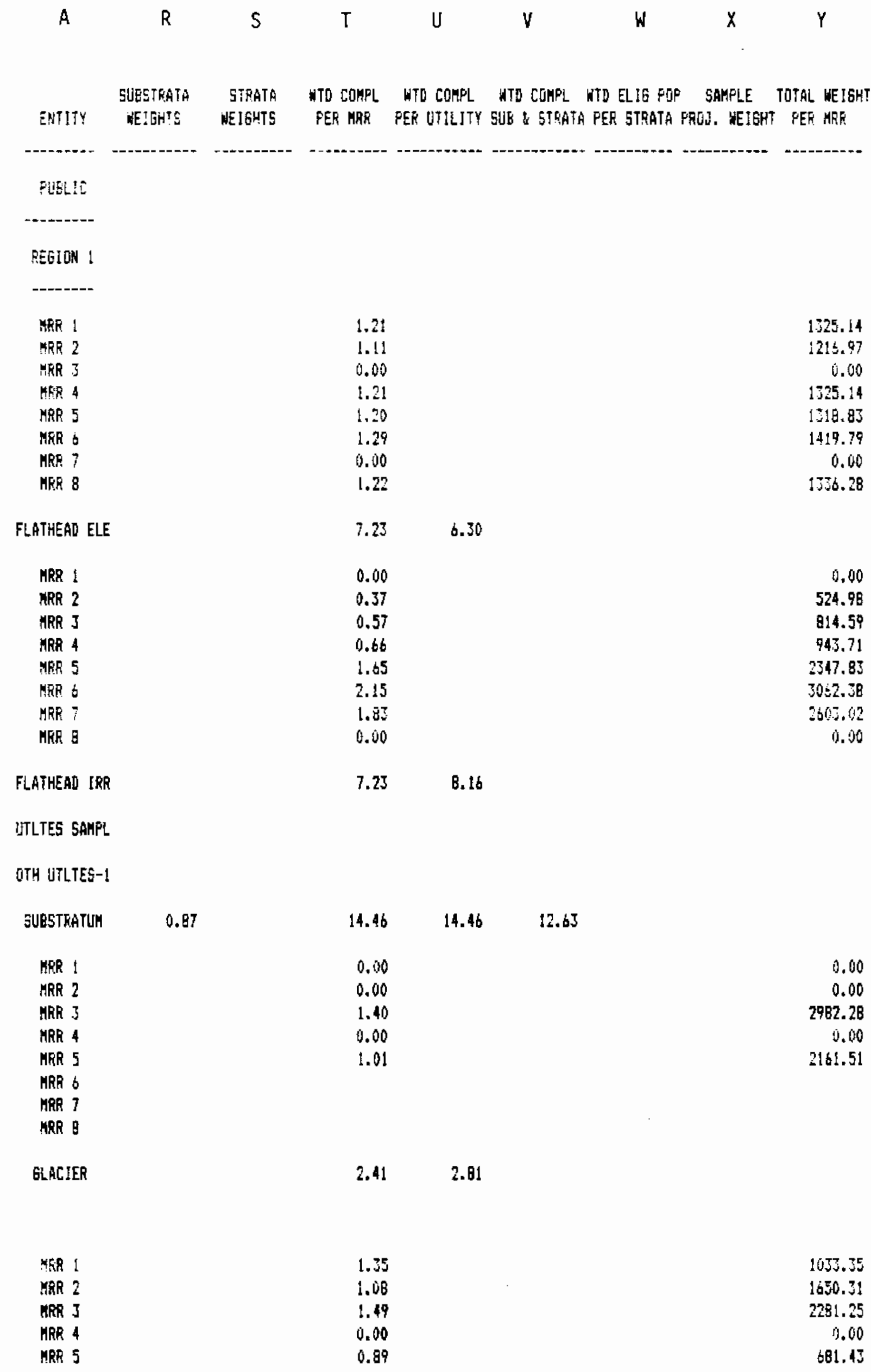




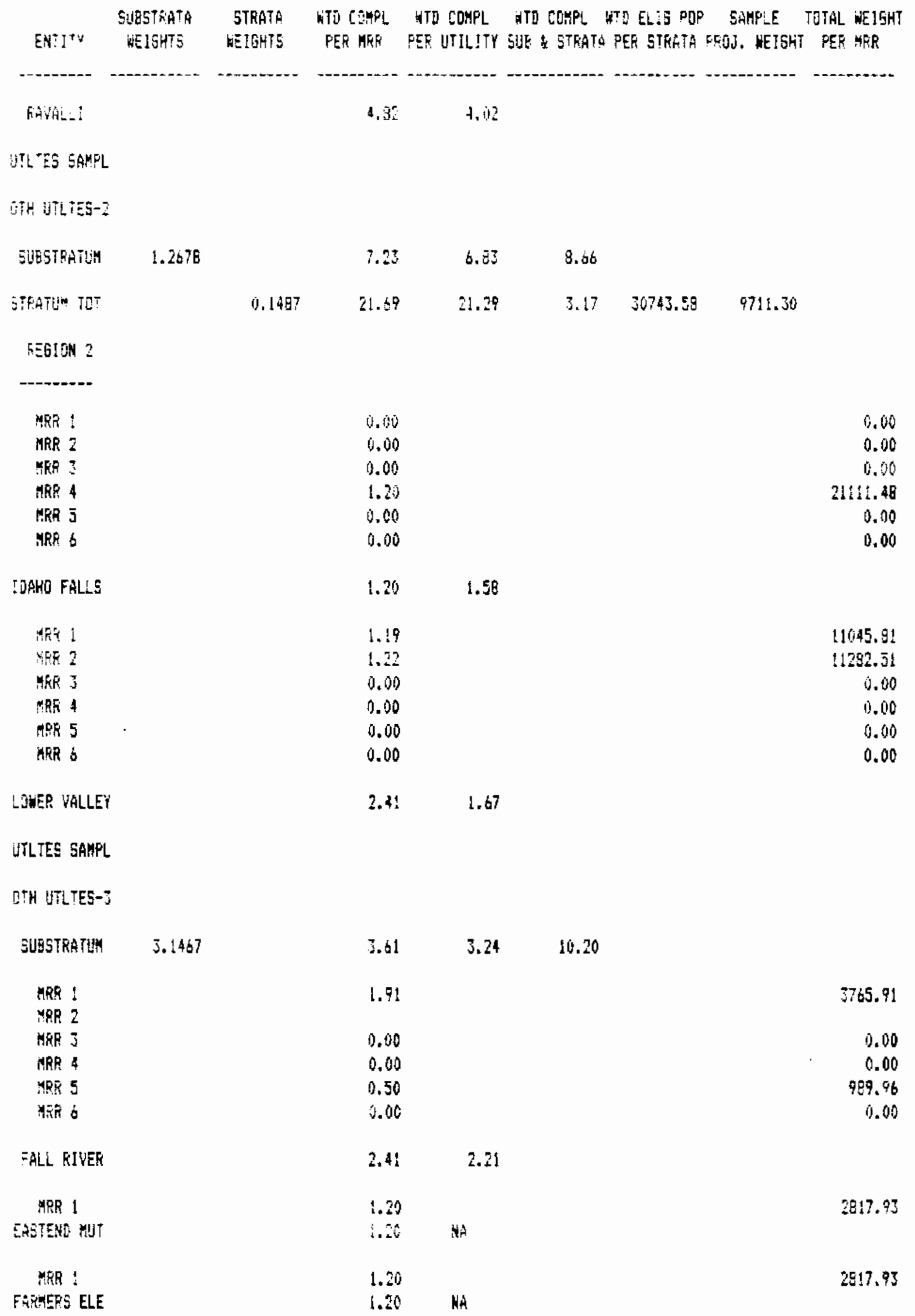




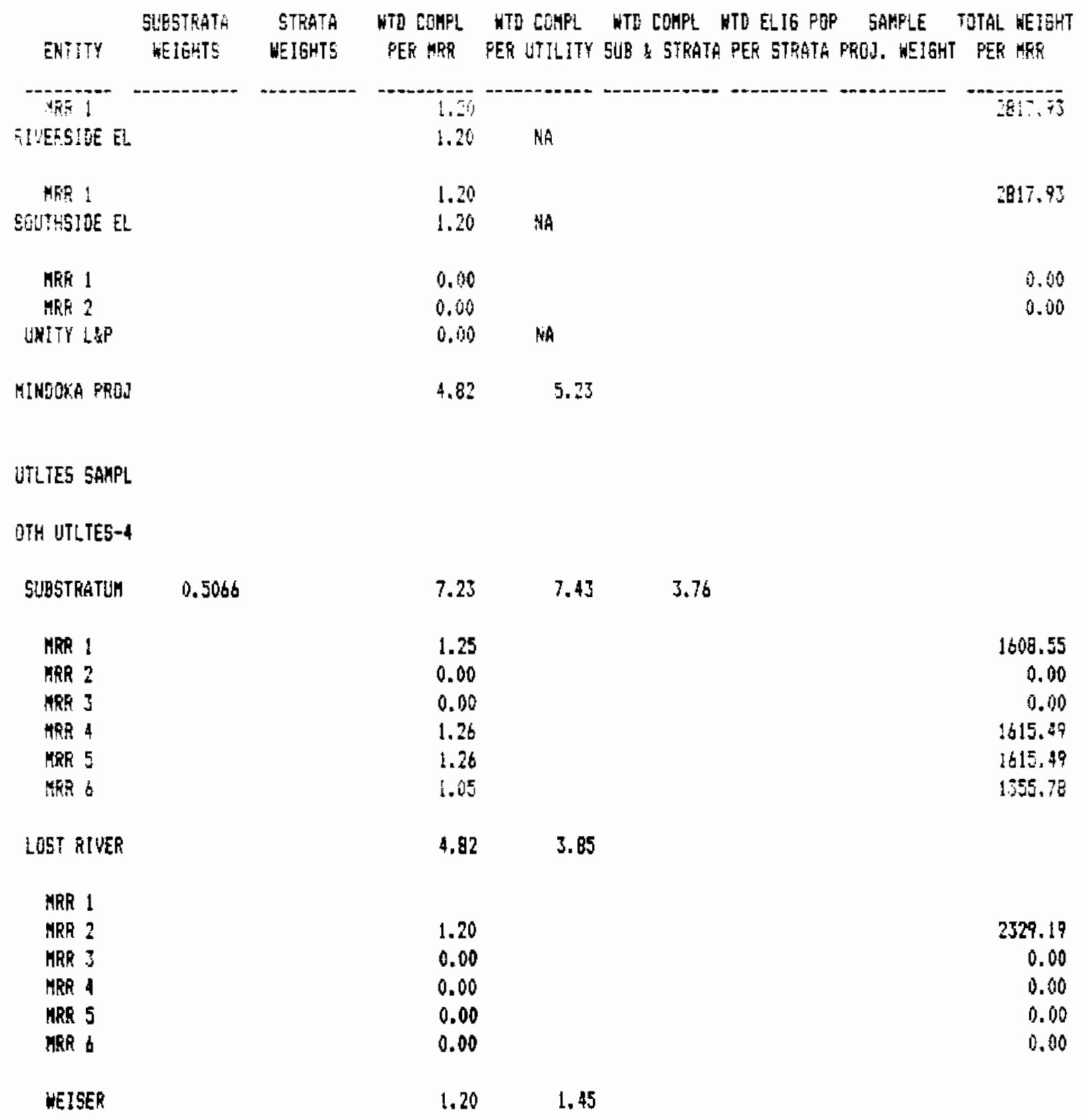

UTLIES SAMPL

OTH UTLTES-4

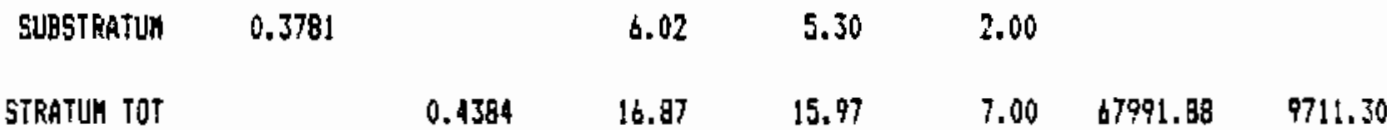

REGIDN 3

$\begin{array}{ll}\text { ARR 1 } & 0.00 \\ \text { MFR 2 } & 0.00 \\ \text { MRR 3 } & 0.00 \\ \text { ARR 4 } & 1.20 \\ \text { ARR 5 } & 0.00\end{array}$

0.00

0.00

0.00

14520.86

0.00 


\begin{tabular}{|c|c|c|c|c|c|c|}
\hline ENTITY & $\begin{array}{l}\text { SUEETEGTA } \\
\text { HEIGHTS }\end{array}$ & $\begin{array}{r}\text { STRATA } \\
\text { MEIGHTS }\end{array}$ & $\begin{array}{l}\text { ATD CDMPL } \\
\text { PEF MFR }\end{array}$ & 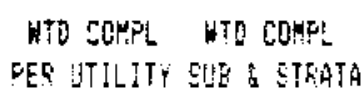 & $\begin{array}{l}\text { WTS ELIG FIP SAMELE } \\
\text { A PER CTRATA PROJ, WEISUTT }\end{array}$ & $\begin{array}{l}\text { POTAL WETEHT } \\
\text { T PEF MPF }\end{array}$ \\
\hline spt & (2) & & 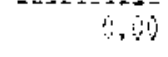 & (2) & 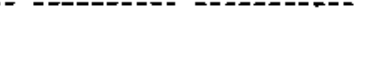 & i. \\
\hline ten: & & & 1.20 & 1.25 & & \\
\hline Wh! & & & 0.97 & & & 9257.15 \\
\hline WFE 2 & & & 0.46 & & & 4047.65 \\
\hline MES 3 & & & 1.49 & & & $157,3,45$ \\
\hline NER 4 & & & 1.90 & & & 2063.15 \\
\hline ARE 5 & & & 0.00 & & & 0.010 \\
\hline CAELIN CTY & & & 4.82 & iA & & \\
\hline MFR 1 & & & 1.20 & & & $127: 4,10$ \\
\hline CASHAEFE & & & 1.20 & NA & & \\
\hline CHELAN-CSHAFE & & & 0.02 & 5.62 & & \\
\hline \multicolumn{7}{|l|}{ WTLTES SAAPL } \\
\hline \multicolumn{7}{|l|}{ CTH UTLTES-2 } \\
\hline SUGSTRATUK & 1.7543 & & 7.23 & 12.12 & & \\
\hline Mha 1 & & & 0.32 & & & 2708.27 \\
\hline Mip $Z$ & & & 9.47 & & & 5.2.5. 07 \\
\hline 45 & & & 0.00 & & & 0.00 \\
\hline$M$ & & & 2.59 & & & 21282.94 \\
\hline MKR 5 & & & 1.43 & & & 11776.45 \\
\hline \multicolumn{7}{|l|}{ ARR a } \\
\hline FPANKLIN & & & 4.62 & 3.87 & & \\
\hline KRR I & & & 0.00 & & & 0.00 \\
\hline Shin 2 & & & 0.00 & & & 6.00 \\
\hline HRR 3 & & & 0.00 & & & $0.0 \%$ \\
\hline$M R R 4$ & & & 1.20 & & & 14728.31 \\
\hline Mth 5 & & & 0.00 & & & 0.00 \\
\hline MRE 6 & & & 0.00 & & & 0.00 \\
\hline RICHLAND & & & 1.20 & 1.44 & & \\
\hline \multicolumn{7}{|l|}{ ITLTES SAMEL } \\
\hline \multicolumn{7}{|l|}{ GTH UTLTES-5 } \\
\hline SigetFATU & 1.5863 & & t. 02 & 5.31 & & \\
\hline$-6 R !$ & & & 0.00 & & & 0.60 \\
\hline $4 \mathrm{TH}$ & & & 0.60 & & & $\dot{1}_{2} 60$ \\
\hline$M \vec{k} \bar{k} j$ & & & 1,01 & & & 2715.96 \\
\hline $\operatorname{ARR} 4$ & & & 1.39 & & & 5734,90 \\
\hline PEW ORIELiE & & & 2.41 & 2.16 & & \\
\hline & & & & B. 36 & & \\
\hline
\end{tabular}




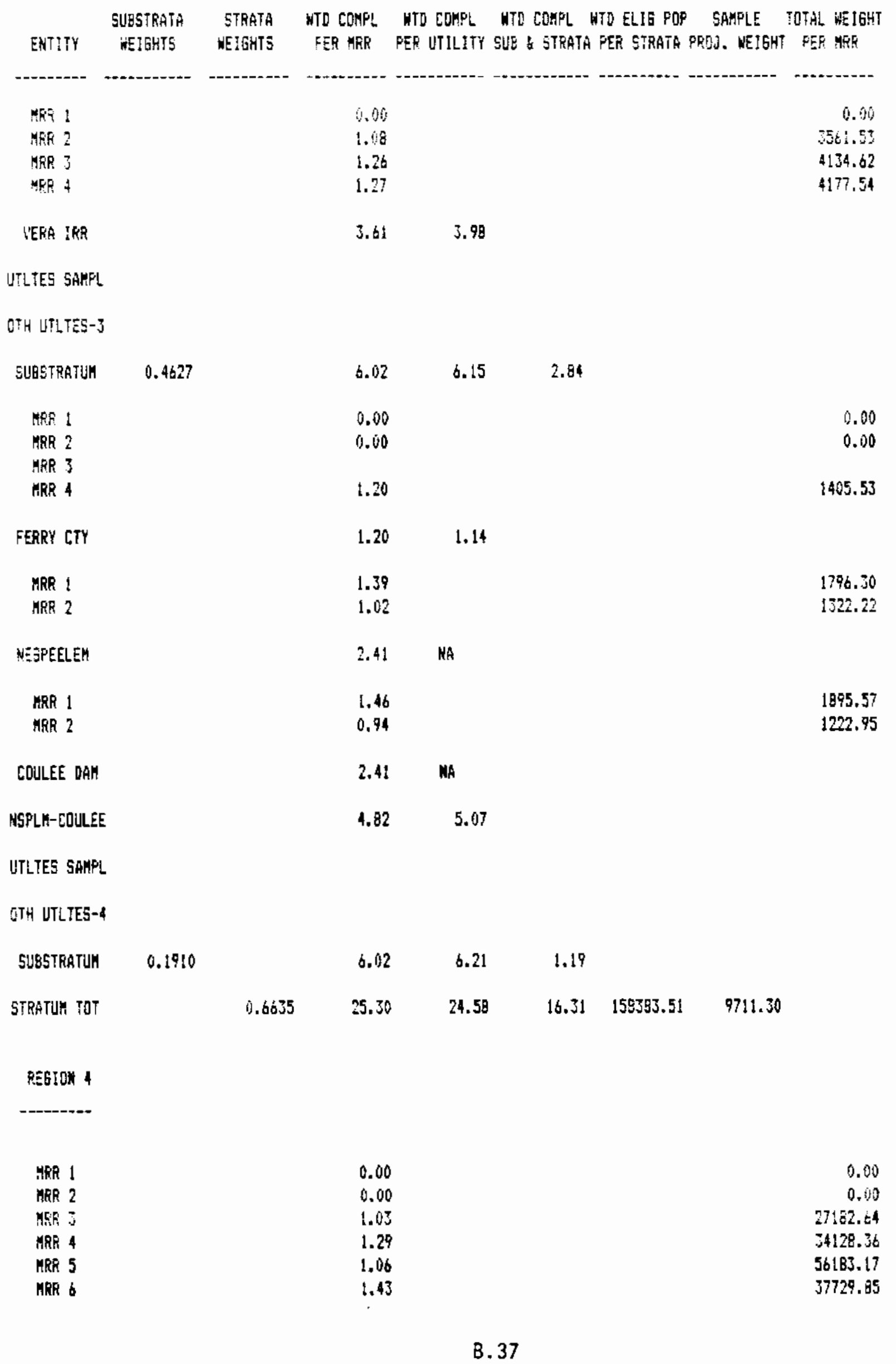




\begin{tabular}{|c|c|c|c|c|c|c|}
\hline ENTITY & $\begin{array}{l}\text { ENBSTRATA } \\
\text { NEIGHTS }\end{array}$ & $\begin{array}{l}\text { STRATA } \\
\text { WEIGHTS }\end{array}$ & $\begin{array}{l}\text { WTI COMPL } \\
\text { PER APR }\end{array}$ & $\begin{array}{l}\text { WTD CEMPL } \\
\text { FES UTILITY }\end{array}$ & $\begin{array}{l}\text { YTD COAFL WTO ELIG POP SAMFLE T } \\
\text { SIJE \& STEGTS PER STRATA EROJ. WEIGHT }\end{array}$ & $\begin{array}{l}\text { TCTAL WEIGHT } \\
\text { PEE HRP }\end{array}$ \\
\hline BEATTEE & +7 & & 4,32 & 6.7 & & \\
\hline 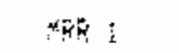 & & & 0.56 & & & $15: 72, E 2$ \\
\hline AfF 2 & & & 1.32 & & & 35716.67 \\
\hline परR 3 & & & 0.60 & & & 0.00 \\
\hline$M R R$ & & & 1.51 & & & 20506.77 \\
\hline MFR 5 & & & 0.00 & & & 0.00 \\
\hline AARR 6 & & & 1.43 & & & 38721.38 \\
\hline SHOHŌMISH & & & 4.82 & 3.27 & & \\
\hline \multicolumn{7}{|l|}{$\begin{array}{l}\text { JTLTES SAMPL } \\
\text { STH UTLTES- }\end{array}$} \\
\hline SUESTRATUM & 3.2947 & & 9.64 & 9.64 & 31.75 & \\
\hline MRR 1 & & & 0.95 & & & 7241.61 \\
\hline MRE 2 & & & 1.27 & & & 19312.30 \\
\hline FRR J & & & 1.35 & & & 20446.60 \\
\hline 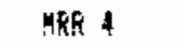 & & & 0.57 & & & 8645.03 \\
\hline rkR 5 & & & 1.89 & & & 28558.57 \\
\hline MER 6 & & & 0.00 & & & 0.00 \\
\hline DLAFE ETY & & & 6.02 & 5.14 & & \\
\hline 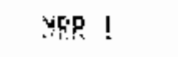 & & & 8.42 & & & 2BOEO. 12 \\
\hline HRE 2 & & & $\{.13$ & & & 23203.90 \\
\hline MRR 3 & & & 1.13 & & & 23047.12 \\
\hline MRR 4 & & & 1.30 & & & 26499.35 \\
\hline $\sin 5$ & & & 1.05 & & & 21424.53 \\
\hline$S R A$ & & & 0.00 & & & 0.00 \\
\hline IACOMA & & & 6.02 & $6.9 !$ & & \\
\hline
\end{tabular}

JTLTES SAMP:

TT! UTLETES-2

ELESTRATUM

1.4700

12.05

$12.05 \quad 17.71$

MS !

Hen 2

Hit? 3

NAR 4

TRP 5

整 $b$

9.00

1.38

0.83

2.02

0.67

1.14

1..00

4170.45

2509.16

12224.13

322.04

3452.75

\$ASรM LTY-3

$6.02 \quad 6.27$

YFR 1

0.90

HFF 2

1.52

NER 3

0.00

ARF 4

0.78

MER 5

0.79

$\dot{0} \cdot 2$

5350.07

0. 60

4382.92

4359.21 


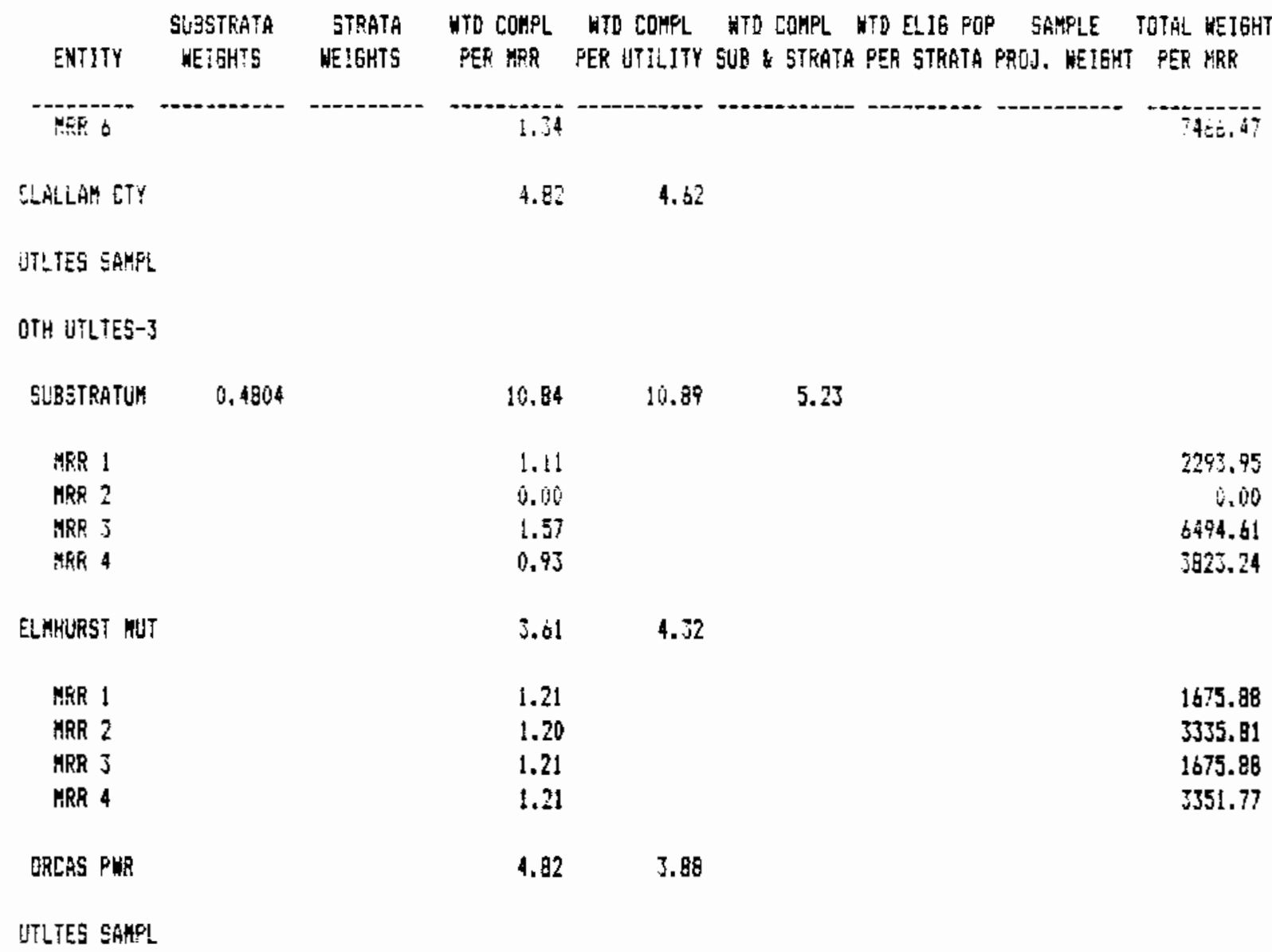

BTH UTLTES-3

\begin{tabular}{|c|c|c|c|c|}
\hline SUBSTRATUN & 0.2847 & 8.43 & 8.20 & 2.33 \\
\hline MRR $!$ & & 0.65 & & 330.93 \\
\hline MRR 2 & & 1.43 & & 722.03 \\
\hline ARR 3 & & 1.45 & & 734.37 \\
\hline MRR 4 & & $\$ .29$ & & 647.98 \\
\hline PARKLAHE LLP & & 4.82 & 4.28 & \\
\hline MRR！ & & 1.31 & & 1656.51 \\
\hline MRR 2 & & 0.95 & & 500.54 \\
\hline MRA 3 & & 1.40 & & 1780.42 \\
\hline MRK 4 & & 1.16 & & 1469.56 \\
\hline SKAHANIA & & 4.82 & 5.36 & \\
\hline
\end{tabular}

DTH UTLTES-2

SUESTRATIN $\quad 0.0940 \quad 9.64 \quad 9.640 .91$

YRR 1

$2.24 \quad 1107.34$

RRR 2

1530.83

HRA 3

0.32

405.71

\section{B. 39}




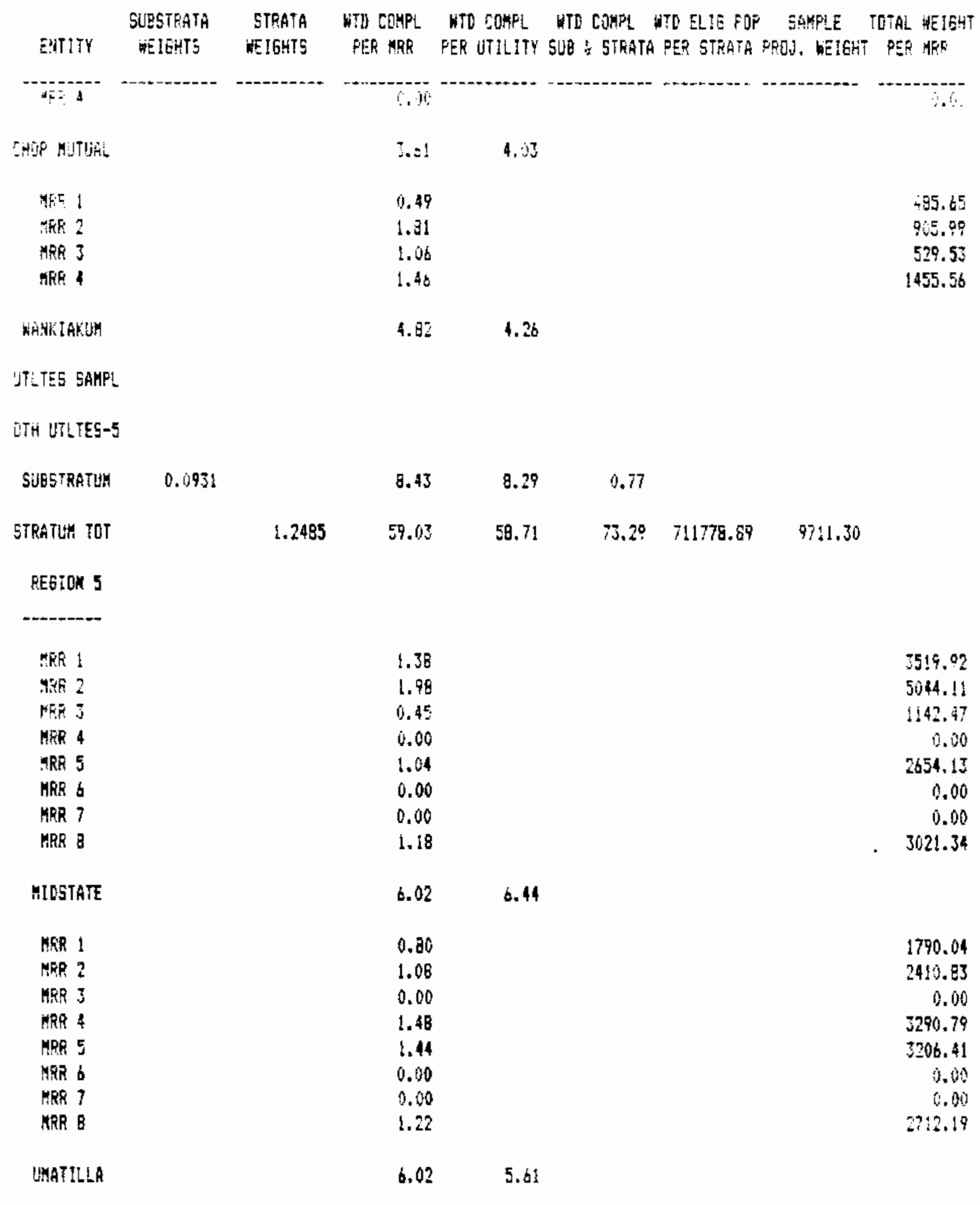

UTLIEE EAFPL

OTH UFLTES-2

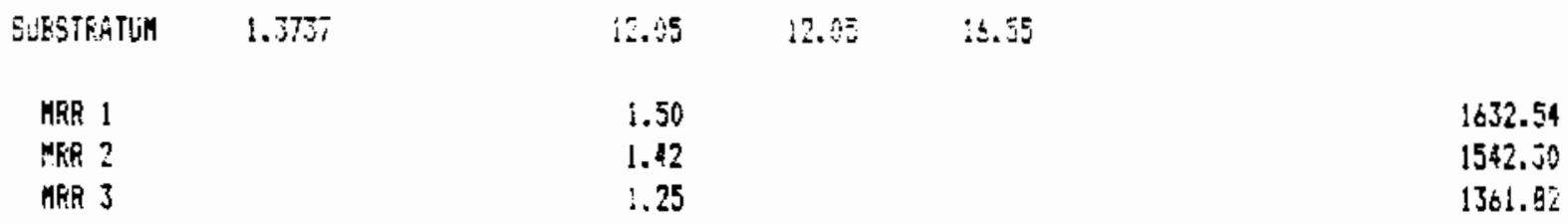




\begin{tabular}{|c|c|c|c|c|c|c|c|}
\hline ENTITY & $\begin{array}{l}\text { SVIESTRATH } \\
\text { WEIEHTS }\end{array}$ & $\begin{array}{l}\text { STRATA } \\
\text { WETGHTS }\end{array}$ & $\begin{array}{l}\text { NTT COHPL } \\
\text { FER MRR }\end{array}$ & $\begin{array}{l}\text { NTD COHPL } \\
\text { PER UTILITY }\end{array}$ & $\begin{array}{l}\text { WTO COAPL } \\
\text { SUB \& STRATA }\end{array}$ & $\begin{array}{l}\text { WTD ELIG POP SAMPLE } \\
\text { PEE STRATA PROJ. WEIGHT }\end{array}$ & $\begin{array}{l}\text { TDTAL WEIGKT } \\
\text { T FES MER }\end{array}$ \\
\hline Net & - & - & 30 & -...-- & n.s. & . & (1) \\
\hline WE 5 & & & 0.44 & & & & 1509.64 \\
\hline HER & & & 1). 10 & & & & 4.07 \\
\hline WRR 7 & & & 0.69 & & & & 745.44 \\
\hline MRR 8 & & & 0.72 & & & & 194.68 \\
\hline COLUMAIA & & & 7.23 & 6.86 & & & \\
\hline KRRR I & & & 0.90 & & & & 1002.51 \\
\hline MRR 2 & & & 1.14 & & & & 1377.57 \\
\hline MFE I & & & 0.00 & & & & 3,00 \\
\hline MRP 4 & & & 0.00 & & & & 0.00 \\
\hline MRR 5 & & & 1.71 & & & & 2059.91 \\
\hline Mif 6 & & & 1.22 & & & & 1471.37 \\
\hline MfR? & & & 1.05 & & & & 1267.64 \\
\hline MRR 8 & & & 0.00 & & & & 10.00 \\
\hline FLTH-FREENTR & & & b. $\dot{1} 2$ & 6.33 & & & \\
\hline \multicolumn{8}{|l|}{ UTLJES SABPL } \\
\hline \multicolumn{8}{|l|}{ OTH SAMPL -5} \\
\hline SUISTRATJH & 0.6587 & & 13.25 & 13.19 & E. 59 & & \\
\hline STRATA taT & & 0.1792 & 25.30 & 25.24 & 4.52 & 43907.67 & \\
\hline \multicolumn{8}{|l|}{ REGIQN } \\
\hline \multicolumn{8}{|l|}{ - } \\
\hline MRA I & & & 1.22 & & & & $4627.7 !$ \\
\hline ARR 2 & & & 1.39 & & & & Lิด89.21 \\
\hline $\operatorname{MRR} 3$ & & & 1.21 & & & & 9185.82 \\
\hline ARR 4 & & & 1.24 & & & & 9411.66 \\
\hline MRR 5 & & & 1.12 & & & & 8409,10 \\
\hline AFR 6 & & & 1.96 & & & & 4048.49 \\
\hline SUGEME WTR & & & 7.23 & 10.65 & & & \\
\hline YRR 1 & & & 1.34 & & & & 3628.14 \\
\hline \multirow{2}{*}{\multicolumn{8}{|c|}{ ARR 2}} \\
\hline \multicolumn{5}{|l|}{ ARR 3} & & & \\
\hline ARR 4 & & & $0.7 t$ & & & & 970.76 \\
\hline HRR 5 & & & 0.00 & & & & 0.00 \\
\hline MRR 6 & & & 1.56 & & & & 4251.73 \\
\hline CNTRL-LMCLN & & & 3.61 & 1.90 & & & \\
\hline \multicolumn{8}{|l|}{$\begin{array}{l}\text { WTLTES SAMPL } \\
\text { OTH WTLIES-O }\end{array}$} \\
\hline SU8STRATUM & 1.1100 & & 10.84 & 12.56 & 13.94 & & \\
\hline RRR I & & & 1.13 & & & & 8538.32 \\
\hline
\end{tabular}




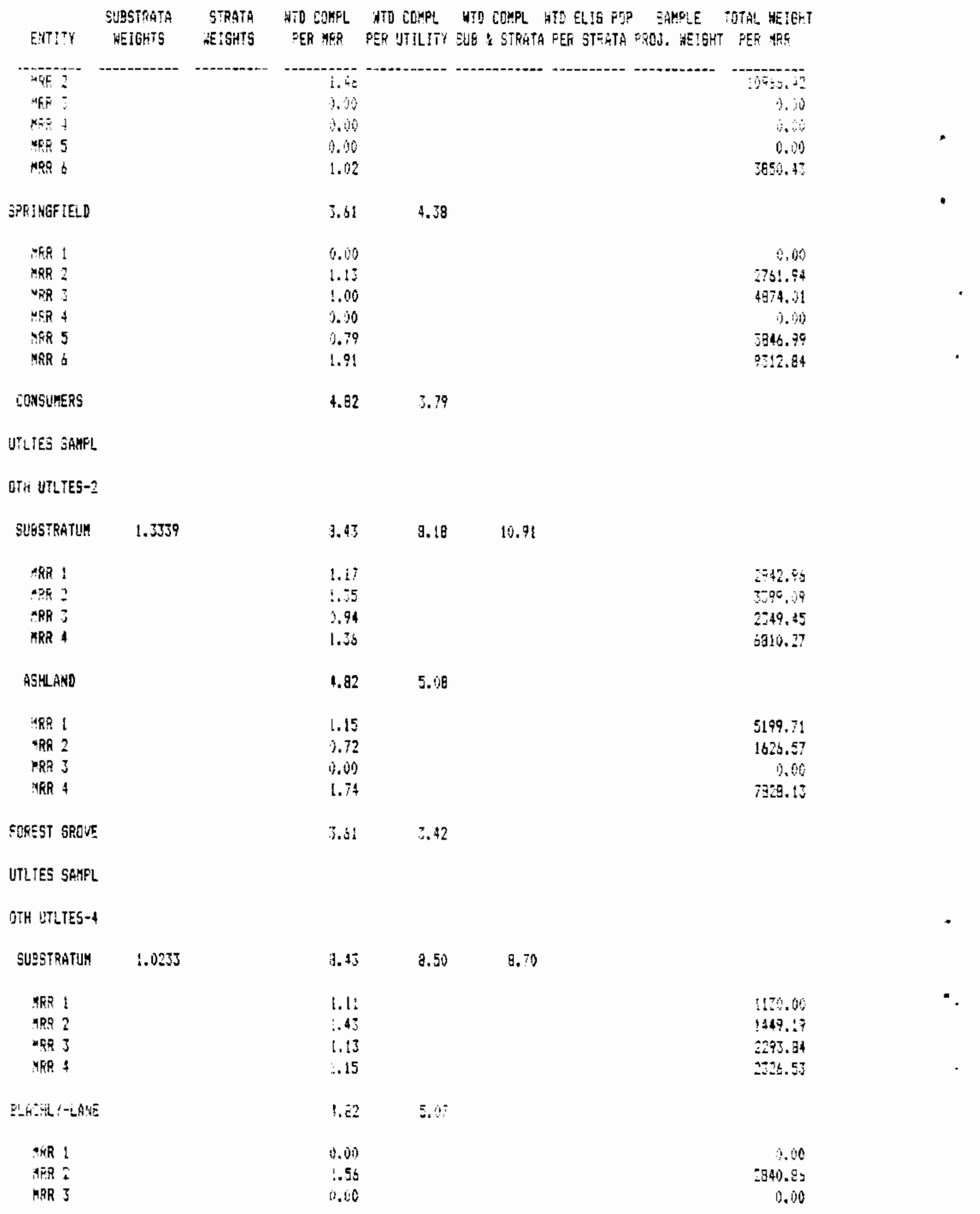




\begin{tabular}{|c|c|c|c|c|c|c|c|c|}
\hline ENTITY & $\begin{array}{l}\text { EUESTKATA } \\
\text { MEIEHTS }\end{array}$ & $\begin{array}{l}\text { STRATA } \\
\text { WEISHTS }\end{array}$ & $\begin{array}{l}\text { HTO CGMFL } \\
\text { PER MAR }\end{array}$ & $\begin{array}{l}\text { WTD COMPL } \\
\text { FER UTILIF! }\end{array}$ & $\begin{array}{l}\text { WTD CONPL } \\
\text { SUE \& ETFATA }\end{array}$ & $\begin{array}{l}\text { HTE ELIG FIP } \\
\text { AER STBATA }\end{array}$ & $\begin{array}{l}\text { P SAMPLE } \\
\text { PRO:. WEIGHT }\end{array}$ & $\begin{array}{l}\text { TETAL WEIGHT } \\
\text { T FER MRK }\end{array}$ \\
\hline 45: & 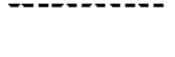 & 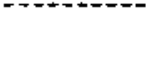 & j, & & & & 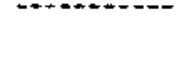 & 79.76 \\
\hline 40;400JTh & & & 2.41 & 2.25 & & & & \\
\hline \multicolumn{9}{|l|}{ UTLTES EAMPL } \\
\hline \multicolumn{9}{|l|}{ OTH UTLTES-4 } \\
\hline SIJESTRATUK & 0.4141 & & 7.23 & 7.36 & 3.05 & & & \\
\hline STRATUH TOT & & 0.4792 & 34.94 & 30.59 & 17.53 & 176274.15 & 971.30 & \\
\hline PUELIC TOT & & 1.00 & 183.12 & 182.37 & 121.83 & 1153075.68 & 9711.30 & \\
\hline \multicolumn{9}{|l|}{ FEIVATE } \\
\hline \multicolumn{9}{|l|}{ FEGION I } \\
\hline \multicolumn{9}{|l|}{ - - - } \\
\hline HRR 1 & & & 0.35 & & & & & 1212.19 \\
\hline MRR 2 & & & 0.00 & & & & & 0.00 \\
\hline MRR 3 & & & 0.55 & & & & & 1880.17 \\
\hline MER 4 & & & 0.00 & & & & & 0.00 \\
\hline MEP 5 & & & 0.24 & & & & & 534.91 \\
\hline HER & & & 0.00 & & & & & $0, n$ \\
\hline KRR 7 & & & 2.42 & & & & & 8302.69 \\
\hline MRR 8 & & & 1.97 & & & & & 6741.78 \\
\hline MRA 9 & & & 1.99 & & & & & 6824.81 \\
\hline MRR 10 & & & 2.26 & & & & - & 7749.59 \\
\hline MRR II & & & 1.55 & & & & & 5321.47 \\
\hline MRR 12 & & & 0.00 & & & & & 0.00 \\
\hline MRR 13 & & & 0.59 & & & & & 2036.04 \\
\hline ARR 14 & & & 0.11 & & & & & 361.25 \\
\hline ARR 15 & & & 0.00 & & & & & 0.00 \\
\hline MRR 16 & & & 0.00 & & & & & 0.00 \\
\hline mONTANA PMR & & & 12.05 & 12.05 & & & & \\
\hline SUBSSTRATUM & 1.00 & & & & & & & \\
\hline
\end{tabular}

MRR 1

MRR 2

HRP?

MRR 4

MRE 5

HR 6

MEF ?

MER 9

H H 9

MRR 10

HaR 11
1.18

1.56

0.00

0.00

0.00

0.76

0.00

0.00

1.12

0.00

0.00
4053.51

5373.88

0.00

0.00

0.00

2604.79

0.00

0.00

3845.59

0.30

0.00 


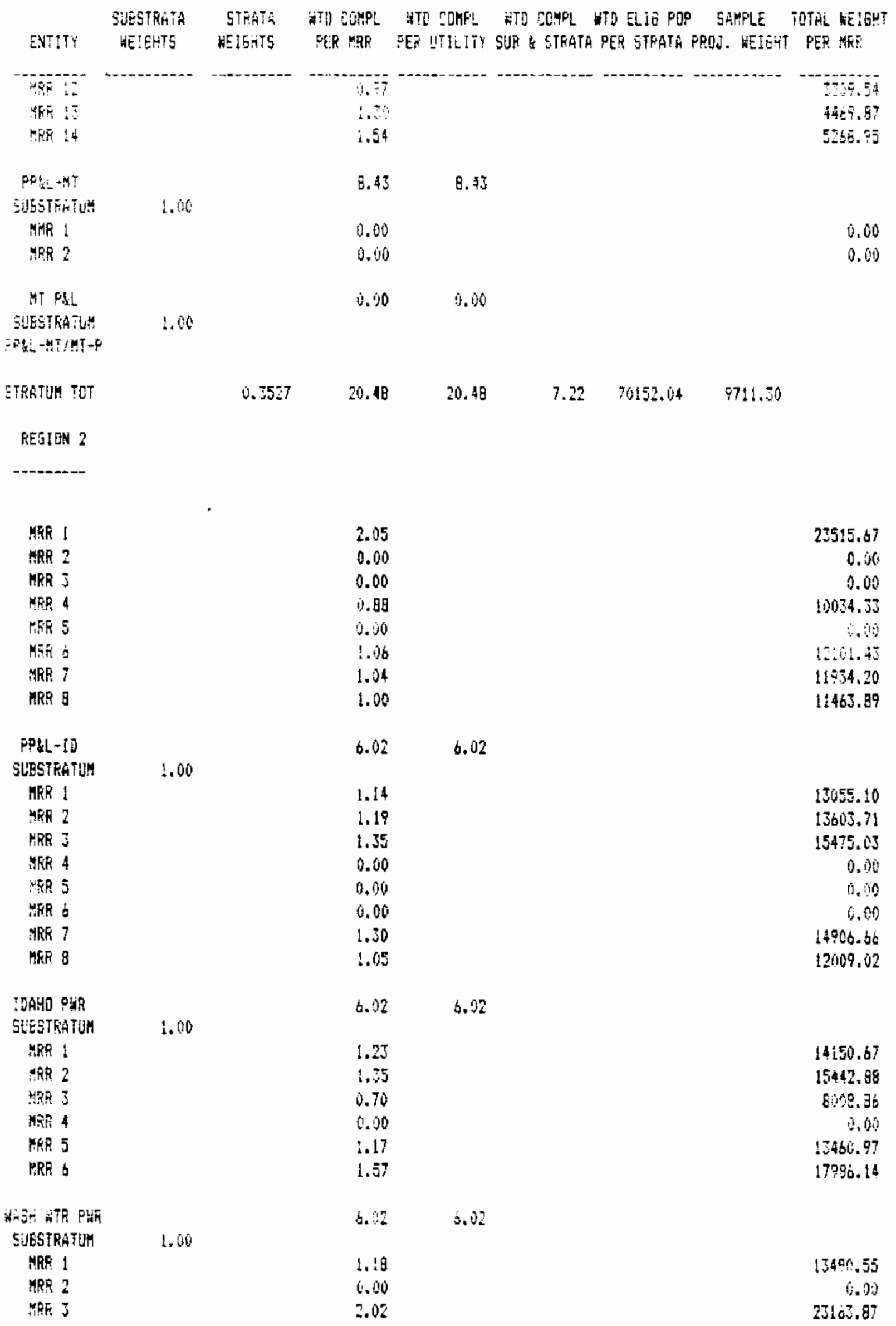




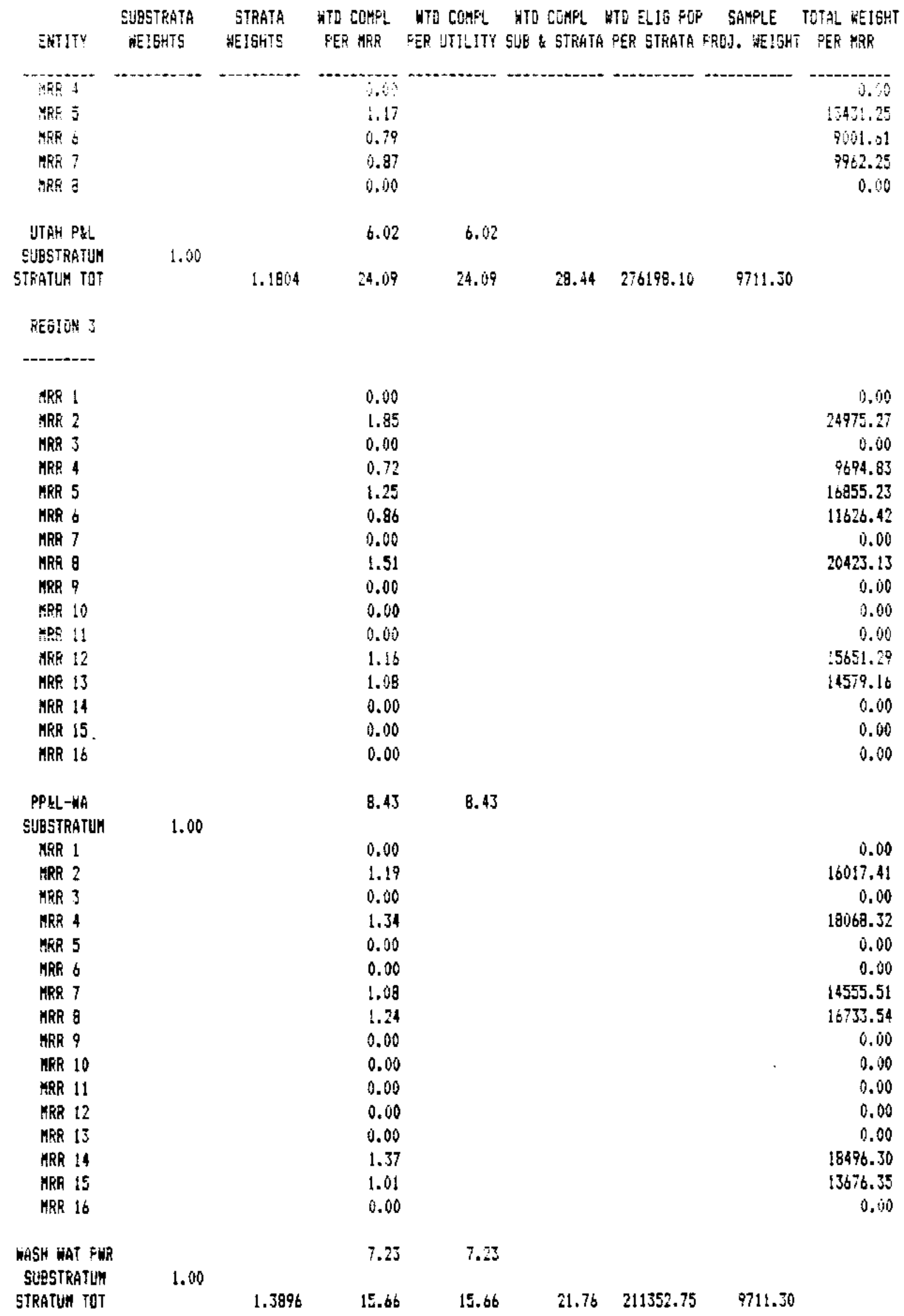




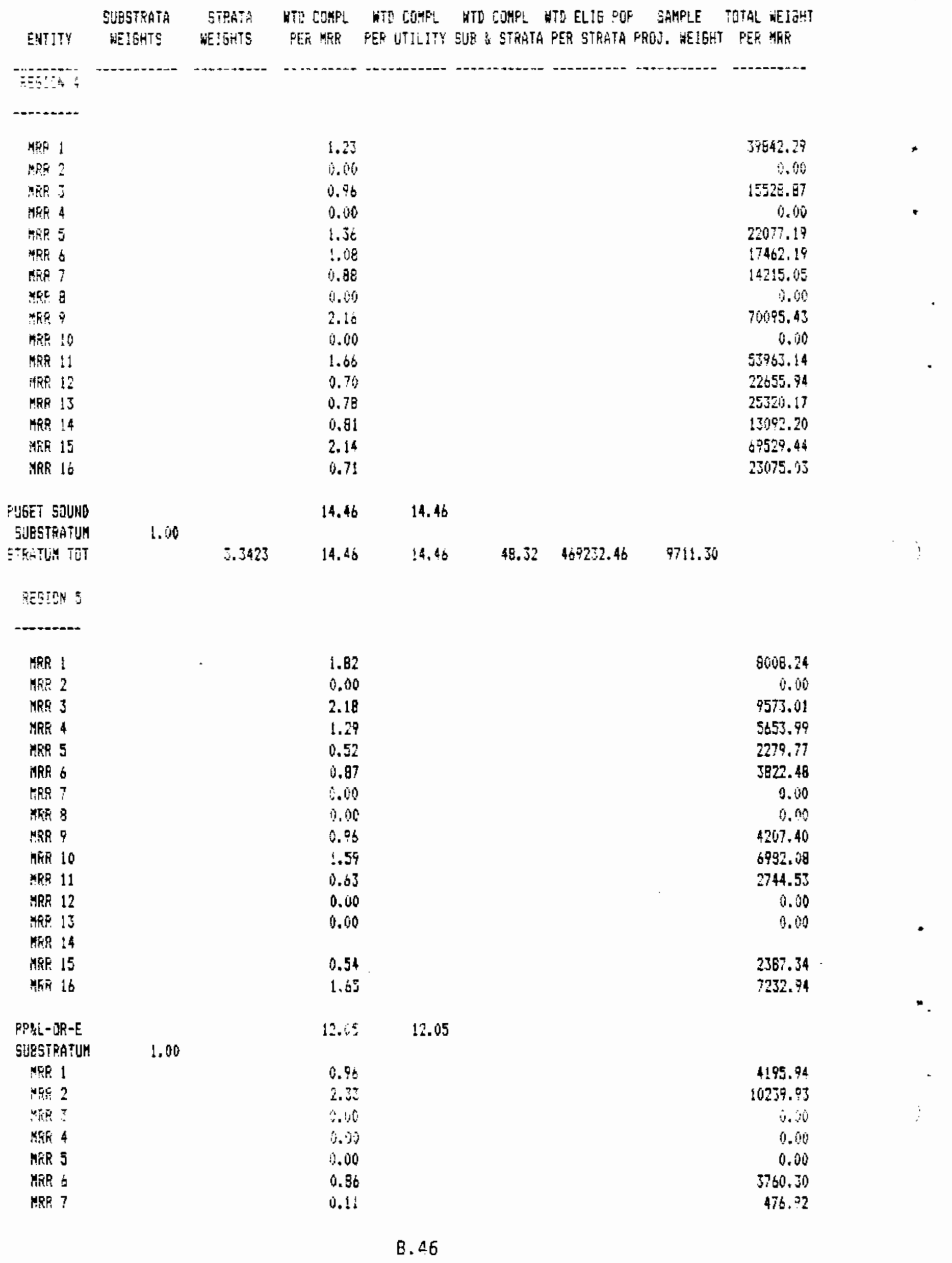




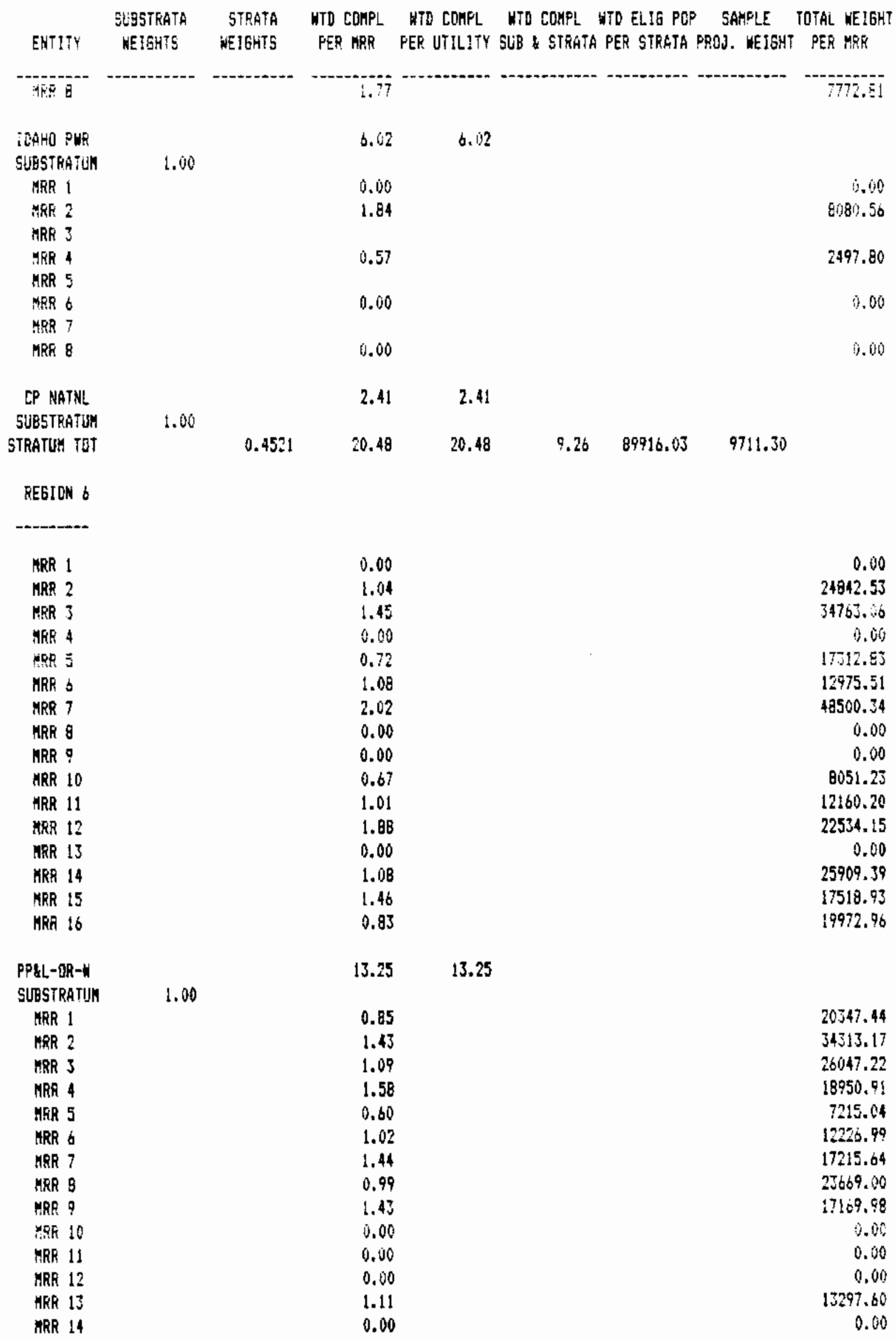




\begin{tabular}{|c|c|c|c|c|c|c|c|c|}
\hline ENTITY & $\begin{array}{c}\text { SURSTRATA } \\
\text { HEIBHTS }\end{array}$ & $\begin{array}{l}\text { STRATA } \\
\text { WEIGHIS }\end{array}$ & 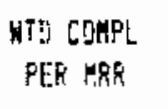 & $\begin{array}{l}\text { WTO CDMFL } \\
\text { PER UTLILITY }\end{array}$ & $\begin{array}{l}\text { ATO COMPL } \\
\text { SUE: STKATA }\end{array}$ & $\begin{array}{l}\text { NTE ELIG POP } \\
\text { PER STFATA }\end{array}$ & $\begin{array}{c}\text { SAMELE } \\
\text { PHOS. HE!GHT }\end{array}$ & $\begin{array}{l}\text { TOTAL WE1GHT } \\
\text { T PER MER }\end{array}$ \\
\hline 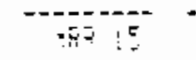 & --- & .... & 17 & --- & & & & 3070,4 \\
\hline 10 & & & 1.55 & & & & & $165 \theta^{2}=5 \overline{5}$ \\
\hline CWT GES ELE & & & 14.46 & 14.46 & & & & \\
\hline EJESTFATH & 1. & & & & & & & \\
\hline ETridum & & 2.4683 & 9.71 & $77.7 !$ & 58.42 & 664451.47 & 9711.30 & \\
\hline ERTVATE TO? & & 1.0000 & 122.50 & 122.68 & 19.5 .43 & 1781302.85 & 9711.30 & \\
\hline GEAAL IDT & & $1.00001)$ & 30.00 & 315.25 & 305.25 & 2764382.32 & 9714.30 & \\
\hline
\end{tabular}




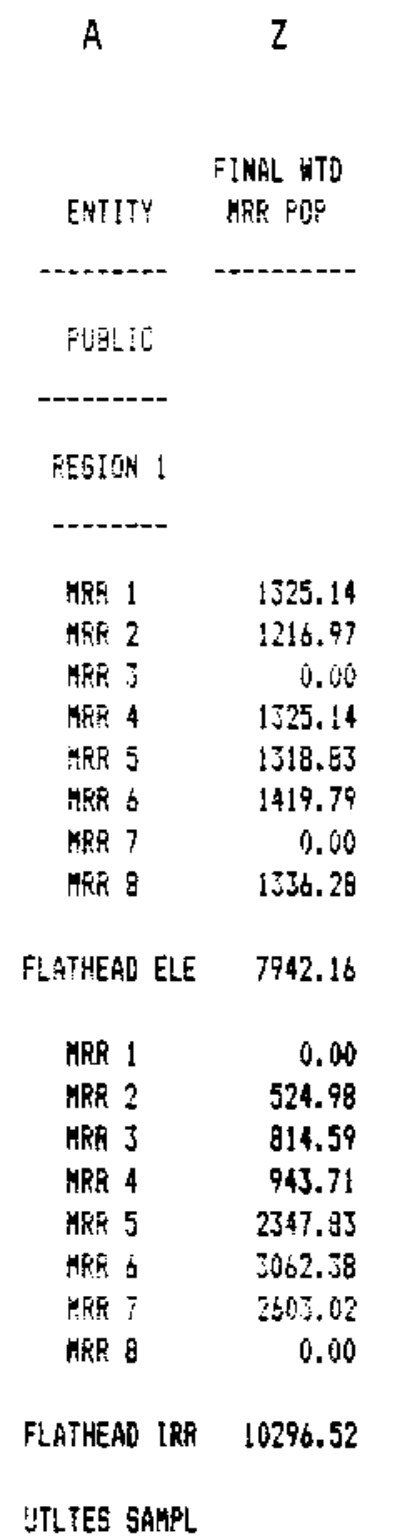

OTH UTLTES-1

SUBSTRATUM $\quad 18238.67$

$\begin{array}{ll}\text { MRR } 1 & 0.00\end{array}$

MRR $2 \quad 0.00$

NIRA $3 \quad 2982.28$

MTh $4 \quad 0.00$

MRR $5 \quad 2161.51$

Map 6

HRR 7

MRR 8

GLACIER $\quad 5143.79$

RRP $1 \quad 2066.59$

$\begin{array}{ll}\text { MKR } 2 & 1650.31\end{array}$

HR $3 \quad 2281.25$

WRR $4 \quad 0.09$

MRR $5 \quad 1362.86$ 


\begin{tabular}{|c|c|}
\hline EHTITY & $\begin{array}{c}\text { FINAL WTD } \\
\text { MRP POF }\end{array}$ \\
\hline - & $\cdots$ \\
\hline BXIMLLI & 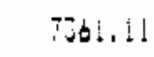 \\
\hline TLTES SAMFL & \\
\hline DTH UTLIE-Z & \\
\hline SUESTRATLIY & 12504.90 \\
\hline grदitun tot & 31743.58 \\
\hline KEGION 2 & \\
\hline -..--.-- & \\
\hline Me 1 & 0.90 \\
\hline Hef 2 & 0.00 \\
\hline MPR 3 & 0.50 \\
\hline ARR 4 & $2111: .48$ \\
\hline MRR 5 & 0.00 \\
\hline MRR 6 & 0.00 \\
\hline INAHO FALLS & 21111.48 \\
\hline RR: & [11/45.91 \\
\hline MRP : & 11262.51 \\
\hline infa 3 & 0.00 \\
\hline ARR 4 & 0.00 \\
\hline MRR 5 & 0.00 \\
\hline FRR 6 & 0.60 \\
\hline
\end{tabular}

LGWER YALLEY 22328.32

UTLTES SAMPL

DTH UTLTES-3

SLIBSTRATUA 43439.80

H65 1

称 2

MER $3 \quad 0.00$

STR 4 D. 0

ARO 5 989.96

称 60

FGLL RIVER $\quad 4755.67$

MPF $1 \quad 2817.92$

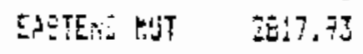

AFS: $\quad 2817.95$

FAFHEOS ELE 2917.93 


\begin{tabular}{|c|c|}
\hline EHTITY & $\begin{array}{l}\text { FINAL WTO } \\
\text { MRR POPP }\end{array}$ \\
\hline 相倩! & 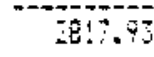 \\
\hline RItUERSIOE SL & 2317.73 \\
\hline MRE I & 2517.93 \\
\hline SOUTHSIDE EL & 2917.93 \\
\hline MRP 1 & 0.10 \\
\hline MEP 2 & 0.00 \\
\hline JNITY LAP & 0.00 \\
\hline BINOOK'A PROJ & $1: 271.71$ \\
\hline UTLTES SAMPL & \\
\hline OTH UFLTES-4 & \\
\hline SLLSTRATUH & 16027.58 \\
\hline ARR ! & 1600.55 \\
\hline MRR 2 & 0.00 \\
\hline MRR 3 & 0.00 \\
\hline$M R R A$ & 1615.49 \\
\hline MER 5 & 1615.49 \\
\hline Mrg b & 1355.79 \\
\hline LOST RIVER & 6195.31 \\
\hline MRR I & \\
\hline GRR 2 & 2329.19 \\
\hline MRR 3 & 0.00 \\
\hline MRR 4 & 10.10 \\
\hline MRR 5 & 0.00 \\
\hline MRR 6 & 0.00 \\
\hline HEISER & 2329.19 \\
\hline UTLTES SAAPL & \\
\hline QTH UTLTES- & \\
\hline SUBSTRATUH & 8524.50 \\
\hline STRATLH TOT & 67991.88 \\
\hline REEION 3 & \\
\hline - & \\
\hline KR? 1 & $\dot{0} .30$ \\
\hline HRR 2 & 0.00 \\
\hline$M R R$ & 0.00 \\
\hline haR 4 & 14520.86 \\
\hline ARR 5 & 0.60 \\
\hline
\end{tabular}

B. 51 


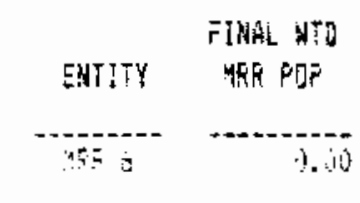

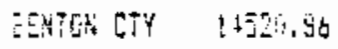
HQE! MORT.15

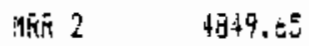
MRF $3 \quad 15736.45$
, the 420033.15
S.9R $5 \quad 0.00$

IHELAN CTH 50956.40

TRR I 12714.10

CAEHAERE 12714.10

CHE.H-ESHMRE SOS70.50

UTLIES SAMPLL

OTH UTLTES-2

FUPSTRATU: 78:51.57

S4 $1 \quad 270.27$

Af 2 30:0.37

MFR $3 \quad 0.00$

ATR $+\quad 21282.94$

KKR 5 11776.45

Min:

FRAMKLIN 39595. IJ

NRR ! $\quad 0.00$

积 $2 \quad 0.00$

MPE $3 \quad 0.00$

MER $4 \quad 14729.31$

STRR 5

KRR \& 0.60

FICHLAND 14729.31

UTLTEE SAMPL

DTY URLTES̈-5

SUBSTRATIHA 5 \$324.04

MRA : B.SB

$\operatorname{tin} 20$

जe? $32 ? 16.96$

FEP : 3734,90

PEVO DHIELLE SA5I.35 


\begin{tabular}{|c|c|}
\hline ENTITY & $\begin{array}{l}\text { FINAL WTD } \\
\text { MFR PIVP }\end{array}$ \\
\hline - & 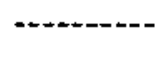 \\
\hline MFF 1 & $0 ., 00$ \\
\hline $\mathrm{YFR} 2$ & $356 i .55$ \\
\hline MES 3 & 4134.52 \\
\hline$M R P$ & 4177.54 \\
\hline VERA IER & 11873.68 \\
\hline UTLTES SAKFL & \\
\hline QTH UTLTES-3 & \\
\hline SLESTRATUA & 18325.54 \\
\hline MRE 1 & 0.00 \\
\hline MRR 2 & 0.00 \\
\hline MRR $\mathrm{j}$ & \\
\hline WRR 4 & 1405.53 \\
\hline FERRY CTY & 1405.53 \\
\hline MRR ! & 1796.30 \\
\hline HRR 2 & 1322.22 \\
\hline NESPEEIEM & 3118.52 \\
\hline MRR I & 1895.57 \\
\hline MRR 2 & 1222.95 \\
\hline COULEE BAH & 3118.52 \\
\hline HSPLH-COULEE & 6237.04 \\
\hline UTLTES SAMPL & \\
\hline UTH UTLTES-4 & \\
\hline SUBSTRATUH & 7642.57 \\
\hline STRATUM TDT & 159383.51 \\
\hline REGION 4 & \\
\hline HRR 1 & 0.00 \\
\hline MRP 2 & 0.00 \\
\hline$M$ & 54365.28 \\
\hline ARA 4 & 68256.72 \\
\hline 5 & 56183.17 \\
\hline MRP o & 75459.69 \\
\hline
\end{tabular}




\begin{tabular}{|c|c|}
\hline ENTITY & $\begin{array}{l}\text { FINAL HTO } \\
\text { MRE PGE }\end{array}$ \\
\hline 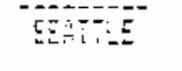 & 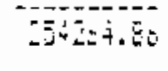 \\
\hline NG 1 & 15273.32 \\
\hline 称贶 2 & 35766.97 \\
\hline NFE & 6.00 \\
\hline M & $40: 5.57$ \\
\hline $\operatorname{mes} 5$ & 600 \\
\hline MES & 88721.38 \\
\hline EGHOHISH & .30724 .40 \\
\hline $\begin{array}{l}\text { ITLTES SAMFL } \\
\text { SL UTLTES-O }\end{array}$ & \\
\hline SDETPATHK & 384957.26 \\
\hline MF 1 & $14493.2 \mathrm{~J}$ \\
\hline MP? & 19712.30 \\
\hline ME & 20446.68 \\
\hline$P R R 4$ & $864 \overline{0} .03$ \\
\hline MAR 5 & $28659.5 ?$ \\
\hline$M E R \quad 6$ & 0.00 \\
\hline EAFT OTH & 91545.81 \\
\hline Wh 1 & 80986.12 \\
\hline MRE 2 & 25203.90 \\
\hline MFR J & 23047.12 \\
\hline ARP 4 & $26499 . \mathrm{J5}$ \\
\hline Mhe 5 & 21424.53 \\
\hline$M R R \quad b$ & 0.00 \\
\hline TACONA & 123161.02 \\
\hline WTLTES SAYYPL & \\
\hline DTH WTLTES-2 & \\
\hline SLESTRATUM & 214706.83 \\
\hline YRR I & 0.10 \\
\hline YRR 2 & 8340.90 \\
\hline MRR 3 & 5018.33 \\
\hline NAR 4 & 12224.13 \\
\hline HER 5 & 4044.07 \\
\hline ARR 6 & 5905.58 \\
\hline MASDA CTY-3 & 36532.94 \\
\hline Nhe 1 & $\dot{3} .50$ \\
\hline Whe 2 & 10701.94 \\
\hline AER 3 & 0.09 \\
\hline NRE 4 & $430 \% .72$ \\
\hline MSR 5 & 4359.21 \\
\hline
\end{tabular}




\begin{tabular}{|c|c|}
\hline ENTITY & $\begin{array}{l}\text { FINAL HTD } \\
\text { MRE FISP }\end{array}$ \\
\hline$* 45$ & 766.47 \\
\hline CLALLAAH CTY & 26910.54 \\
\hline JULTES SAMPL & \\
\hline OTH UTLTES-J & \\
\hline SUBCTRATUH & 63443.49 \\
\hline KES 1 & 4587.89 \\
\hline MrR 2 & 0.60 \\
\hline MER 3 & 6494.61 \\
\hline MRR 4 & 3823.24 \\
\hline ELMHURST MUT & 14905.74 \\
\hline MRR I & 3351.77 \\
\hline MRR 2 & 3335.81 \\
\hline MRR 3 & 3351.77 \\
\hline MRR 4 & 3551.77 \\
\hline ORCAS PHR & 13391.11 \\
\hline UTLTES SAMPL & \\
\hline OTH UTLTES-3 & \\
\hline SUESTRATUK & 28296.85 \\
\hline 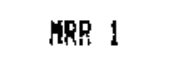 & 661.86 \\
\hline MRR 2 & 1444.06 \\
\hline MFR 3 & 1468.75 \\
\hline NRR A & 1295.95 \\
\hline PAEKKLANOLLP & 4670.63 \\
\hline MRR ! & 1656.51 \\
\hline MR 2 & 1201.08 \\
\hline MRR 3 & 1780.42 \\
\hline ASG 4 & 1469.56 \\
\hline SKAHANIA & 6107.57 \\
\hline UTLTES SAMFL & \\
\hline OTHH UTLTES-2 & \\
\hline SUESTRATH & 10979.20 \\
\hline MRE 1 & 2815.47 \\
\hline MRR 2 & [330.93 \\
\hline MRR 3 & 405.71 \\
\hline
\end{tabular}

B. 55 


\begin{tabular}{|c|c|}
\hline ENITY & $\begin{array}{l}\text { FINAL WTO } \\
\text { WRR POP }\end{array}$ \\
\hline Whe 4 & 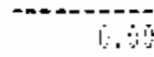 \\
\hline ERLP MSIDSTL & 4552.63 \\
\hline MER ! & 425.65 \\
\hline MKS 2 & 1911.97 \\
\hline AFE 3 & 1059.07 \\
\hline MFF 4 & $\$ 455.56$ \\
\hline
\end{tabular}

WAFKIAKUM $45: 2.24$

WTLEE SAMPL

GTH LTLTES-S

SUBSTEATUM 9364.26

ETRATUH TET 711778.99

REGION 5

\begin{tabular}{|c|c|}
\hline MER ! & $35 ! 9.92$ \\
\hline AEE 2 & 544.11 \\
\hline 4 & 1142.17 \\
\hline MPR 4 & 0.00 \\
\hline NRR 5 & $2654.1 j$ \\
\hline MRR 6 & 0.00 \\
\hline ARP $T$ & 0.00 \\
\hline AR 8 & 3022.34 \\
\hline MIOSTATE & 15381.97 \\
\hline$M \& R !$ & 1790.04 \\
\hline ARR 2 & 2410.95 \\
\hline Hex 3 & 0.00 \\
\hline MVT & 3290.79 \\
\hline HRR 5 & 3206.41 \\
\hline IAR 6 & 0.00 \\
\hline MRR 7 & 0.00 \\
\hline MPR a & 2712.19 \\
\hline JNGTILLA & 17410.26 \\
\hline ILTES SAYPL & \\
\hline TH !ITLIES-? & \\
\hline BLBSTRATLH & 29792.33 \\
\hline MR ! & $1 \mathrm{j} 32.54$ \\
\hline MRE 2 & 1542.30 \\
\hline ATR 3 & 1361.82 \\
\hline
\end{tabular}

B. 56 


\begin{tabular}{|c|c|}
\hline ENTTTY & $\begin{array}{l}\text { FINAL HTO } \\
\text { MER FUF }\end{array}$ \\
\hline$M 4=4$ & 6.0 \\
\hline IRf 5 & 1565.64 \\
\hline MRR D & 0.00 \\
\hline MRR 7 & 745.44 \\
\hline$Y K R$ & 1004.68 \\
\hline COLUABEIA & $7956.4 \mathrm{~J}$ \\
\hline AER ! & 1082.51 \\
\hline MRE 2 & 1377.59 \\
\hline M裸 3 & 0.010 \\
\hline MFR 4 & 0.00 \\
\hline MRR 5 & 2059.91 \\
\hline MFR 6 & 1471.37 \\
\hline ARR 7 & 1267.64 \\
\hline$M R R$ & 0.00 \\
\hline MTN-FREEATR & 7259.01 \\
\hline \multicolumn{2}{|l|}{ UTLTES SAKFL } \\
\hline \multicolumn{2}{|l|}{ DFH SAMPL -5} \\
\hline SUBSTRATUM & 15115.44 \\
\hline STRATA TOT & 43907.67 \\
\hline \multicolumn{2}{|l|}{ REGIGN 6} \\
\hline ARR I & 9255.42 \\
\hline MRR 2 & 10589.21 \\
\hline $\operatorname{MR} 3$ & 9185.82 \\
\hline MRR 4 & 9411.66 \\
\hline HRR 5 & 0499.00 \\
\hline MEP 6 & 8096.98 \\
\hline EUGEHE UTR & 55038.08 \\
\hline MRR 1 & $362 B .14$ \\
\hline MRR 2 & \\
\hline MRR 3 & \\
\hline WRR 4 & 1941.51 \\
\hline ARR 5 & 0,100 \\
\hline HRR 6 & 4251.73 \\
\hline CXTRL-LACEN & 9821.37 \\
\hline UTLIES SAAPL & \\
\hline OTH UTLTES-O & \\
\hline SLBSTRATUH & 64859,45 \\
\hline KKR t & 8538.32 \\
\hline
\end{tabular}




\begin{tabular}{|c|c|}
\hline ENTITY & $\begin{array}{l}\text { FIMAL WTD } \\
\text { MFR TER }\end{array}$ \\
\hline $4=0$ & 1096. \\
\hline J: & 1. \\
\hline $4 n=4$ & 0.80 \\
\hline XFi 5 & 0.100 \\
\hline ACE & 7700.96 \\
\hline SFRIAGFIELD & 27205.59 \\
\hline MPF 1 & 0.00 \\
\hline 4 TR 2 & 5525.88 \\
\hline HRF 3 & 9074.01 \\
\hline MRR 4 & 0.00 \\
\hline HARH 5 & 3845.99 \\
\hline Mre of & 9312.84 \\
\hline CONSUHERS & 23557.72 \\
\hline JTLES SAHPL & \\
\hline OTH :TLTES-2 & \\
\hline SUBSTRATUM & 50763.31 \\
\hline$M k \bar{n} !$ & 5985.92 \\
\hline MeP 2 & 6799.19 \\
\hline ABR 3 & 4698,90 \\
\hline hiR 4 & 6710.27 \\
\hline ASHLAKKO & 24193.27 \\
\hline MFE 1 & $5: 99.71$ \\
\hline KRR 2 & 3253.15 \\
\hline MRR 3 & 0.00 \\
\hline MrR 4 & 7828.13 \\
\hline FGREST GROVE & 16290.98 \\
\hline UTLTES SAMPL. & \\
\hline OTH UILTSS-4 & \\
\hline SLJBSTRATUH & $40474.2 b$ \\
\hline HER 1 & 2260.00 \\
\hline$K \in R$ & 2899,38 \\
\hline MPR 3 & 2293.84 \\
\hline HKE 4 & 2326.53 \\
\hline BLACGLY-LAHE & 9775.75 \\
\hline MRA I & 0.00 \\
\hline Hik 2 & 2840.86 \\
\hline MKR 3 & 0.00 \\
\hline
\end{tabular}




\begin{tabular}{|c|c|}
\hline EHTITY & $\begin{array}{l}\text { FINAL WTD } \\
\text { MRE POT }\end{array}$ \\
\hline 4 & 15750 \\
\hline MENGJiTh & 4355.38 \\
\hline \multicolumn{2}{|l|}{ JILLTES SAMPL } \\
\hline \multicolumn{2}{|l|}{ DTH UTLIES-4 } \\
\hline SLBBSTEATUM & 14177.13 \\
\hline STFATUM TUT & 170274.15 \\
\hline PUELIC TOT & 1183079.68 \\
\hline \multicolumn{2}{|l|}{ PFIVATE } \\
\hline \multicolumn{2}{|l|}{ REEION $\mathrm{I}$} \\
\hline -........ & \\
\hline MRR! & 1212.19 \\
\hline MRR 2 & 0.00 \\
\hline MRR 3 & 1880.17 \\
\hline MRP 4 & 0.00 \\
\hline MRR 5 & G.? \\
\hline MFE 5 & $a \hat{0}$ \\
\hline ARR 7 & 8302.69 \\
\hline MRR 8 & 6741.78 \\
\hline MRR 9 & 6824.81 \\
\hline MRR 10 & 7748.59 \\
\hline MRR 11 & 5321.47 \\
\hline GRR 12 & 0.00 \\
\hline MRR 13 & 2036.04 \\
\hline HRR 14 & 361.25 \\
\hline MRR 15 & 0.00 \\
\hline MRR is & 0.100 \\
\hline $\begin{array}{l}\text { MENTANA PHR } \\
\text { SUBSTRATEH }\end{array}$ & 41265.91 \\
\hline
\end{tabular}

\begin{tabular}{|c|c|}
\hline HRP: I & 4053.51 \\
\hline NFE 2 & 533.88 \\
\hline WRR 3 & 0.00 \\
\hline Maf 4 & 0.00 \\
\hline IRR 5 & 0.00 \\
\hline MER 6 & 2604.79 \\
\hline NER 7 & 0.00 \\
\hline RRA & 0.00 \\
\hline MRR 9 & 3945.59 \\
\hline HRR :0 & 0.00 \\
\hline MER 11 & 0.00 \\
\hline
\end{tabular}




\begin{tabular}{|c|c|}
\hline ENTITY & $\begin{array}{c}\text { FEAL WTA } \\
\text { MRE POP }\end{array}$ \\
\hline 40 & 30.54 \\
\hline $45 \div 13$ & 4459,97 \\
\hline MGR is & $=205.95$ \\
\hline$F=1 L-M T$ & gete6.:4 \\
\hline EUESTKATLA & \\
\hline$M R$ & 9.00 \\
\hline$M Q R 2$ & 2.60 \\
\hline $\begin{array}{c}\text { AT FUL } \\
\text { SLESTRATUM } \\
\text { COU -MTIMT-3 }\end{array}$ & 0.60 \\
\hline COLL-MTIHT-P & \\
\hline STRATUM TOT & 70152.04 \\
\hline REGION 2 & \\
\hline+- & \\
\hline MRR ! & 23515.67 \\
\hline$M R R 2$ & 0.00 \\
\hline MRA 3 & 0.00 \\
\hline KSP 4 & 1004.33 \\
\hline RR 5 & 3.00 \\
\hline ARE 5 & 12101.43 \\
\hline ARR ? & 19934.20 \\
\hline MRR $B$ & 11463.99 \\
\hline PP & 69049.52 \\
\hline SUESTRATUM & \\
\hline ME I & 13255.10 \\
\hline MRR Z & 13603.71 \\
\hline ARR 3 & 15475.03 \\
\hline NRR 4 & 0,00 \\
\hline MRR 5 & 0.00 \\
\hline MRR 6 & 0.00 \\
\hline MeF 7 & 14706.66 \\
\hline MRR G & 120199.02 \\
\hline IDAHO PN & 69049.52 \\
\hline SLEETRATUK & \\
\hline MFR 1 & $: 4150.67$ \\
\hline FER 2 & 15442.88 \\
\hline MES 3 & 8605.86 \\
\hline MER 4 & 11.50 \\
\hline ARP 5 & 13460.97 \\
\hline 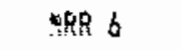 & 17986.17 \\
\hline
\end{tabular}

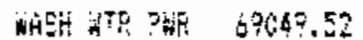
BuEGRATH

HF⿱ 13480.55

MER $2 \quad 0.00$

MRR $3 \quad 23163.87$ 


\begin{tabular}{|c|c|}
\hline EHTTY & $\begin{array}{l}\text { FHAL WTD } \\
\text { KFE POP }\end{array}$ \\
\hline 48 & iviv \\
\hline$\times 85$ & 13431.25 \\
\hline MFi & 9001.61 \\
\hline $\operatorname{maR} 7$ & 9962.25 \\
\hline$A P R$ & 1). 00 \\
\hline IJTAH PLL & 690949.52 \\
\hline 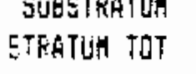 & 276198.10 \\
\hline \multicolumn{2}{|l|}{ BEGIOA 3} \\
\hline -- & \\
\hline MRR 1 & 0.00 \\
\hline MFR 2 & 24975.27 \\
\hline KRR 3 & 0.00 \\
\hline MaP 4 & 9694.83 \\
\hline HFR 5 & 16855.23 \\
\hline ARR 6 & 11626.42 \\
\hline INR 7 & 0.60 \\
\hline MRR g & 20423.13 \\
\hline MRR 9 & 0.00 \\
\hline MRR 10 & 0.00 \\
\hline HRE II & 0.00 \\
\hline HRR 12 & 15651.29 \\
\hline HRR 13 & 14579.16 \\
\hline HRR 14 & 0.00 \\
\hline MRR 15 & 0.00 \\
\hline MRR 16 & 0.00 \\
\hline$P P E L-H A$ & 113805.33 \\
\hline \multicolumn{2}{|l|}{ SURSTRATUH } \\
\hline MRR ! & 0,00 \\
\hline MRR 2 & 16017.41 \\
\hline Heg 3 & 0.00 \\
\hline ARR 4 & $\{8068.32$ \\
\hline MRR 5 & 0.00 \\
\hline MRR 6 & 0.00 \\
\hline 积 7 & 14555.5! \\
\hline ARR B & 16773.54 \\
\hline HRR 9 & 0.00 \\
\hline ARA 10 & 0.150 \\
\hline MRR If & 0.00 \\
\hline MRR 12 & 0.00 \\
\hline MRR 13 & 0.00 \\
\hline MRT I4 & 18496.30 \\
\hline MRR IS & 13676.35 \\
\hline MER 16 & 0.10 \\
\hline $\begin{array}{l}\text { WASH WET PUF } \\
\text { SUBSTRATUM }\end{array}$ & .97547 .42 \\
\hline ETKATUA TOT & 211352.75 \\
\hline
\end{tabular}

B. 61 


\begin{tabular}{|c|c|}
\hline हागए & $\begin{array}{l}\text { FINAE UTD } \\
\text { MPR PIS }\end{array}$ \\
\hline \multicolumn{2}{|l|}{ os 4} \\
\hline \multicolumn{2}{|l|}{--_- } \\
\hline AER I & 59942.34 \\
\hline पदी : & 3,10 \\
\hline 45 & 31057.76 \\
\hline$=4$ & 0.00 \\
\hline knk 5 & $4+545.38$ \\
\hline $\operatorname{sen}_{0}$ & 34924,37 \\
\hline$M C_{R}=$ & $2843 \pi .10$ \\
\hline 985 3 & 3.00 \\
\hline MER & 70095.47 \\
\hline KR 10 & 0.00 \\
\hline$A P R 11$ & 53963.14 \\
\hline 56 & 22655.74 \\
\hline MER 13 & 25320.17 \\
\hline$\forall R R: 4$ & 26184.41 \\
\hline HER 15 & 09527.44 \\
\hline MGR 16 & 23075.03 \\
\hline $\begin{array}{l}\text { FJGET SOUND } \\
\text { SUBSTRATUY }\end{array}$ & 469232.46 \\
\hline STRATUA ToT & 467232.46 \\
\hline \multicolumn{2}{|l|}{ GEGIDN 5} \\
\hline \multicolumn{2}{|l|}{----...... } \\
\hline HFR I & 8008.24 \\
\hline HRR 2 & 0.00 \\
\hline ARR 3 & 9573.01 \\
\hline HRR 4 & 3653.99 \\
\hline $\operatorname{MRR} 5$ & 2279.77 \\
\hline MRR 6 & 3822.48 \\
\hline FRe 7 & 0.00 \\
\hline F积 3 & 0.60 \\
\hline YKR 9 & 4207.40 \\
\hline HRR 10 & 6982.08 \\
\hline MEA 11 & 2744.53 \\
\hline XRR 12 & 0.00 \\
\hline$M R R$ & 0.00 \\
\hline HRR :4 & \\
\hline MRR 15 & 2387.34 \\
\hline NRR is & 7232.94 \\
\hline TPLL-9R-5 & 52891.78 \\
\hline \multicolumn{2}{|l|}{ SLESTEATUM } \\
\hline MRE I & 4195.94 \\
\hline Mef 2 & 10239.93 \\
\hline MEP 3 & 5.100 \\
\hline Fef 4 & 0.00 \\
\hline 散保 5 & 0.60 \\
\hline ज़:RR & 3760.30 \\
\hline SRP ? & 476.92 \\
\hline
\end{tabular}

B. 62 


\begin{tabular}{|c|c|}
\hline & FINAL YTD \\
\hline ENTITY & KER PIP \\
\hline $4 f_{3}$ & PYTE! \\
\hline INAHO PIR & $2 t+45.39$ \\
\hline SLESTRATLH & \\
\hline MRE I & 6.00 \\
\hline Mes 2 & $60 \mathrm{D} 1.56$ \\
\hline HFR 3 & \\
\hline MRR 4 & 2497.80 \\
\hline MRE 5 & \\
\hline MRR b & 0.00 \\
\hline WRR 7 & \\
\hline MER 9 & 0.00 \\
\hline $\begin{array}{l}\text { CP NATNL } \\
\text { SHAETFATUM }\end{array}$ & 10578.36 \\
\hline STRATUM TOT & 89916.03 \\
\hline REEION 6 & \\
\hline - & \\
\hline MRR 1 & 0.00 \\
\hline HRR 2 & 24842.53 \\
\hline HRR 3 & 34763.06 \\
\hline ARR 4 & 0.100 \\
\hline MRR 5 & 17212.85 \\
\hline MRR 6 & 25951.01 \\
\hline HRR 7 & 48500.34 \\
\hline HRP 8 & 0.00 \\
\hline HRR 9 & 0.00 \\
\hline HRR 10 & 16102.45 \\
\hline MRR II & 24320.40 \\
\hline MRR 12 & 15068.31 \\
\hline SRR 13 & 0.00 \\
\hline HRR 14 & 25909.39 \\
\hline APR 15 & 35037.66 \\
\hline MRR 16 & 19972.96 \\
\hline PP\&L-OR-I & 317781.14 \\
\hline SUESTRATLH & \\
\hline MRR I & 20347.44 \\
\hline MER 2 & 34313.17 \\
\hline MRR 3 & 26047.22 \\
\hline MRR 4 & 37901.81 \\
\hline ARR 5 & 14430.09 \\
\hline MRR b & 24453.98 \\
\hline IRR 7 & 34431.29 \\
\hline MRR B & 23669,00 \\
\hline FRR 9 & 34339.96 \\
\hline MRE 10 & 0.00 \\
\hline NFR II & 0.00 \\
\hline MRR 12 & 0.00 \\
\hline MRR 13 & 26595.20 \\
\hline KRRP 14 & 0.00 \\
\hline
\end{tabular}




\begin{tabular}{|c|c|}
\hline EUTITY & $\begin{array}{l}\text { FINAL UTD } \\
\text { MER FOOF }\end{array}$ \\
\hline बदि & : \\
\hline 3516 & 9174.13 \\
\hline
\end{tabular}

FER: EEN ELE 346670.33

SLESTRMTUA

ETGATUM TOT 604451.47

DR:VATE TDT 1781302.85

GeANE TU? 2964352.55 
APPENDIX $C$

ROST RESPONDENTS SPREADSHEET CONTENTS 


\begin{tabular}{|c|c|c|c|c|c|c|c|c|}
\hline$A$ & B & C & D & $E$ & $F$ & G & $\mathrm{H}$ & I \\
\hline EHTITY & MFR CODE & $\begin{array}{c}\text { KNOHN } \\
\text { INE:IGELS }\end{array}$ & $\begin{array}{l}\text { JNAELE TO } \\
\text { CONTACT }\end{array}$ & $\begin{array}{l}\text { MRR SANFLE } \\
\text { ORAUN }\end{array}$ & $\begin{array}{c}\text { ELIGIBLLITY } \\
\text { RATIO }\end{array}$ & $\begin{array}{l}\text { TOTAL } \\
\text { INELIGELES }\end{array}$ & $\begin{array}{l}\text { TOTAL HRR } \\
\text { POPULATIOK }\end{array}$ & $\begin{array}{l}\text { ELIGIELE MRE } \\
\text { POPULATION }\end{array}$ \\
\hline --------- & --- & .......... & - n-n-- & -------- & ------- & 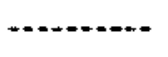 & -.......... & ......... \\
\hline \multicolumn{9}{|l|}{ PUELiC } \\
\hline ------ & & & & & & & & \\
\hline \multicolumn{9}{|l|}{ REg1ON ! } \\
\hline n.nen. & & & & & & & & \\
\hline HRR ! & 104101 & & 0 & 1 & 0.9333 & 0.0000 & 680 & 634.67 \\
\hline$M R=$ & 104102 & & 0 & 1 & 0.8571 & 0.0000 & 680 & 582.86 \\
\hline He? & 104103 & & 0 & 1 & 1.0000 & 0.0000 & 580 & 680.00 \\
\hline NRR 4 & 104194 & & 1 & 1 & 0.9333 & 0.0000 & 680 & i. 60 \\
\hline KRR 5 & 104105 & & 0 & 1 & 0.9289 & 6.0000 & 680 & 631.64 \\
\hline MRR 6 & 104106 & & 0 & 1 & 1.0000 & 0.0000 & 680 & 680.00 \\
\hline MRR 7 & 104107 & & 1 & 1 & 0.5462 & 0.0000 & 680 & 0.00 \\
\hline MRR 8 & t04108 & & 0 & 1 & 0.9412 & 0.0000 & 680 & 640.00 \\
\hline FLATKEAD ELE & 1041 & & 2 & $B$ & 0.9322 & 0.0000 & 5440 & 3849.17 \\
\hline MRR 1 & 105101 & & 1 & 1 & 0.7143 & 0.0000 & 30 & 0.00 \\
\hline MRA 2 & 105102 & & 0 & 1 & 0.8571 & 0.0000 & 120 & 102.86 \\
\hline MRR 3 & 105103 & & 0 & 1 & 0.8400 & 0.0000 & 190 & 159.60 \\
\hline HRR 4 & 105104 & & 0 & 1 & 0.7704 & 0.0000 & 240 & 184.90 \\
\hline MRR 5 & 105105 & & 0 & 1 & 1.0000 & 0.0000 & 460 & 460.00 \\
\hline MRR 6 & 105106 & & 0 & 1 & 1.0000 & 0.0000 & 560 & 600.00 \\
\hline MFA 7 & 105107 & & 0 & 1 & 0.6000 & 0.0000 & 850 & 510.00 \\
\hline$M R R B$ & 105108 & & $i$ & 1 & 0.8667 & 0.0000 & 690 & 0.00 \\
\hline FLATHEAD IRR & $105:$ & & 2 & $B$ & 0.8324 & 0.0000 & 3180 & 2017.36 \\
\hline UTLTES SAMPL & & & 4 & 16 & 0.8827 & 0.0000 & 8620 & 5966.52 \\
\hline OTH UTLTES-I & & & & NA & & & 0 & 0.00 \\
\hline SUBSTRATUH & & & & NA & & 0.0000 & 9620 & 5866.52 \\
\hline ARR 1 & 106101 & & 0 & 1 & 1.0000 & 0.0000 & 117 & 117.00 \\
\hline HRR 2 & 106102 & & 1 & 1 & $\$ .0000$ & 0.0000 & 78 & 0.00 \\
\hline ARR 3 & 106103 & & 0 & 1 & 0.9375 & 0,0000 & 130 & 121.88 \\
\hline KRR 4 & 106504 & & 1 & 1 & 0.9235 & 0.0000 & 102 & 0.00 \\
\hline MRR 5 & 106105 & & 0 & 1 & 0.8333 & 0.0000 & 106 & 88.33 \\
\hline MRR 6 & 1061106 & & 0 & & 0.8542 & & & 0.00 \\
\hline NRR 7 & 106107 & & 0 & & 1.0000 & & & 0.00 \\
\hline MRR B & 106108 & & 0 & & 0.8889 & & & 0.00 \\
\hline BLACIER & 1061 & & 2 & 5 & 0.9312 & 0.0000 & 533 & 327.21 \\
\hline MER 1 & 107161 & & 0 & 2 & 0.9130 & 0.0000 & 801 & $75 ! .35$ \\
\hline ARR 2 & 107102 & & 0 & i & 1.0000 & 0.0000 & 584 & 534.00 \\
\hline MRR 3 & 107103 & & 1 & 2 & 0.9091 & 0.0000 & 888 & 807.27 \\
\hline MRR 4 & 107104 & & $i$ & 1 & 0.9132 & 0.0000 & 370 & 0.00 \\
\hline ARF 5 & 107105 & & 0 & 2 & 0.9531 & 0.0000 & 506 & 482.28 \\
\hline
\end{tabular}




\begin{tabular}{|c|c|c|c|c|c|c|c|c|}
\hline$\equiv \mathrm{NTITY}$ & MFS COSE & $\begin{array}{c}\text { MDOWN } \\
\text { [NEEIGESS }\end{array}$ & $\begin{array}{l}\text { SNABLE TO } \\
\text {-JNTACT }\end{array}$ & $\begin{array}{c}\text { MER SIMPLE } \\
\text { DFAHN }\end{array}$ & $\begin{array}{c}\text { ELISIEILITY } \\
\text { AATIO }\end{array}$ & $\begin{array}{c}\text { TDTAL } \\
\text { IMELIEELLES }\end{array}$ & $\begin{array}{l}\text { TOTAL MRR } \\
\text { FOPULATION }\end{array}$ & $\begin{array}{l}\text { EIIGIBLE MRR } \\
\text { POFISLATION }\end{array}$ \\
\hline - & 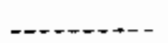 & -....--- & ----------- & -------- & - - & - & ---- & ------ \\
\hline r. & $107 !$ & & 2 & 8 & 0.9379 & 6,0000 & 3149 & 2604.90 \\
\hline JTLIES SAMEL & & & 4 & 13 & 0.9347 & 0.0000 & 3682 & 2932.11 \\
\hline JTH UTLTES-2 & & & & SA & & & & \\
\hline SHESTKATUH & & & & NA & & 0.0000 & 35E? & 2932.11 \\
\hline STPATUN TOP & & & & NA & & 0.1000 & 12302 & 8798.63 \\
\hline \multicolumn{9}{|l|}{ SEGION 2} \\
\hline \multicolumn{9}{|l|}{-........ } \\
\hline MFR 1 & $1: 2201$ & & 1 & $i$ & 1.3000 & 0.0000 & 148 & 0.60 \\
\hline MR 2 & 112202 & & 0 & 1 & 1.0000 & 0.0000 & 256 & 256.00 \\
\hline YRE 3 & 12203 & & $!$ & \pm & 0.3667 & 0.0000 & $27 b$ & 0.60 \\
\hline NARR 4 & 112204 & & 0 & 1 & 0.9333 & 0.0000 & 187 & $\$ 74.53$ \\
\hline MER 5 & 112265 & & 0 & 1 & 0.9335 & 0.0000 & 175 & 163.33 \\
\hline MRP & $1 \pm 2206$ & & 0 & 1 & 1.0000 & 0.0000 & 84 & 84.00 \\
\hline DAtiO FALIS & 1172 & & 2 & 6 & 0.9551 & 0.0000 & 1126 & 677.87 \\
\hline MKR I & $1: 3201$ & & 0 & 1 & 0.7692 & 0.0000 & 130 & 101.00 \\
\hline$\Delta$ & $1: 30 \%$ & & ij & $\vdots$ & 1.9286 & 0.0000 & $1 ! 0$ & 102.14 \\
\hline ARA 3 & 113203 & & 0 & 1 & 0.8571 & 0.0000 & 125 & 107.14 \\
\hline men 4 & 113204 & & 1 & 1 & 0.9299 & 0.0000 & 94 & 0.00 \\
\hline MRR 5 & 113205 & & $t$ & 1 & 0.9231 & 0.0000 & 304 & 0.00 \\
\hline HRR 6 & 113206 & & 1 & 1 & 0.9333 & 0.0000 & 240 & 0.00 \\
\hline LOHER VALLEY & 1132 & & 3 & $b$ & 0.8921 & 0.0000 & 1085 & 309.29 \\
\hline UTLIES SAMFL & & & 5 & 12 & 0.9245 & 0.0000 & 2209 & 987.15 \\
\hline GTH UTLTES-3 & & & $\vartheta$ & MA & & & & \\
\hline SUESTRATUH & & & & NA & & 0.0000 & 2269 & 987.15 \\
\hline HFR I & $11420 !$ & & 0 & $\mathrm{i}$ & 0.7143 & 0.0000 & 351 & 250.71 \\
\hline MRR 2 & $11 \div 202$ & & $\theta$ & & & & & \\
\hline Het 3 & 114203 & & 0 & 1 & 1.0000 & 0.0000 & 737 & 739.60 \\
\hline KRT 4 & 114204 & & 1 & 1 & $0.357 t$ & 0.0000 & 119 & 0.00 \\
\hline NRR 5 & 114205 & & 0 & 1 & 0.5672 & 0.0000 & 76 & $65.9 !$ \\
\hline MFR S & 114206 & & 1 & 1 & - 0.06007 & 0.9000 & 389 & 0.00 \\
\hline FALL RIVER & $1: 42$ & & 2 & $\xi$ & 0.7229 & 0.0000 & 1673 & 1055.62 \\
\hline MFT 1 & 115201 & & 0 & 1 & $0.929 \mathrm{~b}$ & 0.0000 & 179 & 160.21 \\
\hline EAGTEND XUT & 1152 & & 0 & 1 & 10.9236 & 1.0000 & 179 & 260.21 \\
\hline HKR ! & $11020 j$ & & 0 & 1 & 0.7533 & 0.6000 & 90 & 84.00 \\
\hline FARHERS ELE & 1162 & & 0 & 1 & 0.9333 & 1.0000 & 90 & 84.00 \\
\hline
\end{tabular}




\begin{tabular}{|c|c|c|c|c|c|c|c|c|}
\hline ENTITY & MAR CODE & $\begin{array}{c}\text { KNOLN } \\
\text { IMEL: GELS }\end{array}$ & $\begin{array}{l}\text { UNABLE TO } \\
\text { COKTACT }\end{array}$ & $\begin{array}{l}\text { MRR SAMPLE } \\
\text { DRALN }\end{array}$ & $\begin{array}{l}\text { ELIGIGILITY } \\
\text { RATHO }\end{array}$ & $\begin{array}{c}\text { TOTAL } \\
\text { INELIGELES }\end{array}$ & $\begin{array}{l}\text { TOTAL KRR } \\
\text { POPULATION }\end{array}$ & $\begin{array}{l}\text { ELIGIELF MRR } \\
\text { PORULATION }\end{array}$ \\
\hline RE & 1941 & -...- & $\bar{j}$ & 1 & 0.7389 & 0.0000 & 350 & 35.11 \\
\hline EIVESGIDE EL & 118 & & i! & $i$ & 0.9289 & 0.10060 & $35 i$ & 325.11 \\
\hline 知P 1 & 119201 & & 1 & $!$ & 1.0000 & 0,0000 & 500 & 1.00 \\
\hline SOUT:GIUE EL & 1192 & & 1 & 1 & 1,0000 & 0.6000 & 500 & 0.00 \\
\hline MER 1 & $\{20201$ & & 1 & 1 & 0.8578 & 9.0000 & 300 & 0.00 \\
\hline MFR 2 & 120202 & & 0 & 1 & 0.7245 & 0.0000 & 310 & 224.59 \\
\hline WRITY LEP & 1202 & & 1 & 2 & 0.7934 & 0.0000 & $\$ 10$ & 224.59 \\
\hline HINDOKA PROJ & & & 6 & 0.00 & $0.897 !$ & 2.0000 & NA & BA \\
\hline UTLTES SAMPL & & & & 1 & 0.8095 & 2.0000 & 3402 & 1855.54 \\
\hline OTH UTLTES-4 & & & & NA & & & & \\
\hline SUBSTRATUM & & & & Nค่ & & 2.0000 & 3402 & 1855.54 \\
\hline MRR I & 121201 & & 1 & 2 & 0.9333 & 0.0000 & 232 & 216.53 \\
\hline MRR 2 & 121202 & & 1 & 1 & 0.8667 & 0.0000 & 233 & 0.00 \\
\hline MRR 3 & 121203 & & 1 & 1 & $0.7 \mathrm{~B} 67$ & 0.0000 & 235 & 0.00 \\
\hline MFin 4 & 121204 & & 0 & 1 & 0.9733 & 0.0000 & 233 & 217.47 \\
\hline ARR 5 & 121205 & & 0 & $i$ & 0.0333 & 0.0000 & 233 & 217.47 \\
\hline MRE 6 & $12: 206$ & & 1 & 1 & 0.7867 & 0.0000 & 232 & 9.00 \\
\hline LOST RIVER & 1212 & & 4 & 7 & 0.8733 & 0.0000 & 1395 & 651.47 \\
\hline MRR 1 & 122201 & & 0 & & 0.8578 & & & 0.00 \\
\hline MRR 2 & 122202 & & 0 & 1 & 0.9299 & 0.0000 & 129 & 119.03 \\
\hline HR 3 & 122203 & & $\mathfrak{1}$ & $i$ & 1.0000 & 0.0000 & 123 & 0.00 \\
\hline MRR 4 & 122204 & & 1 & 1 & 0.9333 & 0.0040 & 166 & 0.00 \\
\hline MRR 5 & 122205 & & 1 & 1 & 1.0000 & 0.0000 & 104 & 0.00 \\
\hline MRR 6 & 122206 & & 1 & 1 & $0.92 \mathrm{Bb}$ & 0.0000 & 169 & 0.00 \\
\hline HEISER & 1222 & & 4 & 5 & 0.9416 & 0.0000 & 691 & 119.83 \\
\hline UTLTES SARFL & & & & 12 & 0.9073 & 0.0000 & 2086 & 771.29 \\
\hline OTH UTLTES-4 & & & & NA & & & & \\
\hline SUBSTRATUR: & & & & :A & & 0.0000 & 2086 & 771.29 \\
\hline STRATUM TOT & & & & HA & & 2.0000 & 7697 & 3613.98 \\
\hline \multicolumn{9}{|l|}{ RESION 3} \\
\hline \multicolumn{9}{|l|}{ - } \\
\hline HRR 1 & 123301 & & 1 & $!$ & 0.9244 & 0.0000 & 92 & 6.50 \\
\hline MER 2 & 125302 & & 1 & 1 & 0.9231 & 0.3000 & 242 & 0.00 \\
\hline $\operatorname{MRR} 3$ & 123303 & & 1 & 1 & 0.7333 & 0.0000 & 819 & 0.00 \\
\hline GPR & 123304 & & $D$ & $!$ & 0.9244 & 0.0000 & 628 & 580.55 \\
\hline MRR 5 & 123365 & & 1 & 1 & 0.7704 & 0.0000 & 675 & 0.00 \\
\hline & & & & C. 3 & & & & \\
\hline
\end{tabular}




\begin{tabular}{|c|c|c|c|c|c|c|c|c|}
\hline ENTITY & MAR CQDE & 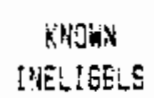 & $\begin{array}{l}\text { HWABLE TO } \\
\text { OON:AC? }\end{array}$ & $\begin{array}{l}\text { MEF SAMPLE } \\
\text { DFATN }\end{array}$ & $\begin{array}{c}\text { ELIGISILITY } \\
\text { RATIO }\end{array}$ & $\begin{array}{l}\text { TOTAL } \\
\text { INELISELES }\end{array}$ & $\begin{array}{l}\text { TUTAL NGH } \\
\text { POPULATIDK }\end{array}$ & $\begin{array}{l}\text { ELIEI8LE MFR } \\
\text { PUPLLATIOH }\end{array}$ \\
\hline 涪F & Sani & $\cdots$ & $i$ & 1 & 6,7244 & 6.000 & 77 & 1.10 \\
\hline EESTC: EO & 1333 & & 5 & b & 0.8005 & 0.1500 & 2783 & $5 E 6.55$ \\
\hline Yद & $12470 !$ & & 0 & $\mathrm{i}$ & 0.7333 & 0.5000 & $3 i s$ & 225.87 \\
\hline AKE 2 & 124302 & & 0 & i & 1.0600 & 0.0000 & 107 & 107.90 \\
\hline MRE 3 & 124303 & & 1 & 1 & 0.9333 & 0.0000 & 372 & 0.60 \\
\hline MES 4 & $124: 04$ & & 0 & 1 & 1.0000 & 0.0000 & 442 & 442.00 \\
\hline ME & 124305 & & 0 & ! & 1.0000 & 0.00000 & 475 & 475.90 \\
\hline GELAN STY & 1243 & & : & 5 & 1.9733 & บ. 0000 & 1704 & 1249.57 \\
\hline MRE 1 & 153301 & & $\hat{0}$ & 1 & 0.9289 & 0.0000 & 2290 & $2: 27.10$ \\
\hline CAEUHEPE & 1253 & & 0 & 1 & 0.7299 & 1.0667 & 2290 & 2127.16 \\
\hline CHELRH-CSHYKE & & & 1 & 6.100 & 0.9326 & 1.0667 & NA & UA \\
\hline JILTES SAMFL & & & & 12 & 0.9001 & 1.0507 & bi77 & 3958 \\
\hline OTH UTLTES-2 & & & & $M A$ & & & & \\
\hline SUBSTRATUH & & & & NA & & 1.0667 & 6777 & 3957.57 \\
\hline mith 1 & 126301 & & 0 & 1 & 0.733. & 0.000 & 49 & 31.97 \\
\hline ME & $126 j i 2$ & & 0 & 1 & 0.9235 & D.0000 & 110 & 151.58 \\
\hline NKR j & 126303 & & $!$ & 1 & 0.8000 & 0.0000 & 1103 & 0.00 \\
\hline MRE 4 & 126304 & & 0 & 1 & 0.9289 & 0.0000 & búg & 564.76 \\
\hline MRe 5 & 126305 & & 0 & ! & 0.3571 & 0.0000 & ถ் 75 & $3: 2.50$ \\
\hline Mas 6 & 126306 & & 0 & & 0.5333 & & & 0.00 \\
\hline FRANKLIN & 1263 & & 1 & 5 & 0.7143 & 0.0000 & 2794 & 1050.71 \\
\hline MKR 1 & 127301 & & 0 & 1 & 1.0000 & 0.0000 & 135 & 135.00 \\
\hline MRR 2 & $12730:$ & & i & i & 0.7143 & 0.0000 & 177 & 0.00 \\
\hline ARE 3 & $127 \mathrm{n} 3$ & & 0 & 1 & 1.0000 & 0.0000 & 164 & 164.00 \\
\hline IFR 4 & 127304 & & 0 & $i$ & 1.0000 & 0.0060 & 138 & 138.00 \\
\hline MFR 5 & 127305 & & 0 & $i$ & 1.0000 & 0.00000 & 194 & 194.00 \\
\hline KFR o & 127306 & & $i$ & 1 & 0.9289 & 0.0000 & 191 & 0.00 \\
\hline RICHLAND & 1275 & & 2 & 6 & 0.9418 & 0.0000 & 999 & 631.60 \\
\hline UTLTES SAKPL & & & & 11 & 0.8274 & 0,0000 & $3793 .(10)$ & 1681.71 \\
\hline WTH UILTES-5 & & & & NA & & & & \\
\hline SUAETRATIM! & & & & its & & 0.0000 & 3793 & 1681.71 \\
\hline YRR .1 & $\mid: 8501$ & & $!$ & : & 1,0000 & 0.2000 & 219 & 0.60 \\
\hline The 2 & 170.302 & & 1 & $i$ & 1.0000 & 0.0010 & 219 & $1, \therefore 0$ \\
\hline mi $j$ & {$[29303$} & & 1 & 1 & 0.9335 & 0.0000 & 53 & 9.00 \\
\hline WhR 4 & 128304 & & 0 & 1 & 1.0000 & 0.0000 & 38 & 66.00 \\
\hline PEHO ORIELLE & $\{293$ & & 3 & 4 & 0.9833 & 0.0000 & 559 & 38.10 \\
\hline
\end{tabular}




\begin{tabular}{|c|c|c|c|c|c|c|c|c|}
\hline ERTITY & MRR CONE & $\begin{array}{c}\text { VNGWH } \\
\text { INELIGBLS }\end{array}$ & $\begin{array}{l}\text { WNABLE TO } \\
\text { CONTACT }\end{array}$ & $\begin{array}{c}\text { MRR SAMPLE } \\
\text { DRANN }\end{array}$ & $\begin{array}{c}\text { ELIGIBILITY } \\
\text { RATID }\end{array}$ & $\begin{array}{l}\text { TOTAL } \\
\text { INEELIGELES }\end{array}$ & $\begin{array}{l}\text { TLTAL MGF } \\
\text { PEPJLAT IOA }\end{array}$ & $\begin{array}{l}\text { ELIETBLE WRA } \\
\text { POPLLAIION }\end{array}$ \\
\hline ------ & - & ......... & .............. & +........ & -..- & --...- & ------- & 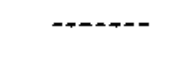 \\
\hline MFE 1 & 130301 & & 5 & 1 & 0.9567 & 1.0000 & 341 & 205.55 \\
\hline MFE 2 & 13002 & & 0 & 1 & $0 . \overline{4} 269$ & 1. & 58 & 249.94 \\
\hline MRR 3 & 130303 & & 1 & 1 & 1.00100 & 0.0000 & 289 & 0.00 \\
\hline ARR 4 & 139354 & & 0 & 1 & 1.0000 & 0,500 & 892 & 292.00 \\
\hline VERA IKR & 1393 & & 1 & 4 & 0.9535 & 0.0000 & 329 & $93 b .48$ \\
\hline LTLTES SAMFL & & & & 8 & 0.9677 & 0.0000 & 988 & $\square 04.48$ \\
\hline DTH UTLTES-3 & & & & NA & & & & \\
\hline SUBSTRATUK & & & & NA & & 0.0000 & 888 & 904.48 \\
\hline MRR : & 131301 & & 0 & 1 & 0.6000 & 0.0000 & 404 & 242.10 \\
\hline MRR 2 & 131302 & & 0 & 1 & 0.6805 & 0.0000 & 404 & 274.91 \\
\hline MRR 3 & 131303 & & 0 & & 0.8889 & & & 0.00 \\
\hline MRR & 131304 & & 1 & 1 & 0.9111 & 0.0000 & 404 & 10.100 \\
\hline FERRY CTY & 1313 & & 1 & 3 & 0.7669 & 0.6000 & 1212 & 517.31 \\
\hline ARR 1 & 132301 & & 1 & 1 & 1.0000 & 0.0000 & 267 & 0.00 \\
\hline MRR 2 & 132302 & & 0 & 1 & 0.7333 & 0.0000 & 268 & 196.53 \\
\hline NESFFEELEM & 1323 & & $!$ & 2 & 0.9667 & 0.6000 & 5.55 & 196.33 \\
\hline MRR ! & 133301 & & 1 & 1 & 0.9333 & 0.0000 & 93 & 0.00 \\
\hline MRR 2 & 133302 & & 0 & 1 & 1.0000 & 0.0000 & 56 & 56.00 \\
\hline COULEE DAH & 1333 & & 1 & 2 & 0.9655 & 0.0000 & 149 & 56.00 \\
\hline NSPLH-CDULEE & & & 4 & 4.00 & 0.9153 & 0.0000 & & \\
\hline UTLIES SAAPL & & & & 7 & 0.8437 & 0.0000 & 1896 & 769.84 \\
\hline OTH UTLTES-4 & & & & NA & & & & \\
\hline SLBSTRATUM & & & & HA & & 0.0000 & 1896 & 769.84 \\
\hline STRATLIM TOF & & & & NA & & 1.0667 & 13354 & 7313.61 \\
\hline \multicolumn{9}{|l|}{ REgIDN 4} \\
\hline - - - & & & & & & & & \\
\hline MRR I & 135401 & & 2 & 2 & 1.0000 & 0.0000 & 399 & 0.00 \\
\hline MRR 2 & $13540 ?$ & & 1 & 2 & 1.0000 & 0.0000 & $4 i 3$ & 403.10 \\
\hline SKR 3 & 15543 & & 2 & 2 & 1.6000 & 0.0000 & 317 & 1.80 \\
\hline MER 4 & 135404 & & 1 & 2 & 1.5000 & 0.0000 & 309 & $39 E .90$ \\
\hline HRR 5 & 135405 & & 1 & 2 & $0.866 ?$ & 0.0000 & 378 & 327.60 \\
\hline MRR 6 & 135406 & & 0 & 2 & 1.0000 & 0.0000 & 440 & 440.00 \\
\hline
\end{tabular}




\begin{tabular}{|c|c|c|c|c|c|c|c|c|}
\hline EITYY & MAX & 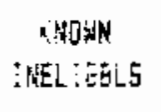 & $\begin{array}{c}\text { SWEE TI } \\
\text { C?MT UCT }\end{array}$ & $\begin{array}{c}\text { MER EAMPLE } \\
\text { OEARA }\end{array}$ & $\begin{array}{l}\text { SLIEIBILITY } \\
\text { FATIO }\end{array}$ & $\begin{array}{c}\text { TOTAL } \\
\text { IKELIGGLES }\end{array}$ & $\begin{array}{l}\text { TOTAL MRF } \\
\text { POPULATION }\end{array}$ & $\begin{array}{c}\text { ELISIELE MRQ } \\
\text { FIPULATIIIN }\end{array}$ \\
\hline ع & 1 & 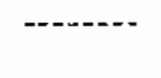 & $\cdots$ & $:=$ & 0.979 & 6.906 & 295 & 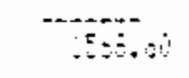 \\
\hline MFE： & 1541 & & ! & 2 & 1.0000 & 6.0000 & 59 & 58.90 \\
\hline 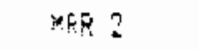 & 136412 & & $\bar{z}$ & 2 & 0.7333 & 0.00000 & 22 & i. 60 \\
\hline Ant 3 & $1: t+6 s$ & & $!$ & $?$ & 1.0373 & 6. Who & 247 & 249.20 \\
\hline pit 4 & $1364 ; 74$ & & 1 & : & $1.000 \mathrm{o}$ & 1.0000 & 20 & 239.30 \\
\hline PAR & 135415 & & 1 & 2 & 1. Boilo) & 9.0000 & 184 & 184.00 \\
\hline Intef 6 & 130400 & & 1 & 2 & 0.9295 & 0.0000 & 243 & 225.04 \\
\hline 三NDHEH:GH & 1354 & & $?$ & 12 & 0.9647 & 0.0000 & 1245 & 986.84 \\
\hline VILIES SAREL & & & & 24 & 0.9711 & 0.10000 & 3500 & 2555.44 \\
\hline Jid :HLIES-6 & & & & $9 \mathrm{~A}$ & & & & \\
\hline SUGSTRATUM & & & & $4 A$ & & 9.0900 & 5580 & 2555.44 \\
\hline MFR I & 137491 & & 0 & 2 & 1.0000 & 0.0000 & 259 & 258.00 \\
\hline ? & 87402 & & 6 & 2 & 9.9053 & 0.0000 & 590 & $34.0 ?$ \\
\hline MER 3 & 13743 & & 0 & 2 & i). 9244 & 0.1000 & 394 & 304.25 \\
\hline MER 4 & 137404 & & 4 & 1 & 0.6675 & 0.0600 & 224 & 154.00 \\
\hline MRR 5 & 137405 & & 1 & 2 & 0.9133 & 0.0000 & 559 & 510.52 \\
\hline MRR S & 137406 & & 1 & 1 & 0.9231 & 0.0000 & 364 & 0.00 \\
\hline LAFS ETY & 534 & & 2 & 10 & 0.8984 & 0,3600 & 3179 & 1630.77 \\
\hline 1 & 137401 & & 0 & 2 & 1. .0333 & 0.0000 & 194 & 171.73 \\
\hline AER 2 & $: 38402$ & & 0 & 2 & 0.9289 & 0.0000 & $14 B$ & 157.48 \\
\hline $\operatorname{Mig} 3$ & 138403 & & ) & 1 & $0.928 \overline{9}$ & 0.0000 & 147 & 936.55 \\
\hline MRR 4 & 158404 & & 1 & 2 & 1.0000 & 0.0000 & 157 & 157.00 \\
\hline YFP 5 & 138405 & & 1 & 1 & 0.9333 & 0.0000 & 136 & 0.00 \\
\hline MER b & 135406 & & 1 & 2 & 9.8571 & 0.0000 & 157 & 134.57 \\
\hline TACOHA & 1384 & & $J$ & 10 & 0.9295 & 0.0000 & 929 & 757.33 \\
\hline UTETES SALPL & & & & 20 & 0.9144 & 6.0000 & 3108 & 336.10 \\
\hline GTH UTLTES-2 & & & & NA & & & & \\
\hline SUGCTRATLM & & & & HA & & 0.0000 & 3108 & 2338.10 \\
\hline MRR 1 & 177401 & & 2 & 2 & 0.9785 & 0. 0000 & $4 ! E$ & 1.00 \\
\hline MRR 2 & 139402 & & 0 & 2 & 1.0000 & 0.6090 & 363 & 363.90 \\
\hline$\forall R$ & 139403 & & 0 & 2 & 0.8667 & 0.0000 & 352 & 218.40 \\
\hline AKR 4 & 123404 & & 1 & $i$ & 1.0005 & $(0.0) 000$ & 52 & $53 \% .60$ \\
\hline ARR 5 & $1374 i 5$ & & 0 & $?$ & 0.8008 & $0.300 \mathrm{i}$ & 220 & 175.00 \\
\hline M:RR b & 139406 & & $i$ & 2 & 0.7551 & 0.6000 & 395 & 300.35 \\
\hline MASON :TYY-3 & $i 374$ & & 4 & 12 & 0. 9921 & 0.6000 & 2183 & +590.93 \\
\hline $\operatorname{Mat} 1$ & itouti & & 2 & 2 & $\therefore \operatorname{sg} 6$ & 0.0000 & $3: ?$ & 0.30 \\
\hline $\operatorname{MeR} 2$ & $1+0402$ & & $\hat{v}$ & 2 & $1, .75 j J$ & $\hat{0}, \dot{00000}$ & 905 & 602.20 \\
\hline GR 3 & 140403 & & $i$ & 2 & 0.8469 & 0.0000 & 548 & 465.19 \\
\hline AfE & 140404 & & $\vdots$ & 2 & $0.8 \leq 00$ & 0.0000 & 339 & 271.20 \\
\hline MRP & $141) 4(65$ & & 1 & 2 & 0.933 & 3.0000 & $2 E 9$ & 26.73 \\
\hline
\end{tabular}

C. 6 


\begin{tabular}{|c|c|c|c|c|c|c|c|c|}
\hline ENTITY & MPR CODE & $\begin{array}{c}\text { KNANA } \\
\text { IHEL IGFLS }\end{array}$ & $\begin{array}{l}\text { WMABLE TO } \\
\text { COMTACT }\end{array}$ & $\begin{array}{l}\text { MRR SAMPLE } \\
\text { DKANN }\end{array}$ & $\begin{array}{l}\text { ELIGIGILITY } \\
\text { RAT!J }\end{array}$ & $\begin{array}{c}\text { TOTÁL } \\
\text { INELIGSLES }\end{array}$ & $\begin{array}{l}\text { TOTAL MRR } \\
\text { PUPULATION }\end{array}$ & $\begin{array}{l}\text { ELIGIELE ARR } \\
\text { POPULATIEH }\end{array}$ \\
\hline MR E & iniage & & 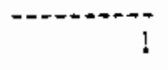 & 2 & 8.840 & 40000 & 50 & 262.90 \\
\hline CLALLAH ETT & 1404 & & 6 & 12 & 1. 8593 & 0.0000 & 3048 & $2 \div 30 \div 2$ \\
\hline UTLTES SARPL & & & & 24 & 0.8755 & 9.0000 & 5231 & 5720.26 \\
\hline OTH ATLTES-3 & & & & NA & & & & \\
\hline SUEETRATUM & & & & NA & & 0,0000 & 5231 & 3720.26 \\
\hline MES！ & 141401 & & 0 & 2 & 0.8667 & 0.0000 & 432 & 374.40 \\
\hline GER 2 & 141402 & & 0 & 2 & 1.0000 & 0.0000 & 250 & 250.00 \\
\hline HRR J & 14140.3 & & 2 & ? & 1.0000 & 0.0000 & 530 & 0.00 \\
\hline MRR 4 & 141404 & & 1 & 2 & 1.0000 & 0.0000 & 312 & 312.00 \\
\hline ELMHURST YUT & 1414 & & 3 & 8 & 0.9667 & 0.0000 & 1524 & 936.40 \\
\hline MRR ! & $\{4240 !$ & & 0 & 2 & 0.9333 & 0.0000 & 1250 & 1106.67 \\
\hline MRR 2 & 142402 & & 1 & 2 & 0.9289 & 0.0000 & 1250 & 1161.11 \\
\hline MRR 3 & 142403 & & 0 & 2 & 0.9333 & 0.0000 & 1250 & 1166.67 \\
\hline MRE 4 & 142404 & & 0 & 2 & 0.9333 & 0.0000 & 1250 & 1166.67 \\
\hline ORCAS PHR & 1424 & & 1 & 8 & 0.9322 & 0.0060 & 5000 & 4661.11 \\
\hline UTLIES SAKP! & & & & is & 0.9474 & 0.0000 & 6524 & 5577.51 \\
\hline DFH UTLTES-3 & & & & NA & & & & \\
\hline SUPSTRATUH & & & & NA & & 0.0000 & 6524 & 5597.51 \\
\hline H 1 & $14] 401$ & & 0 & 2 & 0.9286 & 0.0000 & 77 & 71.50 \\
\hline MRR 2 & 143402 & & 2 & 2 & $0.928 b$ & 0.0000 & 168 & 0.00 \\
\hline MRR 3 & 143403 & & 0 & 2 & 0.9333 & 0.0000 & 170 & 158.67 \\
\hline MRR A & 143404 & & 0 & 2 & 1.0000 & 0.0000 & 140 & 140.00 \\
\hline PARKLLAMD LIP & 1434 & & 2 & 8 & 0.9493 & 0.0000 & 555 & 370.17 \\
\hline MQR ! & 144401 & & 0 & 2 & 0.9231 & 0.0000 & 127 & 417.23 \\
\hline MRP 2 & 144402 & & 0 & 2 & 1.0000 & 0.0000 & 85 & 85.00 \\
\hline MER 3 & 144403 & & 0 & 2 & 0.9333 & 0.0000 & 135 & 126.00 \\
\hline MRR \& & 144404 & & 1 & 2 & 1.0000 & 0.0000 & 104 & 104.00 \\
\hline SKAMANIIA & 1444 & & 1 & 8 & 0.9655 & 0.0000 & 451 & 432.23 \\
\hline UTLTES SAMPL & & & & 16 & 0.9569 & 0.0000 & 1006 & 802.40 \\
\hline OTH UTLTES-2 & & & & NA & & & & \\
\hline 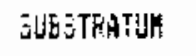 & & & & HA & & 0.0000 & 1000 & 902.40 \\
\hline MRR 1 & 145401 & & 0 & 2 & 1.00000 & 0.0000 & 421 & 421.00 \\
\hline MKR 2 & 145462 & & 2 & 2 & 1.0000 & 0.0000 & 199 & 0.00 \\
\hline MER J & $\{45403$ & & 1 & 2 & 0.9333 & 0.0000 & 65 & 69.67 \\
\hline
\end{tabular}




\begin{tabular}{|c|c|c|c|c|c|c|c|c|}
\hline EntTi & MSP SOLE & $\begin{array}{c}\text { RNOHA } \\
\text { IHEE!IGSLS }\end{array}$ & $\begin{array}{l}\text { LNFELE TO } \\
\text { CLNTACT }\end{array}$ & $\begin{array}{c}\text { Wh SAMPIS } \\
\text { PRiHW }\end{array}$ & $\begin{array}{c}\text { ELESIOHLITY } \\
\text { RATS? }\end{array}$ & $\begin{array}{l}\text { TUTA } \\
\text { MWELIGgLES }\end{array}$ & $\begin{array}{l}\text { TOTEL WGP } \\
\text { POPULEIIOK }\end{array}$ & $\begin{array}{l}\text { ELI6IRLE HES } \\
\text { DONELLATION }\end{array}$ \\
\hline 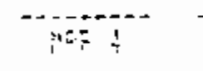 & $1+34 !$ & - & : & ? & $=\quad 1.8$ & anto & 15 & $1: 50$ \\
\hline$[H \mathrm{HF}$ & 1.54 & & 4 & 2 & 5.5233 & D. 1000 & 790 & $5 B t .67$ \\
\hline UP ! & Inblig! & & 0 & 3 & 0.7303 & 90000 & 72 & 52,90 \\
\hline 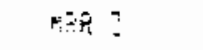 & 146402 & & $!$ & 2 & 1.5000 & 0.090 & 177 & $177.6 \%$ \\
\hline MRE 3 & $14640\}$ & & 0 & 2 & 0.9256 & 0.000 & $: 24$ & 115.14 \\
\hline MST 4 & 146494 & & $!$ & 2 & 0.7500 & i. 0000 & $2 i !$ & 138.25 \\
\hline 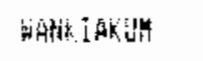 & 1464 & & 2 & 9 & $0 . \overline{4519}$ & 0.6000 & 504 & 523.19 \\
\hline OTTES SAHPt & & & & 16 & 6.9211 & 0.6000 & 1394 & 1109.86 \\
\hline ST4 2TLTES-5 & & & & $N A$ & & & & \\
\hline SUEETRATUM & & & & NA & & 0.0000 & 1354 & 1109.86 \\
\hline STKATUH TOT & & & & $N A$ & & 0.0000 & $20 E 43$ & 15153.56 \\
\hline \multicolumn{9}{|l|}{ AESIOH 5} \\
\hline 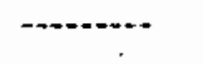 & & & & & & & & \\
\hline MPR I & $14: 50 !$ & & 0 & 1 & 0.7500 & 0.0000 & 584 & 938.00 \\
\hline 证柴 ? & 148502 & & 0 & 1 & 0.7673 & 0.0000 & 518 & 27.66 \\
\hline$\times F$ & 14951 & & 0 & : & 0.8616 & 0.0900 & 165 & 14215 \\
\hline ARA 4 & 142504 & & 0 & i & 1.0000 & 0.0000 & 598 & 399.00 \\
\hline MRR 5 & 148505 & & 0 & 1 & 0.8975 & 0.0000 & 368 & 330.27 \\
\hline Bifk 3 & 148506 & & 1 & 1 & 1.0000 & 0.0000 & 1058 & 0.00 \\
\hline MRR 7 & 148567 & & 0 & 1 & 0.9375 & 0.0000 & 466 & $43 t .8 B$ \\
\hline Men 8 & 148508 & & 0 & 1 & 0.7800 & 0.00010 & 482 & 375.96 \\
\hline MIDSTATE & 1495 & & 1 & 9 & 0.8872 & 0.0000 & 4540 & 2949.93 \\
\hline ARA： & 149501 & & 1 & 1 & 0.7333 & 0.0000 & $8 i$ & 0.60 \\
\hline MYP? & 14002 & & 0 & 1 & .0000 & 0.0000 & 80 & 80.00 \\
\hline MRT ? & 179503 & & 1 & 1 & 0.9333 & 0.0000 & 152 & ง. 90 \\
\hline MFR 4 & 149504 & & 0 & 1 & 0.8667 & 0.0000 & 126 & 169.20 \\
\hline MRR 5 & 149505 & & 0 & : & 0.9333 & 0.0000 & 114 & 100.40 \\
\hline MRR $\$$ & 149506 & & $!$ & 1 & 0.9533 & 0.0000 & 82 & 0.00 \\
\hline$m 7$ & 249507 & & $\vdots$ & & 0.9289 & 0.0000 & 105 & 9.00 \\
\hline MFR 9 & 149508 & & 0 & 1 & 1.0000 & 0.0000 & 90 & 90.00 \\
\hline UMATILLA & 1495 & & 4 & 8 & 0.0161 & 0.0000 & $84 !$ & 385.60 \\
\hline LTUTES SAHFL & & & & 16 & 0.8980 & 0.0000 & $538 !$ & 3355.53 \\
\hline UTH UTLTES-2 & & & & NG & & & & \\
\hline JUESTFATH & & & & NG & & 3.6000 & 5081 & 33.5 .53 \\
\hline MER I & 150501 & & 0 & 1 & 1.0000 & 0.0000 & 199 & 199.00 \\
\hline MhS 2 & 150.502 & & 0 & 1 & 1.0000 & 0.0090 & 198 & 288.00 \\
\hline MRF 3 & 150503 & & 0 & : & 1.6000 & 0.10000 & !os & $16 n .60$ \\
\hline
\end{tabular}




\begin{tabular}{|c|c|c|c|c|c|c|c|c|}
\hline ENTITY & ARP CODE & $\begin{array}{c}\text { KNOHA } \\
\text { INELIGELS }\end{array}$ & $\begin{array}{l}\text { JJHAELE TO } \\
\text { CONTACT }\end{array}$ & $\begin{array}{l}\text { MKR SAMPLE } \\
\text { JRAWN }\end{array}$ & $\begin{array}{l}\text { ELIGIEILITY } \\
\text { RATIO }\end{array}$ & $\begin{array}{c}\text { TOFAL } \\
\text { INELIGELES }\end{array}$ & $\begin{array}{l}\text { TOTAL KRR } \\
\text { POPLLLATIOK }\end{array}$ & $\begin{array}{l}\text { ELIGIGLE HRR } \\
\text { PGPULATIDN }\end{array}$ \\
\hline 的的 & 1eses & -------- & i & $\cdots-\cdots+{ }_{1}$ & - & 9. & 150 & $\cdots, 0$ \\
\hline $\mathrm{m} F$ & 151505 & & 0 & 1 & 0.9337 & 3.1000 & 205 & 91.3 \\
\hline MER & 150500 & & 0 & $i$ & 0.7857 & 0.0000 & 71 & 55.79 \\
\hline siti 7 & 150507 & & 1 & $i$ & 0.6444 & 0. & 141 & 0.00 \\
\hline RP 8 & 159508 & & 0 & 1 & 0.7333 & 0.6000 & 167 & 122.47 \\
\hline CDALABIA & 1505 & & 2 & 8.110 & 0.8764 & 0.0000 & 1267 & 922.59 \\
\hline MRR 1 & $15 ! 501$ & & 0 & 1 & 0.9286 & 0.0000 & 103 & 95.54 \\
\hline MER 2 & 151502 & & 0 & 1 & 0.8571 & 0.0000 & 142 & $12 ! .71$ \\
\hline MRR I & $15150 ?$ & & 1 & 1 & 1.0000 & 0.0000 & 112 & 0.00 \\
\hline MRR 4 & 151504 & & $!$ & 1 & 1.0000 & 0.0000 & 1.34 & 0.60 \\
\hline MFR 5 & 151505 & & 0 & 1 & 1.0000 & 0.0000 & $1 B 2$ & 182.00 \\
\hline MRR 6 & 151506 & & 0 & 1 & 1.0000 & 0.0000 & 130 & 130.00 \\
\hline MRE 7 & 151567 & & 0 & 1 & 1.0000 & 0.0000 & 112 & 112.00 \\
\hline ARP 8 & 151500 & & 1 & 1 & 1.0000 & 0.0000 & 99 & 0.00 \\
\hline HLTN-FREEKTR & 1515 & & 3 & $B$ & 0.9741 & 0.0000 & 1014 & 641.36 \\
\hline UTLTES SARPL & & & & 16 & 0.9253 & 0.0000 & 2281 & 1563.94 \\
\hline OTH SAMPL-5 & & & & WA & & & & \\
\hline SLESTRATUM & & & & $\mathrm{NA}$ & & 0.0000 & 2281 & 1563.94 \\
\hline STRATA TOT & & & & $N F$ & & 0.0000 & $7 b b 2$ & 4899.47 \\
\hline \multicolumn{9}{|l|}{ REGIOH b } \\
\hline ------ & & & & & & & & \\
\hline MRR 1 & 153601 & & 0 & 2 & 0.9289 & 0.0000 & 2741 & 2546.08 \\
\hline MRR 2 & 153602 & & 1 & 2 & 1.0000 & 0.0000 & 2913 & 2913.00 \\
\hline HRR 3 & 153603 & & 2 & 2 & 0.8367 & 0.0000 & 3020 & 0.00 \\
\hline MPA 4 & 153604 & & 0 & 2 & 0.9333 & 0.0000 & 2774 & 2589.07 \\
\hline MRR 5 & 153605 & & $i$ & 2 & 1.0000 & 0.0000 & 2538 & 2378.00 \\
\hline HRR 6 & 153606 & & 1 & 2 & 0.9235 & 0.0000 & 2412 & 2227.41 \\
\hline EUGENE UTR & 1536 & & 5 & 12 & 0.9384 & 0.0000 & 16199 & 12613.56 \\
\hline MRR ! & 154001 & & ij & 2 & 0.9289 & 0.0000 & 192 & 179.35 \\
\hline MRE 2 & 154602 & & 0 & & 0.5833 & & & 0.00 \\
\hline MRR 3 & 154603 & & 0 & & 0.8929 & & & 0.00 \\
\hline MRR 4 & 154604 & & 0 & 2 & 0.7515 & 0.0000 & 127 & 95.44 \\
\hline MRR 5 & 154605 & & 2 & 2 & 0.9289 & 0.0000 & 136 & 0.00 \\
\hline KiR 6 & 154606 & & 1 & 2 & 1.0000 & 0.0000 & 209 & 209.00 \\
\hline CNTRL-LMCLK & 1546 & & 3 & 8 & 0.9533 & 0.0000 & 664 & 482.78 \\
\hline UALJES SAMPL & & & & 20 & 0.8983 & 0.0000 & 16862 & 13095.34 \\
\hline DTH UTLTES-O & & & & RA & & & & \\
\hline SUBSTRATUH & & & & $\mathrm{HA}$ & & 0.0000 & 16862 & 13096.34 \\
\hline MRR I & 155601 & & 0 & 2 & 0.9244 & 0.0000 & 379 & 350.36 \\
\hline
\end{tabular}

C. 9 


\begin{tabular}{|c|c|c|c|c|c|c|c|c|}
\hline ENTITY & MAE CODE & $\begin{array}{c}\text { MrJWh } \\
\text { MEL IGESS }\end{array}$ & $\begin{array}{l}\text { DHABE TA } \\
\text { CEATELT }\end{array}$ & $\begin{array}{l}\text { MER SAKELE } \\
\text { DKAWN }\end{array}$ & $\begin{array}{c}\text { ELIGI3LLITY } \\
\text { Rüito }\end{array}$ & $\begin{array}{c}\text { TDTAL } \\
\text { INELIGBLES }\end{array}$ & $\begin{array}{l}\text { TOTAL MRR } \\
\text { FEPULATIOH }\end{array}$ & $\begin{array}{l}\text { SLIGIELE ARP } \\
\text { FTPULATCON }\end{array}$ \\
\hline P & 法 & $\cdots$ & $i$ & & 1.40 & Binging & 450 & 750.10 \\
\hline 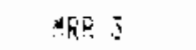 & izjoit? & & $i$ & & 1.200 & 3.500 & $\approx 15$ & 0.00 \\
\hline the 4 & Etetil & & 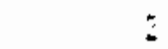 & & 1.5000 & 0.900 & 76 & 0.00 \\
\hline MFin 5 & 155505 & & 1 & 3 & 0.8357 & 0.0000 & 470 & 393.27 \\
\hline Mfe & $15: 5690$ & & 0 & & 1.0090 & 0.0000 & 316 & 316.00 \\
\hline SPEIIGEF IELD & 1556 & & 5 & 12 & 0.9616 & 0.0000 & 2206 & 1509.63 \\
\hline M⿰R口 1 & ¿5bou! & & 2 & & 0.8489 & 0.0000 & 53 & 0,00 \\
\hline MFR 2 & $\{5660\}$ & & 1 & & ij. 9333 & 0.0000 & 58 & 53.47 \\
\hline 訨是 & $15 b t e ?$ & & 1 & & 0.8000 & 0.0900 & 70 & 56.00 \\
\hline $\operatorname{Pen} 4$ & 15 secil & & 2 & & 0.9533 & 0.0000 & 157 & 0.00 \\
\hline MFP 5 & 156005 & & 1 & & 0.8667 & 0.0000 & $5 !$ & 44.20 \\
\hline PRR ó & 150606 & & 0 & 2 & 1.9000 & 0.0000 & 107 & 107.00 \\
\hline COHSUHERS & $15 b 6$ & & 7 & $1:$ & 0.8970 & 0.0000 & 506 & 270.67 \\
\hline WTLTES SAMPL & & & & 24 & 0.9291 & 0.6000 & 2712 & 1760.30 \\
\hline OTH UTLTES-2 & & & & WA & & & & \\
\hline SUBSTKAFUH & & & & $N A$ & & 0.0000 & 2712 & 1780.30 \\
\hline MaE I & 157601 & & 0 & & 1.9289 & 6.0000 & $: 07$ & 99.39 \\
\hline NF 2 & 157502 & & $\hat{\jmath}$ & 2 & 0.9194 & 9.0000 & 125 & 114.60 \\
\hline SARR J & 157603 & & 0 & & i. 6265 & 0.0000 & 96 & 79.35 \\
\hline MRR A & 157604 & & 0 & : & 1.0000 & 0.0000 & 115 & 115.00 \\
\hline ASHLAND & 1576 & & 0 & & 0.9186 & 0.0000 & 443 & 408.53 \\
\hline KER I & 158601 & & 1 & : & 0.8667 & 0.0000 & 105 & 91.00 \\
\hline ARR 2 & 158602 & & 0 & & 0.9333 & 0.0000 & 61 & 56.93 \\
\hline MRR 3 & 158603 & & 2 & : & 0.5667 & 0.0000 & 152 & 0.00 \\
\hline$M R R \quad 4$ & 150604 & & $!$ & & 1.8000 & 0.0000 & 137 & 177.00 \\
\hline FUREST GROVE & 1586 & & 4 & 8 & 0.7167 & 0.0000 & 455 & 284.93 \\
\hline UTLTES SAMPL & & & & 1 & 0.9175 & 0.10000 & 898 & 693.47 \\
\hline OTH LTLTEE-4 & & & & NA & & & & \\
\hline SUBSTRATUM & & & & $N A$ & & 0.0060 & 899 & 690.47 \\
\hline $\operatorname{MRR} 1$ & 159601 & & 0 & 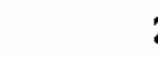 & 0.9134 & 0.0000 & 450 & 415.27 \\
\hline Nen 2 & 159602 & & 0 & & 1.0060 & 0.0000 & 530 & 570.00 \\
\hline MRR 3 & 159603 & & 2 & : & 0.9578 & 0,5000 & 499 & 0.00 \\
\hline HiR \& & 157694 & & ! & ; & 0.9289 & 0.0000 & 458 & 425.43 \\
\hline SEAnẼHLi-LÂHE & 1596 & & $\vdots$ & 8 & 6.595 & 2.0000 & 1927 & 1360.70 \\
\hline Hisk ! & $160 \leq 01$ & & $i$ & & 0.9578 & $0.00,0$ & 138 & 375.71 \\
\hline WRR 2 & 100602 & & $\vartheta$ & ; & 0.9333 & 0.9000 & 299 & 279.07 \\
\hline Mac 3 & 160603 & & $z$ & 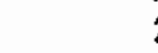 & 0.9269 & ). 0000 & 413 & 0.20 \\
\hline
\end{tabular}




\begin{tabular}{|c|c|c|c|c|c|c|c|c|}
\hline EATITY & MRF CGDE & $\begin{array}{c}\text { KMOHW } \\
\text { IMELIGBLS }\end{array}$ & $\begin{array}{l}\text { UNABELE TO } \\
\text { CONTACT }\end{array}$ & $\begin{array}{c}\text { MFE SAMFLE } \\
\text { DRAHN }\end{array}$ & $\begin{array}{l}\text { ELIGIBILITY } \\
\text { RATIU }\end{array}$ & $\begin{array}{l}\text { TOTAL } \\
\text { INELIGELES }\end{array}$ & $\begin{array}{l}\text { TOTAL MRR } \\
\text { PIPIJLATION }\end{array}$ & $\begin{array}{l}\text { ELIGIBLE MRR } \\
\text { POPULATISN }\end{array}$ \\
\hline 4 & isidgis & & 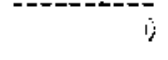 & 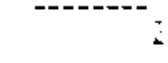 & 1. $)$ & 0.1300 & 155 & 15.90 \\
\hline Makguts & $: 506$ & & 3 & 8 & 0.9360 & 0.9060 & 1303 & 007.77 \\
\hline UFLTES SFHEL & & & & 16 & 0.9270 & 0.6000 & .3230 & 2176.47 \\
\hline OTH UTLTES-A & & & & :A & & & & \\
\hline SUESTRATIJM & & & & NA & & 6.0000 & .330 & 2176.47 \\
\hline STRÁTIH TOT & & & & $N A$ & & 0.0000 & 23702 & 17746.59 \\
\hline PUALIE TOT & & & & $N A$ & & 3.0667 & 85560 & 58525.83 \\
\hline \multicolumn{9}{|l|}{ FRIVATE } \\
\hline \multicolumn{9}{|l|}{ KESION I } \\
\hline -------" & & & & & & & & \\
\hline MRR 1 & 101401 & & 0 & 1 & 1.0000 & 0.0000 & 73 & 73.00 \\
\hline MRR 2 & 101102 & & 1 & 1 & 0.7333 & 0.0000 & 55 & 0.00 \\
\hline MRR 3 & 101103 & & 0 & 1 & 0.8578 & 0.0000 & 132 & 113.23 \\
\hline MRR 4 & 101104 & & 0 & 1 & 0.8000 & 0.0600 & 121 & 96.80 \\
\hline MRR 5 & 101105 & & 0 & 1 & 0.9333 & 0.0000 & 54 & 50.40 \\
\hline Mep $b$ & 101106 & & 1 & ! & $! .6000$ & 0.3000 & 156 & 1.09 \\
\hline MikR 7 & 101107 & & 0 & 1 & 1.0000 & 0.0000 & 500 & 500.00 \\
\hline MRR 8 & 101108 & & 0 & 1 & 0.9333 & 0.0000 & 435 & 406.00 \\
\hline HRR 9 & 101109 & & 0 & 1 & 1.0000 & 0.0000 & 411 & 411.00 \\
\hline MRR 10 & 101110 & & 0 & 1 & 0.8578 & 0.0000 & 544 & 466.63 \\
\hline MRR 11 & 101111 & & 1 & 1 & 0.9289 & 0.0000 & 345 & 0.00 \\
\hline GRR 12 & 101112 & & 0 & 1 & 0.9244 & 0.0000 & 153 & 141.44 \\
\hline MRR 13 & 101113 & & 0 & 1 & 0.9289 & 0.0000 & 132 & 122.61 \\
\hline MRR 14 & 101114 & & 0 & 1 & 0.9367 & 0.0000 & 26 & 21.76 \\
\hline MKR 15 & 101115 & & 1 & 1 & 0.9286 & 0.0000 & 358 & 0.00 \\
\hline MRR 16 & 101116 & & 1 & 1 & $0.755 !$ & 0.0000 & 167 & 0.100 \\
\hline $\begin{array}{l}\text { MONTANA PHK } \\
\text { SUBSTRATUMH }\end{array}$ & 1011 & & 5 & 16 & 0.8996 & 0.0000 & 3662 & 2402.87 \\
\hline
\end{tabular}

\begin{tabular}{|c|c|c|c|c|c|c|c|}
\hline HRR ! & 102101 & 0 & 1 & 0.8367 & 0.0000 & 194 & 162.33 \\
\hline MRR 2 & 102102 & 0 & 1 & 0.8000 & 0.0000 & 267 & 213.60 \\
\hline HRR 3 & 102103 & 0 & 1 & 0.8667 & 0.0000 & 262 & 227.07 \\
\hline MRR 4 & 102104 & 0 & 1 & 0.7867 & 0.0000 & 121 & 95.19 \\
\hline Ka 5 & 102105 & 1 & 1 & 0.6667 & 0.0000 & 79 & 0.10 \\
\hline MER 6 & 102106 & 0 & 1 & 0.7398 & 0.00000 & $14 !$ & 104.31 \\
\hline ARR 7 & 102107 & 0 & 1 & 0.7353 & ป. 0000 & i7) & 158.67 \\
\hline MPR 8 & 102108 & 0 & 1 & 0.7957 & 0.0000 & 211 & 165.79 \\
\hline MRR ? & 102109 & 0 & 1 & 1.0000 & 0.0000 & 154 & 154.60 \\
\hline MRR 10 & 102110 & 1 & 1 & 0.9333 & 0.0000 & 317 & 0.00 \\
\hline HRR I! & 102111 & 0 & 1 & 0.9289 & 0.0000 & 208 & 197.21 \\
\hline
\end{tabular}

C. 11 


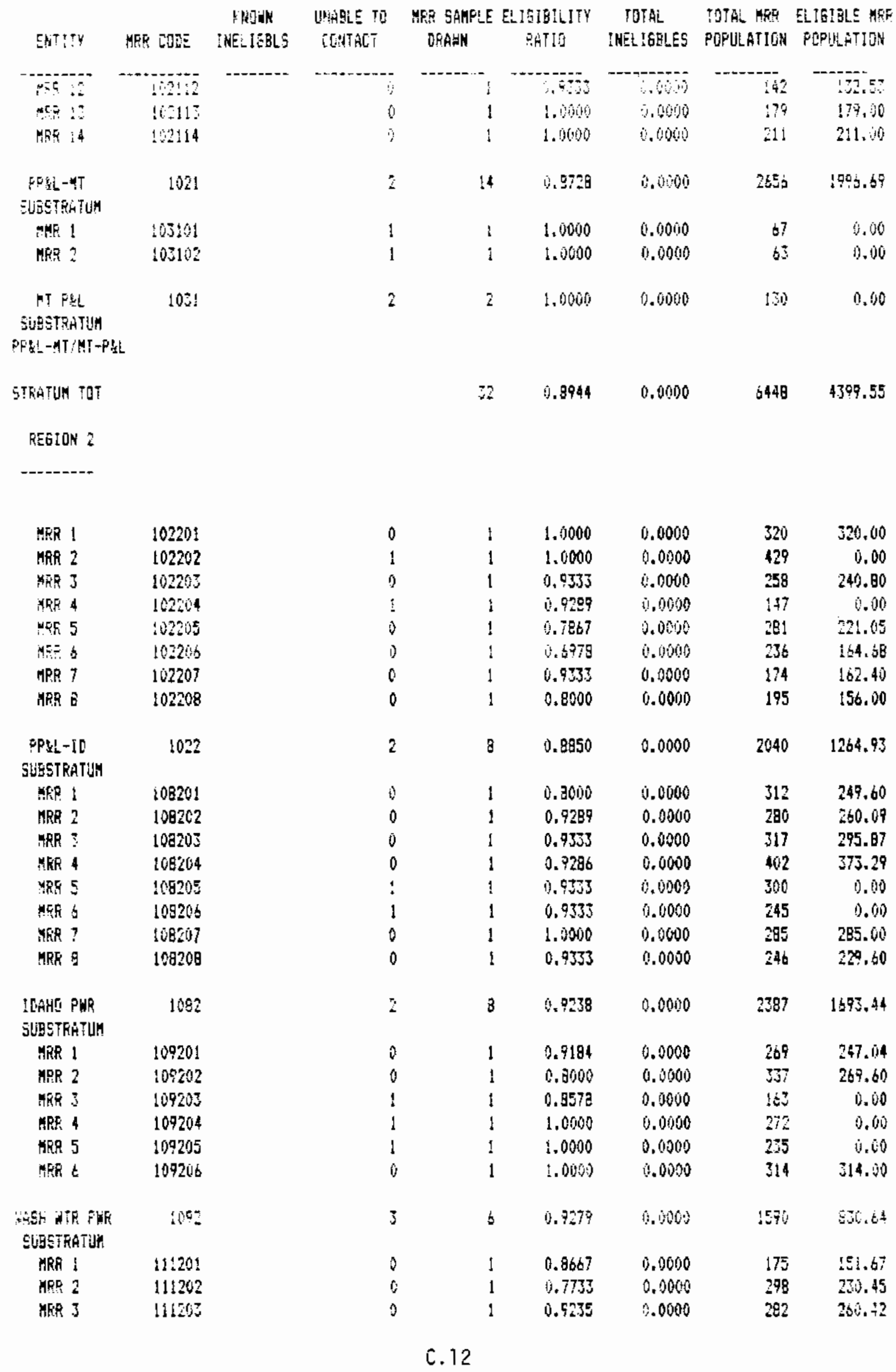




\begin{tabular}{|c|c|c|c|c|c|c|c|c|}
\hline ENTITY & Hā $\quad$ CDLE & $\begin{array}{c}\text { RNOWN } \\
\text { INELIGALS }\end{array}$ & $\begin{array}{c}\text { URABLE TO } \\
\text { CONIACT }\end{array}$ & $\begin{array}{c}\text { MRE SARFLE } \\
\text { ORASHA }\end{array}$ & $\begin{array}{c}\text { ELLEISILITY } \\
\text { PATIO }\end{array}$ & $\begin{array}{c}\text { TOTAL } \\
\text { INELIGBLES }\end{array}$ & $\begin{array}{l}\text { TOTAL BER } \\
\text { POPLLATIOH }\end{array}$ & $\begin{array}{l}\text { ELIGIELE KRR } \\
\text { POPLIATIINN }\end{array}$ \\
\hline-4 & 1134 & $\cdots$ & ? & 1 & 3800 & 9. & 432 & 4.90 \\
\hline MET 5 & $11: 205$ & & $\theta$ & 1 & 1.0000 & 1. 6000 & 151 & $(51.6)$ \\
\hline a & $\pm: 1216$ & & 0 & 1 & 0.7354 & 0.5610 & 138 & 101.20 \\
\hline MRP 7 & 111207 & & 1 & 1 & 0.7353 & $0.9001 j 0$ & 120 & 0.20 \\
\hline WRE 8 & 111208 & & 0 & 1 & 8.8067 & 0.9000 & 116 & 100.53 \\
\hline $\begin{array}{l}\text { UTAH PHL } \\
\text { SLBCTRATUH }\end{array}$ & $1 ! ! 2$ & & 2 & 8 & 0.8616 & 0.0000 & 1712 & 995.27 \\
\hline ETRATIM TIJT & & & & 30 & 0.8975 & 0.6000 & 7729 & 4784.28 \\
\hline
\end{tabular}

kegIon:

\begin{tabular}{|c|c|c|c|c|c|c|c|}
\hline ARR 1 & 102301 & 0 & 1 & 1.0000 & 0.0000 & 413 & 413.90 \\
\hline MER 2 & 102302 & 1 & 1 & 1.0000 & 0.0000 & 406 & 0.00 \\
\hline MRR 3 & 102303 & 1 & 1 & 1.0000 & 0.0000 & 291 & 0.60 \\
\hline $\operatorname{MRR} 4$ & 102304 & 0 & 1 & 0.9000 & 0.0600 & 197 & 257.60 \\
\hline MRR 5 & 102305 & 0 & 1 & 1.0000 & 0,0000 & 274 & 274.10 \\
\hline MRR 6 & 102306 & 0 & 1 & 1.0000 & 0.0000 & 189 & 189.90 \\
\hline MRR 7 & 102307 & 0 & 1 & 1.0000 & 0.0000 & 186 & 186.00 \\
\hline MRR $\mathrm{g}$ & 102308 & 0 & 1 & 1.0000 & 0.0000 & 332 & 332.00 \\
\hline HRR 9 & 102309 & 0 & 1 & 0.9333 & 0.0000 & 390 & 364.00 \\
\hline HRR 10 & 102310 & 0 & 1 & 0.9333 & 0.0000 & $16 !$ & $15 \% .27$ \\
\hline HER $\pm !$ & 102311 & 1 & 1 & 0.9667 & 0.10000 & 212 & 0.00 \\
\hline HEE 12 & 102312 & 0 & 1 & 0.9296 & 0.0000 & 274 & 254.43 \\
\hline AKR 13 & 102313 & 0 & 1 & 1.0000 & 0.0000 & 237 & 237.00 \\
\hline MRR 14 & 102314 & 0 & 1 & 1.0000 & 0.0000 & 226 & $22 b .00$ \\
\hline MRR 15 & 102315 & 0 & 1 & 0.7733 & 0.0000 & 314 & 242.83 \\
\hline MRR 16 & 102316 & 1 & 1 & 1.0000 & 0.0000 & 373 & $i .00$ \\
\hline$P P \& L-H A$ & 1023 & 4 & 16 & 0.9521 & 0.0000 & 4475 & 3026 \\
\hline \multicolumn{8}{|l|}{ SUBSTRATUH } \\
\hline MRR I & 109301 & 1 & 1 & 0.9333 & 0.0000 & 63 & 0.00 \\
\hline MRR 2 & 109302 & 0 & 1 & 1.0000 & 0.0000 & 252 & 252.00 \\
\hline WRE 3 & 109303 & 0 & 1 & 1.0000 & 0.0000 & 254 & 254.00 \\
\hline MRR 4 & 109304 & 0 & 1 & 1). B667 & 0.0000 & 328 & 284.27 \\
\hline MRR 5 & 109305 & $!$ & 1 & 0.8667 & 0.0090 & 172 & 0.00 \\
\hline MRR 6 & 109306 & 1 & 1 & 1.0000 & 0.0000 & 357 & 0.00 \\
\hline KRR 7 & 109307 & 0 & 1 & 1.0000 & 0.0000 & 229 & 229.00 \\
\hline MRP 9 & $10930 \mathrm{~B}$ & 0 & 1 & 0.7333 & 0.0000 & 359 & 26.3 .27 \\
\hline MFK 9 & 109309 & 1 & 1 & 1.0000 & 0.0000 & 272 & $\ddot{0} .00$ \\
\hline MRR 10 & 109310 & 1 & 1 & 0.8311 & 0.0000 & 404 & 0.00 \\
\hline MRR 11 & 109311 & 1 & 1 & 0.9333 & 0.0000 & 424 & 0.00 \\
\hline KRR 12 & 109312 & 0 & 1 & 0.9235 & 0.0000 & 135 & $\{24,6\}$ \\
\hline MRR IJ & 109313 & 1 & 1 & 1.0000 & 0.0000 & 323 & 0.00 \\
\hline MRR 14 & 109314 & 0 & 1 & 1.0000 & 0.0000 & 291 & 291.00 \\
\hline MER 15 & 109315 & 1 & 1 & 0.9235 & 0.0000 & 233 & 0.00 \\
\hline KRR 16 & 169316 & 1 & 1 & 0.7735 & 0.0000 & 193 & 0.00 \\
\hline $\begin{array}{l}\text { HASH WAT PUR } \\
\text { SUESTRATUG }\end{array}$ & 1093 & 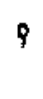 & 16 & 0.9234 & 0.0000 & 4289 & 1698.20 \\
\hline STRATUM TOT & & & 32 & 0.9378 & 0.0000 & 4617 & 4724.32 \\
\hline
\end{tabular}




\begin{tabular}{|c|c|c|c|c|c|c|c|c|}
\hline ENTITY & MRR COSE & $\begin{array}{c}\text { INOHA } \\
\text { INELISELS }\end{array}$ & $\begin{array}{l}\text { UNABLE Ti } \\
\text { CENTACT }\end{array}$ & $\begin{array}{c}\text { MFR SAHFLE } \\
\text { OFA木AN }\end{array}$ & $\begin{array}{c}\text { ELIGISILITY } \\
\text { RATLO }\end{array}$ & $\begin{array}{l}\text { IOTAL } \\
\text { IKELIGBLES }\end{array}$ & $\begin{array}{l}\text { TOTAL MFR } \\
\text { PUPLIATIION }\end{array}$ & $\begin{array}{l}\text { ELIGIELE MRP } \\
\text { POPULATIOH }\end{array}$ \\
\hline 影赫 4 & --- & $\cdots$ & & --- & - & $x+\infty-\infty-\infty$ & & \\
\hline$M R R$ & 154401 & & : & 2 & 9.9286 & 0.6000 & 344 & 317.43 \\
\hline HFR 2 & 134422 & & 2 & 2 & 1.9060 & b. Dingo & 299 & 0.00 \\
\hline 貿 3 & $1344 i)^{7}$ & & $\theta$ & 2 & 1.0000 & 0.1000 & 249 & 247.90 \\
\hline MER 4 & 134404 & & 2 & 2 & 0.9735 & 6. & 262 & 0.00 \\
\hline Papk 5 & 174405 & & 0 & $z$ & 1.0000 & 0.0000 & 354 & 354.00 \\
\hline a & 134406 & & 0 & 2 & 1.0000 & $0.0,000$ & 280 & 280.00 \\
\hline 斯 7 & 134407 & & 1 & $?$ & 0.8667 & 0.9000 & 263 & 227.97 \\
\hline YRE & 134400 & & $!$ & $\frac{2}{2}$ & 1.0000 & 0.10000 & $14 !$ & 141.00 \\
\hline KRP ? & 134409 & & 1 & 2 & 0.9289 & 9.6000 & 205 & 561.58 \\
\hline ME 10 & $1 ; 4410$ & & 1 & 2 & 0.9286 & 0.6000 & 227 & 210.79 \\
\hline MRR II & $1344 i 1$ & & 1 & 2 & 0.9244 & 0.0000 & 468 & 432.64 \\
\hline$M+2$ & 134412 & & ! & 2 & 0.7600 & 0.0000 & 239 & 181.64 \\
\hline KRR 13 & 134413 & & 1 & 2 & 1.0000 & 0.0000 & 203 & 205.00 \\
\hline AF 14 & 13414 & & 0 & 2 & 0.9259 & 0.0000 & 226 & 279.93 \\
\hline MFt 15 & 134415 & & 1 & 2 & 0.9244 & 0.0000 & 603 & 557.44 \\
\hline GRR 16 & 134416 & & $:$ & 2 & 1.0000 & 0.0000 & 185 & 185.00 \\
\hline $\begin{array}{l}\text { FUGET SOLNE } \\
\text { SLESTEATUNA }\end{array}$ & 1344 & & 14 & 32 & 0.9441 & 0.0000 & $494 B$ & 4113.77 \\
\hline STRGTLH TIT & & & & 32 & 0.9441 & 0.0600 & $494 \mathrm{~B}$ & 4113.77 \\
\hline
\end{tabular}

TEeron 5

\begin{tabular}{|c|c|}
\hline MRR 1 & 102501 \\
\hline MeR 2 & 102502 \\
\hline MRR 3 & 102503 \\
\hline MRS 4 & 102504 \\
\hline MER 5 & 102505 \\
\hline rRR o & 102506 \\
\hline$M R R 7$ & 102507 \\
\hline MES 5 & 10250 \\
\hline WhP & 102509 \\
\hline YKR 10 & 102510 \\
\hline MRR 11 & $10251 !$ \\
\hline MRR 1 ? & 102512 \\
\hline MRR 13 & 107513 \\
\hline GRR I4 & 102514 \\
\hline MFR IS & 102515 \\
\hline ARP is & 102516 \\
\hline$F P L=D R-\bar{c}$ & $! 025$ \\
\hline \multicolumn{2}{|l|}{ SUDSTRATUY } \\
\hline MeP 1 & 108501 \\
\hline MET 2 & 169502 \\
\hline 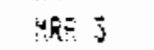 & inescis \\
\hline 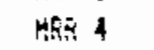 & 218504 \\
\hline $\operatorname{MR} 5$ & 100505 \\
\hline Mat 6 & $10950 \mathrm{c}$ \\
\hline AR? & 108507 \\
\hline
\end{tabular}




\begin{tabular}{|c|c|c|c|c|c|c|c|c|}
\hline EMTITY & MRP CULE & $\begin{array}{c}\text { MNIWN } \\
\text { IHELLEELS }\end{array}$ & $\begin{array}{l}\text { WNAELE TD } \\
\text { CONTACT }\end{array}$ & $\begin{array}{l}\text { MER SAMPLE } \\
\text { DRALHA }\end{array}$ & $\begin{array}{c}\text { ELIGIRILITY } \\
\text { RRTIO }\end{array}$ & $\begin{array}{c}\text { TD'AL } \\
\text { INELIGELEE }\end{array}$ & $\begin{array}{l}\text { TOTAL MFR } \\
\text { POPILATIIDY }\end{array}$ & $\begin{array}{l}\text { ELIGIBLE MRR } \\
\text { PQPULATIST }\end{array}$ \\
\hline 4695 & $-\operatorname{lig} 5$ & & 6 & 1 & 1,6 into & 10.00 & 39 & 78.96 \\
\hline 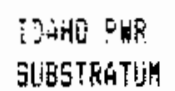 & 1925 & & 2 & 8 & 6.9739 & 0.0100 & $2 \% 52$ & 1449.27 \\
\hline MFR 1 & 147801 & & 1 & $!$ & 0.9235 & 0.0000 & 523 & 0.00 \\
\hline MFR 2 & 147502 & & 0 & $:$ & 0.7639 & 0.0000 & E47 & 647.01 \\
\hline MER 3 & 147503 & & 0 & & 0.6173 & & & 0.00 \\
\hline MFR 4 & 2475,14 & & 0 & 1 & 1.0000 & 0.0000 & 200 & 200.00 \\
\hline ARP 5 & 147505 & & 0 & & 0.1467 & & & 19.00 \\
\hline MFiR 6 & 147506 & & 1 & 1 & 0.9789 & 0.0000 & 206 & 0.00 \\
\hline MER 7 & 147507 & & 0 & & 1.0000 & & & 10.00 \\
\hline MER 8 & $! 47508$ & & 1 & 1 & 0.5733 & 0,0000 & 232 & 0.00 \\
\hline $\begin{array}{l}\text { CP NATHL } \\
\text { SUBSTRATUHA }\end{array}$ & 1475 & & 3 & 5 & 0.7410 & 0.0000 & 2008 & $8 \mathrm{a} 7.01$ \\
\hline STRRTIM TOT & & & & 28 & 0.8444 & 0.0000 & 7728 & 4600.98 \\
\hline REEION 6 & & & & & & & & \\
\hline MRR 1 & 102601 & & 0 & 1 & 0.7704 & 0.0000 & 121 & 93.22 \\
\hline MPR ? & 102602 & & 0 & 1 & 0.4000 & 0.0000 & 452 & 180.80 \\
\hline MRR S & $10260 \mathrm{~J}$ & & 1 & 2 & $\therefore .0000$ & 0.0000 & 253 & 253.60 \\
\hline MFit 4 & 102604 & & 2 & 2 & 1.0000 & $\dot{0} .0000$ & 297 & 0.100 \\
\hline M 5 & 102005 & & 1 & 2 & 1.0000 & 0.0000 & 126 & $12 t .00$ \\
\hline HRR & 102606 & & 0 & 2 & 0.8469 & 0.0000 & 223 & 188.87 \\
\hline MRR 7 & 102607 & & 1 & 2 & 0.9289 & 0.0000 & 390 & 352.98 \\
\hline MRR 8 & $10260 \mathrm{~B}$ & & 1 & 2 & 1.0000 & 0.00010 & 368 & 368.00 \\
\hline HRR 9 & 102609 & & $!$ & 2 & 0.9289 & 0.0000 & 377 & 350.19 \\
\hline MER to & 102610 & & 1 & 2 & 0.9156 & 0.0000 & 128 & 117.19 \\
\hline MRR 11 & 102611 & & 0 & 2 & 1.0000 & 0.0000 & 177 & 177.00 \\
\hline HRR 12 & 102612 & & 1 & 2 & 1.0000 & 0.0000 & 328 & 328.00 \\
\hline MRR 13 & 102613 & & 1 & 2 & 0.0553 & 0.0000 & 427 & 398.53 \\
\hline MRR 14 & 102614 & & 1 & 2 & 0.9289 & 0.0000 & 203 & 188.56 \\
\hline MER 15 & 102615 & & 0 & 2 & 1.0000 & 0.0000 & 255 & 255.00 \\
\hline MRR 16 & 102616 & & 2 & 2 & 0.9200 & 0.0000 & 158 & 0.00 \\
\hline PPAL-OR-W & 1026 & & 13 & 30 & 0.9102 & 0.0000 & 4273 & 5377.34 \\
\hline SUAGSTRATUH & & & & & & & & \\
\hline MRP I & 152601 & & 1 & 2 & 0.9244 & 0.0000 & 241 & 222.79 \\
\hline$M R R 2$ & 152602 & & 2 & 2 & 0.9578 & 0.0000 & 438 & 0.00 \\
\hline MRR 3 & 152603 & & 0 & 2 & 0.9200 & 0.0000 & $3 i 0$ & 285.20 \\
\hline HRR 4 & 152604 & & 0 & 2 & 1.0000 & 0.0000 & 415 & 415.00 \\
\hline MRR 5 & 152605 & & 0 & 2 & 1.0000 & 0.0000 & 158 & 159.00 \\
\hline MRR 6 & 152606 & & 0 & 3 & 0.8367 & 0.00040 & 320 & 207.75 \\
\hline KRR 7 & 152607 & & 1 & 3 & 0.9286 & 0.6000 & 406 & 377.00 \\
\hline MRR 8 & 152608 & & 0 & 2 & 0.9299 & 0.0000 & 279 & 259.16 \\
\hline MRR ? & 152609 & & 1 & 3 & 1.0000 & 0.0000 & 376 & 376.00 \\
\hline MRR 10 & 152610 & & $!$ & 2 & 1.00000 & 4.566 & 446 & 445.60 \\
\hline MRA II & $1526 ! 1$ & & 1 & 2 & 1.0000 & 0.0000 & 392 & 395.60 \\
\hline MRR 12 & 152612 & & 1 & 3 & 1.0000 & 0.0000 & 366 & 366.00 \\
\hline HRR 13 & 152613 & & 1 & 2 & $0.933 \mathrm{~J}$ & 0.0000 & 312 & 291.20 \\
\hline WRE 14 & 152614 & & 2 & 2 & 0.9244 & 0.0000 & 190 & 0.00 \\
\hline
\end{tabular}




\begin{tabular}{|c|c|c|c|c|c|c|c|c|}
\hline ENITY & YQR CODE & $\begin{array}{c}\text { KNONH } \\
\text { IMELIG6LS }\end{array}$ & $\begin{array}{l}\text { DNFELE TI } \\
\text { CONTACT }\end{array}$ & $\begin{array}{l}\text { WHR SAMPLE } \\
\text { ORAWN }\end{array}$ & $\begin{array}{c}\text { ELIEIBILITY } \\
\text { RATI:O }\end{array}$ & $\begin{array}{l}\text { TOTAL } \\
\text { IHELIGYLES }\end{array}$ & $\begin{array}{l}\text { TOTAL ARE } \\
\text { POPGLATISON }\end{array}$ & $\begin{array}{c}\text { ELIG1ELE MER } \\
\text { POPILATIDN }\end{array}$ \\
\hline म5: & 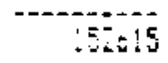 & & $i$ & 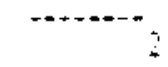 & i. & 40 & ! & 41,0 \\
\hline 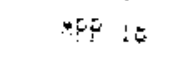 & !5oth & & 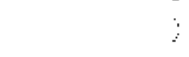 & $z$ & $\therefore 609$ & 9.000 & 47 & 407,00 \\
\hline $\begin{array}{l}\text { DOET GEX ELE } \\
\text { JUESTRATUH }\end{array}$ & 1526 & 0 & 12 & 32 & $i .9530$ & 0.0000 & 5419 & 4625.11 \\
\hline ETKATINH & & $\hat{v}$ & & 62 & 0.9315 & 0. 0000 & 9691 & 8002.45 \\
\hline FRIVATE TOT & & is & & NA & & 0.0000 & $4: 161$ & 50631.36 \\
\hline STAUE TET & & 2 & & $\begin{array}{r}21 E \\
344.60 \\
542.00\end{array}$ & $\begin{array}{l}0.9032 \\
0.9079 \\
0.9060\end{array}$ & $\begin{array}{l}3.0607 \\
0.0000 \\
3.0667\end{array}$ & 126721 & $39: 57.19$ \\
\hline
\end{tabular}

C. 16 


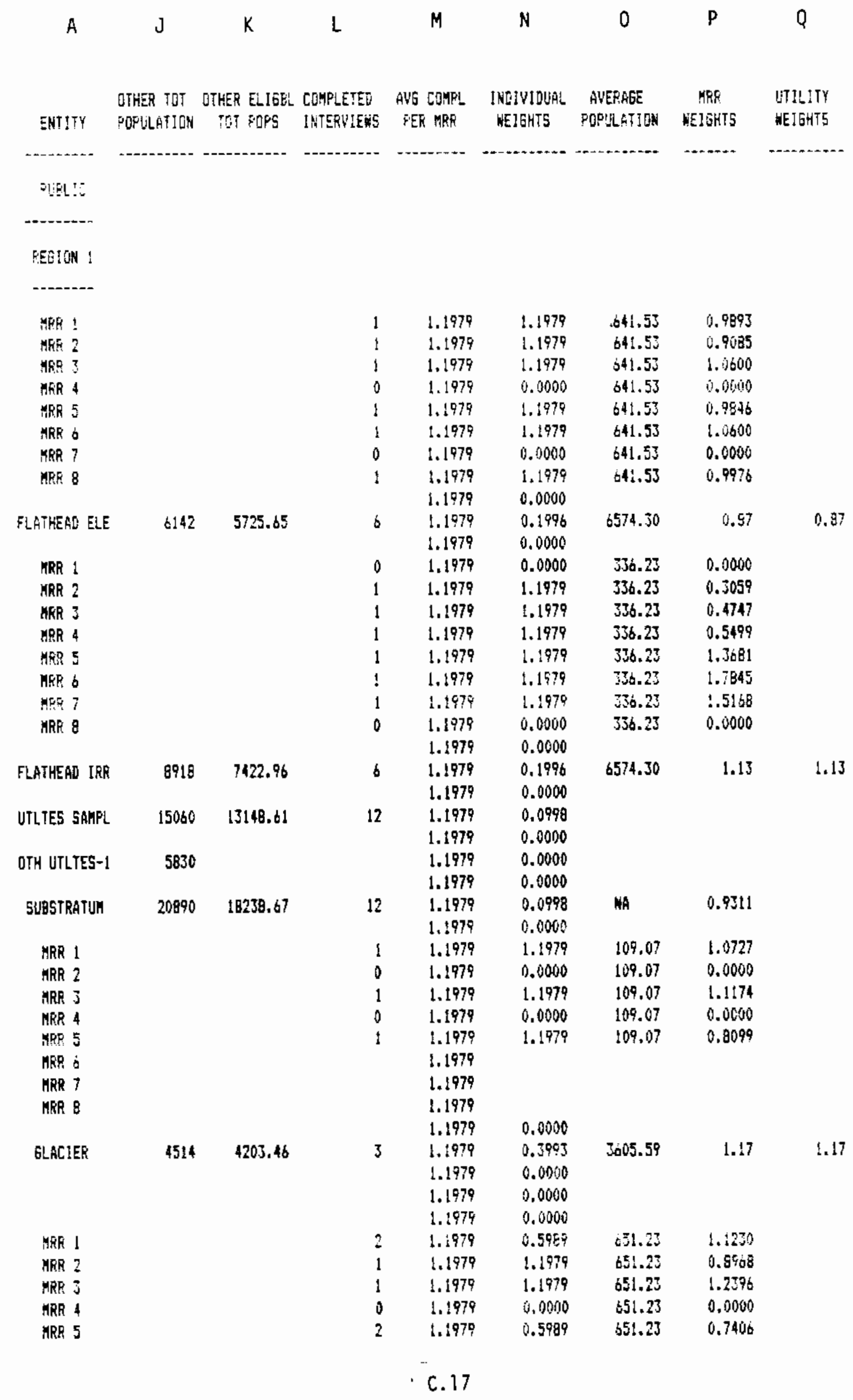




\begin{tabular}{|c|c|c|c|c|c|c|c|c|}
\hline ENTITY & $\begin{array}{l}\text { DTHEF TOT } \\
\text { PQPULATION }\end{array}$ & $\begin{array}{c}\text { OTHES EIIEE } \\
\text { TDT FGPS }\end{array}$ & $\begin{array}{l}\text { COMPLETED } \\
\text { INTERUIENS }\end{array}$ & $\begin{array}{l}\text { AVG CDNEL } \\
\text { FER MEF }\end{array}$ & $\begin{array}{l}\text { INE!VINUAL } \\
\text { HEIGHTS }\end{array}$ & $\begin{array}{l}\text { AVERAGE } \\
\text { POEEUATION }\end{array}$ & $\begin{array}{c}\text { MRG } \\
\text { HEISHTS }\end{array}$ & $\begin{array}{l}\text { UTLLIYY } \\
\text { WEIEATS }\end{array}$ \\
\hline mancon & 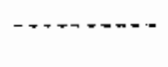 & ................ & - nom & 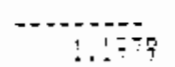 & mo & $\cdots$ & ----- & n...... \\
\hline FARLi & 207 & 257.71 & 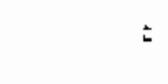 & $\begin{array}{l}1.1979 \\
1.996\end{array}$ & $\begin{array}{l}3.154 t \\
0.0600\end{array}$ & 50.58 & $\therefore 33$ & 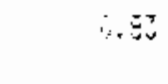 \\
\hline STLTES SAMPL & 7721 & 7211.17 & 9 & $\begin{array}{l}1.1979 \\
1.1979\end{array}$ & $\begin{array}{l}0.1301 \\
0.60000\end{array}$ & & & \\
\hline JiH UIDEE-2 & 5648 & & & $\begin{array}{l}1.1979 \\
1.1979\end{array}$ & $\begin{array}{l}0.0000 \\
0.0000\end{array}$ & & & \\
\hline SLESTRATUH & 17589 & 12504.90 & 9 & $\begin{array}{l}1.1979 \\
1.1979\end{array}$ & $\begin{array}{l}0.1331 \\
0.0000\end{array}$ & NA & 1.1209 & \\
\hline STATIUK TOT & 34279 & 30747.58 & 21 & $\begin{array}{l}1.1979 \\
1.1979\end{array}$ & $\begin{array}{l}0.0570 \\
6.0000\end{array}$ & NA & 0.16 & \\
\hline \multirow[t]{2}{*}{ GEETON 2} & & & & 1.1979 & 9. 6000 & & & \\
\hline & & & & $\begin{array}{l}1.1974 \\
i .1979\end{array}$ & $\begin{array}{l}0.0060 \\
0.0000\end{array}$ & & & \\
\hline MSI & & & 0 & 1.1979 & 0.0000 & 169.47 & 0.0000 & \\
\hline MRT 2 & & & 1 & 1.1974 & 1.1979 & 169.47 & 1.5106 & \\
\hline MPR 3 & & & 0 & 1.1979 & 0.0000 & 169.47 & 0.2000 & \\
\hline HRK 4 & & & 1 & \pm .1979 & 1.1979 & 169.47 & 1.0299 & \\
\hline$A R F 5$ & & & 1 & 1.1479 & 1.1979 & 169.47 & 0.9630 & \\
\hline \multirow[t]{2}{*}{ MER 6} & & & 1 & 1.1979 & 1.1979 & 169,47 & 0.4957 & \\
\hline & & & & 1.1979 & 0.0000 & & & \\
\hline \multirow[t]{2}{*}{ IDHHO FALLS } & 15243 & 14557.92 & 4 & 1.1979 & 0.2995 & 11128.21 & 1.31 & 1.31 \\
\hline & & & & 1.1979 & 0.0000 & & & \\
\hline MRE । & & & i & 1.1979 & 1.1979 & 103.10 & 0.7700 & \\
\hline 裸? & & & I & 1.1979 & 1.5979 & 103.10 & 0.7958 & \\
\hline MAR 3 & & & $:$ & 1.1979 & 1.1979 & 103.10 & 1.0593 & \\
\hline Mak $\neq$ & & & 0 & 1.1979 & 0.01030 & 103.10 & 0.0000 & \\
\hline MRR 5 & & & 0 & 1.1979 & 0.0000 & 103.10 & 0.0000 & \\
\hline \multirow[t]{2}{*}{ MRR 6} & & & 0 & 1.1979 & 0.0000 & 103.10 & 0.0000 & \\
\hline & & & & 1.1979 & 0.0000 & & & \\
\hline \multirow[t]{2}{*}{ LOAER YALLEY } & 8650 & 7698.51 & 3 & 1.1979 & 0,5903 & 11128.21 & 0.69 & 0.39 \\
\hline & & & & 1.1079 & 0.0000 & & & \\
\hline \multirow[t]{2}{*}{ LTLTES SAMPL } & 23973 & $22256.4 \mathrm{~J}$ & 7 & 1.1979 & 0.1711 & & & \\
\hline & & & & 1.1979 & 0.0000 & & & \\
\hline \multirow[t]{2}{*}{ DITH GTLIEE-3 } & 22722 & & & 1.1979 & 0.0000 & & & \\
\hline & & & & 1.1979 & 0.0000 & & & \\
\hline \multirow[t]{2}{*}{ SUSSTRÁTLY } & 46595 & 43435.90 & 7 & 1.1979 & $0.17 ! 1$ & WA & 1.5728 & \\
\hline & & & & 1.1979 & 0.0000 & & & \\
\hline MRR I & & & 1 & 1.1979 & 1.1979 & 351.87 & 0.7125 & \\
\hline $\operatorname{MRh} 2$ & & & & 1.1974 & & 501.97 & & \\
\hline ARE J & & & 1 & 1.1979 & 1.1979 & 351.87 & 2.1002 & \\
\hline MRR 4 & & & 0 & 1.1979 & 0.0000 & 351.87 & 0.0060 & \\
\hline 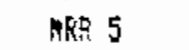 & & & 1 & 1.1979 & 1.1979 & 301.87 & 0.1973 & \\
\hline \multirow[t]{2}{*}{ Bisk } & & & 0 & 1.1979 & 0.9000 & 351.97 & 0.0100 & \\
\hline & & & & 1.1979 & $\overline{0}, 10010$ & & & \\
\hline \multirow[t]{2}{*}{ FALLL RIVER } & 8344 & 3501.92 & 3 & 1.1979 & 0.3993 & 3825.90 & 0.92 & 0.92 \\
\hline & & & & 1.1079 & 0.6000 & & & \\
\hline MEA 1 & & & 1 & 1.159 & 1.1979 & 166.21 & 1.0000 & \\
\hline \multirow[t]{2}{*}{ ERSTEND WUT } & $H_{i}$ & Hit & 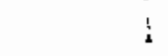 & $\therefore 1 \div 79$ & $\therefore .1979$ & 375.30 & 模 & iA \\
\hline & & & & 1.1979 & $0 . \cos 0$ & & & \\
\hline \multirow{3}{*}{$\begin{array}{l}\text { WRE I } \\
\text { FARMEES ELE }\end{array}$} & & & 1 & 1.1479 & $1 . ! 979$ & 84.60 & 1.0000 & \\
\hline & $N A$ & $\mathrm{HA}$ & 1 & 1.1979 & 1.1979 & 1275.30 & NA & NA \\
\hline & & & & $\therefore 1079$ & 0.0000 & & & \\
\hline
\end{tabular}




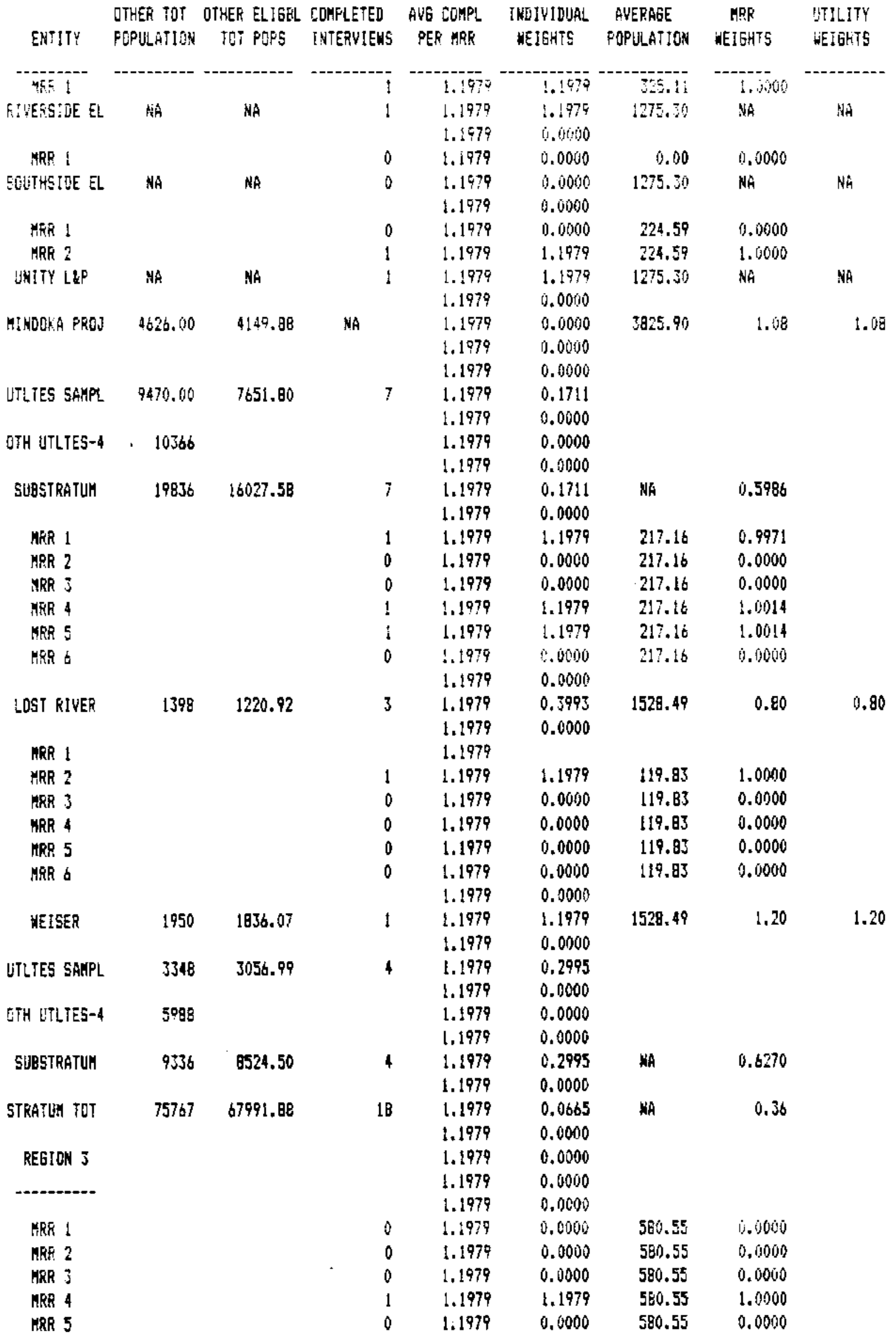




\begin{tabular}{|c|c|c|c|c|c|c|c|c|}
\hline EHTITY & $\begin{array}{l}\text { JTHER TUT } \\
\text { PLPILAT!GN }\end{array}$ & $\begin{array}{c}\text { OTHER ELISEL } \\
\text { TIT POFS }\end{array}$ & $\begin{array}{l}\text { CTAP:ETEC } \\
\text { ITEALEKS }\end{array}$ & $\begin{array}{l}\text { AVG CEMFL } \\
\text { FEF MRK }\end{array}$ & $\begin{array}{l}\text { LROIVIYUAL } \\
\text { WEISHTE }\end{array}$ & $\begin{array}{l}\text { AVERAGE } \\
\text { PEFLIATIDH }\end{array}$ & $\begin{array}{c}\text { MFR } \\
\text { ZEIEHTS }\end{array}$ & $\begin{array}{l}\text { UTLITYY } \\
\text { MEIEHTS }\end{array}$ \\
\hline 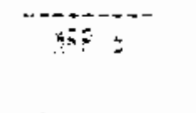 & & $\cdots$ & $\because$ & $\begin{array}{l}1.570 \\
1.199\end{array}$ & 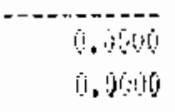 & Sto. & , & \\
\hline ENTEK ETS & 20531 & 23075.87 & $!$ & $\begin{array}{l}1.5079 \\
1.1979\end{array}$ & $\begin{array}{l}1.1975 \\
0.91800\end{array}$ & $314+9.20$ & 1,87 & 1.67 \\
\hline $4 F 91$ & & & $!$ & 1.1779 & 1.1979 & 312.47 & 0.7279 & \\
\hline 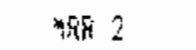 & & & 1 & 1.1979 & 1.1979 & 312.47 & 0.3424 & \\
\hline MRP j & & & 0 & 1.1979 & 0.6000 & 312.47 & 0.0000 & \\
\hline 4 & & & 1 & 1.1979 & 1.1979 & 312.47 & $1.414 t$ & \\
\hline MRP 5 & & & 1 & $\begin{array}{l}1.1979 \\
1.1979\end{array}$ & $\begin{array}{l}1.1974 \\
0.0900\end{array}$ & 312.47 & 1.5702 & \\
\hline DAELAN CT: & :iÂ & 1).60 & 4 & $\begin{array}{l}1.1979 \\
1.1979\end{array}$ & $\begin{array}{l}\text { 1) } 29995 \\
0.6000\end{array}$ & 战A & NA & $\mathrm{HA}$ \\
\hline WRF 1 & & & $!$ & 1.1979 & 1.1979 & 2127.16 & 1.0000 & \\
\hline CAGHAERE & NA & 0.00 & 1 & $\begin{array}{l}1.1979 \\
1.1979\end{array}$ & $\begin{array}{l}1.1979 \\
0.0000\end{array}$ & NA & NA & NA \\
\hline CHELN-LSHYARE & 21005.00 & 20204.61 & 5.60 & $\begin{array}{l}1.1979 \\
1.1979\end{array}$ & $\begin{array}{l}0.2396 \\
0.0000\end{array}$ & 21640.22 & 0.93 & 0.93 \\
\hline JTLTES GAMFL & 48296 & 43280 & $b$ & $\begin{array}{l}1.1979 \\
1.1979\end{array}$ & $\begin{array}{l}0.1976 \\
0.0000\end{array}$ & & & \\
\hline OTH UTLTES-2 & 39845 & & & $\begin{array}{l}1.1979 \\
1.1979\end{array}$ & $\begin{array}{l}0.0000 \\
0.00000\end{array}$ & & & \\
\hline SLBSTRATEM & 87141 & 28091.37 & $b$ & $\begin{array}{l}1.1979 \\
1.1979\end{array}$ & $\begin{array}{l}0.1796 \\
0.0000\end{array}$ & $N A$ & 1.8862 & \\
\hline MAR: & & & i & 1.1979 & 1.1979 & 33.68 & 0.2736 & \\
\hline 485 ? & & & 1 & 1.1979 & 1.1975 & 262.65 & $0 .-3 \mathrm{Eg} 7$ & \\
\hline MEF 3 & & & $\hat{\jmath}$ & 1.1979 & 0.0000 & 262.68 & 0.0000 & \\
\hline $\operatorname{MeR} 4$ & & & 1 & 1.1979 & 1.1979 & $262.6 B$ & 2.1500 & \\
\hline MKR 5 & & & 1 & 1.1579 & 1.1979 & 232.68 & 1.1897 & \\
\hline HAR 5 & & & & $\begin{array}{l}1.1979 \\
1.1979\end{array}$ & 0.0000 & & & \\
\hline FEANKLIN & 11628 & 8390.34 & 4 & $\begin{array}{l}1.1579 \\
1.1979\end{array}$ & $\begin{array}{l}0.2995 \\
0.0000\end{array}$ & 10332.55 & 0.50 & 0.80 \\
\hline Kat 1 & & & 1 & 1.1979 & $\$ .1979$ & 157.75 & 0.8558 & \\
\hline HRR 2 & & & 0 & 1.:979 & 0.9000 & 157.75 & 0.0000 & \\
\hline MEF 3 & & & 1 & 1.1979 & 1.1979 & 157.75 & 1.0396 & \\
\hline Mas 4 & & & 1 & 1.1979 & 1.1979 & 157.75 & 0.8748 & \\
\hline MER 5 & & & 1 & 1.1479 & 1.1979 & 157.75 & 1.2279 & \\
\hline MAR 6 & & & 0 & $\begin{array}{l}1.1979 \\
1.1979\end{array}$ & $\begin{array}{l}0.0000 \\
0.0000\end{array}$ & 157.75 & 0.0000 & \\
\hline STEHLAND & 13123 & 12358.75 & 4 & $\begin{array}{l}1.1979 \\
1.1979\end{array}$ & $\begin{array}{l}0.2995 \\
0.0000\end{array}$ & 10372.55 & 2.20 & 1.70 \\
\hline UTLTÉS SAMPL & 24751.00 & 20665.69 & 8 & $\begin{array}{l}1.1979 \\
1.1979\end{array}$ & $\begin{array}{l}0.1497 \\
0.0000\end{array}$ & & & \\
\hline UTA WRLES-5 & $4(j 314$ & & & $\begin{array}{l}1.1979 \\
1.1979\end{array}$ & $\begin{array}{l}0.0000 \\
0.0000\end{array}$ & & & \\
\hline BUBSTRRTUM & $50 t=$ & 54224.04 & $\Xi$ & $\begin{array}{l}1.1979 \\
1.1979\end{array}$ & $\begin{array}{l}0.1497 \\
0.0000\end{array}$ & NA & 0.9406 & \\
\hline MER 1 & & & 0 & 1.1979 & i. 1000 & 62.00 & 0.0600 & \\
\hline $\operatorname{miz}$ & & & $\hat{0}$ & 1.1579 & 0. 6000 & 68.40 & 0.6000 & \\
\hline Hस 3 & & & i) & 1.1979 & 0.0000 & 68,00 & 0.0000 & \\
\hline 154 4 & & & 1 & $\begin{array}{l}1.1979 \\
1.1979\end{array}$ & $\begin{array}{l}1.1979 \\
0.0600\end{array}$ & 88.00 & 1.0000 & \\
\hline DEND A AELLLE & 38.58 & 3774.03 & 1 & 1.1979 & 1.1979 & 4202.20 & 0.90 & $0 . \overline{90}$ \\
\hline
\end{tabular}




\begin{tabular}{|c|c|c|c|c|c|c|c|c|}
\hline ENTITY & $\begin{array}{l}\text { OTHER TST } \\
\text { POPILET!OHE }\end{array}$ & $\begin{array}{c}\text { OTHES ELIES } \\
\text { TOT FOPS }\end{array}$ & $\begin{array}{l}\text { COHPLETED } \\
\text { IHIERUIENS }\end{array}$ & $\begin{array}{l}\text { AVG CUAPL } \\
\text { PER MRP }\end{array}$ & $\begin{array}{l}\text { INDIVIOUAL } \\
\text { WEIGHTS }\end{array}$ & $\begin{array}{l}\text { AVERAGE } \\
\text { PGPIHATION }\end{array}$ & $\begin{array}{c}\text { MER } \\
\text { WEISHTS }\end{array}$ & $\begin{array}{l}\text { UIILITY } \\
\text { WEIGHTS }\end{array}$ \\
\hline------- & - & - - - & -- & 1.1979 & 0,0010 & - & 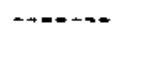 & $\cdots$ \\
\hline MEF! & & & 1 & 1.1579 & 1.1975 & $270 . \overline{8}$ & 1.1)549 & \\
\hline $\operatorname{MR} 2$ & & & $!$ & 1.1974 & 1.1979 & 278.87 & 0.9528 & \\
\hline Hek 3 & & & f) & 1.1979 & 0.0000 & 278.83 & 0.10000 & \\
\hline \multirow[t]{2}{*}{ SEF 4} & & & 1 & 1.1979 & 1.1979 & 278.83 & 1.0475 & \\
\hline & & & & 1.1979 & 0.0000 & & & \\
\hline \multirow[t]{2}{*}{ VERA IRR } & 4856 & 4630.37 & 3 & 1.1979 & 0.3993 & 4202.20 & 1.10 & 1.10 \\
\hline & & & & 1.1979 & 0.0000 & & & \\
\hline \multirow[t]{2}{*}{ IITLTES JAMPL } & 8074 & 8404.40 & 4 & 1.1979 & 0.2995 & & & \\
\hline & & & & 1.1979 & 0.0000 & & & \\
\hline \multirow[t]{2}{*}{ OTH UTLTES-J } & 10363 & & & 1.1979 & 0.0000 & & & \\
\hline & & & & 1.1979 & 0.0000 & & & \\
\hline \multirow[t]{2}{*}{ SUESTRRIUH } & 18957 & 18325.54 & 4 & 1.1979 & 0.2995 & NA & 0.6038 & \\
\hline & & & & 1.1979 & 0.0000 & & & \\
\hline MER I & & & $!$ & 1.1979 & 1.1979 & 259.66 & 0.9572 & \\
\hline ARR 2 & & & 1 & 1.1979 & 1.1979 & 258.66 & $1.062 B$ & \\
\hline MRR 3 & & & & 1.1979 & & $25 B .66$ & & \\
\hline \multirow[t]{2}{*}{ HRR \& } & & & ) & 1.1979 & 0.0000 & 258.66 & 0.0000 & \\
\hline & & & & 1.1979 & 0.0000 & & & \\
\hline \multirow[t]{2}{*}{ FERRY CTY } & $174 \delta$ & 1339.01 & 2 & 1.1979 & 0.5989 & 1412.23 & 0.95 & 0.95 \\
\hline & & & & 1.1979 & 0.0000 & & & \\
\hline WRR ! & & & 0 & 1.1979 & 0.0000 & 196.53 & 0.0000 & \\
\hline \multirow[t]{2}{*}{ HEk 2} & & & 1 & 1.1979 & 1.1979 & 196.53 & 1.0000 & \\
\hline & & & & 1.1979 & 0.0000 & & & \\
\hline \multirow[t]{2}{*}{ HCSPEELEH } & NA & NA & 1 & $1.197 \overline{4}$ & 1.1979 & NA & $\mathrm{NA}$ & tiA \\
\hline & & & & 1.1979 & 0.0000 & & & \\
\hline MRR ! & & & 0 & 1.1979 & 0.0000 & 56.00 & 0.0000 & \\
\hline \multirow[t]{2}{*}{ HRR 2} & & & 1 & 1.1979 & 1.1979 & 58.00 & 1.0000 & \\
\hline & & & & 1.1979 & 0.0000 & & & \\
\hline \multirow[t]{2}{*}{ COULEE DAH } & NA & NA & 1 & 1.1979 & 1.1979 & NA & $N A$ & NA \\
\hline & & & & 1.1979 & 0.0000 & & & \\
\hline \multirow[t]{2}{*}{ NSPLH-CDULEE } & 1623.00 & 1485.46 & & 1.1979 & 0.0000 & 1412.23 & 1.05 & 1.05 \\
\hline & & & & 1.1979 & 0.0000 & & & \\
\hline \multirow[t]{2}{*}{ UTLTES SAMPL } & 3369.00 & $2 B 24.46$ & 4 & 1.1979 & 0.2995 & & & \\
\hline & & & & 1.1979 & 0.0090 & & & \\
\hline \multirow[t]{2}{*}{ OTH UTLFES-4 } & 5747 & & & 1.1979 & 0.0000 & & & \\
\hline & & & & 1.1979 & 0.0000 & & & \\
\hline \multirow[t]{2}{*}{ SUBSTRATUA } & 9116 & 7642.57 & 4 & 1.1979 & 0.2995 & HA & 0.2647 & \\
\hline & & & & 1.1979 & 0.0000 & & & \\
\hline \multirow[t]{3}{*}{ STRATHM TOT } & 180279 & 158383.51 & 22 & 1.1979 & 0.0544 & NA & 0.39 & \\
\hline & & & & 1.1979 & 0.0000 & & & \\
\hline & & & & 1.1979 & 0.0000 & & & \\
\hline \multirow[t]{4}{*}{ REGIOH 4} & & & & 1.1979 & 0.0000 & & & \\
\hline & & & & 1.1979 & 0.0000 & & & \\
\hline & & & & 1.1979 & 0.0000 & & & \\
\hline & & & & 1.1979 & 0.0000 & & & \\
\hline MRR 1 & & & 0 & 1.1979 & 0.0000 & 392.15 & 0.0000 & \\
\hline MRR 2 & & & ! & 1.1979 & 1.1979 & 392.15 & 1.0277 & \\
\hline Meti & & & $\dot{v}$ & 1.1579 & $0.900 \%$ & 357.15 & 0.0600 & \\
\hline NRR 4 & & & 1 & 1.1979 & 1.1979 & 392.15 & 1.0149 & \\
\hline ARR 5 & & & 1 & 1.1779 & 1.1979 & 392.15 & 0.6354 & \\
\hline \multirow[t]{2}{*}{ MPR : } & & & 2 & 1.1979 & 0.5989 & 392.15 & 1.1220 & \\
\hline & & & & 1.1979 & 0.0000 & & & \\
\hline
\end{tabular}




\begin{tabular}{|c|c|c|c|c|c|c|c|c|}
\hline EYT & $\begin{array}{l}\text { DTHER TQT } \\
\text { PEFULATSG }\end{array}$ & $\begin{array}{l}\text { QTHEF ELIGEL } \\
\text { TIT SOFS }\end{array}$ & $\begin{array}{l}\text { COMFETED } \\
\text { IHIEEVIEHE }\end{array}$ & $\begin{array}{l}\text { AVE COMFL } \\
\text { FER MRR }\end{array}$ & $\begin{array}{l}\text { THDIVIDUAL } \\
\text { WEIEHTS }\end{array}$ & $\begin{array}{l}\text { AVEFGGE } \\
\text { POFULATIOK }\end{array}$ & $\begin{array}{c}\text { MFF } \\
\text { WELARTS }\end{array}$ & $\begin{array}{l}\text { LTIIIYY } \\
\text { REIGHi5 }\end{array}$ \\
\hline 活 & .60179 & $25+204.60$ & $\vdots$ & $\begin{array}{l}0.1779 \\
1.19 .8\end{array}$ & i. & 10247.25 & 1.32 & (1) \\
\hline mif ! & & & ! & 1.1979 & 1.1979 & 197.37 & 6.45 .979 & \\
\hline SF 2 & & & ij & 1.1979 & 0.0000 & 197.37 & 1.0000 & \\
\hline MPE : & & & $!$ & 1.9970 & 1.1079 & 197.37 & 1.2626 & \\
\hline AfR 4 & & & 1 & 1.1979 & 1.1790 & 197.37 & 1.2106 & \\
\hline MFR 5 & & & $!$ & 1.1979 & 1.1779 & {$[47.37$} & 0.9 .323 & \\
\hline MRE 6 & & & 1 & $\begin{array}{l}1.1979 \\
1.1979\end{array}$ & $\begin{array}{l}1.1979 \\
0.00609\end{array}$ & 197.37 & 1.1473 & \\
\hline Skg ONISH & 125507 & 136724.40 & 5 & $\begin{array}{l}1.1979 \\
1.1979\end{array}$ & $\begin{array}{l}0.209 \\
0.0060\end{array}$ & 172494.65 & 6.68 & 0.68 \\
\hline $\begin{array}{l}\text { ULTES SAAPL } \\
\text { OTH UTLITS-l }\end{array}$ & 375685 & 354989.26 & ii) & $\begin{array}{l}1.1979 \\
1.1979\end{array}$ & $\begin{array}{l}0.1198 \\
0.1000\end{array}$ & & & \\
\hline EUESTRATUM & 395685 & 384989.26 & 10 & $\begin{array}{l}1.1979 \\
1.1979\end{array}$ & $\begin{array}{l}0.1950 \\
0.00000\end{array}$ & NA & 3.9837 & \\
\hline KER I & & & 2 & 1.1979 & 0.5999 & 326.15 & 0.7910 & \\
\hline$M R 2$ & & & 2 & 1.1979 & 0.5999 & 326.15 & $1.054 \mathrm{~B}$ & \\
\hline MP : & & & 2 & 1.1979 & 0.5989 & $32 b .15$ & 1.1167 & \\
\hline MRR 4 & & & 1 & 1.1979 & 1.1979 & 326.15 & 0.4722 & \\
\hline KRP 5 & & & 1 & 1.1979 & 1.1979 & 326.15 & 1.5653 & \\
\hline MRR 6 & & & 0 & 1.1979 & 0.0000 & 326.15 & 0.0000 & \\
\hline & & & & 1.1979 & 0.0000 & & & \\
\hline ILARK CTY & 74392 & $6683 b .32$ & 8 & 1.1979 & 0.1497 & 78377.24 & 0.85 & 0.85 \\
\hline & & & & 1,1579 & 0.0000 & & & \\
\hline ARR 1 & & & 2 & 1.1975 & 0.5999 & 147.27 & 1.1546 & \\
\hline $\operatorname{MrR} 2$ & & & 2 & 1.1779 & 0.5959 & 147.47 & 0.9323 & \\
\hline AfR 3 & & & 1 & 1.1979 & 1.1979 & 147.47 & 0.9260 & \\
\hline int 4 & & & 1 & 1.1979 & 1.1979 & 147.47 & 1.0647 & \\
\hline MRE 5 & & & 0 & 1.1979 & 0.0000 & 147.47 & 0.0000 & \\
\hline$M E R$ & & & 1 & 1.1979 & 1.1979 & 167.47 & 0.9126 & \\
\hline & & & & 1.1979 & 0.0000 & & & \\
\hline TACOHA & $9673 B$ & 89918.16 & $\bar{i}$ & 1.1979 & 0.1711 & $7 B 377.24$ & 1.15 & 6.15 \\
\hline & & & & 1.1979 & 0.0060 & & & \\
\hline LĨLTES SAKPL & $971131)$ & 158754.48 & $! 5$ & 1.1979 & 0.0790 & & & \\
\hline & & & & 1.1979 & 0.0006 & & & \\
\hline WTH STLTES-2 & 63267 & & & 1.1979 & 0.0000 & & & \\
\hline & & & & 1.1979 & 0.0000 & & & \\
\hline ELESTRATMM & 234397 & 214706.87 & $! 5$ & 1.1979 & 0.0799 & Ne & 1,4926 & \\
\hline & & & & 1.1979 & 0.0000 & & & \\
\hline AGP! & & & 0 & 1.1979 & 0.6000 & 317.99 & 0.0000 & \\
\hline $\operatorname{MRR} 2$ & & & 2 & 1.1979 & 0.5589 & 317.90 & 1.1416 & \\
\hline MER 3 & & & 2 & 1.1979 & 0.5989 & 317.99 & 0.6868 & \\
\hline MeR 4 & & & 1 & 1.1879 & 1.1979 & 317.99 & 1.6730 & \\
\hline PRP $\bar{j}$ & & & 2 & 1.1979 & 0.5999 & 317.99 & 0.5535 & \\
\hline hiñ & & & 1 & 1.1979 & 1.1979 & 317.09 & 0.9451 & \\
\hline & & & & 1.1979 & 0.0000 & & & \\
\hline *GSül CiY-? & 16253 & 14481.45 & 8 & 1,1979 & 0.1497 & 13907.72 & 1.04 & 1.1 .4 \\
\hline & & & & 1.1077 & 0.0000 & & & \\
\hline AlkP ! & & & 0 & $\therefore 197 \%$ & U. 000 & 476.60 & 0.0 .00 & \\
\hline MFF 2 & & & 2 & 1.1979 & 0.5989 & 425.126 & 1.5542 & \\
\hline A AR 3 & & & $!$ & 1.1979 & 1.1979 & 426.06 & 1.6919 & \\
\hline 致R 4 & & & \pm & 1.1979 & $\llbracket .1979$ & 426.66 & 0.6565 & \\
\hline MPR 5 & & & $!$ & 1.1974 & 1.1979 & 426.36 & 0.0351 & \\
\hline & & & & C. .22 & & & & \\
\hline
\end{tabular}




\begin{tabular}{|c|c|c|c|c|c|c|c|c|}
\hline ENT!TY & $\begin{array}{l}\text { OTHER TOT } \\
\text { FUEULATION }\end{array}$ & $\begin{array}{c}\text { OTHEF EL:GBL } \\
\text { TIJT FDPE }\end{array}$ & $\begin{array}{l}\text { COMFLETED } \\
\text { INTERVIELS }\end{array}$ & $\begin{array}{l}\text { AVG CDMPL } \\
\text { PEF MFR }\end{array}$ & $\begin{array}{l}\text { INOIVIDUAL } \\
\text { HEIGATS }\end{array}$ & $\begin{array}{l}\text { AVERAGE } \\
\text { POPUIATIOH }\end{array}$ & $\begin{array}{c}\text { HFR } \\
\text { WEIGHTS }\end{array}$ & $\begin{array}{l}\text { JTILITY } \\
\text { XETSTTS }\end{array}$ \\
\hline \multirow[t]{2}{*}{ apto } & 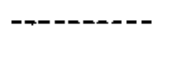 & --.-- & 1 & 1.1079 & $1.1^{0} \overline{4}$ & 420.70 & $1, i 6 \div j$ & \\
\hline & & & & 1.1779 & t) & & & \\
\hline \multirow[t]{2}{*}{ SLELLAM CTS } & 15519 & 13033.99 & $b$ & 1.1979 & 1.1990 & 13707.72 & 9.96 & 1.76 \\
\hline & & & & 1.1979 & 0.0000 & & & \\
\hline \multirow[t]{2}{*}{ MLYES SIYPL } & 31751 & 27915.44 & 14 & 1.1979 & 6.9956 & & & \\
\hline & & & & 1.1979 & 0.0000 & & & \\
\hline \multirow[t]{2}{*}{ CTH UTLTES-3 } & 40669 & & & 1.1979 & 0.0000 & & & \\
\hline & & & & 1.1979 & 0.0000 & & & \\
\hline \multirow{2}{*}{ SIJSTRATUH } & 72420 & 63443.48 & 14 & 1.1979 & 0.0856 & $N A$ & 0.4410 & \\
\hline & & & & 1.1979 & 0.0000 & & & \\
\hline MER ! & & & 2 & 1.1579 & 1. 5999 & 312.13 & 1.1995 & \\
\hline NER 2 & & & 2 & 1.1979 & 0.5999 & 32.13 & 3.8009 & \\
\hline HER 3 & & & 0 & 1.1979 & 0.0000 & 312,13 & 0.0000 & \\
\hline \multirow[t]{2}{*}{ MER 4} & & & 1 & 1.1979 & 1.1979 & 312.13 & 0.9996 & \\
\hline & & & & 1.1779 & 0.0000 & & & \\
\hline \multirow[t]{2}{*}{ ELHHURST MUT } & 7225 & 6984.17 & 5 & 1.1979 & 0.2396 & 5845,01 & 1.19 & 1.19 \\
\hline & & & & 1.1979 & 0.0090 & & & \\
\hline WRR 1 & & & 2 & 1.1979 & 0.5999 & 1165.73 & 1.0012 & \\
\hline MRE 2 & & & 1 & 1.1979 & 1.1979 & 1165.28 & 0.9964 & \\
\hline MRR 3 & & & 2 & 1.1979 & 0.5989 & 1165.28 & 1.0012 & \\
\hline \multirow{2}{*}{ MRR 4} & & & 2 & 1.1979 & 0.5969 & $1165.2 B$ & 1.0012 & \\
\hline & & & & 1.1979 & 0.0000 & & & \\
\hline \multirow[t]{2}{*}{ DREAO PUR } & 5048 & 4705.86 & 7 & 1.1979 & 0.1711 & 5845.01 & $\dot{1}, 81$ & 0. 31 \\
\hline & & & & 1.1979 & 0.0000 & & & \\
\hline \multirow[t]{2}{*}{ WTZIES SAHPL } & 12273 & 11450.02 & 12 & 1.1979 & 0.19998 & & & \\
\hline & & & & 1.1979 & 0.0000 & & & \\
\hline \multirow[t]{2}{*}{ OTH UTLTES-3 } & 17435 & & & 1.1979 & 0.0060 & & & \\
\hline & & & & 1.1979 & 0.0000 & & & \\
\hline \multirow[t]{2}{*}{ SURSTRATU } & 29708 & 28296.85 & 12 & 1.1979 & 0.0998 & $\mathrm{HA}$ & 0.2991 & \\
\hline & & & & 1.1979 & 0.0000 & & & \\
\hline HRR ! & & & 2 & 1.1979 & 0.5989 & 123.39 & 0.5795 & \\
\hline MRP 2 & & & 0 & 1.1979 & 0.0000 & 123.39 & 0.0000 & \\
\hline MPR $J$ & & & 2 & $\$ .1979$ & 0.5989 & 123.39 & 1.2059 & \\
\hline \multirow[t]{2}{*}{ MER 4} & & & 2 & 1.1979 & 0.5989 & 123.39 & 1.1346 & \\
\hline & & & & 1.1979 & 0.0000 & & & \\
\hline \multirow[t]{2}{*}{ PARKLANB LEP } & 2794 & 2649.48 & 6 & 1.1979 & 0.1996 & 2985.91 & 0.89 & 0.89 \\
\hline & & & & 1.1979 & 0.0000 & & & \\
\hline MPR 1 & & & 2 & 1.1979 & 0.5989 & 109.06 & 1.0949 & \\
\hline MQR 2 & & & 2 & 1.1979 & 0.5989 & 108.06 & 0.7866 & \\
\hline MRE J & & & 2 & 1.1979 & 0.5999 & 108.06 & 1.1660 & \\
\hline \multirow[t]{2}{*}{ MRT 4} & & & 1 & 1.1979 & 1.1979 & 108.06 & 0.9624 & \\
\hline & & & & 1,1979 & 0.0000 & & & \\
\hline \multirow[t]{2}{*}{ SKAMARIP } & 3441 & 3322.34 & 7 & 1.1979 & 0.1711 & 2985.91 & 1.11 & 1.11 \\
\hline & & & & 1.1979 & 0.0000 & & & \\
\hline \multirow[t]{2}{*}{ UTLIES SAHPL } & 6235 & 5971.83 & 13 & 1.1979 & 0.0121 & & & \\
\hline & & & & 1.1979 & 0.0000 & & & \\
\hline \multirow[t]{2}{*}{ DFH UZLTES-2 } & 5227 & & & 1.1979 & 0.0000 & & & \\
\hline & & & & 1.1979 & 0.0000 & & & \\
\hline SHESTRATUA & 11462 & 10778.20 & 13 & 1.1979 & 0.19921 & NA & 0.1073 & \\
\hline & & & & 1.1979 & 0.0000 & & & \\
\hline MRR ! & & & 2 & 1.1979 & 0.5999 & 195.56 & 2.1528 & \\
\hline HRP 2 & & & 0 & 1.1979 & 0.0000 & 195.56 & 0.0000 & \\
\hline HRP 3 & & & 1 & 1.1979 & 1.1979 & 195.56 & 0.3102 & \\
\hline
\end{tabular}




\begin{tabular}{|c|c|c|c|c|c|c|c|c|}
\hline ENTITY & $\begin{array}{l}\text { OTHEQ TOT } \\
\text { PEPLLATIBK }\end{array}$ & $\begin{array}{c}\text { PTAEF ELIEES } \\
\text { TOT PGFS }\end{array}$ & $\begin{array}{l}\text { COHFLETED } \\
\text { IWHERIIEKS }\end{array}$ & $\begin{array}{l}\text { SUG COMPL } \\
\text { PER MIF }\end{array}$ & $\begin{array}{l}\text { IYDIVINHAL } \\
\text { WEIGHTS }\end{array}$ & $\begin{array}{l}\text { AVERAGE } \\
\text { POFILATIOH }\end{array}$ & $\begin{array}{c}\text { MRP } \\
\text { WE:GKTS }\end{array}$ & $\begin{array}{l}\text { HILITY } \\
\text { HEIGHTS }\end{array}$ \\
\hline 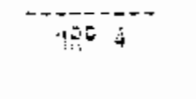 & & & 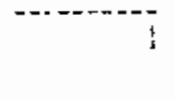 & $\begin{array}{l}1.1970 \\
1.1979\end{array}$ & $\begin{array}{l}1.1070 \\
0.5000\end{array}$ & 19 & .5959 & \\
\hline 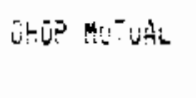 & 102 & 1624,47 & 4 & $\begin{array}{l}1.1779 \\
1.1979\end{array}$ & $\begin{array}{l}0.275 e \\
0.00000\end{array}$ & 145.25 & 1.12 & 1.12 \\
\hline ME 1 & & & 2 & 1.1579 & 0.5989 & 130.80 & i. 40,3 ? & \\
\hline$M$ & & & $!$ & 1.1979 & 1.1979 & 130.80 & 1.501 & \\
\hline MER 3 & & & 2 & 1.1979 & 8.5999 & $13 i j .80$ & 0.8803 & \\
\hline YFiR 4 & & & 1 & $\begin{array}{l}1.1979 \\
1.1599\end{array}$ & $\begin{array}{l}1.1979 \\
0.0000\end{array}$ & 130.80 & 1.2099 & \\
\hline WANA I A & 1512 & 1288.06 & b & $\begin{array}{l}1.1979 \\
1.1779\end{array}$ & $\begin{array}{l}0.1596 \\
0.00606\end{array}$ & 1456.23 & 0.38 & ). 58 \\
\hline JTLTES SAMEL & 3104 & 2912.47 & to & $\begin{array}{l}1.1979 \\
1.1979\end{array}$ & $\begin{array}{l}10.1193 \\
0.0000\end{array}$ & & & \\
\hline OTH UILIES-כ & $700 \overline{4}$ & & & $\begin{array}{l}1.1979 \\
1.1979\end{array}$ & $\begin{array}{l}0.0000 \\
0.0000\end{array}$ & & & \\
\hline SUESTSATUH & 10173 & 9364.26 & 10 & $\begin{array}{l}1.1979 \\
1.1979\end{array}$ & 0.1198 & HA & 0.6946 & \\
\hline jTRAILA TOT & 753845 & 711778.94 & 74 & $\begin{array}{l}1.1970 \\
1.1979\end{array}$ & $\begin{array}{l}0.0162 \\
0.0000\end{array}$ & NA & 1.36 & \\
\hline REGION 5 & & & & $\begin{array}{l}1.1979 \\
1.1979 \\
1.1979\end{array}$ & $\begin{array}{l}0.0000 \\
0.0000 \\
0.0000\end{array}$ & & & \\
\hline RRR: : & & & 1 & 1.1979 & $1 .: 979$ & $42 !, 4 ?$ & 1.0393 & \\
\hline min? & & & 1 & 1.1977 & 1.1978 & $42: .42$ & 1.4874 & \\
\hline $\operatorname{sen} 3$ & & & 1 & 1.1979 & 1,1979 & 421.42 & 9.530 & \\
\hline Anth 4 & & & 1 & 1.1979 & 1.1979 & $42 ! .42$ & $1 . \$ 214$ & \\
\hline $\operatorname{men} 5$ & & & 1 & 1.1979 & 1.1979 & 421.42 & 0.7857 & \\
\hline MPQ 6 & & & 0 & 1.1979 & 0.0000 & 421.42 & 0.0000 & \\
\hline MRT 7 & & & $\vdots$ & 1.1979 & 1.1979 & 421.42 & 1.0567 & \\
\hline HRF 5 & & & 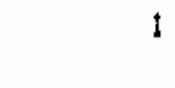 & $\begin{array}{l}1.1979 \\
1.5979\end{array}$ & $\begin{array}{l}1.1979 \\
0.1000\end{array}$ & 421.42 & 0.8921 & \\
\hline MIOSTATE & 7797 & 6878.58 & 7 & $\begin{array}{l}1.1979 \\
1.1979\end{array}$ & $\begin{array}{l}0.1711 \\
0.0000\end{array}$ & 6457.72 & 1.07 & 1.07 \\
\hline MRR 1 & & & 0 & 1.1977 & 0.0000 & 96.40 & 0.0000 & \\
\hline K & & & $\mathfrak{l}$ & 1.1979 & 1.1979 & 96.40 & 0.3999 & \\
\hline MRR I & & & 0 & 1.1879 & 0.0000 & $9 i .40$ & $0.00 \% 9$ & \\
\hline MRF 4 & & & $!$ & 1.1979 & 1.1979 & 96.40 & 1.1328 & \\
\hline HeR 5 & & & 1 & 1.1979 & 1.1979 & 95.40 & 1.10 .37 & \\
\hline 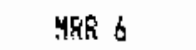 & & & 0 & 1.1979 & 0.0000 & 96.10 & 0.0000 & \\
\hline MRR 7 & & & 0 & 1.1779 & 0.0000 & $: 6,40$ & 0.0000 & \\
\hline SER $B$ & & & 1 & $\begin{array}{l}1.1979 \\
1.1779\end{array}$ & $\begin{array}{l}1.1979 \\
0.0000\end{array}$ & 96.40 & 0.9336 & \\
\hline MATILEA & 6546 & 5996.56 & 4 & $\begin{array}{l}1.1979 \\
1.1979\end{array}$ & $\begin{array}{l}4.2995 \\
0.0000\end{array}$ & 6437.72 & 0.95 & 0.93 \\
\hline JTLTES SAMPL & 14343 & 12975.44 & 11 & $\begin{array}{l}1.1979 \\
1.1979\end{array}$ & $\begin{array}{l}0.1089 \\
0.0000\end{array}$ & & & \\
\hline VTH UTLFES-2 & 17731 & & & $\begin{array}{l}1.1979 \\
1.1979\end{array}$ & $\begin{array}{l}0.0000 \\
0.0000\end{array}$ & & & \\
\hline EVsTimith & 52634 & 28742.23 & ii & $\begin{array}{l}1.1979 \\
1.1779\end{array}$ & $\begin{array}{l}\text { i, } 1069 \\
0,6000\end{array}$ & NA & 1.2904 & \\
\hline NaR L & & & 1 & 1.1979 & 1.1979 & 153.76 & 1.7942 & \\
\hline GAñ 2 & & & 1 & 1.1979 & 1.1979 & 153.76 & 1.2227 & \\
\hline MRE 3 & & & i & 1.1779 & $1 . ! 579$ & $15: 76$ & 1.17796 & \\
\hline
\end{tabular}




\begin{tabular}{|c|c|c|c|c|c|c|c|c|}
\hline EnTITY & $\begin{array}{l}\text { OTHER TOT } \\
\text { PJPULATION }\end{array}$ & $\begin{array}{c}\text { CTHER ELIEEL } \\
\text { TOT PJPS }\end{array}$ & $\begin{array}{l}\text { COHFLETED } \\
\text { IHTERVIEHS }\end{array}$ & $\begin{array}{l}\text { AVG COMPL } \\
\text { PER MER }\end{array}$ & $\begin{array}{l}\text { INDIVIGUAL } \\
\text { WEIGHTS }\end{array}$ & $\begin{array}{l}\text { AYEFAGE } \\
\text { PDPULATIOH }\end{array}$ & $\begin{array}{c}\text { MER } \\
\text { WEIGHTS }\end{array}$ & $\begin{array}{l}\text { UTILITY } \\
\text { WEIEHTS }\end{array}$ \\
\hline MEQ & & & i & 0.1570 & T.juco & 150.76 & 0,900 & \\
\hline Nifig & & & 1 & 1.1979 & 1.1979 & 153.76 & 1.2443 & \\
\hline$M$ & & & $!$ & 1.1979 & 1.1979 & 153.76 & 0.3628 & \\
\hline $\operatorname{MER} 7$ & & & 0 & 1.1979 & 0.0000 & 153.76 & 0.0000 & \\
\hline \multirow[t]{2}{*}{ MP } & & & 1 & 1.1979 & 1.1979 & 153.76 & 0.7965 & \\
\hline & & & & 1.1979 & 0,0000 & & & \\
\hline \multirow{2}{*}{ COLUKFBIA } & 2855 & 2502.23 & 6 & 1.1979 & 0.1996 & 2638.29 & 0.95 & 0.95 \\
\hline & & & & 1.1979 & 0.0000 & & & \\
\hline MRR 1 & & & 1 & 1.1979 & 1.1979 & 129.27 & 0.7456 & \\
\hline MSR 2 & & & 1 & 1.1979 & 1.1979 & $\{28.27$ & 0.9489 & \\
\hline MRT 3 & & & 0 & 1.1979 & 0.0000 & 126.27 & 0.0000 & \\
\hline MRE 4 & & & 0 & $1.1977^{\circ}$ & 0.0000 & 128.27 & 0.0000 & \\
\hline MRR 5 & & & 1 & 1.1979 & 1.1979 & 128.27 & 1.4189 & \\
\hline MRP 6 & & & 1 & 1.1979 & 1.1979 & 126.27 & 1.0135 & \\
\hline MAR 7 & & & 1 & 1.1979 & 1.1979 & 129.27 & 0.9731 & \\
\hline \multirow[t]{2}{*}{ MRR 8} & & & 0 & 1.1979 & 0.17000 & 128.27 & 0.0000 & \\
\hline & & & & 1.1970 & 0.6000 & & & \\
\hline \multirow[t]{2}{*}{ MLTW-FREEHTR } & 2848 & 2774.34 & 5 & 1.1979 & 0.2396 & 2638.29 & 1.05 & 1.05 \\
\hline & & & & 1.1979 & 0.0000 & & & \\
\hline \multirow[t]{2}{*}{ UTLTES SAMPL } & 5703 & 5276.57 & 11 & 1.1979 & 0.1089 & & & \\
\hline & & & & 1.1979 & 0.0000 & & & \\
\hline \multirow[t]{2}{*}{ OTH SAMPL -5 } & 10634 & & & 1.1979 & 0.0000 & & & \\
\hline & & & & 1.1979 & 0.0000 & & & \\
\hline \multirow[t]{2}{*}{ SUESTRATUK } & 16337 & 15115.44 & 11 & 1.1979 & 0.1089 & 21.953 .83 & 0.6966 & \\
\hline & & & & 1.1979 & 0.0000 & & & \\
\hline \multirow[t]{2}{*}{ STRATA TOT } & 48411 & 43907.67 & 22 & 1.1979 & 0.0544 & $N A$ & 0.19 & \\
\hline & & & & 1.1979 & 0.0000 & & & \\
\hline \multirow[t]{3}{*}{ REGION $b$} & & & & 1.1979 & 0.0000 & & & \\
\hline & & & & 1.1979 & 0.0000 & & & \\
\hline & & & & 1.1979 & 0.0000 & & & \\
\hline RRR ! & & & 2 & 1.1979 & 0.5989 & 2522.71 & 1.0093 & \\
\hline MRA 2 & & & 1 & 1.1979 & 1.1979 & 2522.71 & 1.1547 & \\
\hline MRR 3 & & & 0 & 1.1979 & 0.0000 & 2522.71 & 0.6000 & \\
\hline MRR 4 & & & 2 & 1.1979 & 0.5989 & 2522.71 & 1.0263 & \\
\hline MRR 5 & & & 1 & 1.1979 & 1.1979 & 2522.71 & $0.92 b 6$ & \\
\hline \multirow[t]{2}{*}{ MRR 6} & & & 1 & 1.1979 & 1.1979 & 2522.71 & 0.8829 & \\
\hline & & & & 1.1979 & 0.0000 & & & \\
\hline \multirow[t]{2}{*}{ EUGENE BTR } & 54494 & 51135.31 & 7 & 1.1979 & 0.1711 & 34692.59 & 1.47 & 1.47 \\
\hline & & & & 1.1979 & 0.0000 & & & \\
\hline MRP 1 & & & 2 & 1.1979 & 0.5939 & 160.73 & $1.10 \mathrm{~B} 2$ & \\
\hline KRR 2 & & & & 1.1979 & & 160.93 & & \\
\hline ARR 3 & & & & 1.1979 & & 160.93 & & \\
\hline MRR 4 & & & 2 & 1.1979 & 0.5989 & 160.93 & 0.5930 & \\
\hline MRR 5 & & & 0 & 1.1979 & 0.0000 & 160.93 & 0.0000 & \\
\hline \multirow[t]{2}{*}{ ARR 6} & & & 1 & 1.1979 & 1.1979 & 160.73 & $1.29 \overline{\mathrm{B}} 7$ & \\
\hline & & & & 1.1979 & 0.0000 & & & \\
\hline \multirow[t]{2}{*}{ CNTRL-ZNCLN } & 21337 & 18249.87 & 5 & 1.1979 & 0.2396 & 34692.59 & 11.53 & 0.53 \\
\hline & & & & 1.1979 & 0.9000 & & & \\
\hline UTLTES SANPL & 75871 & 69385.18 & 12 & 1.1979 & 0.1989 & & & \\
\hline ОТИ JFLTES-O & & & & 1.1979 & 0.0000 & & & \\
\hline \multirow{2}{*}{ SUESTRATUM } & 75831 & 64859.45 & 12 & 1.1979 & 0.0999 & NA & 1.2772 & \\
\hline & & & & 1.1979 & 0.0000 & & & \\
\hline \multirow[t]{2}{*}{ MFR : } & & & 2 & 1.1979 & 0.5989 & $377 . \$ 1$ & 1). 9233 & \\
\hline & & & & C. 25 & & & & \\
\hline
\end{tabular}




\begin{tabular}{|c|c|c|c|c|c|c|c|c|}
\hline EHTITY & 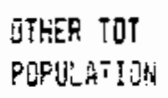 & $\begin{array}{l}\text { OTHER ELISEL } \\
\text { TOT POPS }\end{array}$ & $\begin{array}{l}\text { CQHEEETED } \\
\text { INFESUIEHS }\end{array}$ & $\begin{array}{l}\text { AVS COMPL } \\
\text { PER HPE }\end{array}$ & $\begin{array}{l}\text { IHOIVIGIJAL } \\
\text { WEISHTS }\end{array}$ & $\begin{array}{l}\text { AVEEAGE } \\
\text { POPHLATION }\end{array}$ & $\begin{array}{c}\text { MIRR } \\
\text { HEIGHTS }\end{array}$ & $\begin{array}{l}\text { LTILITY } \\
\text { WE TGHS }\end{array}$ \\
\hline 泾: & $\cdots$ & 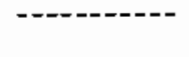 & 8 & 1.te: & 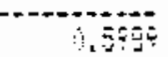 & 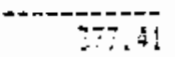 & 1.992 & $=-$ \\
\hline ME 3 & & & : & $\therefore 1779$ & 0. Hulu & 3794 & $\therefore$. 000 & \\
\hline$M B R+4$ & & & is & 1.1979 & 1) 500 & 377,41 & $0.0 \% 0$ & \\
\hline HRE $\overline{3}$ & & & $!$ & 1.1979 & 1.1979 & 377.41 & 1.6420 & \\
\hline \multirow[t]{2}{*}{ 足 } & & & 2 & 1.1979 & i. $5 \div 99$ & 377.41 & 0.873 & \\
\hline & & & & 1.1879 & 0.0000 & & & \\
\hline \multirow[t]{2}{*}{ GFRINGFIELD } & 18520 & 17900.53 & 7 & 1.1579 & i. 1711 & 14687.01 & 1.21 & 1.21 \\
\hline & & & & 1.1979 & 0.0000 & & & \\
\hline MRR 1 & & & 0 & 1.1979 & i. iono & 67.67 & 0.0000 & \\
\hline 鶖 2 & & & $!$ & 1.1979 & 1.1979 & 67.67 & $0.937^{\circ}$ & \\
\hline 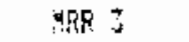 & & & 1 & 1.1579 & 5.1979 & 67.67 & $9.92 \% 6$ & \\
\hline M的 4 & & & 8 & 1.1979 & 0.0000 & 67.67 & 0.0000 & \\
\hline MEF 5 & & & 1 & 1.1979 & 1.1979 & 07.67 & 0.5527 & \\
\hline \multirow[t]{2}{*}{ 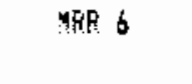 } & & & 2 & 1.1979 & 0. 5989 & 67.67 & 1.5813 & \\
\hline & & & & 1.1979 & 0.6000 & & & \\
\hline \multirow[t]{2}{*}{ CONSUMERS } & 12893 & 11565.50 & 5 & 1.1979 & 0.2396 & 14687.01 & 0.79 & 0.79 \\
\hline & & & & 1.1979 & 0.0000 & & & \\
\hline \multirow[t]{2}{*}{ UTLTES SAMFL } & 31413 & 29574.03 & i? & 1.1979 & 0.0998 & & & \\
\hline & & & & 1.1979 & 0.0000 & & & \\
\hline \multirow[t]{2}{*}{ OTH UTLTES-2 } & 22874 & & & 1.1979 & 0.0000 & & & \\
\hline & & & & 1.1979 & 0.0000 & & & \\
\hline \multirow[t]{2}{*}{ SIJESTRATUK } & 54287 & 50763.31 & 12 & 1.1979 & 0.0998 & $M A$ & 1.1191 & \\
\hline & & & & 1.1779 & 0.0000 & & & \\
\hline 希R！ & & & 2 & 1.1979 & $0.5 \div 29$ & 102.13 & 0.9731 & \\
\hline $5 ?$ & & & 2 & 1.1979 & 0.5999 & 102.13 & 1.124 & \\
\hline $\operatorname{TRR} 3$ & & & 2 & 1.1979 & 0.5989 & 102.13 & 0.7759 & \\
\hline \multirow[t]{2}{*}{$\operatorname{MRR} 1$} & & & 2 & 1.1979 & 0.5989 & 102.13 & 1.1260 & \\
\hline & & & & 1.1979 & 0.0000 & & & \\
\hline \multirow{2}{*}{ ASHLAND } & 5714 & 5249.05 & 8 & 1.1979 & 0.1497 & 4979.44 & 1.05 & 1.05 \\
\hline & & & & 1.1979 & 0.0000 & & & \\
\hline ARR I & & & 1 & 1.1979 & 1.1979 & 94.98 & 0.9581 & \\
\hline MRR 2 & & & 2 & 1.1079 & 0.5799 & 94.98 & 0.5994 & \\
\hline GRR 3 & & & 0 & 1.1979 & 0.0000 & $94.9 B$ & 0.0060 & \\
\hline \multirow[t]{2}{*}{$M$} & & & 1 & 1.1979 & 1.1979 & 94.98 & 1.4424 & \\
\hline & & & & 1.1979 & 0.0000 & & & \\
\hline \multirow[t]{2}{*}{ TOREST GRGVE } & 5158 & 4709.83 & 4 & 1.1079 & 0.2995 & 4979.44 & 1). 95 & 0.95 \\
\hline & & & & 1.1579 & 0.0000 & & & \\
\hline \multirow[t]{2}{*}{ UTLTES SAHPL } & 10952 & 9958.98 & 12 & 1.1979 & 0.0998 & & & \\
\hline & & & & 1.1979 & 0.0000 & & & \\
\hline \multirow[t]{2}{*}{ QT: UTLTES-4 } & 33252 & & & 1.1979 & 0.9000 & & & \\
\hline & & & & 1.1979 & 0.0000 & & & \\
\hline \multirow[t]{2}{*}{ SUESTRATUH } & 44104 & 4 (1) 474.26 & 12 & 1.1979 & 0.0999 & $N A$ & 1.0110 & \\
\hline & & & & 1.1579 & 0.0000 & & & \\
\hline ARF : & & & 2 & 1.1979 & 0.5999 & 456.23 & 0.5058 & \\
\hline MER 2 & & & 2 & 1.1979 & 0.5989 & 456.23 & 1.1617 & \\
\hline$M R R$ & & & ? & 1.1979 & 0.0000 & 456.23 & 0.0000 & \\
\hline \multirow[t]{2}{*}{ YRR 4} & & & 1 & 1.1979 & 1.1779 & 456.23 & 0.9325 & \\
\hline & & & & 1.1979 & 0.0000 & & & \\
\hline \multirow[t]{2}{*}{ LLAERLY-LEME } & 5528 & 258.39 & 5 & 1. 1779 & 0.2555 & 2218.13 & 1,25 & $1, i^{2}$ \\
\hline & & & & $1 .\{979$ & 1.00000 & & & \\
\hline WhR I & & & 1 & $\therefore .1979$ & $1.197^{\circ}$ & 269.26 & $1.305 \%$ & \\
\hline MPL 2 & & & 2 & 1.1979 & 0.5999 & 269.26 & 1.0364 & \\
\hline MRE 3 & & & 0 & 1.1579 & 0.0000 & 259.26 & 0.0000 & \\
\hline
\end{tabular}




\begin{tabular}{|c|c|c|c|c|c|c|c|c|}
\hline & $\begin{array}{l}\text { DTHEF TOT } \\
\text { OBPEY ATINH }\end{array}$ & $\begin{array}{l}\text { DTHER ELIGBL } \\
\text { TIT FAPS }\end{array}$ & $\begin{array}{l}\text { COMPLETED } \\
\text { INTEPUIEHS }\end{array}$ & AVE COMFL & IHDIVIOUAL & $\begin{array}{l}\text { ANERAGE } \\
\text { POPUHATION }\end{array}$ & MER & UTILITY \\
\hline ENIIT & & & & & & & & \\
\hline MES & & & 2 & 1,1579 & 0,5750 & 209.26 & (j. 582 & \\
\hline & & & & 1.1979 & 0.0060 & & & \\
\hline MLEML!JTH & 2559 & 21011.87 & 5 & 1.1479 & 9.2396 & 2918.13 & 3.95 & 0.95 \\
\hline & & & & 1.1979 & 1). 00000 & & & \\
\hline UTLTES SAMPL & 4797 & 4430.20 & 10 & 1.1979 & 0.1199 & & & \\
\hline & & & & 1.1979 & 0.6000 & & & \\
\hline OTH UTLTES-4 & 10511 & & & 1.1979 & 0.0000 & & & \\
\hline & & & & 1.1979 & 0.0000 & & & \\
\hline SLBSTRATUN & 15298 & 14177.13 & 10 & 1.1979 & 0.1198 & HA & 0.4163 & \\
\hline & & & & 1.1979 & 0.0000 & & & \\
\hline STEATÜH TOT & 189520 & 170274.15 & 46 & 1.1979 & 0.0260 & HA & 0.54 & \\
\hline & & & & $1 . ! 979$ & 0.0000 & & & \\
\hline PUELIC TOT & 1282101 & 1183079.68 & 203 & 1.1979 & 0.0059 & & $\therefore 0060$ & \\
\hline & & & & 1.1979 & 0.0000 & & & \\
\hline PRIVATE & & & & 1.1979 & 0.0000 & & & \\
\hline & & & & 1.1979 & 0.0000 & & & \\
\hline RE6ION 1 & & & & 1.1979 & 0.0000 & & & \\
\hline 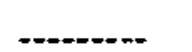 & & & & 1.1979 & 0.0000 & & & \\
\hline & & & & 1.1979 & 0.0000 & & & \\
\hline MRR 1 & & & 1 & 1.1979 & 1.1974 & 218.44 & 0.3342 & \\
\hline MRR 2 & & & 0 & 1.1979 & 0.0000 & 218.44 & 0.0000 & \\
\hline HR 3 & & & 1 & 1.1979 & 1.1979 & 218.44 & $0.51 \mathrm{B3}$ & \\
\hline HER 4 & & & 1 & 1.1979 & 1.1979 & 218.44 & 0,4431 & \\
\hline MRE 5 & & & 1 & 1.1979 & 1.1979 & 218.44 & 0.2307 & \\
\hline MRR i & & & 0 & 1.1979 & 0.000 & 218.44 & 0.0000 & \\
\hline KRR 7 & & & 1 & 1.1979 & 1.1979 & 218.44 & $2.2 B E 9$ & \\
\hline KRR E & & & 1 & 1.1979 & 1.1979 & 219.44 & 1.8586 & \\
\hline MRR 9 & & & 1 & 1.1979 & 1.1979 & 218.44 & $1 . \mathrm{BB} 15$ & \\
\hline MRR 10 & & & 1 & $1.1979^{\circ}$ & 1.1979 & 218.44 & 2.1362 & \\
\hline MRR 11 & & & 0 & 1.1979 & 0.0000 & 218.44 & 0.0000 & \\
\hline WRR 12 & & & 1 & 1.1979 & 1.1979 & 218.44 & 0.6475 & \\
\hline HRR I3 & & & 1 & 1.1979 & 1.1979 & 218.44 & 0.5613 & \\
\hline MRR 14 & & & 1 & 1.1979 & 1.1979 & 218.44 & 0.0996 & \\
\hline ARR 15 & & & 0 & 1.1979 & 0.0000 & 218.44 & 0.0000 & \\
\hline MRP 16 & & & 0 & 1.1979 & 0.0000 & 218.44 & 0.0000 & \\
\hline & & & & 1.1979 & 0.0000 & & & \\
\hline MONTANA GNR & 55982 & 50372.31 & 11 & 1.1979 & 0.1089 & 23364.01 & 1.0000 & 1.60 \\
\hline SLESTRATUN & & & & 1.1979 & 0.0000 & & & \\
\hline & & & & 1.1979 & 0.0000 & & & \\
\hline & & & & 1.1979 & 1,9000 & & & \\
\hline & & & & 1.1979 & 0.0000 & & & \\
\hline & & & & 1.1979 & 0.0000 & & & \\
\hline MRR 1 & & & 1 & 1.1979 & 1.1979 & 160.39 & 0.9756 & \\
\hline MRR 2 & & & 1 & 1.1979 & 1.1979 & 166.39 & 1.2877 & \\
\hline $\operatorname{MRR} \overline{\mathrm{S}}$ & & & 1 & 1.1979 & 1.1979 & 166.39 & 1.3647 & \\
\hline$M R R \&$ & & & 1 & $\$ .1979$ & 1.1979 & $166.3 P$ & 0.5721 & \\
\hline MRR 5 & & & 0 & 1.1979 & 0.0000 & 160.39 & 0.0060 & \\
\hline Mfen & & & 1 & 1.1979 & 1.1979 & 166.39 & 0.5269 & \\
\hline akn 7 & & & $!$ & $\therefore .1979$ & 1.1979 & $: t c .37$ & $\dot{6} .95 .36$ & \\
\hline MRE $g$ & & & 1 & 5.1979 & 1.1979 & 166.39 & 0.9954 & \\
\hline HRR : & & & \pm & 1.1979 & 1.1979 & 166.39 & 0.9255 & \\
\hline MRR 10 & & & 0 & $\$ .1979$ & 0.0000 & 166.39 & 0.6000 & \\
\hline HQR 11 & & & 1 & 1.1979 & 1.1979 & 166.39 & 1.1612 & \\
\hline
\end{tabular}




\begin{tabular}{|c|c|c|c|c|c|c|c|c|}
\hline ENTIT: & 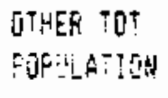 & $\begin{array}{l}\text { OTHEF ELIGEt } \\
\text { TOT POPS }\end{array}$ & $\begin{array}{l}\text { CDMPIETEN } \\
\text { DHEFiVIERS }\end{array}$ & $\begin{array}{l}\text { ANE COMF: } \\
\text { PES MSE }\end{array}$ & $\begin{array}{l}\text { INEIUILIAL } \\
\text { WEIFTTS }\end{array}$ & $\begin{array}{l}\text { AVESAEE } \\
\text { FOFULATION }\end{array}$ & $\begin{array}{c}\text { Hapk } \\
\text { HEISHTS }\end{array}$ & $\begin{array}{l}\text { WTILTTY } \\
\text { WEIEHTS }\end{array}$ \\
\hline 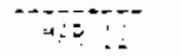 & & & i & 1.1408 & 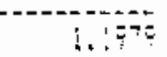 & 16,3 & 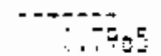 & \\
\hline$x=1:$ & & & ! & 1.6979 & $\therefore 1794$ & $1 \leq 6.39$ & 1,1 & \\
\hline \multirow[t]{2}{*}{$y=k: 4$} & & & 1 & 1.157 & $\therefore 150$ & 165.37 & $\therefore .2091$ & \\
\hline & & & & 1.1979 & 1.. 06010 & & & \\
\hline 垃 & $219: 7$ & 19139.73 & 12 & 1.1074 & 9.990 & 9859.97 & 1.00 & 1.000 \\
\hline SUESTEATUH & & & & 1.1970 & 1). 6000 & & & \\
\hline MRE I & & & 0 & 1.1979 & 0.0000 & 0.00 & 0.0000 & \\
\hline Mak 2 & & & 0 & $\begin{array}{l}1.1979 \\
1.1979\end{array}$ & $\begin{array}{l}0.0000 \\
0.00000\end{array}$ & 0.10 & 0.0060 & \\
\hline MT PSi & $\leq 41$ & 541.00 & 0 & 1.1979 & 0.0000 & $9689 . \pm 7$ & 1.00 & 1.3009 \\
\hline SLESTRATIM & & & & 1.1979 & $4.500 \%$ & & & \\
\hline \multirow[t]{2}{*}{ FELL-MT:MT-F } & & 19777.73 & & 1.1979 & 0.6000 & & & \\
\hline & & & & 1.1979 & 0.0000 & & & \\
\hline \multirow[t]{2}{*}{ STEATUM TOT } & 78550 & 70152.014 & 23 & 1.1979 & 0.19521 & NA & 0.2916 & \\
\hline & & & & 1.1979 & 0.0000 & & & \\
\hline \multirow[t]{2}{*}{ Region 2} & & & & 1.1979 & 0.0090 & & & \\
\hline & & & & 1.1979 & $0.009 \%$ & & & \\
\hline \multirow[t]{2}{*}{ 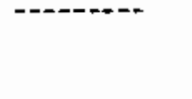 } & & & & 1.1979 & 0.0010 & & & \\
\hline & & & & 1.1979 & 0.6010 & & & \\
\hline KRR I & & & 1 & $\therefore .1979$ & 1.1979 & 210.82 & 1.5179 & \\
\hline $\operatorname{MRR} 2$ & & & 0 & 1.1979 & 0.0000 & 210.82 & 0.0000 & \\
\hline MAP 3 & & & i & 1.1979 & 1.1979 & 210.82 & 1.1422 & \\
\hline MLp 4 & & & 0 & 1.1979 & 0,0000 & 210.82 & 0.0000 & \\
\hline Sk: 5 & & & $i$ & 1.1979 & 1.1975 & 210.92 & 1.0485 & \\
\hline tक & & & i & 1.1979 & 1.1979 & 210.92 & (1), $76 ! 1$ & \\
\hline MRR 7 & & & ! & 1.1979 & 1.1979 & 210.92 & 0.7703 & \\
\hline \multirow[t]{2}{*}{ AR 8} & & & 1 & 1.1979 & 1.1979 & 210.82 & 0.7400 & \\
\hline & & & & 1.1979 & 0.6000 & & & \\
\hline CPLL-ID & 6055 & $5889.6 B$ & 6 & 1.1979 & 0.1956 & 69049.52 & 1.00 & 1.0000 \\
\hline SUBSTRATUM & & & & 1.1979 & 0.0000 & & & \\
\hline MER I & & & 1 & 1.1979 & 1.1979 & 282.24 & 0.8844 & \\
\hline Yh 2 & & & $i$ & 1.1979 & 1.1979 & 282.24 & 0.9215 & \\
\hline Mrp 3 & & & $i$ & 1.1979 & 1.1979 & 282.24 & $1.04 B J$ & \\
\hline his a & & & 1 & 1.1979 & $\therefore 1979$ & 282.24 & 1,3226 & \\
\hline 4 & & & 0 & 1.1979 & 0.0000 & 282.24 & 0.0600 & \\
\hline MRP 6 & & & 0 & 1.1979 & 0.0000 & 282.24 & 0.10000 & \\
\hline KRf 7 & & & 1 & 1.1979 & 1.1979 & 282.24 & 1.0098 & \\
\hline \multirow[t]{2}{*}{ MRR 3} & & & 1 & 1.1979 & 1.1979 & 282.24 & 0.8135 & \\
\hline & & & & 1.1979 & D. 1000 & & & \\
\hline IDAHO PUA & 262647 & 197209,89 & $\delta$ & 1.1079 & 0.1996 & 69049.52 & 1.00 & 1.0000 \\
\hline SUBSTRATLH & & & & $\therefore .1977$ & 0.0000 & & & \\
\hline MRP I & & & 1 & 1.1979 & 1.1979 & 276.89 & 0.8922 & \\
\hline MRP? 2 & & & 1 & 1.1979 & 1.1979 & 276.88 & 0.9727 & \\
\hline Aks 3 & & & 0 & $1 .: 579$ & 0.0000 & 276.78 & 0.0000 & \\
\hline AFE 4 & & & 0 & 1.1979 & 0.0000 & $27: .88$ & 1.6000 & \\
\hline$M P R$ & & & $\hat{\theta}$ & $\therefore .1779$ & 0.0000 & $275 . E B$ & 0.0000 & \\
\hline \multirow[t]{2}{*}{ Mat } & & & f & 1.1579 & 1.1979 & 276.65 & $1.134 !$ & \\
\hline & & & & $1.157^{\circ}$ & 0.6000 & & & \\
\hline 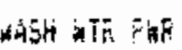 & $587 ;$ & 3449,43 & 3 & $\therefore \div 979$ & 0.5993 & 69049.52 & 1.60 & 1.0000 \\
\hline SUESTRATIM & & & & 1.1979 & 0. 6060 & & & \\
\hline MER ! & & & ! & 1.1979 & 1.1979 & 165.88 & 0.9143 & \\
\hline 虽? & & & 1 & 1.1579 & 1.5979 & 165.96 & 1.3893 & \\
\hline MFi 3 & & & 1 & 1.1979 & 1.1079 & 165.86 & $\$ .5699$ & \\
\hline
\end{tabular}




\begin{tabular}{|c|c|c|c|c|c|c|c|c|}
\hline ENTITY & $\begin{array}{l}\text { OTHER TOT } \\
\text { FOPLILATIOA }\end{array}$ & $\begin{array}{c}\text { OTHEE ELIGQL } \\
\text { TOT FOFS }\end{array}$ & $\begin{array}{l}\text { COHPLETED } \\
\text { INTERVIEHS }\end{array}$ & $\begin{array}{l}\text { AVS CGMFL } \\
\text { PER HRA }\end{array}$ & $\begin{array}{l}\text { INDIVIDUAL } \\
\text { SHEIGHTS }\end{array}$ & $\begin{array}{l}\text { AUERAGE } \\
\text { POPULATION }\end{array}$ & $\begin{array}{c}\text { MRR } \\
\text { DEIBHTS }\end{array}$ & $\begin{array}{l}\text { UTILITY } \\
\text { WEIGHTS }\end{array}$ \\
\hline 政 & $+\infty+\infty-\infty$ & - - & $\cdots+$ & 1.1579 & 0,0 & 165.09 & 5000 & \\
\hline MRE 5 & & & 1 & \pm .1979 & 1.1579 & $! 6=39$ & 0.9163 & \\
\hline ing ín & & & 1 & 1.1979 & 1.1979 & $165 . \overline{8} \overline{8}$ & ). 6101 & \\
\hline MSH 7 & & & 0 & 1.1979 & 0.0000 & 165.88 & 0.0000 & \\
\hline \multirow[t]{2}{*}{ MFE $\&$} & & & $!$ & 1.1779 & 1.1979 & 165.88 & 0.6061 & \\
\hline & & & & 1.1979 & 0.0000 & & & \\
\hline UTAH P\&L & 33205 & 28608.92 & 6 & 1.1979 & 0.1996 & 69049.52 & 1.00 & 1.0000 \\
\hline SUBSTFATUM & & & & 1.1979 & 0.0000 & & & \\
\hline \multirow[t]{2}{*}{ STERTIM TQT } & 301236 & 276198.10 & 21 & 1.1979 & 0.0570 & NA & 1.26 & \\
\hline & & & & 1.1979 & 0.0000 & & & \\
\hline \multirow[t]{3}{*}{ REGION 3} & & & & 1.1979 & 0.0000 & & & \\
\hline & & & & 1.1979 & 4.1000 & & & \\
\hline & & & & 1.1979 & 0.0000 & & & \\
\hline HFR I & & & 1 & t.1979 & 1.1979 & 252.18 & 1.6377 & \\
\hline 数 2 & & & 0 & 1.1979 & $0.00 \%$ & 252.18 & 0.0000 & \\
\hline $\operatorname{MRR} 3$ & & & 0 & 1.1979 & 0.0000 & 252.18 & 0.0000 & \\
\hline KER 4 & & & 1 & 1.1979 & 1.1970 & 252.18 & 0.6250 & \\
\hline MRR 5 & & & $!$ & 1.1979 & 1.1979 & 252.18 & 1.0865 & \\
\hline ARR 6 & & & 1 & 1.1979 & 1.1979 & 252.18 & 0.7495 & \\
\hline HeR 7 & & & 1 & 1.1979 & 1.1979 & 252.18 & 0.7376 & \\
\hline MRR B & & & 1 & 1.1979 & 1.1979 & 252.18 & 1.3165 & \\
\hline MRR 9 & & & 1 & 1.1979 & 1.1979 & 252.18 & 1.4434 & \\
\hline Mp 10 & & & 1 & 1.1979 & 1.1979 & 252.18 & 0.5759 & \\
\hline MER if & & & 0 & 1.1979 & 0.0000 & $252.1 \mathrm{E}$ & 0.0000 & \\
\hline HFo 1? & & & 1 & 1.1579 & 1.1979 & 252.18 & 1.0099 & \\
\hline ARR 13 & & & 1 & $\$ .1979$ & 1.1979 & 252.18 & 0.9398 & \\
\hline MRR 14 & & & 1 & 1.1979 & 1.1979 & 252.18 & 0.8962 & \\
\hline MRR 15 & & & 1 & 1.1979 & 1.1979 & 252.18 & 0.9629 & \\
\hline \multirow[t]{2}{*}{ MRR 16} & & & 0 & 1.1979 & 0.0000 & 252.18 & 0.0000 & \\
\hline & & & & 1.1979 & 0.0000 & & & \\
\hline PPLL-HA & 81702 & 77780.54 & $! 2$ & 1.1979 & 0.0998 & 105676.38 & 1,00 & 1.0000 \\
\hline SUBSTRATLH & & & & 1.1979 & 0.0000 & & & \\
\hline MFR 1 & & & 0 & 1.1979 & 0.0000 & $2 \notin 2.60$ & 0.0000 & \\
\hline NaR 2 & & & 1 & 1.1979 & 1.1979 & 242.60 & 1.0387 & \\
\hline MRR J & & & 1 & 1.1979 & 1.1979 & 242.60 & 1.0970 & \\
\hline MRR 4 & & & 1 & 1.1979 & 1.1979 & 242.60 & 1.1717 & \\
\hline ARR 5 & & & 0 & 1.1979 & 0.0000 & 242.60 & 0.0000 & \\
\hline MRR 6 & & & 0 & 1.1979 & 0.0000 & 242.60 & 0.0000 & \\
\hline $\mathrm{MRR} 7$ & & & $t$ & $\$ .1979$ & 1.1979 & 242.60 & 0.9439 & \\
\hline MRR g & & & 1 & 1.1979 & 1.1979 & 242.60 & 1.0852 & \\
\hline MRR 9 & & & 0 & 1.1979 & 0.0000 & 242.60 & 0.0000 & \\
\hline HKR 10 & & & 0 & 1.1979 & 0,0000 & 242.60 & 0.0000 & \\
\hline MRR II & & & 0 & 1.1979 & 0.0000 & 242.60 & 0.0000 & \\
\hline $\operatorname{MRR} 12$ & & & 1 & 1.1979 & 1.1979 & 242.60 & 0.5139 & \\
\hline ARR 13 & & & 0 & 1.1979 & 0.0000 & 242.60 & 0.01000 & \\
\hline MRR 14 & & & 1 & 1.1979 & 1.1979 & 242.60 & 1.1995 & \\
\hline KRR 15 & & & 0 & 1.1979 & 0.0600 & 242.60 & 0.00010 & \\
\hline \multirow[t]{2}{*}{ NRR 16 } & & & 0 & 1.1979 & 0.0000 & 242.60 & 0.0000 & \\
\hline & & & & 1.1970 & 0.4000 & & & \\
\hline \multirow{2}{*}{$\begin{array}{l}\text { WASH WAT PUF } \\
\text { SUBSTRATUH }\end{array}$} & $\{44643$ & 137564.21 & 7 & 1.1979 & $0.17: 1$ & 105576.350 & 1.00 & 1.0000 \\
\hline & & & & 1.1979 & 0.0000 & & & \\
\hline \multirow[t]{2}{*}{ STERTUM TOT } & 226345 & 211352.75 & 19 & 1.1979 & 0.0630 & NA & 1.06 & \\
\hline & & & & 1.1979 & 0.0000 & & & \\
\hline
\end{tabular}




\begin{tabular}{|c|c|c|c|c|c|c|c|c|}
\hline EMTITY & $\begin{array}{l}\text { DTHES TST } \\
\text { PUEOLATISN }\end{array}$ & $\begin{array}{c}\text { UTHE ELIEEL } \\
\text { TOT POPS }\end{array}$ & $\begin{array}{l}\text { CDMPLETEI } \\
\text { IAHESUIE'S }\end{array}$ & $\begin{array}{l}\text { AVG CDMF! } \\
\text { PER MPK }\end{array}$ & $\begin{array}{l}\text { INC:WWDUAL } \\
\text { AEIGHTE }\end{array}$ & $\begin{array}{l}\text { AUERAGE } \\
\text { FOPGLATIIONJ }\end{array}$ & $\begin{array}{c}\text { NFS } \\
\text { WEIGHTS }\end{array}$ & $\begin{array}{l}\text { WTILITY } \\
\text { WEIBHTS }\end{array}$ \\
\hline 4 & & & & $\begin{array}{l}1.179 \\
1.1979 \\
1.1979\end{array}$ & $\begin{array}{l}0.000 \\
0.0000 \\
0.0 .00\end{array}$ & & - n-a... & \\
\hline Whe 1 & & & 1 & 1.1979 & 1.1979 & 273.54 & 1.6871 & \\
\hline MFi $=$ & & & $\hat{0}$ & 1.197 & $0.30 \mathrm{in}$ & 20.3 .34 & 19000 & \\
\hline risk 3 & & & 2 & 1.1979 & 0.5959 & F9.2.E4 & 0.8474 & \\
\hline Me 4 & & & 0 & 1.1979 & 0.0600 & 293.84 & 0.00100 & \\
\hline FER 5 & & & 2 & 1.1979 & 0.5489 & 293.84 & 1.2047 & \\
\hline YGT & & & 2 & 1.1979 & 0.5999 & $29 \% .84$ & 0.7529 & \\
\hline 7 & & & 1 & 1.1979 & 1.1979 & 273.84 & $\dot{1} .7757$ & \\
\hline 35 & & & 1 & 1,1977 & 1.1979 & 24.84 & 3.4799 & \\
\hline MET ? & & & 1 & 1.9979 & 1.1979 & 253.84 & 1.9125 & \\
\hline ARE 10 & & & 1 & 1.1974 & 1.1979 & 703.94 & $0.7 ! 73$ & \\
\hline MFR 11 & & & 1 & 1.1979 & 1.1979 & 293.84 & 1.4724 & \\
\hline 解 12 & & & $!$ & 1.1979 & 1.1779 & 29.5 .94 & 0.5192 & \\
\hline MER 13 & & & 1 & 1.1979 & 1.1979 & 293.84 & 0.6900 & \\
\hline$M 0914$ & & & 2 & 1.1979 & 0.5989 & 293.94 & $0.7: 44$ & \\
\hline HFR 15 & & & 1 & 1.1979 & $\$ .1979$ & 295.84 & $\therefore .897:$ & \\
\hline MFE !́ & & & 1 & 1.1979 & 1.1979 & 243.84 & 0.6296 & \\
\hline & & & & 1.1979 & 0.0000 & & & \\
\hline WGET SOUND & 496995 & $4692 \mathrm{~J} 2.46$ & 19 & 1.1979 & 0.0665 & 469232.46 & 1.00 & 1.0000 \\
\hline SUSSTFATUAH & & & & 1.1979 & 0.0000 & & & \\
\hline STRATUM TGT & $4 \% 995$ & 469232.46 & 18 & 1.1979 & 0.0665 & $N A$ & 3.20 & \\
\hline & & & & 1.1979 & 9.0600 & & & \\
\hline EEG!CN S & & & & 1.1979 & 9.0060 & & & \\
\hline -......--- & & & & 1.1979 & 0.0000 & & & \\
\hline 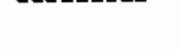 & & & & 1.1979 & 0.0000 & & & \\
\hline MPR 1 & & & 1 & 1.1979 & 1.1979 & 231.97 & 1.4605 & \\
\hline HRR ? & & & 1 & 1.1979 & 1.1979 & 231.97 & 1.2449 & \\
\hline YPR 3 & & & 0 & 1.1979 & 0.0000 & 231.97 & 0.0000 & \\
\hline MER 4 & & & ! & 1.1979 & 1.1974 & 231.97 & 1.9312 & \\
\hline MSล 5 & & & 0 & 1.1979 & 0.0000 & 231.97 & 0.6900 & \\
\hline MRE i & & & $!$ & 1.1979 & 1.1979 & 231.97 & 0.6971 & \\
\hline MGR 7 & & & 0 & 1.1979 & 0.0000 & 231.97 & 0,0000 & \\
\hline MPR 8 & & & 1 & 1.1979 & 1.1979 & 251.97 & $\therefore 406$ & \\
\hline MER ? & & & 1 & 1.1979 & 1.1979 & 231.97 & 0.7673 & \\
\hline MKR: $: 0$ & & & 1 & 1.1979 & 1.1979 & 231.97 & 1.2734 & \\
\hline Hin 11 & & & 0 & 1.1979 & 0.0000 & 231.57 & 0.6000 & \\
\hline$Y P R 12$ & & & ij & 1.1979 & 0.0000 & $=1.97$ & 0.0060 & \\
\hline सात 13 & & & 1 & 1.1979 & $\therefore 1979$ & 231.97 & $0.292 \mathrm{~J}$ & \\
\hline Hip 14 & & & & 1.1979 & & 231.97 & & \\
\hline ANP 15 & & & $!$ & 1.1979 & 1.1979 & 231.97 & 0.4354 & \\
\hline Man : : & & & f & 1.1979 & 1. $1977^{\circ}$ & 231.97 & $1.31 \% \mathrm{i}$ & \\
\hline & & & & 1.1979 & 0.0000 & & & \\
\hline$F O 4-3 R-E$ & $7: 835$ & bJ192.58 & 10 & 1.1979 & 1). 1198 & 29072.01 & 1.00 & 1.0000 \\
\hline SLISTRATUA & & & & 1.1979 & 0.0000 & & & \\
\hline MER ! & & & 1 & 1.1079 & 1.1979 & $24 \% .04$ & 0.7624 & \\
\hline MER 2 & & & 1 & 1.1979 & 1.1979 & 240.104 & 1.5065 & \\
\hline Min & & & 0 & 1.1979 & 0.00100 & 240.04 & j.6.50 & \\
\hline $\operatorname{mañ} \alpha$ & & & t & 1.1579 & 1.1979 & 240.04 & 1.1951 & \\
\hline What 5 & & & 0 & 1.1979 & 0.0000 & 240.04 & 6.6000 & \\
\hline and & & & $i$ & 1.1979 & 1.1979 & 240.64 & 0.1302 & \\
\hline HAF? & & & 1 & 1.1979 & i. 1979 & $24 i, 04$ & 0.2557 & \\
\hline
\end{tabular}




\begin{tabular}{|c|c|c|c|c|c|c|c|c|}
\hline EMTITY & $\begin{array}{l}\text { OTHER TOT } \\
\text { PUPULATION }\end{array}$ & $\begin{array}{c}\text { DTHER ELIEEL } \\
\text { TOT POPS }\end{array}$ & $\begin{array}{l}\text { CDAPLETED } \\
\text { INTERVIENS }\end{array}$ & $\begin{array}{c}\text { AVG COUPL } \\
\text { PER HBR }\end{array}$ & $\begin{array}{l}\text { IMDIVIDUAL } \\
\text { WEIGHTS }\end{array}$ & $\begin{array}{l}\text { AVERAGE } \\
\text { POPLAATION }\end{array}$ & $\begin{array}{c}\text { MRR } \\
\text { MEIGHTS }\end{array}$ & $\begin{array}{l}\text { UTILITY } \\
\text { WEIGHTS }\end{array}$ \\
\hline pang & & n. & $i$ & $\begin{array}{l}1.1979 \\
1.1979\end{array}$ & $\begin{array}{l}1.1579 \\
0,6000\end{array}$ & 240,14 & 1,4122 & \\
\hline $\begin{array}{l}\text { ILIAHHO PUR } \\
\text { SUBSTRATTUK }\end{array}$ & 11675 & 10203.36 & $b$ & 1.1979 & $0 . ! 996$ & $290: 2.51$ & 1.00 & 1. jision \\
\hline SUESTRATUK & & & & 1.1979 & Q. Düiou & & & \\
\hline MRI & & & 0 & 1.1979 & 9. & $423.5 !$ & 0.0060 & \\
\hline$M E R 2$ & & & $!$ & 1.1979 & 1.1979 & 423.51 & 1.5278 & \\
\hline MFR 3 & & & & 1.1979 & & $423.5 !$ & & \\
\hline MRP 1 & & & 1 & 1.1979 & 1.1979 & 423.51 & 0.4722 & \\
\hline ARE 5 & & & & $\therefore 1979$ & & 423.51 & & \\
\hline MAF 6 & & & 0 & 1.1979 & $\dot{0} .0000$ & 423.51 & 0.0000 & \\
\hline $\operatorname{MRE} 7$ & & & & 1.1979 & & 423.51 & & \\
\hline \multirow[t]{2}{*}{ MPF $\mathrm{E}$} & & & 0 & 1.1979 & 0.9000 & $423.5 !$ & 0.6000 & \\
\hline & & & & 1.1979 & 0.9000 & & & \\
\hline CP MATNI & 22295 & 16520.09 & 2 & 1.1979 & 0.5989 & 29972.01 & 1.60 & 1.00 \\
\hline SUESTRATUV & & & & 1.1979 & 0.9000 & & & \\
\hline \multirow[t]{2}{*}{ STRATUA TOT } & 105805 & 89916.03 & 1B & 1.1979 & 0.0665 & NA & 0.48 & \\
\hline & & & & 1.1979 & 0.0000 & & & \\
\hline \multirow[t]{3}{*}{ REGIDN 6} & & & & 1.1979 & 0.0000 & & & \\
\hline & & & & 1.1979 & 0.0000 & & & \\
\hline & & & & 1.1979 & 0.0000 & & & \\
\hline MRR 1 & & & 1 & 1.1979 & 1.1979 & 241.24 & 0.3864 & \\
\hline MRR 2 & & & 1 & 1.1979 & 1.1979 & 241.24 & 0.7495 & \\
\hline ARR 3 & & & 1 & 1.1979 & 1.1979 & 241.24 & 1.0488 & \\
\hline $\mathrm{ARR} 4$ & & & $\hat{v}$ & 1.1979 & $0 . \cos 0$ & 241.24 & 0.0000 & \\
\hline SRR 5 & & & 1 & 1.1979 & 1.1979 & 241.24 & 0.525 & \\
\hline MRR i & & & 2 & 1.1979 & 0.5989 & 241.24 & 0.7929 & \\
\hline MAR 7 & & & 1 & 1.1979 & 1.1979 & 241.24 & 1.46 .52 & \\
\hline MFR 8 & & & 1 & 1.1979 & 1.1979 & 241.24 & 1.5255 & \\
\hline ARR 9 & & & 1 & 1.1979 & 1.1979 & 241.24 & 1.4516 & \\
\hline AER 10 & & & 1 & 1.1979 & 1.1979 & 241.24 & 0.4858 & \\
\hline MER II & & & 2 & 1.1979 & 0.5989 & 241.24 & 0.7337 & \\
\hline MRR 12 & & & 1 & 1.1979 & 1.1979 & 241.24 & 1.3596 & \\
\hline MRR 13 & & & 1 & 1.1979 & 1.1979 & 241.24 & $\$ .6520$ & \\
\hline MRR if & & & 1 & 1.1979 & $1 .: 979$ & 241.24 & 0.7817 & \\
\hline WAP 15 & & & 2 & 1.1979 & 0.5989 & 241.24 & 1.0570 & \\
\hline \multirow[t]{2}{*}{ MRR i6 } & & & 0 & 1.1979 & 0.0000 & 241.24 & 0.0000 & \\
\hline & & & & 1.1979 & 0.0000 & & & \\
\hline$P P E L-O R-1$ & 270257 & 245957.46 & 17 & $1 . ! 979$ & 0.0705 & 332225.74 & 1.00 & 1.0000 \\
\hline SUBSTRATUH & & & & 1.1979 & 0.0000 & & & \\
\hline MRT 1 & & & 1 & 1.1979 & 1.1979 & $.37\} .36$ & 0.6744 & \\
\hline HKR 2 & & & 0 & 1.1979 & 0.0000 & 330.36 & 0.0060 & \\
\hline MRR 3 & & & 2 & 1.1979 & 0.5989 & 330.36 & 0.8633 & \\
\hline ARR 4 & & & 2 & 1.1979 & 0.5989 & 530.36 & 1.2562 & \\
\hline MER 5 & & & 2 & 1.1979 & 0.5989 & 330.36 & 0.4783 & \\
\hline MRR 6 & & & 2 & 1.1979 & 0.5989 & 330.36 & 0.8105 & \\
\hline HRR ? & & & $!$ & 1.1979 & $\$ .1979$ & 330.36 & 1.1412 & \\
\hline AFR 8 & & & 2 & 1.1979 & 0.5989 & 330.36 & 0.7845 & \\
\hline MRA 9 & & & 1 & 1.1979 & 1.1979 & 330.36 & 1.1381 & \\
\hline MER $: 0$ & & & 1 & 1.1779 & $1.197^{\circ}$ & 370.36 & 1.3500 & \\
\hline MER 11 & & & 1 & 1.1979 & 1.1979 & 330.36 & 1.1896 & \\
\hline MRR 12 & & & 1 & 1.1979 & 1.1979 & 330.36 & 3.1079 & \\
\hline MRR 13 & & & 1 & 1.1979 & 1.1979 & $330.3 b$ & $0.8 B 15$ & \\
\hline MAR 14 & & & 0 & 1.1979 & 0.0060 & 330.36 & 0.0000 & \\
\hline
\end{tabular}




\begin{tabular}{|c|c|c|c|c|c|c|c|c|}
\hline EYTiTYY & $\begin{array}{l}\text { JTHEF TOT } \\
\text { FIFULATION }\end{array}$ & $\begin{array}{c}\text { WTHER ELIJBL } \\
\text { TOT COPS }\end{array}$ & $\begin{array}{l}\text { COHSLETED } \\
\text { OU"EEUIEHS }\end{array}$ & $\begin{array}{l}\text { AVG CSHEA } \\
\text { FEF MRE }\end{array}$ & $\begin{array}{c}\text { INDPVIBUAL } \\
\text { HETBHTS }\end{array}$ & $\begin{array}{l}\text { AUEFAEE } \\
\text { PDPLLATION }\end{array}$ & $\begin{array}{c}\text { YPR } \\
\text { WE ISHTS }\end{array}$ & $\begin{array}{l}\text { JTILITY } \\
\text { HETEHTS }\end{array}$ \\
\hline 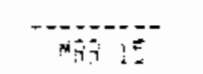 & 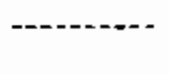 & 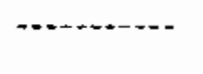 & $\vdots$ & 3.1799 & 1.1979 & 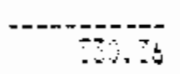 & 4.997 & \\
\hline Mtrits & & & $z$ & $\begin{array}{l}1.1979 \\
1.1979\end{array}$ & $\therefore 5799$ & Mt. & 1.3729 & \\
\hline $\begin{array}{l}\text { POKT GEN ELE } \\
\text { S!ESIRATUM }\end{array}$ & $4391 j 0$ & 419454.61 & 20 & $\begin{array}{l}1.1979 \\
1.1979\end{array}$ & & 332225.74 & 1.60 & 1.8000 \\
\hline STRETER TOT & 709573 & 604451.47 & 37 & $\begin{array}{l}1.1970 \\
1.1979\end{array}$ & & Kin & 2.27 & \\
\hline FRlVAFE TIT & 1918364 & 1781305 & 136 & & & 1781303 & 1.60 & \\
\hline OFAlij TOT & 3200405 & 2964385 & 39 & & & 2934303 & 1.00 & \\
\hline
\end{tabular}




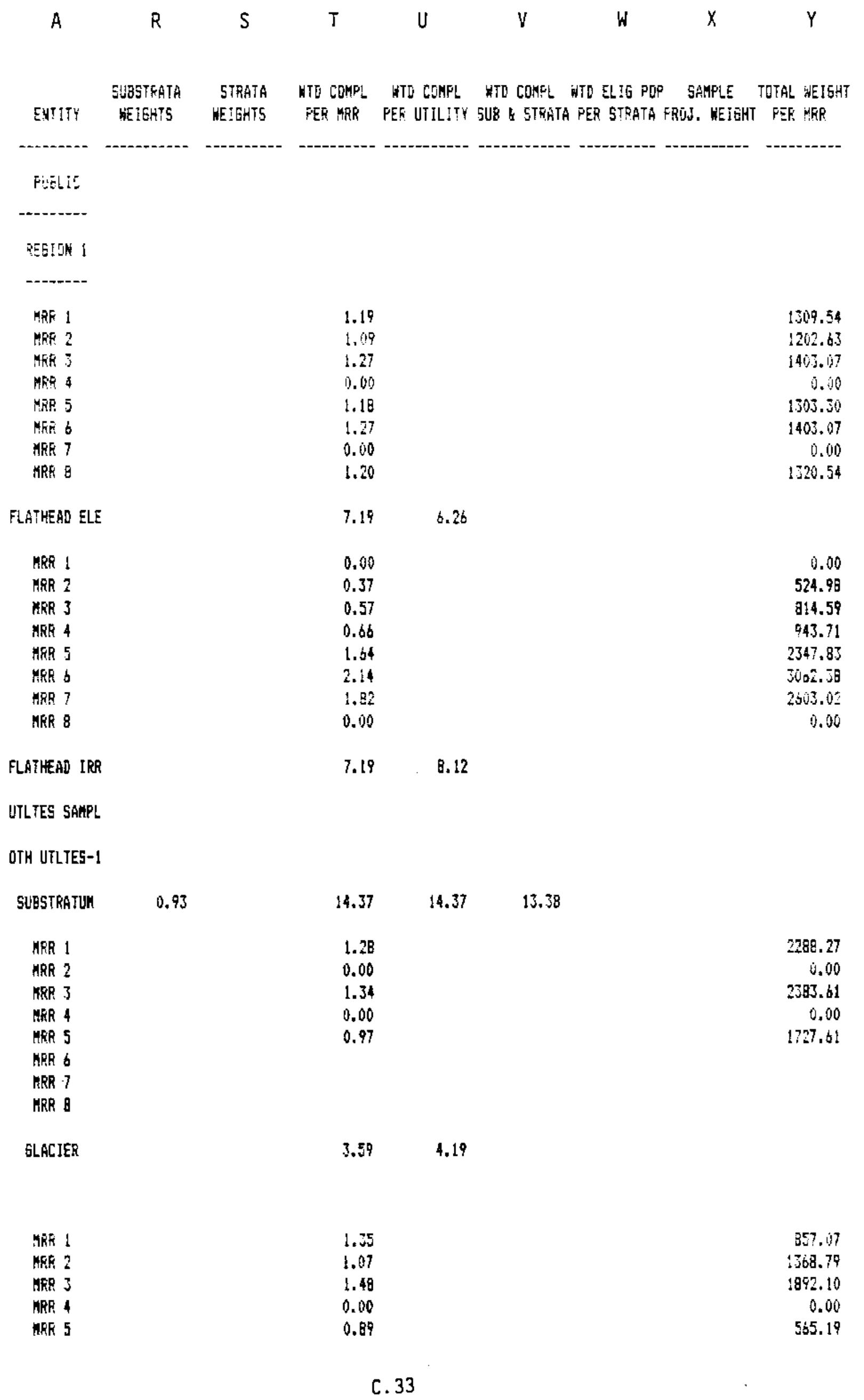




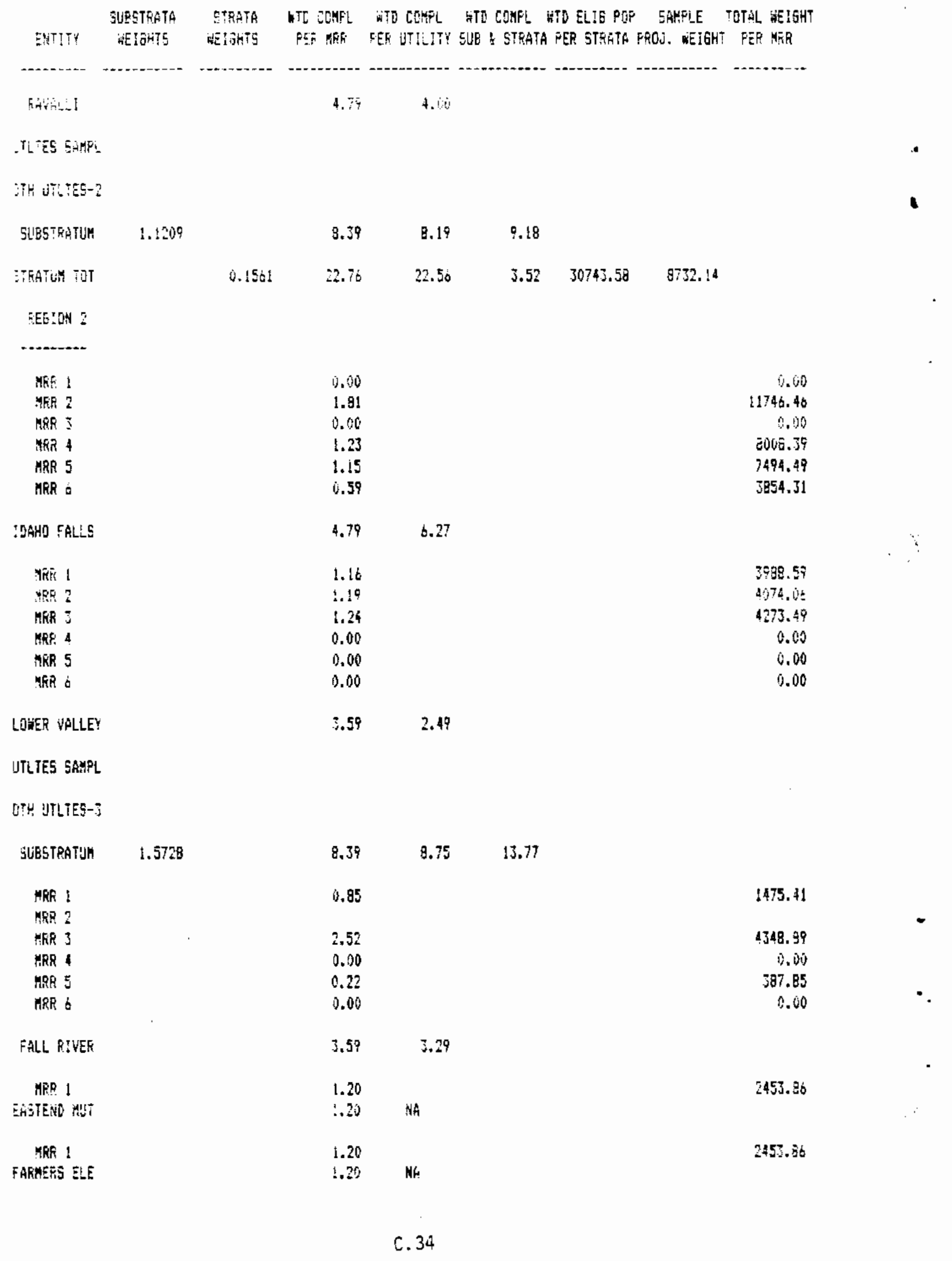




\begin{tabular}{|c|c|c|c|c|c|c|}
\hline ENTITY & $\begin{array}{l}\text { SUFSTKETA } \\
\text { WEIEHTS }\end{array}$ & $\begin{array}{r}\text { STRATA } \\
\text { HEIEHTS }\end{array}$ & $\begin{array}{l}\text { WTO COMPL } \\
\text { PER MRR }\end{array}$ & $\begin{array}{l}\text { WTO COKPL } \\
\text { PER UTILITH }\end{array}$ & $\begin{array}{l}\text { WTO COMPL HTD ELIS POP SAMELE } \\
\text { SUB \& STRATA FER STRATA PROJ. WEIEHT }\end{array}$ & $\begin{array}{l}\text { TOTAL WEIGHT } \\
\text { T WEF HER }\end{array}$ \\
\hline He: ! & & & 1.0 & & & 450 \\
\hline RIVESTIE E & & & 1.20 & Nis & & \\
\hline MFR ! & & & 0.00 & & & 0.90 \\
\hline COUTHSIDE EL & & & 9.90 & $N A$ & & \\
\hline MRE I & & & 0.00 & & & 0.60 \\
\hline MRP 2 & & & 1.20 & & & 2453.86 \\
\hline UNITY LLP & & & 1.20 & $\mathrm{NA}$ & & \\
\hline MINOGKA PROJ & & & 4.79 & 5.30 & & \\
\hline \multicolumn{7}{|l|}{ UTLIES SAMPL } \\
\hline \multicolumn{7}{|l|}{ QTH UTLTES-4 } \\
\hline SUESTRATU: & 0.5986 & & B. 39 & 8.19 & 5.68 & \\
\hline MRR 1 & & & 1.19 & & & 1887.30 \\
\hline MRR 2 & & & 0.00 & & & 0.00 \\
\hline FRR 3 & & & 0.00 & & & 0.00 \\
\hline MRR 4 & & & 1.20 & & & 1895.43 \\
\hline MR 5 & & & 1.20 & & & 1255.43 \\
\hline mRR b & & & 0.00 & & & 9.60 \\
\hline LOST RIVER & & & 3.59 & 2.87 & & \\
\hline \multicolumn{7}{|l|}{ MRR I } \\
\hline MRR 2 & & & 1.20 & & & 2846.35 \\
\hline MRR 3 & & & 0.00 & & & 0.00 \\
\hline MRR 4 & & & 0.00 & & & 0.00 \\
\hline HRR 5 & & & 0.00 & & & 0.00 \\
\hline ARR 6 & & & 0.00 & & & 0.00 \\
\hline HEISER & & & 1.20 & 1.44 & & \\
\hline
\end{tabular}

UTLTES SAAPL

OTH UILLES-4

\begin{tabular}{|c|c|c|c|c|c|}
\hline SUESTRATUH & 0.6270 & 4.79 & 4.31 & 2.70 & \\
\hline STRATUM TOT & 0.3613 & 21.56 & 21.55 & 7.79 & 67991.88 \\
\hline
\end{tabular}

REEIOA 3

$\begin{array}{ll}\text { HAR I } & 0.00 \\ \text { MRF 2 } & 0.00 \\ \text { MRR 3 } & 0.00 \\ \text { MFR 1 } & 1.20 \\ \text { MRR 5 } & 0.00\end{array}$

$$
\begin{array}{r}
5.00 \\
0.00 \\
0.00 \\
14520.06 \\
0.00
\end{array}
$$

C. 35 


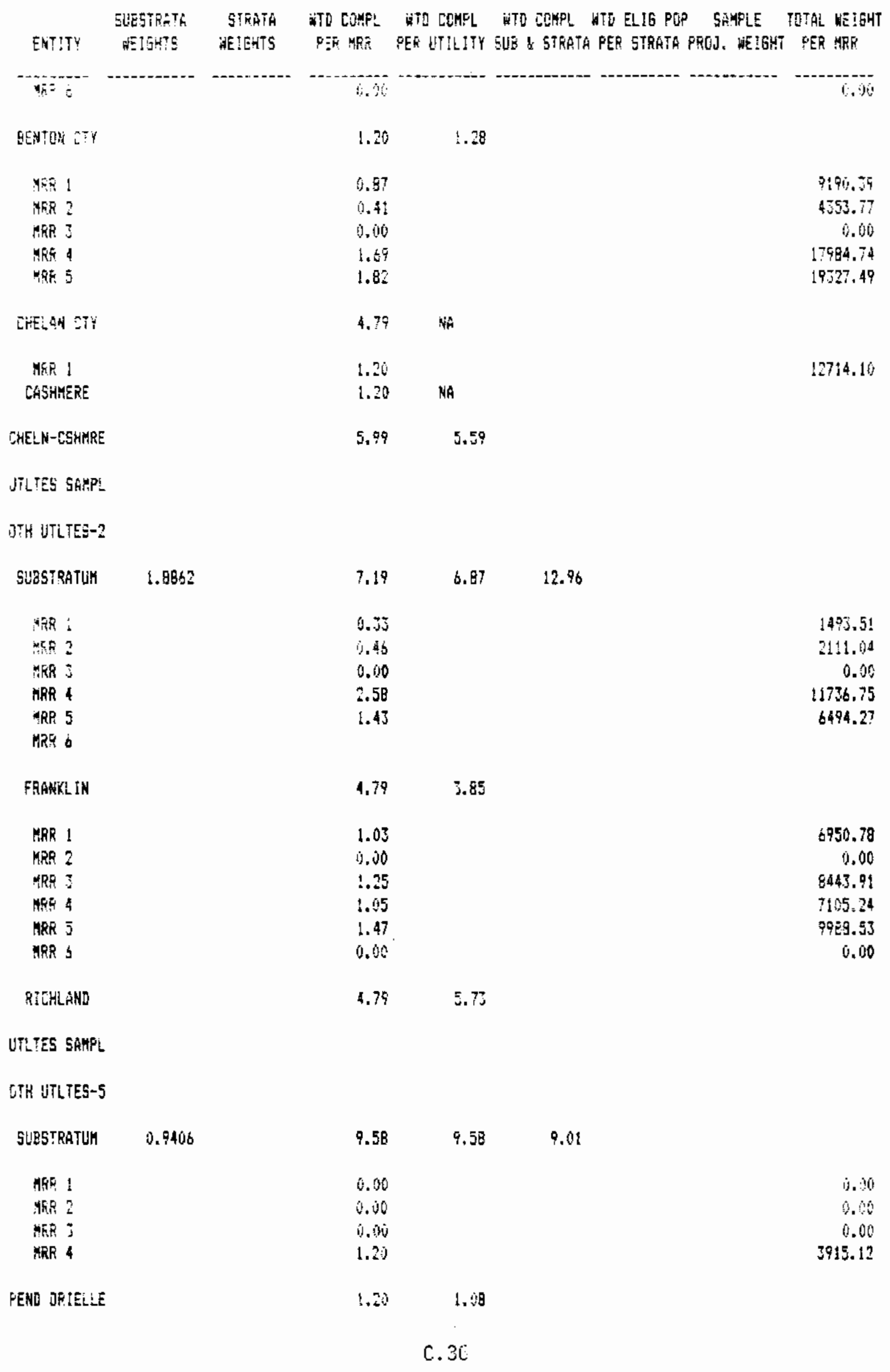




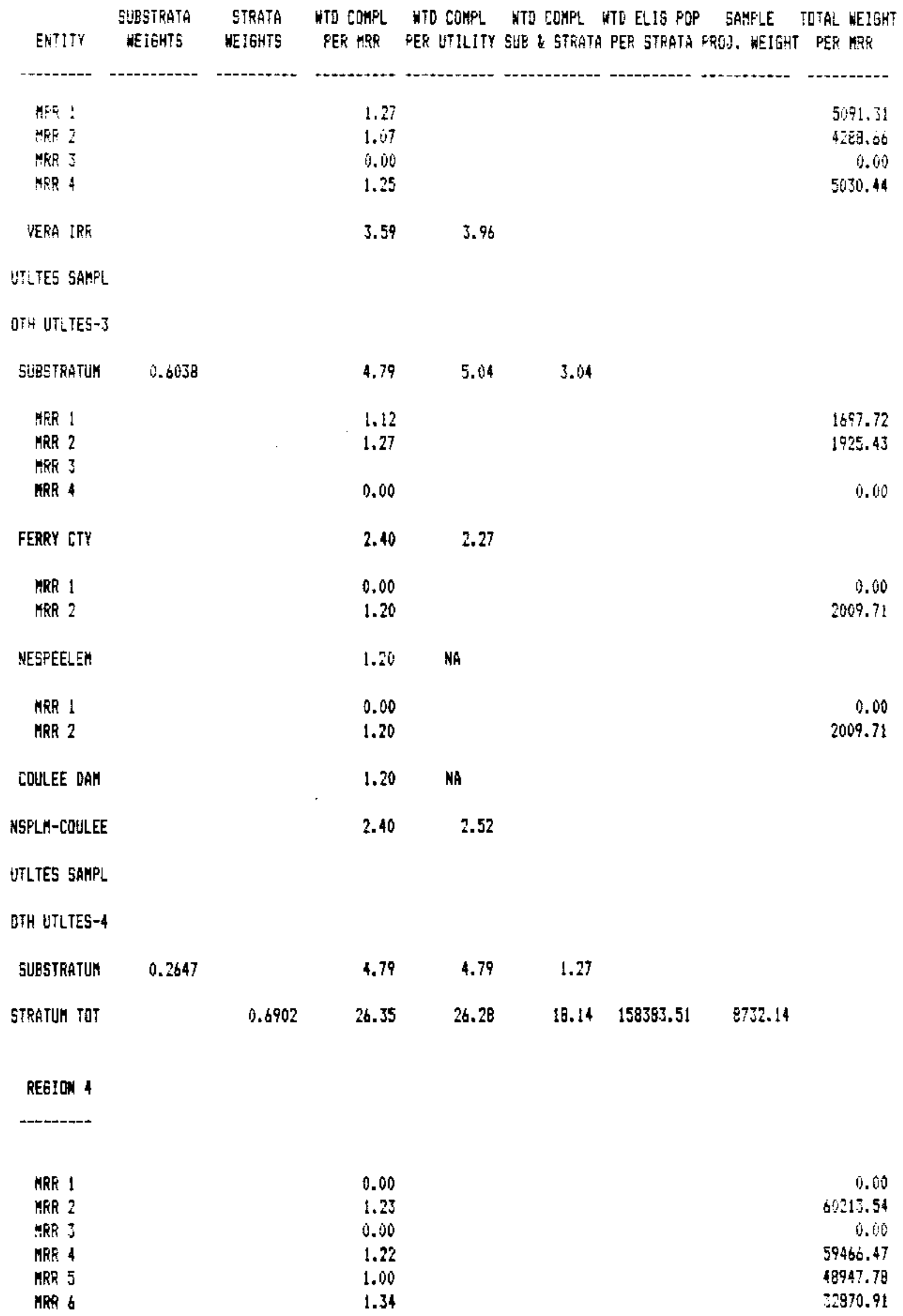




\begin{tabular}{|c|c|c|c|c|c|c|}
\hline EHTITY & $\begin{array}{l}\text { SLESTRETTA } \\
\text { WEISHTS }\end{array}$ & $\begin{array}{l}\text { STR.ATA } \\
\text { WEIGHTS }\end{array}$ & 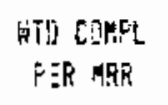 & $\begin{array}{l}\text { WTE COMPL } \\
\text { PER STILITY }\end{array}$ & $\begin{array}{l}\text { HTC COMPL WTE ELIG PGP SAMPLE } \\
\text { SUE \& STRATA PEF STEATA ERDJ. WEIGHT }\end{array}$ & $\begin{array}{l}\text { TOTAL 䜣IEHT } \\
\text { FER WAR }\end{array}$ \\
\hline BET & - & - & 4.78 & 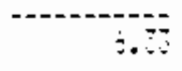 & (n) & -........- \\
\hline MEP 1 & & & 0.54 & & & 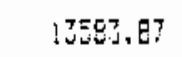 \\
\hline MEN 2 & & & 0.60 & & & 0.100 \\
\hline जिए & & & 1.51 & & & 32074.85 \\
\hline HER 4 & & & 1.45 & & & 30478.04 \\
\hline RRR 5 & & & 1.12 & & & 28083.51 \\
\hline Mafi of & & & 1.37 & & & 34439.37 \\
\hline SAGGOHISH & & & 5.49 & 4.07 & & \\
\hline \multicolumn{7}{|l|}{$\begin{array}{l}\text { !JTLTES SANGL } \\
\text { IJTH ITLTES- }\end{array}$} \\
\hline SEETFGTH & 3.0837 & & 10.78 & 10.40 & 32.06 & \\
\hline HRR I & & & 0.95 & & & 7241.61 \\
\hline צp 2 & & & 1.26 & & & 9656.15 \\
\hline ME 3 & & & 1.34 & & & 10223.34 \\
\hline MER 4 & & & 0.57 & & & 3645.03 \\
\hline KRT 5 & & & 1.97 & & & 28658.57 \\
\hline Mre 6 & & & 0.00 & & & 0.00 \\
\hline CLAfí ETY & & & 5.99 & 5.11 & & \\
\hline MSE : & & & 1.90 & & & 14342.97 \\
\hline WRR 2 & & & 1.12 & & & 11481.76 \\
\hline hes 3 & & & 1.11 & & & 22808.57 \\
\hline MRR 4 & & & $1.2 B$ & & & 26224.84 \\
\hline MRR 5 & & & 0.00 & & & 0.00 \\
\hline MRR b & & & 1.09 & - & & 22478.43 \\
\hline TACDMA & & & 5.99 & 6.87 & & \\
\hline \multicolumn{7}{|l|}{ UTLIES SRĨPL } \\
\hline \multicolumn{7}{|l|}{ DTH UTLTES-2 } \\
\hline SUESTRATUM & 1.4926 & & 11.98 & 11.98 & 17.88 & \\
\hline MER 1 & & & 0.00 & & & 0.00 \\
\hline MER 2 & & & 1.37 & & & 3770.61 \\
\hline NER 3 & & & 0.82 & & & 2268.60 \\
\hline FRR 4 & & & 2.60 & & & 11052.45 \\
\hline MAP 5 & & & 17.66 & & & 182E.17 \\
\hline 425 & & & 1.13 & & & 6243.44 \\
\hline MAEOH CTY-J & & & 5.99 & 6.24 & & \\
\hline the 1 & & & 4,60 & & & 0.30 \\
\hline we? 2 & & & 1.30 & & & $472 \hat{s} .98$ \\
\hline MKR 3 & & & 1.31 & & & 6041.20 \\
\hline MFR 4 & & & 0.76 & & & 3871.77 \\
\hline At 5 & & & 4.76 & & & 3850.79 \\
\hline
\end{tabular}




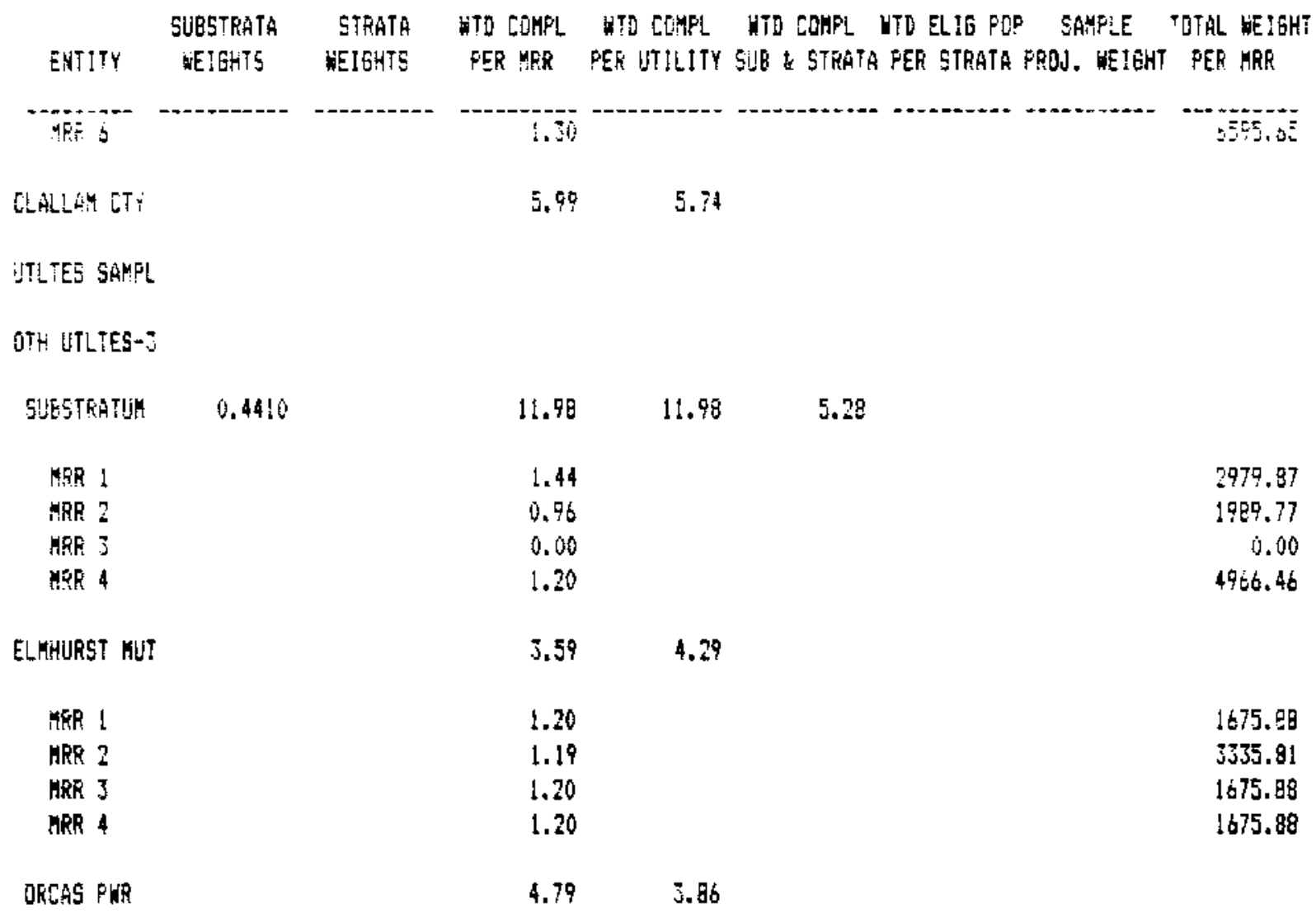

WTLES SAMPL

OTY UTLTES-3

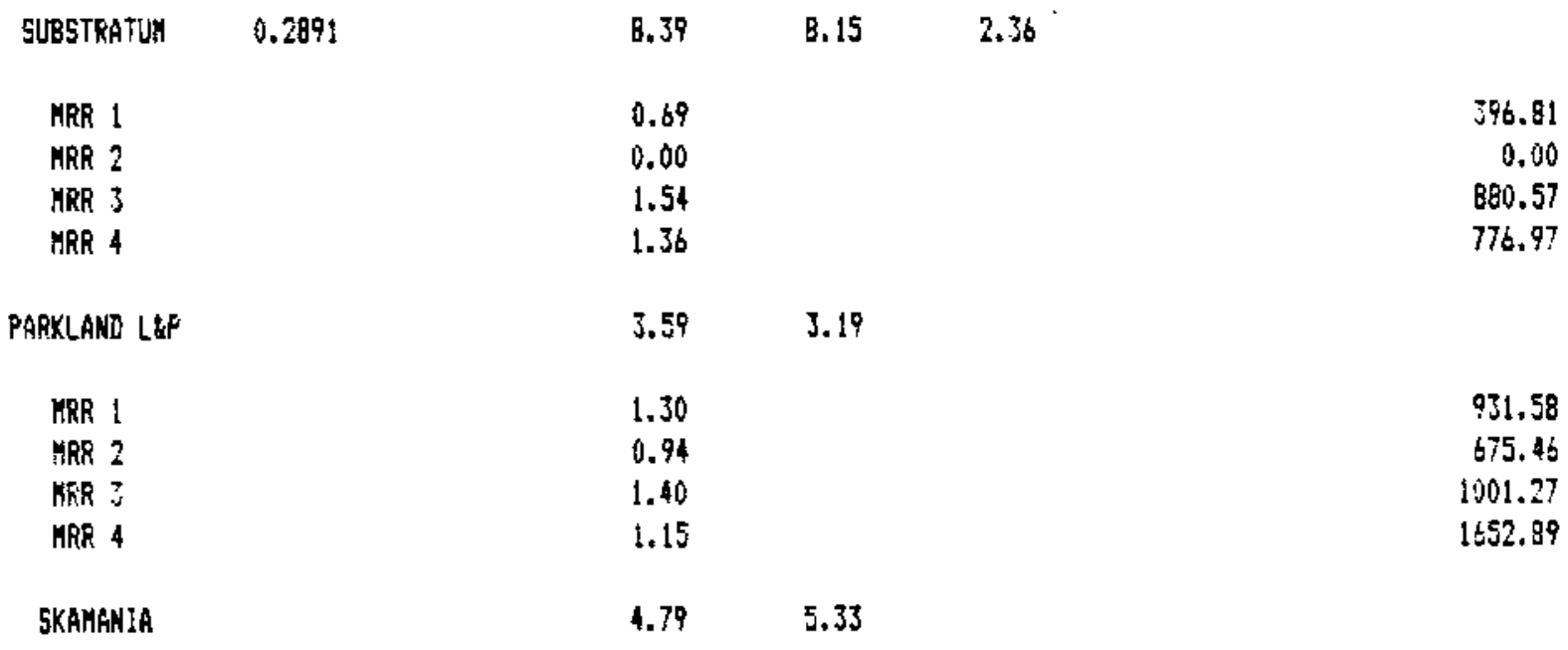

UTLTES SAMPL

OTH UTLTES-2

$\begin{array}{lllll}\text { SU85TRATUA } & 0.1073 & 8.39 & 8.52 & 0.91\end{array}$

HAR I 2.59

IRR 3

0.37

470.72 


\begin{tabular}{|c|c|c|c|c|c|c|c|}
\hline ENTITY & $\begin{array}{l}\text { BUSOTEATA } \\
\text { WEIEHTE }\end{array}$ & $\begin{array}{l}\text { STRATA } \\
\text { WEIGHTS }\end{array}$ & $\begin{array}{l}\text { HD COMPL } \\
\text { PEK MRP }\end{array}$ & $\begin{array}{l}\text { WTD COMFL } \\
\text { FER UTHLITY }\end{array}$ & $\begin{array}{l}\text { WTE COMPL } \\
\text { SUE \& STHATA }\end{array}$ & $\begin{array}{l}\text { WTO ELIG FOP SAMPLE T } \\
\text { PER STRATA FFOU. WEIGHT }\end{array}$ & $\begin{array}{l}\text { TOTAL WEIGHT } \\
\text { PEQ KFR }\end{array}$ \\
\hline Ff & 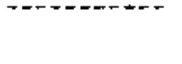 & - & 9.54 & 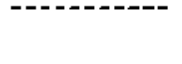 & & n. & $314,7 !$ \\
\hline 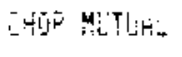 & & & $3.5 \overline{7}$ & 4.01 & & & \\
\hline NFF： & & & 0.48 & & & & 242.82 \\
\hline ks & & & 1.80 & & & & 1611.57 \\
\hline MER J & & & 1.05 & & & & 529.53 \\
\hline MER 4 & & & 1.45 & & & & 1455.56 \\
\hline Whzik IAKidy & & & 4.79 & 4.24 & & & \\
\hline \multicolumn{8}{|l|}{ UILTES SAMPL } \\
\hline \multicolumn{8}{|l|}{ STHE UTLTES-J } \\
\hline SLESTFRTUM & 0.0946 & & 8.39 & 8.25 & 0.78 & & \\
\hline STRATUH TOT & & 1.3752 & 59.89 & 59.27 & 81.51 & 711778.89 & \\
\hline \multicolumn{8}{|l|}{ RESION 5} \\
\hline \multicolumn{8}{|l|}{ +....--- } \\
\hline W 1 & & & 1.25 & & & & 2653.47 \\
\hline FE 2 & & & $1.7 B$ & & & & $400 \%, 4$ \\
\hline $\sin 3$ & & & 0.40 & & & & 72612 \\
\hline $\operatorname{Man} 4$ & & & 1.70 & & & & 3902.35 \\
\hline MRR 5 & & & 0.94 & & & & 2151.61 \\
\hline MRR 6 & & & 0.00 & & & . & 0.00 \\
\hline HFR ? & & & 1,24 & & & & 2846.15 \\
\hline 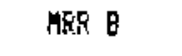 & & & 1.07 & & & & 2449.30 \\
\hline MIDSTATE & & & 8.39 & 8.96 & & & \\
\hline MKR ! & & & 0.00 & & & & 0.00 \\
\hline Mro 2 & & & 0.99 & & & & 1986.33 \\
\hline MRR 3 & & & 0.00 & & & & 0.50 \\
\hline FRA 4 & & & 1.36 & & & & 2711.34 \\
\hline HRP 5 & & & 1.32 & & & & 2641.82 \\
\hline MRR 6 & & & 0.00 & & & & 0.00 \\
\hline MER i & & & 0.00 & & & & 0.60 \\
\hline MFR E & & & 1.12 & & & & 2234.62 \\
\hline URATI!LAA & & & 4.79 & 4.46 & & & \\
\hline \multicolumn{8}{|l|}{ LTLTES SAMPL } \\
\hline \multicolumn{8}{|l|}{ OFH USLTES-2 } \\
\hline EUS5TRATHA & 1.2964 & & 13.18 & 1.42 & 17.40 & & \\
\hline MER 1 & & & 1.55 & & & & 1694.62 \\
\hline MES 2 & & & 1.46 & & & & 1600.74 \\
\hline$M R Q j$ & & & 1.29 & & & & $1413 . ; 0$ \\
\hline
\end{tabular}




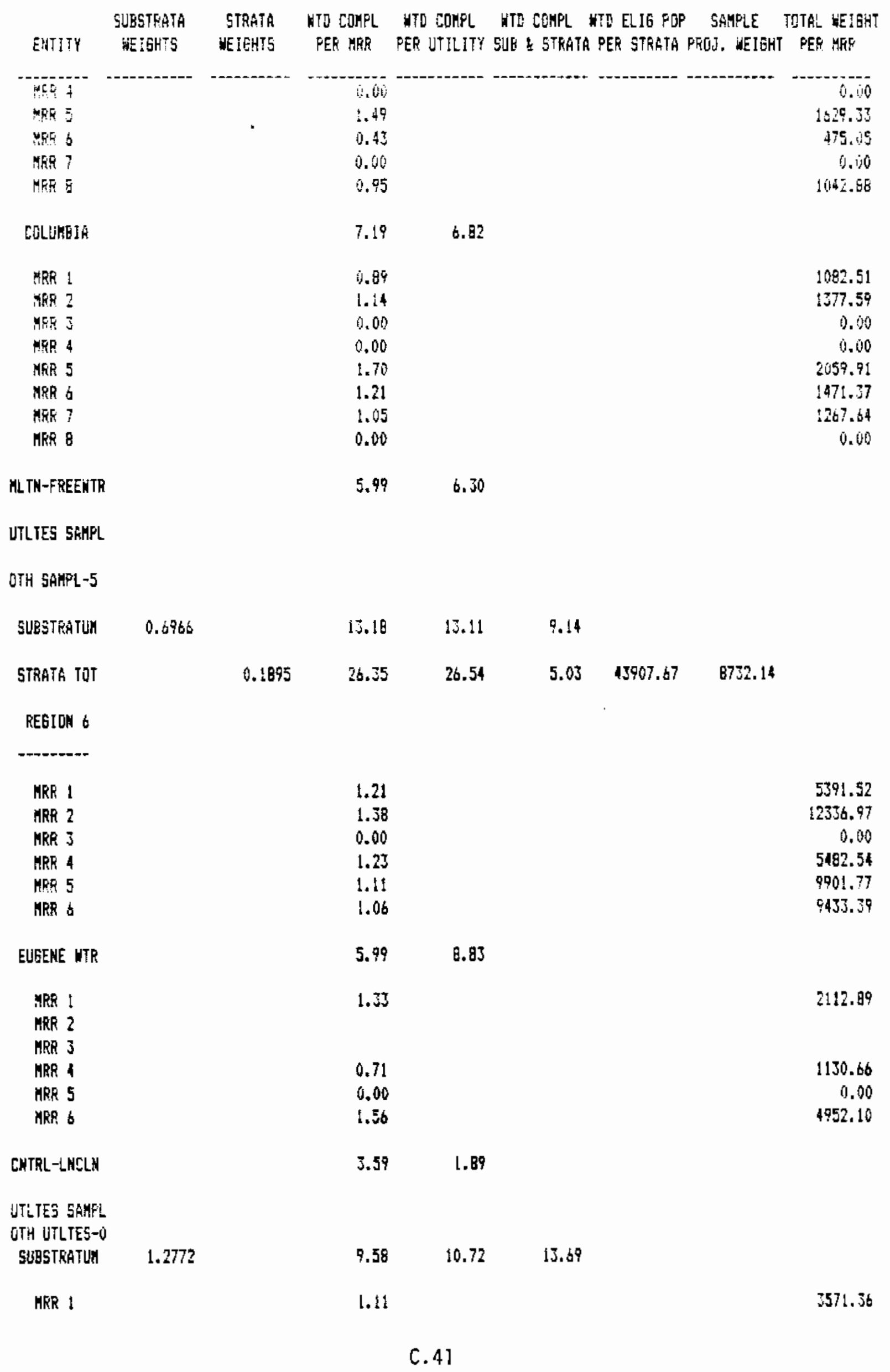




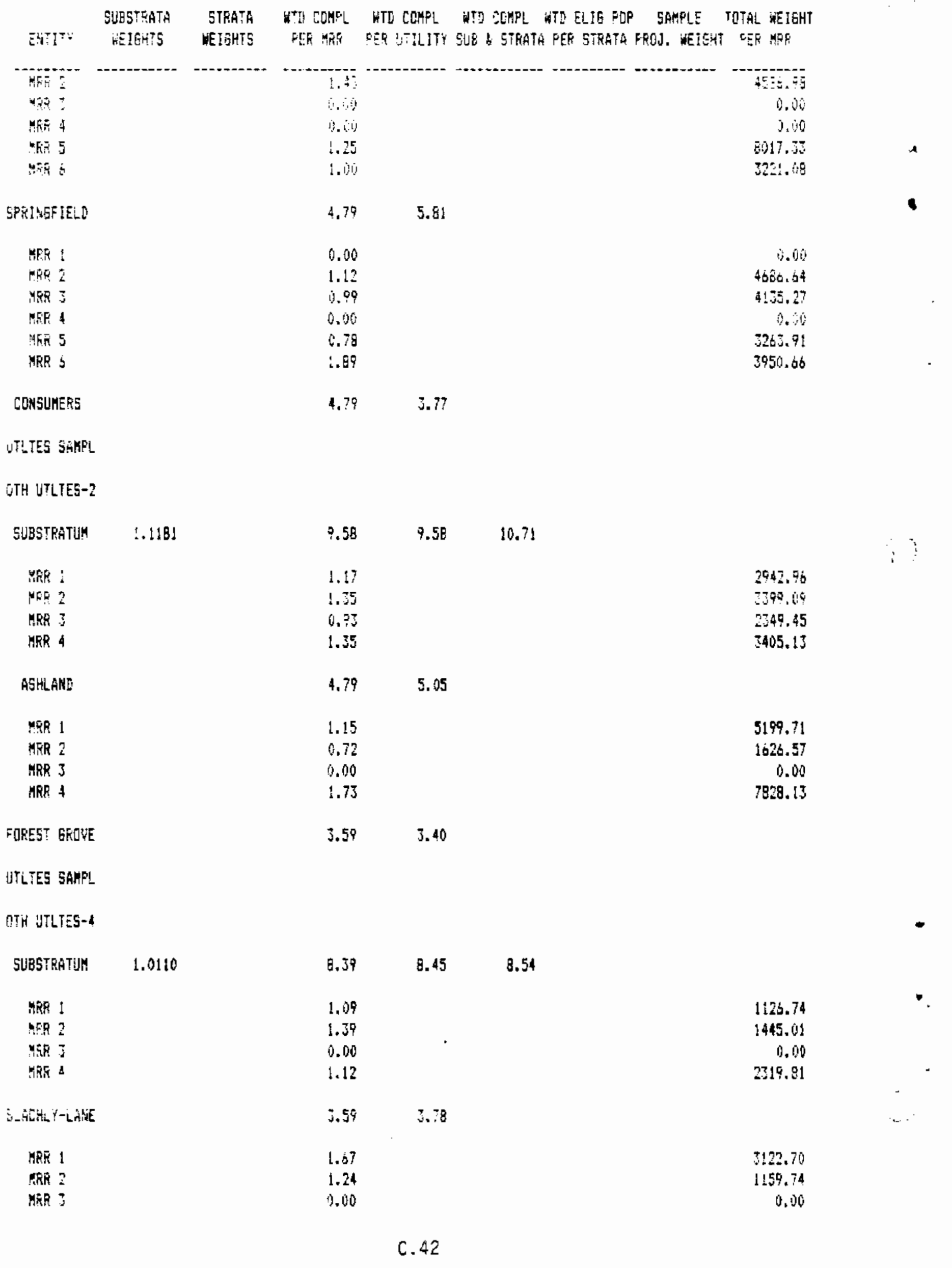




\begin{tabular}{|c|c|c|c|c|c|c|c|c|}
\hline ENTIIY & $\begin{array}{l}\text { SUESTRATA } \\
\text { WEIGHTS }\end{array}$ & $\begin{array}{l}\text { STRATA } \\
\text { HE!EHTS }\end{array}$ & $\begin{array}{l}\text { HTD COHPL } \\
\text { FER MFF }\end{array}$ & $\begin{array}{l}\text { WTD COMPL } \\
\text { FER UTILITY }\end{array}$ & $\begin{array}{l}\text { UTD CGMPL } \\
\text { SUE \& STRATA }\end{array}$ & $\begin{array}{l}\text { WTO ELI6 PGF } \\
\text { FER STRATA }\end{array}$ & $\begin{array}{l}\text { FAMFLE } \\
\text { FE(0), HEIGHT }\end{array}$ & $\begin{array}{l}\text { TOTAL WEIEHT } \\
\text { T PEE MRE }\end{array}$ \\
\hline$x 6 \times 4$ & & & 0.06 & & & & & 53 \\
\hline MOMHUTH & & & 3.55 & 3.40 & & & & \\
\hline \multicolumn{9}{|l|}{ UTLTES SAHPL } \\
\hline \multicolumn{9}{|l|}{ DTH UTLTES-4 } \\
\hline SUESTRATHM & $9.4 ! 63$ & & 7.19 & 7.19 & 2.90 & & & \\
\hline STRATIA TUT & & 0.5426 & 34.74 & 35.94 & 17.50 & 170274.15 & 8732.14 & \\
\hline FUGLIC TOT & & 1.00 & 191.66 & 192.14 & 135.48 & 1183079.68 & 8732.14 & \\
\hline \multicolumn{9}{|l|}{ PRIVATE } \\
\hline \multicolumn{9}{|l|}{ PESION I } \\
\hline \multicolumn{9}{|l|}{-...... } \\
\hline MER 1 & & & 0.40 & & & & & 1019.29 \\
\hline MRR 2 & & & 0.00 & & & & & 0.00 \\
\hline MER 3 & & & 0.62 & & & & & 1580.97 \\
\hline MRO \& & & & 0.53 & & & & & 1551.61 \\
\hline Math 5 & & & 0.28 & & & & & 705.73 \\
\hline HaR & & & 0.60 & & & & & 0.00 \\
\hline MRR 1 & & & 2.74 & & & & & 6981.45 \\
\hline MRR 9 & & & 2.23 & & & & & 5666.94 \\
\hline MRR 9 & & & 2.25 & & & & & 5738.75 \\
\hline MRR 10 & & & 2.56 & & & & & 6515.52 \\
\hline HRR 11 & & & 0.00 & & & & & 0.00 \\
\hline MFR 12 & & & 0.78 & & & & & 1974.91 \\
\hline $\operatorname{MRR} 13$ & & & 0.67 & & & & & 1712.04 \\
\hline HRA 14 & & & 0.12 & & & & & 305.76 \\
\hline MRR 15 & & & 0.00 & & & & & 0.00 \\
\hline RRR 16 & & & 0.00 & & & & & 0.60 \\
\hline MDHJANA PUR & & & 13.18 & 13.18 & & & & \\
\hline SUBSTRATUH & 1.00 & & & & & & & \\
\hline
\end{tabular}

HRR 1

HRR 2

ARR 3

WAR 4

IRR 5

MRR 6

維 7

NFR 8

MER 9

MPR 10

HRP II
1.17

1.54

$1.6 \mathrm{~J}$

0.69

0.00

0.75

1.14

1.19

1.11

0.00

1.39
2975.59

$3915.4 \mathrm{~B}$

4162.34

1744.86

0.010

1912.12

2700.50

3039.09

$2 B 22.96$

0.00

3541.69 


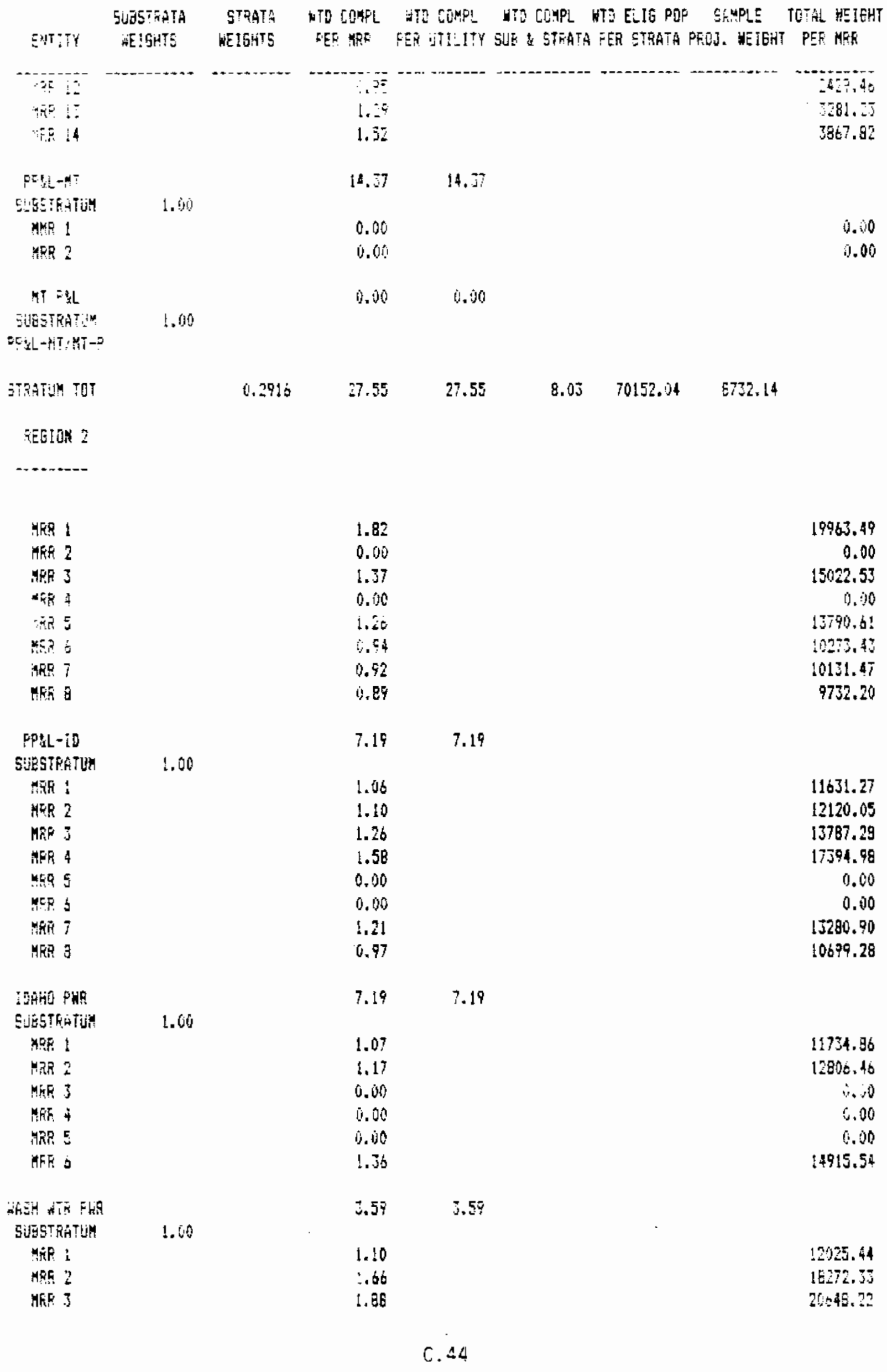




\begin{tabular}{|c|c|c|c|c|c|c|c|}
\hline ENTITY & $\begin{array}{l}\text { SUESTRGTA } \\
\text { HE:GHTS }\end{array}$ & $\begin{array}{l}\text { STRATA } \\
\text { dETGHTS }\end{array}$ & $\begin{array}{l}\text { HTO COMPL } \\
\text { PER WRR }\end{array}$ & $\begin{array}{l}\text { NTO CEMPL } \\
\text { PER UTILITY }\end{array}$ & $\begin{array}{l}\text { HTD COAFL } \\
\text { SUP \& STRATA }\end{array}$ & $\begin{array}{l}\text { HTÜ ELIG POP SAMPLE } \\
\text { A PER STRATA PRDJ. WEIGKT }\end{array}$ & $\begin{array}{l}\text { TDTAL MEIGHT } \\
T \text { FEF MFF }\end{array}$ \\
\hline 465 & - & - & ing & & 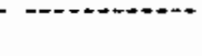 & & ing \\
\hline पर & & & 1.199 & & & & 11972.58 \\
\hline S & & & i). 73 & & & & 8024.05 \\
\hline HER ? & & & 0.00 & & & & 0.60 \\
\hline PtPg & & & 0.73 & & & & 7971.15 \\
\hline UTAH PLL & & & 7.19 & 7.19 & & & \\
\hline SUBSTKATUK & 1.00 & & & & & & \\
\hline STRATUH TOT & & 1.2574 & 25.16 & 25.16 & 31.63 & 276198.10 & \\
\hline \multicolumn{8}{|l|}{ REGLON 3} \\
\hline \multicolumn{8}{|l|}{-........- } \\
\hline MRR ! & & & 1.96 & & & & 18217.94 \\
\hline MRR ? & & & 0.00 & & & & 0.00 \\
\hline MRR 3 & & & 0.00 & & & & 0.00 \\
\hline $\mathrm{MRR} 4$ & & & 0.75 & & & & 6051.93 \\
\hline TRR 5 & & & 1.30 & & & & 120B6.4B \\
\hline MRR 6 & & & 0.90 & & & & 8337.02 \\
\hline MRR 7 & & & 0.66 & & & & 8204.69 \\
\hline MRR 8 & & & 1.58 & & & & 14644.93 \\
\hline KRR 9 & & & $1.7 \mathrm{~J}$ & & & & 16056.49 \\
\hline MRF 10 & & & 0.71 & & & & 6628.45 \\
\hline MFR II & & & 0.00 & & & & 0.00 \\
\hline $\operatorname{MR}: 2$ & & & 1.21 & & & & 11225.16 \\
\hline MRR 1] & & & 1.13 & & & & 10454.36 \\
\hline MRR It & & & 1.07 & & & & 9969.14 \\
\hline KKR 15 & & & 1.15 & & & & 10711.38 \\
\hline MRR 16 & & & 0.00 & & & & 0.00 \\
\hline PPLLLWA & & & 14.37 & 14.37 & & & \\
\hline SUESTRATUH & 1.00 & & & & & & \\
\hline KRR 1 & & & 0.00 & & & & 0.00 \\
\hline MRR 2 & & & 1.24 & & & & 11554.83 \\
\hline KRR 3 & & & $\$ .25$ & & & & 11646.54 \\
\hline$M R R$ & & & 1.40 & & & & 13034.34 \\
\hline MRR 5 & & & 0.00 & & & & 0.10 \\
\hline MRR b & & & 0.00 & & & & 0.00 \\
\hline SRR 7 & & & 1.13 & & & & 10500.22 \\
\hline MRR 8 & & & 1.30 & & & & 12071.44 \\
\hline MRR 9 & & & 0.00 & & & & 0.00 \\
\hline MRF 10 & . & & 0.00 & & & & 0.00 \\
\hline MRR 11 & & & 0.00 & & & & 10.00 \\
\hline MER 12 & & & 0.62 & & & & 5716.36 \\
\hline MER is & & & 0.00 & & & & 0.00 \\
\hline MRR 14 & & & 1.44 & & & & 13345.06 \\
\hline MRR 15 & & & 0.00 & & & & 0.00 \\
\hline MRR 16 & & & 0.00 & & & & 0.00 \\
\hline WASH WAT PUR & & & 8.39 & 8.39 & & & \\
\hline SUESTRATUH & 1.00 & & & & & & \\
\hline STRATUM TOT & & 1.0635 & 22.76 & 22.76 & 24.20 & 211352.75 & \\
\hline
\end{tabular}




\begin{tabular}{|c|c|c|c|c|c|c|c|}
\hline EMT!TY & $\begin{array}{c}\text { SLESTKLTA } \\
\text { HEISHTE }\end{array}$ & $\begin{array}{l}\text { ¿TKATA } \\
\text { MEIGHTS }\end{array}$ & $\begin{array}{l}\text { HTi COMFL } \\
\text { PCA MR }\end{array}$ & $\begin{array}{l}\text { WOD CONEL } \\
\text { PER ITILITY }\end{array}$ & $\begin{array}{l}\text { HTS COMFL I } \\
\text { SUE \& ETRATA }\end{array}$ & $\begin{array}{l}\text { WT ELIE PJP SAMPLE } \\
\text { AER STRGTA PRDS. HEIGHT }\end{array}$ & $\begin{array}{l}\text { TDTAL MEIGHT } \\
\text { PER MRK }\end{array}$ \\
\hline \multicolumn{8}{|l|}{ 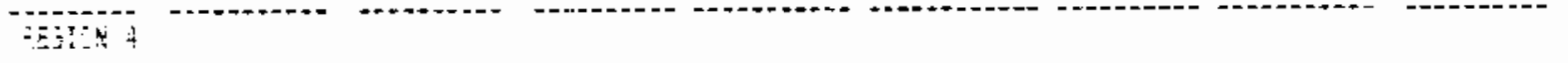 } \\
\hline \multicolumn{8}{|l|}{$-\ldots+\ldots$} \\
\hline MER ! & & & 1.30 & & & & $364 \div 3.22$ \\
\hline the 2 & & & 0.30 & & & & 9.00 \\
\hline MKi? & & & 1.0 & & & & 14200.73 \\
\hline NFF 4 & & & 0.00 & & & & 0.00 \\
\hline M69 5 & & & 1.44 & & & & $20189.2 \mathrm{~B}$ \\
\hline$x 406$ & & & 1.14 & & & & 15968.72 \\
\hline $\operatorname{Mag} 7$ & & & $0.5^{2}$ & & & & 25598.47 \\
\hline MFF E & & & 0.57 & & & & 16092.99 \\
\hline MES ? & & & 2.29 & & & & 54101.28 \\
\hline 10 & & & 6.86 & & & & 24043.01 \\
\hline Afp 11 & & & 1.76 & & & & 49.348 .53 \\
\hline WhF $1:$ & & & $\therefore .74$ & & & & 20718.54 \\
\hline$\triangle R_{R} 13$ & & & 0.83 & & & & 23154.94 \\
\hline MER 14 & & & 0.86 & & & & 11972.64 \\
\hline Kes 15 & & & 2.27 & & & & 60583.69 \\
\hline MRP 16 & & & 0.75 & & & & 21101.79 \\
\hline FIEET SOLHD & & & 16.77 & 16.77 & & & \\
\hline SUESTRATUM & 1.10 & & & & & & \\
\hline ETEATIM TQ? & & 3.2042 & 8.77 & 16.77 & 53.74 & 469232.46 & \\
\hline \multicolumn{8}{|l|}{$5=10 \times 5$} \\
\hline \multicolumn{8}{|l|}{--------- } \\
\hline MRP ! & & & 1.75 & & & & 7295.87 \\
\hline akn 2 & & & 1.49 & & & & 6318.94 \\
\hline Mag 3 & & & 0.00 & & & & 0.20 \\
\hline AER 4 & & & 1.24 & & & & 5151.04 \\
\hline NRR 5 & & & 0.00 & & & & 0.00 \\
\hline HRR 6 & & & 0.84 & & & & 3482.45 \\
\hline $\operatorname{MER} 7$ & & & 0.00 & & & & 0.00 \\
\hline KER & & & 1.77 & & & & 756.31 \\
\hline HPS 9 & & & 9.92 & & & & 3933.13 \\
\hline HeR 10 & & & $1.5 \mathrm{~J}$ & & & & 6360.96 \\
\hline WE 11 & & & 0.00 & & & & 0.00 \\
\hline AFR 12 & & & 0.00 & & & & 0.00 \\
\hline M & & & 0.35 & & & & $\{460.23$ \\
\hline \multicolumn{8}{|l|}{ MER 14} \\
\hline MAR 15 & & & 0.52 & & & & 2174,98 \\
\hline$M P R$ 15 & & & 1.58 & & & & 6599.54 \\
\hline$P F H L-B R-E$ & & & 11.78 & 11.98 & & & \\
\hline SUESTRATIM & 1.00 & & & & & & \\
\hline MF ! & & & $0.9 !$ & & & & 3309.24 \\
\hline 粗 2 & & & 2.73 & & & & 989.77 \\
\hline MkT & & & 3.00 & & & & 3.60 \\
\hline HRF & & & 1.43 & & & & $5069.7 !$ \\
\hline MFR & & & 0.00 & & & & v. 00 \\
\hline MRP $=$ & & & 0.82 & & & & 3412.85 \\
\hline MER 7 & & & 5.10 & & & & 432.85 \\
\hline
\end{tabular}




\begin{tabular}{|c|c|c|c|c|c|c|c|}
\hline ENTIT: & $\begin{array}{l}\text { SUPSTFATA } \\
\text { HETGHTS }\end{array}$ & $\begin{array}{l}\text { STRATA } \\
\text { WEIGHTS }\end{array}$ & $\begin{array}{l}\text { WTD COMPL } \\
\text { PER MSR }\end{array}$ & $\begin{array}{l}\text { WTD COAPL } \\
\text { FEF UTHLITY }\end{array}$ & $\begin{array}{l}\text { WTD COMFL } \\
\text { SUE \& STKATA }\end{array}$ & $\begin{array}{l}\text { WTO ELIG PGP SAMPLE } \\
\text { A PER STRATA PROJ. WEIGHT }\end{array}$ & $\begin{array}{l}\text { TOTAL WEIGHT } \\
\text { T PER KRR }\end{array}$ \\
\hline Mg & $\cdots$ & & 1.98 & & & & 7040 \\
\hline $\begin{array}{l}\text { IDEHO PHA } \\
\text { SUBSTRATLA }\end{array}$ & 1,00 & & 7.19 & 7.19 & & & \\
\hline Min ! & 10,0 & & 0.00 & & & & 0.00 \\
\hline $\begin{array}{l}\text { ARF } 2 \\
\text { ARP } 3\end{array}$ & & & 1.87 & & & & 76.51 .64 \\
\hline MER 4 & & & 0.57 & & & & 2359.03 \\
\hline MER 5 & & & & & & & \\
\hline MRE 6 & & & 0.00 & & & & 0.00 \\
\hline ME 7 & & & & & & & \\
\hline HRR 8 & & & 0.00 & & & & 0.00 \\
\hline CP MATHL & & & 2.40 & 2.40 & & & \\
\hline EUESTRATUM & 1.00 & & & & & & \\
\hline STRATUM TOT & & 0.4776 & 21.56 & 21.56 & 10.30 & 29916.03 & \\
\hline \multicolumn{8}{|l|}{ REGIOH 6} \\
\hline \multicolumn{8}{|l|}{ …....... } \\
\hline MRR 1 & & & 10.46 & & & & 9169.99 \\
\hline ARR 2 & & & 0.90 & & & & 17785.10 \\
\hline MRR 3 & & & 1.26 & & & & 24887.34 \\
\hline Ha 4 & & & 0.60 & & & & 0.50 \\
\hline$n t$ & & & 0.63 & & & & 12394.48 \\
\hline $\operatorname{ARR} \&$ & & & 0.94 & & & & 9289.34 \\
\hline MRR 7 & & & 1.75 & & & & 34722.04 \\
\hline GFR \& & & & 1.83 & & & & 36199.76 \\
\hline MRR 9 & & & 1.74 & & & & 34447.92 \\
\hline GRR 10 & & & 0.58 & & & & 11527.96 \\
\hline MRR 11 & & & 0.98 & & & & 8705.65 \\
\hline MRR 12 & & & 1.63 & & & & 32265.01 \\
\hline KRA 13 & & & 1.99 & & & & 39203.29 \\
\hline MRR 14 & & & 0.94 & & & & 18540.98 \\
\hline MRR $: 5$ & & & 1.27 & & & & 12542.04 \\
\hline MRR 16 & & & 0.00 & & & & 0.00 \\
\hline PPELLOR-H & & & 16.77 & 16.77 & & & \\
\hline SUESTRATUH & 1.00 & & & & & & \\
\hline MRA I & & & 0.81 & & & & 16003.30 \\
\hline MRR 2 & & & 0.00 & & & & 0.50 \\
\hline MRR 3 & & & 1.03 & & & & 10243.09 \\
\hline MRR 4 & & & 1.50 & & & & 84904.92 \\
\hline MaR 5 & & & 0.57 & & & & 5674.64 \\
\hline MRR 6 & & & 0.97 & & & & 9616.55 \\
\hline MRR 7 & & & 1.57 & & & & 27080.27 \\
\hline KFR E & & & 0.74 & & & & 9.307 .85 \\
\hline MRR ? & & & 1.36 & & & & 27008.43 \\
\hline Min 10 & & & 1.62 & & & & 32030.00 \\
\hline MRA 11 & & & 1.42 & & & & 28229.56 \\
\hline HRR 12 & & & 1.33 & & & & 26290.12 \\
\hline MRR 13 & & & 1.06 & & & & 20917.17 \\
\hline MRR 14 & & & 0.00 & & & & 0.00 \\
\hline
\end{tabular}




\begin{tabular}{|c|c|c|c|c|c|c|c|c|}
\hline ENTITY & $\begin{array}{l}\text { ELSSTRATA } \\
\text { HEIGH:S }\end{array}$ & $\begin{array}{r}\text { STEATA } \\
\text { WEIBHTS }\end{array}$ & $\begin{array}{l}\text { UTI COMEL } \\
\text { O: MRR }\end{array}$ & $\begin{array}{l}\text { WTO COMPL } \\
\text { PER UTILITH }\end{array}$ & $\begin{array}{l}\text { NTO CONFL } \\
\text { ELS \& STFATA }\end{array}$ & $\begin{array}{l}\text { WD ELIG PIP } \\
\text { A PER STRHIA }\end{array}$ & $\begin{array}{c}\text { SAMPIE } \\
\text { FROS. HEIEHT }\end{array}$ & $\begin{array}{l}\text { TOTAL HE!GHT } \\
\text { FEP HAP }\end{array}$ \\
\hline 积 & - & $\cdots+\cdots$ & 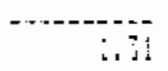 & 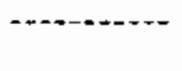 & - & - rensen & --------- & 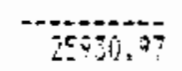 \\
\hline प5: is & & & $1 .+3$ & & & & & 14517.00 \\
\hline $\begin{array}{l}\text { PUIT GEN ELE } \\
\text { SIJETRATILY }\end{array}$ & $\therefore 30$ & & $16.7 ?$ & 10.77 & & & & \\
\hline SIRETU: TIT & & $2.269 ?$ & 33.54 & 33.54 & 76.09 & $66445 ! .47$ & 9732.14 & \\
\hline PRIVATE TOT & & 1. 0000 & 147.34 & 147.34 & 203.99 & $1781302.8 \mathrm{z}$ & 875.14 & \\
\hline GEMHJ TVI & & 1.0000 & 337.60 & 339.48 & 339.48 & 2964382.55 & $37: 2.14$ & \\
\hline
\end{tabular}




\begin{tabular}{|c|c|}
\hline A & $Z$ \\
\hline EHTITY & $\begin{array}{l}\text { EINAL WTD } \\
\text { YRP FOP }\end{array}$ \\
\hline 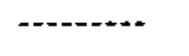 & ------ \\
\hline PLEIC & \\
\hline 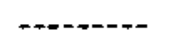 & \\
\hline EEGION ! & \\
\hline -........... & \\
\hline SRP 1 & 1309.54 \\
\hline MRR 2 & 1202.63 \\
\hline MGR 3 & 140.3 .07 \\
\hline MES 4 & 0,60 \\
\hline MES & 1303.30 \\
\hline WRA 6 & 1403.07 \\
\hline ARR 7 & 0.00 \\
\hline ARR 8 & 1320.54 \\
\hline FLATHEAD ELE & 7942.16 \\
\hline MRR 1 & 0.00 \\
\hline MRR 2 & 524.98 \\
\hline MRP 3 & 814.59 \\
\hline MRR 4 & 943.71 \\
\hline MRR 5 & 2347.83 \\
\hline MeRR ó & 30162.38 \\
\hline$M R R$ & 2603.02 \\
\hline$A R E$ a & 0.00 \\
\hline FLATHEAD IAR & 10296.52 \\
\hline UTLTES SAHPL & \\
\hline DTH UFLTES-1 & \\
\hline SUBSTRATUM & 48238.67 \\
\hline MRR 1 & 2289.27 \\
\hline HRR 2 & 0.00 \\
\hline HRR 3 & 2383.61 \\
\hline HRR \& & 0.60 \\
\hline MRR 5 & 1727.61 \\
\hline MRR 6 & \\
\hline MRR 7 & \\
\hline MRR 8 & \\
\hline ELACIER & 6399.50 \\
\hline MFIR ! & 1714.14 \\
\hline MER ? & 1368.79 \\
\hline MFR J & 1892.10 \\
\hline MER 4 & 0.00 \\
\hline MRE 5 & 1130.38 \\
\hline
\end{tabular}




\begin{tabular}{|c|c|}
\hline EHTIY & $\begin{array}{c}\text { FINAL WTD } \\
\text { MEE FDE }\end{array}$ \\
\hline - - & - - - \\
\hline 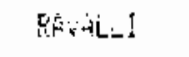 & 5195.41 \\
\hline TTLTES SAHF'L & \\
\hline STA UTLTES-? & \\
\hline SUBSTRATUK & 12504.70 \\
\hline STRATLA TUT & 30743.58 \\
\hline RESION 2 & \\
\hline . & \\
\hline 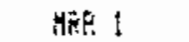 & 0.00 \\
\hline NRP 2 & 11746.46 \\
\hline MRR 3 & 0.06 \\
\hline Aris 4 & 8608.39 \\
\hline HFR 5 & 7494.49 \\
\hline ARR 6 & 3854.31 \\
\hline IDAHO FALLS & 31103.65 \\
\hline MFI L & 3905.59 \\
\hline $\operatorname{res} 2$ & $4074.9 \mathrm{E}$ \\
\hline MER j & 4273.49 \\
\hline FRP 4 & 0.00 \\
\hline HRR 5 & 0.00 \\
\hline KRR 6 & 0.00 \\
\hline WUER VALLEY & $12336 .: 5$ \\
\hline UTLTES SAMPL & \\
\hline OA LILTES-J & \\
\hline SUBSTRATLH & 43439.80 \\
\hline MRS 1 & 1475.41 \\
\hline KRP 2 & \\
\hline MR: & 4348.89 \\
\hline MRE 4 & 0.00 \\
\hline $\mathrm{Nh} s$ & 367.65 \\
\hline MER 6 & 0.00 \\
\hline FALL TIVER & 6212.14 \\
\hline 䋦 I & 2453.96 \\
\hline ENEAESO KUT & $3-86$ \\
\hline Wfr t & 2453.86 \\
\hline FABWERS ELE & $245 ., 86$ \\
\hline
\end{tabular}

C. 50 


\begin{tabular}{|c|c|}
\hline ERT!TY & $\begin{array}{l}\text { FINAL WTO } \\
\text { MOF POF }\end{array}$ \\
\hline m!! & $2+50$ \\
\hline RIVEREIDE EL & $24=5.80$ \\
\hline MRR I & 0.00 \\
\hline EOUTHSIOE EL & 0.00 \\
\hline MFE 1 & 0.00 \\
\hline M质 2 & 2953.86 \\
\hline UHITY L\&P & 245.86 \\
\hline MLNEDEA PROJ & 9515.44 \\
\hline UTLEES SAMFL & \\
\hline OTH UTLTES-A & \\
\hline SUBSTRATUA & 16027.58 \\
\hline MRR I & 1987.30 \\
\hline MRR 2 & 0.00 \\
\hline KRR 3 & 0.00 \\
\hline MRR 4 & 1895.43 \\
\hline$M R R 5$ & 1895.43 \\
\hline MRR b & 0.00 \\
\hline
\end{tabular}

$\begin{array}{lr}\text { LOST RIVER } & 5678.16 \\ \text { MRR 1 } & \\ \text { MRR 2 } & 2946.35 \\ \text { MER 3 } & 0.00 \\ \text { MRR 4 } & 0.00 \\ \text { MRR 5 } & 0.00 \\ \text { MRR 6 } & 0.00 \\ \text { MEISER } & 2946.35 \\ \text { JTLTES SAMPL } & \end{array}$

UTH UFLTES-4

\begin{tabular}{|c|c|}
\hline SU85TGATUK & 8524.50 \\
\hline STRATUR TOT & 67991.88 \\
\hline \multicolumn{2}{|l|}{ REGION 3} \\
\hline - & \\
\hline SFR ! & 0.00 \\
\hline MRR 2 & 0.00 \\
\hline MRR 3 & 0.00 \\
\hline MRR 4 & 14520.86 \\
\hline MRR 5 & 0.00 \\
\hline
\end{tabular}




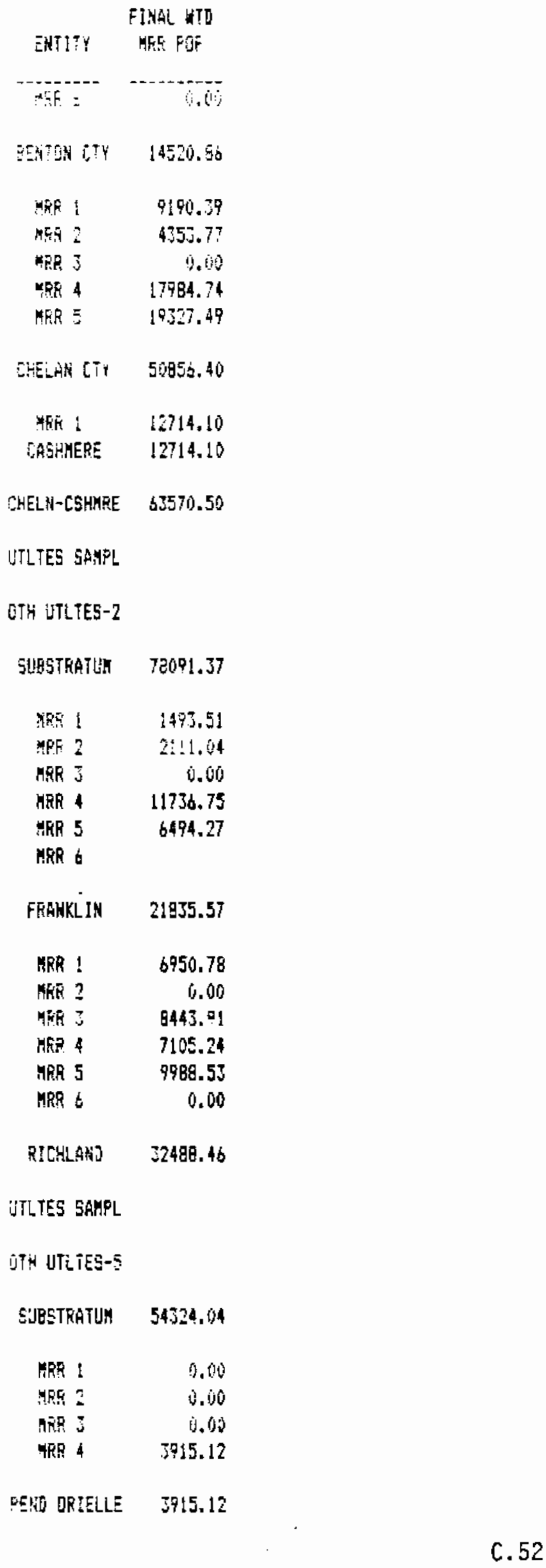




\begin{tabular}{|c|c|}
\hline EHTITY & $\begin{array}{l}\text { FINAL WTD } \\
\text { MRR FAP }\end{array}$ \\
\hline +costo-s- & ------n... \\
\hline$m 1$ & 5091.31 \\
\hline MES 2 & 4288.66 \\
\hline ARR J & 0.60 \\
\hline Fro 4 & 5030.44 \\
\hline VERA IRR & 14410.41 \\
\hline UTLTES SAMFL & \\
\hline OTH UTLTES-J & \\
\hline SUESTFATLM & 19325.54 \\
\hline MRP 1 & 1697.72 \\
\hline URR 2 & 1925.43 \\
\hline AHR 3 & \\
\hline IRP 4 & 0.00 \\
\hline FERRY CTY & 3623.15 \\
\hline MRR 1 & 0.00 \\
\hline MKR ? & $2009.7 !$ \\
\hline NESPEELEH & $990^{\circ} .71$ \\
\hline MRR! & 0.00 \\
\hline MER 2 & 2009.71 \\
\hline COULEE DAM & 2009.71 \\
\hline HSPLK-COULEE & 4019.42 \\
\hline UTLTES SAMPL & \\
\hline OTH UTLTES-4 & \\
\hline SUESTRATUH & 7642.57 \\
\hline STRATLM TOT & $15 \mathrm{EBB} 3.51$ \\
\hline REgion 4 & \\
\hline -......-- & \\
\hline MRR I & 0.00 \\
\hline HR 2 & 60213.54 \\
\hline HFF 3 & 0.00 \\
\hline ARR 4 & 59466.47 \\
\hline MRR 5 & 48947.78 \\
\hline HKR b & $6574 ! .83$ \\
\hline
\end{tabular}

C. 53 


\begin{tabular}{|c|c|}
\hline ERTTT: & 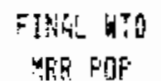 \\
\hline SETHE & 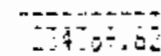 \\
\hline ARE 1 & $1355.5 ?$ \\
\hline HFE 2 & ¿.60 \\
\hline$X \in R 3$ & 5644.95 \\
\hline 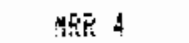 & 38478.94 \\
\hline MSF 5 & 28093.51 \\
\hline MGR o & 34459.37 \\
\hline JMgHONISH & $15061 \div .65$ \\
\hline $\begin{array}{l}\text { LILTES ECMPL } \\
\text { UTh UTITES-i }\end{array}$ & \\
\hline jisstkatUH & 384999.26 \\
\hline Mा⿰ 1 & 1443.23 \\
\hline$M \bar{P}=$ & 19312.50 \\
\hline ARR 3 & 20446.58 \\
\hline HRR 4 & 8645.93 \\
\hline AFR 5 & 26655.57 \\
\hline$M F R$ & 0.10 \\
\hline OARE CTY & 71545,81 \\
\hline W: & $2 B s 35.8=$ \\
\hline MeF 2 & 22960.53 \\
\hline An 3 & 22858.57 \\
\hline MkR 4 & 26224.84 \\
\hline MRA 5 & 8.00 \\
\hline MER 6 & $22478.4 J$ \\
\hline TACOMA & 123161.02 \\
\hline & \\
\hline
\end{tabular}

DT: UTLTES-2

\begin{tabular}{|c|c|}
\hline SUBSTRATUH & 214706.85 \\
\hline FRF 1 & 00 \\
\hline$A B F ?$ & $754 ! .22$ \\
\hline $4 R E 3$ & 4537,20 \\
\hline 쓴 & $1955=.5$ \\
\hline 445 & 3550.05 \\
\hline Miti 6 & 5.43 .41 \\
\hline MASOH CTI-3 & 37630.35 \\
\hline 64: ! & 0.51 \\
\hline AR 2 & 9453.76 \\
\hline Mhî 3 & $E 041.20$ \\
\hline ERR 4 & 3871,73 \\
\hline Mgt 5 & 5356,76 \\
\hline
\end{tabular}




\begin{tabular}{|c|c|}
\hline ENTITY & $\begin{array}{l}\text { FINAL WTD } \\
\text { MER PONE }\end{array}$ \\
\hline 460 & $25=55$ \\
\hline SLALIAK LTY & 30413.15 \\
\hline LTLTES SAMPL & \\
\hline OTH UTLTES-J & \\
\hline SUESTGATLH & 63443.48 \\
\hline SRR I & 5959.75 \\
\hline KRR 2 & 3079.53 \\
\hline HRR I & 0.00 \\
\hline FRR 4 & 4966.46 \\
\hline ELMHURST MUT & 14905.74 \\
\hline MRR 1 & 3351.77 \\
\hline MRR 2 & $5335.9 !$ \\
\hline HRR 3 & $335: .77$ \\
\hline MRR 4 & 3351.77 \\
\hline ORCA5 PWR & 13391.11 \\
\hline UTLIES SAKFL & \\
\hline OTH UTLTES-3 & \\
\hline SUBSTRATUK & $2 \mathrm{~B} 296.85$ \\
\hline MRR I & 793.62 \\
\hline MRR 2 & 0.00 \\
\hline MRR 3 & 1761.13 \\
\hline $\operatorname{MrR} 4$ & 1553.94 \\
\hline FARXLAND LLP & 4108.69 \\
\hline KRR 1 & 1863.17 \\
\hline MRR 2 & 1354.92 \\
\hline MRR 3 & 2002.54 \\
\hline MRR 4 & 1652.89 \\
\hline SKANANIA & 6869.51 \\
\hline & \\
\hline
\end{tabular}

OTH UTLTES-?

sliestrintth 10978.20

MRR $1 \quad 3266.59$

ARR 20.00

$\begin{array}{ll}\text { MRR } 3 & 470.72\end{array}$ 


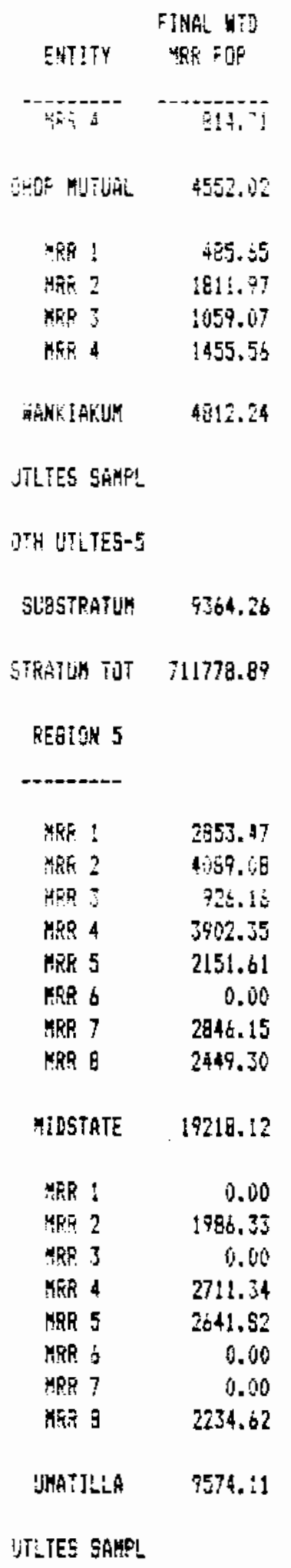

ITH !JFLES-2

\begin{tabular}{|c|c|}
\hline Sazerthidu & 29792.23 \\
\hline MER ! & 1694.62 \\
\hline MPR 2 & 1500,74 \\
\hline AR 3 & $14 ! 3.00$ \\
\hline
\end{tabular}




\begin{tabular}{|c|c|}
\hline EHTITY & $\begin{array}{l}\text { FIHAL WTD } \\
\text { MRE POF }\end{array}$ \\
\hline पị & 4. \\
\hline 455 & 1629.53 \\
\hline$* R R$ b & 475.65 \\
\hline MKR 7 & 0.00 \\
\hline MER & 1042.88 \\
\hline COLUMBIA & 7856.43 \\
\hline MFE ! & 1082.51 \\
\hline$A R R 2$ & 1377.59 \\
\hline ARR 3 & 0.00 \\
\hline$\because R R 4$ & 0.00 \\
\hline MRR 5 & 2059.91 \\
\hline Whe o & 1471.37 \\
\hline WR 7 & 1267.64 \\
\hline MRR 9 & 0.00 \\
\hline MLTN-F KEEWTR & 7259.01 \\
\hline UTLTES SAKFL & \\
\hline
\end{tabular}

OTH SAMPL-5

SUESTKATUM 15115.44

STRATA TOT 43907.67

REGIDN 6

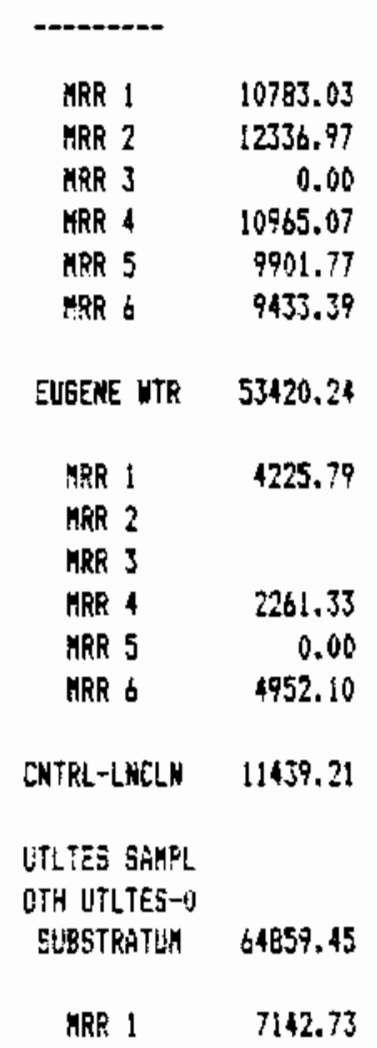




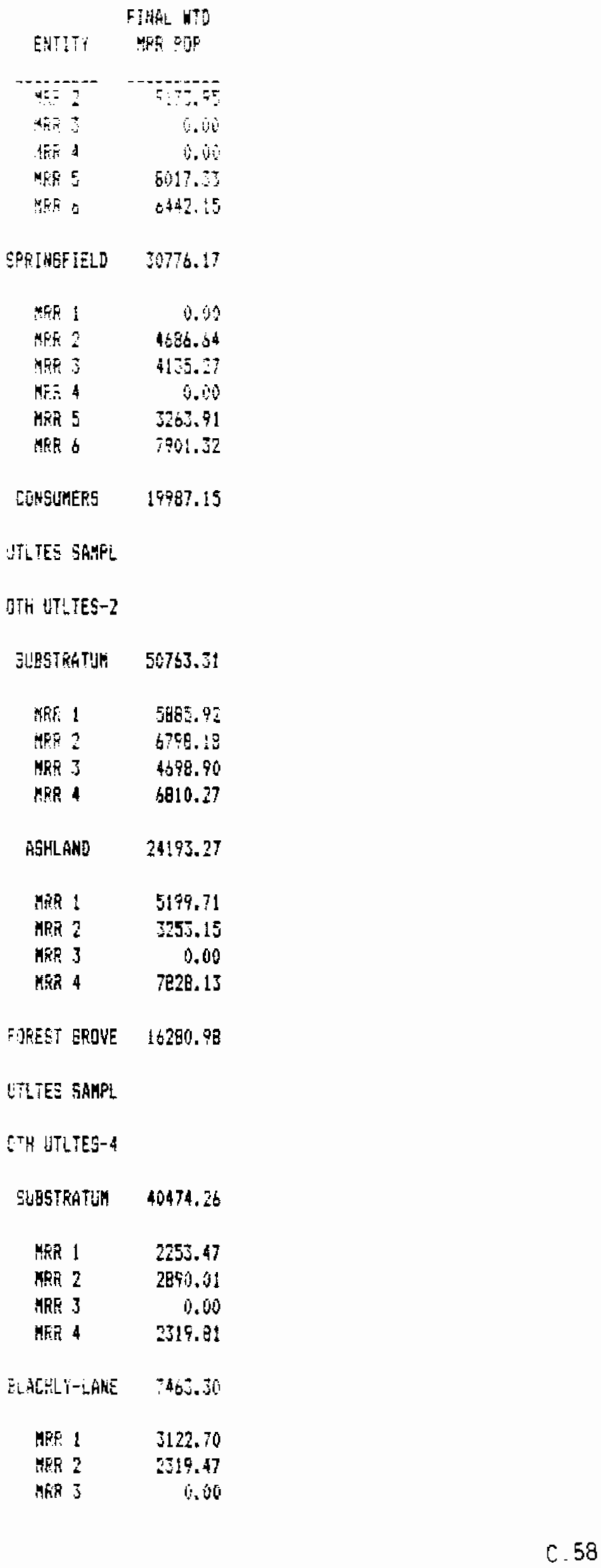




\begin{tabular}{|c|c|}
\hline ENTITY & $\begin{array}{l}\text { FINAL WTJ } \\
\text { WFP PQF }\end{array}$ \\
\hline 854 & $1771,=6$ \\
\hline SINWOUTH & 5713.83 \\
\hline UTLTES SAMPL & \\
\hline OTH UTLTES-A & \\
\hline SUASTRATUK & 14177,13 \\
\hline GTFATUE TOT & 170274.15 \\
\hline PUALIC TOT & 1183079.68 \\
\hline PRIVATE & \\
\hline PEEION 1 & \\
\hline MRR ! & 1019.29 \\
\hline MRR 2 & 0.00 \\
\hline ARR 3 & 1580.97 \\
\hline MRR 4 & 1351.61 \\
\hline Mes 5 & 703.73 \\
\hline MRR $b$ & 0.00 \\
\hline Mkg 7 & 6981.45 \\
\hline MRR 8 & 5668.94 \\
\hline MRA 9 & 578.75 \\
\hline MFR 10 & 6515.52 \\
\hline MRP II & 0.00 \\
\hline MRR 12 & 1974.91 \\
\hline MRR 13 & 1712.04 \\
\hline MPR 14 & 303.76 \\
\hline HRR 15 & 0.00 \\
\hline MRP i6 & 0.00 \\
\hline $\begin{array}{l}\text { FONTANA FHR } \\
\text { SUESTKATIJH }\end{array}$ & 33550.98 \\
\hline
\end{tabular}




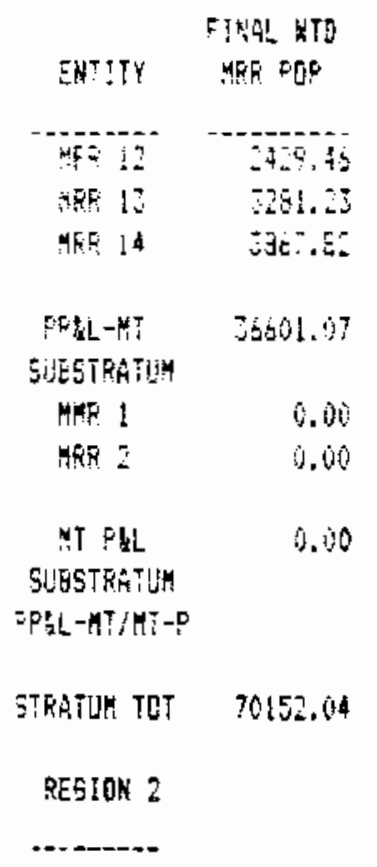

\begin{tabular}{|c|c|}
\hline MRR 1 & 19963.49 \\
\hline ARR 3 & 0.00 \\
\hline HER 3 & 15072.55 \\
\hline$N$ Af & (i). 00 \\
\hline MFF & 13790.61 \\
\hline $4 R^{2}$ & {$[1273,43$} \\
\hline MRR 7 & $\$ 0131.17$ \\
\hline MRR o & 9732.20 \\
\hline FPALLIi & 79913.74 \\
\hline \multicolumn{2}{|l|}{ SUESTRATUA } \\
\hline MRR 1 & 11631.27 \\
\hline MRF 2 & 12120.05 \\
\hline BRR 3 & 13757.23 \\
\hline MRR A & 17394.98 \\
\hline YRR 5 & 0.60 \\
\hline Fin 6 & $0.0 \mathrm{v}$ \\
\hline HER 7 & 13280.90 \\
\hline ARR 8 & 10659.28 \\
\hline IDFifH PNE & 78913.74 \\
\hline \multicolumn{2}{|l|}{ SLESTRRTUM } \\
\hline ARR I & 11734.86 \\
\hline Men 2 & 12306.46 \\
\hline$x+3$ & $\hat{A} \hat{0}$ \\
\hline MkR 4 & 9.30 \\
\hline MRR 5 & 30 \\
\hline Men & 14915.54 \\
\hline 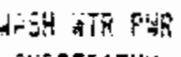 & 97455.87 \\
\hline \multicolumn{2}{|l|}{ SUSSIRATL'A } \\
\hline Mer: & 12125.44 \\
\hline AKR 2 & 12272.35 \\
\hline SRR $\mathrm{j}$ & 20648.22 \\
\hline
\end{tabular}




\begin{tabular}{cr} 
ENTITY & \multicolumn{1}{c}{ FINAL WTD } \\
MRR EOP \\
AFF
\end{tabular}

REGION 3

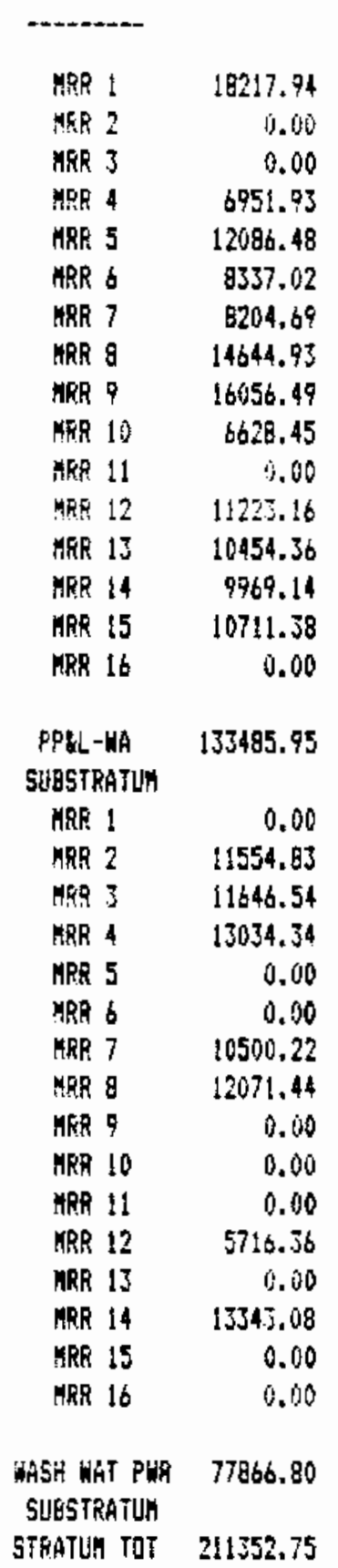




\begin{tabular}{|c|c|}
\hline ENTITY & $\begin{array}{l}\text { FINAL HTD } \\
\text { KRF FIF }\end{array}$ \\
\hline \multicolumn{2}{|l|}{ 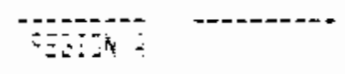 } \\
\hline \multicolumn{2}{|l|}{-------. } \\
\hline$A R F !$ & 3445.28 \\
\hline ME? & 3. \\
\hline 4 & 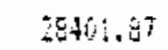 \\
\hline Sing 4 & 0.00 \\
\hline HiR 5 & $40079.5 t$ \\
\hline ME a & 31537.55 \\
\hline$\therefore F$ & 25998,73 \\
\hline 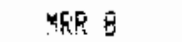 & $: \pm 82.96$ \\
\hline A6 9 & 64101.28 \\
\hline MEF id & $24 ; 43 \times 1$ \\
\hline A $k$ EF 11 & $49348.5 j$ \\
\hline WE 12 & 20718.54 \\
\hline rif 13 & 23154.94 \\
\hline m? 14 & 23945.27 \\
\hline ME 15 & 53583.64 \\
\hline$m=16$ & 21601.79 \\
\hline PUGE i SPUND & 467232.46 \\
\hline SUASTRATIJK & \\
\hline STERTLK TGT & 469232.40 \\
\hline \multicolumn{2}{|l|}{ SESTEN } \\
\hline \multicolumn{2}{|l|}{ - } \\
\hline 滔 1 & 7295.87 \\
\hline HFR 2 & 6218.84 \\
\hline MRE 3 & 0,00 \\
\hline MAR 4 & 5151.04 \\
\hline MRA 5 & 0.00 \\
\hline KER 6 & 3482,45 \\
\hline MKS 7 & 0.00 \\
\hline HFo $\mathrm{g}$ & 7386.31 \\
\hline $\mathrm{PL} 9$ & 3833.13 \\
\hline MER IS & 63.61. 98 \\
\hline ARR 11 & 0.00 \\
\hline MFR :2 & 0.00 \\
\hline$M P 13$ & 1460.23 \\
\hline SF & \\
\hline 15 & 2174.98 \\
\hline Afe ts & 6889.54 \\
\hline$F$ & 19953.35 \\
\hline \multicolumn{2}{|l|}{ SUASTRATUA } \\
\hline Asp! & 8908.24 \\
\hline $4 h 2 ?$ & $\$ 293.77$ \\
\hline mis: : & $\therefore m$ \\
\hline $\mathrm{RRR} 4$ & 5069.71 \\
\hline PFE 5 & 0.00 \\
\hline Mes 6 & 3412.85 \\
\hline Mig 7 & 432.85 \\
\hline
\end{tabular}




\begin{tabular}{|c|c|}
\hline EATYY & $\begin{array}{l}\text { FiNAC DTO } \\
\text { FEF }\end{array}$ \\
\hline 9 & 354,06 \\
\hline $\begin{array}{l}\text { Whid Fin } \\
\text { Sugethetidy }\end{array}$ & 29972.01 \\
\hline MER！ & $\dot{0}, 60$ \\
\hline $\begin{array}{l}\text { MER } 2 \\
\text { MFR } 3\end{array}$ & 7631.64 \\
\hline $\begin{array}{l}\text { WEF } 4 \\
\text { WFT } 5\end{array}$ & 2559.03 \\
\hline $\begin{array}{l}\text { MER } 6 \\
\text { MFE } 7\end{array}$ & ํ. \\
\hline MRP 9 & 10.00 \\
\hline $\begin{array}{l}\text { CP NATNL } \\
\text { SUASTRATUM }\end{array}$ & 97961.67 \\
\hline STRATUH TOT & 89916.03 \\
\hline
\end{tabular}

\section{REgION 6}

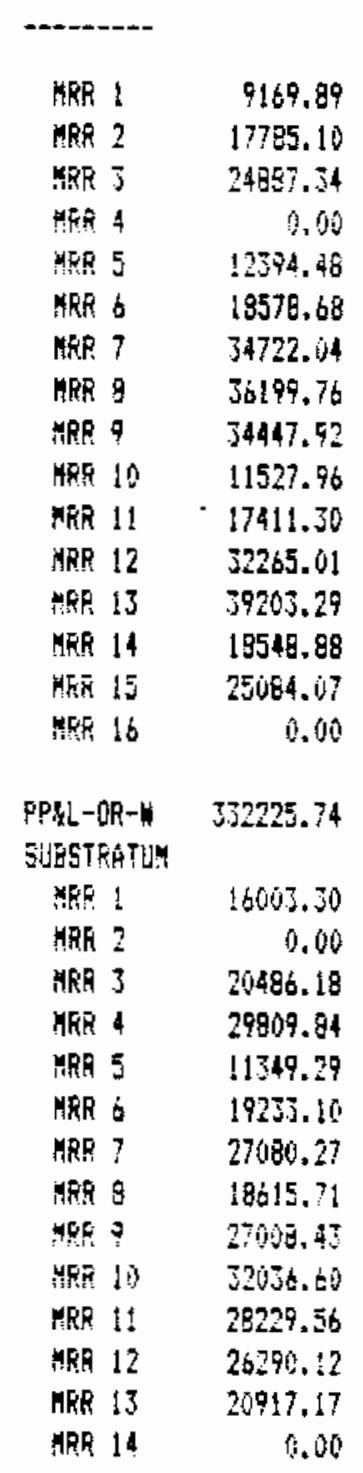




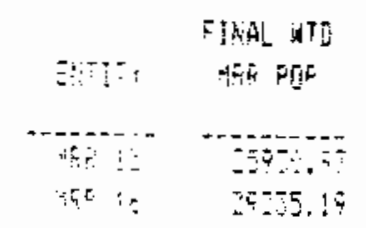

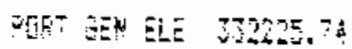
SUET⿰亻丨丁口灬

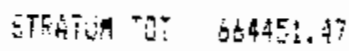

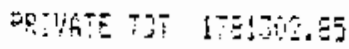

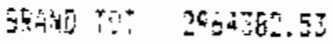




\section{DISTRIBUTION}

No. of

Copies

OFFSITE

2 DOE/Office of Scientific and Technical Information

2 R. A. Gillman

Bonneville Power Administration End-Use Research Section

P.0. Box 3621-RPEE

Portland, OR 97208

M. E. Taylor

Bonneville Power Administration

End-Use Research Section

P.0. Box 3621-RPEE

Portiand, OR 97208

S. G. Hauser

Battelle Portland office

500 NE Multnomah, Suite 650

Portland, OR 97232
No. of

Copies

W. M. Warwick

Battelle Portland office

$500 \mathrm{NE}$ Multnomah, Suite 650

Portland, OR 97232

\section{ONSITE}

12 Pacific Northwest Laboratory

C. C. Connor

R. G. Pratt

W. F. Sandusky (4)

G. M. Stokes

Publishing Coordination

Technical Report Files 
